\title{
"Facci Lei!": Subtitling Humour in Fantozzi (1975)
}

\author{
Rory McKenzie
}

A thesis submitted to the Victoria University of Wellington

in fulfilment of the requirements for the degree of

Doctor of Philosophy in Literary Translation Studies

Victoria University of Wellington 


\section{Acknowledgements}

First and foremost, I would like to thank my supervisors, Dr Marco Sonzogni and Dr Claudia Bernardi, who were not only invaluable in providing expertise related specifically to this project but, alongside Dr Sally Hill, the Head of School of the School of Languages and Cultures at Victoria University, sparked my interest in Italian language and culture. I will always be grateful for their hard work, enthusiasm and support throughout my studies.

I would like to thank Victoria University of Wellington for funding my doctoral research and for being supportive throughout my years of study here. To the Faculty of Humanities and Social Sciences, I am incredibly grateful for the funding you provided me to attend international conferences and carry out archival research abroad.

Many thanks to His Excellency Fabrizio Marcelli, Ambassador of Italy in New Zealand, to Martina Depentor and the Embassy of Italy and to Sydney Shep from Wai-te-ata Press, thank you for the support in organising the screening of Fantozzi with my English subtitles as part of La settimana della lingua italiana in 2017.

I am grateful to Elena Scarfi and the staff of the Biblioteca Istituto Nazionale "Ferruccio Parri" in Milan, allowing me to undertake archival research while on study leave.

And finally, to all my family and friends who have supported me throughout my many years at university, this thesis is a result of the hard work that I would have not been able to handle both physically and emotionally without your love and friendship. 


\begin{abstract}
Subtitling provides scholars and translators alike with the challenge of negotiating meaning across languages and cultures in an extremely limited space. The subtitler faces many restrictions than can severely affect a translation. However, subtitles are central to making films more widely and easily accessible. These difficulties are challenging at the best of times and are compounded by the specific difficulties of translating comedy. Humour is both universal and at the same time culturally specific. Anthropologists, sociologists, literary theorists and scholars have amply demonstrated how deeply intertwined humour, culture, and language are. It is for this reason that the current project will expand on the literature of subtitling humour, applying the relevant theories associated with both subtitling and translating humour to the Italian film classic Fantozzi (1975).

The character Ugo Fantozzi has been a cult figure in Italian culture and society since his appearance in Italian cinema and literature in the late 1960s and early 1970s. In the films in particular he has come to represent the average Italian of the post-economic miracle, whose life does not quite match the dreams of wealth and prosperity emphasized by the media. Fantozzi epitomises the average, and while his character has received little academic attention to date more credible academic studies are emerging since the death of his creator, Paolo Villaggio, in 2017. Fantozzi, therefore, provides the perfect cultural product for a discussion of what it means to translate Italian culture and humour, combining this with considerations about the emerging field of translation studies of subtitling.

By providing a complete translation of Fantozzi in English, accompanied by a critical commentary, in this thesis I attempt to show how, despite all the restrictions imposed by the field of subtitling, as well as the difficulties of translating humour, a subtitler can still produce well thought out and reliable subtitles that convey the cultural and comedic aspects of film, and more specifically of this beloved Italian icon.
\end{abstract}




\section{Table of Contents}

Introduction........................................................................................................................13

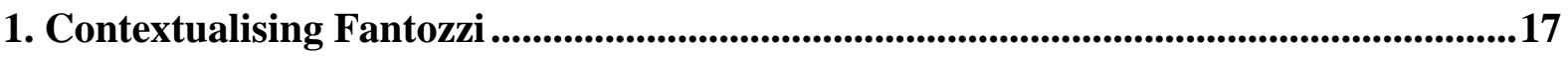

1.1 Vita, morte e miracoli di un pezzo di merda: Fantozzi, Villaggio and Salce ...........................17

1.2 Ugo Fantozzi and l'italiano medio..................................................................................24

1.3 Fantozzi and other international comedic figures...........................................................27

1.4 The Fantozzi series: patterns of humour ...............................................................................................31

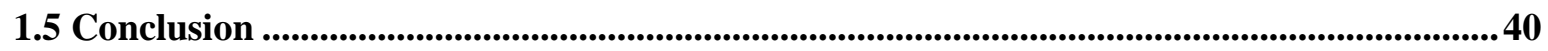

2. Translating humour in an audiovisual context ............................................................43

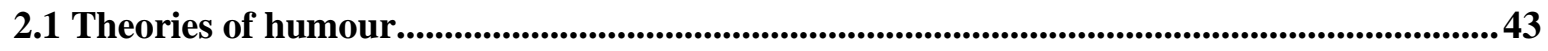

2.2 Translating humour across borders...............................................................................................47

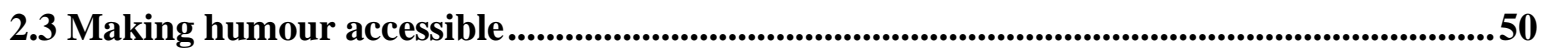

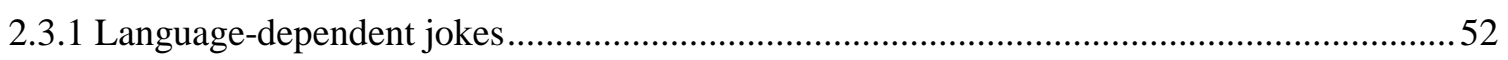

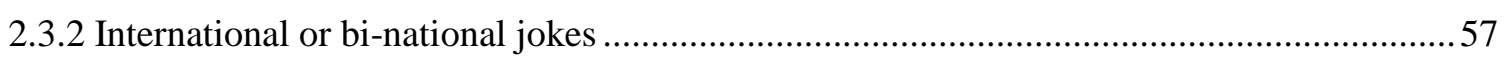

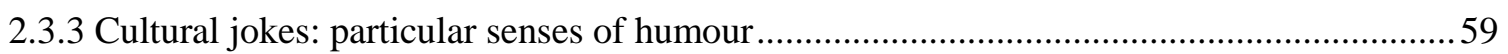

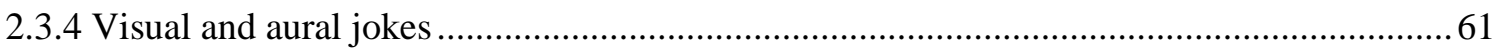

2.4 Conclusion ..................................................................................................................................................62

3. Subtitling as inter-semiotic translation .............................................................................65

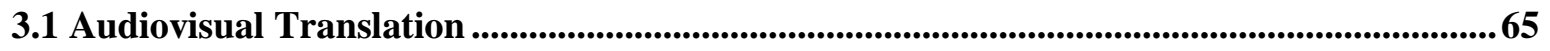

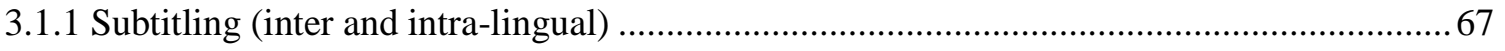

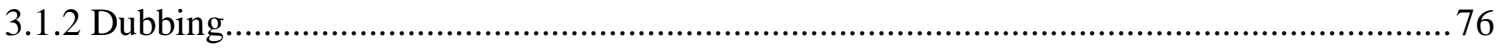

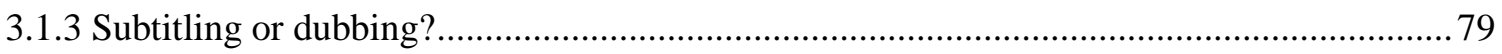

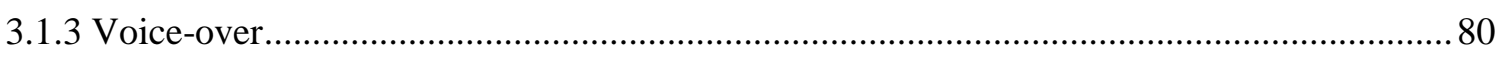

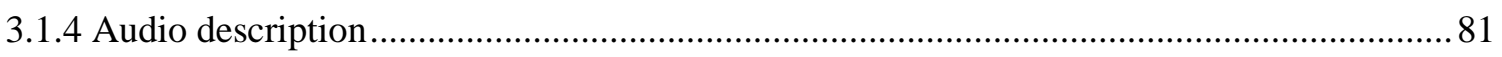

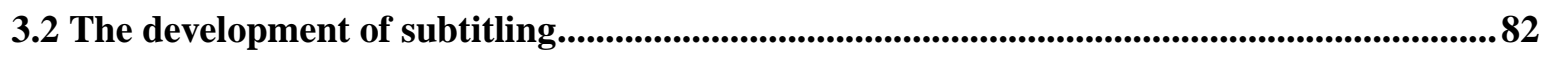

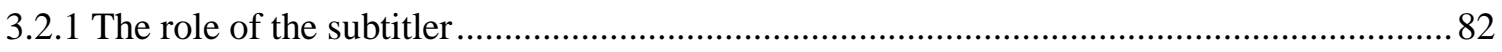

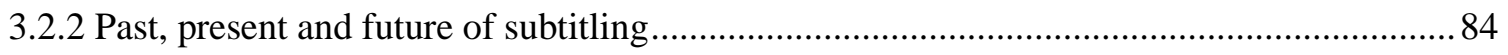

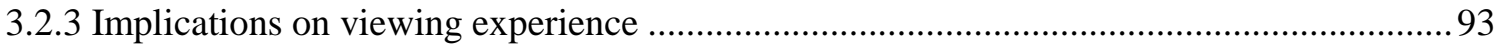

3.3 The theory and practice of subtitling: overcoming the challenges .........................................95

3.3.1 Subtitling techniques: time, space and content restrictions................................................99

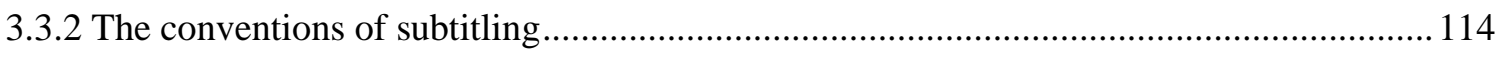

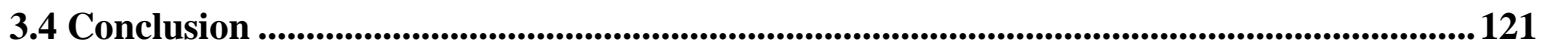

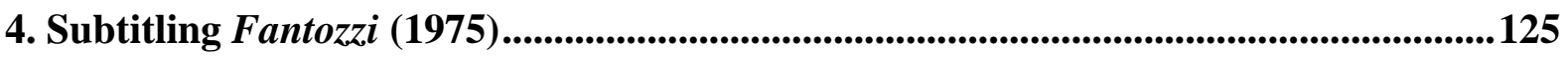

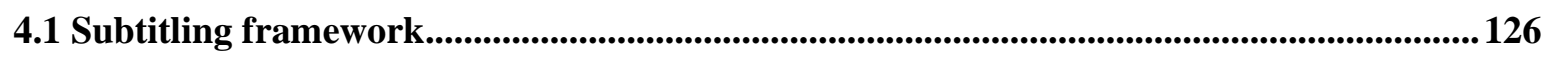


4.2 Fantozzi (1975) subtitles........................................................................................................................... 129

4.3 Specific difficulties in translation ........................................................................................................2232

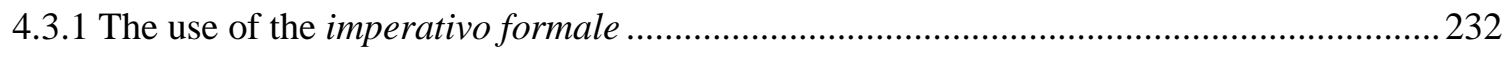

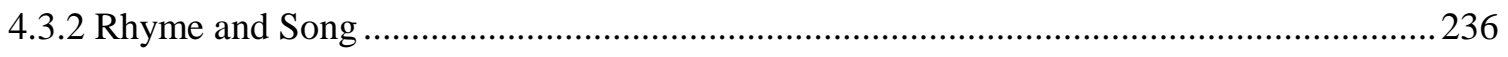

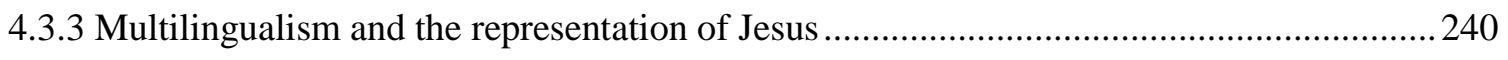

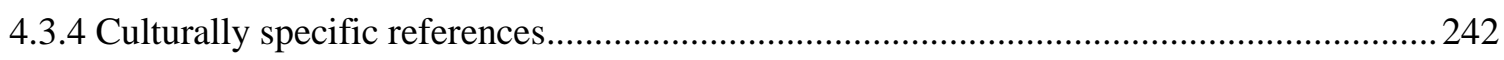

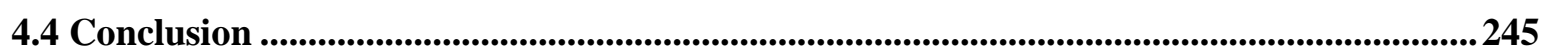

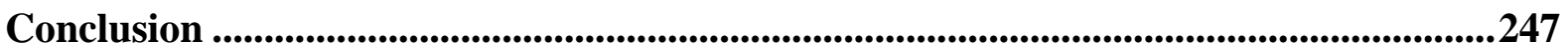

Bibliography and Filmography ..............................................................................................................251

Publications by Paolo Villaggio .....................................................................................................251

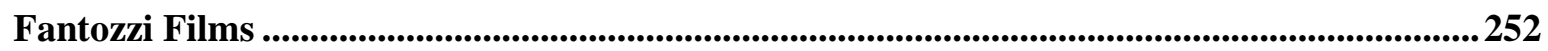

Referenced texts ..........................................................................................................................................................253

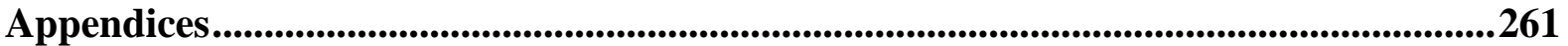




\section{List of Figures}

Figure 1: Director of the first two Fantozzi films, Luciano Salce

Figure 2: A timeline highlighting the important moments in the history of the Fantozzi series...........20

Figure 3: Fantozzi's famous line "Com'è umano Lei" expressed as a meme .........................21

Figure 4: Elisabetta Villaggio's tribute to her father, posted online. $\left(3^{\text {rd }}\right.$ July 2017)..................22

Figure 5(a-d): Headlines from Italy's biggest newspapers, celebrating Villaggio and Fantozzi.........23

Figure 6(a-b): Fantozzi and Signorina Silvani (left), Reginald Perrin and Joan (right).................28

Figure 7(a-f): Scenes from the Fantozzi films the style of The Simpsons ................................29

Figure 8: An example of the misuse of the 'imperativo formale' from the first Fantozzi film...........32

Figure 9(a-d): Examples of the biblical characters that appear in Fantozzi.............................35

Figure 10(a-b): The Mega-direttore Galattico's office in the first two Fantozzi films.................36

Figure 11(a-d): Examples of Mariangela' comparison to a monkey throughout the series..............38

Figure 12(a-d): The representation of other languages and cultures in the Fantozzi series.............40

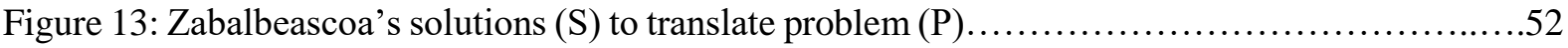

Figure 14: Example of language-dependent humour in Benvenuti al Sud .........................57

Figure 15: Example of an International joke from Benvenuti al Sud..............................59

Figure 16: Example of a cultural joke in Benvenuti al Sud ......................................61

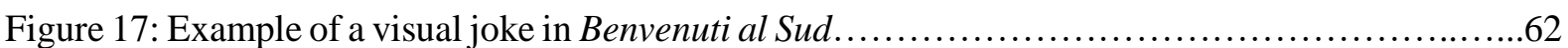

Figure 18: Gambier and Di Giovanni’s model for subtitling research..............................67

Figure 19: Susan Boyle subtitled despite the SL and TL being the same......................... 74

Figure 20: Skiptrace presented with bilingual Chinese/English subtitles..........................75

Figure 21: Screenshot of WinCAPS Q4untum, the software being used for this project...............88

Figure 22(a-d): Examples of integrated subtitles on television and in film........................93

Figure 23: The Filefish, used in the translation of Pasta alla Montecristo........................244

Figure 24: The use of "dollars" in translation, rather than the retention of "lira" ......................245 
You have to be happy when there's the opportunity to talk about Fantozzi. He is an accountant, the most typical Italian product. He is an employee, the type of individual that yesterday was an incarnation of normality and today seems a curious residual survivor, like telephones on walls or school desks with the hole for the ink well. ${ }^{1}$

Stefano Bartezzaghi

\footnotetext{
${ }^{1}$ Bartezzaghi, Stefano in Paolo Villaggio, Fantozzi, rag. Ugo (Milano: RCS Libri S.p.A, 2013), 1. [My translation] Original Italian: "Bisogna sempre essere contenti di avere l'occasione per parlare di Fantozzi. È un ragioniere, prodotto italiano dei più tipici. Ė un impiegato, individuo di una specie che ieri era l'incarnazione della normalità e oggi pare una curiosa sopravvivenza residuale, come i telefoni a muro o i banchi di scuola con il foro per il calamaio."
} 


\section{Introduction}

Despite the amount of research that has been undertaken in the field of both humour translation and subtitling, as we will see in the next chapters, there are quite simply no right or wrong answers or ways as to how a translator can go about subtitling humour. Indeed, humour is deeply cultural and jokes, wordplay, specific references or allusions that are funny in one culture may not be easily accessible, or may not even make sense in another. This is where translation, particularly in the case of subtitling, becomes an especially arduous task; humour has to inevitably change, or may even be lost, when shifting from one language to another given the cultural, linguistic and technical, medium-specific restrictions that the subtitler faces. Because of this, the subtitler must have a deep understanding of the intricacies of the source text (ST) and source language (SL) in order to be able to negotiate between the two. Each text must be dealt with on a case-by-case basis and must always take into consideration the target language (TL) and target culture (TC) for whom the text is being translated while still attempting to retain the idiosyncrasies of the original content. Given the added challenges inherent to subtitling, the translation of humour in an audio-visual (AV) context is often all the more difficult.

The choice of the film Fantozzi (1975), directed by Luciano Salce, as my case study is based on its compelling exemplarity: to start with, this film, as well as the rest of the films in the series, $I l$ secondo tragico Fantozzi, directed by Luciano Salce, Fantozzi contro tutti (1980), Fantozzi subisce ancora (1983), Superfantozzi (1986), Fantozzi va in pensione (1988), Fantozzi alla riscossa (1990), Fantozzi in paradiso (1993) and Fantozzi - il ritorno (1996) all directed by Neri Parenti, and finally Fantozzi: la clonazione (1999) directed by Domenico Saverni, all have acquired cult status in Italy and remain relevant to this day. The main character of the series, Ugo Fantozzi, has become deeply engrained into Italian culture. Italians young and old alike recognise, quote and enjoy the Fantozzi series on a regular basis, thus presenting a unique and intriguing case of Italian comedy and culture. Fantozzi still graces the "palinsesto" of Italian broadcasting, forty four years since the first film and twenty years since the last film was released. The death of Paolo Villaggio, the creator of Fantozzi, in July 2017 has undoubtedly revitalised the series' popularity. In fact, when I started this project in 2016, I attempted to find the first Fantozzi book in many new and second-hand book stores across Florence to no avail. In late 2017, all of the books in the series (on which the earlier films are predominantly based) could be found at the central book displays of Feltrinelli stores across Milan, which had been republished after Villaggio's death. The reactions to Paolo Villaggio's death, which will be discussed in the next chapter, have undoubtedly confirmed how Fantozzi is cherished as a national icon, still relevant both in terms of its comedy and as a satirical commentary on the contemporary Italian audience. Due to the culturespecific humour and references present, the temporal and historical displacement, as well as cultural displacement, this film undoubtedly provides an intriguing and difficult challenge in translation. 
A further reason for choosing the first film of the Fantozzi series as a case study is that it has never been translated commercially into English. ${ }^{2}$ A translation therefore allows an English-speaking audience access to an important product of Italian culture and comedy to which they have not had access so far; a relative surprise given that the films are a staple part of Italian cinematic culture but have had very limited exposure to the rest of the world, with little to no exposure to an English-speaking context given the themes, humour and content present are so quintessentially Italian. Interestingly, Paolo Villaggio was, at one point, in conversation with American producers about selling the rights for an American remake of the film, starring Danny DeVito as Fantozzi. ${ }^{3}$ These conversations fell through and no remake was ever produced, perhaps fortunately, as the comedy would have run the risk of going from a scathing social commentary mixed with aspects of visual and slapstick humour, to a purely slapstick, low-brow production.

Translating this film is not only a challenging task given the nature of the content, but also due to the fact that it is not being translated in a traditional sense, but rather it is being subtitled, which requires its own unique set of skills and practical solutions. In 1959 Roman Jakobson proposed three different typologies of translation: inter-lingual translation, inter-semiotic translation and intra-lingual translation. ${ }^{4}$ Subtitling could be considered an intersection of all three forms of translation, given that it is an intra-lingual translation from spoken to written communication, a translation from SL to TL in traditional inter-lingual subtitles, and a translation from TL to TL in the case of captioning for deaf and hard of hearing audiences. As Patrick Zabalbeascoa explains, "[i]f we have two types of signs and two different channels of communication, we get four different types of signs: audio-verbal (words uttered), audio-nonverbal (all other sounds), visual-verbal (writing), visual-nonverbal (all other visual signs)." All of these 'channels' must therefore work together in order for any audiovisual translation (AVT) to be successful - subtitles must cater to both the dialogue and the images on-screen at all times. Due to the restrictions that are imposed on the translation of audiovisual (AV) material, subtitling is an especially difficult area of translation studies that requires not only linguistic and cultural competency, but also technological expertise. The subtitler must tailor the translation to a new culture and language, but also bear in mind that the content is being changed from an audial form to a written form, which is why subtitling is referred to as 'diagonal translation' by Hencrick Gottlieb, or more generally as 'intersemiotic translation', as seen above. Added to this, the very restrictions, particularly those associated with time and space, which are characteristic to subtitling, often lead subtitlers to be exposed to criticism: indeed, subtitling is often labelled a 'recreation' of the original, rather than a translation. Although recent studies on subtitling have attempted to put an end to this point of view, providing

\footnotetext{
${ }^{2}$ The film does have an English title, White Collar Blues, and fairly poor quality fan subtitles have been created and are available for download online, presumably produced by a non-English native speaker given the grammatical errors and lack of English fluency.

${ }_{3}^{3}$ Alessandra Rota, "Paolo Villaggio - Il ragionier Ugo è un vendicatore," La Repubblica, 5 Luglio 1999.

${ }^{4}$ Roman Jakobson, "On Linguistic Aspects of Translation," On Translation (1959).

${ }_{5}^{5}$ Patrick Zabalbeascoa, "The nature of the audiovisual text and its parameters," in The Didactics of Audiovisual Translation, ed. Jorge Diaz-Cintas (Amsterdam: John Benjamins, 2008), 24.
} 
evidence that subtitling should be considered a legitimate academic focus in the field of inter-semiotic, or multi-modal, translation, there are still many translation scholars who are unfamiliar with, or who simply dismiss, subtitling as translation. In fact, an important scholar in the advancement of subtitling, Yves Gambier, believes that certain concepts of translation studies should be rethought when it comes to AVT - one of these being the very definition of translation as a practice. ${ }^{6}$ It is unquestionable, however, that subtitling is an important practise for a multitude of different reasons: its contribution to globalising the film industry and promoting cultural and linguistic information specific to certain linguistic and cultural contexts, as well as its usefulness in providing access to content for deaf and hard of hearing audiences are just two important examples as to why subtitling is an essential part of today's film industry. Despite the unavoidable difficulties and challenges of both of these practices (subtitling and translating humour), both need to be undertaken and, at times, co-exist within the same translation process when subtitling comedy films.

Before conducting this research my experience in translating humour was rather limited; my interest in translation, and in particular subtitling, however, has been a constant presence since taking up a second language at high school. I have always taken an interest in subtitled films, and in 2014 I received the opportunity to write the Italian subtitles for the New Zealand film The Dark Horse (2014) as part of a Victoria University of Wellington Summer Scholarship research project. ${ }^{7}$ That particular case study opened my eyes to the unique difficulties and intricacies of the field of subtitling, particularly when attempting to translate the deeply cultural New Zealand content of the film, and has led me to pursue subtitling from a scholarly, pedagogical and technological perspective. ${ }^{8}$ This thesis expands on that research from a slightly different perspective, that of negotiating Italian humour in an Englishspeaking context.

This translation studies project is comprised of four chapters that, together, provide an essential analysis of both the cultural implications and translation challenges in order to be able to adequately render the content of this case study from the original Italian into English. The first part of chapter one will introduce both the character of Ugo Fantozzi and his creator Paolo Villaggio, placing Fantozzi in the context of Italian society and culture in the 1970s, exploring his introduction into Italian comedy. This is followed by an analysis into the exact types of humour one expects to find throughout the film and series. It is important to bear in mind that this is a project in Translation Studies, so the discussion of the Fantozzi series is limited to an outline of the overall cultural aspects and themes present in the film. The character, as well as the films in general, are incredibly rich with cultural specificity, but a more holistic view of the character and series is essential in understanding the implications that inevitably exist in translation.

\footnotetext{
${ }^{6}$ Yves Gambier, "Challenges in research on audiovisual translation," in Translation Research Projects 2, ed. Anthony Pym and Alexander Perekrestenko (Tarragona: Intercultural Studies Group, 2009), 19.

7 James Napier Robertson, "The Dark Horse," (Transmission Films, 2014).

${ }^{8}$ Rory McKenzie, "A Dark Horse in Translation Studies: Subtitling Kiwi Culture," Journal of Italian Translation 11, no. 2 (2016).
} 
Chapter two looks at translating humour in an audiovisual context in general, highlighting particular theories of humour and presenting the subsequent challenges of retaining this humour in translation. Techniques that aid the translator are proposed and discussed, and presented in the form of a contemporary case study of humour translation from Italian to English.

Chapter three then provides an overview of AVT, and more specifically of subtitling, with a discussion of both the development of subtitling as an industry, as well as the practical and theoretical challenges with which a subtitler is faced, as well as possible ways in which these challenges can be overcome according to the literature on subtitling.

The fourth chapter draws heavily on the analysis undertaken in the first two chapters of this thesis. First of all, a framework for the creation of subtitles is provided, followed by the full translation of the film, which bears in mind both the cultural and technical components and requirements discussed in detail in the previous chapters. The translation is accompanied by a commentary, which functions as an insight into the thought processes undertaken throughout the translation. This is then followed by a breakdown of specific complex scenes and dialogues that proved especially challenging, as well as the decisions made to overcome these challenges.

This project has been undertaken as an original research project from start to finish. No transcription of the film existed and therefore the dialogue had to be transcribed to begin with. I then translated the text, turned it into subtitles.

The purpose of this research project is to build on the literature of AVT, in particular subtitling, highlighting its importance first of all in understanding other languages and cultures, and also showing that it is an essential form of inter-semiotic translation that requires a deep cultural, linguistic and technological knowledge. I also aim to add academic credibility to an often dismissed film (and series as a whole) that seems to be a fixture in Italian comedy, film and culture. This project aims to provide an English-speaking audience with the opportunity to learn about and enjoy a staple figure in Italian cinema and society. In subtitling this film, I hope to prove that translating the humour of Fantozzi, while not a simple task, is certainly possible when the translator is aware of its cultural implications, alongside an in-depth knowledge of the AVT industry and its practices. 


\section{Contextualising Fantozzi}

Paolo Villaggio's most famous character, Fantozzi, is the quintessential modern Italian mask of physical pain and moral humiliation. Importantly, the audience is not invited to empathise with such pain and humiliation (that is, to identify with the main character and therefore feel humiliated). On the contrary, through comic expedients, a sapient use of language, especially by the voice-over, and the constant recurrence of characters, episodes and formulaic expressions, the audience is invited to laugh freely at Fantozzi, while at the same time receiving an uncanny, grotesquely exaggerated reminder of the inhumane and alienating nature of capitalism, bureaucracy, and consumerism. ${ }^{9}$

Francesco Ricatti

The Fantozzi series has, to date, received very little academic attention, presumably due to its rather crude and immature slapstick-style of humour, although a growing number of critics and academics alike agree that the themes and humour present in the first Fantozzi film, as well as the series as a whole, go beyond this. Indeed, scholarship on the characters and series has been on the rise since Paolo Villaggio's death in 2017. This chapter will, first of all, explore the life of the creator, Paolo Villaggio, one of Italy's most prestigious comics. It will then introduce the character Ugo Fantozzi, placing him in both an Italian and global context; then it will provide an analysis of the types of humour present across the series. The aim is not to critique the Fantozzi series or Salce's filmmaking abilities from a film studies point of view (which would be a very interesting and deserving topic of study in and of itself), but rather to contextualise the linguistic and cultural challenges that the subtitler faces when translating the type of humour which is presented in this case study. A particularly challenging aspect in writing this chapter is that, until now, there has been very little scholarly secondary literature on Fantozzi (in both Italian and English), and the sources that do exist tend to be in the form of interviews, film reviews or creative pieces. This fact is rather surprising, given Fantozzi's continued popularity in Italy, and proves problematic when wanting to discuss Fantozzi from a scholarly perspective.

\subsection{Vita, morte e miracoli di un pezzo di merda: Fantozzi, Villaggio and Salce ${ }^{10}$}

Paolo Villaggio (1932-2017) was one of the most recognized and appreciated contemporary Italian comic writers and performers of his era. He was an actor, director, writer and comedian whose work was not only well received for its comedic and satirical elements, but for its commentary on working and low-middle class Italy in the 1970s. Born in 1932, Villaggio grew up in Genoa, in the North of Italy. He initially studied Law at the University of Genoa, but never completed his degree, instead

\footnotetext{
${ }^{9}$ Francesco Ricatti, "Humiliation and Love: Villaggio, Benigni and the cultural politics of emotions," Incontri: Rivista europea di studi italiani 29, no. 2 (2014): 11.

${ }^{10}$ This is also the title of an autobiography written by Paolo Villaggio in 2002. Given that I will be discussing Villaggio's life, death and his character that is often referred to as "La nostra merdaccia", the heading seemed apt.
} 
emabarked on a writing career. He started by writing short stories for the magazine $L{ }^{\prime}$ Europeo. ${ }^{11}$ His part-time position (before he embarked on his career as a writer) and the people with whom he worked at the Italsider steel works were the inspiration behind his most famous character, Ugo Fantozzi, as explained by Fabrizio Buratto:

The character of Fantozzi very much derives from the characteristics of a series of people Villaggio met at the time in which he worked at the Italsider in Genoa [...] Italsider represented for Italy at the time an example of an avant-garde company in terms of the organization of work and of the experimentation of new dynamics of collaboration between workers. ${ }^{12}$

As well as creating the character of Fantozzi, Villaggio played Fantozzi in the film adaptations of his short stories and in the further original film versions. Villaggio won numerous awards for his acting, writing and contribution to Italian cinema. Such awards included the Davide di Donatello, the Leone d'Oro, the Nastro d'Argento, the Pardo d'onore, an Aquila d'Oro, and two Grolle d'Oro. In 2011, on the occasion of the 150th anniversary of Italy's unification, his first collection of stories, Fantozzi, written in 1971 and on which the first film was based, was included by the Centro per il libro e la lettura among the top 150 most influential books that marked Italian history. When first published, Rizzoli produced a conservative 10,000 copies of the book. It went on to sell over 1.5 million copies nationwide.

The first film, Fantozzi, directed by Luciano Salce (pictured in Figure 1) in 1975, draws from the books initially published in 1971. The film, with its episodic structure, is made up of numerous short stories involving stereotypical aspects of Italian culture and society from football to food, countryside and mountainous holidays, Christmas and New Year's Eve celebrations, religion, workplace hierarchy and everything in between.

Salce's personal experiences also undoubtedly add to the richness of the character Ugo Fantozzi and the humour of the first two films. Salce, who grew up in Rome, was an actor, director, screenwriter, television host, playwright and lyricist. His first appearance as an actor came in 1946 in the film Un americano in vacanza and his final work as a director came in 1987 with his film Quelli del casco. In February 1943, before his career in cinema had started, Salce was called to undertake military service, and on September 8 he was taken prisoner by German forces and deported to a labour camp for prisoners of war, where he remained for two years until the end of the conflict. ${ }^{13}$ It is therefore, perhaps, no coincidence that the first Fantozzi film takes aim at Germans for comedic effect, particularly the scene

\footnotetext{
${ }^{11}$ The short stories that Villaggio wrote for L'Europeo can be found in the appendix of this thesis, courtesy of the Biblioteca Istituto Nazionale "Ferruccio Parri" in Milan.

${ }^{12}$ Fabrizio Buratto, Fantozzi: Una maschera italiana (Torino: Lindau, 2003), 32. [My translation]

Original Italian: "Il personaggio di Fantozzi deriva molte sue caratterstiche da una serie di persone conosciute da Villaggio ai tempi in cui lavorava all'Italsider di Genova [...] L'Italsider rappresentò per l'Italia di quegli anni un esempio di azienda all'avanguardia in tema di organizzazione del lavoro e di sperimentazione di nuove dinamiche di collaborazione fra lavoratori."

${ }^{13}$ Andrea Maori, Luciano Salce prigioniero n. 120842. Storia di un intellettuale internato 1943-1945 (Perugia: Tozzuolo, 2018). Andrea Pergolari and Emanuele Salce, Luciano Salce: Una vita spettacolare (Roma: Edilazio, 2009).
} 
at 'Le Magnolie' diet therapy clinic, which is very reminiscent of a prison, in which the German directors of the centre starve their patients and exploit them by charging obscene amounts of money for their food. In fact, this hypothesis is supported by Salce's own son - in his book detailing the life of his father, Emanuele Salce states, "[h] is two years in German prison were horrifying: they imprinted forever on his life (even his artistic one)."14

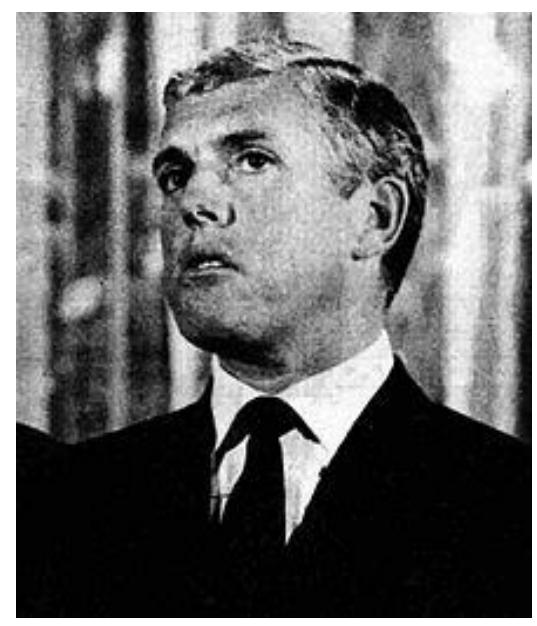

Figure 1: Director of the first two Fantozzi films, Luciano Salce ${ }^{15}$

The Fantozzi books and films are regarded as staples of Italian culture. Popular with young and old audiences alike, they appear relevant to understanding Italian society, history, humour and traditions. The Fantozzi character first graced Italian television (albeit with the name 'Fantocci') in 1968, on the programme Quelli della domenica on Rai. ${ }^{16}$ Fantozzi then hit the national box office in 1975 and is frequently screened, even to this day, on national television. Il ragionere (accountant) Ugo Fantozzi is the protagonist of eight books and ten films. The films, at least the early ones, draw heavily on the content originally written by Villaggio - whether that be through particular descriptions, comedic elements or specific language used throughout, the similarities between the two are clear. In fact, the Fantozzi series is as popular as ever in today's Italy, as exemplified by Figure 2, which shows a timeline of the release of films and the earlier collections of short stories, a visual example of the longevity of the Fantozzi series.

\footnotetext{
${ }^{14}$ Luciano Salce: Una vita spettacolare, 46. (My translation, original as a timeline and therefore adapted for context) Original Italian: "I due anni di prigionia in Germania sono terrificanti: segneranno per sempre la sua vita (anche artistica)." ${ }^{15}$ Picture found on: https://nl.wikipedia.org/wiki/Luciano_Salce. Accessed 15 $5^{\text {th }}$ November, 2018.

${ }^{16}$ Marco De Simone, Memorie dal sottoscala (Rieti: Amarganta, 2015), 23.
} 


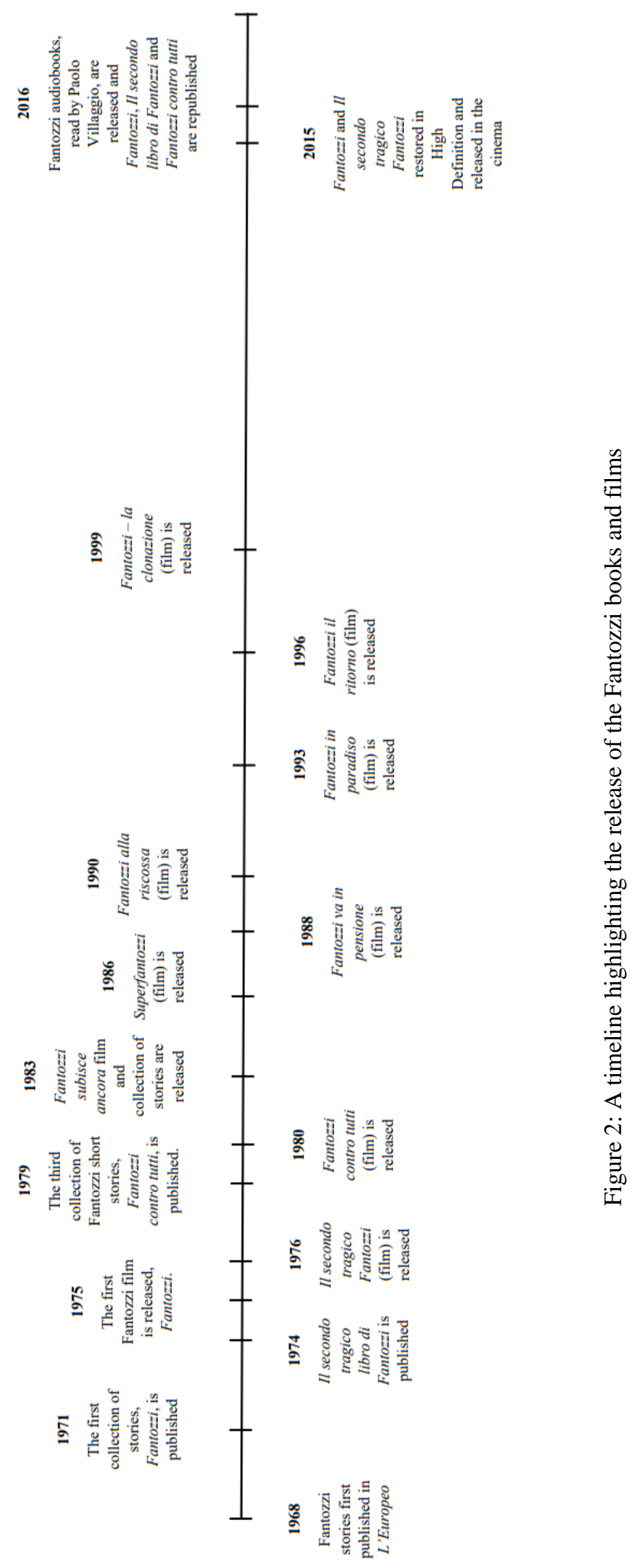


In the early 1990s the character was adapted into a comic series in the children's magazine Il Corrierino; a new graphic novel Fantozzi Forever was published in 2014; the audiobooks of the short stories, read by Paolo Villaggio himself, were released in 2016, graded reader adaptations of the first and second film for Italian language learners were published in $2018 .{ }^{17}$ The first, second and third books were republished in 2017 by Rizzoli, and the first and second films were restored in High Definition for Italian cinema in 2015. Recently, a group of Italians even erected a plaque (Figure 3) onto the building in which Fantozzi jumps off in one of the early scenes from the film, upon which appears Fantozzi’s now famous quote: "Non l'ho mai fatto, ma l'ho sempre sognato" ("I have never done it, but I have always dreamed of it"). ${ }^{18}$

Fantozzi even appears in the relatively new social media craze known as 'memes' where a still from a film, for example, is presented alongside a caption, usually the quote from the part of the film where the still is taken, as shown in Figure 3. This undoubtedly proves that, despite being a part of Italian culture for the best part of fifty years, the characters continue to hold cult status, a status that has continued into the age of social media.

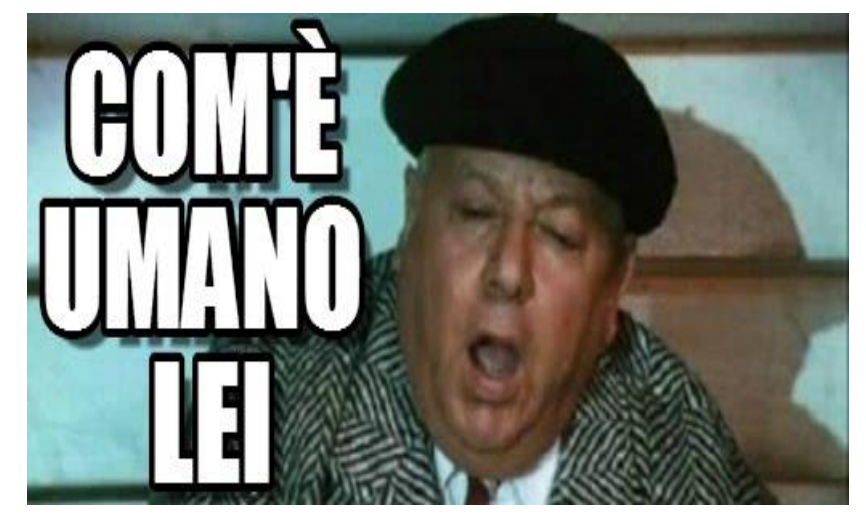

Figure 3: "Com'è umano Lei" ("how compassionate of you") expressed as a meme. ${ }^{19}$

On July $3^{\text {rd }} 2017$, at the age of 84 , Paolo Villaggio passed away in Rome through complications due to his diabetes. His daughter, Elisabetta, announced the news to the public through social media, posting to her Facebook page: "Ciao papà ora sei di nuovo libero di volare" ("Goodbye, Dad, now you are free to fly again"), as illustrated in Figure 4.

\footnotetext{
${ }^{17}$ Paolo Villaggio, Fantozzi: L'audiolibro inedito (Volume S.R.L., 2016).

$18 \mathrm{https}: / /$ www.webstagram.one/media/BtL3bv5n1hb. Accessed on $20^{\text {th }}$ February 2019.

${ }_{19}$ Image found on: http://network-marketingteam.blogspot.com/2016/03/smetti-di-parlare-come-fantozzi-se-vuoi.html Accessed $18^{\text {th }}$ January, 2018.
} 


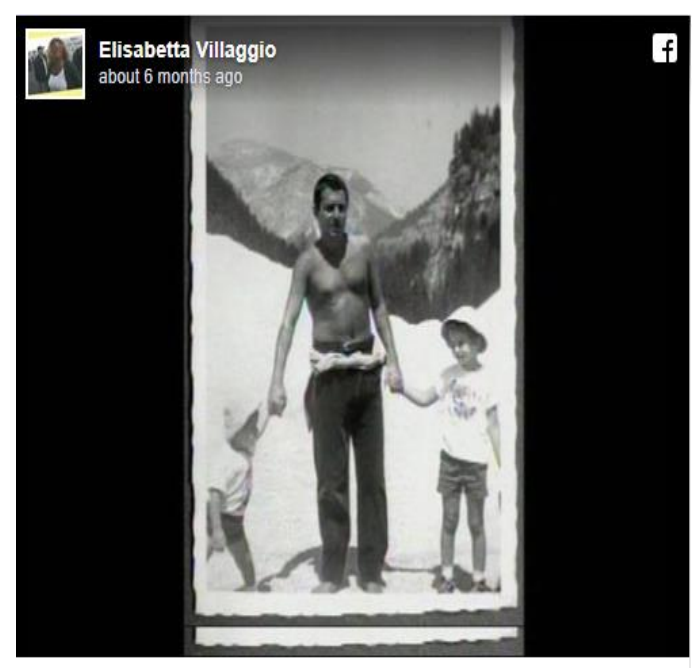

Ciao papà ora sei di nuovo libero di volare $\bullet$

Figure 4: Elisabetta Villaggio's tribute to her father, posted online. ( ${ }^{\text {rd }}$ July 2017)

Paolo Villaggio's funeral, held at La casa del cinema di Roma, was attended by many of Italy's screen idols: in addition to his wife Maura Albites and their children Pierfrancesco and Elisabetta, were singer Dori Ghezzi (wife of Fabrizio De Andrè and great friend of Paolo Villaggio), directors and actors such as Ricky Tognazzi, Lino Banfi, Renzo Arbore, Paolo Sorrentino, Neri Parenti (the director of the majority of the Fantozzi films), and his Fantozzi co-stars Plinio Fernando (Mariangela) and Milena Vukotic (Pina). Images of Villaggio, as well as references to Fantozzi, were plastered across all Italian media in both the private and public eye: my own Facebook newsfeed had numerous posts and pictures by Italian friends for whom Fantozzi was a big part of their childhood, all saying goodbye to Villaggio and his ragionier (Fantozzi). As can be seen in the newspaper front pages (Figure 5a-d), taken from the day after his passing, Italian media emphasised his influence on Italian culture from the 1970s to the present day. Many journalists bemoaned the fact that his work had not been the object of academic discussion. 


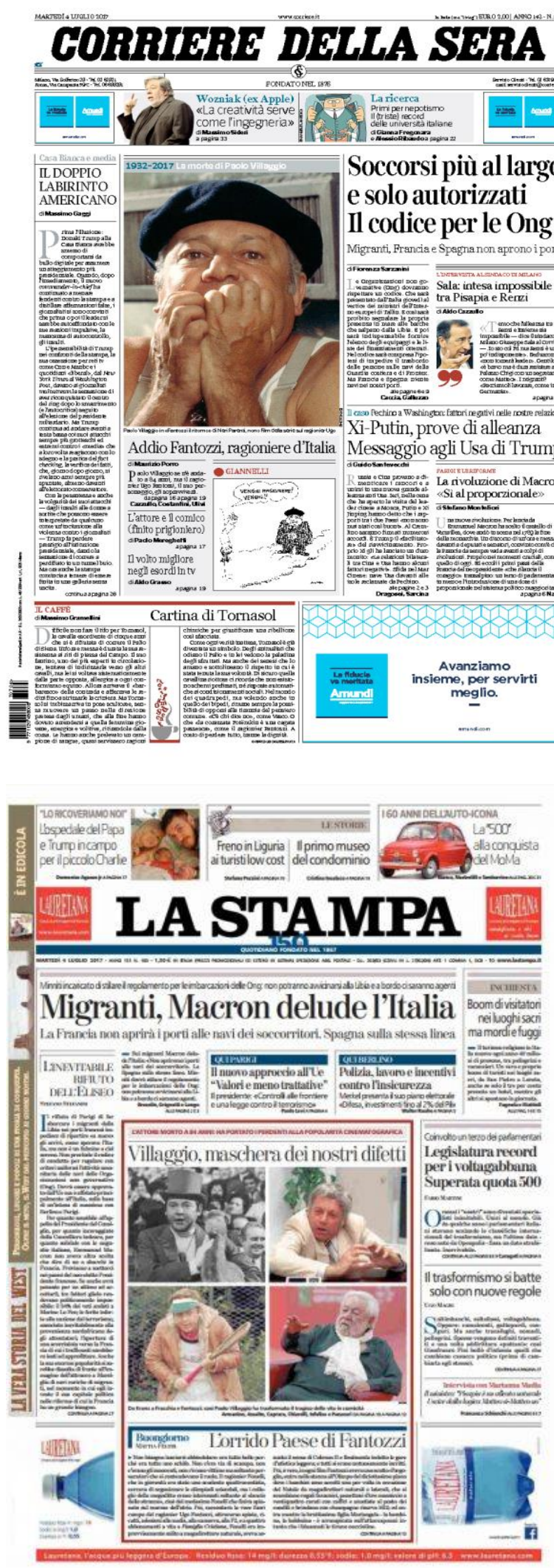

\section{laRepubblica $=$}
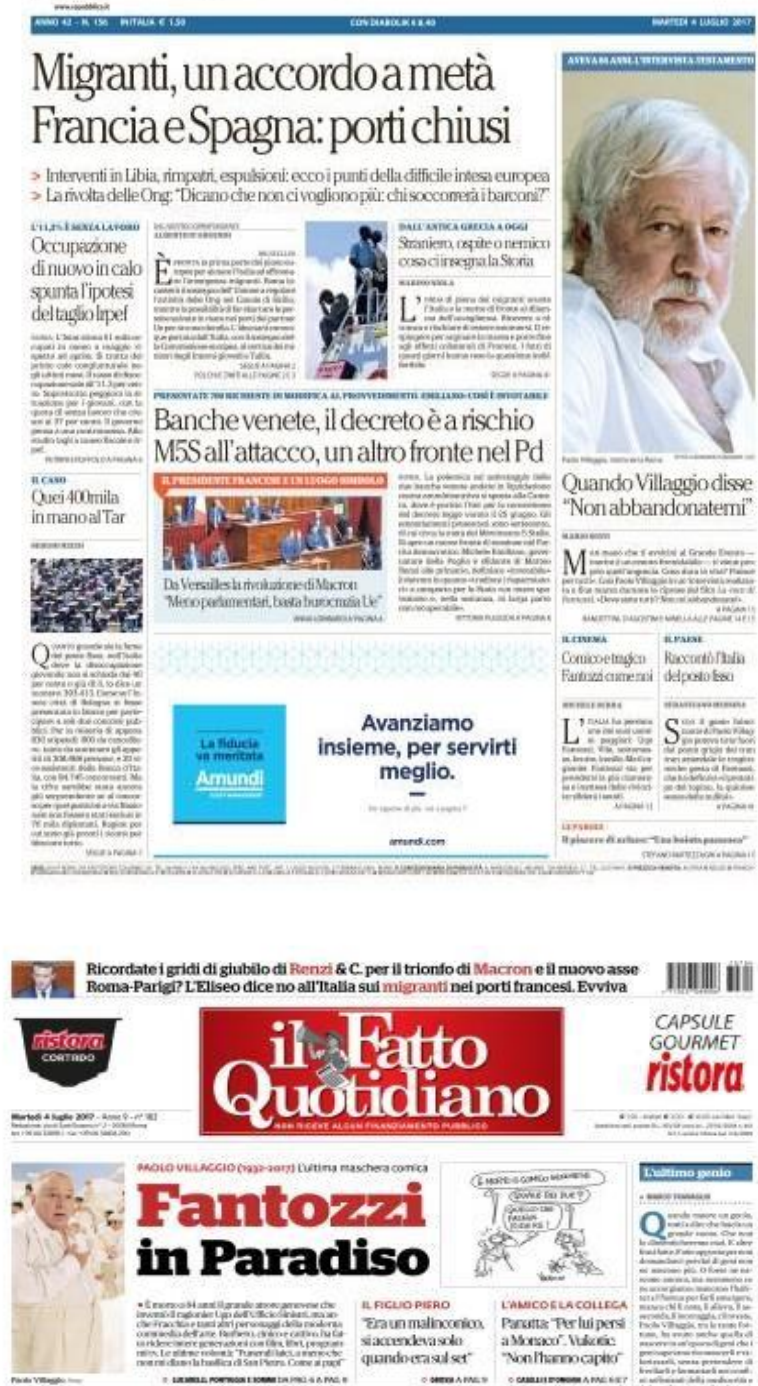

RENZUSCONI Interrogazione Pd contro il Noeeil pm di Consip. Etruria, tutto ferm

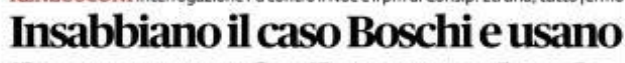

il Senatoper fucilare Woodcock
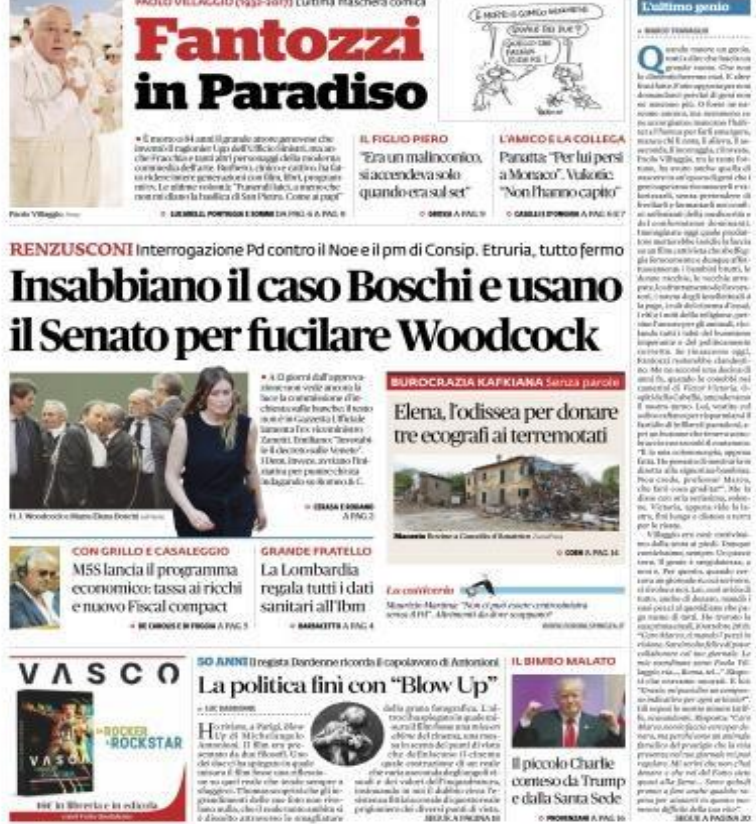

Figure 5(a-d): Headlines from Italy's biggest newspapers, celebrating Villaggio and Fantozzi ${ }^{20}$

${ }^{20}$ Images found on: http://www.dire.it/04-07-2017/131630-prime-pagine-quotidiani-4-luglio-2017/. Accessed 20 ${ }^{\text {th }}$ August, 2018. 


\subsection{Ugo Fantozzi and l'italiano medio}

Fantozzi was, in a nutshell, the portrait of some Italians, an insignificant bourgeois in constant pursuit of redemption. Archetype of the 'average Italian', employed without a qualification or friend (think about the relationship with Filini that never goes past the 'Lei'), that puts to the forefront of the publics' minds their own anxieties and failings. In every office there exists a two-faced seductress like Silvani, a gameplayer who is filled with ambition [Calboni], and, above all, we have all had the thought, at least once, of being persecuted by bad luck. ${ }^{21}$

Massimiliano Romualdi

At the time of Fantozzi's creation, Italy had experienced over a decade of economic prosperity, in a period known as the 'economic miracle', which had revolutionised the Italian economy and society in the 1960s and transformed the country's cultural landscape. The transformation was caused by fast economic expansion, aided by financial aid from the United States of America, which induced an influx of workers from the South to the North in the post-war years. The Italian economy grew rapidly due to new-found productivity. According to Paolo Scrivano, "in the five years between 1958 and 1963 the gross domestic product increased an average of $6.3 \%$ per year (it had been $5.5 \%$ between 1951 and 1958)." ${ }^{22}$ Industrial areas had especially benefited from this growth; Milan, Turin and Genoa, where Villaggio grew up, became the 'capitals' of the Italian economic boom. Italy thus became one of the success stories of the post-World War II era, mainly thanks to the availability of cheap labour. It changed from being the least developed nation of the founding members of the European Community to becoming one of the world's leading industrial countries. Italy reshaped itself, creating prosperity and previously unobtainable access to consumer goods. ${ }^{23}$ This emphasis on industrialisation, commercialisation and economic prosperity are what laid the social foundations for the character of Ugo Fantozzi, the decidedly average worker living in an Italy where prosperity and social image became increasingly fashionable: he is a character who tries his utmost to fit in with societal norms but always seems to be on the outside, a character who appears to always be let down by the promise of the economic miracle and the happiness associated with it.

As far as Italian cinema is concerned, some critics believe that the 1970s marked the beginning of a decline in quality in the industry, where low brow humour and serials became prevalent. Slapstick

\footnotetext{
${ }^{21}$ Massimiliano Romualdi, "41 anni di Fantozzi: com'è cambiata l’Italia?," Artspecialday, http://www.artspecialday.com/9art/2016/03/27/41-anni-di-fantozzi-come-cambiata-litalia/. accessed 25th January 2018. [My translation]

Original Italian: "Fantozzi era, in poche parole, il ritratto di una parte degli italiani, un borghese piccolo piccolo alla continua ricerca di un riscatto. Archetipo dell'italiano medio dunque, impiegato senza una laurea e senza un amico (pensiamo al legame con Filini, un rapporto che non va oltre il lei), che mette il pubblico di fronte alle proprie ansie e ai propri fallimenti. In tutti gli uffici è esistita una seduttrice un po' doppiogiochista come la Silvani, un collega arrivista e, soprattutto, tutti abbiamo almeno una volta pensato di essere perseguitati dalla sfortuna."

${ }^{22}$ Paolo Scrivano, "Signs of Americanization in Italian Domestic Life: Italy's Postwar Conversion to Consumerism," Journal of Contemporary History 40, no. 2 (2005): 321.

${ }^{23}$ Ibid.
} 
comedies by writers and directors such as Villaggio, Ciccio Ingrassi and Franco Franchi became popular. As Carlo Celli and Marga Cottino-Jones argue, even well respected commedia all'italiana directors such as Dino Risi began to create films that bordered on soft porn. ${ }^{24}$ It is generally accepted, however, that l'italiano medio became the subject of Italian film comedy in a post-economic miracle society, given that, as argued by Vittorio Spinazzola, the italiano medio reflected the socially shared values and common experiences of "ordinary" Italian people. ${ }^{25}$

Villaggio chooses to show the path of this anti-hero, the unfortunate buffoon that is Ugo Fantozzi. Other literary figures are born around the same time that also reflect the idea of the Italian anti-hero: Italo Calvino's Marcovaldo (1963), for example, shares similar servile and almost dim-witted traits, as well as a comparable narrative structure, as both characters are originally presented in the form of several short stories that made up a larger collection. Marcovaldo is a labourer, his relationship with his wife is very dull and his mind is full of big dreams that will never eventuate into reality. In fact, many of the Marcovaldo short stories could easily star Fantozzi: in the story Funghi in città, for example, Marcovaldo finds a patch of mushrooms and cultivates them to take home to his family only for them to end up sick, an action that I can certainly see Fantozzi undertaking. ${ }^{26}$ While there is no academic literature that shows a direct influence of Calvino's Marcovaldo on Villaggio's Fantozzi, , it would appear that some similarities and overarching themes would warrant further attention, especially in their shared critique of the social costs of the economic miracle and Italy's industrial development.

Ugo Fantozzi (also known, by his colleagues and superiors at least, as Fantocci, Pupazzi, Zanfozzi, Bambocci, Bacarozzi, Puccettino and perhaps most famously, 'la nostra merdaccia') is an exaggerated and comedic personification of the italiano medio of the 1970s in this post-economic boom society. However, the purpose of Fantozzi is not so much to show a comical version of a typical lowermiddle class Italian citizen, as to portray a comical take on the disparity between social classes that existed and the ultimate failure of the economic miracle to provide happiness for many Italians. This is achieved precisely through the introduction of the "average" in the form of an anti-hero into Italian cinema. As Paolo D’Agostini argues:

[t]he "average" [medietà] is precisely the soul, the solid base of the "commedia all'italiana". A double-sided average: a source of extraordinary vivacity and of real contact with the climate and the general feelings of the society... Average is the person "fabricated" by the authors of the comedy, "average" (in the sense of least), for many years, is the commercial benchmark, "average" is the position, the setting that for more than twenty years this type of cinema occupies in the Italian movie industry, which only then begins to assume less imprecise contours. Between the mid-1950s and the mid-1970s, comedy becomes its true backbone. No wonder then, that the average becomes its ideology. ${ }^{27}$

\footnotetext{
${ }^{24}$ Carlo Celli and Marga Cottino-Jones, A New Guide to Italian Cinema (New York: Palgrave Macmillan, 2007), 117.

${ }^{25}$ Vittorio Spinazzola, Cinema e pubblico: Lo spettacolo filmico in Italia 1954-1965 (Rome: Bulzoni, 1985), 289.

${ }^{26}$ Italo Calvino, Marcovaldo (Torino: Giulio Einaudi editore, 1963).

${ }^{27}$ Paolo D'Agostini, Romanzo popolare: Il cinema di Age e Scarpelli (Napoli: Edizioni Scientifiche Italiane, 1991), 37.
} 
I use the term 'anti-hero' to describe Fantozzi because he is certainly no hero in the traditional sense: he tries to be brave but inevitably backs down when things do not go his way, he has very few redeeming qualities and demands very little respect from the viewers. The audience are encouraged to feel sorry for him, perhaps understand him, but never want to be him. This point is argued by Sergio Rigoletto, who, on the subject of l'italiano medio in Italian comedy, notes that "[o]rdinariness is its most persistent attribute." ${ }^{28}$ Villaggio himself describes Fantozzi in a similar fashion: "an anti-hero, he isn’t funny, he is a show-off." 29 In fact, the word 'fantozziano' is now used in Italian as an adjective meaning "a mentally timid and servile attitude attributed to a depressing average employee, alienated and persecuted by bad luck", "a situation characterised by unfortunate and grotesque events", "clumsy, grotesque.", "a person who is clumsy and servile with his superiors." ${ }^{30}$ This is the very nature of Fantozzi. As Paolo Mereghetti describes him:

Fantozzi is weak and servile as only the little bourgeois can be, always terrorized by his superiors, shy and clumsy to the point of catastrophe, he presents himself as the natural victim of mass media, of consumerism and of advertising, tragically incapable of adapting to the social models of his daily myths. ${ }^{31}$

While at the time of his creation there would have certainly been people who could relate to Fantozzi and his struggles through life, it is important to note that he is not necessarily a realistic example of life in post-economic miracle Italy, but rather he is an exaggeration of this italiano medio, as discussed by Francesco Ricatti:

[h]e does not offer a realistic representation of the average Italian, as is usually the case with the majority of standard characters in the commedia all'italiana. While he is the quintessential weak and mediocre comic character who experiences an incredible series of physical and psychological abuses and humiliations, his world is of an exaggerated, at times surreal and often grotesque nature, and his painful and humiliating, yet stubborn, survival is comparable to that of a comic character in a children's cartoon. ${ }^{32}$

Fantozzi himself works in the corporate world as an accountant and lives a decidedly average life. $\mathrm{He}$ reflects particular traits of the italiano medio through his obsession with certain stereotypical Italian

\footnotetext{
${ }^{28}$ Sergio Rigoletto, "The Italian Comedy of the Economic Miracle," in Italy On Screen: National Identity and Italian Imaginary, ed. Lucy Bolton and Christina Siggers Manson (Bern: Peter Lang, 2010), 31.

${ }^{29}$ Paolo Villaggio and Luca Sommi, Non mi fido dei santi: autobiografia bugiarda (2011), 15. [My translation] Original Italian: "un eroe negativo, non è simpatico, è un millantatore."

30 ""fantozziano, adj."," La Repubblica, https://dizionari.repubblica.it/Italiano/F/fantozziano.html. [My translation] Original Italian: "di atteggiamento e mentalità pavidi e servili, attribuiti a un deprimente tipo di impiegato medio, alienato e perseguitato dalla sfortuna", "di situazione caratterizzata da eventi grottescamente sfortunati”, or "goffo, grottesco." "fantozziano," Trecanni, http://www.treccani.it/vocabolario/fantozziano/. [My translation] Original Italian: "Di persona, impacciato e servile con i superiori: quel collaboratore è proprio una figura fantozziana." ${ }^{31}$ Paolo Mereghetti, Dizionario dei film 2011, Il Mereghetti (Milano: B.G Dalai editore, 2010), 1214. [My translation] Original Italian: Fantozzi è debole e servile come sa esserlo solo il piccolo-borghese, sempre terrorizzato dai superiori, timido e impacciato fino al catastrofico, si presenta come vittima naturale dei mass media, del consumismo e della pubblicità televisiva, tragicamente incapace di adeguarsi ai modelli sociali che mitizza quotidianamente."

${ }^{32}$ Ricatti, "Humiliation and Love: Villaggio, Benigni and the cultural politics of emotions," 12.
} 
pastimes like football, billiards or cycling. ${ }^{33} \mathrm{He}$ is the very opposite of the stereotypical Italian/Latin lover, both in looks and in character; he is married to an emphatically unattractive wife, Pina, with whom, for the most part, he does not appear to be in love, while he is actually infatuated with a work colleague, Signorina Silvani; he has an ugly daughter, Mariangela, whom he loves, but of whom he is also very embarrassed. ${ }^{34}$ Other aspects of Italian culture are all reflected throughout the series, including the way Italians deal with life's problems, the unhappiness associated with personal and professional lives, the idea of the 'dongiovanni' Fantozzi wishes he could be (represented in the film series by the equally grotesque Calboni), but very clearly is not, and the failed aspirations of prestige and status, shown through the use and misuse of language by both Fantozzi and his colleague, Filini, which will be discussed in detail in the following pages.

\subsection{Fantozzi and other international comedic figures}

Fantozzi played an important role in the representation and critique of Italian culture and society during the 1970s. This is partially achieved through the plots of the films themselves, but equally central to this are the comedic elements present in the films, and specifically through the use of language, as well as a rather low-brow, slapstick humour. Globally, Fantozzi is not alone in this: there are many other characters, series and films that use these types of humour to touch on important cultural, social and political issues relevant to their times and cultural contexts - particularly issues surrounding the representation of both workplace and family. These comparisons are valuable to this project as they can be used as inspiration in finding comedic solutions to challenges in translation, as well as showing that there is an audience for Fantozzi in the English-speaking world, given the many similarities that exist between him and the other very well-known characters.

One series, and character, that is very similar to Fantozzi, which was in fact released at a similar time (1976), albeit in a British context, is Reginald Perrin in the television series The Fall and Rise of Reginald Perrin (1976-1979). Both of these series were initially released as books (Perrin in 1975, compared to Fantozzi in 1968), both use voice-overs to help develop the story (in the case of Perrin we also have text that appears on screen, used as a narrative device of sorts), and both adopt a rather slapstick style of humour, particularly around bodily functions: for example, Reginald's boss, CJ, has chairs which continually let out flatulent noises, while in the Fantozzi series, the main character is notorious for the noise and chaos produced by his burps. Despite being from rather different social backgrounds (Perrin from the upper-middle class and Fantozzi more lower-middle class), there is

\footnotetext{
${ }^{33}$ Giacomo Baresi, "Fantozzi e lo sport: il ciclismo e la coppa Cobram," http://www.giacomobaresi.com/category/fantozzie-lo-sport/. accessed 20th July, 2017.

${ }^{34}$ In saying this, Pina is not always the subservient wife. Indeed, she uses interesting language such as 'ti stimo moltissimo' instead of 'ti amo', and also threatens to leave Fantozzi at certain parts of the series.
} 
undoubtedly a similarity in that both characters want to escape the mediocrity that they are living in. ${ }^{35}$ As Hilary Dannenberg reminds us,

[m]any sitcoms represent their protagonists as involved in a struggle to escape the cultural norms of their particular class or occupation. Two 1970s sitcoms in particular - The Fall and Rise of Reginald Perrin and The Good Life - constructed a comic dynamic which opposed the protagonists' desire to escape with ongoing entrapment in social class and lifestyle. ${ }^{36}$

There are also content-specific similarities between the series: both male characters work in very menial jobs where they do not want to be (Fantozzi in a chemical plant and Perrin for an ice-cream company); they both use repetition as a source of humour (the same jokes are repeated, albeit in different contexts across both series); they both have rather politically incorrect styles of humour (particularly around the role of women); both Fantozzi and Reginald are obsessed, bordering on in love with, their younger colleagues as both characters fantasize over them (Fantozzi over Signorina Silvani and Perrin over Joan, his secretary, as presented in Figure 6); there are comparisons of family members with animals (in the Fantozzi series, Mariangela is often compared to a monkey, while in The Fall and Rise of Reginald Perrin Reginald's mother-in-law is compared to a hippopotamus); and even the plot of the first season of the TV show is relatively similar to Il secondo tragico Fantozzi, particularly where Fantozzi attempts to abandon his life and start over with Signorina Silvani, only to end back up with his family - similar to what happens to Reginald Perrin when he fakes his own suicide to start afresh, only to end up in his previous job, as if nothing had changed.
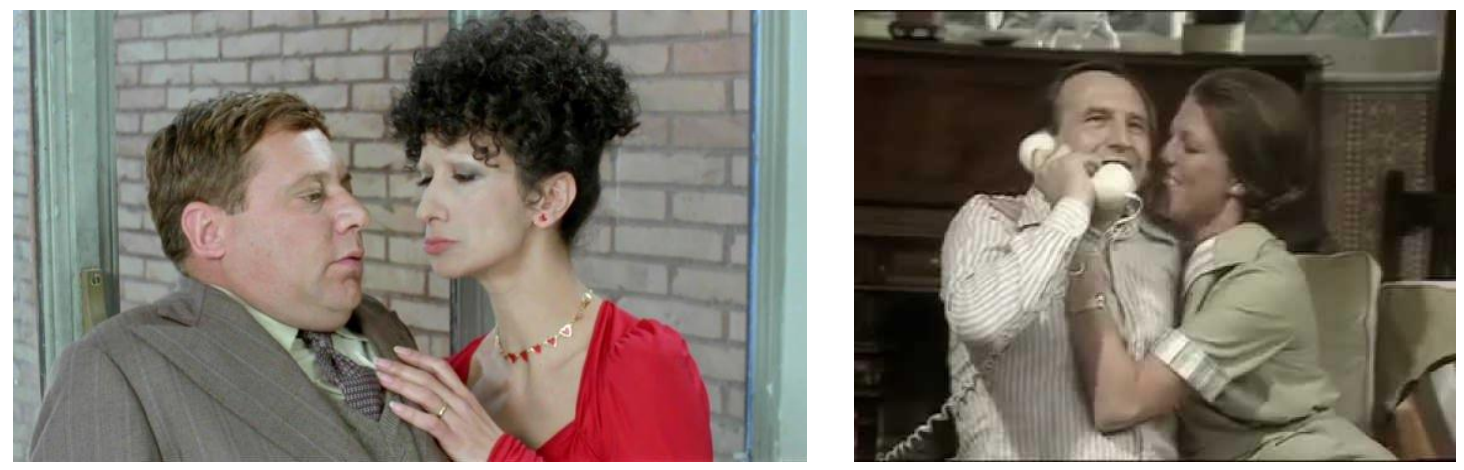

Figure 6(a-b): Fantozzi and Signorina Silvani (left), Reginald Perrin and Joan (right) ${ }^{37}$

One other more recent television series that shows similarities with the Fantozzi series is The Simpsons. Figure 7(a-f) are scenes taken from Fantozzi and Il secondo tragico Fantozzi, next to which are pictures

\footnotetext{
${ }^{35}$ Frank McDonough, "Class and Politics," in British Cultural Identities, ed. Mike Storry and Peter Childs (London: Routledge, 2007), 184.

${ }^{36}$ Hilary Dannenberg, "Marketing the British Situation Comedy," Journal for the Study of British Cultures 11, no. 2 (2004): 176.

${ }^{37}$ Reginal Perrin image found on: https://www.youtube.com/watch?v=8vUdhAAMldc. Accessed 20 $0^{\text {th }}$ October, 2018.
} 
drawn by the online artists 'Dan e Dav', who are clearly fans of both Fantozzi and The Simpsons, depicting the characters from the Fantozzi films in the style of The Simpsons. ${ }^{38}$ The first image recreates a scene from the second Fantozzi film, Il secondo tragico Fantozzi (1976), where Pina (in this case Marge Simpson) is dreading to tell Fantozzi (Homer) that they are being forced to watch the Battleship Potemkin by Fantozzi's superiors, instead of watching the Italy versus England football match; the second image recreates the final scene of the first film where Fantozzi and the Mega-Direttore Galattico (Mr Burns) are speaking in front of the L'aquario dei sorteggiati which, instead of containing Fantozzi's colleagues Fonelli, Peissi, Molli, Tritti and Bulzoni, contains The Simpsons' characters Lenny and Karl; and the final image depicts Fantozzi announcing that the 'Corazzata Kotiomkin' (Potemkin) is "una cagata pazzesca" ("a crazy piece of shit”), while Guidobaldo Riccardelli (Smithers) looks on in dread.
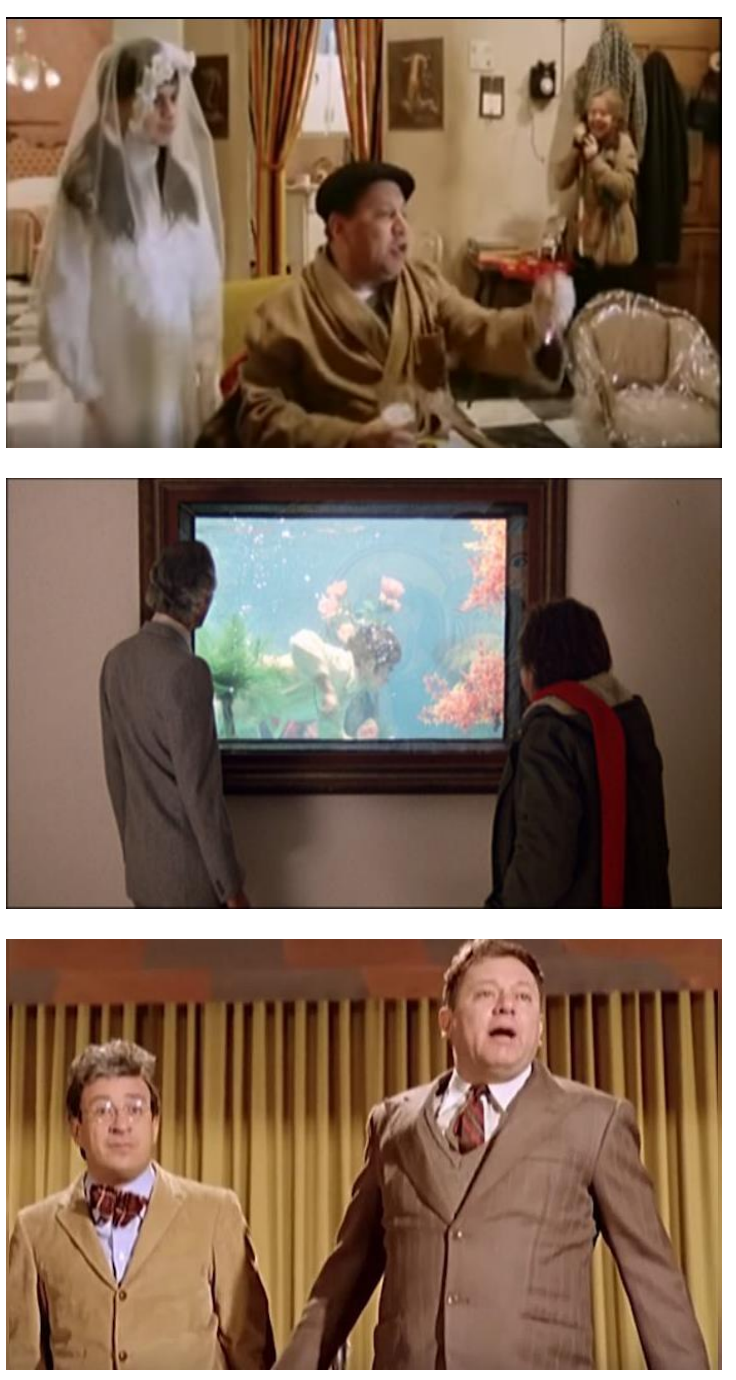
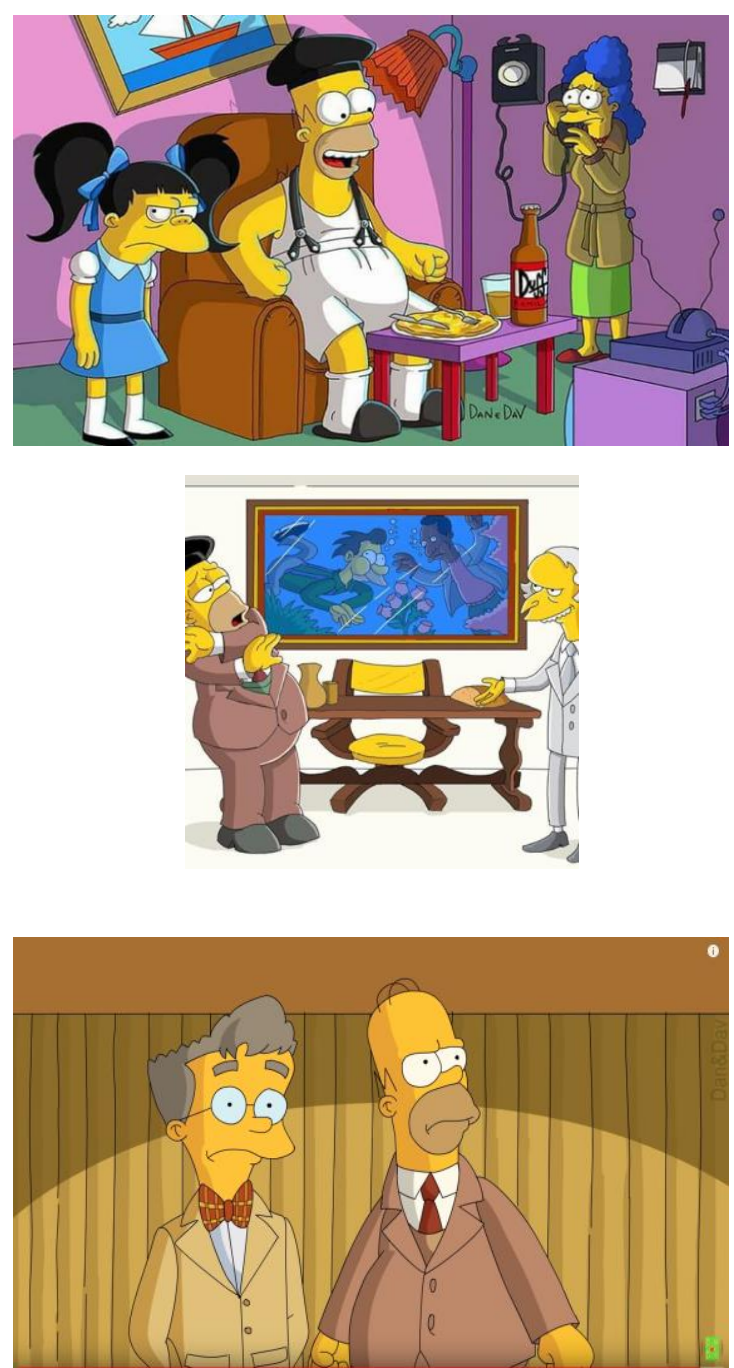

Figure 7(a-f): Scenes from the Fantozzi films the style of The Simpsons

\footnotetext{
${ }^{38}$ Dan and Dav, "Simpson Tribute to Paolo Villaggio (Fantozzi)," 9GAG, https://9gag.com/gag/agYvjV6/simpson-tributeto-paolo-villaggio-fantozzi. accessed 20th September, 2017.
} 
Seeing these images prompted me to compare the character of Fantozzi to Homer Simpson, Marge to Pina, Il Mega-Direttore Galattico to Mr Burns, etc. The pictures emphasise the similar nature of these characters, but also the comedic styles of the two comedy series, which use similar patterns of humour to comment on their respective cultures in different historical contexts. Since its introduction to American television in 1989, The Simpsons has become a worldwide phenomenon and the longest running television programme in history; in 2019 it continues with its $30^{\text {th }}$ season. From the very start the programme became popular for its satirical commentary on contemporary American society, incorporating current events, popular culture references and humour that were accessible nationwide in the United States of America, as well as internationally. This is exemplified through the number of special guest appearances of iconic figures in society: Michael Jackson, Meryl Streep, Buzz Aldrin, Stephen Hawking, Tony Blair, Venus and Serena Willams, and New Zealand's own Jemaine Clement and Bret McKenzie are just a few examples of how influential the programme has been in attracting guests from a variety of different backgrounds and societies. In its early years The Simpsons was described in Entertainment Weekly as "a wicked satire masquerading as a prime-time cartoon." 39 Possibly the most important role that The Simpsons has played across its many years on-screen has been its commentary on some of contemporary American society's more important and controversial social and cultural issues, which includes race, class, gender, sexuality and religion. As Matthew Henry states:

The Simpsons exposes the hypocrisy and ineptitude of pop psychology, corporate greed, commercialism, consumerism, and modern child-rearing. The Simpsons appears to embody a progressive politics, and it most commonly offers its satire from a leftist political position; from this vantage point, the show works to expose the dangers of religious fundamentalism, homophobia, xenophobia, racism and sexism. ${ }^{40}$

Although perhaps not to the same extent, given its comparative lack of global popularity, the Fantozzi series does aim to comment on some similar social issues as well. In fact, Henry's statements above could be applicable to Fantozzi: the idea of corporate greed is emphasised through the hierarchy within Fantozzi's workplace (and indeed we also have the recurring pattern of both Mr Burns and the Direttori constantly forgetting Homer and Fantozzi's names respectively); the idea of commercialism and consumerism is shown through the character of La Contessa Serbelloni Mazzanti Vien Dal Mare and her over the top parties and famous guests; we even get a small taste of 'leftist politics' from an Italian perspective, albeit very briefly and superficially unlike in The Simpsons where it is central to the series, through the lens of Folagra at the end of the film, where Salce gives us a satirical commentary of the standing of leftist politics in a multinational corporation of the time. Racist and xenophobic undertones are present throughout the film: the Japanese, Germans and French are all targets of what could be read

\footnotetext{
${ }^{39}$ Joe Rhodes, "The Making of The Simpsons," Entertainment Weekly, http://ew.com/article/1990/05/18/making-simpsons/. accessed 29th January, 2018.

${ }^{40}$ Matthew Henry, The Simpsons, Satire, and American Culture (New York: Palgrave Macmillan, 2012$), 7$.
} 
as a stereotypical racist, or at least culturally insensitive, commentary at multiple points throughout the film and series, and in later films we see rather blatant and stereotypical uses of homosexuality as a source of humour (particularly in the casino scene in Il secondo tragico Fantozzi). It must be pointed out, however, that in Fantozzi the use of homophobia and racism are used as comedic devices that accept the cultural prejudices, rather than as satirical commentary about these prejudices as is the case in The Simpsons.

One of the important values that The Simpsons puts at the forefront of every episode, which is also consistent across the Fantozzi franchise, is the depiction of family. Both Ugo Fantozzi and Homer Simpson play the respective breadwinner for their families, both work very low down in big corporations in similar positions - Homer in a nuclear power plant and Fantozzi in a chemical company, both Marge and Pina play the role of the subservient wives who stay at home to look after the children (their similarities are of course emphasised by the fact that, in the Italian dubbed version of the television series, the voice actress for Marge Simpson, Liù Bosisio, plays Pina in three of the Fantozzi movies), and while the children are very different in both the comedies, they play a very important role in the stories as a whole, impacting how we view the main protagonists of each series in terms their values, nature and humility, particularly surrounding them and their families. From the first Fantozzi film we see similar sketches that echo the seriality of The Simpsons: skiing holidays, camping holidays, being stranded in the middle of the ocean as well as frequenting a Japanese restaurant, to give just a few examples.

\subsection{The Fantozzi series: patterns of humour}

Having outlined the history and significance of the character in Italian culture and society, I would now like to provide a more in-depth discussion of the different types of humour present in the first Fantozzi film, as well as the series in general. While each Fantozzi film stands alone in its own right, there are certain elements that recur through the series that will pose challenges in terms of the translation, where retaining the cultural, comedic and historical content of the original becomes problematic. I will highlight some of the similarities that exist between the various films in the series in terms of language, cultural content and social commentary in order to emphasize and exemplify the humour that develops across the series. This discussion will not cover all of the similarities, however it will highlight a few of the key examples of how the translation of the films requires a careful approach, particularly given that some of the translation choices may need to be replicated over a variety of different contexts. Moreover, the patterns we are about to explore are intrinsically related to Fantozzi - retaining these in a way where none of the humour is lost or no connotations are altered is important to the quality of the translation. Although some of the comedic devices are of a more visual nature and therefore do not pose too difficult a challenge in translation, it is important to discuss them in order to understand the character, the humour, and even the success of the franchise. Certain jokes throughout the series remain 
prevalent, however, in my opinion, the quality of the films does deteriorate: the clever social commentary is, gradually, replaced by rather vulgar and low-brow references, particularly in the latter films; however they all seem to retain aspects of the original humour, as we shall see. This discussion will be followed in the next chapter by an analysis of theories of humour and humour translation in general, as well as more specifically in relation to audiovisual texts, all of which come into play when translating this rather challenging content.

The first, and most obvious, pattern that occurs throughout the Fantozzi series is undoubtedly the recurring use (and misuse) of language for comedic purposes. From the misuse of relatively complex grammatical structures like the imperativo formale (formal imperative, used for giving commands or instructions), to the repetition of several expressions and catch phrases, the linguistic humour has played a big part in the success of the series as a whole. In fact, language plays a key part of the Fantozzi series in both the films and the novels, so much so that in the republished versions of the Fantozzi books, a 'Fantozzi glossary' has been written by Stefano Bartezzaghi.

The first example I want to highlight is the misuse of the imperativo formale/congiuntivo. This is something with which Fantozzi is deeply associated, as illustrated in Figure 8, where an example of dialogue from the film is depicted in a meme from the website cinepanettone.it).

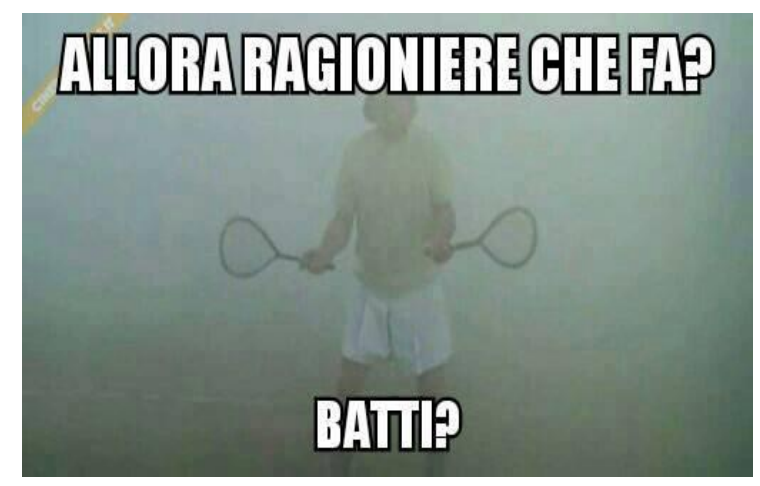

Figure 8: An example of the misuse of the 'imperativo formale' from the first Fantozzi film ("So, accountant, what are you doing? Serve?") ${ }^{41}$

In wanting to sound well-educated, Fantozzi attempts to use this rather complex grammatical structure, but in doing so he gets the conjugation completely wrong. As Giuseppe Patota and Valeria Della Valle argue, Villaggio uses this linguistic humour "[t]o comically highlight the deep ignorance of all of the characters that make up the micro-bourgeois universe." ${ }^{42}$ In total, there are 14 such instances of incorrect imperatives in the first Fantozzi film, made by a variety of characters, but mainly Fantozzi and Filini. In the second film the joke is taken even further, with 22 instances of using the wrong conjugation of the imperativo formale. However, in the later Fantozzi films the misuse of the

\footnotetext{
${ }^{41}$ Image found on: http://cinepanettoni.it/scuola-cult-curiosita-di-fantozzi/. Accessed on $20^{\text {th }}$ January, 2017.

42 Valeria Della Valle and Giuseppe Patota, Viva il congiuntivo! (Sperling \& Kupfer, 2011), 11. [My translation] Original Italian: "a sottolineare comicamente la profonda ignoranza di tutti i personaggi che compongono quell'universo microborghese."
} 
congiuntivo becomes less of a distinction between classes, rather turning into a running joke by all of the characters, thus losing its aspect of social satire. In the final film, Fantozzi 2000: La Clonazione (1999), the misuse of the congiuntivo is abundant in the very first scene alone, and is even adopted by one of the directors of the Megadita where Fantozzi works, who says: "Non mentischi!", as opposed to the correct "Non mentisca!" ("Don't lie!"). Other characters continue to adopt the very common fantozziane expressions "vadi", "venghi" and "facci" throughout this same scene. In this final film of the series we also see the misuse of congiuntivo in a more general sense, not just as the formal imperative, when in a conversation with the Duca-Conte, Fantozzi says, "“" as opposed to "spero che abbia dormito bene, eh?" ("I hope you slept well"). These examples show how quintessential this linguistic error is to Fantozzi's humour throughout the series, albeit in a much more absurd, and ultimately less effective, way in the later films.

The second example of a linguistic pattern that occurs throughout the entire series appears in the first scene of the first film, where Fantozzi hits his head on the ceiling of his 'office' (under the stairs) after becoming nervously excited that Signorina Silvani has come to speak with him. Silvani asks him: "Si è fatto male?" ("Did you hurt yourself?"). This expression is particularly fantozziana: it is mostly used after someone has inflicted pain upon him, or caused him to inflict pain upon himself. This pattern emphasises the place Fantozzi holds in society: those above him do not realise they are the ones hurting him or causing his pain, or if they do then they do not care for the damage they have caused. All in all the expression is repeated three times in the first film, the first instance in the above example with Signorina Silvani in the office, then when his fingers are shattered in the car door by Signorina Silvani, and finally in the New Year's celebrations, when he gets thrown through a glass panel while dancing. It is repeated twice more in the second film (as well as there being one instance of the informal "ti sei fatto male"), again when his fingers are crushed, this time by Count-Duke Semenzara, and the second time during the hunting scene where he accidentally shoots himself in the crotch with a pistol he has acquired. ${ }^{43}$ This slapstick humour is usually associated with him being hit on the head, or rather commonly involves his hands being crushed or burnt, but the humour is always brought home by this particular phrase.

Another example of recurring language used for comedic purposes, occurs through descriptions of characters' clothes by the voice-over narrator (Paolo Villaggio). The films use this device to comment on absurd fashion choices made by both Filini and Fantozzi, and in the later films by Uga, Fantozzi's granddaughter. These depictions also show Fantozzi's lower to middle-class nature as they are clothes that he has owned, and never replaced, for a number of years. All of these scenes use a similar structure: in Fantozzi and Il secondo tragico Fantozzi we see the dialogue introduced with "abbigliamento di

\footnotetext{
${ }^{43}$ In researching for this project I came across the following news article, by an unspecified writer, published on an online Italian news page which recounts the story of a New Zealander who shot himself in the crotch, comparing him to Ugo Fantozzi. "Il "Fantozzi" neozelandese: nasconde la pistola nei pantaloni e si spara," Blitz Quotidiano, https://www.blitzquotidiano.it/cronaca-mondo/fantozzi-nuova-zelanda-pistola-pene-986897/. accessed 23rd August, 2018.
} 
Filini:" (“Fallini's attire:”), followed by "Fantozzi:", and a description of their respective clothes. In both the films there are mentions of Filini's rich aunt, as well as references to the rather outdated nature of all of their possessions. Moreover, these two scenes also mirror each other later on when the voiceover describes the technique used to serve a tennis ball in the first film, and fire a slingshot in the second: "Per una buona battuta..." ("for the perfect serve...") versus "per un perfetto tiro con la fionda da caccia..." ("for the perfect slingshot:..."). In Superfantozzi (1986) we see a slight variation of these descriptions: Fantozzi has entered a jousting tournament to win the love of the Principessa (Serbelloni Mazzanti Vien Dal Mare), so the attire is introduced instead as "Armatura di Fantozzi:" ("Fantozzi's armour:"). Finally in Fantozzi: La Clonazione, there is a description of Uga's absurd modern fashion choices, followed by a young man crashing his motorbike when he sees her unattractive face. These scenes, rather interestingly, cause problems in translation given some of the references that are made through the descriptions, as shown below, that are both comedic and historical. In the first film, for example, as we will discuss in further detail, we see the cultural reference to "GIL". In some of the other descriptions we have references to historical figures such as Pipino il Breve (Pepin the Short) that may well be lost on the target audience in translation:

\section{Fantozzi (1975)}

Abbigliamento di Filini: gonnellino pantalone bianco di una sua zia ricca, maglietta Lacosta pure bianca, scarpa da passeggio di cuoio grasso, calza scozzese e giarrettiere; doppia racchettina Liberty da volano.

Fantozzi: maglietta della “GIL”, mutanda ascellare aperta sul davanti e chiusa pietosamente con uno spillo da balia, grosso racchettone 1912 , elegante visiera verde con la scritta: "Casino Municipale di Saint Vincent".

\section{Il secondo tragico \\ Fantozzi (1976)}

Abbigliamento di Filini:
berrettone Sherlock
Holmes con penna alla
Robin Hood, poncho
argentino di una sua zia
ricca, scarpe da tennis con
sopra galoche, carte
topografiche, e trombone
da brigante calabrese.

Fantozzi: berretto bianco alla marinara di sua figlia Mariangela, giacca penosamente normale stretta in vita da gigantesca cartucciera da mitragliatrice, residuato della seconda guerra mondiale, fionda elastica, siero antivipera a tracolla, gabbietta con canarino da richiamo, e gatto randagio da riporto, sùbito fuggito durante le operazioni di partenza.

\section{Superfantozzi (1986)}

Armatura di Fantozzi: banderuola 4 venti in funzione di pennacchio, pauroso elmo vichingo con visibilità azzerata, sospensorio in bronzo sottratto alla statua di Pipino il Breve e, ai piedi, ferroni da stiro a carbonella di piombo fuso. Peso complessivo armatura Fantozzi: 4 quintali, 32 chili e 7 etti e mezzo.

\author{
Fantozzi: La \\ clonazione (1999) \\ Come tutte le \\ adolescenti, anche \\ Uga la nipote di \\ Fantozzi si era \\ adeguata all'ultima \\ moda: Scarpettone \\ ginniche con zeppone \\ multistrato, pantalone \\ a zampa di mammut e \\ mini-top ultra-succinto \\ ad effetto sexy \\ garantito.
}


importance of religion in Italian culture, an element that Villaggio, perhaps, adopts rather controversially for comedic effect. In the first film, Fantozzi sees Jesus walk across water (Figure 9a) when he is severely dehydrated, with Jesus asking him if he has fish or bread to multiply. Furthermore, the Jesus figure uses a rather strong accent from the Lazio region, it is almost a hippy representation of Jesus, used to demystify and, to a certain extent, mock the Catholic church and religion, which will inevitably prove difficult in translation should the connotations want to be replicated in the English translation. He also sees San Pietro above the crossbar at the end of the football match (Figure 9b) after taking a severe knock on the head. In the second film Fantozzi also hallucinates after being ferociously burned by Pina's family remedy; in the hallucination we see the Archangel Gabriel who has come to tell him that he is pregnant (Figure 9c). In Fantozzi contro tutti religion is again at the forefront when Fantozzi, while watching a strip tease on his television, pretending to undress the woman on-screen, inadvertently changes the channel to a religious programme, just as Pina walks in, and we see Fantozzi kissing a priest. Perhaps the most obvious religious imagery, used to show Fantozzi's mediocrity, appears in Superfantozzi, where God creates man, the first man being Fantozzi, only for him to realise his mistake, blaming it on tiredness, and recreating man in his own image the next day, this time in the form of the hunk movie star of the time, Luc Merenda (Figure 9d) ${ }^{44}$
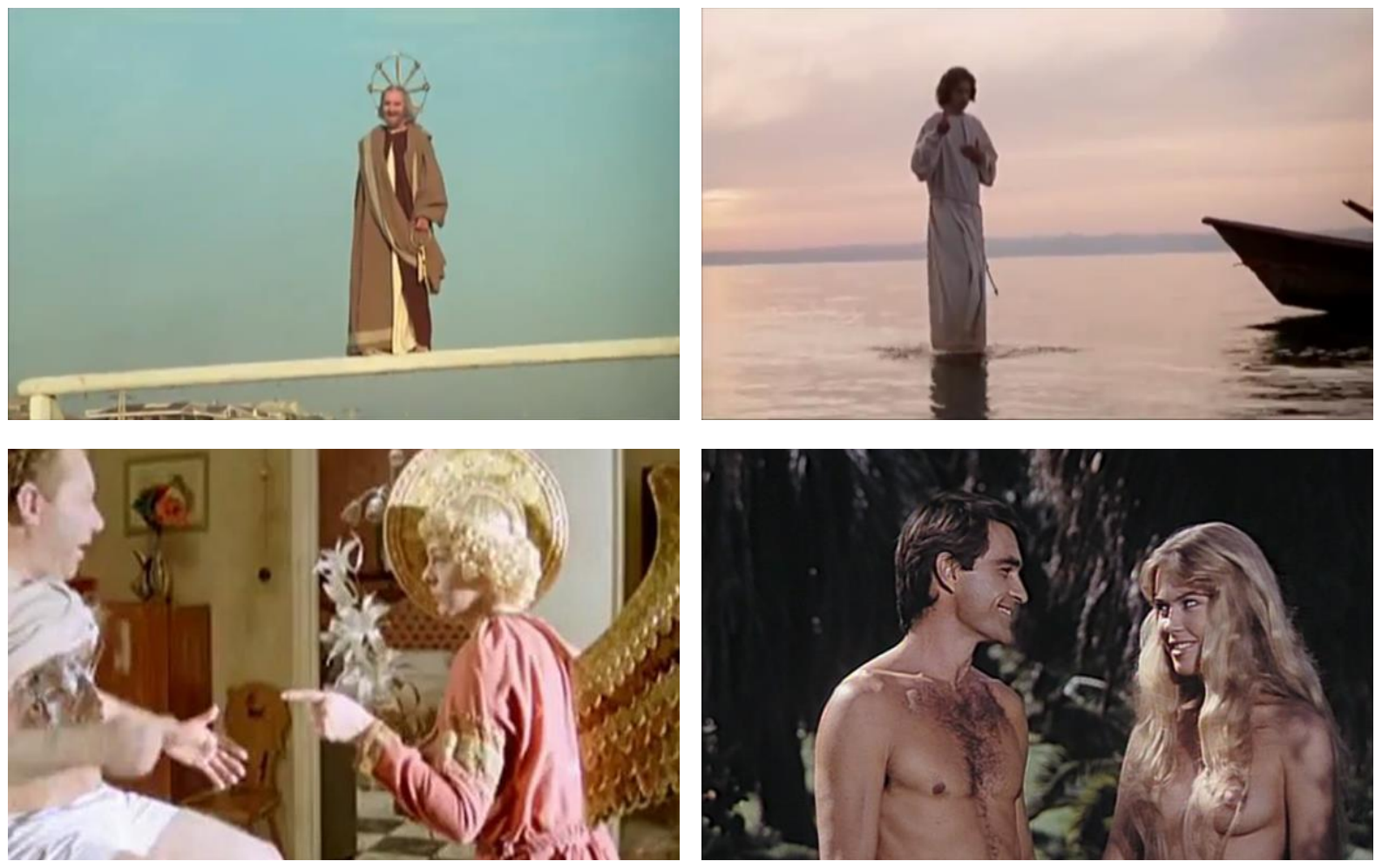

Figure 9(a-d): Examples of the biblical characters that appear in Fantozzi including Jesus, Saint Peter, the Archangel Gabriel and Adam and Eve

\footnotetext{
${ }^{44}$ For more detail on the different aspects of religion in Fantozzi, and indeed other symbolic features of the films including sex, homosexuality, politics and sport, see: Simone, Memorie dal sottoscala.
} 
Another example that depicts particular religious connotations, which also exemplifies the ever-present theme of classism, is the representation of the relationship between employee and employer in the entire series. As previously discussed, Fantozzi represents the average office employee of the 1970s and beyond. Because of this, he embodies the struggling and somewhat depressing anti-hero that became a character through whom Italian audiences could reflect upon their own lives. The depiction of the employers, on the other hand, is almost God-like. In both the final scenes of the first two movies we see the Mega-Direttore Galattico referred to as "Altezza" ("Your Highness"), "Santità" ("Your Holiness") and "Onnipotente" ("God Almighty"): the colour scheme of the set design is pure white, his speech formulated in a very high register, and the chair made out of human skin is very prominent, emphasising the place employees hold in the big corporations (see Figure 10). Fantozzi bows down to him in both contexts, feeling unworthy to be in his presence. The social distinction and disparity is also amplified through Fantozzi's superiors, not just the Mega-direttori, who inevitably call him Fantocci, Pupazzi or other variations of these names. This recurring gag provides an effective satirical commentary of the social inequality that existed between employers and employees, as well as the workplace hierarchy that existed in 1970s Italy and beyond. In translation the challenge is undoubtedly capturing the difference in register between the characters, particularly the distinction between the worshipping nature of the "Lei" (formal "you") Fantozzi uses, compared to fake respect that the Mega-Direttore gives Fantozzi when he uses the "Lei" form.
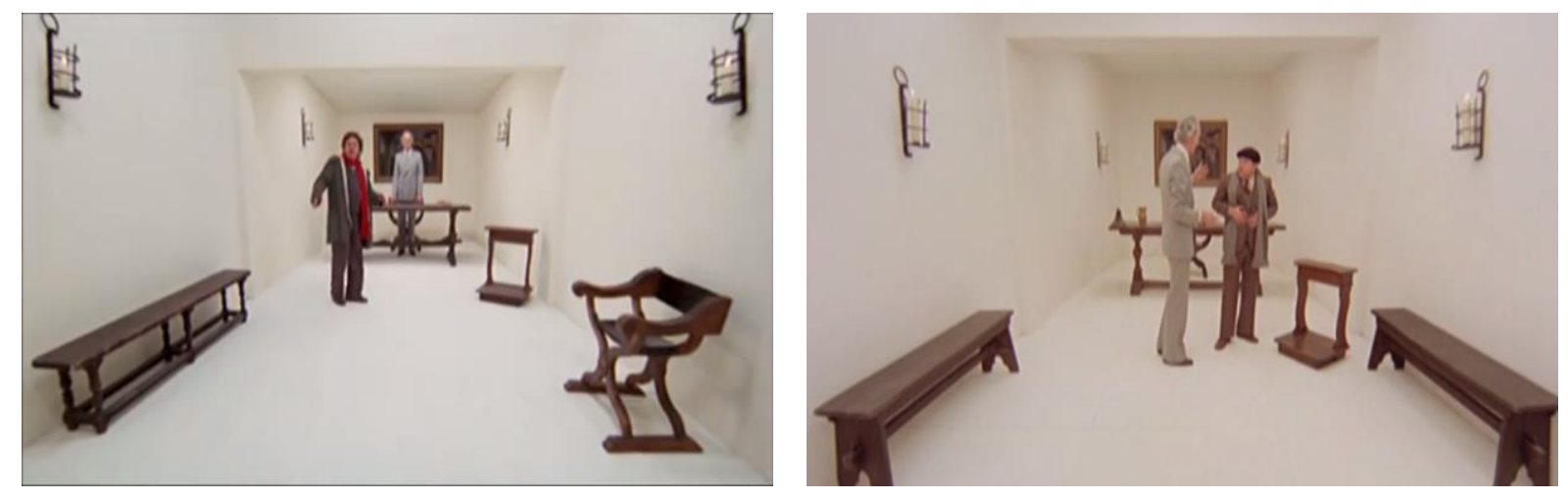

Figure 10(a-b): The Mega-Direttore Galattico's office in the first two Fantozzi films.

Another example of the classist, hierarchical society and the importance of social status is provided through mentioning particular high-standing members of Italian society. In the first film this takes place at Contessa Serbelloni Mazzanti Vien Dal Mare's party that she hosts at the ski resort. Names of many high profile members of Italian society including the aristocratic winemakers Riccadonna, as well as the heirs to the Sambuca family are referred to in the elitist conversations that take place there. In the second film we see a similar event take place at the launch of the boat, where Serbelloni Mazzanti Vien Dal Mare, one of the stakeholders in the firm, is tasked with smashing the champagne bottle against the vessel as a ceremonial ritual. In the process she knocks several people into the water, all of them high 
profile figures in Fantozzi's workplace, or from the Italian community; then again at the subsequent party, Fantozzi is introduced to many high-profile figures from Italian society including the German Ambassador. Furthermore, during this scene he is repeatedly referred to by Serbelloni Mazzante Vien Dal Mare as "impiegato" ("employee"), clearly showing the social distinction between the employer and the employee, particularly from the perspective of those higher in the social hierarchy. And finally in Superfantozzi we see the same pattern when medieval knights are jousting for the hand of the Principessa Serbelloni Mazzanti Vien Dal Mare in marriage. Each high profile knight's sigil is read out as they are defeated. Each of these scenes uses the same structure of dialogue, introducing the list of names, both real and fictional, with the phrase "Nell'ordine..." (“In order...") followed by a list of the fallen knights' names. Serbelloni Mazzanti Vien Dal Mare, with her absurdly long and pretentious name, undoubtedly embodies Villaggio's commentary on the contrast between social classes in 1970s Italy and beyond, one that no 'economic miracle' was able to overcome. In translation one of the challenges may well be to ensure that these high profile members of society are portrayed in a way that reflects their status. This is the biggest challenge with a temporal displacement as references from the original content were applicable fifty years ago and may not be accessible, even to an Italian audience, today. ${ }^{45}$

\section{Fantozzi (1975)}

Dopo quella diamantata pazzesca la contessina Serbelloni Mazzanti Vien Dal Mare gli fece conoscere alcuni amici. E gli presentò nell'ordine: La Signora Bolla, i Coniugi Bertani, la Contessa Ruffino, i Fratelli Gancia, Donna Folonari, il Barone Ricàsoli, il Marchese Antinori, $i$ Serristori, Branca e i Moretti, quelli della birra. A metà di quel giro di presentazioni, Fantozzi era già completamente ubriaco.

\section{Il secondo tragico Fantozzi Superfantozzi (1986)} (1976)

Qui lo raggiunsero nell'ordine: sindaco con fascia tricolore, ministro della marina mercantile, centoduenne baronessa Filiguelli de Bonchamp, mascotte a vita della società. Tutte le autorità vennero poi furtivamente varate a parte. Finita la riserva di champagne, fu deciso di cambiare il rituale della cerimonia: taglio di un cavetto metallico che avrebbe messo in moto il meccanismo del varo. Riparte da settantasei metri la Serbelloni Mazzanti Viendalmare!
Caddero nell'ordine: torro, unicorno, idra, acquila. $E$ arrive finalmente il turno di Fantozzi. Era l'insegna della locanda il girarrosto. Sponsor ufficiale di quell concorrente...

\footnotetext{
45 The representation of the white-collar worker and their relationship with their superiors, looking specifically at Fantozzi, is the subject of a doctoral dissertation by Paolo D'Amora, "Cinematic Representations of Italian Office Workers From the Death of the Travet to Fantozzi, 1952-1983" (The University of Texas at Austin, 2017).
} 
Another reccurent comedic gag is the comparison between Fantozzi's daughter, Mariangela, and a variety of different primates, by Fantozzi himself, as well as a variety of different colleagues. A rather interesting comparison given Mariangela's name would imply an angelic nature and appearance. In Fantozzi this is arguably the foundation for the most touching and sad scene, when Fantozzi's superiors bully Mariangela, calling her "Cheeta", in reference to Tarzan's monkey, asking her if she would like a banana, or if she could do a somersault. This offers a rare moment in the satire when Fantozzi stands up for himself and his family, one of only two instances in the first film where this is the case. This leads to the rather humorous quip where Fantozzi tells his daughter that his superiors must have meant "Cheeta Hayworth", the very famous and beautiful American actress [i.e. Rita Hayworth]. In the second film, however, the humour based around Mariangela's looks is taken to new heights when Fantozzi takes his daughter to the circus, where he leads a monkey by the hand, believing it to be Mariangela. In Fantozzi contro tutti (1980), Fantozzi goes to kiss a soft toy monkey, not realising that it isn't his daughter, and in Fantozzi subisce ancora (1983), Fantozzi picks Mariangela up from her workplace, the rather aptly named pet shop "Casa degli animali" ("House of animals"), only to guide a monkey out of the shop, rather than Mariangela (see Figure 11a-d). A joke that starts to appear later in the series involves Fantozzi often saying "mia babbuina" ('my baboon') instead of "mia bambina” (my child). The trend continues with Mariangela's daughter, Ughina, who is also often referred to as 'la babbuina' by Fantozzi. Mariangela's husband, Bongo, is also portrayed as a gorilla, likely another use of racism for comedic purposes.
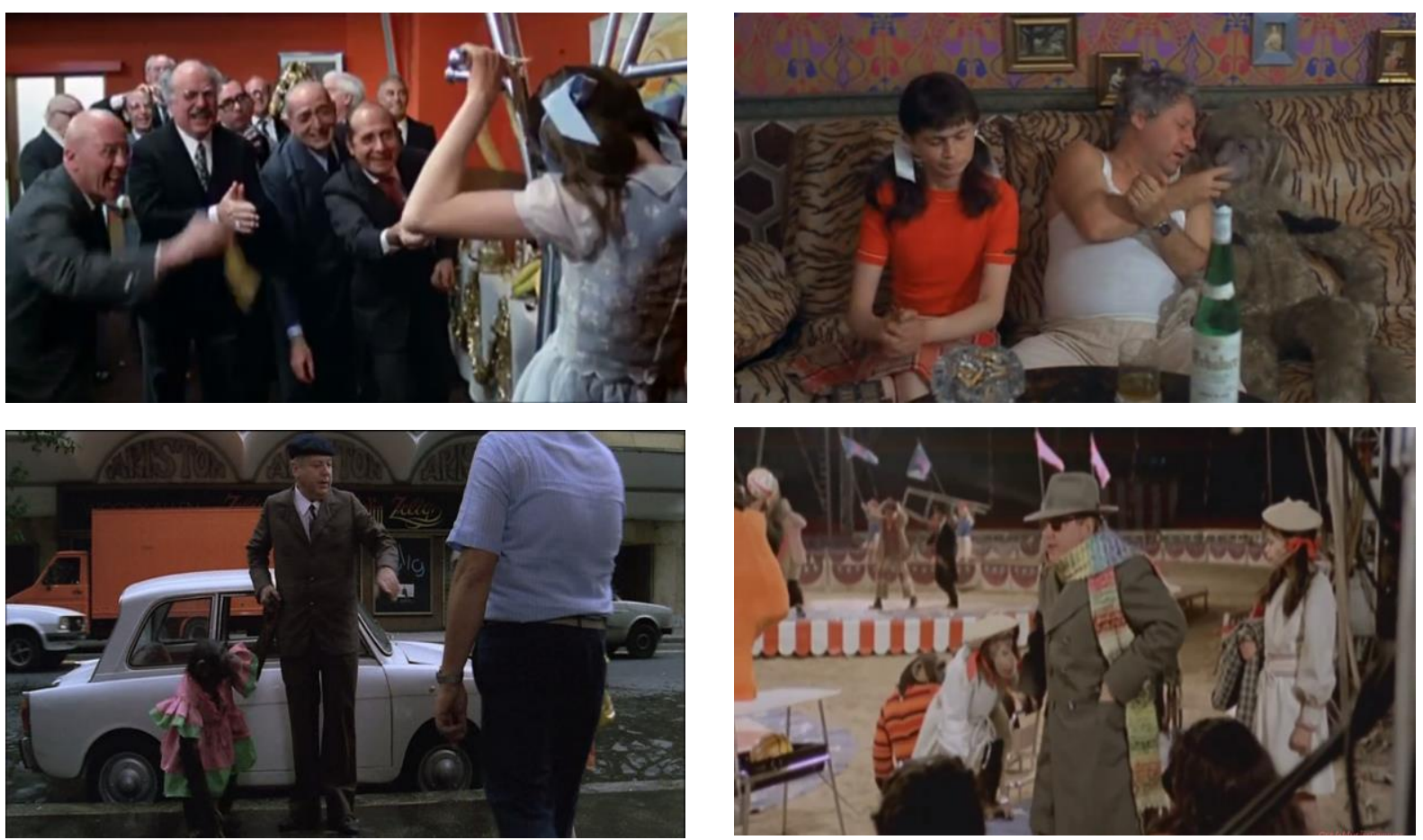

Figure 11(a-d): Examples of Mariangela's comparison to a monkey throughout the series 
As previously discussed, just as with The Simpsons, the Fantozzi series often uses other languages, cultures or societies as a source of humour. Most notable is the attention that the Germans and Japanese garner throughout the entire Fantozzi series. The Germans, in particular, just as in the case of many English films, are often portrayed as the antagonists due to the negative connotations associated with their military history, and in particular due to the memory of Nazi occupation during World War II. One scene that takes place in the first film is eerily reminiscent of a jail, where Fantozzi is imprisoned and starved in a 'diet therapy' clinic (Figures 12a-b). In fact, the location of the set is an actual prison in Rome, the Carcere minorile di San Michele. The directors of this centre in the film are German, a clear and strong reference to the horrific past events of World War II, a way for director Luciano Salce to exorcise his experience as a prisoner of war in a German prison camp. In fact, this scene is effectively repeated in Fantozzi contro tutti, changing small details but using an identical set design. In this scene, for example, the German doctor, Dr Birkermaier, forces the starving patients to sit and watch him eat, and should they attempt to eat, they are whipped. Arguably in this scene there is an even greater emphasis on the evil nature of the German doctor, who seems to take pleasure from torturing the Italians that he has under his care.

The references to Japanese culture are quite different. Salce appears to be representing the exotic as comical to the Italian audience and exaggerating the stereotypes associated with it. This is particularly prevalent in the Japanese restaurant scene from the first film, which is riddled with exaggerated stereotypes including the restaurant cooking dog, staff speaking exclusively in Japanese, Italian customers only being allowed to use chopsticks, and Signorina Silvani going to the restaurant wearing traditional Japanese attire in "yellow face" (Figure 12c). In fact, to this very day these themes and stereotypes associated with the Japanese, and their cuisine in particular, remain relevant as a source of racist humour. In his stand-up comedy show titled Il meglio d'Italia (2014) (The Best of Italy), Italian actor and comedian Enrico Brignano uses very similar themes as the subject of his comedy, including the language used by the wait staff, the customary rituals such as bowing on entry, the type of food one eats, as well as references to the samurai and other Japanese customs, showing that the humour in Fantozzi remains pertinent to the contemporary Italian audience. In Superfantozzi the Japanese are the targets of humour yet again when a Fantozzi, a.k.a 'Tozzi-Fan', is selected to be a kamikaze bomber over Hiroshima in one of the historical representations that comprise the film (Figure 12d). While the racism towards Germans is more clear-cut in the sense that they are stereotypically portrayed as antagonists in film, and that Luciano Salce himself was held prisoner in a German prison camp, the racism towards Japanese culture seems, sadly, to be solely for the sake of racism. 

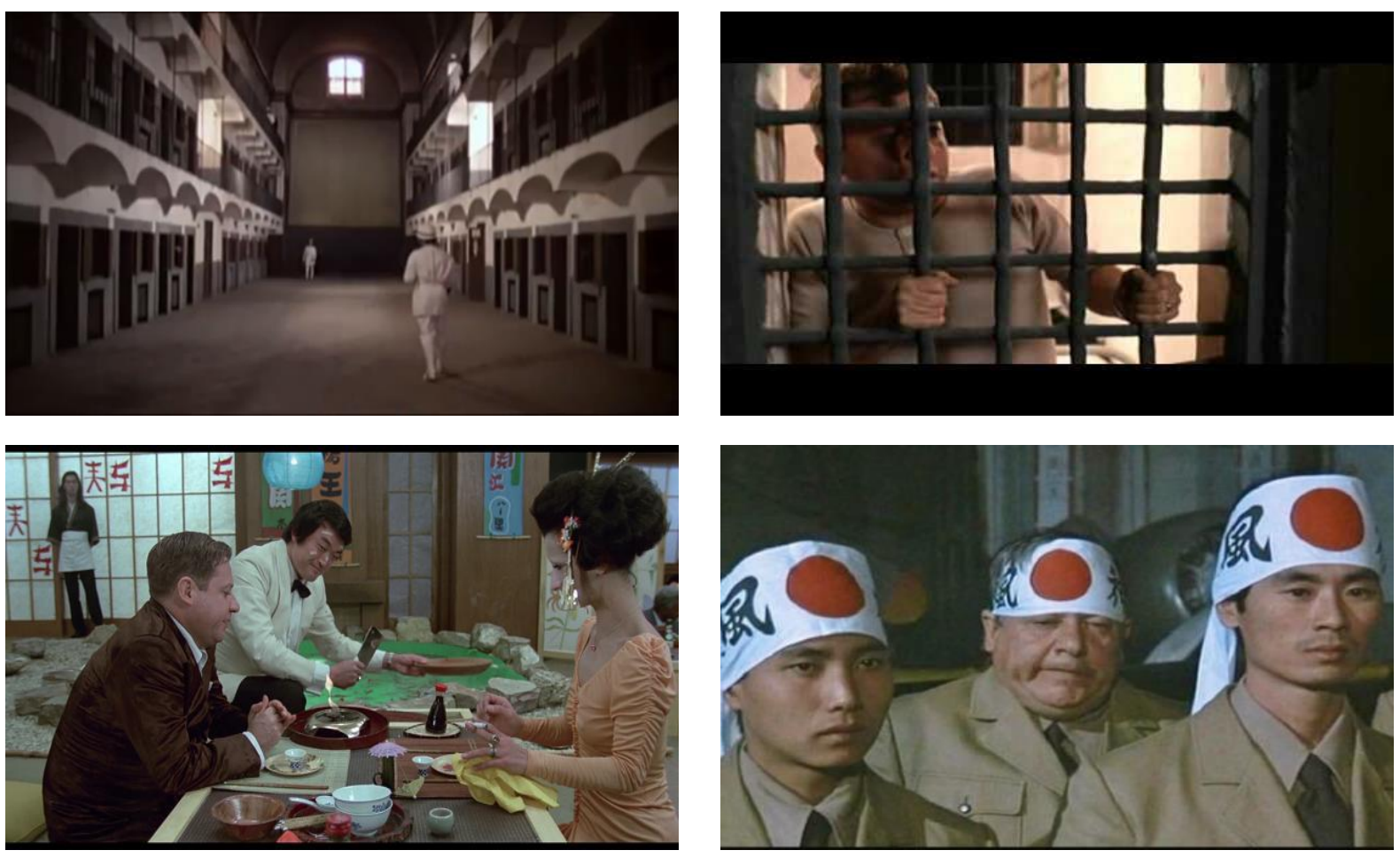

Figure 12(a-d): The representation of other languages and cultures in the Fantozzi series, from references to World War II to racist stereotypes.

\subsection{Conclusion}

Paolo Villaggio was one of modern day Italy's finest comedic authors and performers. His work is recognised and remembered by a very diverse audience for both its comedic value, but also its social commentary of a post-economic boom society. His numerous awards were a testament to his popularity and success. While his character, Fantozzi, may not be the stereotypical, likeable protagonist, the slapstick nature of the humour, and the variety of humour and cultural references that exist within his universe, keep the books and films entertaining and light-hearted, while commenting on important social issues of 1970's Italy. Untranslated into English, the Fantozzi series offers an exciting challenge for a translator and subtitler of audiovisual humour. The theoretical analysis of the next chapter on translating humour will provide us with the appropriate tools to complete the translation of Fantozzi into English. Given that various linguistic jokes are also replicated, particular themes are almost identical across the series, and the content itself remains fairly similar, the translator must also be wary of this and therefore must translate the text with this in mind: translation solutions need to have the ability to be replicated across a variety contexts. This only adds to the difficulty of the translation given the pre-existing difficulties that are present in multi-modal translation.

This chapter has touched on a variety of aspects that make Fantozzi, and subtitling Fantozzi, the unique case study that it is. Each of those aspects is, in and of itself, a case study in Italian culture and its national and international appeal and reception, and would thus warrant a more thorough 
discussion which goes beyond the scope of this thesis. However, without exploring, albeit briefly, the character and context, the task at hand would have been far more difficult. The contribution of this chapter to the thesis as a whole is, instead, one of signposting all the issues, some general and some specific, of which the subtitler ought to be aware in order to attempt to do justice to this iconic comedic figure. The comparison with similar examples of humour in English has the same function: to test the linguistic and cultural viability and translatability of Fantozzi outside the sociocultural cauldron of posteconomic boom Italy out of which this character has emerged. Researching and writing this chapter, then re-reading it as the thesis and the translation progressed, has undoubtedly deepened my understanding of 1970s Italy and sharpened my strategies and skills as a subtitler who maintains that even under taxing limitations such as those imposed by audiovisual translation (as will be discussed indepth in chapter 3), every effort ought to be made to retain as much as possible of the original content, function and effect on the audience. 


\section{Translating humour in an audiovisual context}

There is one idea among translation scholars that is hardly disputed at all nowadays; and it is that translation studies is an interdisciplinary field of research. So is humor studies; and both draw from linguistics, psychology and sociology, among other disciplines, for their descriptions and their theoretical models and constructs. It is not surprising, then, that humor and translation studies overlap, and the findings of one must be of interest to the other. ${ }^{46}$

Patrick Zabalbeascoa

Having outlined the character of Ugo Fantozzi, the patterns of humour that are present throughout the film and series, and the challenges that these could pose in translation, I would now like to discuss the general issues pertaining to translating humour before embarking on the translation project itself. First, I will explore the leading theories, as proposed by different scholars, that engender comic amusement. This will help us explain why Fantozzi in particular has been so successful in Italy and guide us through the essential elements of the film that must be retained in translation. Then I will explore translating humour across borders in a more general sense, highlighting some of the success stories of translating humour across languages and cultures. Finally, I will look into how different types of humour can be translated by analysing different techniques adopted by translators and scholars of comedic content. I will then use these techniques, in conjunction with the specific techniques highlighted in the next chapter on subtitling, to produce my subtitles for Fantozzi.

\subsection{Theories of humour}

What humour is and why particular texts are humorous has been a large focus of humour studies. As Delia Chiaro states, “[j] okes and humour are natural companions. However, while we all know what humour is, the concept itself is not only difficult to pin down, but also to unequivocally define. It is unlikely that there is or has ever been an eminent philosopher or intellectual who has not attempted to produce a definition for humour." ${ }^{47}$ Several theories have been drawn up by philosophers and psychologists alike to help us try to understand what humour is, and why we find particular things funny. These main theories that have established themselves in the field of study are known as the superiority theory, the incongruity theory, the relief theory, the play theory and the dispositional theory all of which will be briefly outlined in this section. Luigi Pirandello's "Sentimento del contrario" ("The feeling of the opposite") will also be outlined as it is particularly relevant to Fantozzi and Italian comedy in general. While theories of humour are not the main focus of this translation project, these theories

\footnotetext{
${ }^{46}$ Patrick Zabalbeascoa, "Humor and translation - an interdiscipline," International Journal of Humor Research 18, no. 2 (2005): 185.

${ }^{47}$ Delia Chiaro, The Language of Jokes in the Digital Age (New York: Routledge, 2018), 9.
} 
will provide some context as to why Fantozzi has been successful and why Italian society has found the character and the stories so entertaining and humorous across the decades. Moreover, this discussion will help us to ground specific issues related to translation and subtitling. It must also be stated that humour is very subjective, so the outline of these theories is not necessarily to explain why Fantozzi is funny, more so an explanation of why an audience could find it funny and why it has achieved the cult status that was explored in Chapter 1.

The superiority theory, in a sense, is synonymous with the German concept of schadenfreude, or taking pleasure in the misfortune of others: we take pleasure because we think we are better than those who are the butt of the humour. This theory is credited to Thomas Hobbes (1588-1679), and as the name would suggest, "results from perceiving infirmities in others which reinforce our own sense of superiority." ${ }^{48}$ Humour, in general, is often made at the expense of an individual or group of individuals, which tends to be targeted at the particularly moronic, vain, greedy, selfish or at people who are deficient in some other respect. This could certainly be said of Fantozzi, and perhaps the superiority theory is the best suited explanation as to why Fantozzi has been so successful in an Italian context: he is the epitome of the average and he is seen as being inferior by the Italian viewer. The humour in Fantozzi is, for the most part, of a slapstick nature, which seems to fall into the type of humour described by the superiority theory as it is very easy to laugh at the way in which a character slams his fingers in a car door, or completely loses control while skiing down a mountain, or how he encourages people to throw items out of their window on New Year's eve only to have those exact items crush their car. There are critics of this theory, however, such as Frances Hutcheson, who point out that humans perceive themselves as being superior to many things, but don't necessarily find them funny. He gives the example of a gentleman riding a coach passing by beggars on the street, inevitably one will feel a sense of superiority, but the action is more likely to encourage pity, rather than humour. ${ }^{49}$

The incongruity theory, on the other hand, emphasises that random, absurd, inappropriate or unexpected events are central to humour, that is, that incongruity is the object of comic amusement. ${ }^{50}$ Incongruity was a concept developed by Immanuel Kant (1724-1804). I would argue that this theory, for the most part (particularly for the very base level of humour that exists in the film) is not relevant to Fantozzi, given that the slapstick humour present in the film tends to be more predictable and not very controversial. However, there are aspects of humour in the Fantozzi series that perhaps do align themselves with the incongruity theory. For example, Fantozzi tends to have deep mystical hallucinations which could be considered absurd; the situations he finds himself in are undoubtedly random and fairly far removed from everyday life; and there are certainly moments that can be considered inappropriate: the Japanese restaurant scene is a prime example (as well as many other

\footnotetext{
${ }^{48}$ Noël Carroll, Humour: A Very Short Introduction (New York: Oxford University Press, 2014), 8.

${ }^{49}$ Frances Hutcheson, Reflections Upon Laughter, and Remarks Upon the Fable of the Bees. (Glasgow1750), 10.

${ }^{50}$ John Morreall, "Philosophy of Humor," in Stanford Encyclopedia of Philosophy, ed. Edward Zalta (California: Metaphysics Research Lab, Stanford University, 2012).
} 
scenes that have racist and xenophobic undertones), particularly when the Japanese chefs end up cooking the dog, Pierugo.

Another theory of humour, however one that does not necessarily apply to Fantozzi, is the relief theory, attributed to Lord Shaftesbury (1801-1885) although revised by other philosophers such as Sigmund Freud (1856-1939) and Herbert Spencer (1820-1903). The relief theory suggests that comedy releases a build-up of nervous energy in the form of laughter. As Noel Carroll argues " $[w]$ hen we are asked a riddle or told a joke, it might be said, naturally enough, that expectation builds as we await the punchline. We are curious about how this comic discourse will end. When the punchline arrives, the pressure of those expectations is released and laughter ensues." ${ }^{51}$ In Fantozzi, there does not tend to be a build up to the jokes, the humour itself is visible on-screen at all times. In fact, the very episodic nature of the Fantozzi series, in which one film is made up of multiple short stories with a very loose overarching plot, means there is very little time to produce a build-up of such nervous energy.

In 1936, Max Eastman proposed the play theory, which works on the premise that humour involves 'playful relaxation'. In a sense, it is similar to the relief theory, where comic amusement results from everyday life. St Thomas Aquinas argues this point stating that "[ $t]$ hose words and deeds in which nothing is sought beyond the soul's pleasure are called pleasure and humorous, and it is necessary to make use of them at times for solace and soul. ${ }^{, 52}$ The ideas of play and humour do seem to go together (e.g. wordplay, to give an obvious example), however humour and play are not necessarily synonymous; many forms of play are not at all humorous in their nature for example chess, cricket or draughts could all be considered a form of play, yet none of these are particularly funny. The play theory can be used to explain particular cases of humour, but, as Carroll explains: "[s]urely the idea that comic amusement has some connection with play is highly plausible, given the frequent correlation of the two. But what that connection or those connections might be remains to be excavated." 53

Similarly, the dispositional theory, proposed by Jerrold Levinson in 1998, explains that something can be considered humorous that:

[h] as the disposition to elicit, through the mere cognition of it, and not for ulterior reasons, a certain kind of pleasurable reaction in appropriate subjects (that is, informationally, attitudinally and emotionally prepared subjects), and where, furthermore, this pleasurable reaction (amusement, mirth) is identified by its own disposition to induce, at moderate or higher degrees, a further phenomenon, namely laughter." 54

This theory plays on the idea that humour and laughter are inherently linked, in fact, that humour predisposes laughter. That being said, often people may find something funny, but that does not necessarily elicit laughter. Put very simply, the dispositional theory seems to state that something is

\footnotetext{
${ }^{51}$ Carroll, Humour: A Very Short Introduction, 39.

52 Ibid., 42.

53 Ibid., 43.

${ }^{54}$ Ibid., 44
} 
humorous because we laugh at it but, as we know, laughter can be dependent on a number of factors whether we are alone or in a group, for example, or other independent circumstances of the humoristic event including personal predisposition.

Another important theory of humour, proposed by Italian dramatist, novelist and poet, Luigi Pirandello and therefore very influential in Italian culture, is known as the "sentimento del contrario" or 'the feeling of the opposite'. The Fantozzi series undoubtedly encapsulates this, where what is portrayed should not necessarily come across as humoristic, but humour, in one of its many forms, is generated precisely by the opposite of what the audience would generally be expecting. Pirandello provides the humour example of an old lady who has had her hair dyed, her face completely made-up in an attempt to look like a young girl. He finds this humorous as it is the complete opposite to how an older lady is stereotypically perceived. Yet if you stop there, the humour is superficial. Pirandello highlights the fact that, upon reflection, perhaps the older lady does not wish to dress up in such a fashion, that it is perhaps external circumstances such the wish to hold on to the attention and love of her husband that define how she dresses and behaves. What initially comes across as comedy may indeed have a deeper meaning behind it, and on this reflection, the audience is subject to this sentimento del contrario, which is the true core of the humour and causes a superficial level of hilarity on the viewer's part. ${ }^{55}$

This is indeed the case with the Fantozzi series as well, where behind the slapstick knocks on the head, fingers being jammed in car doors or falling out of a boat, deeper issues are at play: Villaggio, and as a result, Salce, show exactly the unfairness that exists due to this new capitalist, consumerist, bureaucratic and classist society, which is at the origin of all of the character's mishaps. As Villaggio himself states:

[w]ith Fantozzi I tried to recount the adventures of those who live in that part of life through which all (except the children of the most powerful) pass or have passed: the moment in which one is under a boss. Many come out of it with honour, many spend twenty years there, others spend thirty, most remain there forever and they are the majority. Fantozzi is one of them. ${ }^{56}$

A clear example of the disparity between upper and lower classes in the first film is when Fantozzi tries to buy a coffee from a coffee machine, yet it will only dispense sugar. When the top-brass 'MegaDirettore' shows up, the machine dispenses a bottle of champagne and champagne flutes from a secret compartment. At first glance it is superficially funny: Fantozzi doesn't get his coffee due to an apparently broken machine, yet upon reflection it becomes a commentary on how difficult it was for

\footnotetext{
${ }^{55}$ Luigi Pirandello, L'umorismo (Milano: Garzanti, 1908). 56 Paolo Villaggio, Fantozzi (Milano: Rizzoli, 1971), 1. [My translation]

Original Italian: Con Fantozzi ho cercato di raccontare l'avventura di chi vive in quella sezione della vita attraverso la quale tutti (tranne i figli dei potentissimi) passano o sono passati: il momento in cui si è sotto padrone. Molti ne vengono fuori con onore, molti ci sono passati a vent'anni, altri a trenta, molti ci rimangono per sempre e sono la maggior parte. Fantozzi è uno di questi.
} 
the average Italian to live in the new corporate Italy, despite the dreams of the upward movement in society offered by the 'economic miracle'.

\subsection{Translating humour across borders}

Verbal humour travels badly. As it crosses geographic boundaries humour has to come to terms with linguistic and cultural elements which are often only typical of the source culture from which it was produced and thereby losing its power to amuse in the new location.

\section{Delia Chiaro ${ }^{57}$}

The translation of humour is by no means an easy task and has been the subject of many an academic study for many years. The fact that academic debate on the topic continues to this very day proves the challenging nature of the discipline. ${ }^{58}$ That being said, it is generally accepted among comedy experts that in order for humour to be successful, a certain level of cultural knowledge is required on the audience's part. Specific references, places, people or language are just a few examples of knowledge that is a key component to understanding humour. These factors make humour intrinsically cultural and difficult to represent in another linguistic system, let alone the fact that a great deal of humour is indeed dependent on language. As Benjamin Lee Whorf argues, something that is accessible and humorous to some cultures, may in fact be completely incomprehensible to others: "No two languages are ever sufficiently similar to be considered as representing the same social reality. The worlds in which different societies live are distinct worlds, not merely the same world with different labels attached." 59

An example of a successful comedy in one culture not faring well when taken abroad, is the hit American television sitcom The Big Bang Theory (2007- present). Giuseppe Balirano examines the Italian dubbed version of the sitcom. ${ }^{60}$ The humour that radiates from the television show is associated with verbally expressed 'nerd-culture' jokes and the inability of normal people to understand them, while similarly, the same nerds on the show struggle to comprehend the normal day to day lives of the rest of society. According to Balirano, the Italian dubbing "seems to entirely re-cast the "context of situation', thus eliminating some fundamental references to the specificity of the language of the ST, and consequently preventing the Italian audience from gaining a full appreciation of its humour." ${ }^{\prime}$ Because of this, the show had little success in an Italian context, notwithstanding Italy's long history

\footnotetext{
${ }^{57}$ Delia Chiaro, ed. Translation, Humour and Literature, vol. 1, Continuum Advances in Translation (London: Continuum International Publishing Group, 2010), 1.

${ }^{58}$ As I complete this thesis a multi-disciplinary publication on humour has just been released: Alleen Nilsen and Don Nilsen, The Language of Humor: An Introduction (Cambridge: Cambridge University Press, 2018).

${ }^{59}$ Benjamin Lee Whorf, "A Linguistic Consideration of Thinking in Primative Communities," in Language, Thought and Reality: Selected Writings of Benjamin Lee Whorf, ed. John Bissell Carroll (Massachusetts: MIT Press, 1956), 69.

${ }^{60}$ Giuseppe Balirano, "The strange case of The Big Bang Theory and its extra-ordinary Italian audiovisual translation: a multimodal corpus-based analysis," Perspectives: Studies in Translatology 21, no. 4 (2013).

${ }^{61}$ Ibid., 564.
} 
and experience of dubbing television programming, as Italian audiences could not access the specific humour of the ST, thus causing the programme to fall flat in the Italian market.

Another example of failure, often cited in literature on translating humour, occurs in Lawrence Kasdan's film The Big Chill (1983). One of the main characters, Sam, is asked by another character, Meg, to father her children. Sam responds with the line "You're giving me a headache," to which Meg responds "You're not gonna use that old excuse, are you? You've got genes!", to which Sam immediately looks down at his trousers. The wordplay on the English 'jeans' and 'genes' is crucial in understanding the joke, however this homophone does not exist in other languages. The translation was simply "Perché hai dei buoni geni!" The Italian audience, therefore, completely loses the double nuance of the language as there is no way of retaining the original English homophone in Italian. So when the actor looks down and touches the jeans he is wearing, the Italian audience would be confused as to what he is doing. Unfortunately, this loss is unavoidable, specifically because of the visual component; even if there was another play on words that could have been used in the TL, the reference could not be changed due to the images that appear on-screen. This is a big factor with subtitling humour. Because the images on-screen cannot be changed, the subtitler must establish some priorities: firstly, is the humour the primary purpose of the dialogue, and, secondly, will the plot be affected by a retention of humour? Once this has been determined the subtitler can put in place some strategies: where humour is a key component, equivalence will be necessary, but where content is more important, a literal translation may be more useful. It can, however, be argued that humour fares better in AVT than in other areas. While spatio-temporal pressures and image constrain a subtitler, the presence of both audial and written information can be of great help as humour "can work through the incongruous juxtaposition of images, for instance, or through the gestures and facial expressions of the speakers." ${ }^{62}$

The issue of humour not travelling well is relevant for programming within the same language as well: one only needs to look at the numerous British television programmes and films that have travelled to the United States, and vice-versa, with little success. For example television shows such as Blackadder (1983-1989) and I'm Alan Partridge (1997-2002) have been popular in the United Kingdom, but had comparatively little following in the United States. As Delia Chiaro explains, "[t]here is no denying that British humour on screen is based on the nation's fixation with class and is conventionally not averse to punning, unlike USA comedy which prefers to play on characterisation of the individual and the gag to punch." ${ }^{63}$

In some cases television programmes are even remade to cater for the new target audience. The Office is a relatively recent example of a British comedy that was completely recreated to cater to an American audience and their sense of humour, so much so that the original British (2001-2002), and

\footnotetext{
62 Jorge Díaz-Cintas and Aline Remael, Audiovisual Translation: Subtitling, Translation Practises Explained (New York: Routledge, 2007), 216.

${ }^{63}$ Delia Chiaro, "Verbally expressed humor and translation: an overview of a neglected field," International Journal of Humour Research 18, no. 2 (2005): 138.
} 
the recreated American version (2005-2013) can be considered two entirely different entities. As Chiaro emphasises:

[t]he intertwining of formal linguistic features and sociocultural elements in a joke is often so specific to a single language community that, beyond its frontiers, the joke is unlikely to succeed. This is why speakers of British English do not necessarily 'get' jokes told in American or Australian English and vice-versa. ${ }^{64}$

The translator of humour must therefore clearly be aware of social or cultural aspects of the text, but also a variety of other factors. In the case of Fantozzi, a film produced in 1970s Italy, we see many examples of politically incorrect humour that would not be acceptable in the film industry today (teasing a girl who looks like a monkey, xenophobic, racist, homophobic or sexist depictions etc.), as well as a forty year temporal displacement that may see some references be lost over time. Brigid Maher argues that both culture and historical displacement play important roles in translation:

[o]ne of the difficulties in writing about humour is defining the very concept. In everyday life, we cannot help but be aware of considerable variation among individuals as to what is considered funny, and what kinds of topics it is legitimate or appropriate to laugh at. This is so even within a single culture and language [...] [and] only becomes more and more evident when we examine humour and its reception in different cultures or, for that matter, historical periods. ${ }^{65}$

Patrick Zabalbeascoa provides a list of 10 'translatability variables' that can affect a translation in a more general sense. These variables are: 1) the languages and cultures one is translating from (including aspects of language variation like dialects and registers); 2) the languages and cultures one is translating into; 3 ) the purpose and justification for the existence of the translated text; 4) the nature of the text, including parameters such as textuality, genre, style and discourse; 5) the clients or translation initiators; 6) the expectations for the translated text and prejudices towards translations and translators; 7) the translators: are they human, machine, fully automatic or computer assisted; 8) the conditions in which the task is carried out, that is, the deadline, materials or motivation and 10) the medium, mode and means of communication: oral, written, audiovisual, private, mass media etc. All of these variables must be well thought out and justified before undertaking the translation. Perhaps the most important variable in the context of this project is number four, the nature of the text, in this case the comedic elements of the ST film, although all are important to bear in mind. ${ }^{66}$

Not only is humour cultural, but it can also take several forms: slapstick, visual, jokes, gesture, sound, puns and wordplay are just a few examples of the great variety present in the field of humour alone. The aim of translating humour should be to remain faithful to the plot, while also maintaining

\footnotetext{
${ }^{64}$ The Language of Jokes: Analysing Verbal Play (London: Routledge, 1992), 78.

${ }^{65}$ Brigid Maher, Recreation and Style: Translating humorous literature in Italian and English, John Benjamin's Translation Library (Amsterdam: John Benjamins Publishing Company, 2011), 2.

${ }^{66}$ Zabalbeascoa, "Humor and translation - an interdiscipline," 186-87.
} 
the humour in the TL. Humour serves several purposes in our daily lives: whether that be to create amusement, ease social tension, re-evaluate established ideas, challenge one's cognitive faculties, help sustain interest in a subject, avoid censorship or become more familiar with another culture or another language, humour plays an important role in personal and cultural understanding. ${ }^{67}$ Because of these factors, the translation of humour is an arduous challenge. In this case study, there are also the added complications of the means of translation, audio-visual, which will be discussed at length in chapter three. The first thing a translator must do is understand the comedic element in the original text, the next challenge is to find a way of representing that humour in another language, or reformulating it so it will have an equivalent effect on the target audience. Once these two steps have been undertaken, a translator gives themselves a better chance to translate the humour successfully.

\subsection{Making humour accessible}

Given the challenges associated with translating humour outlined above, we will now explore ways in which translations of humour can be undertaken by firstly looking at some of Zabalbeascoa's 'translational priorities' of humour, followed by some more general solutions, then finally an analysis of the different types of humour that are often present in AV material, as well as provide some strategies to deal with those challenges. These are just a few of the main types of humour that are commonly encountered in AVT. Given the enormity of the field, I will not be covering all aspects of humour, just the ones I believe are most relevant to the Fantozzi series. The techniques presented here are supported by Díaz-Cintas and Remael, who expand on Zabalbeascoa's classifications of AV humour. Zabalbeascoa proposes that there are seven different types of jokes that are used in dubbed television in particular, all equally relevant to subtitled content. Namely these are: language-dependent jokes, international or bi-national jokes, jokes reflecting a community's sense of humour, and visual and aural jokes, as well as complex jokes which are a combination of two or more of the other classifications. In this discussion I will explore each of these types of humour, and I will present examples from Benvenuti al Sud (2011) by Luca Miniero, which focuses on the divide between the North and South of Italy at a linguistic and cultural level. The humour of this film focuses primarily on the representation of language and stereotypes, in this case Southern dialect(s) and Standard Italian. This film is a contemporary example of how Italian comedy, in my opinion, has been successfully translated for an English-speaking audience. I have chosen to use this film as an example over one of Fantozzi's contemporary films given its similar comedic features to Fantozzi (namely the linguistic humour that centres around dialect and regional varieties, as well as aspects of wordplay), and the fact that it is a commercial example of an Italian film being translated into English where the translator has been able to draw solutions from the many years of subtitling trends in both theory and practice since Fantozzi's introduction into Italian

\footnotetext{
${ }^{67}$ Thorsten Schröter, "Language-play, Translation and Quality - with Examples from Dubbing and Subtitling," in Translation, Humour and The Media, ed. Delia Chiaro (London: Continuum International Publishing Group, 2010), 140-41.
} 
cinema. This will lay the foundations for my translation of the film, where a combination of the translation theory associated with translating humour will be combined with the techniques proposed to overcome the challenges of subtitling (which will be explored in the next chapter), to provide a reasoned and original translation of the film.

As far as translating humour is concerned, Zabalbeascoa bases his translations on three planes of 'translational priorities'. ${ }^{68} \mathrm{He}$ first suggests a 'vertical scale of importance' where a particular instance of humour can have top priority, low priority, or something in between. An example of top priority would be a comedy TV show, whereas a low priority instance would be a tragedy or horror context where humour is unnecessary. He then suggests a 'horizontal scale', whereby he determines whether or not the humour is relevant on a global scale or as a rhetorical device at a local level. Finally he proposes a 'scale of equivalence-non-equivalence', by which he determines whether the ST or the TT should have priority for the translation. This is a similar approach to that of many other scholars, including Lawrence Venuti, whose 'foreignization' or 'domestication' strategies are well known in the field of translation studies. ${ }^{69}$ As alluded to earlier, the genre can also effect the type of translation that is carried out. For example a classic film or a culturally sensitive film will need to be treated carefully by the translator, however one could expect a looser or free translation for a genre such as soap opera or comedy. Ironically, the translator is left with an interesting dilemma, as described by Delabastita: "the only way to be faithful to the original text (i.e. to its verbal playfulness) is paradoxically to be unfaithful to it (i.e. to its vocabulary and grammar)" ${ }^{70}$ In the case of this project, I would argue that on the vertical scale there is a high priority, on the horizontal scale, given the cultural content present, it is at a local level, and that the ST should take priority in an attempt to retain this exact content.

Zabalbeascoa also provides a set of very general solutions for a translator to follow when attempting to translate humour. Figure 13 shows a visual representation of his binary, where solution 1 involves a direct translation from source to TL (the joke is relevant to both languages and cultures), solution 2 is where the translator replaces the SL joke with another that functions in the TL (for example an aspect of wordplay is translated as a different type of wordplay), solution 3 substitutes the SL humour with a different type of humour in the TL (for example irony to satire), solution 4 is where a translator replaces an aspect of humour with another device (such as metaphor, simile or hyperbole), and finally solution 5 involves a direct translation where the aspect of humour is simply translated directly, even if the humour is not retained in the TL. ${ }^{71}$

\footnotetext{
${ }^{68}$ Patrick Zabalbeascoa, "Translating Jokes for Dubbed Television," The Translator 2, no. 2 (1996): 243-48.

${ }^{69}$ Lawrence Venuti, The Translator's Invisibility: A History of Translation, 2nd ed. (New York: Routledge, 2008).

${ }^{70}$ Dirk Delabastita, "Wordplay and Translation," Special Issue of The Translator 2, no. 2 (1996): 135.

${ }^{71}$ Zabalbeascoa, "Humor and translation - an interdiscipline," 201.
} 


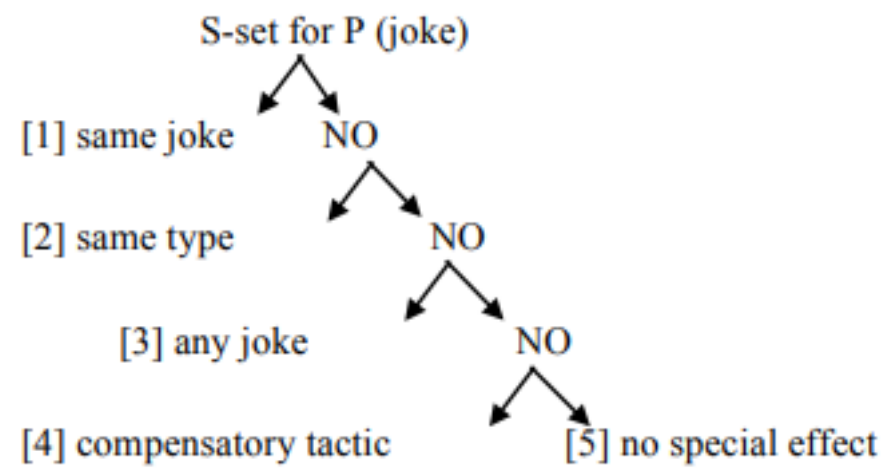

Figure 13: Zabalbeascoa's solutions (S) to translate problem (P)

On the last strategy proposed by Zabalbeascoa, it could be argued that a moral issue comes into play when translating humour: Is a faithful translation of the original required even if it doesn't capture the SL humour, or should the joke be replaced by a different one that is accessible to the TC and thus potentially changing the original content and adding or taking away certain connotations? It is a difficult balancing act for a translator: a joke in the SL may be untranslatable in the TL, yet an adaptation, in the case of subtitling, may clash with images on-screen. There are times when laughter is more important than plot, or vice-versa.

\subsubsection{Language-dependent jokes}

Also known as 'verbally expressed humour', language-dependent jokes challenge the translator in many ways. In fact, as Schröter explains, "not so long ago, the general view had been that language-play, or at least wordplay, the most illustrious subcategory of language-play, is impossible or virtually impossible to translate into another language." 72 These jokes include language specific references such as puns and wordplay, homophones, homonyms, homographs, paronyms, spoonerisms, oxymorons, palindromes and neologisms that may not feature, or have the ability to be replicated, in other languages. Chiaro defines wordplay in particular as "every conceivable way in which language is used with the intent to amuse." 73 When attempting to translate instances of language dependent jokes, the subtitler must ensure that the text still reads organically, and in order to do this may need to use a certain degree of creative license. A well-known example of pun, attributed to Groucho Marx, is the phrase "time flies like an arrow, fruit flies like a banana". This pun plays on the double meanings of the words "fly" as a verb and a noun, and "like" as a verb and a conjunction, which gives the phrase its humoristic properties. ${ }^{74}$ The literal translation into Italian of "Il tempo vola come una freccia, ai moscerini da frutta

\footnotetext{
${ }^{72}$ Schröter, "Language-play, Translation and Quality - with Examples from Dubbing and Subtitling," 141.

${ }^{73}$ Chiaro, The Language of Jokes: Analysing Verbal Play, 2.

${ }^{74}$ Schröter, "Language-play, Translation and Quality - with Examples from Dubbing and Subtitling," 139.
} 
piace una banana" has none of the ST double meaning and actually makes very little sense to the target Italian audience. There can rarely be a literal translation for this type of joke as humour will inevitably be lost in the transfer from ST to TT, as Schauffler argues:

[w] hen it comes to translation of wordplay, a central dilemma resides between the retention of clarity and credibility and the maintenance of formal fidelity to the source text, as well as the consequent prioritisation of the transfer of information on the one hand, and the preservation of comedic value by selecting the funniest option, even if this entails a move away from the original, on the other hand. Subtitled comedy films represent a genre in which these two priorities meet and indeed often clash. ${ }^{75}$

One further problem which burdens the English translator when working from a language such as Italian is the issue of formal and informal language. English does not have the same distinction as Italian ( $t u$ and Lei) therefore translating this meaning is nearly impossible. The translator must therefore use markers of informal or formal language such as 'please', 'sir' or ma'am', although these do not always solve the problem. As we will see in the upcoming pages, this is a particular problem that comes up in the translation of Fantozzi.

Chiaro claims that many translators in such instances opt for a substitution or compensation to account for this type of humour, particularly if the humour is key to the AV material. ${ }^{76}$ In other words, the humour component that does not exist (or make sense) in the TL is replaced by a similar one that does exist. This may cause a change in meaning from the original as a semantic shift occurs. However, provided they are used sparingly and do not detract from or change the content of the film, these substitutions are a widely accepted method. Another well regarded scholar in the field of audio-visual translation and translating humour, Henrik Gottlieb, proposes other methods of dealing with instances of wordplay in audiovisual material: $:^{77}$

- Render the wordplay verbatim, with or without the humorous effect

- Adapt the wordplay to the local setting and thus retain the humorous effect

- Replace with non-wordplay

- Not rendered, using the space for neighbouring subtitles

- Insert wordplay into different textual positions, where the TL renders it possible

In the case of puns, for example, Díaz Pérez argues that, "a pun in the source text can naturally be translated by means of a target text (TT) pun. The TT pun can be based on the same type of structural

\footnotetext{
${ }^{75}$ Svea Schauffler, Subtitling Wordplay - An Audience Study: Approaches to the Translation of Linguistic Humour (Saarbrücken: Scholars' Press, 2012), 8.

${ }^{76}$ Chiaro, "Verbally expressed humor and translation: an overview of a neglected field," 136.

${ }^{77}$ Henrik Gottlieb, "You Got the Picture? On the Polysemiotics of Subtitling Wordplay," in Traductio: Essays on Punning and Translation, ed. Dirk Delabastita (New York: Routledge, 1992), 210.
} 
relations as the source pun; it may or may not reproduce the formal structure of its original; and it can or cannot share the semantic organisation of the source text pun." ${ }^{78}$ Dirk Delabastita provides an alternative systematic approach, specifically for the translation of puns ${ }^{79}$ He provides eight different ways of dealing with a pun in the SL, all justifiable in their own right:

- Pun to pun: the ST pun is translated by an existing pun in the TL that is similar in terms of structure or function.

- Pun to non-pun: this strategy salvages the meaning of the dialogue, potentially at the expense of the humour.

- Pun to related rhetorical device: the pun is replaced by another form of wordplay (repetition, alliteration, rhyme, referential vagueness, irony, paradox etc.) which may be successful in capturing the initial humour from the wordplay.

- Pun to zero: the pun is simply omitted from the text.

- Pun ST = pun TT: no translation occurs, the translator transfers it across languages rather than translating.

- Non-pun to pun: the translator may introduce a pun where it otherwise did not exist in order to make up for the lack of translatability in other parts of the text.

- Zero to pun: new textual information is added which contains wordplay. This is used as a means of compensation as well.

- Editorial techniques: explanatory footnotes or endnotes are used to justify the translation, possible alternatives may be included.

Every now and then the subtitler will be fortunate in that a pun or wordplay can be translated literally, or be substituted by something very similar, and have no repercussions on the wordplay itself, or even the content of the wordplay. Let us look at the following English joke:

Where do cats go when they die?

To purrrgatory

The joke is a play on the noise a cat makes, purring. However if we look to translate this directly into Italian we find it impossible. The verb 'to purr' in Italian is fare le fusa, and the word for 'Purgatory' being Purgatorio, thus the wordplay would become lost in translation. However, if instead of translating the joke literally, we change the reference of the joke we can end up with a possible solution, where the joke still works due to the reference to gatto or 'cat', rather than to purring. In this situation the reference

\footnotetext{
${ }^{78}$ Francisco Javier Díaz Pérez, "Worldplay in film titles: Translating English puns into Spanish," Babel 54, no. 1 (2008): 39.

${ }^{79}$ Delabastita, "Wordplay and Translation."
} 
is displaced rather than substituted, but the joke still works from both a comedic, and a linguistic perspective, while still retaining the original reference.

Dove vanno i gatti quando muiono?

In purgattorio.

The Fantozzi series is riddled with such aspects of humour, that is, specific elements of wordplay. Notwithstanding the repeated misuse of language, another play on words is presented below, taken from Fantozzi va in pensione (1988):

\section{Fantozzi va in pensione (1988)}

- Le casalingue... Ugo ma c'è un errore di ortografia...

- Ehm, ma no scusami perché, perché è plurale, son più di una no? Cioè, singolare casalinga, plurale casalinghe!...gue!

- Ah certo!

- Eh poi scusami, è il film che fa per te adesso che mi sei diventata femminista, no? È il problema di due casalinghe... lingue...

It is a particularly memorable scene where Pina and Fantozzi mistake "casalingua" (the name of a pornography film that they subsequently watch, "casa" meaning "house" and "lingua" meaning "tongue") with "casalinghe", the plural of "housewife". A translation into English would prove difficult given that the words that make up the wordplay are distinct in each language. Perhaps finding a rhyme to a synonym of 'housewife' could be a starting point, substituting the wordplay for another. Although rather vulgar, homemaker' ('taker'), 'stay at home mum' ('slay') or simply 'lady of the house' do all offer some potential.

Another example of a language dependent joke, particularly in the case of Fantozzi, comes in the form of dialect and non-standard language. In her discussion of the translation of dialect, Manuela Perteghella presents a variety of translation methods for this niche market. ${ }^{80}$ She looked specifically at G.B Shaw's Pygmalion (1913), which was adapted to become Alan Jay Lerner's successful musical My Fair Lady (1956). The translation in question is from Cockney Rhyming slang into Italian. She proposes five different strategies for the "theatrical transposition" of dialect and slang, which she names dialectal compilation, pseudo-dialect translation, parallel dialect translation, dialect localisation and standardisation. ${ }^{81}$

Dialectal compilation is a technique that translates a single dialect into a mixture of target dialects. Retaining the dialectal element of the ST can prove problematic as regionalisms may only be accessible

\footnotetext{
${ }^{80}$ Manuela Perteghella, "Language and Politics on Stage: Strategies for Translating Dialect and Slang with References to Shaw's Pygmalion and Bond's Saved," Translation Review 64, no. 1 (2002): 50.

${ }^{81}$ Ibid.
} 
to a limited audience (those familiar with the translated dialect) and "a wide audience reaction can be hampered." 82

Pseudo-dialect translation is a similar technique to dialectal compilation, but instead of using pre-existing dialects, a fictitious dialect is created, while proper names and cultural references are retained. This allows for a greater promotion of the original text as it becomes accessible to a much larger audience, without being regionally specific or creating the 'feedback effect', a phenomenon where a bilingual audience is distracted by subtitles that do not read the same as the original content.

Parallel-dialect translation is when a dialect is translated into another dialect, which is geographically similar, or has connotations that are similar to the ST dialect. As with the previous technique, it is difficult for an audience to suspend their disbelief when using this strategy and the feedback effect may be unavoidable.

A dialect is localized to the target audience and often the characters' names, place names, settings, and cultural and topical references are changed to target source forms. This is described as 'domesticating', and a strategy that might "hamper reception on a national basis, especially in those countries in which regionalisms are stronger." ${ }^{\prime 3}$ On the other hand the standardisation technique substitutes slang, dialect and jargon into standard language. This is more suitable in scholarly work because domestic references are avoided. However, whenever this technique is used the characterisation weakens, and the colourfulness of the original is lost.

One example of translating language dependent humour in Benvenuti al Sud occurs when the main protagonist, Alberto Colombo, is in a restaurant with his colleagues (Figure 14). He, a Northerner, is slowly coming to grips with the Southern dialect of Castellabate. His colleagues explain to him some features of the local dialect, specifically the reduction in vowels and how those vowels become words in their own right. The humour is based on the language used and how it differs from standard Italian:

\section{Orignal Italian}

Comunque, non è difficile parlare come noi. Togliete l'ultima lettera a una parola ed è fatta.

Ad esempio, "telecomando" diventa

"telecomand"

-"bicchiere" diventa "bicchier"

- Se è così, è facilissimo

Piatto diventa "o piatt"

Forchetta diventa " a forchett"

Il coltello diventa " o coltell"

\section{English Subtitles:}

It's not hard to talk like us, just take off the last vowel.

For example, "banana"

becomes "bana-n"

-"Sofa" becomes "so-f"

- Then it's easy

Plate becomes "pla-t" this would be "for-k",

"kni-f".

${ }^{82}$ Ibid.

${ }^{83}$ Ibid., 51. 


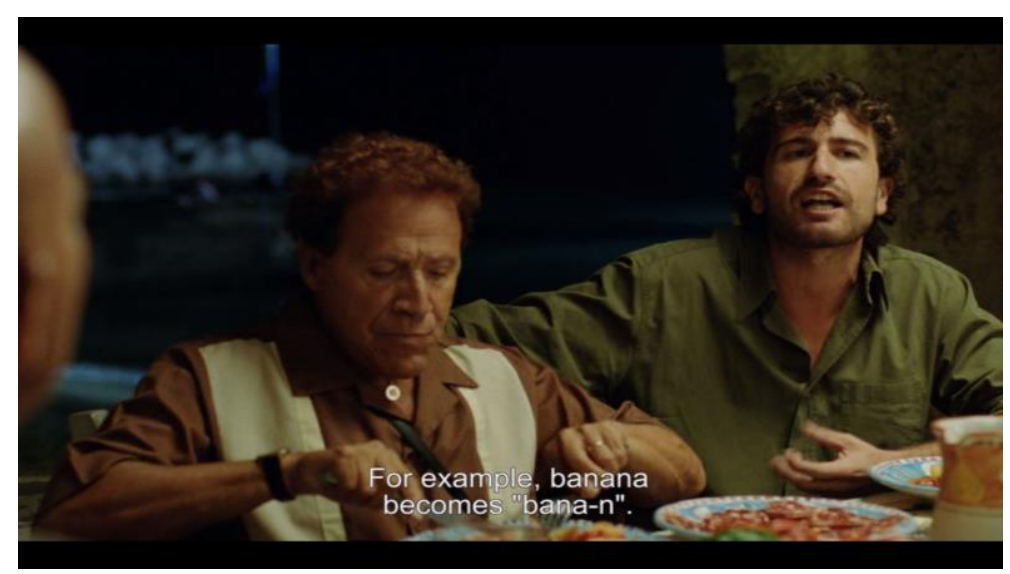

Figure 14: Example of language-dependent humour in Benvenuti al Sud

The English subtitler has used substitution in this case, replacing the references with a different ones in English. This was done as the dialogue would otherwise not make sense in an English context. The subtitler chose to translate "lettera" as "vowel" instead of the direct translation "letters". The subtitler has then had to use substitutions for the rest of the dialogue. The word English for "telecomando", "remote control", does not end with a vowel; in order to retain the humour it has been translated as "banan[a]". Similarly, "bicchiere", or "glass" in English, has been substituted with "sof[a]" to accommodate the idea that the last vowel of the word is removed in the dialect of Castellabate. Conversely, the reason why the last three examples of "piatto", "forchetta" and "coltello" were translated literally is because the images appeared on-screen as they are referenced, and had they been translated into something other than the objects that appeared on-screen, then that would have created an obvious credibility gap in the subtitles, something that a subtitler should avoid at all costs. The only continuity error with this substitution is the fact that "fork" does not end in a vowel and the in-text explanation does not account for this. Perhaps a better option from the translator would have been to translate the passage of dialogue directly, saying instead that one simply deletes the last letter from the end of the word.

\subsubsection{International or bi-national jokes}

An international or bi-national joke is something that is more likely to be humorous the world over given that the specific references are more accissuble. The same comedic effect can possibly be retained in the TL through direct translation or a calque ${ }^{84}$ Put simply, the target audience has significant knowledge of the referent for it to be retained in a direct translation. Examples of such a joke could be a reference to international film stars, famous tourist attractions, politicians, worldwide news headlines, bodily functions that are found to be funny across cultures, or ridiculous hyperbole as these are not

\footnotetext{
${ }^{84}$ Díaz-Cintas and Remael, Audiovisual Translation: Subtitling, 202.
} 
culturally specific references, and they can be accessed by a cross-cultural audience. A topical example, at the time of writing this, would be the joke:

\section{What is Donald Trump's favourite nation?}

\section{Discrimination.}

In the Italian translation, there is no need to change any reference, or any of the linguistic humour as both are accessible to, in this instance, an Italian audience. Donald Trump is a well-known figure across the world, and given English and Italian's shared Indo-European language heritage, both can convey the same pun. The translation would therefore be:

\section{Qual è la nazione preferita di Donald Trump? \\ La discriminazione.}

This type of humour is not particularly challenging for a subtitler as a direct translation is usually adequate, provided the space and time constraints allow for it. Given the constraints inherent to subtitling, there are times when the dialogue will have to be adapted or reformulated to retain the humour. This can lead to two different outcomes: there can be a loss in the strength of the joke, whether that be a reduction in hyperbole or exaggeration, or a more simplified manner of representation that takes away features of the humour such as word play, which can mean that the jokes may not have the desired impact. At other times the humour in the subtitles will need to become more explicit, and therefore less subtle, in order to pass on the literal message of the joke. It can be difficult to retain the humour in such limited space, so a joke may need to be effectively spelt out with nothing left to the imagination.

An example of an international joke from Benvenuti al Sud is where the stereotypical traits of Southern Italians are explained to Alberto Colombo by a fellow Northerner, one of the most prominent being the reference to the Camorra (Figure 15). The subtitler chose a direct translation strategy. The humour is based on the absurdity of the statements that are being made. For example, the reference to the Camorra is retained because it is well known worldwide, meaning that a literal translation and transference of the original is an acceptable strategy. Here, the subtitler also changed the word-class from an adjective to a noun ("camorristi" to "Camorra") which makes both the humour and reference more explicit to an English speaking audience (while also saving space as will be discussed in the next chapter). 


\section{Original Italian:}

Tutti terroni. Capito?

Anche gli animali: uei i gatti sono terroni, i cani sono terroni.

Le vacche, I pollastri, I vitelli.

Tutti sono terroni.

E parlano solamente il terùn. Capito. Tu non capisci niente.

Quando credi di aver capito qualcosa

Capisci che ti stanno prendendo pe' i ciapp' capito?

Perché davanti sono tutti gentili. Buoni.

Come dicono loro "l'ospitalità'...

'Pito sono palle.

Sono tutti camorristi e basta.

\section{English Subtitles:}

they're all shitkickers.

Even the animals: cats, dogs,

cows, chickens, calves, all of them!

They only talk shitkick

and you can't understand a word.

When you think you understand, you know they are fucking with you.

They're nice to your face,

"hospitality"

but they're all in the Camorra.

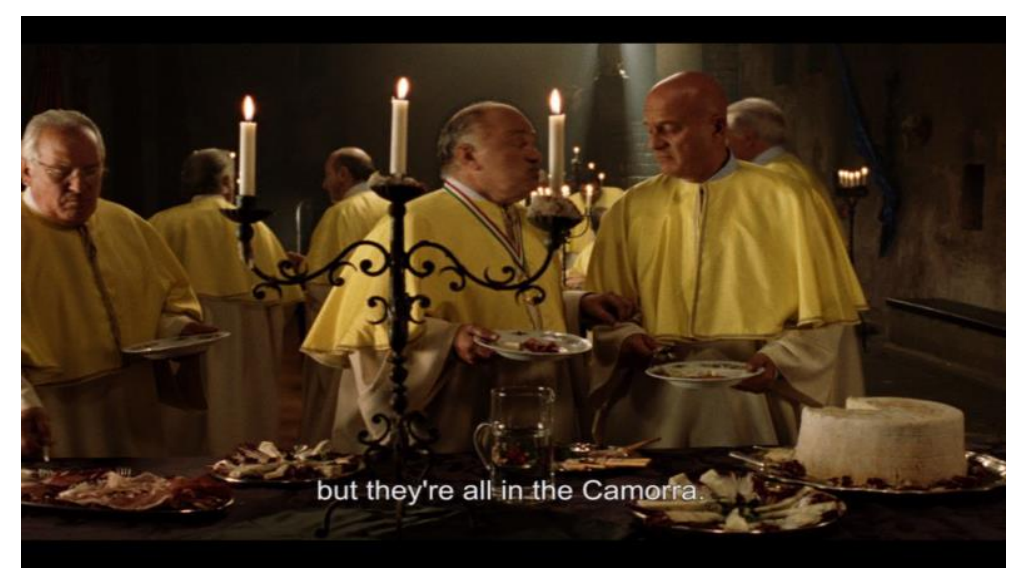

Figure 15: Example of an International joke from Benvenuti al Sud

\subsubsection{Cultural jokes: particular senses of humour}

As highlighted earlier, humour can be engrained in a culture, and different cultures can have particular senses of humour. A lot of these types of jokes come about at the expense of minorities in a specific community, as they poke fun at other nationalities. These jokes partially rely on intertextuality, that is to say, in order to understand the joke, the TL audience must be familiar with the original reference whether it has to do with particular religious beliefs, historical events or prejudices between different communities. This type of humour makes up a large part of the comedy of Fantozzi, not only in the first film but across the series. As I have previously discussed, the Germans and Japanese in particular are targets of the jokes and comedy during the film. Several strategies can be adopted in this instance. A direct translation requires a certain level of knowledge on the audience's part; alternatively an 
adaptation or substitution of the TT may also be used, although this means that the initial ST cultural connotations are lost. An example of a joke which would be fairly specific to a New Zealand audience would be the following:

\section{Four Australians walked into a bar.}

The other one ducked.

The joke's humour is based on the rivalry between New Zealand and Australia and the arrogant assumption that Kiwis are better than their Trans-Tasman rivals. While other countries may find it entertaining due to these connotations, they may not fully understand the cultural implications at play, hence it is a cultural-specific comedic reference. In saying this, the joke could be easily changed to retain those connotations for different cultures. For example the English could say the same about the French, the Germans about the Dutch, or the Americans about Mexicans or Canadians. It is important to note that these jokes rely on cultural prejudice to work from a comedic perspective, and are not necessarily told with racist or xenophobic intentions. This should always be kept in mind when translating this type of humour. In some instances the racism, however, can be more evident, and who the racism is aimed toward tends to be reflective of who has historically held the position of power.

In the case of the joke above, a translation into Italian would be very difficult. The verb 'to walk into a place' and 'to walk into something' are different in Italian (entrare vs. andare adosso a qualcosa), and the word for bar as in 'pub' is different from the one for the metal object (bar vs. barra). Therefore, the reference would need to be replaced, or substituted, by something different. In the following example, the reference to a bar/pub is retained, while the joke is still poking fun an another culture, although completely unrelated to the original joke:

Due tedeschi vanno al bar e dicono: "due Martini, prego."

Il barista dice: "Dry?"

I tedeschi rispondono: "No, zwei."

Of course, humour can also be used to poke fun at those in power: continuing with the Donald Trump theme, the Libertarian presidential nominee, Gary Johnson, made the quip: "I hear that Donald Trump is watching the Olympics tonight. He's seeing how high the Mexican pole vaulters go" in reference to Trump's plan to build a wall to separate Mexico and America, and using Trump's xenophobic attitude as the source of humour. ${ }^{85}$ Such a joke, as mentioned on the previous page, is relatively easy to translate given the reference is accessible world-wide:

\footnotetext{
${ }^{85}$ Rebecca Savransky, "Gary Johnson: Trump watching Olympics to see how high Mexican pole vaulters go," The Hill, http://thehill.com/blogs/ballot-box/presidential-races/290625-gary-johnson-trump-watching-olympics-to-see-how-high. accessed 29th January, 2018.
} 
Ho sentito che Donald Trump ha guardato gli Olimpiadi stasera.

Vedeva quanto in alto sanno saltare i saltati con l'asta messicani.

In Benvenuti al Sud the word "terrone" (a term used to refer to southern Italians) is used. While it is a racist term, its comedic purpose is to make fun of the speaker's prejudice, rather than to express any illfeeling towards the Southerners. The humour here is also visual as the Northern characters are dressed in $\mathrm{Ku}$ Klux Klan-esque attire, while using racist terminology (Figure 16). The subtitler chose an adaptation strategy, but removed all of the connotations associated with the word by going for the fairly neutral "shitkicker". A more comparable expression could be the 'n-word' in English, although it is understandable why the subtitler would decide against that option given its implications. However, in translating it this way, the subtitler toned down the racism present in the ST.

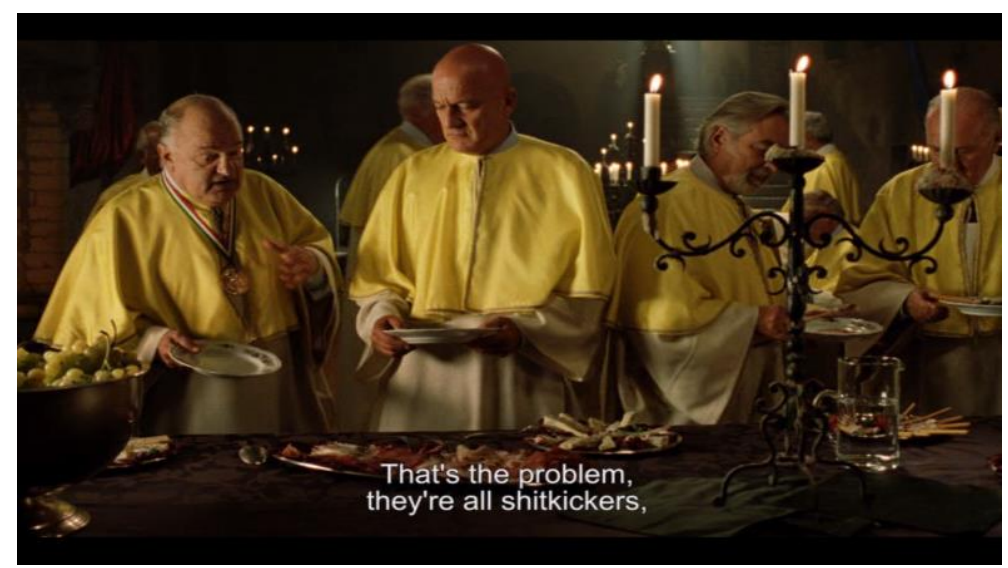

Figure 16: Example of a cultural joke in Benvenuti al Sud

\subsubsection{Visual and aural jokes}

As well as through language, comedy can also be conveyed visually. A visual joke is one which occurs out of the information conveyed on screen. This is usually portrayed through gesture, facial expressions or actions presented by characters on screen rather than the actual content of their dialogue. Similarly, an aural joke is one that is signalled by sounds (other than the dialogue itself). Despite cultural differences, visual and aural jokes are usually accessible to most audiences as a lot of gestures are considered universal, at least within Western cultures. Because the humour does not rely on dialogue, no translation needs to be made, although in the case of captioning sounds related to the image may need to be rendered. ${ }^{86}$

An interesting example from Benvenuti al Sud to show this type of humour is when Alberto Colombo is trying to get a transfer to the desirable Milan posting and fakes a disability in order to be

\footnotetext{
${ }^{86}$ Díaz-Cintas and Remael, Audiovisual Translation: Subtitling, 227.
} 
transferred there (Figure 17). The comedy has nothing to do with dialogue; it revolves around the character's demeanour, his gesture, his actions and the sounds he makes. While not particularly politically correct, the aim of the scene is to not insult disabled people (although it could be argued that the comedic effect lies, in part, on the stereotypical notions of physical disability), but to portray Alberto Colombo's sense of opportunism and ultimate stupidity, particularly when he stands up at the end of the interview with his superior, revealing his plan. For a subtitler in this and similar cases, no intervention is required leaving the interpretation of the humour to the audience. As we have already seen, this type of humour is prevalent in Fantozzi due to its slapstick and at times immature nature, particularly the very low-brow humour of bodily functions.

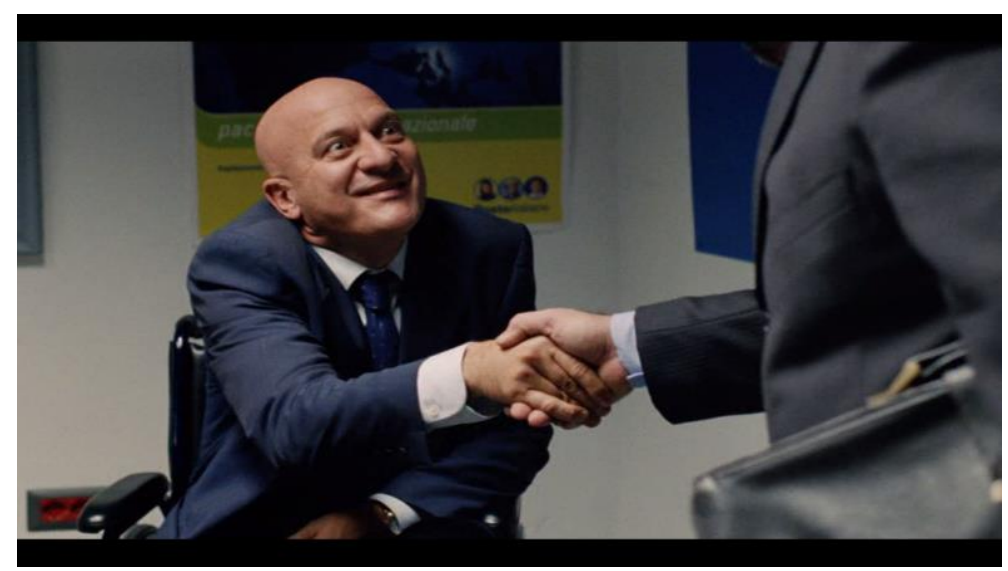

Figure 17: Example of a visual joke in Benvenuti al Sud

\subsection{Conclusion}

In this chapter, I have shown that there is great diversity within the field of translating humour, which makes the translation of Fantozzi a very interesting undertaking. After outlining a number of common theories of humour, we looked at four of the most common types of humour that exist in AV material: language-dependent jokes, international jokes, culturally specific jokes and visual and aural jokes. Each different type of humour needs to be negotiated on a case-by-case basis, and often there is more than one strategy that can be put in place to convey the original humour into the TL. A variety of techniques have been exemplified and illustrated through examples from the hugely successful Italian film Benvenuti al Sud, helping to shed light on the diversity of humour that can exist within one film alone, and subsequently highlighting the difficult nature of translating humour in an AV context that belongs to the same cultural context as Fantozzi. The techniques proposed have undoubtedly served me well in undertaking the translation, although not all of the strategies will need to be implemented. It is, however, necessary to delve into them all in order to give myself the best opportunity to translate this challenging content successfully. 
The next chapter will examine the more technical and practical aspects of AVT, starting with a brief introduction to its four different, yet related forms: subtitling, dubbing, voice over and audio description, followed by a more detailed analysis of the development of subtitling, and the practical difficulties that exist when attempting to translate audiovisual material. This will be followed by the full translation and creation of subtitles in English for the first film of the Fantozzi series, Fantozzi, which will draw on the theoretical discussion just presented on translating humour, as well as the upcoming discussion on AVT. 


\section{Subtitling as inter-semiotic translation}

Subtitling is an amphibian: it flows with the current of speech, defining the pace of reception; it jumps at regular intervals, allowing a new text chunk to be read; and flying over the audiovisual landscape, it does not mingle with the human voices of that landscape: instead it provides the audience with a birds-eye view of the scenery. ${ }^{87}$

Henrik Gottlieb

This chapter will outline some of the key developments of subtitling, firstly by looking at the discipline of AVT in general (not just subtitling, but dubbing, voice-over and audio-description as well), then moving on to the more practical challenges of subtitling in particular. The analysis of the literature on this subject will allow us to discuss the practical and ethical responsibilities of the subtitler (the role they should play in translation), the history of subtitling and how the technology associated with subtitling has developed, and current trends and future prospects of subtitling. I will also discuss how subtitles are perceived by the audience, including an attempt to debunk one of the main misconceptions associated with watching subtitled content - that the viewing experience is hindered by the need to watch the content while simultaneously reading subtitles. I will also highlight the different field-specific challenges faced by a subtitler, how the subtitler may overcome these difficulties with different techniques proposed by leading scholars in the field of subtitling, the conventions that exist within the practice (specifically the punctuation and grammar of subtitling and how this differs from traditional written texts), as well as the placement and aesthetics of subtitles on-screen such as the number of lines that should be used, the colour of subtitles, and particular fonts that should be used.

\subsection{Audiovisual Translation}

AVT, also referred to as 'constrained translation', 'subordinate translation', 'film translation', 'cinema translation' and 'screen translation', ${ }^{88}$ is a fast growing area of research within translation studies that was, for years, neglected by both the translation industry and by academics within translation studies. ${ }^{89}$ While this statement may seem bold, one needs only look at some of the poor quality of subtitles that have been produced commercially for films, broadcasts or television programmes that are riddled with grammar, spelling, punctuation errors or even mistranslations. ${ }^{90}$

\footnotetext{
${ }^{87}$ Henrik Gottlieb, "Subtitling: Diagonal Translation," Perspectives: Studies in Translatology 2, no. 1 (1994): 101.

${ }^{88}$ Sara Corrizzato, Spike Lee's Bamboozled: A Contrastive Analysis of Compliments and Insults from English into Italian (Cambridge: Cambridge Scholars Publishing, 2015), 39.

${ }^{89}$ Yves Gambier states that it was not until 1995 that AVT began to flourish due to a variety of reasons including technological developments, language policy and awareness, an increase in publications and a consistent number of seminars/conferences in the area. Yves Gambier, "Introduction. Screen Transadaptation: Perception and Reception," The Translator 9, no. 2 (2003): 171.

${ }^{90}$ See Wang Kaihao, "Poor subtitles mar prospects of Chinese cinema abroad," China Daily, 24 March 2016; Sarah Wescott, "When words fail BBC's subtitles: Ironic mess up on Breakfast show," in Express (London: Express, 2015); Hansjörg Bittner, "The Quality of Translation in Subtitling " trans-kom 4, no. 1 (2011); Szu-Yu Kuo, "Quality in Subtitling: Theory
} 
AVT is comprised of a variety of practices, namely subtitling (both inter-lingual and intra-lingual), dubbing, voice-over and audio description. This discussion will focus primarily on the field of subtitling as a means of inter-semiotic translation. Some features are shared by all of these practises that are unique to AVT as a whole, which is why it is important to discuss them together. The main feature that connects them is that there are always two codes present in the content: sound and image. The translator, caption producer, voice-over artist or audio describer is confined by these parameters, which can severely affect the final product.

A prime example of this is highlighted by Zoe De Linde and Neil Kay, who cite a particular example of closed captioning (a form of intra-lingual AVT) in the chat show Oprah, where "the meaning of an utterance is subtly altered by the removal of a few cohesive elements": ${ }^{91}$

Dialogue: It's what I call the vicious cycle syndrome. You start with drug A and then they put you on drug B, and drug $\mathrm{C}$, and pretty soon you are taking a handful of pills, all because of the first drug.

Subtitle: It's a vicious cycle. You start with drug A, then drug B, then soon you are taking a handful of drugs.

By removing "they put you on" from the caption, De Linde and Kay argue that the meaning of the sentence changes from the fact that drugs are prescribed to a patient, to placing the responsibility directly on the patient, as it implies an addiction. By omitting part of the original content, the meaning is significantly altered and a negative connotation is added to the text. A discussion around options the subtitler has, particularly given the time and space constraints, will be presented in the upcoming pages.

Although a rather convoluted and over-complexdiagram, Gambier and Di Giovanni break AVT up into four sub-categories worthy of research: language policy, descriptive studies, accessibility and applied research (Figure 18). As the diagram below shows, Gambier and Di Giovanni define specific results to these distinct types of research within the field: 1) "products" refers to research undertaken based on the problems or constraints of the field, in other words the content, that is humour, swearwords, discourse markers, language register, cultural items or the technical problems such as time and space, oral to written etc.; 2) "process" focuses on translation work in terms of strategies, norms, conventions, technical constraints, from script writing to dialogue, interplay visual/verbal/sound etc.; 3) "technology" research focuses on how the AVT landscape have changed in regards to automation and quality, working conditions, fees, ethics, copyright, as well as how technologies have developed and how production, distribution and broadcasting has changed; and 4) "effects" refers to studies on reading

and Professional Reality" (Imperial College London, 2014); Thorsten Schröter, "Quantity and quality in screen translation," Perspectives: Studies in Translatology 11, no. 2 (2003).

${ }^{91}$ Zoe De Linde and Neil Kay, "Processing Subtitles and Film Images," The Translator 5, no. 1 (1999): 50. 
skills, reading habits and language learning of the audience. ${ }^{92}$ My analysis focuses mainly on descriptive studies by looking at content-specific restrictions as well as solutions to overcome them; however the other categories are referred to at various points throughout the discussion.

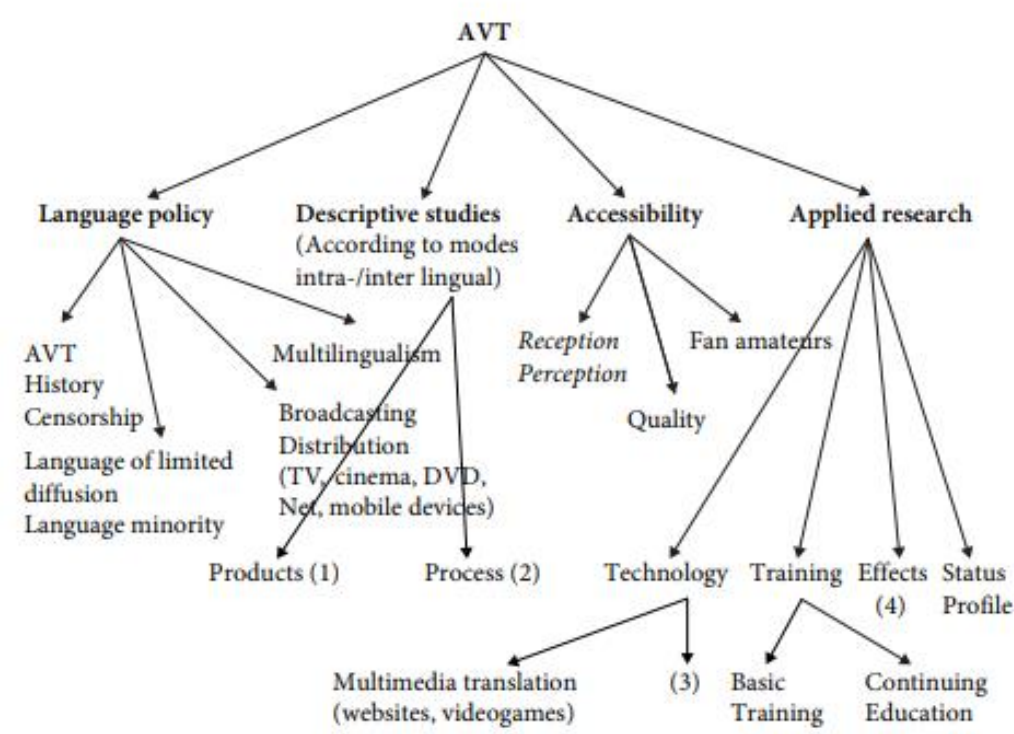

Figure 18: Gambier and Di Giovanni's model for audiovisual translation research

\subsubsection{Subtitling (inter and intra-lingual)}

Subtitling is gaining traction and credibility within academic studies in translation, albeit it is a relatively recent phenomenon. In this form of AVT spoken language becomes textualized and, depending on the function of the subtitles, audio-elements outside of spoken dialogue can become represented as well as in the case of captioning/intra-lingual subtitling, particularly for a deaf and hard of hearing audience. Subtitles may be defined as "a written text, generally on the lower part of the screen, that endeavours to recount the original dialogue of the speakers, as well as the discursive elements that appear in the image, and the information that is contained on the soundtrack." 93 While this definition is acceptable to the layman, Henrik Gottlieb proposes a much more comprehensive definition, emphasising how subtitling differentiates itself from other forms of translation; he defines subtitling as "[p]repared communication using written language acting as an additive and synchronous semiotic channel, and as part of a transient and polysemiotic text." ${ }^{94}$ Another definition, proposed by the rather controversial Urban Dictionary, provides a rather different and humorous

\footnotetext{
92 Elena Di Giovanni and Yves Gambier, eds., Reception Studies and Audiovisual Translation, Benjamins Translation Library (Amsterdam: John Benjamins Publishing Company, 2018), IX.

${ }^{93}$ Díaz-Cintas and Remael, Audiovisual Translation: Subtitling, 8.

${ }^{94}$ Henrik Gottlieb, Screen Translation: Six Studies in Subtitling, Dubbing and Voice-over (Cph. : Centre for Translation Studies, Department of English, University of Copenhagen, 2001), 15. [emphasis in original text]
} 
interpretation, labelling them as "a firewall that keeps stupid and impatient people from watching foreign films." 95

There are three main components to this unique form of inter-semiotic translation: dialogue, image and text, all of which must work simultaneously on-screen. Subtitling is therefore a form of intersemiotic translation, as defined by Roman Jakobson, which is not simply a translation of ST to TT, but also a translation across sign systems. In the case of subtitling, this shift happens from the visual and audial to the written form, as explained by Carol O'Sullivan:

[s]ubtitling is 'diagonal translation': not merely between languages, but also as a translation of the oral into the written. Subtitling selects, condenses and organises discourse into discrete syntactically, spatially and temporally delimited units. ${ }^{96}$

These three components - dialogue, image and text - must work together, in conjunction with the audience's ability to read and process information, in order to be successful in conveying the appropriate content. Herein lies the problem: subtitling is exposed to several external factors that other forms of translation are not, such as the spatio-temporal constraints of the AV medium, the need to cater to the reading rates of viewers, as well as content specific restrictions (these limitations will be discussed in the following sections). Moreover, the fact that SL and TL co-exist within the same environment leaves the subtitles vulnerable to criticism. From a technical perspective these issues can plague a subtitler and make subtitling a laborious task. In fact, as Abé Mark Nornes quite rightly remarks, "nothing is simple when it comes to subtitles; every turn of phrase, every punctuation mark, every decision the translator makes holds implications for the viewing experience for foreign spectators". ${ }^{97}$

Despite all this, subtitling serves a variety of important functions: it allows diverse cultures and communities to access materials that were previously unreachable (this is particularly important for the deaf and hard of hearing audiences); many studies have proven that watching subtitled films can be a useful means of language learning, particularly for children; other studies have proven that writing subtitles has also benefited second language acquisition. Although these are not necessarily the primary purposes of subtitling, they are undoubtedly positive and important by-products of the field.

However, subtitling has often been dismissed within Translation Studies where it has tended to be labelled as a recreation or an adaptation of the original text, rather than translation in the more traditional sense. ${ }^{98}$ The implication is that in transferring the message across languages and cultures the original content must be heavily adapted for the target audience due to the complex nature of subtitling itself. As Jorge Díaz-Cintas and Aline Remael explain,

\footnotetext{
95 "Texts, Translation and Subtitling - In Theory, and in Denmark," Screen Translation (2001): 165.

${ }^{96}$ Carol O'Sullivan, Translating Popular Film (Basingstoke: Palgrave MacMillan, 2011), 102.

${ }^{97}$ Mark Abé Nornes, "For an Abusive Subtitling," Film Quarterly 52, no. 3 (1999): 17.

${ }^{98}$ Although this is not necessarily a bad thing - in Schauffler's study on the reception of humour from two different forms of subtitles, an audience targeted (labelled 'skopos-oriented' in the study) text was received better by the viewers than a text prioritizing 'formal equivalence': Schauffler, Subtitling Wordplay - An Audience Study: Approaches to the Translation of Linguistic Humour, 217.
} 
[f]or some, this activity falls short of being a translation proper because of all the spatial and temporal limitations imposed by the medium itself which constrain the end result. They prefer to talk about adaptation - an attitude that has stymied debate about AVT and could be tainted as having been one of the main reasons why the whole area has been traditionally ignored by scholars in translation until very recently. ${ }^{99}$

Indeed, the scholar Markus Nornes draws attention to the corruptness of a subtitler in their acceptance of "a vision of translation that violently appropriates the source text, and in the process of converting speech into writing within the time and space limits of the subtitle", by doing so, Nornes argues that subtitlers "conform the original to the rules, regulations, idioms and frame of reference of the TL and its culture." $" 100$

These negative opinions towards subtitling are based not only on the technical constraints of the field (predominantly the spatio-temporal constraints, where part of the ST must often be left out, adapted, or substituted in order to provide a solution), but also on poor quality of some subtitles produced since their inception into film, where they have often been perceived as a distraction, rather than being helpful to the viewer. This is particularly the case when incorrect grammar or punctuation is used, or if the subtitles do not read fluently, rendering the subtitles 'abusive' both in terms of the translation itself and their imposing nature on-screen . ${ }^{101}$ Jorge Díaz-Cintas summarises this issue accurately:

[f]or a long time now there has been growing concern among many professionals about the relatively low levels of quality that can be found in some subtitled programs. Although it is clear that subjectivity can play a big role in identifying what is wrong or of low quality in subtitling, it is my opinion undeniable that quality standards in subtitling have seen a sharp decline in recent years. ${ }^{102}$

Díaz-Cintas also explores some other contributing factors as to why, at times, the quality in subtitles has left a lot to be desired. He highlights the spike in demand for subtitles as a key issue, stating that this demand has led to translators who are not trained in AVT practise undertaking the work. Poor working conditions could also be a contributing factor, as well as a lack of comprehensive and standardized guidelines when it comes to AVT. As well as poor quality translations, poor subtitles often present incorrect spotting (or timing), wrong choice of fonts, or other issues around their level of readability.

As far as translation itself is concerned, it is often impossible to find a perfect lexical equivalent, or an exact way of saying what appears in the ST, as Mona Baker reflects:

\footnotetext{
${ }^{99}$ Díaz-Cintas and Remael, Audiovisual Translation: Subtitling, 9.

${ }^{100}$ Mark Abé Nornes, Cinema Babel: Translating Global Cinema (Minneapolis: University of Minnesota Press, 2007$), 155$.

${ }^{101}$ Bittner, "The Quality of Translation in Subtitling "; Kuo, "Quality in Subtitling: Theory and Professional Reality."; Schröter, "Quantity and quality in screen translation."

102 Jorge Díaz-Cintas, "Back to the Future in Subtitling" (paper presented at the MuTra Conference: Challenges of Multidimensional Translation, Saarbrücken, 2-6 May 2005), 4.
} 
[t]here is no shortage of discussions on the shortcomings and failures of translation as a tool of language mediation across cultures. The literature abounds with theoretical arguments which suggest that translation is an impossible task, that it is doomed to failure because (a) languages are never sufficiently similar to express the same realities and (b) even worse, 'reality' cannot be assumed to exist independently of language. ${ }^{103}$

This is all the more pertinent to subtitling, where the inescapable restrictions constrain and effect a subtitlers translation decisions. That being said, subtitles allow for the consumption of material across cultures and languages, and do so based on the original language content. Indeed, given the amount of time, energy and work that goes into making a film or television series, it is not just in the translators' best interest to render the original content adequately, but it is also in the best interest of all those who had an input on the creation of the original content.

In the case of subtitles in particular, on top of the fact that there is very rarely full equivalence across languages, content also needs to be re-worked or adapted. Subtitles are often not, and probably should never be, considered a perfect representation of the original spoken dialogue. The notion that what they are reading is exactly the same as what the characters are saying is a common misconception with film-goers. All one needs to do is watch a film or television programme in their own language and with the captions or subtitles on to find that even when the SL and TL are the same, there will be a difference between the two texts: often subtitles need to be reduced in order to adhere to the AV format and the restrictions of time and space, while negotiating particular cultural references that may be difficult to render across languages and cultures, as is the case in other forms of translation. As is summarised by Łukasz Bogucki, "[s]ubtitling, as a cross-medium activity (spoken to written) is much more complex, therefore translational loss is practically an occupational hazard." ${ }^{104}$ Díaz-Cintas and Remael also argue, "[t]he one to one translation approach loses all validity in our field and the concept of formal equivalence must be understood from a much more flexible perspective than in other spheres of translation." 105 Yves Gambier even argues that concepts in Translation Studies should be "revised, extended and rethought" when applied to audiovisual translation. These are the concept of text (given that screen texts are short lived and multimodal), the concept of authorship (the fact that AVT can involve an individual, group, or institution), the concept of sense (since in AVT sense is produced neither in a linear sequence nor with a single system of signs), translation (the very idea of translations versus adaptation/manipulation/transfer or remake/rewriting), translation strategy (does subtitling necessarily and systematically imply foreignizing given that the SL and TL are both present?), norms (the necessity to look at the links between translation norms and technical constraints), and the relationship between written and oral (that is to say, the sociolinguistic role of the subtitler). ${ }^{106}$

\footnotetext{
${ }^{103}$ Mona Baker, In Other Words: A Coursebook on Translation (Oxon: Routledge, 2018), 8.

${ }^{104}$ Łukasz Bogucki, "The Constraint of Relevance in Subtitling," The Journal of Specialised Translation, no. 1 (2004): 72.

105 Díaz-Cintas and Remael, Audiovisual Translation: Subtitling, 11.

${ }^{106}$ Gambier, "Challenges in research on audiovisual translation," 19-20.
} 
Subtitling can happen at an inter-lingual level, where a translation occurs from one SL to a TL, or at an intra-lingual level, where all elements of sound from the ST are portrayed in a written form of the same language. Intra-lingual subtitles involve a shift from oral to written communication within one language. This is why some scholars are reluctant to label this practice as translation, as there is no transfer across languages, simply across mediums. ${ }^{107}$ It could be argued, however, that it is a form of inter-semiotic translation as envisaged by Roman Jakobson, as it shifts communication from oral to written form. By undergoing this shift, the syntax and register will often need to be modified to compensate with change in media, given that reading a text and listening to a dialogue are processed at different speeds in the human brain. This form of subtitle is becoming more and more important in contemporary society given the growing importance and emphasis on accessibility. Inter-lingual subtitles, on the other hand, are translations from a SL to a TL, in other words they are a more traditional form of cross-cultural translation. There is also a third type of subtitle, known as bilingual titles, where the SL is translated into two TLs, both of which appear simultaneously on-screen. These tend to be used at international film festivals, where the 'home' language is presented alongside the translated language.

According to Díaz-Cintas and Remael, intra-lingual subtitles, also known as 'closed captions', are used to serve four distinct purposes: for the deaf and hard of hearing, for language learning, for karaoke effect and for expressing dialects, accents and regional variations within the same language. ${ }^{108}$

Subtitles for the deaf and hard of hearing are often available on television via teletext, although in more recent times subtitle tracks for the deaf and the hard of hearing have been a feature of DVDs across many countries. These subtitles, or more accurately, captions, can often change colour depending on the character speaking in order to offer verbal queues, like accent and pitch, which are easily traceable to a hearing audience, but are not accessible to a non-hearing audience. They include all information that is overt in the soundtrack. As Díaz-Cintas explains, intra-lingual subtitles are:

achieved by changing the actors' dialogue into written speech which also incorporates, among other things, all the paratextual information that contributes to the development of the plot or the creation of atmosphere that the deaf and hard-of-hearing are unable to access through the soundtrack, e.g. a telephone ringing, the knock on a door or a car revving. ${ }^{109}$

This practise has grown rapidly worldwide: captions are becoming readily available on national television stations all around the globe. As of 2014, the BBC (British Broadcasting Corporation) was one of the world leaders for providing accessibility to deaf and hard of hearing audiences. Since 2008, all of their broadcasts have had subtitles for the deaf and hard of hearing. In Canada, the Global Television Network has subtitled their programmes 24 hours a day, 7 days a week since January 2005, and in Spain the public station TVE increased the availability of subtitled programming by $73 \%$ in

\footnotetext{
${ }^{107}$ Díaz-Cintas and Remael, Audiovisual Translation: Subtitling, 14.

108 Ibid., 13-14.

${ }^{109}$ Díaz-Cintas, "Back to the Future in Subtitling," 4.
} 
regard to 2002-2003. ${ }^{110}$ While this trend is promising, there is still a long way to go to ensure the deaf and hard of hearing have access to all forms of AV material. An interesting, and unfortunate, by-product of the preference for dubbing over subtitling in countries such as Spain, France, Germany and Italy has been the lack of availability of content that the deaf and the hard of hearing have to AV material produced overseas. By contrast, in other countries that have a history of subtitling, the deaf and hard of hearing communities have had greater access to foreign content. ${ }^{111}$ This situation is fortunately changing, particularly since the introduction of the DVD, where multiple subtitle tracks, and multiple dubbed versions for that matter, can be included in the same package. Indeed, Díaz-Cintas reminds us that:

[t]he growing pre-eminence of the audiovisual media in our society has been clearly visible in the proliferation of television stations, the academic interest in film and television studies, the arrival of the DVD, and the potential of digitising the image and enhancing interactivity between broadcasters and viewers. ${ }^{112}$

The situation in New Zealand is regrettably determined by political priorities. In a conversation with Wendy Youens, Chief Executive of ABLE, the only company in New Zealand that produces captions for television, I discovered that currently only about $60 \%$ of TVNZ's main channels (TVNZ1 and TVNZ2), and only $30 \%$ of New Zealand's other main channel, Three, are captioned. For New Zealand, this will become a matter of equity, with the deaf and hard of hearing having already made huge ground in 2006, since the establishment of New Zealand Sign Language (NZSL) as an official national language alongside English and te reo Māori. It would therefore stand to reason that providing full access to all broadcast television programmes should only be a matter of time, although a lot depends on the importance placed upon this by the ruling government.

Intra-lingual subtitles have also proven to have a positive influence on second language learning. Several studies have highlighted the positive effects that captioned programming have on language learners, in particular children. ${ }^{113}$ By watching a programme with intra-lingual subtitles, a language learner can both read and listen to the dialogue in the same language as it occurs, allowing for better comprehension and retention of the language. Similar results have been confirmed in inter-lingual subtitling as well, where both the SL and TL are represented. As well as linguistic development, the

\footnotetext{
${ }^{110}$ Díaz-Cintas and Remael, Audiovisual Translation: Subtitling, 15.

${ }^{111}$ Díaz-Cintas, "Back to the Future in Subtitling," 4.

112 "Teaching and learning to subtitle in an academic environment," in The Didactics of Audiovisual Translation, ed. Jorge Diaz-Cintas (Amsterdam: John Benjamins, 2008), 89.

113 Judy Chai and Rosemary Erlam, "The Effect and the Influence of the use of Video and Captions on Second Language Learning," New Zealand Studies in Applied Linguistics 14, no. 2 (2008); Susan B. Neuman and Patricia Koskinen, "Captioned Television as Comprehensible Input: Effects of Incidental Word Learning from Context for Language Minority Students," Reading Research Quarterly 27, no. 1 (1992); Lekkai Ina, "Incidental Foreign-Language Acquisition by Children Watching Subtitled Television Programs," TOJET 13, no. 4 (2014); Cees M. Koolstra and Johannes W.J. Beentjes, "Children's Vocabulary Acquisition in a Foreign Language through Watching Subtitled Television Programs at Home.," Educational Technology Research and Development 47, no. 1 (1999).
} 
production of subtitles also aids in the cross-cultural competency of students, as is argued by Claudia Borghetti:

[t]his complexity [the creation of subtitles] constitutes an opportunity to stimulate the intercultural acquisition process, since, given the quality of the operations which must be undertaken, it first induces inquiry, doubt and modesty, and then requires definitive decisionmaking, thus fostering responsibility. ${ }^{114}$

One study, undertaken in New Zealand by Judy Chai and Rosemary Erlam, tested the vocabulary retention of 20 students of English as a Second Language across two language schools in Auckland. ${ }^{115}$ The students were rated as being at an upper-intermediate level of English competency. The subjects interviewed were shown 10 minutes of Hayao Miyakazi's Howl's Moving Castle (2004). Participants were split across two groups: one watched the video with captions, the other without. The results showed a positive correlation between language acquisition and the presence of captions. This does not come at a surprise, as confirmed by Díaz-Cintas and Remael:

[w]atching and listening to films and programmes subtitled from other languages helps us not only to develop and expand our linguistic skills, but also to contextualize the language and culture of other countries. We familiarise ourselves with the foreign language through the soundtrack (vocabulary, intonation, pronunciation) and the images bring us into contact with the mannerisms and behaviours of other cultures (gesticulation, way of dressing, interpersonal relationships, geographical spaces). ${ }^{116}$

For language learners, like myself, it is often very difficult to overstate the importance of subtitles that not only allow access to another medium of language learning, but also to a culture and people that would otherwise be impossible to reach. In my own experiences tutoring Italian at Victoria University of Wellington, I have tried to incorporate aspects of subtitling in my teaching. In fact, as an assignment I tasked the students with subtitling the second Fantozzi film in its entirety, a project which was a resounding success based on student feedback and one with which the students engaged very well and led them to produce very high quality work. ${ }^{117}$

Another branch of intra-lingual subtitling that is gaining steady ground is the use of subtitles for karaoke in cinema. Díaz-Cintas and Remael cite the movie The Sound of Music (1965) directed by Robert Wise, which was advertised in London as "the classic film musical, now with subtitles so everyone can enjoy it!" This popularity has led to subtitles being written for other productions such as The Rocky Horror Picture Show, ABBA Live in Concert and Joseph and the Amazing Technicolor

\footnotetext{
${ }^{114}$ Claudia Borghetti, "Intercultural Learning Through Subtitling: The Cultural Studies Approach," in Audiovisual Translation - Subtitles and Subtitling, ed. Laura Incalcaterra-McLoughlin, Marie Biscio, and Máire Aine Ní Mhainnín (Bern: Peter Lang, 2011), 9.

${ }^{115}$ Chai and Erlam, "The Effect and the Influence of the use of Video and Captions on Second Language Learning."

${ }^{116}$ Díaz-Cintas and Remael, Audiovisual Translation: Subtitling, 15.

${ }^{117}$ Rory McKenzie, "Subtitling in the Classroom: Il secondo tragico Fantozzi (1976)," Neke: The New Zealand Journal of Translation 1, no. 1 (2018).
} 
Dreamcoat so that an audience can sing along with the characters on-screen, enhancing viewer participation and enjoyment.

Intra-lingual subtitles are also used in movies or television for characters whose speech patterns are difficult to understand, despite being the same SL for the viewers. This is the case for the more widely spoken languages around the world such as English, Spanish or French as the pronunciation, syntax and other linguistic components can often depend on different factors such as age, geography, gender and social status. An example of these intra-lingual subtitles in English comes from a 2009 episode of The Oprah Winfrey Show (1986-2011). In the episode, the Scottish singer, and former contestant on the popular show Britain's Got Talent, Susan Boyle, appeared. ${ }^{118}$ As shown in Figure 19, the singer's words were subtitled due to the perceived American audience's inability to understand her strong Scottish accent.

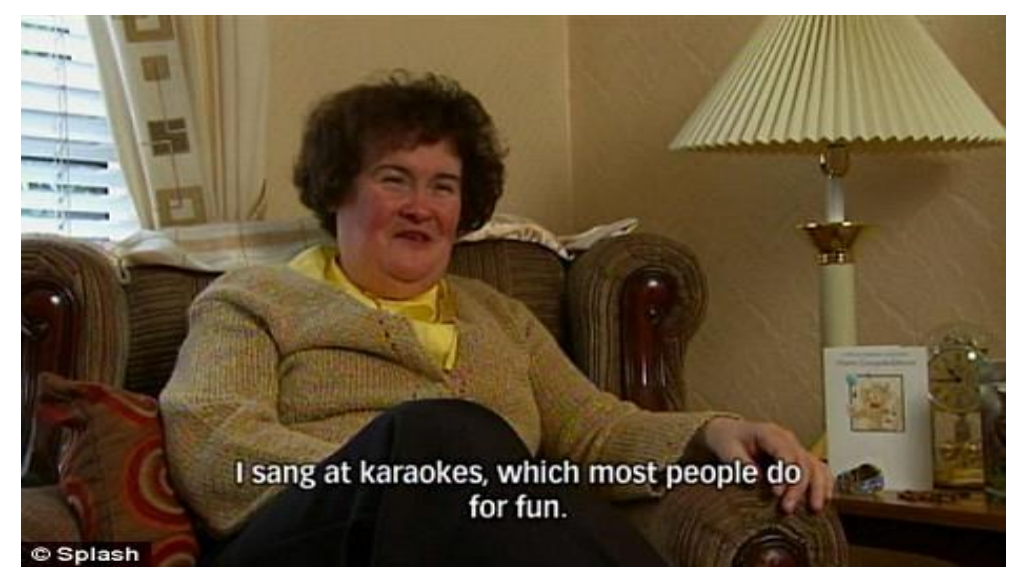

Figure 19: Susan Boyle subtitled despite the SL and TL being the same

The other category of subtitling in relation to linguistic factors is known as inter-lingual subtitling, or as scholars such as Henrik Gottlieb and Carol O'Sullivan call it 'diagonal translation'. It is a translation that not only takes place across mediums but also across languages. ${ }^{119}$ It is exactly this feature of interlingual subtitling that creates difficulties for a subtitler. Kajingulu Somwe Mubenga summarises the issue in these terms:

[t]he rendering of the verbal message from one language to another and from the oral mode of communication to the written one is a challenge in itself. It is submitted to a host of both linguistic and technical constraints. These constraints are the major sources of most problems encountered by subtitlers because they relate to differences in the logic of image, speech and writing. ${ }^{120}$

\footnotetext{
${ }^{118}$ Ben Leach, "Susan Boyle of Britain's Got Talent gets subtitles on Oprah Winfrey," The Daily Mail 2009.

${ }^{119}$ Gottlieb, "Subtitling: Diagonal Translation."

${ }^{120}$ Kajingulu Somwe Mubenga, "Investigating Norms in Interlingual Subtitling: A Systematic Functional Perspective,"

Perspectives 18, no. 4 (2010): 253.
} 
In the context of AVT, inter-lingual translation can take two forms: intra-diegetic, where the translation is contained within the narrative structure of a film, and extra-diegetic where the translation comes outside the narrative and the audience need to understand what is being said, which is usually done through use of subtitles. ${ }^{121}$ Michael Cronin uses the example of the original Star Wars trilogy (19771983), where the robot C3PO is used to provide translations or interpretations within the film itself as part of the narrative (intra-diegetic) and therefore no subtitles are needed, however moments exist throughout the film where subtitles appear as to provide a narration outside the confines of the action (extra-diegetic). ${ }^{122}$

In the context of inter-lingual subtitles, bilingual subtitles tend to be produced in territories where two languages are spoken. These subtitles tend to cover only two lines of dialogue to avoid the screen being polluted with text (that is, one line per language, rather than the traditional 2 lines of subtitles in the TL). I would argue that the downside to this practice is that the subtitles have an even more constrained screen time, which places additional pressure on the audience to comprehend them. These subtitles are used in international film festivals to attract a wider audience; one line of subtitle tends to be in English and the other line would be in the language of the country where the festival is being held. This allows both the international audience and domestic audience to have access to the subtitles and therefore, the film. Below is an example of bilingual subtitles, taken from Renny Harlin's film Skiptrace (2016), transcribed into Mandarin Chinese where English subtitles are also present (Figure 20).

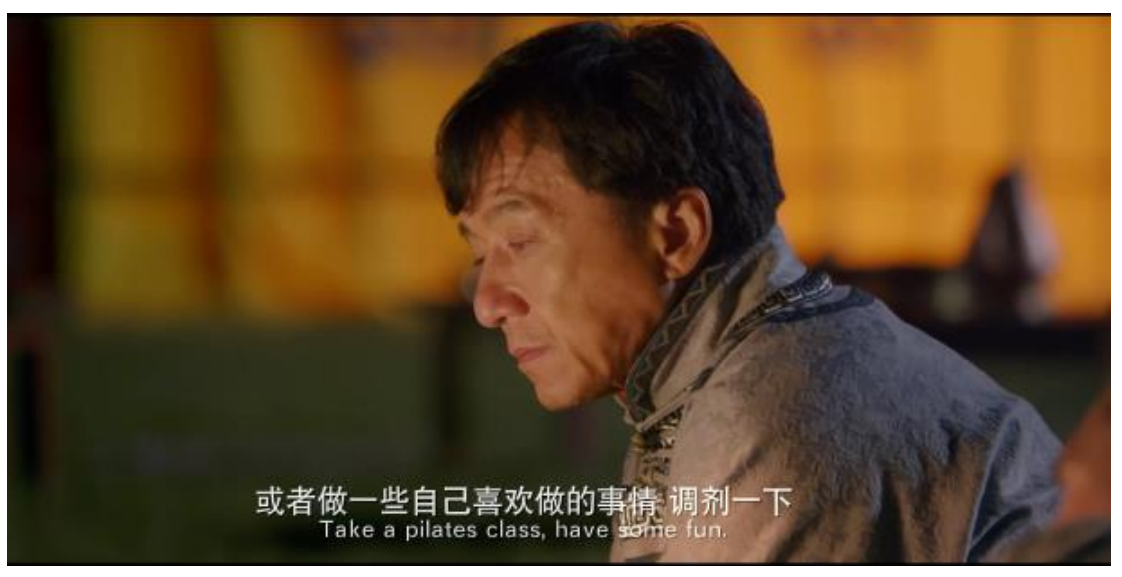

Figure 20: Skiptrace presented with bilingual Chinese/English subtitles

\footnotetext{
${ }^{121}$ Michael Cronin, Translation Goes to the Movies (New York: Routledge, 2009), 116.

${ }^{122}$ Ibid., 108-33.
} 


\subsubsection{Dubbing}

Both dubbing and subtitling were introduced to cinema in the 1920 s, coinciding historically with the birth of spoken cinema. They can both be considered a form of constrained AVT used for the same purpose: to deliver previously inaccessible content to a new target audience. Each discipline is considerably affected by challenges that are specific to AVT. For example, both dubs and subtitles must be in synchrony with the on-screen image and must not differ drastically from what the characters are saying. In the case of subtitles, the text must synchronise with the dialogue being spoken. In the case of dubs, the length of the utterances in the ST and TT must be similar to avoid creating disbelief in the audience when watching dubbed programming. That is, the purpose of dubbing is to provide the audience with a form of AVT that they believe is being spoken by the characters on-screen, despite the fact that the SL and TL could be significantly different. The TL dialogue must sync up with the movement of the character's lips, although different countries place different levels of importance on this. For example Italy chooses to remain faithful to the dialogue, rather than the synchronisation between sound and image. ${ }^{123}$ Despite these similarities, subtitling and dubbing "can be considered to represent two ends of the scale as far as the visibility or overtness of the translation is concerned." 124 Subtitles are always presented alongside the original soundtrack, whereas dubbing provides the possibility of a more 'free' translation that can be accommodated to the TL, giving the illusion that the dialogue is taking place in the TL.

As well as the general restrictions of AVT, dubs must "comply with the phonetic and spoken language synchronies." ${ }^{125}$ It is generally agreed that matching the place of articulation of sounds is the main focus of dubbed programming in order to ensure visual harmony. For example, sounds that are articulated behind the lips are not greatly off-putting to an audience if they are not replaced by similar sounds in the TL, however labial and approximant consonants, such as /p/, /b/, /m/, /f/, /v/ and /w/, when dubbed can cause disharmony in the viewers experience. ${ }^{126}$ While this may seem like a near impossible task for the translator, it is not as difficult to achieve as one might think: Thorsten Schröter believes that often, lip sync is not the be all and end all of a successfully dubbed programme, that there is an 'indefinable threshold' between imprecise yet still acceptable synchrony, and a violation of norms. ${ }^{127}$ Of course, dialogue can also occur without the audience seeing the character's face, in these instances the dubber has a much simpler task and can dub more freely. It is also important to note that the movement of lips plays only a part in expressing spoken dialogue: eye movement, eyebrow movement and hand gestures are just a few of the other ways in which communication is achieved; this is another

\footnotetext{
123 Jorge Díaz-Cintas, "Dubbing or Subtitling: The Eternal Dilemma," Perspectives 7, no. 1 (1999): 33.

${ }^{124}$ Schauffler, Subtitling Wordplay - An Audience Study: Approaches to the Translation of Linguistic Humour, 18.

${ }^{125}$ Díaz-Cintas, "Dubbing or Subtitling: The Eternal Dilemma," 33.

126 Thorsten Schröter, "Shun the Pun, Rescue the Rhyme? The Dubbing and Subtitling of Language-Play in Film" (Karlstad University, 2005), 19.

${ }^{127}$ Ibid., 21.
} 
argument as to why, according to some practitioners and scholars of dubbing, synchrony is not always necessary.

It is not only the visual transposition of the phonetics or the place of articulation about which the dubber must worry. One does not need to look far to find a contemporary example of how dubbing for content, while also taking into account the original SL phonetic sounds, can be a difficult task. In season six of the hit HBO programme Game of Thrones (2011-2019), for example, the audience learns about the derivation of the name of one of the characters, Hodor. It is a contraction of the English phrase 'Hold the door'. Under normal circumstances this may not be an issue, the translator may substitute the name of the character to a contraction of a similar phrase in the TL. However this is a character that has been present from season one of the series, which was released in 2011, and whose name has been retained across the various languages into which the show has been dubbed with no prior insight as to the derivation of the name. Because of this, the dubbers of the various languages were challenged to find phrases that retained both the message of 'holding a door', as this was an integral part of the scene visually, while also maintaining the phonetic sounds of the name 'Hodor'. As Diego Castelli has discussed in detail, this was achieved with varying success across different languages. ${ }^{128}$ The Italian translators, for example, settled on trova un modo or 'find a way' which retained the vowel sounds and the /d/ sound, but missed the specific context of the original English version. The Spanish version was slightly more successful by translating the phrase as ocluye el corredor or 'block the corridor' which kept both the 'o' sound at the beginning 'Hodor', and the 'dor' part at the end, while also remaining somewhat faithful to the on-screen context. This is just one example of how difficult a task AVT in the case of dubbing can be, not just due to the technical limitations of the field, but also to the actual content in need of translation.

Dubbed programming, unlike subtitled content, does not allow the audience the opportunity to compare the original film to the translation. Although this can result in the audience of a dubbed programme being constantly reminded that the images belong to a completely different culture and social environment. This is the case when cultural references are overt in the image, such as money, landmarks, signs or food.

There are also doubts as to the effectiveness of dubs given that markers of regional variety, such as dialects or lexicon, are often erased. For example, no dialect from the TL could be substituted for the original without introducing or eliminating specific connotations. ${ }^{129}$ This often means that regional varieties are often dubbed into standard language. In the case of The Simpsons in Italian, Groundskeeper Willy, in English, speaks with a Scottish accent, in the Italian dubbed version of The Simpsons he speaks as if he were from Sardinia.

\footnotetext{
${ }^{128}$ Diego Castelli, "Hold the Door: ecco il doppiaggio nelle varie lingue," http://www.serialminds.com/2016/05/31/hold-thedoor-doppiaggio-lingue/. accessed 1st June, 2016.

${ }^{129}$ Schröter, "Shun the Pun, Rescue the Rhyme? The Dubbing and Subtitling of Language-Play in Film," 13.
} 
One other feature of dubbing is that the literacy levels of the audience, or the quality of their vision will have little to no effect on the viewing experience, meaning the likes of children as well as visionimpaired people can readily access the content.

Frederic Chaume, in his comprehensive study on dubbing, suggests that there are six distinct areas which dubbed programming needs to meet to be successful. ${ }^{130}$ The first of these is that the lipsync of the translation is acceptable, i.e. the timing of the lip movements, the length of the utterance being equal in length to the original, and that other visual cues like body movements are taken into account. The second criteria is that the translation must be credible, so the register is similar to the original text, while the translation makes sense in the TL (i.e. it is realistic and plausible); the translation needs to be, as Chaume calls it, 'false spontaneous', where the audience is able to overlook the severe limitations of dubbing. The next area where quality is achieved is through the coherence between words and images: the translation must take into account both the original dialogue and the images on-screen to produce an acceptable translation. The fourth criteria set out is that the translation must be faithful to the original. While this almost goes without saying, given the limitations of AVT this can sometimes prove to be a challenge. Chaume outlines some 'thresholds of acceptability' in a dubbed translation, where particular changes can be accepted by an audience, and others cannot. ${ }^{131}$ Having clear sound quality on a dubbed translation is also outlined as being crucial to a quality dubbed translation. Dubs must be recorded in a sound-proof studio, there must be no remnants of the original dialogue, the volume should be higher than that of normal speech, and sound effects need to be used appropriately. The final quality assurance measure is to ensure that the dubs are neither over nor under-acted, as this can take away from the content of the programme or film.

One of the negative aspects of dubbing is undoubtedly the cost that it takes to produce the new soundtrack of the film, particularly when it is dubbed into a variety of different languages. In fact, subtitling only costs around a tenth to a twentieth the price of dubbing. ${ }^{132}$ In Italy, for example, "the cost of dubbing a film is somewhere in between twenty thousand and one hundred thousand euros depending on the number and quality of the actors, while subtitling is between three and eight thousand euro." ${ }^{133}$ The costs of dubbing are accrued through the numerous speech actors that need to be contracted, as well as the creation of a dubbed script and the technical team that must synchronise the new dubbed audio with the original images. Hence countries with smaller film industries tend to subtitle as it is a much cheaper, more easily produced and faster alternative, usually involving just one or two contractors to produce the translations and subtitles.

\footnotetext{
${ }^{130}$ Frederic Chaume, Audiovisual Translation: Dubbing (New York: Routledge, 2012), 17-21.

${ }^{131}$ Ibid., 14-20.

132 Cees Koolstra, Allerd Peeters, and Herman Spinhof, "The Pros and Cons of Dubbing and Subtitling," European Journal of Communication 17, no. 3 (2002): 329.

${ }^{133}$ Alessandra Catania, "La traduzione audiovisiva: techniche, strategie e difficoltà: proposta di traduzione di quattro articoli tecnico-informativi" (Università Ca' Foscari Venezia, 2013), 45. [My translation]

Original Italian: "il costo per il doppiaggio di un film di circuito è compreso tra i ventimila e i centomila euro in base al numero e alla qualità degli attori, mentre quello dei sottotitoli varia tra i tre e gli ottomila euro."
} 
Despite the best efforts of dubbing companies, who may follow strict ethical guidelines and take pride in the services they offer, "they are also for-profit organizations that will always be forced to strike a balance between the minimally required standards of quality and the maximally allowed costs." ${ }^{134}$ They will always be looking to save money, particularly if the prescribed appropriate standards have been met. Eventually this could lead to the standards being reduced in order to save money resulting in poor quality dubs produced at cheap rates.

\subsubsection{Subtitling or dubbing?}

The choice between the two main AVT practices, dubbing and subtitling, varies greatly across Europe alone, with countries such as Germany, France, Austria, Spain and Italy all preferring to dub incoming content rather than to subtitle it, while countries such as Belgium, Denmark, Finland, Luxemburg, the Netherlands, Portugal, and Sweden have a subtitling, rather than dubbing, tradition. The United Kingdom and Ireland have a history of both, although the cheaper alternative of subtitling is more common. ${ }^{135}$ For some countries it was an argument around cost, but for others there were political factors associated with the decision. ${ }^{136}$ For example, in Italy the decision to dub, rather than subtitle films, dates back to the Fascist regime, although other factors such as the analphabetism of Italy, as well as the need to create new jobs, certainly influenced the decision to dub rather than subtitle incoming content: ${ }^{137}$

[t]he dubbing of films in Italy was first adopted because fascist censorship (1929-1946) forbade the use of foreign languages in all films screened in Italy. This has had far-reaching effects on the way cinema is offered to the Italian public: subtitling and other means of language transfer are not accepted at all, regardless of genre and mode of presentation. ${ }^{138}$

While content translated from other languages into English tends to be subtitled, some examples of dubbed translations of international films include Roberto Bengini's Life is Beautiful (1997) from Italian and Ang Lee's Crouching Tiger Hidden Dragon (2000) from Mandarin Chinese. Broadly speaking, "large countries dub and small countries subtitle"139, possibly representing the costeffectiveness of each practice. In modern times, Netflix has released content dubbed into English, practice that may well continue to develop in the future.

\footnotetext{
${ }^{134}$ Schröter, "Shun the Pun, Rescue the Rhyme? The Dubbing and Subtitling of Language-Play in Film," 25.

${ }^{135}$ Bartholomäus Wissmath, David Weibel, and Rudolf Groner, "Dubbing or subtitling?: Effects on spatial presence, transportation, flow, and enjoyment," Journal of Media Psychology: Theories, Methods, and Applications 21, no. 3 (2009). ${ }^{136}$ Tessa Dwyer, Speaking in Subtitles: Revaluing Screen Translation (Edinburgh: Edinburgh University Press, 2017), 1952.

${ }^{137}$ Paola Guardini, "Decision-Making in Subtitling," Perspectives: Studies in Translatology 6, no. 1 (1998): 95.

${ }^{138}$ Ibid., 1.

${ }^{139}$ Peter Fawcett, "Translating film," in Translating French Literature and Film, ed. Geoffrey Harris (Amsterdam: Rodopi, 1996).
} 
New Zealand has tended to develop a subtitling tradition, probably due to the fact that most international films that are screened here will have already been marketed to American or British audiences prior to arriving in this country, meaning that the subtitles will already exist in English. The exception to this practice is the dubbing of content into New Zealand's indigenous language, te reo Māori; with the introduction of Māori television, many programs, particularly cartoons, SpongeBob Squarepants (1999 - present) for example, are being dubbed into te reo. ${ }^{140}$ Recently, the very successful Disney film Moana (2016), which is based on Pacific culture and traditions, has been dubbed into te reo Māori, which is particularly relevant given the Pacific cultural content of the film and is a way to promote the use of this endangered national language. ${ }^{141}$

\subsubsection{Voice-over}

Linked to dubbing, voice-over is the third of the AVT practises, although it is used in a rather limited context. It is predominantly used, at least in English, for documentaries or news broadcasts where a narrator will be describing the action on-screen.

Voice-over is defined as a narrative technique in which the voice of a faceless narrator is heard over different images and for different purposes. Compare, for example, the expository voice in a documentary about the life of animals and the accusing voice of the narrator towards one of the murder suspects in a thriller film. ${ }^{142}$

However this is not its only purpose: radio or television programming will often have a voice-over for an interview in another language. Instead of completely re-voicing the programme, the SL is dimmed down to background noise, and the TL is spoken over the top of it. In fact, there are a variety of uses for voice-over which have some advantages over dubbing and subtitling, as outlined by Eliana Franco, Anna Matamala and Pilar Orero. ${ }^{143}$ For example, unlike dubbing, voice-over has the benefit of not needing lip-synchronisation, as a programme that has been voiced-over is not attempting to hide the fact that it is not spoken in the original language and therefore does not require this synchrony. The process of voice-over is therefore much simpler than its other AVT counterpart in dubbing. Another benefit from the practice is the fact that it can render content very closely to the original given that there are no external factors to consider.

\footnotetext{
${ }^{140}$ Chaume, Audiovisual Translation: Dubbing, 10.

141 "There's going to be a Te reo Maori version of Moana," Stuff,

http://www.stuff.co.nz/entertainment/film/93512377/theres-going-to-be-a-te-reo-maori-version-of-moana. accessed 13th June, 2017.

${ }^{142}$ Eliana Franco, Anna Matamala, and Pilar Orero, Voice-Over Translation: An Overview (Bern: Peter Lang, 2013$), 18$.

${ }^{143}$ Ibid.
} 


\subsubsection{Audio description}

Audio description (AD) is a relatively new field within Translation Studies. The main purpose of audio description is to provide accessibility to the blind or visually impaired to audiovisual content by narrating descriptions of the events as they unfold, in between the dialogue. Examples of descriptions could include the action of the programme/film, body language of particular characters, facial expressions, scenery, costumes, on-screen text or any other important feature of the material that will help a person with a sight problem follow the plot of the story. ${ }^{144}$ Audio description has been defined by Andrew Salway as "a description of visual information delivered via an audio channel and it is crucial for improving media accessibility for blind and visually impaired people"145, and by Bernd Benecke as "the description [that] fits in between the dialogue and does not interfere with important sound and music effects... it consists of an additional narration [that] describes the action, body language, facial expressions, scenery and costumes". ${ }^{146}$

An example of audio description applied to feature film is a scene taken from Gary Ross' dystopian film The Hunger Games (2012). As can be seen, aspects of plot, as well as textual elements from the film, are described by the narrator, allowing the blind or the visually impaired audience access to the action that is taking place:

Narrator: Signs on a tall wire fence read "District Boundary. No access beyond this point" and "High Voltage." Katniss steps through a gap in the wires and heads into the woods beyond. She glances around before reaching into the hollow of a fallen tree. She draws out a wooden bow. From another tree, she plucks out a sheaf of arrows and straps it over her shoulder. Katniss makes her way through thick, green vegetation. Bow and arrow at the ready, she walks over a fallen tree, suspended over the forest floor. She pauses. Her gaze locked on a deer in the distance. Leaning against a tree trunk, she aims the bow and arrow. The deer sniffs the air and moves out of sight. ${ }^{147}$

The field of AD is in constant growth and development, attracting scholars to the field; in 2011, for example, Christopher Taylor and Elisa Perego from the Università di Trieste set up a project named ADLAB (Audio Description: Lifelong Access for the Blind) and were able to secure funding for three years:

[t]he basic motivation for the launching of ADLAB was the need to define and create, in cooperation with industrial partners and service providers, a series of effective and reliable, practical and educational guidelines for the practice of audio description (AD), usable throughout Europe. Their aim is to make most audiovisual products (e.g. films, television

\footnotetext{
144 Jill Whitehead, "What is audio description," International Congress Series 1282 (2005): 960.

145 Andrew Salway, "A corpus-based analysis of audio description," in Media for all: Subtitling for the deaf, audio description and sign language, ed. Jorge Diaz-Cintas, Pilar Orero, and Aline Remael (Amsterdam: Rodopi, 2007), 151.

${ }^{146}$ Bernd Benecke, "Audio-description," Meta: Translators' Journal 49, no. 1 (2004): 78.

${ }^{147}$ Centre for Inclusive Design, "The Hunger Games with audio description: Katniss hunting,"

https://www.youtube.com/watch?v=B8BD9txkGL4. accessed 1st November, 2018.
} 
programmes, documentaries, advertisements, but also such audiovisual phenomena like art galleries, museums, dance performances, city tours, live events) available to the blind and visually impaired community. ${ }^{148}$

The number of people that such a service can help is phenomenal: in New Zealand alone there are 12,272 people registered with the blind foundation as of July 2016. ${ }^{149}$ In New Zealand the service does exist on selected programmes; the same company that produces captions, Able, also produces audio descriptions. This service has been available on New Zealand television since 2011, originally provided by TVNZ Access Services.

\subsection{The development of subtitling}

Despite the evolution of AVT and the growth of its many forms and technologies, the subtitling profession has often been overlooked and, as already discussed, this has led to the production of poor quality subtitles (or, perhaps, the poor quality of subtitling has led to the discipline being overlooked). This is particularly evident in university departments where other strands of translation have received a lot of attention, yet, for several reasons (such as lack of interest, exorbitant prices of software, limited expertise among academics) subtitling seems to have been neglected as a discipline. The situation is fortunately changing with some universities beginning to take up the challenge of teaching AVT, mainly in the disciplines of subtitling, dubbing and voice-over. For example, at Victoria University of Wellington a new course has been offered in Translation Studies at Honours level since 2017, which includes a unit on AVT. Many world-class universities around the world boast prestigious AVT courses and degrees. Initiatives like these across the world will undoubtedly lead to a higher prestige being placed upon AVT, resulting in a more professional approach toward, and understanding of, this form of translation.

\subsubsection{The role of the subtitler}

Subtitlers are dealt the unenviable task of trying to translate AV material faithfully, while contending with the severe restrictions imposed on them by the medium itself. It is a rather unforgiving form of translation practice given the amount of interference the subtitler will have to engage with in the original text.

However, what is a subtitler's responsibility as a translator? Within translation studies a lot of attention has been paid to the issue of the 'invisibility' of a translator. In subtitling, this appears as an

\footnotetext{
148 Anna Maszerowska et al., "From source text to target text: The art of audio description," in Audio Description : New perspectives, ed. Anna Maszerowska, Anna Matamala, and Pilar Orero (Amsterdam: John Benjamins Publishing Company, 2014), 1.

${ }^{149}$ Blind Foundation, "Latest stats at a glance," https://blindfoundation.org.nz/eye-info/latest-statistics/. accessed 1st November, 2018.
} 
oxymoron: it is physically impossible to remain invisible when both the ST and TT are presented simultaneously and therefore are being compared at all times, let alone the fact that the subtitles are quite literally imposed upon to the original text on-screen. Therefore, it is the responsibility of a subtitler to ensure that the subtitles do not take away from the viewing experience, that is to say, that despite their being 'in the way' subtitles are written in a manner that is an accurate representation of the film, written with correct grammar and spelling, and consistent across the entire film. If the subtitles are written poorly, the audience's focus will shift from the content of the film to the subtitles themselves.

The subtitler may also have to negotiate several other difficulties that perhaps go over and above their immediate role. In a recent subtitling project, for example, I faced a difficult decision, while translating into Italian from the English subtitles of a film where the SL was Tok Pisin, a native language of Papua New Guinea. Translating from the English subtitles of another, not known language, is not an ideal practice, given that the translation to English is already one step removed from the original dialogue (and the English is likely to be a reduced version of the original). The English subtitles, moreover, were full of punctuation errors, a few misspellings and some other grammatical errors. In such a case, is the role of a subtitler to play editor as well? Alternatively, should they translate exactly what they are commissioned to, mistakes and all? Given the quality of the original subtitles, comprehension for an English-speaking audience could have been compromised and therefore I felt obligated to edit the English subtitles. Ideally, the subtitles should have already been of good quality standard, however, someone who had had no training or experience in subtitling had produced the English subtitles of this film. Furthermore, when I attended the screening of the film, the filmmaker used the original subtitle file, mistakes and all, and acknowledged me for them in the final credits of the film. This is an example of the status that subtitling enjoys in both film-making and translation studies. Subtitles are quite often the last thing a filmmaker or production company thinks about, and often need to be produced quickly and on a minimal budget. This undoubtedly lends itself to inaccuracies in translation or legibility.

Subtitlers tend to work as freelance translators. There is no specific pay rate for a subtitler's work; remuneration tends to vary from company to company where subtitlers can either be paid per whole programme, per minute of programme, per number of words, or per number of subtitles. A subtitler should also be given sufficient time, and therefore be paid more, depending on the specific circumstances of each translation, such as whether they are provided with the original dialogue in written form, or just the video itself, or how much research they must undertake in regards to culturallyspecific terminology and content. However, recognition of this extra work is not always forthcoming and it may come down to the bargaining power of the individual subtitler. ${ }^{150}$ Luyken et al. suggested that in 1991 a subtitler was paid around $15 \%$ of the overall costs of subtitling a programme and, on average, the cost of one hour of programming in Europe was around $€ 1,500$; the subtitler would

\footnotetext{
${ }^{150}$ Díaz-Cintas and Remael, Audiovisual Translation: Subtitling, 36.
} 
therefore expect to be paid around $€ 225$ for every subtitled hour. ${ }^{151}$ Unfortunately, the situation does not seem to have improved since then, as attested to by Subtle, the Subtitler's Association based in the United Kingdom. They highlight the poor working conditions subtitlers face in this day and age. They are often paid less than minimum wage for the work they do, earning as little as $£ 7-£ 13$ per hour, whereas for other, less-technically demanding work, translators can earn around $£ 30$ per hour: this leads experienced, qualified translators away from the field of subtitling. ${ }^{152}$ Improvements in technology, as well as shorter deadlines, have also meant that, according to Andrew Lambourne, the time available for preparation of one hours' worth of subtitles has decreased from 40 hours in 1980 to only 10 hours today. ${ }^{153}$

Despite this, subtitlers still need to uphold professional standards. In an attempt to create consistency across the field of subtitling, aiding the subtitler in his or her work, in 1998 Jan Ivarsson and Mary Carroll proposed a 'Code of Good Subtitling Practice'. ${ }^{154}$ Following this code is not mandatory, and subtitling companies tend not to enforce it, but it does provide an exemplary subtitling standard. The code outlines what subtitlers should be aspiring towards in their translations, some of the technical norms that should be followed, as well as suggestions associated with editing and revising subtitles. Although relatively old given the recent developments in subtitling, the Code does provide some guidance to an uninformed subtitler.

\subsubsection{Past, present and future of subtitling}

Compared to more traditional forms of translation, subtitling is a relatively modern form of translation and is arguably "the fastest growing strand within translation studies". ${ }^{155}$ This seems to not only be the case in both a practical and academic setting, but also given the rapid advancements in the technology available to a subtitler. Subtitles were first developed from intertitles or 'title cards', which were used in silent movies, allowing the audience to understand sequences of silent action. These title cards were not used exclusively, and there were some filmmakers who "ostracize[d] any written elements, including intertitles, regarding them as an obstacle to the obligatory "visuality" of their films." ${ }^{156}$ Cards would be printed and inserted between film sequences (as opposed to being projected onto the screen simultaneously with the video component, as subtitles are today). When films became audible in the late 1920s, intertitles became redundant and were phased out as newer technologies began to develop; these technologies will be discussed in more detail in the upcoming pages.

\footnotetext{
${ }^{151}$ Georg-Michael Luyken

Thomas Herbst, Overcoming Language Barriers in Television: Dubbing and Subtitling for the European Audience (Manchester: The European Institute for the Media, 1991), 95-96.

152 "Hard Facts," The Subtitler's Association,

http://www.subtitlers.org.uk/ajax.php?modulo=paginas\&accion=sitio_ver\&idpaginas=16. accessed 23rd June, 2016.

${ }^{153}$ Andrew Lambourne, "Future Trends in Subtitling," in 9th Languages in the Media Conference (Berlin2006).

154 Jan Ivarsson and Mary Carroll, Subtitling (Simrishamn: TransEdit HB 1998), 157-59.

${ }^{155}$ Luis Pérez-González, Audiovisual Translation: Theories, Methods and Issues (New York: Taylor and Francis, 2014$), 1$.

${ }^{156}$ Rosemary Brant, "The History and Practise of French Subtitling" (The University of Texas, 1986), 10.
} 
Several problems existed with intertitles, the most prominent being the lack of continuity they created within the film. Directors also faced the problem of determining where to place the cards in the sequence, with the function of intertitles often becoming a narrative device:

[i]n their anticipation of inserting intertitles into the shot continuity, traditional directors arranged it so that the image that followed each shot did not go with the one before. The intertitle simply took the place of events that were supposed to take place at the same time. It was therefore impossible to take out the intertitle, for fear of a jump. ${ }^{157}$

To cope with the demand for international exposure, filmmakers began to shoot their films in different languages. This meant that bilingual actors were cast, or production companies would have entire casts from different language backgrounds to re-shoot the films, however the cost of this was immense. This led to the creation of the modern practise of dubbing; instead of re-shooting the entire film with different cast members, voice actors are employed, and the new audio replaces the original soundtrack and is played alongside the existing images.

Around the same period in which dubbing gained popularity, the field of subtitling was developed as a cheaper and more easily produced alternative. Subtitling into English, however, did not become popularized until around 1931 partly due to the plentiful supply of English language content coming out of the United States. ${ }^{158}$ However subtitling eventually established itself as a common means of translating film, particularly in English speaking and Scandinavian countries, due to their comparatively inexpensive nature. Since their creation, subtitles were white due to their "photographic reality' and appeared at the bottom of the screen as it was recognised that in movies, the bottom of the screen tended to be darker than the top. This was problematic as it meant that, for scenes where there was white at the bottom of the screen, the subtitles were therefore invisible. This created similar concerns to those associated with intertitles, where the action would be broken up and the lack of visible subtitle hindered continuity. Since then, subtitling has continued to develop to accommodate this issue. Subtitles are now produced digitally, the colour can be changed, a black box can be inserted as a background, or a black outline can be placed around the subtitles themselves. Yellow has also been used as an alternative to white subtitles, particularly in black and white film. Not only can the colour be changed, but also the overall accessibility to change subtitles has also markedly increased due to the developments in subtitling technology - whether further editing is required, or whether the subtitles need to be modified in any other way, nowadays subtitlers can easily access and change them, due to the technological progress of the field.

Although from the start huge benefits associated with the production of subtitles were apparent, most notably the reduction in production costs, subtitles were not always received positively. In a 1929

\footnotetext{
${ }^{157}$ Ibid., 9.

${ }^{158}$ For a history of the introduction of subtitles into both the USA and UK see: Carol O'Sullivan, "'A splendid innovation, these English titles!' The invention of Subtitling in the USA and the UK," Proceedings of the British Academy 218 (2018).
} 
New York Times article, a columnist described the film Broadway Melody (1929) as having been "translated by the now despised subtitle" and noting, "[t]he French follow the story by means of occasional captions tacked at the foot of the film itself." 159 This seems a rather narrow perspective, considering that French audiences would otherwise not have had access to the content in the first place without these subtitles, but illustrates that from its very inception into cinema, subtitling was met with some negativity and the experience of viewing a subtitled film could be seen as being somewhat diminished. A lot of technical developments and experimentations were subsequently undertaken in an attempt to alleviate precisely these issues, resulting in the new technology that we have available to us today.

Throughout the history of subtitling, a variety of different methods has been used to project subtitles on-screen, ranging from physically imprinting the subtitles onto the film reel, to superimposing them in post-production. A brief historical overview of this technical component of subtitling will allow us to consider both the positives and negatives that were associated with each of these practices, as well as how modern technologies have improved the experience of watching subtitled content. As noted by Díaz-Cintas and Remael, "The technical process of transferring the subtitles to actual film or programme has undergone a considerable evolution, which has led to an improvement in the presentation and stability on-screen." ${ }^{160}$ Since the introduction of subtitling, mechanical and thermal subtitling, photochemical subtitling, optical subtitling, laser subtitling and electronic subtitling have all been used as projection methods, with varying degrees of success. ${ }^{161}$

The process of mechanical subtitling can be accredited to a Norwegian inventor, Leif Eriksen, whose technique, which he patented in 1930, was to soften the emulsion layer and stamp the titles directly onto the positive copy of the film. The subtitles were firstly typeset in the traditional manner on paper. Small letterpress plates (around $0.8 \mathrm{~mm}$ high) were produced and placed in a printing press of sorts, and one by one were pressed against the emulsion layer of the individual frames that corresponded to the respective utterances and segments of speech. The plates pushed the emulsion layer aside leaving the film to produce white letters when projected onto a screen. The process was repeated from beginning to end of the film and the 'moist' film was then put through a drier. In 1935, this technique was progressed by Hungarian inventor O. Turchanyi who heated the plates to a high temperature, melting the emulsion layer rather than softening it. The results of both of these processes were unpredictable: definition was very poor, the ridges of the emulsion were susceptible to wear, and the letters accumulated dirt very quickly. Despite this, thermal subtitling is still used in some parts of Eastern Europe and South America today.

\footnotetext{
${ }^{159}$ Morris Gilbert, "PICTORIAL ENTERTAINMENT IN PARIS.; "Broadway Melody" Attracts Huge Crowds, the Dialogue Being Translated by the Now Despised Subtitle A Study in Contrasts. Royalty at Film Opening.," New York Times, 1st December 1929.

${ }^{160}$ Díaz-Cintas and Remael, Audiovisual Translation: Subtitling, 22.

161 The methods of projection are analysed in detail by both Ivarsson and Carroll, Subtitling; Díaz-Cintas and Remael, Audiovisual Translation: Subtitling.
} 
An improved technique was developed in 1932 when R. Huskra in Budapest and Oscar Ertnaes of Oslo simultaneously took out patents on a new method known as photochemical subtitling. Their improvements involved adding a thin wax coating to the emulsion side of the film copy. The plates with the individually written subtitles were fed through a printing press nearing 100 degrees Celsius. The wax was melted and displaced which left the emulsion layer exposed. The film was then put through a bleach bath, dissolving the emulsion layer and leaving only the nitrate or acetate film. The characters produced were white and clearly readable. The edges were at times ragged due to the variability in consistency of the wax layer and the penetration strength of the etching fluid. The process was later automated where plates were fed forward and the frames of the film were all counted, ensuring the subtitles matched the dialogue of the film.

At around the same time, optical subtitling became popular. Individual subtitles that were printed in black were positioned between the negative and positive film reels while the film was being copied. This led to white subtitles being printed onto the positive print. This process was developed so that a short roll of film was used with each subtitle photographed, and a blank space where no subtitle appeared. The subtitles were then copied from the negative to the positive print. Unfortunately, film negatives were not always available, meaning that the entire film had to be photographed to obtain an inter-negative, resulting in a loss of focus and an increase in the noise level of the sound track. To increase efficiency, the titles were photographed onto a spate film of the same length as the original film. The film negative and the reel with subtitles were then copied simultaneously.

Laser subtitling is one of the current methods of projection that is used in the production of subtitles for film. It was introduced in the 1980s and overtook previous technologies associated with the field. With this method, the subtitles are synchronised perfectly with the actor's speech thanks to a time code. A very precise laser burns the emulsion of the positive copy of the film reel, printing the subtitles. These subtitles become part of the film (i.e. open subtitles) and cannot be removed. They will always appear white on the lower part of the screen since they are burned in. This method allows for a more precise definition of letters, and it means that the subtitles will not move or shake throughout the projection of the film. It takes roughly 10 times as long as the actual film footage to laser subtitle a film, a fairly timeconsuming exercise.

Electronic subtitling is the other practise that is commonly used in film today. Its great advantage over laser subtitling is that the subtitles are superimposed onto the screen, rather than being permanently burned into the film reel. A time code system is also used to ensure synchrony with the film. The advantage of this type of subtitle is that it can be projected in any colour, in any language and does not damage the original film reel, while it can also be easily revised and modified between projections of the film. These subtitles are also cheaper to produce than laser subtitles, which is why they are most commonly used in television and on DVD. This method is also becoming readily available to the general public as software becomes more accessible. I will be using an electronic subtitling software called WinCAPS Qu4ntum to produce the subtitles for Fantozzi in this research project. 
Although not overtly obvious in this project, part of my understanding of subtitling as a form of translation, and the associated technology, has come about through teaching myself how to use WinCAPS, which has undoubtedly led to a far deeper understanding of the restrictions and processes of the industry. As can be seen by the image of the software (Figure 21), it allows the subtitler absolute precision - it provides the subtitler with audio cues as to when dialogue starts, it gives the exact number of words per minute as well as the duration of the subtitler, and the characters per line are also limited so the subtitler must adhere to particular restrictions put in place.

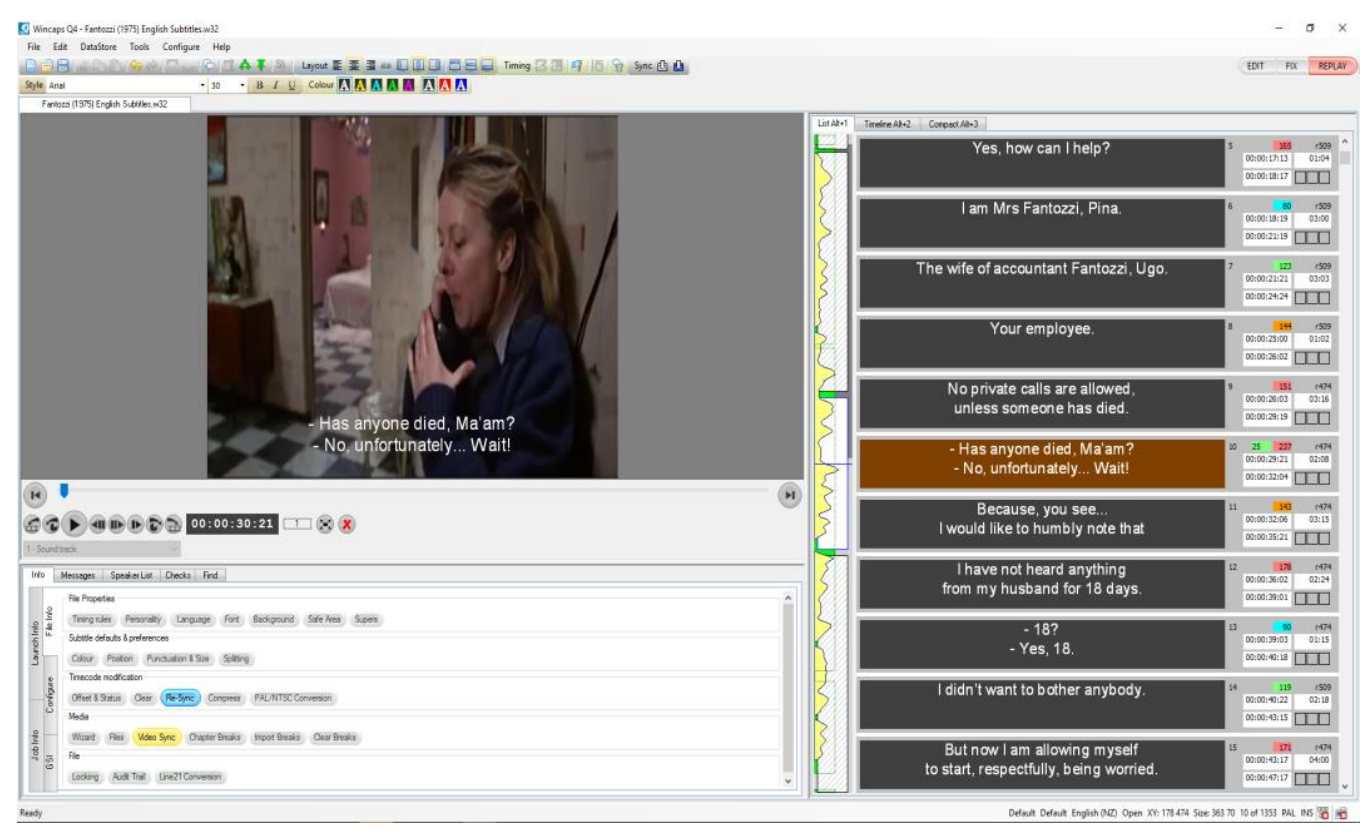

Figure 21: Screenshot of WinCAPS Q4untum, the software being used for this project.

Technology is rapidly advancing in the field of AVT, and as Díaz-Cintas reminds us, "[w]hat is today considered innovative and advanced may soon cease to be so." ${ }^{\prime 62}$ The creation of technology that instantly allows for a subtitler to simply use a computer and the appropriate subtitling software to convert a text file into subtitles complete with spotting and spell-checking has made the process much more efficient and cost-effective, as opposed to even just a few years ago when subtitling required a computer, video player, an external television monitor and a lot more time to complete the same job. Possibly the most influential piece of technology that has led to the development in the field of subtitling is the introduction of the DVD. It has allowed up to 32 different subtitle tracks to be added to a disc, as well as up to 8 different dubbed tracks. ${ }^{163}$

One of the modern trends in subtitling is undoubtedly the introduction of 'fansubs', produced by groups or individuals who voluntarily create these subtitles with the aim of sharing such material

${ }^{162}$ Díaz-Cintas, "Back to the Future in Subtitling," 1.
${ }^{163}$ Ibid., 3. 
with other fans of the content. Díaz-Cintas uses the term 'cybersubtitles', which not only encapsulates 'fansubs', but also 'guerrilla' subtitles and 'altruist' subtitles. He defines 'fansubs' as subtitles that are voluntarily produced and freely distributed (via the internet) by a 'fan' or 'fanatic' who has an interest in or enthusiasm for the content, 'guerrilla' subtitles as the types of subtitles that are produced to avoid censorship and whose objective is "to contest and challenge hegemonic practices in society". And finally he defines 'altruist' subtitles as a "less militant, belligerent version of these initiatives ('guerrilla' subtitles)". ${ }^{164}$ Their popularity has undoubtedly come about through the ease at which new materials can be distributed across the world: "the rapid development of communication technologies together with the pervasiveness of the internet and the ease with which audiovisual materials circulate in the ether have catapulted the practice of subtitling, both interlingual and intralingual, to the centre stage of the digital world." 165 The practice certainly has some positive effects: they have increased the exposure of international materials and thus created more of an appetite in the market for subtitled content, they are produced incredibly quickly (particularly in the case of crowd-sourcing) meaning that content is readily available to the audience. The negative side of 'cybersubs' is that they do not necessarily follow subtitling conventions, their creative or unconventional nature of translation means the content is not always rendered appropriately or faithfully and the subtitles can indeed become distractions from the material.

In researching for this project, I found some fansubtitles of the first Fantozzi film written in English. ${ }^{166}$ In some parts they are not too bad - they summarise the content adequately enough, however they do come across as rather stilted at times and, every so often, do not make sense in English. Below I have provided an example segment of these fan subtitles, alongside the original dialogue (left) and my own subtitles (right). There are a few translation issues - the translation of "più" as 'over', for example is slightly problematic, as well as the misuse of the idiom "split seconds" in English, and the last line of dialogue not being an adequate representation at all of the original content.

\section{Original Italian}

Per arrivare a timbrare il cartellino d'entrata alle 8:30 precise,

Fantozzi, 16 anni fa,
cominciò col mettere la
sveglia alle 6:15.
Oggi, a forza di esperimenti e
perfezionamenti continui,

\section{Fansubtitles}

In order to stamp his punch card exactly at 8:30

$$
\begin{aligned}
& \text { Fantozzi used to wake up at } \\
& \text { 06:15. } \\
& \text { That was } 16 \text { years ago. } \\
& \text { Today, after many experiments } \\
& \text { and improvements,... }
\end{aligned}
$$

\section{My Translation}

To arrive punctually and punch his timecard at 8.30am sharp

Fantozzi, 16 years ago, started setting his alarm for $6.15 \mathrm{am}$.
Today, through continuous experimenting and improvements

164 "'Subtitling's a carnival': New practices in cyberspace," The Journal of Specialised Translation, no. 30 (2018): 133-34. 165 Ibid., 127.

${ }^{166}$ Fantozzi subtitles - English, (OpenSubtitles, 2010). 
è arrivato a metterla alle 7:51.

Vale a dire al limite delle possibilità umane.

Tutto è calcolato sul filo dei secondi:

5 secondi per riprendere conoscenza;

4 secondi per superare il quotidiano impatto con la vista della moglie;

più 6 per chiedersi, come sempre senza risposta,

cosa mai lo spinse un giorno a sposare quella specie di curioso animale domestico;

3 secondi per bere il maladetto caffè della Signora Pina...

3000 gradi Fahrenheit! Dagli 8 ai 10 secondi per stemperare la lingua rovente sotto il rubinetto;

Due secondi e mezzo per il bacino a sua figlia Mariangela;

caffelatte con pettinata incorporata.

spazzolata dentifricio mentolato su sapore caffè

provocante funzioni fisiologiche che può così espletare

nel tempo di valore europeo di 6 secondi netti. he wakes up at 07: 51

stretching the limits of human possibilities.

Everything is calculated in split seconds:

5 seconds to come to.

4 seconds to bear his wife's appearance...

...over 6 seconds to ask himself, as usual, and with no answer,...

...why he married that domesticated creature in the first place

3 seconds to drink his wife's abominable coffee

... at 3000 degrees Fahrenheit. 8 to 10 seconds to cool off his burning tongue.

$21 / 2$ seconds to kiss his daughter Mariangela.

Simultaneous combing and latte-drinking

Brushing teeth with mint toothpaste and coffee flavor,..

...causing a physiological response

requiring another 6 seconds European time. he has started

setting it for 7.51am.

Pushing it to the limit of what is humanly possible.

Everything is calculated down to the last second:

Five seconds to regain consciousness;

Four seconds to get over the daily shock of his wife's face;

Six more seconds to ask himself, always in vain,

why he married this species of curious domestic animal;

Three seconds to drink Mrs Pina's insufferable coffee.

3000 degrees Fahrenheit! Between eight and ten seconds to cool down his burning tongue;

Two and a half seconds to kiss his daughter, Mariangela;

Latte with a comb;

Mint toothpaste to cover the taste of the coffee.

Provoking bodily functions that he could complete

in the European record time of six seconds flat. 
As far as the future of subtitling is concerned, one of the more innovative and interesting developments is an attempt to improve the viewing experience by testing different areas onto which subtitles can be placed on-screen. A study undertaken recently by Wendy Fox, from the University of Mainz in Germany, has looked at the development of integrated titles, using eye-tracking software to determine the effectiveness of the practice. ${ }^{167}$ These titles appear in distinct areas of the screen, not in the traditional allocated space at the bottom. Fox argues that, "traditional subtitling can be seen as a strong intrusion into the original image composition that may disrupt or even destroy the director's intended shot composition and focal points." 168 Therefore, the hypothesis behind integrated subtitles is that, because the subtitles appear in the middle of the screen where the action is predominantly taking place, the viewer would spend less time changing their gaze to and from subtitles and more time focusing on the action of the film; thus, this new method of subtitling would "not only express respect towards the original film version but also the translator's work." 169

Fox undertook this study in Germany, a country where dubbing, rather than subtitling, is prevalent. However, the practice of subtitling is becoming more and more common given that programmes can be released more quickly in English with German subtitles than a dubbed version can be produced as well as the comparative costs of each option. German viewers are not necessarily used to subtitled programming, and as Fox explains, "long saccades between focal points and subtitles affect the viewer's information intake and in particular the German audience, who are not used to subtitles, seems to prefer to wait for the next subtitle instead of looking up again." 170

Fox's research sets out to address the issue of whether the placement of subtitles affects the viewers reading time, by measuring the time spent focused on the image, rather than the subtitle, and also by measuring the effectiveness of the overall viewing experience. As a case study, Fox used the short documentary Joining the Dots (2012), created by Pablo Romero-Fresco. Being a documentary, it provided a very good case study for the placement of integrated titles, as the images that were present in the film were often static. Fox, first of all, measured the eye tracking data of 14 English native speakers to determine where the most common focus points were for an English speaking audience, this helped, in turn, to determine where the integrated titles should predominantly appear on-screen for the German audience. She then had 15 native speakers of German, with little to no knowledge of English, watch the film with traditional subtitles, and 16 German native speakers with little to no knowledge of English watch the film with integrated subtitles. The study measured the reaction times of the native German audience, as well as their reading times, and the general distribution of gaze between the image and the subtitles.

\footnotetext{
${ }^{167}$ Wendy Fox, "Integrated titles: An improved viewing experience?," in Eye-tracking and Applied Linguistics, ed. Silvia Hansen-Schirra and Sambor Grucza (Berlin: Language Science Press, 2016).

${ }^{168}$ Ibid., 6.

169 Ibid.

${ }^{170}$ Ibid.
} 
The results from the study backed up Fox's hypothesis. She found that there was a reduction of $14.4 \%$ in reading time for integrated titles compared to traditional subtitles and that, on average, the integrated subtitle viewers were looking at the subtitles for $47.5 \%$ of subtitle duration, rather than $51.6 \%$ for traditional subtitles, thus providing evidence that viewers who watched the film with integrated titles spent less time reading subtitles and more time exploring the images on-screen. She also found that participants watching the film with traditional subtitles tended to focus on the area where subtitles were appearing, even when subtitles were not visible on-screen. Other results concluded that the reaction times of the integrated title audience were much faster than those of the traditional subtitle audience. To measure the effectiveness of the integrated titles, Fox also asked her participants to rank the following statements from 1 (fully agree) to 4 (completely disagree):

- I could easily read all integrated titles.

- I received all necessary information through integrated titles.

- I would prefer integrated titles to traditional subtitles.

- I could spend more time exploring the image compared to traditional titles.

- Due to the integrated titles, I was aware of more details in the image.

- The integrated titles didn't cover important elements in the image.

- The integrated titles distracted me less from the image, compared to traditional subtitles.

The results of the survey showed that more than half of the participants agreed or fully agreed to all of the above statements, showing positive results in terms of reception and positive attitudes towards integrated subtitles. The overall results from Fox's study, both in terms of the eye-tracking data and the evaluation of the aesthetic experience, point to integrated subtitles as a viable alternative to traditional subtitles.

I have noticed very recently on New Zealand television that integrated subtitles have slowly started to appear, albeit in slightly different contexts. For example, in an advertising campaign for the 2017 edition of New Zealand Sign Language (NZSL) week, the NZSL advertisement had no spoken dialogue, but open captions were produced in the form of speech bubbles next to each actor's face, rather than appearing on the bottom of the screen (Figure 22a). This is not only the case in New Zealand, as a certain type of integrated subtitle can be found in the hit BBC television series Sherlock (2010present), where they are often used to convey text messages, emails etc. that the different characters are reading (Figure 22b). Although these are not subtitles for the dialogue present in the programme, there is a preference to place these integrated titles on-screen, as opposed to traditional subtitles or simply filming the message and projecting it. Integrated subtitles have also been used in mainstream cinema, as is the case in Jaume Collet-Serra's Non-Stop (2014), when the text messages sent to Liam Neeson's character, Bill Marks, are presented as mid-screen integrated subtitles (Figure 22c). In David Leitch 
and Chad Stahelski's John Wick (2014), the subtitles, translated from Russian, appear integrated into the image. All of these examples show that integrated titles are already making their way into mainstream media (Figure 22d).
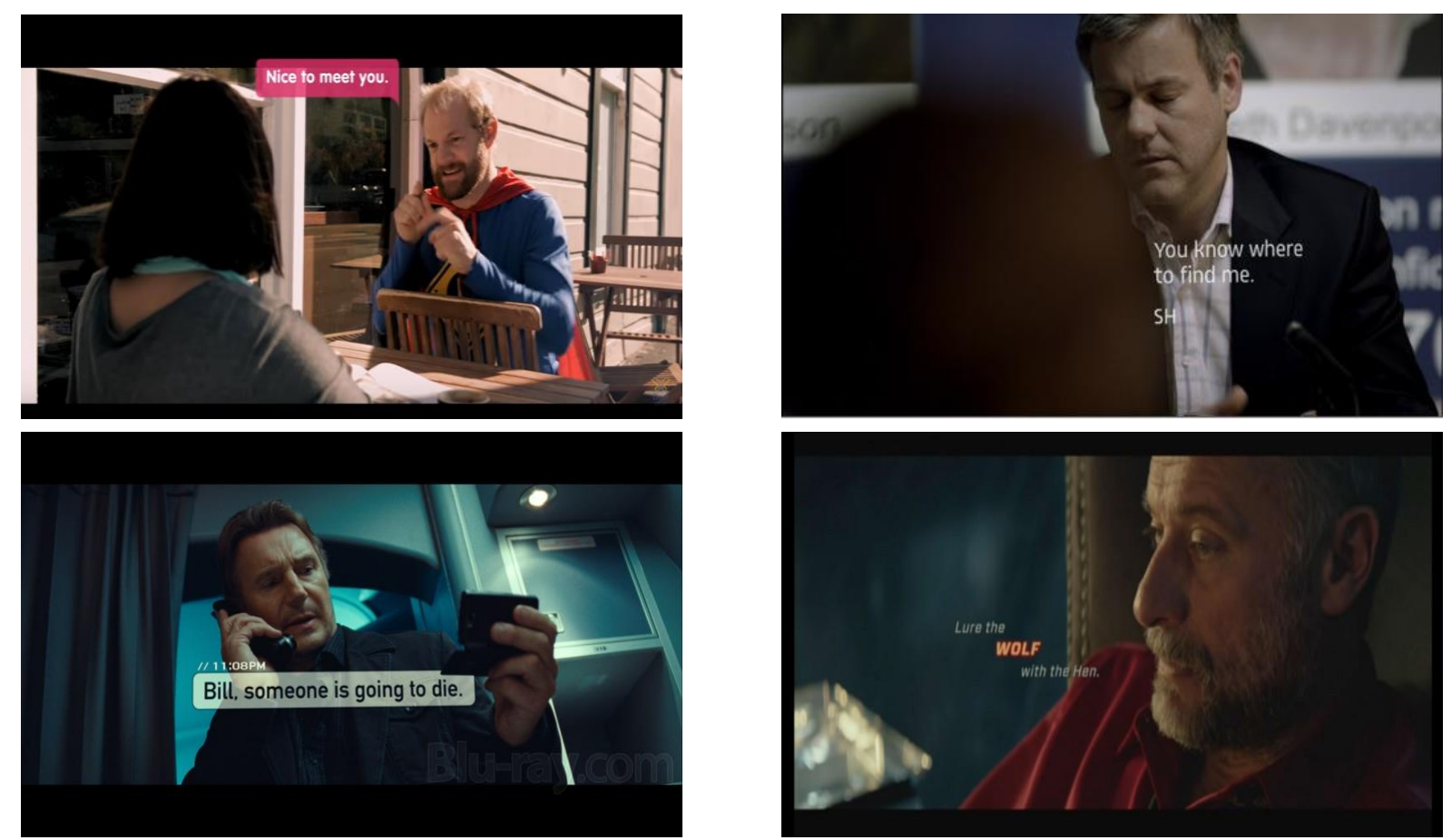

Figure 22(a-d): Examples of integrated subtitles on television and in film

\subsubsection{Implications on viewing experience}

Watching $\mathrm{AV}$ material requires the processing of information from three different sources (images, text and sound). The taxing nature of having to shift between text and image, and the fact that the viewer has to listen to a soundtrack in another language, are just a few of the concerns that viewers associate with watching subtitled content. Literature on subtitling can be rather contradictory as to whether these concerns are valid. In terms of the taxing nature of shifting from text to image, there are two sides to the argument. The first is that there must be a trade-off between visual processing and subtitle processing. ${ }^{171}$ When the viewer is focused on the film, rather than the subtitles, their comprehension of the subtitles will be limited, and where they focus on the subtitles rather than the film, they may miss particular important plot features:

\footnotetext{
${ }^{171}$ See Géry d'Ydewalle and J. Van Rensbergen, "Developmental Studies of Text-Picture Interactions in the Perception of Animated Cartoons with Text," in Knowledge Acquisition from Text and Pictures, ed. Heinz Mendl and Joel R. Levin (Amsterdam: Elselvier Science, 1989); Annie Lang, "Defining Audio/Video Redundancy from a Limited Capacity Information Processing Perspective," Communication Research 22, no. 1 (1995); Anne Treisman, "Strategies and Models of Selective Attention," Psychological Review 76, no. 3 (1969).
} 
Human communication research and cognitive psychology have been clear about the subject of parallel processing: Humans have poor parallel processing skills. Those skills are finite, fairly inflexible, and therefore quite limited in where and how they can be applied. Parallel processing becomes even more difficult when different messages are conveyed via different information channels, such as through visual and auditory channels. ${ }^{172}$

The other side of the argument is that visual processing and reading are so engrained in human nature and developed from a young age that the task can be considered, overall, effortless. ${ }^{173}$ As Gery d'Ydewalle and Ingrid Gielen propose,

When people watch television, the distribution of attention between different channels of information turns out to be an effortless process. Viewers seem to have developed a strategy that allows them to process these channels without problems and in which reading the subtitles occupies a major place. ${ }^{174}$

If the latter argument is the case, then there should be no subtractive trade-off between the visual processing of subtitles and the visual processing of image on-screen. An interesting consideration to take into account in this debate is that the ability to process several media of information at once could be considered a generational skill. In an article written for the New York Times in 1990, the creator of the TV network MTV, Robert Pittman, suggested that 'TV babies' have developed the ability to process parallel complex information, whereas older generations of Americans cannot:

In 1981, when developing MTV, we identified this profound difference [between generations]. The pre-TV adults are the "one thing at a time" generation. They read a magazine article straight through...then they make a phone call or watch TV. The TV babies, by contrast, seem to be happy processing information from different sources almost simultaneously. They really can do their homework, watch TV, talk on phone, and listen to the radio all at the same time. It's as if information from each source finds its way to a different cluster of thoughts. ${ }^{175}$

Given these contradictory beliefs as to whether or not a trade-off exists when processing visual and written information, Perego et al, undertook a study, adopting a methodological approach, which included eye movement testing, word recognition, and visual scene recognition, in an attempt to set the record straight. ${ }^{176}$ The study observed 41 undergraduate and postgraduate students from the Universities of Trieste and Pavia: 23 female and 18 male students with an average age of 25 . All subjects were native Italian speakers with normal, or corrected to normal, vision. The video that they were shown was taken from a Hungarian drama, Szerelmesfilm (Love Film), with Italian subtitles. None of the participants had

\footnotetext{
${ }^{172}$ Lori Bergen, Tom Grimes, and Deborah Potter, "How Attention Partitions Itself During Simultaneous Message Presentations," Human Communications Research 31 (2005): 314

${ }^{173}$ David LaBerge and S. Jay Samuels, "Toward a Theory of Automatic Information Processing in Reading," Cognitive Psychology 6, no. 2 (1974); Géry d'Ydewalle and Ingrid Gielen, "Attention Allocation with Overlapping Sound, Image, and Text," in Eye Movements and Visual Cognition, ed. Keith Rayner (New York: Springer New York, 1992).

174 "Attention Allocation with Overlapping Sound, Image, and Text," 425.

175 Robert Pittman, "We're talking the wrong language to 'TV Babies.'," New York Times 1990.

${ }^{176}$ Elisa Perego et al., "The Cognitive Effectiveness of Subtitle Processing," Media Psychology 13, no. 3 (2010).
} 
any knowledge of the Hungarian language. The participants were given two questionnaires: one to determine whether they paid attention to the subtitles, and the other a multi-choice questionnaire to see if they understood the gist of the film. This determined whether or not the students read the subtitles, and whether or not they understood the plot of the movie.

The next phase of testing was to determine subtitle recognition and scene recognition. Another multi-choice questionnaire was given to the students about the specific wording of target subtitles. Out of three subtitles provided that were all semantically close, the students had to choose which subtitle they thought was the one they had read previously on-screen. The scene recognition test was completed by showing the students freeze frames from both the film and other sources. They had to determine whether the frame had appeared during the video they had watched by pushing one of two keys on the computer: only 30 of the 60 frames that they were shown had actually appeared in the video. The freeze frames, like the subtitles, were very similar to action that actually occurred in the film.

The results proved that there was little to no comprehension loss on any part, whether it be comprehension of subtitles or the ability to recognise the images that appeared in the film. The students did however find it much harder to recognise the precise subtitles that were present in the film compared to the images: for the first part of the film, the students managed a $61 \%$ success rate, and for the second part a 70\% success rate. For the scene recognition test, the participants managed much more accurate results: $90 \%$ for the first part of the film and $87 \%$ for the second. The overall correlation between the two measures was not overly significant; however, there was a slightly positive correlation between subtitle recognition and gist comprehension. Interestingly, the study showed a rather large positive correlation between word recognition and scene recognition for the second part of the film.

Ultimately, the findings of this study were "at odds with the hypothesis of a trade-off between subtitle processing and scene processing, and consistent with the idea that watching a subtitled movie is cognitively effective." 177 Although this was just one study, it goes some way to dispelling some of the negative assumptions about subtitled programming. It would appear that it is actually in human nature to be able to process multiple sources of information simultaneously, which may well be influenced by generational attitudes to technology and their habits. Despite the fact that the viewer is processing multiple sources of information, this has no substantial implications on comprehension, and some viewers' reasoning for not watching or appreciating subtitled programming may indeed just be a preconception, rather than due to any pitfalls of subtitling itself.

\subsection{The theory and practice of subtitling: overcoming the challenges}

This following section draws from literature around both inter and intra-lingual subtitles, as both share similar concerns when it comes to the limitations of each practice. The biggest difficulties to this unique

\footnotetext{
${ }^{177}$ Ibid., 258.
} 
form of inter-semiotic translation, as acknowledged in all literature on the subject, are the pressures of time and space. The spatio-temporal pressures undoubtedly constrain and affect a subtitler's final decision. The subtitler is limited to a maximum number of characters on-screen at any one time; DíazCintas and Remael suggest that this figure is between 32 and 41 characters per line depending on the type-face and the size of font used, for a maximum of two lines per subtitle. ${ }^{178}$ Subtitles tend to appear in what is known as a 'safe area' on-screen, which is the area that subtitles will fit comfortably onscreen without taking away from the audience's viewing experience, traditionally at the bottom of the screen, as it is the least intrusive position (although as we have seen, other alternatives are still being explored). Díaz-Cintas and Remael argue that where there is only one line of subtitling, it should appear in the position that the second line of subtitles would normally occupy in a standard two line subtitle.

Depending on the medium in which the subtitles are being viewed, the distribution format, and therefore the associated restrictions of the subtitles can change. It is possible that three or four different versions of the subtitles are produced for each method of distribution on the market, whether that be cinema, television, video, DVD or internet. Cinema subtitles tend to have a higher density of words than their television counterparts - presumably due to the better definition of the subtitles on-screen, larger screen dimensions, and possibly the greater levels of concentration by the audience when watching a film at the cinema as opposed to at home on television.

Different conventions exist for different media; an example of this is in cinema subtitling, where subtitles tend to be centred on-screen. Generally DVDs also have longer subtitles due to the audience's ability to rewind the programming at will. Movies screened in theatres tend to be at a frame rate of 24 frames per second, whereas television is usually broadcast around 25 frames per second. The film must therefore be sped up when converted across mediums, leading to the reduction of time available for subtitles to remain on-screen. "Generally speaking," Díaz-Cintas and Remael remind us, "a movie lasting approximately 90 minutes contains 900 subtitles in the cinema, 750 on video or DVD, and 650 in the televised version." ${ }^{\prime 179}$

Secondly, the translation is affected by, and therefore must be catered to, the reading rates of an audience. Zoe De Linde and Neil Kay propose that the reading speed of an average viewer is between 150 and 180 words per minute, or 70-74 characters in 6 seconds according to Díaz-Cintas and Remael. ${ }^{180}$ This limitation, on top of the already existing spatio-temporal constraints, place a huge burden on the subtitler trying to remain as faithful to the original content as possible as the subtitles must also remain on-screen for a long enough duration for the audience to firstly read, and then be able to comprehend the spoken dialogue. ${ }^{181}$ Some subtitling companies and practitioners follow the ' 6

\footnotetext{
${ }^{178}$ Díaz-Cintas and Remael, Audiovisual Translation: Subtitling, 9.

${ }^{179}$ Ibid., 25.

${ }^{180}$ Zoe De Linde and Neil Kay, The Semiotics of Subtitling (Manchester: St Jerome Publishing, 1999), 6.; Díaz-Cintas and Remael, Audiovisual Translation: Subtitling, 23.

${ }^{181}$ Peter Fawcett argues however that there seems to be no basis in the literature on subtitling as to where these numbers have come from, and that the 'average viewer' is presumably House's 'educated middle-class speaker' classification. He also argues that there is fluctuation within academic research as to the agreed reading rate of an audience from 8 characters
} 
second rule', which is the calculated time it would take for a viewer to read two lines of dialogue with 35 to 37 characters in each line. If the subtitles remain on-screen for too long, the reader will have the chance to re-read them, which can break the audience's reading rhythm. ${ }^{182}$ Conversely, Ivarsson and Carroll argue that, "as a general rule, the minimum time for even a very short subtitle on a television screen is at least one and a half seconds and the maximum time for a full two liner should not exceed five to six seconds." ${ }^{" 183}$ Díaz-Cintas and Remael, however, propose that the minimum duration of a subtitle should be one second or 24 to 25 frames. ${ }^{184}$

Peter Fawcett also highlights the fact that subtitles need to have some degree of timesynchronisation between spoken utterance and written title (as is the case in dubbing where synchrony is key to the success or detriment of the translation), which relates to the above two constraints and leads to the need to reduce dialogue as will be discussed in the upcoming pages. There is some debate within the field itself as to the exact conventions that should be followed - whether the subtitles should appear slightly before or slightly after the dialogue starts, whether the subtitles should be left on for the duration of the utterance, or whether it should remain on screen after the dialogue has been finished. ${ }^{185}$

De Linde and Kay also suggest that the effects of camera angles and cutting, particularly when a speaker is talking off-screen, can also be a difficulty with which a subtitler may need to contend. ${ }^{186}$ Normally a viewer is able to determine who is speaking by a character's tone of voice, accent or other distinguishing features when the camera is not on them. However, with subtitles this is not the case. This can often leave a viewer feeling confused and frustrated. It can also be difficult for a viewer who is unfamiliar with the SL to pick out regional varieties and accents, particularly if the subtitles do not adequately reflect the variation in language that can often detract from the viewing experience. Representing multiple voices on-screen is therefore a very difficult challenge with which a subtitler must contend. Subtitles, being written text, are presented sequentially and therefore only express dialogue exchanges that occur one after another and the 'layering' of speech cannot be recreated in text form. When the dialogue is overlapping and spoken at a fast pace, the subtitler must not only keep up with the dialogue, but also be able to portray quickly and effectively who is speaking. The 'spotting' or 'timing' of the subtitles becomes crucial in this instance as the viewer is otherwise unaware of who is speaking and relevant dialogue may be lost. Where there are two speakers, subtitles are often introduced with a dash, however this tends to limit each speaker to only one line of subtitles per exchange.

per second to 70-72 characters per title to 80 characters depending on which study one looks at. Peter Fawcett, "The Manipulation of Language and Culture in Film Translation," in Apropos of Ideology: Translation Studies on Ideologyideologies in Translation Studies, ed. Maria Calzada Perez (London: Routledge, 2014), 147-48.

182 Díaz-Cintas and Remael, Audiovisual Translation: Subtitling, 23. This figure is predominantly used when calculating reading speeds for audiences on television. The reading speed in other mediums tends to be slightly faster. Cinema subtitling, for example, uses a maximum of 41-43 characters per line.

${ }^{183}$ Ivarsson and Carroll, Subtitling, 65.

${ }^{184}$ Díaz-Cintas and Remael, Audiovisual Translation: Subtitling, 90.

${ }^{185}$ Fawcett, "The Manipulation of Language and Culture in Film Translation," 148.

${ }^{186}$ Linde and Kay, The Semiotics of Subtitling, 32. 
Another challenge that is specific to subtitling is the necessity to remain as faithful as possible to the images that are appearing on-screen. Because image and text must work in tandem due to subtitles' nature as 'diagonal translation', references cannot always be changed to accommodate the TC. For certain culturally specific references a subtitler can struggle to find an accessible alternative, particularly if the references that appear on-screen are ingrained in a culture (for example money, food, native animals, national icons, places etc.). In a similar vein, remaining faithful to the original dialogue and soundtrack features can prove problematic: rendering the emotion of $\mathrm{AV}$ material (at both a semantic and sentence level) in text form is very difficult given that aural features such as emotion, intonation, volume, irony and other characteristics of speech are difficult to convey in written form. ${ }^{187}$ What's more, related to Fawcett's discussion on the timing of subtitles, the subtitles cannot pre-empt the dialogue - the art and thought process behind script-writing must be respected. For example, some dialogue will look to build suspense, and subtitles should not interfere with that process. ${ }^{188}$

Representing multiple languages, dialects or accents on-screen can also pose a particular challenge for the subtitler due to the fact that oral elements are very difficult to render in written text. Moreover, multilingualism in film is often used as a narrative device:

[1]anguage has the power to symbolise both understanding and misunderstanding either by covering common ground (normally using the same language) or by emphasising Otherness (usually by confronting different languages). Languages are clear signposts of geographical and political borders and have the potential to portray the different social, cultural and personal dimensions of the various characters. ${ }^{189}$

The subtitler will often have to decide whether to adopt a foreignization approach, to retain the original language variation that is present, or a domestication approach in which the language variation is simply removed. ${ }^{190}$ Other options have been discussed by Perteghella, presented earlier in chapter two. As a general rule, however, Díaz-Cintas argues that a second or third language should be left in the SL when the dialogue serves no narrative function: the general rule is to translate solely the main language of the ST. ${ }^{191}$

It is also a difficult challenge for a subtitler to try and retain the oral 'flavour' of the film, while switching between semiotic spheres, particularly given that spoken and written language can vary so greatly. Subtitling in its very nature is not merely between languages, but also as a translation of the oral into the written. It selects, condenses and organises discourse into discrete syntactically, spatially

\footnotetext{
${ }^{187}$ Suzanne Ayonghe, "Translation of Emotions in Subtitling of the Film Paris àtout prix," International Journal of English Language \& Translation Studies 4, no. 2 (2016). Allira Hanczakowski, "Translating Emotion from Italian to English: A Lexical-Semantic Analysis," The AALITRA Review 13 (2018).

${ }^{188}$ Bogucki, "The Constraint of Relevance in Subtitling," 77.

189 Jorge Díaz-Cintas, "Dealing with Multilingual Films in Audiovisual Translation," in Translation - SprachvariationMehrsprachigkeit: Festschrift für Lew Zybatow zum 60. Geburtstag (English, German and Russian Edition), ed. Wolfgang Pöckl, Ingeborg Ohnheiser, and Peter Sandrini (2011), 216.

${ }^{190}$ Claire Ellender, Dealing with Difference in Audiovisual Translation: Subtitling Linguistic Variation in Films, ed. Jorge Diaz-Cintas, vol. 14, New Trends in Translation Studies (Bern: peter Lang, 2015), 15.

${ }^{191}$ Díaz-Cintas, "Dealing with Multilingual Films in Audiovisual Translation," 220.
} 
and temporally delimited units. ${ }^{192}$ Hence, the syntax, lexicon and register can vary greatly not only across languages, but also across modes of communication. Díaz-Cintas and Remael also support this argument: "The transition from oral to written mode obviously means that some of the typical features of spoken language will have to disappear, no matter what sub-genre a dialogue belongs to." 193

All of the above challenges emphasise how subtitling must be treated as a multi-disciplinary, multi-modal, inter-semiotic form of translation: the subtitler must draw on particular skills and expertise across a wide range of translation disciplines to create high-quality subtitles. While having to deal with the technical limitations of subtitling, the subtitler must also negotiate the content specific challenges, for example humour, song, poetry and cultural specific content that could all be present in a film, yet each of these content-specific examples have their own place within translation studies literature. As Bogucki confirms,

[w]hile all translation is necessarily constrained, subtitling is constrained in a more intricate and complex way. The technical restrictions on the length of the subtitle, the additive nature of subtitling and the visual complementation of the text on screen mean that the traditional approach to translational constraints, though an excellent starting point, cannot possibly account for all the facets of this unique type of interlingual translation. ${ }^{194}$

I have so far outlined some of the field-specific challenges that are ever present within the field of AVT, particularly subtitling. While daunting, these challenges must be faced in order to produce quality subtitles. Luckily, several techniques have been developed that a subtitler can bear in mind when undertaking a translation project. Next, I will outline these techniques, providing examples of how they can be used in practice, before undertaking my translation of Fantozzi.

\subsubsection{Subtitling techniques: time, space and content restrictions}

Subtitling is conditioned by a vast number of variables, so much so that in order to establish an objective, valid and incontestable classification of the norms that govern it is nearly impossible. Among the factors that most influence the strategies adopted, are the nature of the original text, the type of audiovisual product to subtitle, the target audience, the structure of the languages involved and the diverse level of affinity between the source and target languages and cultures.

- Alessandra Catania ${ }^{195}$

\footnotetext{
192 O'Sullivan, Translating Popular Film, 102.

${ }^{193}$ Díaz-Cintas and Remael, Audiovisual Translation: Subtitling, 61.

194 Bogucki, "The Constraint of Relevance in Subtitling," 73.

${ }^{195}$ Catania, "La traduzione audiovisiva: techniche, strategie e difficoltà: proposta di traduzione di quattro articoli tecnicoinformativi," 27. [My translation]

Original Italian: "La sottotitolazione è condizionata da un numero vastissimo di variabili, tanto che stabilire una classificazione oggettiva, valida e incontestabili delle norme che la governano risulta quasi impossibile. Tra $\mathrm{i}$ fattori più influenti sulle strategie utilizzate vanno ricordati la natura del testo originale, il genere del prodotto audiovisivo da sottotitolare, i destinatari, la struttura delle lingue coinvolte e il diverso grado di affinità tra lingua e cultura sorgenti e lingua e cultura riceventi."
} 
Given the demanding nature of subtitling, specific techniques have been developed by a variety of scholars to help accommodate in particular the spatio-temporal constraints illustrated earlier, as well as techniques to aid the rendering of cultural content from ST to TT. Díaz-Cintas and Remael call their suggestions text reduction strategies, the three main techniques of which are condensation, reformulation, and omission. However, there are several sub-categories of methodology within these three practise, these reductions can be made at a word level, or a sentence level. ${ }^{196}$ Other scholars, such as Jan Pedersen and Henrik Gottlieb, present equally useful strategies centred on translating particular cultural references, which will also be discussed. It is important to emphasise that there is no one 'trick' or technique that a subtitler can use; but rather the dialogue should be looked at on a case-by-case basis. As Ayonghe reminds us, "the wrong use of AVT techniques and strategies leads to communication problems for the receiving audience. For example, omissions, loss and lack of emotions in the TL of the audience, makes the reading of subtitles very difficult and incomprehensible." ${ }^{197}$ The purpose of this discussion is to be able to use the suggestions present in existing literature on subtitling to adequately convey the original content of Fantozzi in translation.

\section{Díaz-Cintas and Remael's strategies}

The examples provided will illustrate these strategies and show the benefits of each individual technique proposed by Díaz-Cintas and Remael. They have been taken from the translation of Fantozzi that appears in full in Chapter four as the practical and creative component to this Translation Studies project. The examples will be presented in three columns: the first is the original Italian dialogue, the second is a literal translation, and the third column will show the subtitle(s) that I have produced using the particular techniques suggested in the literature. The number of characters are presented in each column to show exactly how much space a subtitler can save by using these varying techniques. It is personal philosophy that if the dialogue does not require simplification or reduction, the subtitles should remain as close as possible to the original dialogue; this is what I have attempted to adhere to throughout the translation project - where the translation fell within the predetermined restrictions they were left unchanged.

\section{Condensation and reformulation at a word-level}

There are several ways in which reworking subtitles can occur at a word level to save space when subtitling. The first is to simplify verbal periphrases: because of the longer nature of idiomatic expressions and colloquial language, a subtitler can replace such occurrences with more simplified solutions provided the TL can oblige. An example of this could be the following dialogue taking place

\footnotetext{
${ }^{196}$ Díaz-Cintas and Remael, Audiovisual Translation: Subtitling, 145-66.

197 Ayonghe, "Translation of Emotions in Subtitling of the Film Paris àtout prix," 178.
} 
when Fantozzi is taking the bus al volo in order to avoid being late for work, pulling everyone off the bus as he attempts to jump on it. Instead of a literal translation, by simplifying the content, the same message is conveyed:

\section{Example from Fantozzi:}

Chi è stato il primo? Si può sapere chi è stato il primo? (57)

\section{Literal Translation: $\quad$ English Subtitle:}

Who was the first? Can I know who the first was?

\section{I want to know who started it! (30)}

A subtitler can also generalise. Generalisations are particularly useful to replace enumerations, or lists of items. However, they can have the unwanted effect of changing the style of the speech, but are a useful tool to save space. The example below, when Fantozzi gives the last of his money to one of his superiors so he can purchase a drink from the vending machine, generalizes 'one hundred lire' to 'coins'. In this example the same message is conveyed without any loss of meaning, and is also relevant given that the currency lire would probably have very little significance for a modern day English speaking audience, as will be discussed later.

\section{Example from Fantozzi: \\ Literal Translation: \\ English Subtitle: \\ Buongiorno, signor Direttore. \\ Good morning, Mr Director. \\ One hundred lire? Right \\ Good morning Director. Coins? \\ Cento lire? Subito, aspetti. \\ away, please wait. (69) \\ Right away, one moment. (53)

The subtitler also has the option of finding a synonym or an equivalent. The easiest way of doing this is to find a shorter synonym in the TL. This can, however, subtly change the content of the text, as synonyms are rarely perfect equivalents of each other and can have varying registers. In the example below, where Fantozzi's colleagues are talking in the background of the scene, replacing difficile (difficult) with 'hard' provides a close enough alternative from a semantic perspective, although the connotation that is implied by the original, the degree of difficulty, is partially lost.

\section{Example from Fantozzi:}

La cosa non è difficile. (24)

\section{Literal Translation:}

The thing is not difficult. (27)

\section{English Subtitle:}

It's not hard. (14)

Another way to shorten a subtitle is to change compound tenses to simple tenses. In some instances, the compound tense can easily be replaced, cutting out the auxiliary verb altogether. This can only happen when it is not necessary to say explicitly that one action occurred before another. In the example below, the voice-over is referring to the debt accrued by Fantozzi and Filini. The tense used is the past 
conditional, however in the subtitles this has simply been changed to the future tense. Because there is a historical displacement, 1984 has also been changed to "for at least 10 years" given that the film was released in 1975, this does lengthen the subtitle compared to a literal translation, but is necessary in conveying the appropriate information to the target audience.

\section{Example from Fantozzi: $\quad$ Literal Translation: English Subtitle: \\ [c]he avrebbero perseguetati fino that would have burdened that will burden them for at least al 1984. (40) \\ them until 1984. (41) \\ 10 years. (44)}

Changing word classes can be useful to shorten the length of a subtitle as well. Turning a verb into a noun, an adjective into a verb, a verb into an adverb or an adjective into a noun, to name a few examples, can allow the subtitler to create shorter alternatives. The example below, an exchange between the administrator at Fantozzi's workplace and his wife, Pina, the noun decessi (deceased) changes to the verbal phrase 'has anyone died'.

\section{Example from Fantozzi: $\quad$ Literal Translation: $\quad$ English Subtitle: \\ Lei ha decessi? (15) Do you have any deceased, Has anyone died, Ma'am? ma'am? (32) (23)}

Using contractions is a simple way to decrease the number of characters that appear on-screen. Contractions are possible in many languages; they are particularly common in English where the subject and the verb are often contracted (I'd, you've, he's etc.). While it does not save the subtitler many characters, it is a useful means of shortening the subtitles that are slightly above the upper limit of characters allowed on-screen.

\section{Example from Fantozzi: $\quad$ Literal Translation: $\quad$ English Subtitle: \\ Non resisto, mi sembra di I cannot take it, I am going I can't take it, I'm going crazy. impazzire. (36) crazy. (35)

Depending on the language, the subtitler could employ a strategy that involves the use of pronouns. Given that pronouns are often a shorter alternative to the noun that they are replacing, provided the context allows then they are an acceptable replacement in the subtitles. As can be seen in the example below, the pronoun 'him' replaces the noun 'the accountant', saving several characters in the English subtitles. 


\section{Example from Fantozzi:}

Attenti, attenti al ragioniere!

(31)

\section{Literal Translation:}

Watch out, watch out for the

accountant! (40)

\section{English Subtitle:}

Watch him, watch him!

(21)

\section{Condensation and reformulation at a sentence-level}

Similarly, the subtitler can use techniques that reduce the time taken for the viewer to read the subtitles, as well as the space taken up by subtitles at a sentence or clause level. This can be achieved in several ways, however, in slight contrast with reformulation at a word level, changing the structure or words within a sentence can lead to the loss or addition of certain connotations to the ST dialogue. The subtitler must tread carefully, but in some circumstances, this type of sentential negotiation is unavoidable.

The first sentence-level technique that a subtitler can put into practice is to change negations into affirmations, or indirect questions into direct question or statements. Changing the way in which the sentence is structured can have the positive effect (for subtitling purposes) of reducing the length of the dialogue. Removing any negative markers from the sentence, such as 'not', creates more space. This is particularly useful for translations from a language such as Italian, where double negatives are not uncommon, to a language such as English where they do not exist. In the first example below, we see a change from a direct question, to a statement; in the second there is a change from a negation to an affirmation:

\section{Example from Fantozzi: $\quad$ Literal Translation: English Subtitle: \\ $\begin{array}{lll}\text { Facciamo un brindisi? Attento. } & \begin{array}{l}\text { Shall we have a toast? Be } \\ \text { careful. (34) }\end{array} & \text { Let's have a toast. Careful. } \\ \text { (30) } & \text { (28) }\end{array}$}

\section{Example from Fantozzi:}

Non si può rinviare la discussione? (35)

\section{Literal Translation:}

Can we not postpone the discussion? (35)

\section{English Subtitle:}

Can we postpone this discussion? (32)

Another way of creating space at a sentential level is to simplify indicators of modality. The use of modal verbs as auxiliaries is quite common to indicate an aspect of uncertainty, possibility or probability, particularly for address or enquiry that is more formal. By removing these, the subtitler can make space. However in doing so some of the emphasis of the original statement can be lost. In the 
example provided below, the modal verb volere (to want) has been removed, although context still implies the inherent desire and therefore it is necessary to convey this explicitly.

\section{Example from Fantozzi: $\quad$ Literal Translation: $\quad$ English Subtitle: \\ Vogliamo andare al bar. (23) We want to go to the bar. Let's go to the bar. (20)}

A subtitler can also change direct speech into indirect speech in order to maximise space. This can eliminate the 'presentative' verb of the ST without drastically changing its meaning. It also minimises the punctuation of the subtitles as speech marks are not included, reducing the number of characters onscreen, while also having the positive effect of making the subtitles more readable to the audience.
Example from Fantozzi:
Literal Translation:
English Subtitle:
Eh cioè praticamente dice che...
She basically said... Well...
She basically said... Insomma ha detto: "Buon She said: "Buon apettito". Enjoy your meal! apettito". (67)

Reducing the number of characters on-screen can also be obtained through changing the subject of the sentence or changing the passive voice to active (or vice-versa). Particularly in a language such as Italian, where the passive voice is more commonly used than in English, changing from passive to active can be a good way of creating space. Depending on the SL, translating from active to passive may have a similar effect. In the case of Fantozzi we have an example from where Filini has invited Fantozzi to the New Year's work party, to which Fantozzi responds using the passive voice, saying that he has been invited by his brother-in-law. By changes the subject of the sentence from Fantozzi to the brother-inlaw, there is a retention of characters.

\section{Italian example: \\ Quest'anno sarei invitato da mio cognato... (43)}

\section{Literal Translation:}

This year I have been invited by my brother-inlaw...(53)

\author{
English translation: \\ This year my brother-in-law \\ invited me... (41)
}

The reduction of compound/complex sentences into simple sentences can also result in space saving, although this is not the primary effect of this technique. By cutting up long chunks of text into smaller pieces, it has the effect of making the subtitles more readable as the audience does not have to rely on memory to understand the entire subtitle. While the example below, taken from the scene in which 
Fantozzi and Signorina Silvani dine at a Japanese restaurant, does not reduce the number of characters greatly, the reduced subtitles become more manageable for the audience.

\begin{tabular}{|c|c|c|}
\hline Example from Fantozzi : & Literal Translation: & English Subtitle: \\
\hline $\begin{array}{l}\text { Le regole dei ristoranti } \\
\text { giapponesi sono tremende. La } \\
\text { prima è che il personale parla }\end{array}$ & $\begin{array}{l}\text { The rules of Japanese } \\
\text { restaurants are unbearable. } \\
\text { The first is that the wait }\end{array}$ & $\begin{array}{l}\text { The rules of Japanese } \\
\text { restaurants are unbearable. }\end{array}$ \\
\hline $\begin{array}{l}\text { esclusivamente il giapponese } \\
\text { stretto. (122) }\end{array}$ & $\begin{array}{l}\text { staff speak exclusively in } \\
\text { Japanese. (115) }\end{array}$ & $\begin{array}{l}\text { The first rule: the wait staff } \\
\text { speak exclusively in Japanese. } \\
\text { (110) }\end{array}$ \\
\hline
\end{tabular}

The final technique that falls under the category of reformulation at a sentence level is to merge phrases from the ST. This also has the added effect of simplifying the dialogue for the target audience as can be seen in the following example, from the same scene, where the content of two sentence is merged into one.

\section{Example from Fantozzi : $\quad$ Literal Translation: English Subtitle:}

Il mio cagnolino adorato. Io, My beloved little dog. I I couldn't live without senza di lui, non posso vivere. could not live without him. my beloved little dog. (47) (61)

\section{Omission}

Another way to create space on screen is by simply omitting particular words or phrases from the dialogue. This decision is always dictated by the relevance of the content to the plot of the AV material. Words that tend to disappear from dialogue, particularly from English, are: question markers (which can simply be included in the grammar of the TL); modifiers, usually adjectives or adverbs (depending on the importance of the modification); phatic words such as 'anyway' and 'you know' that mark spoken language; interpersonal elements such as greetings or interjections; formulaic expressions of politeness; hesitations and false starts.

Entire sentences or clauses may be left out, although this is not a recommended practise. If the sentence presents little information, for example, isolated sentences, it may not require a subtitle. As we saw in the discussion on condensing and reformulating, phrases can also often be absorbed into other subtitles rather than being deleted completely, omitting yet not compromising the content. It cannot be emphasised enough that the only content that should be omitted needs to bear little relevance to the overarching plot of the film, or as a very last option where content is impossible to translate. Omission plays a very important role in AVT, as Díaz-Cintas explains, "The degree of excellence of an 
audiovisual translation has to be measured against what the subtitlers decide to translate as well as what they decide not to translate because they prefer to exploit other semiotic dimensions of the film." 198

\section{Pedersen's strategies}

Jan Pedersen also proposes a variety of strategies, which are based on the taxonomies of many scholars, including Lawrence Venuti, Henrik Gottlieb and Andrew Chesterman to name just a few, who conduct research in the fields of both translation studies and/or subtitling. These strategies have a slightly different focus to those of Díaz-Cintas and Remael, more so on the content of AV material and the rendering of cultural content in the TT, with issues on reduction of characters or text being secondary. $\mathrm{He}$ is, however, hesitant to call them strategies, as the term is rather misleading given that the rendering of what he defines as extra linguistic cultural references (ECRs) may not involve a translation at all. Moreover the term 'strategy' implies a global, rather than micro, level decision (for simplicity here, however, we will use 'strategies' as a blanket term). Instead, he proposes that 'translation tactic' is a more accurate name for describing how ECRs may be rendered in the TL. Nevertheless, he proposes six main headings for these 'tactics': retention, specification, direct translation, generalisation, substitution and omission. ${ }^{199}$

\section{Retention}

This strategy, or 'tactic' allows for cultural references that are present in the ST to be retained in the TT, in other words, it is similar to Venuti's 'foreignization' approach or Friedrich Schleiermarcher's notion of taking the reader to the source culture. He proposes that these could be further subdivided into complete retention or TL adjusted retention. He explains that while this technique is common practise among translators, and in particular subtitlers, as it requires very little effort, it does have its flaws when the ECR is monocultural and the reference is not accessible to the target audience.

An example of retention in the translation of Fantozzi is in the Magnolia diet clinic where the names of traditional Italian dishes are retained (the difficulties associated with the translation of this scene will be discussed in detail in chapter 4). In short, the audience has some knowledge of the dishes present in the ST, and thus these references have been retained through a mixture of both complete retention (for 'tagliatelle alla boscaiola' and TL adjusted retention (for 'Four cheese pasta', 'Carbonara' and 'Bolognese'):

\footnotetext{
198 Jorge Díaz-Cintas, "The value of the semiotic dimension in the subtitling of humour," in Aspects of specialised translation, ed. Lucile Desblache (Paris: La Maison du Dictionnaire, 2001), 189-90.

199 Jan Pedersen, Subtitling Norms for Television: An Exploration Focusing on Extralinguistic Cultural References (Amsterdam: John Benjamins, 2011), 70-103.
} 
Subtitle

No. and

Timecode

0853 - Tagliatelle alla boscaiola.

01:05:41:14 - Paglia e fieno ai 4 formaggi.

01:05:44:16

0854

01:05:44:18 - Rigatoni alla bella Bologna.

01:05:48:21

$0853 \quad-$ Tagliatelle alla boscaiola.

01:05:41:14 - Paglia e fieno ai 4 formaggi.

01:05:44:16
English Subtitles

- Tagliatelle alla boscaiola!

- Four cheese pasta!

- Carbonara!

- Bolognese!

- Tagliatelle alla boscaiola!

- Four cheese pasta!

\section{Specification}

Specification, also referred to by other scholars as explicitation, is where the SL is retained but additional information is given to the viewer that is not present in the ST. Examples of such a strategy could be spelling out an acronym or abbreviation, or adding someone's first name to the subtitles to ensure that the audience is aware of the referent. The purpose of this strategy is to remove any ambiguity that may be present for the viewer. As with any strategy there is quite often a trade-off, in this case the length of text will inevitably grow, meaning that the technique can only be used in certain circumstances. This strategy could also be seen as patronizing to the audience, so a subtitler must tread with caution when applying it. In Fantozzi, this strategy has been used for a particular cultural and historical reference, GIL, used to describe the clothing worn by Fantozzi during this particular scene on the tennis court. GIL is a reference to Gioventù Italiana del Littorio, the youth movement of the Italian fascist party. It has therefore been replaced with "Fascist Youth" as this effectively spells out what GIL represents:

Subtitle

No. and

Timecode

0791

01:00:54:15

01:00:57:08
Original Italian

Fantozzi: maglietta della "GIL",

(5)

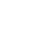




\section{Direct translation}

Pedersen refers to his next technique as direct translation, although others, including Díaz-Cintas and Remael, refer to it by other means such as literal translation, or calque. The purpose of this technique is to replicate the ST content in the TL, that is, no content is added, changed or subtracted, nor are any connotations added to or taken away from the original content. This strategy is particularly useful for proper names, or, as Pedersen puts it, for names constructed by common nouns such as companies, official institutions or the names of electronic gadgets etc. A prime example of where this technique is used in this project is right at the beginning of the film when Pina is calling Fantozzi's workplace in an attempt to find out where he has been for the last 18 days. The rather long and complex company name in Italian is made up of a variety of partial nouns, which translate roughly in English to "Italian", "petrol", "cement", "thermal", "textile", "pharmaceutical", "metal" and "chemical". Each of these has been translated and joined, just like the original Italian, as partial nouns forming a long-winded company name:

\section{Subtitle No. Original Italian English Subtitles \\ and \\ Timecode}

0004

00:00:15:06

00:00:18:03

00:00:15:09

00:00:17:22
Parlo con lo Spettabile Centralino della illustre Società

"Italpetrolcemeter motessilfarmometalchimica"?
Am I speaking to the respected
call-centre of the illustrious

"Italpetrolcemethermotextil

pharmometalchemical"?

\section{Generaliation}

This strategy, as the name would suggest, generalises content from a specific reference to something more general. This is done in one of two ways: either the item is replaced by a superordinate or is paraphrased. With this strategy the 'uniqueness' of the original text is lost, particularly when using a superordinate, however the content becomes more accessible to the target audience. In the case of paraphrasing, the subtitler may well be able to retain a particular connotation, however in doing so the subtitlers will inevitably be slightly longer. This technique is also proposed by Díaz-Cintas and Remael, which we saw earlier in the discussion when looking at the translation of "cento lire" into "coins". 


\section{Substitution}

Substitution of content can happen in a variety of ways. For example, it can be replaced by a different reference from the source culture (SC), with a different reference from the TC or by something that works in the context of that particular scene or dialogue (also known as situational substitution or, I would argue, a case of intertextuality). The reference may also come from a 'third party' culture, so to speak, where a reference is used that can be understood by both the SC and TC. This strategy can remove any exoticisms from the original content and replace them with more neutral terms, or alternatively substitution can cause the references that are used to replace the original ones to add new connotations to the ST: either way a subtitler must tread with caution when using this particular technique. The subtitler must also be wary of not creating a credibility gap, where the translated content is so far removed from the original that the TC is put off by it. A prime example of this in Fantozzi is at the end of the diet therapy scene, where Fantozzi escapes by sawing his way out of the cell. The dish he orders is known as "Spaghetti alla Montecristo", a reference to the Count of Montecristo. In my translation, this dish has been substituted with the name of a real type of fish, the "filefish", as it is arguably unlikely that the English speaking audience of today would immediately understand this intertextual reference.

\section{Subtitle \\ No. and \\ Timecode}

0887

01:08:23:08

$01: 08: 27: 18$

0888

01:08:27:20

01:08:30:12

\section{9}

01:08:30:14

\section{Quella stessa notte, Fantozzi firmò due} cambiali giganti.

01:08:33:21

\section{English Subtitles}

\section{That same night, Fantozzi \\ signed two enormous cheques.}

\section{He bought the most expensive dish on the тепи:}

Filefish Spaghetti.

\section{Omission}

Finally, just like Díaz-Cintas and Remael, Pedersen also makes the case for omitting content, arguing that it "can be the most target-oriented strategy available, as it stops a problematic foreign item from entering the TT in any form at all. ${ }^{201} \mathrm{He}$ also claims that it is the easiest technique for a subtitler to apply, given that it involves doing nothing. However he does highlight the need to use omission

\footnotetext{
${ }^{201}$ Pedersen, Subtitling Norms for Television: An Exploration Focusing on Extralinguistic Cultural References, 96.
} 
responsibly and that alternative strategies should be tested before settling on omitting content. Omission is a particularly important strategy given the ever-present spatio-temporal pressures inherent to subtitling, and as a result, as summarised by Katja Pelsmaekers and Fred Van Eesien, parts of language that can be omitted from subtitles include: ${ }^{202}$

i. current expressions with an international character;

ii. interjections and formulae of greeting, politeness, affirmation, negation, amazement, exclamation; question tags;

iii. forms of address with a proper noun;

iv. forms of address with a common noun with a particular sound;

v. incomplete sentences;

vi. explanations already known by the viewer, or explanations that are clear from the image;

vii. phatic dialogue;

viii. repetitions that are clear from the context.

An important, and somewhat controversial, example of omission that takes place in Fantozzi is simply leaving out his, and many of his colleagues, formal title (the removal of the common noun). In Italian, it is not uncommon to address someone by their occupation (in the case of Fantozzi, his title is 'ragionier' or 'accountant'). Because this does not occur in English, I have decided to leave it out as to not alienate the English speaking audience. Referring to the characters by their title occurs throughout the entire film, where I believed a title was important, I replaced 'ragionier' with ' $\mathrm{Mr}$ ', or alternatively I either omitted it completely, or replaced it with the name of the character themselves. During the football match scene, for example, I have decided to leave the title out altogether:

\section{Subtitle No. Original Italian English Sulbtitles \\ and \\ Timecode}

0266

Ragioniere, passi la palla!

Pass the ball!

00:20:37:16

$00: 20: 38: 21$

${ }^{202}$ Katja Pelsmaekers and Fred Van Eesien, "Subtitling Irony," The Translator 8, no. 2 (2002); ibid. 
Henrik Gottlieb, one of the founding fathers of research in audiovisual translation, has also provided a variety of different strategies that can be put in place in the translation of audiovisual material. ${ }^{203}$ While these strategies were developed in 1992, they are still relevant. He developed ten distinct strategies: expansion, paraphrase, transfer, imitation, transcription, dislocation, condensation, decimation, deletion and resignation. As some of these strategies are also proposed by Díaz-Cintas and Remael and by Pedersen, I will only provide examples for the strategies not yet discussed.

\section{Expansion}

The idea of expansion is that the meaning of the original content is retained through an expanded translation. That is, the translator must provide extra information to the viewer in order for the content to be adequately rendered across languages. This strategy is the same as what Pedersen calls specification, as discussed earlier.

\section{Paraphrase}

Paraphrase is a technique whereby the language is altered somewhat to achieve equivalence. This strategy is predominantly used for longer segments of dialogue that need to be broken down, as well as particular idioms that may not be able to be replicated across languages. Again, Pedersen supports this strategy under the title of generalization.

\section{Transfer}

Through transfer is the content of the ST can simply be translated word-for-word across languages and no alterations need to be made in order to adequately convey the original message. Petersen presents this as Direct Translation.

\section{Imitation}

Imitation is a fairly rare strategy used particularly with proper names, titles, quotations in other languages, greetings, song lyrics etc. The content of the original language is rendered through identical segments of text in the TL. In a sense, it is equivalent to non-translation, retention, or a foreignization approach. An example of this from Fantozzi would be the use of the Italian phrase "Bravo!", which can

\footnotetext{
${ }^{203}$ Henrik Gottlieb, "Subtitling - A New University Discipline," in Teaching Translation and Interpreting: Training Talent and Experience. Papers from the First Language International Conference, Elsinore, Denmark, 1991, ed. Cay Dollerup and Anne Loddegaard (Amsterdam: John Benjamins Publishing Company, 1992).
} 
be rendered in the TL relatively easily given that the phrase is widely known, whether the viewer is proficient in Italian or not.

\author{
Subtitle No. Original Italian English Subtitles \\ and
}

Timecode

0038 Perfetto. Ho solo un leggero appetito. Perfect, just a bit hungry...

00:03:10:17

00:03:14:08

0039 Bravo! Bravo!

00:03:14:20

00:03:16:12

\title{
Transcription
}

Transcription consists of the rendering of non-standard language, including dialects, accents, wordplay and other means of humour, which requires a rather creative approach on the translator's behalf. This strategy could be considered a recreation of the original, very similar to the substitution strategy proposed by Pedersen. In the example below, taken from the tennis scene, we have linguistic humour (the misuse of the imperativo formale) rendered as wordplay in English:

$\begin{array}{lll}\begin{array}{l}\text { Subtitle } \\ \text { No. and } \\ \text { Timecode }\end{array} & \text { Original Italian } & \text { English Sulbtitles } \\ \text { 0800 } & \text { Ma, mi dà del tu? } & \text { Did you just call me "sir"? } \\ 01: 01: 34: 05 & & \\ 01: 01: 35: 21 & & \text { No, I said "serve!" } \\ 0801 & \text { No, dicevo: batti lei? } & \\ 01: 01: 35: 23 & & \\ 01: 01: 38: 06 & & \text { Ah! My mistake. At your service. } \\ 0802 & \text { Ah, congiuntivo. } & \\ 01: 01: 38: 08 & & \\ 01: 01: 41: 02 & & \end{array}$




\section{Dislocation}

Dislocation is a strategy where the subtitler translates original content that features aspects of rhyme and rhythm, for example, with words or phrases that retain these aspects of language in the TT. That is, the content is adjusted in translation to account for particular linguistic features. In Fantozzi we see this through the short poem Mariangela recites to Fantozzi's superiors, where aspects of rhyme were taken into account in the translation:

\section{Subtitle \\ No. and}

Timecode

$0442 \quad$ Un cigliegio grande e bello, disse a un

00:34:28:19 piccolo alberello:

$00: 34: 33: 23$

0443 Sì, sei bravo, hai messo i fiori, coi lor

00:34:34:00 petali a colori.

$00: 34: 38: 24$

0444 Ma cambiarli con i frutti, alberello non

00:34:39:01 saprai.

$00: 34: 43: 04$

\section{English Subtitles}

\section{A cherry tree, pretty and tall \\ told a sapling ever so small:}

Yes, bravo, you're about to flower.

Your petals are anything but dour

\section{How to get fruit from flower, little sapling, you'll never know.}

\section{Condensation}

Condensation is a very common technique used in subtitling and is proposed by Díaz-Cintas and Remael, as well as Pedersen. The variation across languages is a matter of form, rather than of the actual content itself, and is ultimately a more concise version of the original text.

\section{Deletion}

Deletion, otherwise known as omission, is the removal of any segments of dialogue deemed superfluous to the target audience. The previous discussed scholars refer to deletion as omission. It must be emphasised that only content that is not important to the overall plot of development of the story should ever be deleted from the translation.

\section{Decimation}

Decimation is where the original text is abridged, meaning there will be drastic cuts from the original text and an extensive reduction in transference of the original message. In these cases, the translation relies on the images on-screen, as well as the quality of the rest of the translation, to convey any lost 
meaning. Personally, I believe that this strategy should be avoided unless absolutely necessary given the connotations that may be added or removed from the original content.

\section{Resignation}

Resignation is a technique used when the content is simply too difficult, or impossible, to translate. In my opinion, there should never be a need to employ this strategy; the translator can always find some way of representing the message from the ST, even if it does not convey all of the different connotations or meanings.

\subsubsection{The conventions of subtitling}

The way subtitles are presented on screen is by no means arbitrary. There are a number of proposed punctuation and grammar conventions of which a subtitler must be aware when translating AV material. For the most part subtitles tend to follow typographical rules that are familiar to an audience, as well as standard rules of punctuation. Departure from normal conventions of punctuation will cause a negative reaction from the audience as noted by Clara Ceron: "Whenever movie TV viewers are watching a subtitled film, they are deciphering a whole set of codes. They may be unaware of it, but they will react immediately to a departure from the norm." ${ }^{204}$ However, specific uses of punctuation marks are required in subtitling, that have been developed due to subtitles' fragmented nature. The subtitles are individual units that must work in conjunction with image on-screen and soundtrack; they should not be re-read, so punctuation plays a large part maintaining the flow and clarity of the subtitled dialogue.

One of the main problems with attempting to standardize punctuation conventions is that film companies, DVD distributors, and even subtitling companies do not always have style guides, often applying contradictory norms across their AV content. This has led to many inconsistencies within the field:

In this climate, attempting to offer a fixed and unequivocal set of guidelines is certainly a daunting task. While some of the rules followed in subtitling today seem to be backed by logic, many are probably applied arbitrarily and may be difficult to justify over others [...] Any attempt to draw up a list of conventions that can be applied universally could even be considered conceited and presumptuous. ${ }^{205}$

Díaz-Cintas and Remael have nevertheless analysed the guidelines of several different subtitling companies in an attempt to find consistency and have proposed a more holistic guide to subtitling conventions in an English context. The following section will provide a summary of these proposed

\footnotetext{
${ }^{204}$ Clara Cerón, "Punctuating Subtitles: Typographical Conventions and their Evolution," in (Multi) Media Translation: Concepts, Practises and Research, ed. Yves Gambier and Henrik Gottlieb (Philadelphia: John Benjamins Publishing Company, 2001), 173.

${ }^{205}$ Díaz-Cintas and Remael, Audiovisual Translation: Subtitling, 104.
} 
conventions, including specific examples of their application taken from my English subtitles of Fantozzi. ${ }^{206}$

\section{Punctuation rules in subtitling}

\section{Commas}

The comma plays an important role in English grammar, and not all of its uses can be covered here. However, its main function is to show the structure of a sentence, breaking up long-winded sections of text to allow for better readability. Because commas elicit a pause in reading, they must be used in an appropriate manner to dictate where the audience should pause while reading subtitles. This means that commas in subtitles may not follow the punctuation rules in Standard English. Rather, they must be used whenever there is a potential for misunderstanding: ${ }^{207}$

\section{The comma is used to retain the flow and meaning of the text:}

Welcome, ma'am, how are you?

\section{Commas are required after words that are used as vocatives:}

Pina, I just wanted to tell you

3. They are also used to signal phrases and clauses inserted into the main sentence. However these clauses should be moved to the beginning or end of the subtitle:

You know, with a bit of garlic,

in a casserole dish.

According to Díaz-Cintas and Remael, the use of commas at the end of a subtitle must be kept to a minimum to avoid any confusion with a full stop, as this could lead the audience to believe that the spoken phrase is completed. A change in subtitle is often considered as a substitute for the comma, as the disappearance of the subtitle elicits a pause in the viewer's reading and therefore makes the presence of the comma redundant. If there is no punctuation at the end of a subtitle then the implication is that the sentence is not yet complete.

In my view, however, if there are enumerations that spread over two subtitles it is important to signal to the viewer that the list is not yet complete, something that the use of commas can emphasise (ellipses could also be used to serve this purpose). The use of commas at the end of a subtitle should be

${ }^{206}$ Ibid., 104-39.

${ }^{207}$ Ibid., 106. 
made on a case-by-case basis, depending on how long the pause between dialogue: the longer the pause, the less necessary the presence of a comma becomes.

Full stops

The full stop works in subtitling in exactly the same way as it does in standard punctuation conventions, signalling the end of a sentence or phrase. This is also a message to the audience to divert briefly their eyes away from the titles to take in the images on-screen. Just as in the case of normal punctuation, the full stop comes directly after the last character of the subtitle, and the following subtitle starts with an upper case letter. Subtitling companies do not always use full stops at the end of their subtitles, although there does not seem to be a basis for this practice. In Clara Cerón's view, this “creates the most confusing and even irritating situation of all, as it may mean two contradictory things: either the sequence stops there, or it goes on. Needless to say, this makes subtitles very difficult to read." ${ }^{208}$ The three examples below show exactly how full stops should work in subtitling, ${ }^{209}$ whether the subtitle is made up of one sentence, as in the case in the first example, several sentences, as in the second example, or several lines like in the third example shows:

\section{Your employee. \\ Yes, that's definitely his. \\ His timecard from 18 days ago. \\ No private calls are allowed, unless someone has died.}

\section{Colons}

As with full stops, in subtitling, the colon has a similar function to its use in Standard English writing. It signals a pause, and announces or introduces something. Some subtitling agencies tend to put a space between the last character before the colon and the colon itself. This is particularly common in French subtitles, for example, as well as intra-lingual captioning. Others follow standard conventions, placing it directly after the word. The uses of colons in subtitling is classified by Díaz-Cintas and Remael as follows: ${ }^{210}$

\section{They can be used to introduce lists, enumerations or explanations.}

\section{Everything is calculated \\ down to the last second:}

${ }^{208}$ Cerón, "Punctuating Subtitles: Typographical Conventions and their Evolution," 176.

${ }^{209}$ Díaz-Cintas and Remael, Audiovisual Translation: Subtitling, 107.

${ }^{210}$ Ibid. 
2. To quote somebody else's words (NB. The word following a colon should always begin with lower case letter, unless that word is a proper noun)

He once had the honour of rushing

a crime fiction book to her titled:

"The Apricot and the Curare"

\section{Exclamation and question marks}

Exclamation and question marks also have the same function in subtitling as they do in Standard English punctuation. They should appear after the dialogue is completed, being placed straight after the last word with no gap in between, followed by a new sentence starting with a capital letter. The presence of an exclamation or question mark indicates that the phrase or query is completed. Díaz-Cintas and Remael emphasise that there is a danger of overusing these symbols in subtitles; for example if a character is shouting there may not necessarily be a need for exclamation marks as the signal is in the audio of the dialogue itself (for inter-lingual subtitles). ${ }^{211}$ The repetition of these symbols should also be avoided, and should there be a question that is exclaimed or asked enthusiastically, then the subtitler needs to make a decision and omit one of the symbols. This is important given the limited space a subtitler is allowed, whereby valuable characters cannot be wasted on unnecessary punctuation.

Again, while I agree with the practise outlined by Díaz-Cintas and Remael, I contend that a subtitler should not diminish any exclamations present in the original dialogue, particularly if this is not clear by the tone of voice alone. The use of exclamation marks is very important to show to the viewer the surprise, anger, intensity etc. of the characters on-screen: exclamations may well be overused in the original film dialogue, and therefore the subtitler should reflect that in their translation. I would also argue that it is important to highlight an aggressive question with both a question and exclamation mark as otherwise the connotations of the original may be lost, particularly on a deaf or hard of hearing audience, on a foreign audience that is unfamiliar with culturally specific speech intonation, exemplified again by examples from the Fantozzi subtitles. The functions of these two symbols are explained by Díaz-Cintas and Remael: ${ }^{212}$

1. Exclamation marks are used to portray anger, scorn, surprise, happiness and disgust. They can also be used to show irony, underline insults and expletives or to command.

- Has anyone died, ma'am?

- No, unfortunately... Wait!

${ }^{211}$ Ibid., 109.

${ }^{212}$ Ibid., 109-11. 
2. As the name suggests, a question mark is used to indicate that a question has just been asked.

Yes, how can I help?

Dashes and hyphens

The dash is used for a variety of functions in subtitling. These are not necessarily consistent with how dashes and hyphens work in Standard English punctuation, as some of the uses in English are not suitable for a subtitling context. The use of dashes at the end of a line to split up a word should be avoided in subtitling, as well as the parenthetical dash, an alternative to brackets. Díaz-Cintas and Remael indicate the uses of dashes in subtitling are as follows: ${ }^{213}$

1. To link compound words or create new adjectival groups

Am I speaking to the respected

call-centre of the illustrious

2. In subtitling, dashes have the specific function of indicating that there are two different speakers over the one subtitle. The first line is for the speaker we hear first, the second for the speaker that interjects, modern conventions dictate that a dash appears in the second line of the subtitle, with a space between it, and the start of the dialogue. Dashes can also occur when two conversations are overlapping, or if one line of subtitles would otherwise not be on-screen for the minimum of 1.5 seconds. In such case, I would argue that good practice would be to present both subtitles on-screen at the same time, for at least the minimum amount of time possible, indicating the two different speakers by starting each line of subtitles with a dash.

- Why not do mine as well?

- With absolute pleasure.

Triple dots/Ellipses

Triple dots, or ellipses, are generally used to indicate that a sentence or phrase is yet to be completed. The three dots have, in the past, been used to bridge the gap across two subtitles. The dots come after the sentence, with no space separating them from the dialogue, then three dots will precede the dialogue in the following subtitle. Díaz-Cintas and Remael argue that in a professional practice where space is premium it is uneconomical to use characters to portray this information, when no punctuation at the

${ }^{213}$ Ibid., 111-12. 
end of a subtitle already indicates pause or incompletion. The three dots do continue, however, to have a purpose in subtitling outside of this function: ${ }^{214}$

1. To indicate a pause or hesitation within the dialogue.

The old bathrooms... Covered

up to work on the new elevators.

2. When a sentence is carried across two subtitles due to a lengthy pause that calls for two subtitles. The dots are not used for the second subtitle.

Miss Silvani...

Did you notice I'm back?

3. When there is a change in thread of the conversation. This is followed by a capital letter

- But... Where is the ball?

- He kicked it...

4. They are used when convening a sentence or idea that goes unfinished

- Sayonara.

- Sayo... You look beautiful...

5. When a character finishes a sentence started by someone else.

he calls his wife "old hag" and...

- "Bitch!"

- "Bitch."

6. When a list of items is deliberately not completed

Fabriani, Fachetti, Falabrino,

Zarotti, Battiferro...

7. To indicate the stutter of a character, which could be repetition of the first letter of the word, or of the word itself, followed by three dots.

The multiplication of... of...of...

\section{Presentation on-screen}

Not only is punctuation important in maintaining a 'flow' in readability, how the subtitles are physically presented on-screen is also important. Depending on the type of film or programming the colour or font may vary, and the number of lines that a subtitler should use has caused discussion amongst subtitling

${ }^{214}$ Ibid., 112-16. 
practitioners in terms of both their aesthetic nature and their usability. Where the subtitle should appear on screen has also been an interesting topic for debate as previously discussed in this chapter in regards to Wendy Fox's study on integrated subtitles.

\section{Colour and font}

In contemporary cinema subtitles tend to be white. However for black and white films, yellow is often used to show a greater contrast between the text and the on-screen images. White characters tend to be shadowed or outlined in black to aid the reader, and should the subtitles appear on a light background, the subtitles may be set in a shadowed box. In terms of the different fonts available to a subtitler, it is common practice to use a font without 'serifs', for example Arial, Helvetica or Times New Roman. ${ }^{215}$ The font used in this project is the suggested Arial with a point size of 30, they appear white in colour. These subtitles have a slight black outline, meaning where there are moments of white background, the subtitles are still legible.

\section{Number of lines}

Depending on the number of characters present on-screen in the subtitles, a subtitler may face the decision of using one or two lines to convey the content. According to studies on viewer's reading speeds, the more words that appear in one line of subtitles, the less time the audience will spend reading each individual word. ${ }^{216}$ By this logic it would make sense to use two lines of subtitles as often as possible. Of course, this should only be the case when the number of words in a subtitle warrants being split across two lines. The rule proposed by Díaz-Cintas and Remael is that "if a relatively short subtitle fits into one line, do not use two. There is no need to make the eyes travel from one line to the next when all the information can be presented in a single line that viewers can read at a glance." ${ }^{217}$ Some scholars, however, tend to favour one-liners due to their readability. ${ }^{218}$ The downside to this practice is that if they are too condensed with information the viewer will not be able to take in all of the content. In regards to two-line subtitles, the placement and length of the two lines is very important on both an aesthetic and practical level. Thorsten Schröter suggests that the first line should be kept shorter than the second, in order to keep eye movements required by a viewer to a minimum. I have attempted to reproduce this throughout the subtitles of Fantozzi, despite the subtitles being centred on screen. This phenomenon is shown in the following example, proposed by Schröter, by where, on the left, the

\footnotetext{
215 Ibid., 84.

216 Ivarsson and Carroll, Subtitling, 64.

${ }^{217}$ Díaz-Cintas and Remael, Audiovisual Translation: Subtitling, 86.

218 See Sylfest Lomheim, "L'écriture sur l'écran: stratégies de sous-titrage à NRK, une étude de cas," Translatio, Nouvelles de la FIT/FIT Newsletter 15, no. 3-4 (1995).
} 
viewer's eyes must track the whole way across the sentence. The opposite effect occurs on the right hand side: ${ }^{219}$

In this case, a longer eye movement

is required
In this case,

the eye movement is much shorter

Position on screen

There are two other aesthetic decisions that a subtitler must make when finalising subtitles. Firstly, whether to place a single line of subtitles in the place where the top line would be in a two-line subtitle, or where the bottom line would be. There are two schools of thought regarding this decision: if the single subtitle goes on the top line, there is consistency across the whole film as to where the subtitles will start. The counter argument is that if the single subtitle appears where the second line should be, the subtitles will interfere less with the image. ${ }^{220}$ The other decision a subtitler needs to make is whether the subtitles are centred or left-aligned. Traditionally, television subtitles have been presented in the latter format. However cinema and DVD subtitles tend to be centred, the reason for this being that, on a larger screen, the spectators sitting on the right of the screen are too removed from the content; moreover, the action of the film also tends to occur in the middle of the screen, so with centred subtitles the eye does not need to move all around the screen to take in content. ${ }^{221}$

\subsection{Conclusion}

Until relatively recently, AVT has had little credibility in the field of translation studies. Scholars such as Frederic Chaume, Jorge Díaz-Cintas, Yves Gambier, Henrik Gottlieb, Jan Pedersen and Aline Remael, to name just a few, have changed this, giving academic credibility to an important industry and therefore increasing its exposure and quality. The four main forms of AVT are subtitling, dubbing, voice-over and audio description. They all come with their own difficulties, sharing specific traits and equally effective in their own right depending on the context.

Subtitling allows an audience to hear the original language soundtrack and is therefore beneficial to language learners and non-speakers of the SL content; it is comparably cheaper; and can also be used for accessibility purposes allowing the deaf and hard of hearing to access the content. Dubbing, on the other hand, is a fairly expensive exercise and can be very difficult to complete given the phonetic restrictions imposed on it, yet when done well it can be less invasive than subtitles for an audience. It also has the potential to face less criticism than subtitles given that no comparison of SL

\footnotetext{
${ }^{219}$ Schröter, "Shun the Pun, Rescue the Rhyme? The Dubbing and Subtitling of Language-Play in Film," 41.

${ }^{220}$ Díaz-Cintas and Remael, Audiovisual Translation: Subtitling, 87.

${ }^{221}$ Ibid., 88.
} 
and TL can be made while watching the content, although faces its own problems particularly when done poorly as it is easily noticeable. Finally, voice-over is used in a slightly different context, mainly in AV material such as documentaries, news reports or for narration, where short snippets of dialogue are placed over the existing soundtrack. Voice-over does not have to face the same challenges as dubbing in the sense that the SL is not being hidden, simply dimmed down in the background, however the contexts for its use are fairly limited.

The subtitler is tasked with the unenviable job of having to translate content while dealing with heavy constraints that have the potential to severely affect a translation. The industry is incredibly important for the promotion of languages, cultures and film around the globe as we have seen in examples of pedagogical uses of this practice, yet as we have found out, subtitlers are not paid particularly well, and subtitles are often the last thing sought after by filmmakers, therefore must be written quickly with limited funds available.

Subtitles were first introduced to cinema in the 1920s and the first processes to create subtitles were unreliable, but technology continued to develop from physically burning the subtitles into the film reel, to contemporary digital subtitle files that are superimposed onto the film. The technology available today has drastically changed the subtitling industry: subtitlers now have access to fairly cheap software that allows them to spot subtitles easily, edit them at the touch of a button, see exactly how many characters are present in a subtitle, measure the words per minute that are present and also allows the subtitler to change the style, font, placement and colour of subtitles with ease. Yet, the subtitling industry continues to develop as researchers attempt to find new ways to make AV content more accessible. Wendy Fox, for example, is attempting to revolutionise the way subtitles are presented on screen and slowly but surely this practise seems to be catching on, albeit in small and isolated contexts. Whether this practise eliminates the audience's feeling that subtitles are intrusive will be interesting to discover as this research continues, although in saying this, subtitles may not be as intrusive as many filmgoers believe they are.

The restrictions placed upon the field of audiovisual translation, and in particular subtitling, are well documented. Time and space restrictions can have a large impact on the translation, and a subtitlers ability to deal with these restrictions is paramount in the quality of translated AV material. While multiple techniques can be used to help alleviate some of these issues, in the context of this thesis we have focused on those proposed by Díaz-Cintas and Remael as well as Jan Pedersen and Henrik Gottlieb as the most useful in aiding a translation of Fantozzi. It is important to emphasise that there is no easy solution and at times multiple techniques will need to be put in place in order to adequately translate particular content. On top of this, how the subtitles are presented on-screen can have a bearing on the audience's ability to adequately process the subtitles. Whether that be through how the subtitles are punctuated, or through the font, colour or size of text that is used, it is important that the subtitler is wary of the different effects that each of these variables can have. 
Having discussed the issues pertaining to both subtitling and translating humour, as well as having explored the cultural aspects to the character of Ugo Fantozzi and its relevance to Italian society, I will now present and comment on the full translation of the first Fantozzi film in the series, Luciano Salce's Fantozzi. 


\section{Subtitling Fantozzi (1975)}

The discussion of the theoretical and practical issues around translating humour and subtitling has been leading towards the translation of the first Fantozzi film. I would now like to present the translation of Fantozzi in full. Before providing the translation, however, I would like to present the framework that I followed throughout the production of my subtitles, which has been based on the previous discussion as well as a number of professional guidelines used in commercial subtitling.

The translation is divided into the twelve different chapters of the film. It is presented in three columns: the first column provides the subtitle number and timecodes for each subtitle, the second column is the original Italian dialogue, the third column is the translation, which has been cut up into the corresponding subtitles. Where appropriate, footnotes have been included to provide a brief insight into particular translation choices. This section is then followed by an analysis of specific scenes and/or parts of dialogue that warrant further discussion. It is important to note that there are four key elements that I have kept in mind throughout this translation: 1) the spatio-temporal pressures of subtitling; 2) the need to retain the original text humour; 3) the need to accommodate for culturally specific references; and 4) to remain faithful to the images on-screen. The 'Original Italian' dialogue has been transcribed by myself, and checked by native Italian speakers as part of this project, as there was no script or dialogue list available.

Given the aforementioned constraints inherent to subtitling, and in an attempt to achieve the criteria that I have set for myself, I have attempted to follow the translation theories highlighted earlier in this discussion around reduction of text for subtitling, and translation techniques specific to issues related to humour based texts for the actual content of the film. I have also kept in mind Mona Baker's theories of equivalence and techniques to overcome these issues in translation, particularly at a wordlevel, above word-level, at a pragmatic level, at a semiotic level, as well as aspects of ethics, particularly important given that some comedic content is relevant to 1970s Italy and can be seen as problematic, or politically incorrect, to a contemporary audience. ${ }^{222}$

So far I have screened this film once publically, as part of the 2017 edition of La settimana della lingua italiana, supported by the Embassy of Italy in Wellington. I invited family and friends as well as members of the Italian community in Wellington along to the screening. The feedback was overwhelmingly positive, and the screening, as well as the many conference presentations where I received invaluable feedback from colleagues in both Italian Studies and Translation Studies, have contributed to the final subtitles.

\footnotetext{
${ }^{222}$ Baker, In Other Words: A Coursebook on Translation.
} 


\subsection{Subtitling framework}

The subtitles have been produced following the basic guidelines I developed for this project, presented below, in order to provide consistency across the translation of the film into English. These guidelines are specific to this project, although they have been based on the Ivarsson and Carrol's Code of Good Subtitling Practise, Netflix's Subtitle Style Guide (September 2018), as well as the British Broadcasting Corporation's subtitling guidelines (version 1.1, September 2009) and Fotios Karamitroglou's Proposed Set of Subtitling Guidelines in Europe. ${ }^{223}$ Some of the practices outlined below are common in subtitling for the deaf and hard-of-hearing, such as the presence of textual information or highlighting that music is being played. I have decided to do this as I would like to enhance the accessibility aspects of my project, allowing a deaf and hard-of-hearing audience to enjoy the film as much as a hearing audience.

\section{Presentation of subtitles on-screen}

\subsection{Position of Subtitles}

Subtitles will appear centred and at the bottom of the screen. This leads to the subtitles being in the least invasive position, requiring the shortest eye-movement on the part of the viewer to read the subtitles as they appear on screen.

\subsection{Maximum number of lines}

The subtitles should be no longer than two lines, with a maximum of 41 characters per line, as suggested by Díaz-Cintas and Remael. The subtitles should endeavour to be under 180 words per minute where possible. The lines should always be broken at appropriate times such as after punctuation marks, before conjunctions or before prepositions, and should never break up an article and a noun, a preposition and the following phrase, a conjunction and the following phrase, a pronoun and a verb, or a verb and its auxiliary. Where there are two lines of text, the lines should be of similar length. Should this not be possible, the second line should be the longer of the two lines, provided the syntax and line breaks allow for it.

\subsection{Colour and font of subtitles}

The subtitles should appear in white using the 'Arial' typeface, at point size 30.

223 "Timed Text Style Guide: General Requirements ", Netflix, https://partnerhelp.netflixstudios.com/hc/enus/articles/215758617-Timed-Text-Style-Guide-General-Requirements. accessed 12th December, 2018; Gareth Ford Williams, "Subtitling Guidelines v1.0," BBC,

https://www.bbc.co.uk/guidelines/futuremedia/accessibility/subtitling_guides/online_sub_editorial_guidelines_vs1_1.pdf. accessed 12th December, 2018; Fotios Karamitroglou, "A Proposed Set of Subtitling Standards in Europe," European Association for Studies in Screen Translation,

http://www.sub2learn.ie/downloads/karamitroglou_fotiosa_proposed_set_of_subtitling_standards_in_europe.pdf. accessed 12th December, 2018. 


\subsection{Multiple speakers}

Where multiple speakers are subtitled at one time, each speaker should be given one line introduced by a dash. Each line should contain no more than 41 characters per line.

\subsection{Voice-over}

Where there is a voice-over (as opposed to the characters speaking), the subtitles should appear in italics.

\subsection{Punctuation}

The punctuation of the subtitles should be based on standard punctuation conventions for subtitles proposed by Díaz-Cintas and Remael, discussed in chapter 3 of this thesis.

\subsection{Numbers}

Numbers between one and ten should be spelled out; any numbers higher than this will appear as numerals. This is common practice among commercial subtitling organisations.

\subsection{Currencies}

Where currencies are present, the symbol for each currency (i.e. $\$, €, £$ ) should be used to retain space. In the case of the 'lira' it should be translated to 'dollar' (see discussion in chapter 4).

\section{Timing/spotting of Subtitles}

\subsection{Minimum and maximum subtitle duration}

Each subtitle should remain on-screen for a minimum of 1 second and for a maximum of 6 seconds.

\subsection{Space between subtitles}

Each subtitle should be separated by a minimum of one frame.

\subsection{Timing to audio and shot changes}

Each subtitle should be timed to the audio, the out-time may be extended should the minimum subtitle duration not be met. The subtitle should not anticipate speech by more than 1.5 seconds, or remain onscreen for more than 1.5 seconds after the dialogue has stopped. Subtitling across shot changes should be avoided where possible. 


\section{Content specific guidelines}

\subsection{Humour}

Where humour exists in the original, the subtitles should endeavour to reflect this humour. Where possible, punchlines should be separated across subtitles and time must be given to the audience to process more complicated and culturally problematic aspects of humour. The translation should reflect the humour of the original content where possible, and should not be changed in order to be accepted by a contemporary audience.

\subsection{Multiple Languages/Dialects}

Subtitles that exist in the original film should be translated and presented in the English subtitles. Where foreign languages are spoken and not translated, no translation will be made, unless the foreign language terms used are well known. Where dialects or accents are used, the translation should attempt to reflect the distinction between the standard language and the other dialect or accent present.

\subsection{Music and lyrics}

Lyrics should be translated into the TL. The translations should attempt to retain the different aspects of the original including rhyme, rhythm and meaning. Any music should appear with the symbol $\boldsymbol{J}$ at the beginning and end of the subtitle to highlight the difference between standard dialogue and music.

\subsection{Culturally specific content}

Where possible, the translation should maintain the original cultural content from the film, provided the content remains accessible. It is up to the subtitler's discretion as to which content needs to be domesticated to the target audience and which content may remain.

\subsection{Images/Signs}

All images, signs, newspaper headings etc. should be translated into the TL. 


\title{
THE ORIGINAL ITALIAN AND THE ENGLISH TRANSLATION HAVE BEEN REDACTED IN THEIR ENTIRETY FOR COPYRIGHT PURPOSES
}

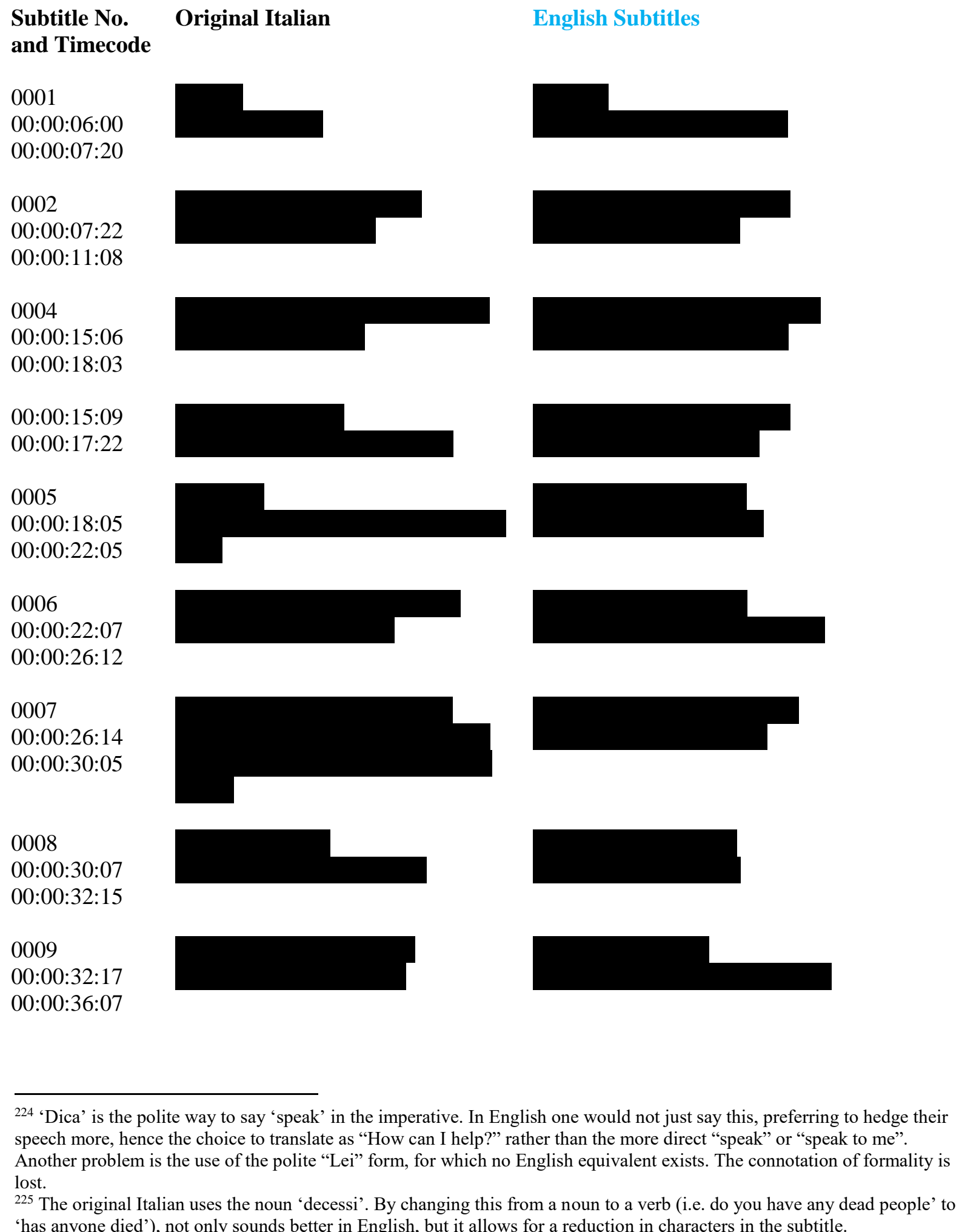

\begin{abstract}
Subtitle No.
\end{abstract}
Original Italian

English Subtitles

0001

00:00:06:00

0002

00:00:07:22

00:00:11:08

0004

00:00:15:06

00:00:18:03

00:00:15:09

00:00:17:22

0005

00:00:18:05

00:00:22:05

0006

00:00:22:07

$00: 00: 26: 12$

\section{8 \\ 00:00:30:07 \\ 00:00:32:15}

0009

00:00:32:17

00:00:36:07

\footnotetext{
224 'Dica' is the polite way to say 'speak' in the imperative. In English one would not just say this, preferring to hedge their speech more, hence the choice to translate as "How can I help?" rather than the more direct "speak" or "speak to me".

Another problem is the use of the polite "Lei" form, for which no English equivalent exists. The connotation of formality is

'has anyone died'), not only sounds better in English, but it allows for a reduction in characters in the subtitle.
} 
0010

00:00:36:13

00:00:39:12

\section{1}

00:00:39:14

00:00:41:04

0012

00:00:41:08

00:00:44:01

0013

00:00:44:03

00:00:48:03

0014

00:00:48:05

00:00:49:13

0015

00:00:49:15

00:00:51:09

0016

00:00:51:11

00:00:55:09

0017

00:00:55:13

00:00:57:05

0018

00:00:57:10

00:00:58:13

0019

00:00:58:17

00:01:01:09

0020

00:01:01:12

00:01:03:04

0021

00:01:03:06

00:01:06:07
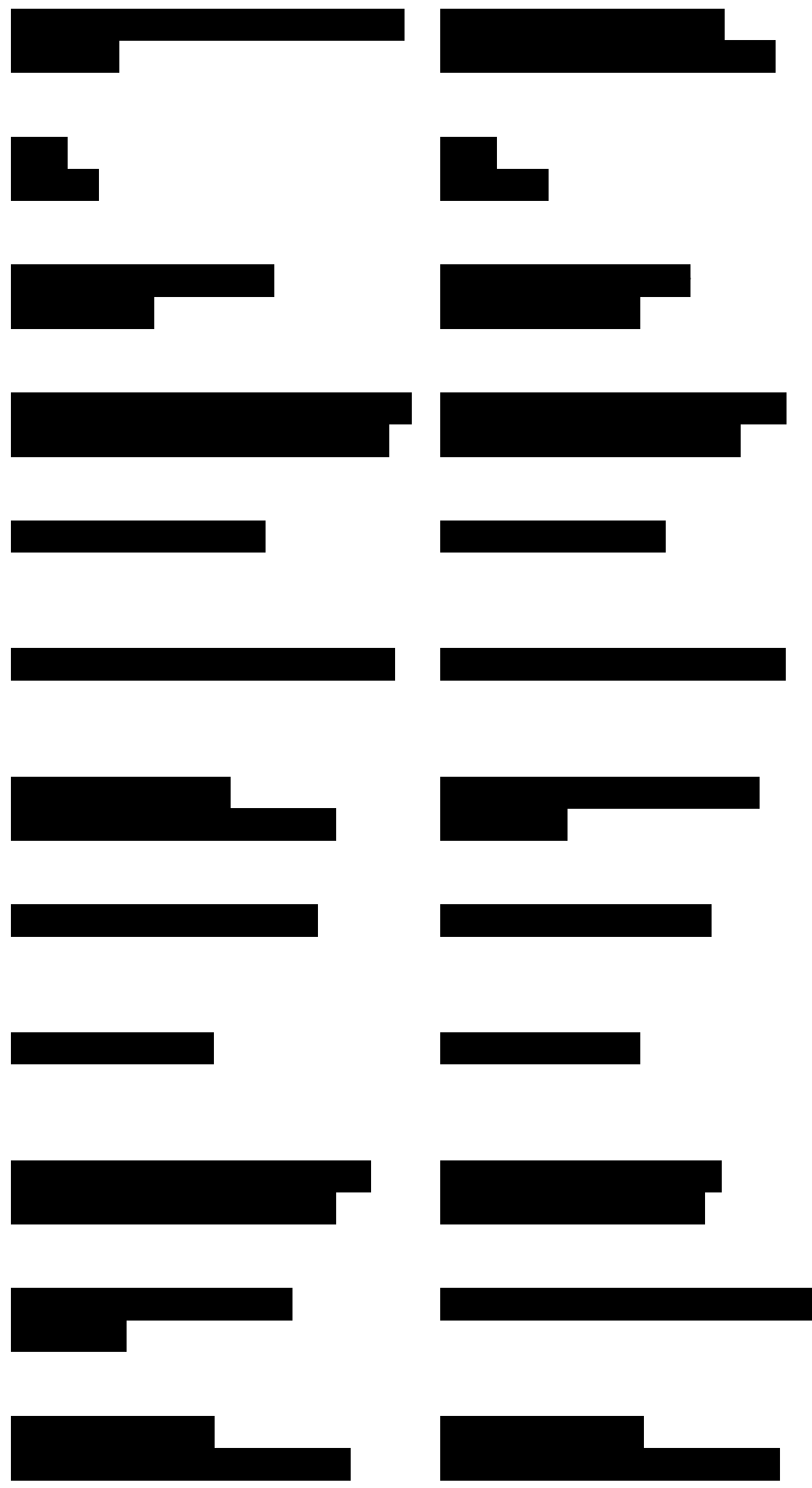

\footnotetext{
${ }^{226}$ In the original Italian, Fantozzi is always referred to as 'ragioniere' or 'accountant'. This is not uncommon in Italian, however in English, a person's occupation is very rarely used as a method of address (with the exception of titles like 'Doctor' or 'Professor'. Unfortunately a lot of the connotations from the original are removed in using an alternative such as 'Mr' or 'Sir'. However it is a necessary decision given the alternative of a literal translation would not work.
} 
0022

00:01:06:09

00:01:08:16

0023

00:01:08:18

00:01:10:08

0024

00:01:10:10

00:01:13:15

0025

00:01:14:07

00:01:16:05

0026

00:01:20:12

00:01:23:10

0027

00:01:23:12

00:01:24:24

0028

00:01:25:01

00:01:27:19

0029

00:02:21:21

$00: 02: 23: 24$

0030

00:02:24:01

00:02:27:04

0031

00:02:27:06

00:02:29:23

0032

00:02:55:22

00:02:58:24

0033

00:02:59:01

00:03:01:08

0034

00:03:01:09

00:03:02:16

0035

00:03:02:17
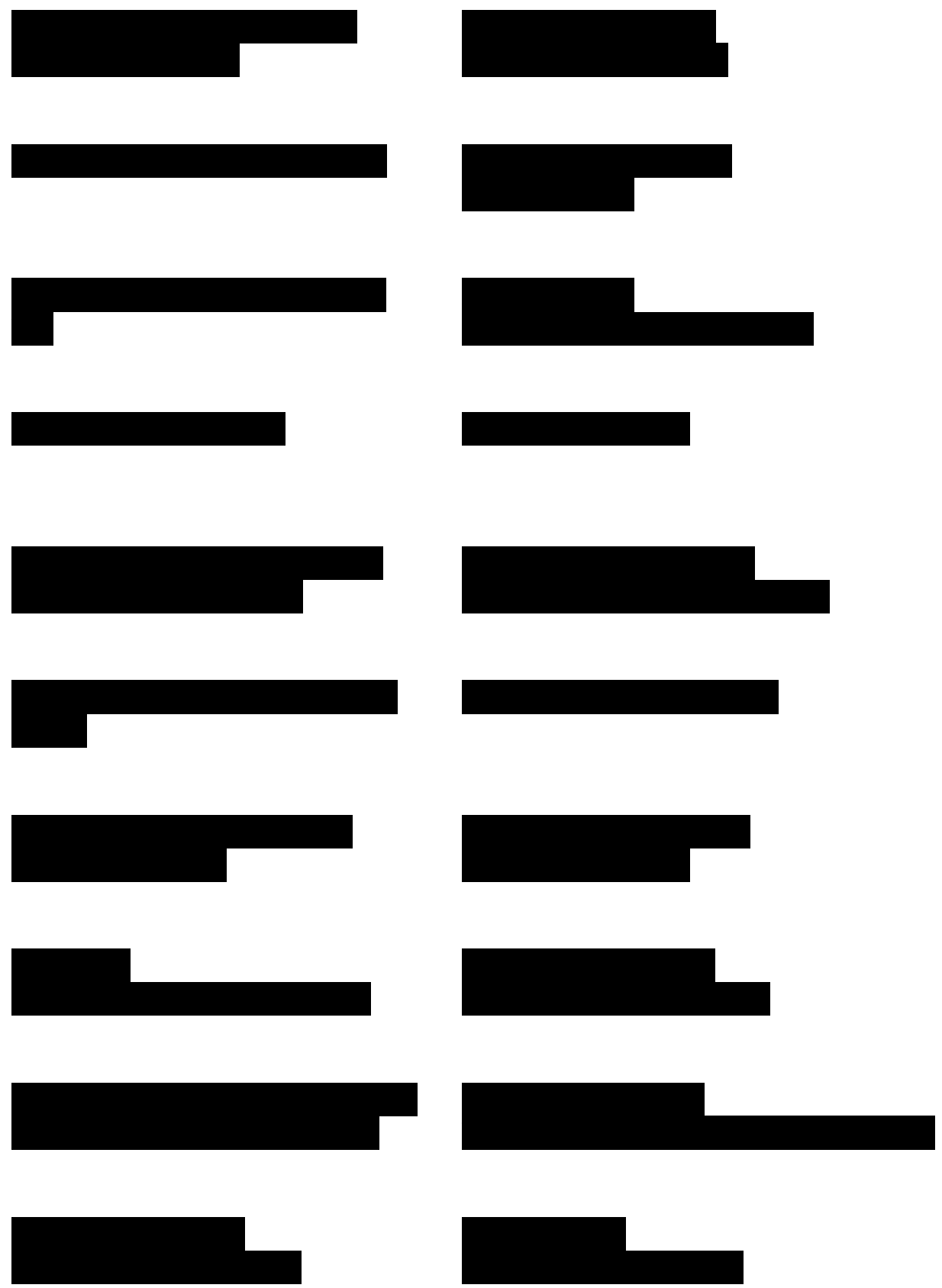

00:03:04:21
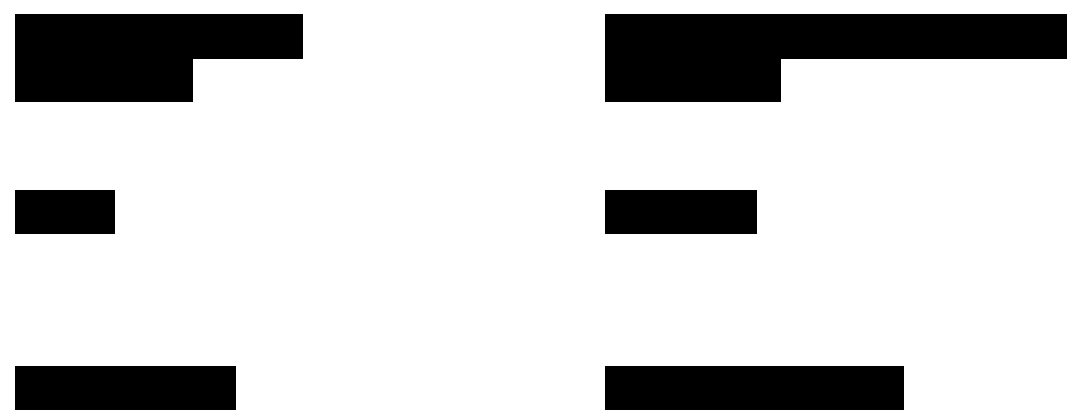
0036

00:03:05:20

00:03:08:17

0037

00:03:09:14

00:03:10:15

0038

00:03:10:17

00:03:14:08

0039

00:03:14:20

00:03:16:12

0040

00:03:24:04

00:03:25:21

0041

00:03:26:07

00:03:27:20

0042

00:03:28:01

00:03:30:08

0043

00:03:34:06

00:03:36:06

0044

00:03:36:12

00:03:38:03

0045

00:03:38:05

00:03:39:07

0046

00:03:39:09

00:03:40:14

0047

00:03:40:16

00:03:41:23

0048

00:03:47:08

00:03:48:19
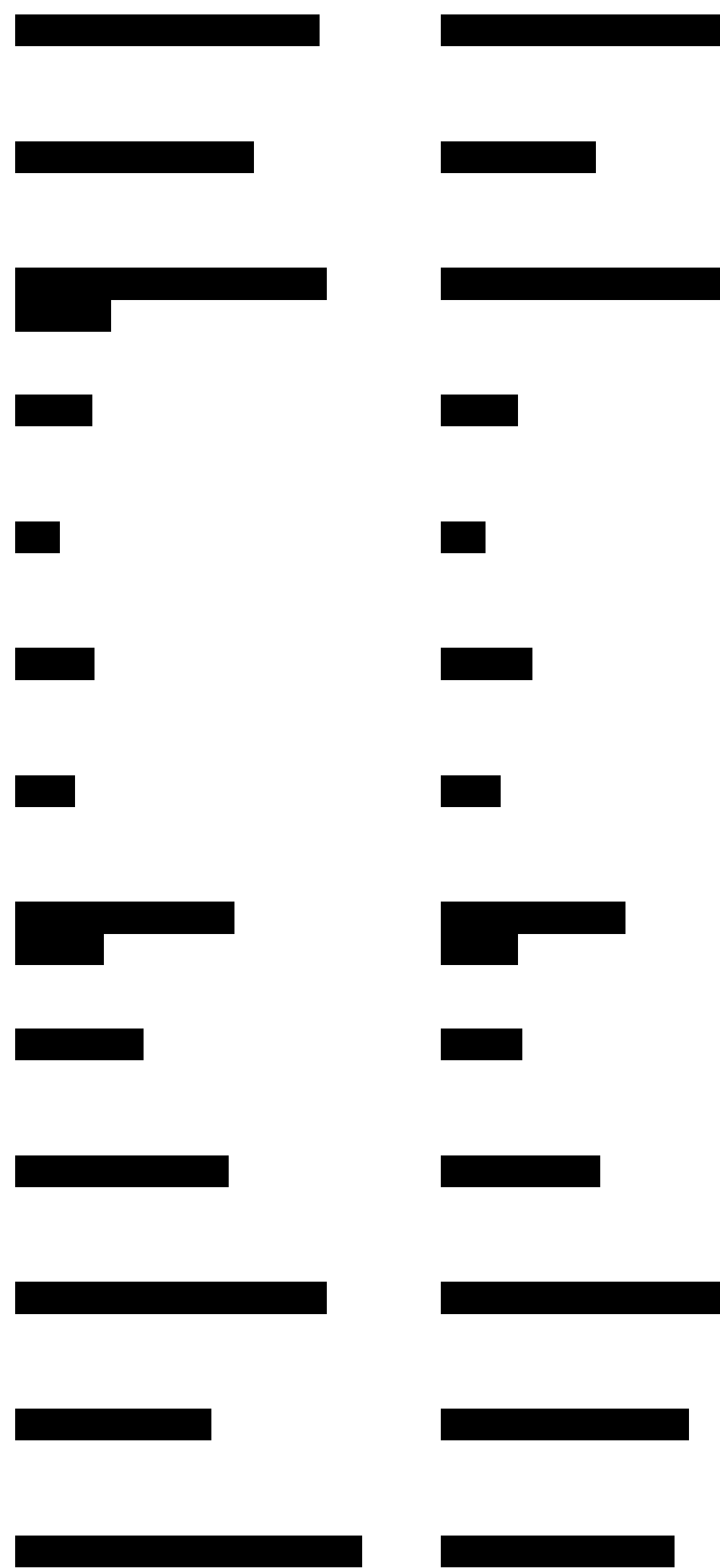

${ }^{227}$ The game being played on-screen exists in English. It is known as Battleships. Instead of saying 'colpito' (hit) and 'acqua' (water), the English version of the game uses the terms 'hit' and 'miss'. 
0049

00:03:48:21

00:03:50:19
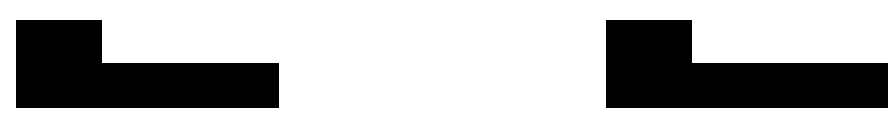

0050

00:03:50:21

00:03:53:12

0051

00:03:55:11

00:03:56:24

0052

00:04:05:13

00:04:06:22
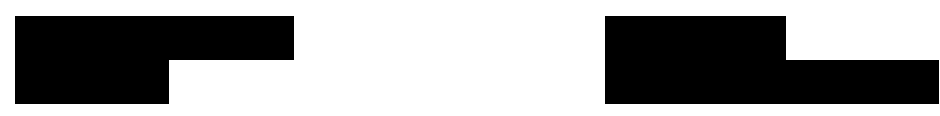

0053

00:04:06:24

00:04:10:21

0054

00:04:10:23

00:04:12:24

0055

00:04:30:13
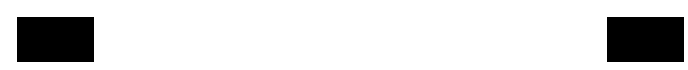

00:04:32:01

0056

00:04:33:00

00:04:35:18

0057

00:04:35:20

00:04:38:12

0058

00:04:38:13

00:04:40:03

0059

00:04:40:04

00:04:41:19

0060

00:04:41:20

00:04:42:20

0061

00:05:00:05
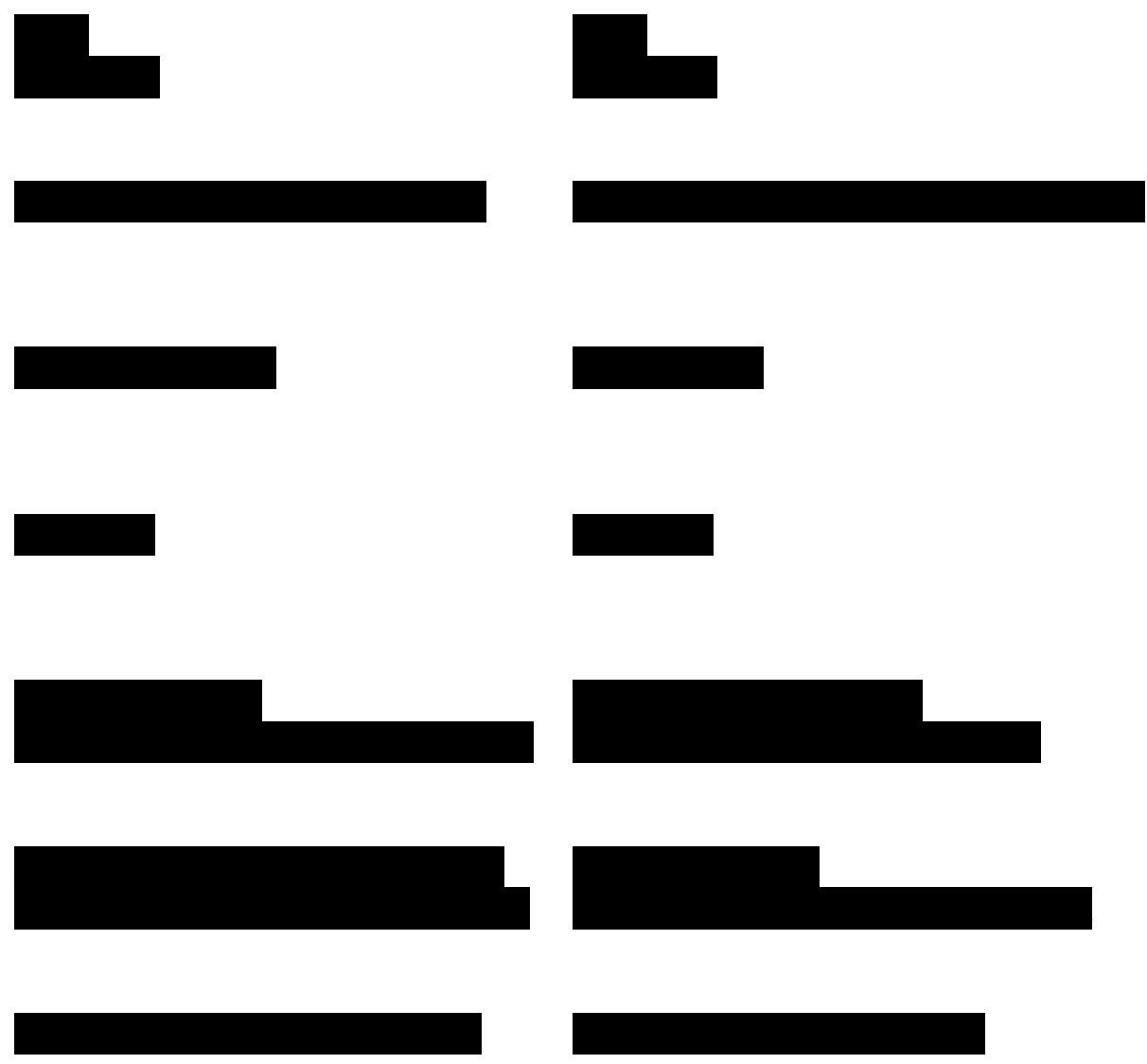

00:05:04:00
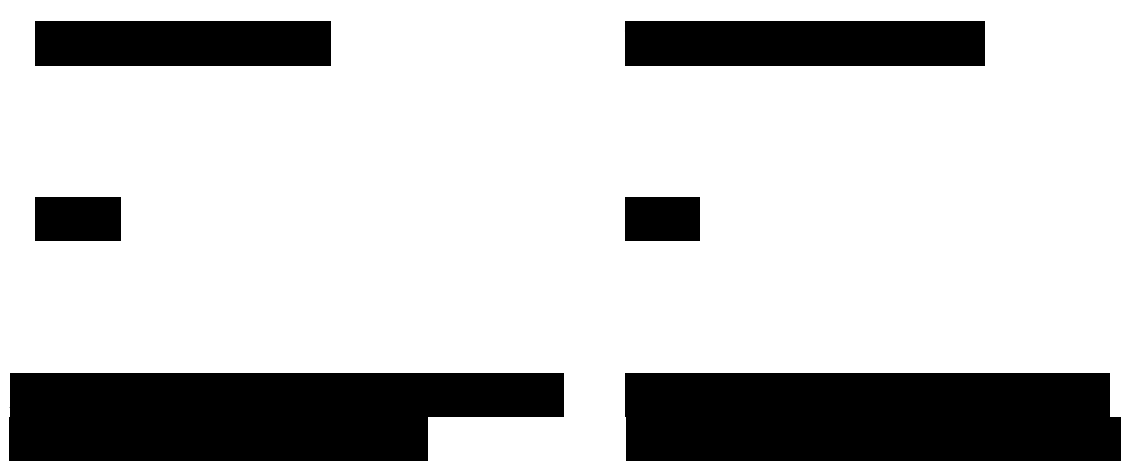

${ }^{228}$ Instead of saying "precisely 8.30am" I have decided on the more idiomatic expression in English "at 8.30am sharp" for its expressiveness. 
0062

00:05:04:02

00:05:08:18

0063

00:05:08:20

00:05:12:15

0064

00:05:12:17

00:05:15:17

0065

00:05:17:00

00:05:21:02

0066

00:05:21:04

00:05:23:08

0067

00:05:23:11

00:05:25:14

0068

00:05:26:04

00:05:30:06

0069

00:05:31:02

00:05:33:19

0070

00:05:34:00

00:05:39:06

0071

00:05:39:08

00:05:42:04

0072

00:05:42:24

00:05:45:09

0073

00:05:47:17

00:05:52:16

0074

00:06:12:23

00:06:16:11

0075

00:06:20:02

00:06:23:09
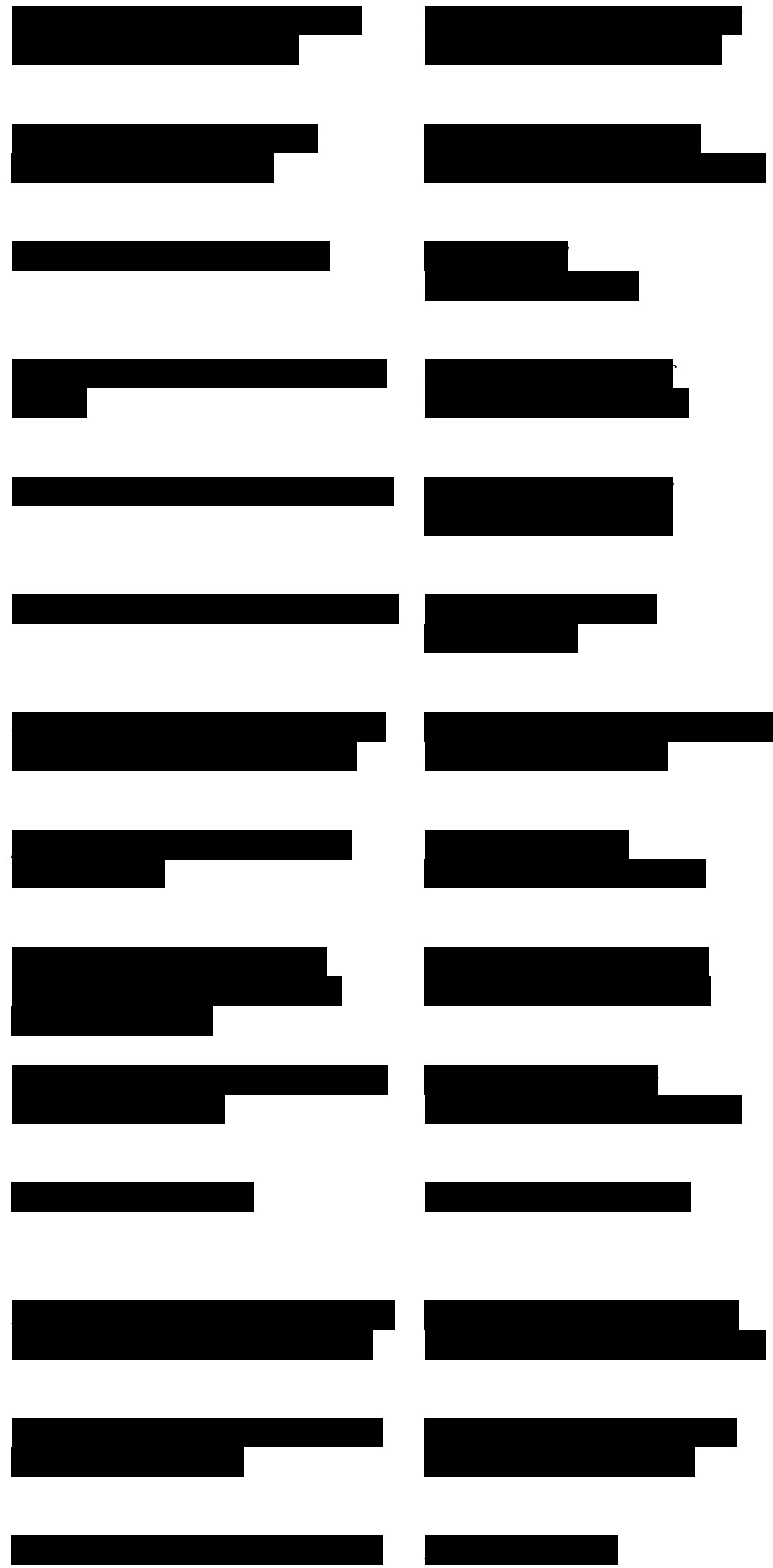
0076

00:06:28:01

00:06:32:03

0077

00:06:33:15

00:06:36:19

0078

00:06:36:22

00:06:40:09

0079

00:06:44:05

00:06:46:22

0080

00:06:46:24

00:06:50:14

0081

00:06:57:09

00:07:02:12

0082

00:07:05:05

00:07:06:16

0083

00:07:17:21

00:07:19:15

0084

00:07:20:15

00:07:22:00

0085

00:07:22:02
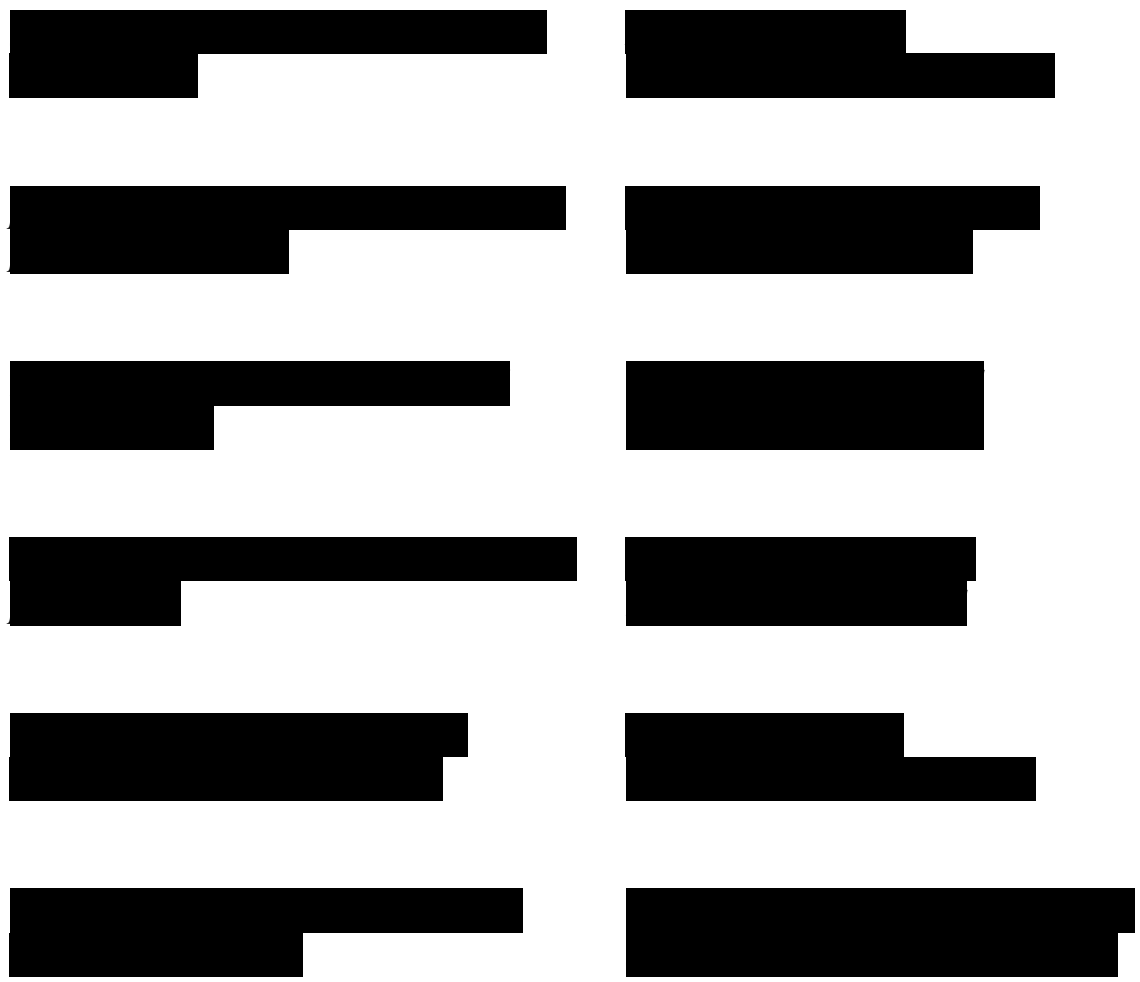

00:07:23:02

0086

00:07:23:04

00:07:24:13

0087

00:07:24:15

00:07:27:00

0088

00:07:27:01
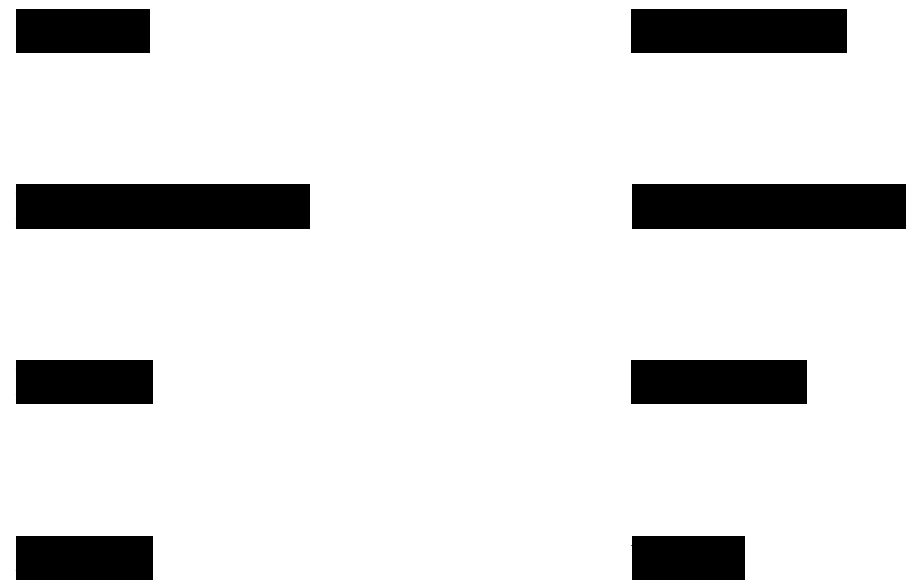

00:07:28:14

${ }^{229}$ The expression 'prendere al volo' literally translates to "catch". It is also an expression used for food, when one quickly eats something 'on the go'. Potential solutions could be 'on the run' or 'on the go' however to retain the idea of 'catching' the bus, as well as connotations of 'volo' or 'flight', I have settled with 'leap'. 
0089

00:07:28:18

00:07:30:10

0090

00:07:30:12

00:07:32:13

0091

00:07:32:15

00:07:34:14

0092

00:07:35:19

00:07:38:13

0093

00:07:44:18

00:07:45:22

0094

00:07:45:24

00:07:48:19

0095

00:07:57:10

00:07:59:03

0096

00:07:59:19

00:08:01:11

0097

00:08:42:09

00:08:44:02

0098

00:08:50:12

00:08:52:07

0099

00:08:52:11

00:08:54:03

0100

00:08:54:06

00:08:56:07

0101

00:08:59:13

00:09:03:11

0102

00:09:05:14

00:09:08:12
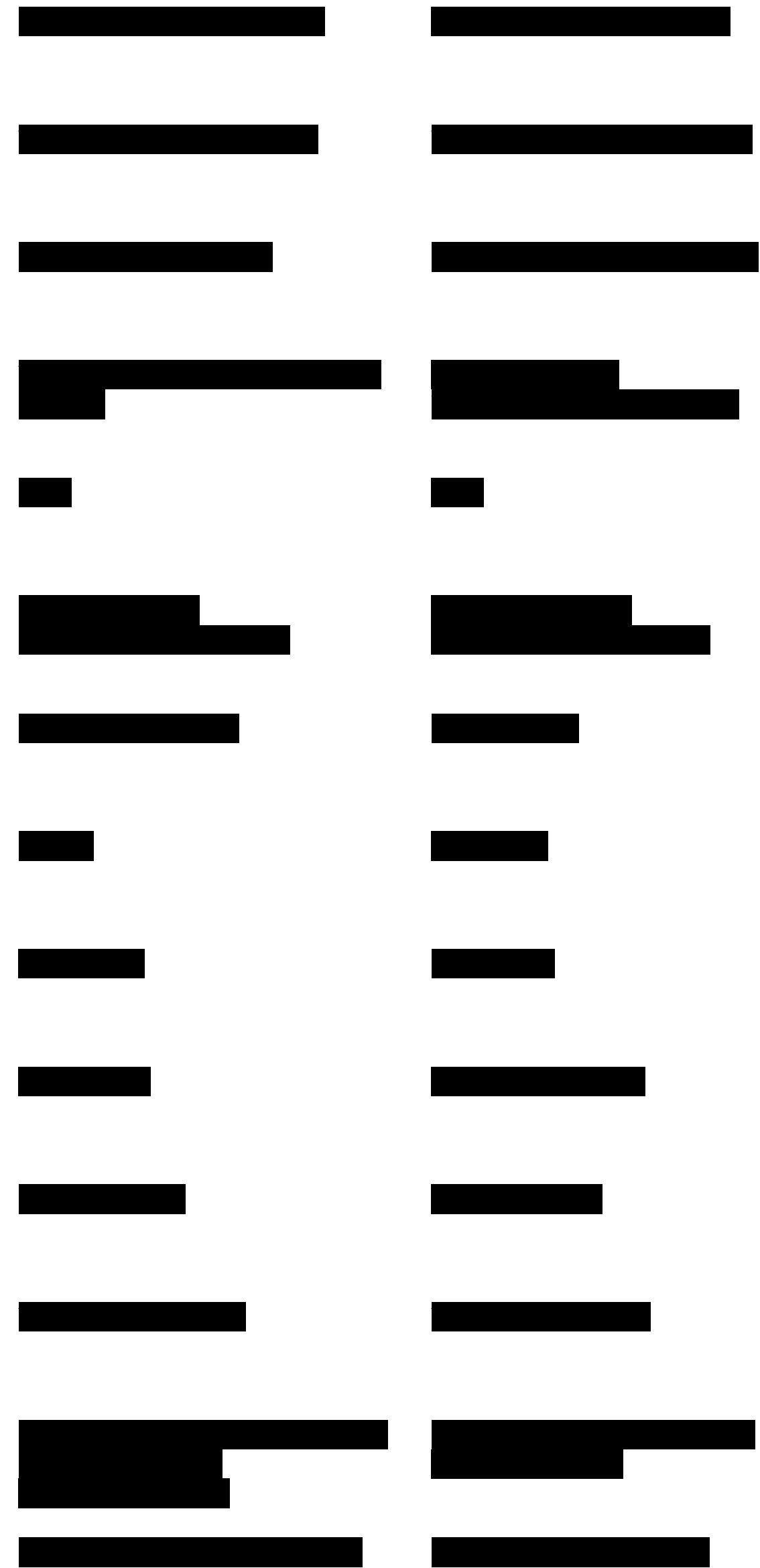
0103

00:09:08:14

00:09:10:09

0104

00:09:10:11

00:09:13:00

0105

00:09:25:00

00:09:27:16

0106

00:09:27:18

00:09:30:07

0107

00:09:30:12

00:09:32:13

0108

00:09:33:04

00:09:34:20

0109

00:09:36:07

00:09:37:24

0110

00:09:45:22

00:09:47:16

0111

00:09:47:18

00:09:51:02

0112

00:09:51:15

00:09:54:10

0113

00:10:00:19

00:10:02:18

0114

00:10:02:20

00:10:04:04

0115

00:10:04:07

00:10:07:05

0116

00:10:07:21

00:10:11:08
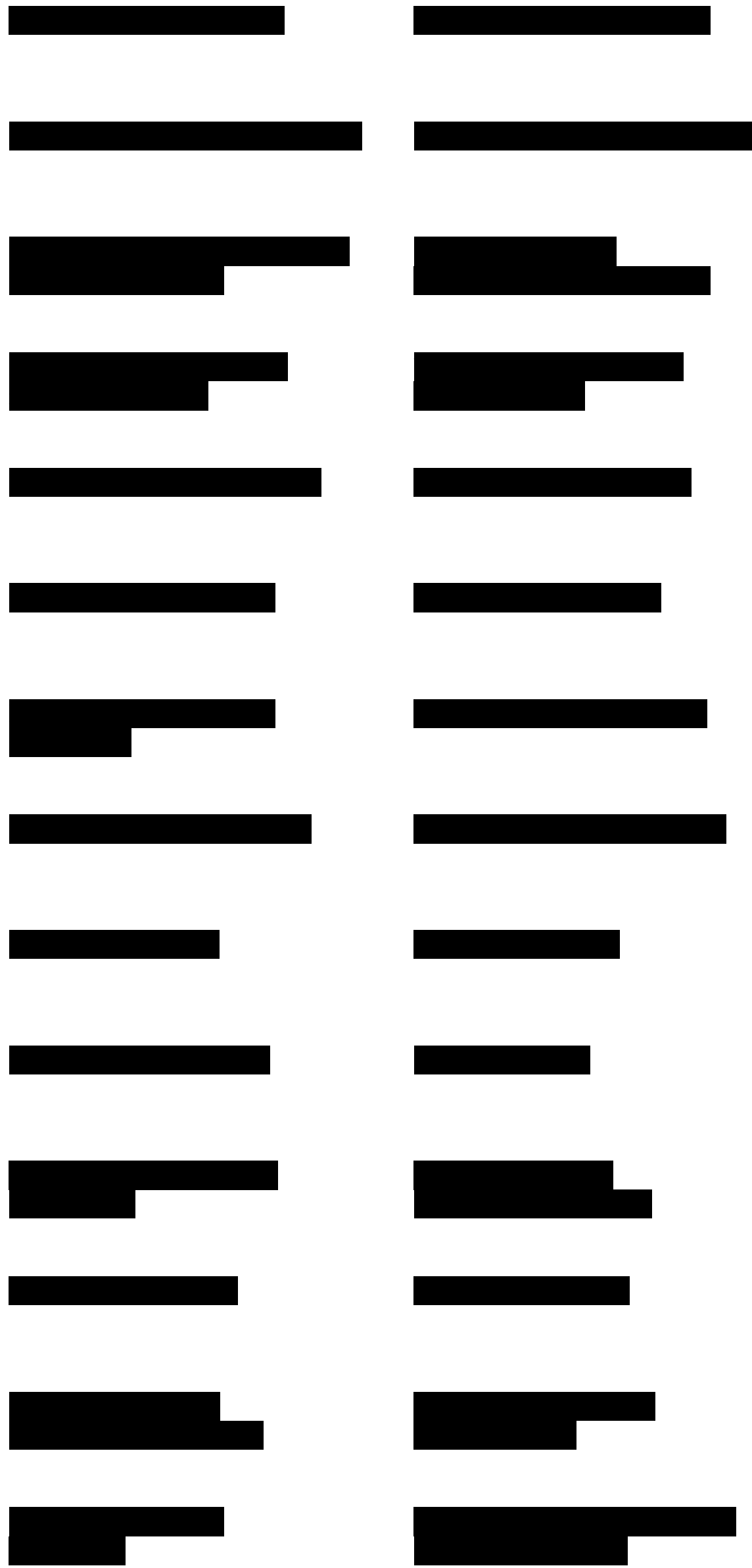
0117

00:10:11:23

00:10:13:22

0118

00:10:14:19

00:10:18:00

0119

00:10:18:02

00:10:20:03

0120

00:10:20:23

00:10:24:05

0121

00:10:41:05

00:10:44:06

0122

00:10:44:07

00:10:48:01

0123

00:11:27:13

00:11:31:14

0124

00:11:31:15

00:11:33:02

0125

00:11:33:03

00:11:37:15

0126

00:11:38:04

00:11:40:02

0127

00:11:40:04 00:11:41:14

0128

00:11:41:15

00:11:43:20

0129

00:11:43:23

00:11:45:18

0130

00:11:46:09

00:11:50:07
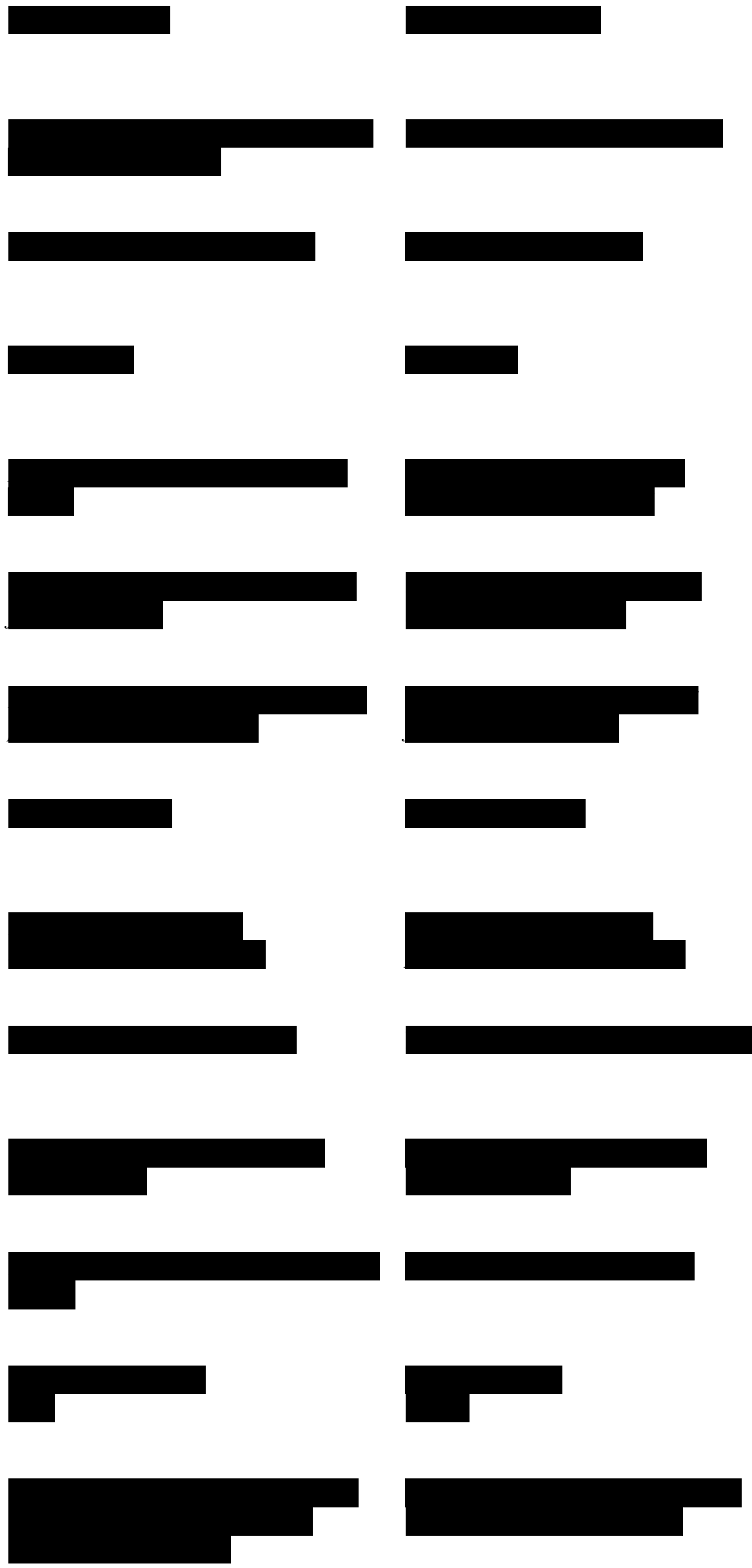
0131

00:11:50:10

00:11:53:13

0132

00:11:53:15

00:11:54:22

0133

00:11:54:24

00:11:57:19

0134

00:11:58:15

00:12:01:01

0135

00:12:01:04

00:12:03:09

0136

00:12:04:07

00:12:06:03

0137

00:12:06:06

00:12:08:01

0138

00:12:08:03

00:12:10:09
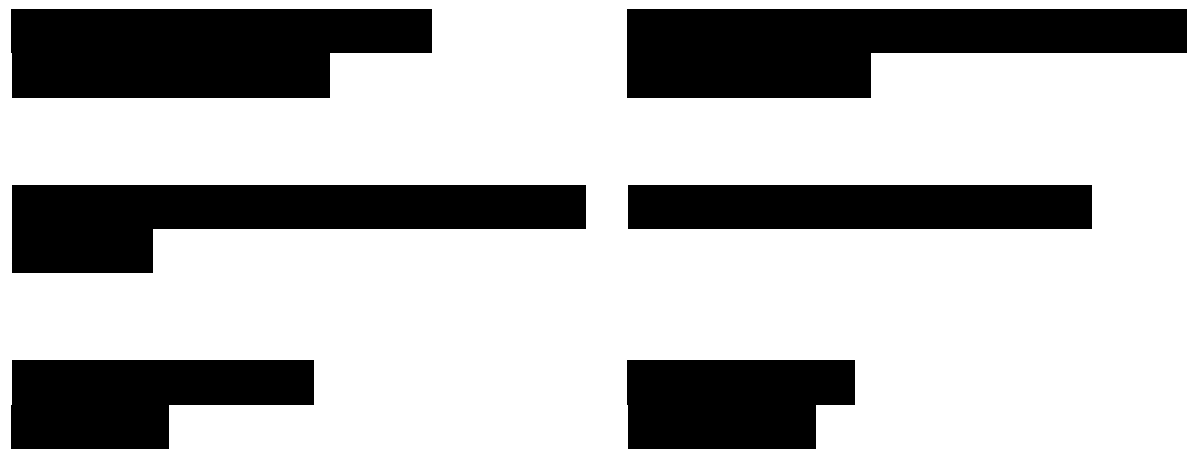

0139

00:12:10:11

00:12:11:11

0140

00:12:11:13
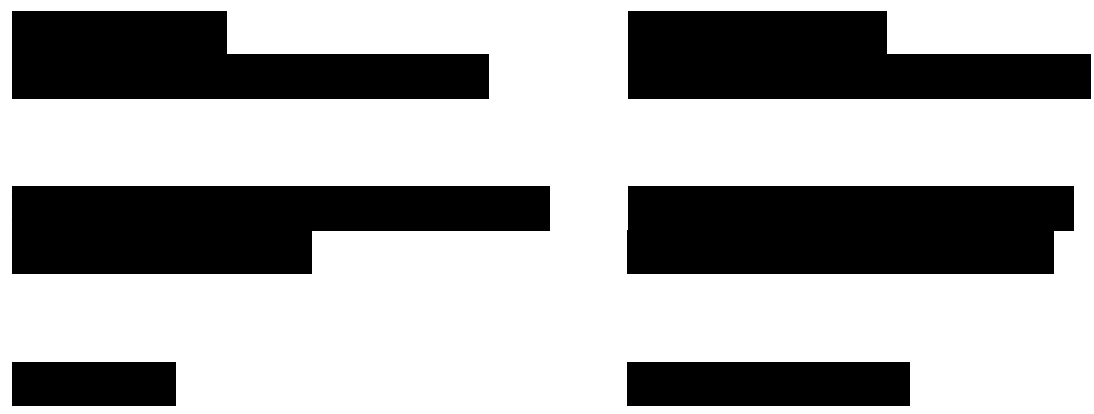

$00: 12: 13: 15$

0141

00:12:13:16

00:12:15:09

0142

00:12:15:12

$00: 12: 17: 13$

0143

00:12:17:24

00:12:20:21
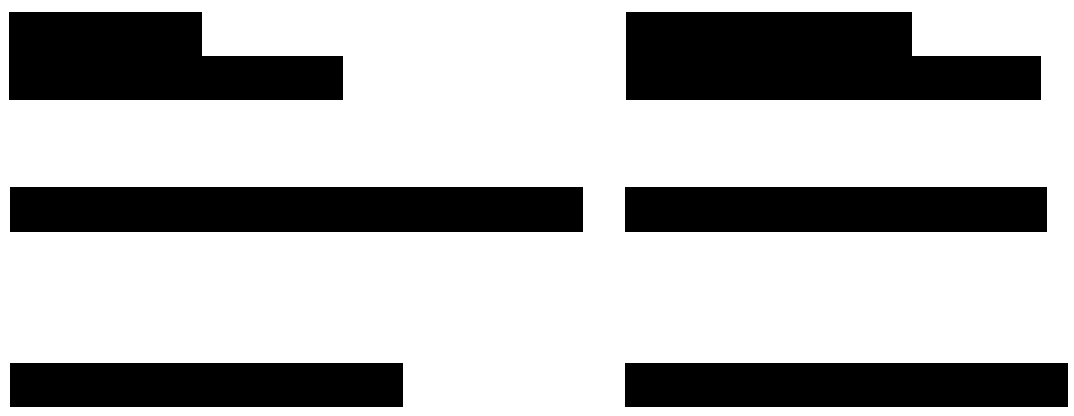

230 'Pratica' has been translated as 'business' here, whereas it was translated as 'file' before. Both translations are acceptable, although the context here supports the translation 'business'. It is also a bit of a play on words as business can refer to the fact that she is about to go to the bathroom. 
0144

00:12:20:23

00:12:23:03

\section{5}

00:12:23:05

$00: 12: 26: 21$

0146

00:12:28:03

$00: 12: 29: 24$

0147

00:12:30:01

$00: 12: 33: 23$

0148

00:12:34:01

$00: 12: 35: 24$

0149

00:12:36:01

$00: 12: 37: 15$

0150

$00: 12: 37: 17$

00:12:41:09

0151

00:12:41:11

$00: 12: 42: 21$

0152

00:12:42:23

00:12:44:21

\section{3}

$00: 12: 44: 23$

$00: 12: 47: 10$

0154

00:12:47:12

00:12:48:20

\section{5}

$00: 12: 48: 22$

00:12:51:09
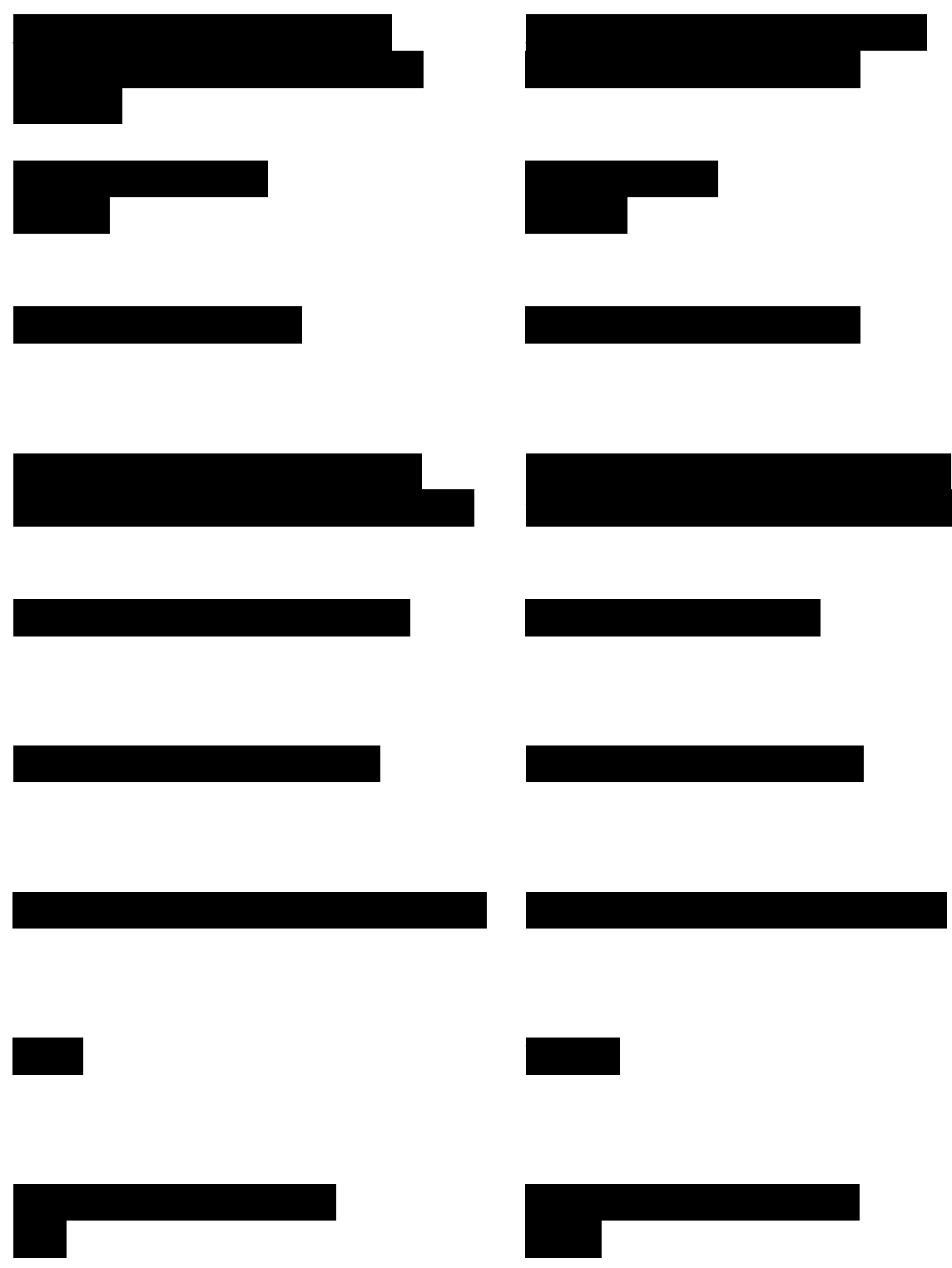

${ }^{231}$ The direct object 'la' here refers to the formal 'you'. 'May I' retains some of the formality that exists in the original. ${ }^{232}$ Here, as we see throughout the film, the characters' title is used (in this example, 'geometra' or 'surveyor). This has been removed as it would not be used in English. Unfortunately this removes some of the subtlety of the original, particularly in placing the characters within a specific social class or status. 
0156

$00: 12: 51: 12$

00:12:53:07

0157

00:12:53:09

00:12:57:00

0158

00:12:57:02

00:12:58:23

0159

00:12:59:00

00:13:01:24

0160

00:13:02:02

00:13:03:13

0161

00:13:04:07

00:13:05:09

0162

00:13:05:11

00:13:08:10

0163

00:13:08:12

00:13:10:02

0164

00:13:10:04

00:13:13:02

\section{5}

00:13:13:05

$00: 13: 14: 22$

0166

00:13:15:00

00:13:16:17

\section{7}

00:13:16:19

00:13:19:10

\section{8}

00:13:19:20

$00: 13: 21: 15$
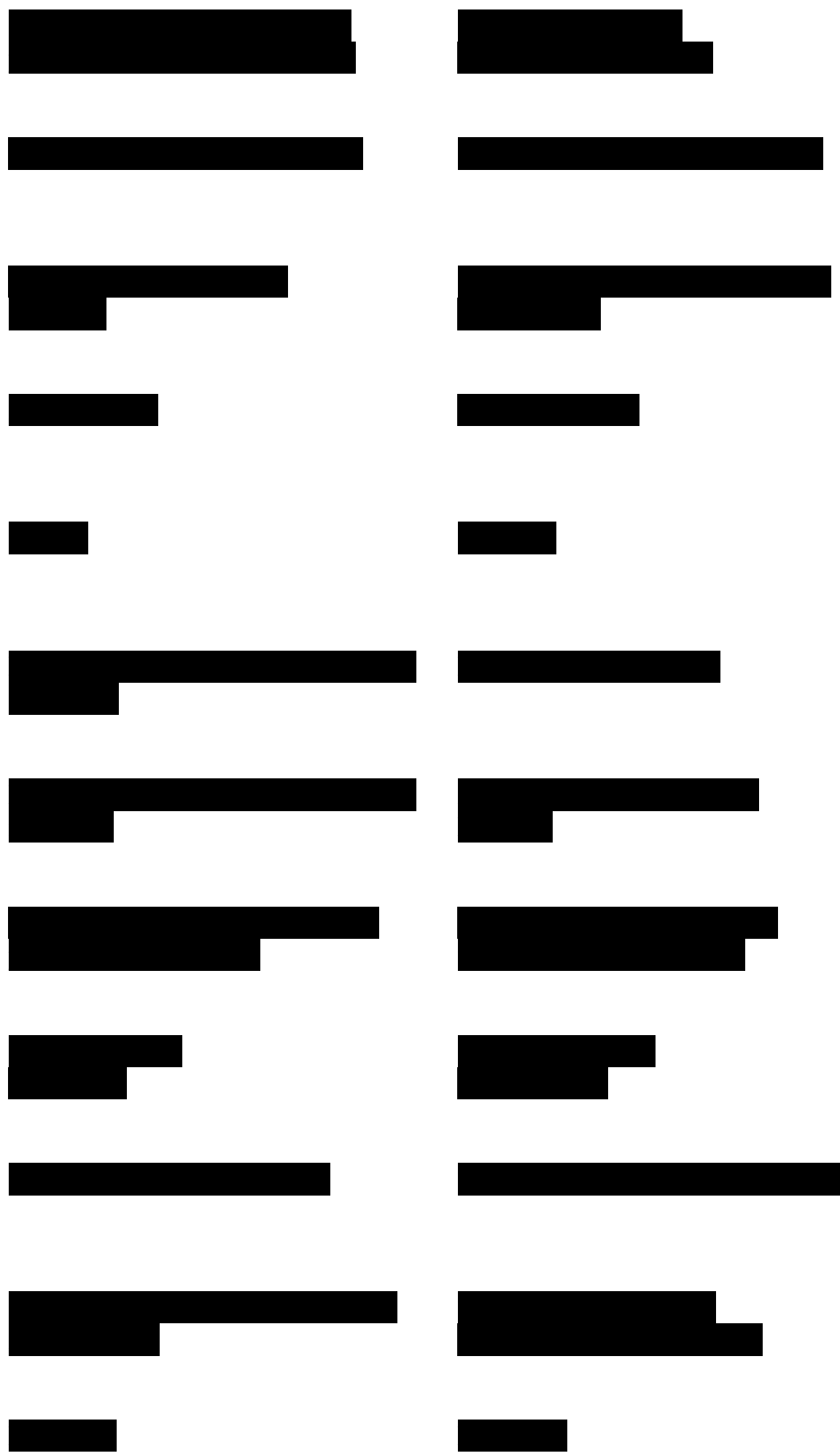

${ }^{233}$ This is the first occasion where we see an improper use of imperatives which will be discussed in depth at the end of this chapter. 
0169

00:13:23:16

00:13:25:17

0170

00:13:25:19

00:13:29:00

\section{1}

00:13:29:12

$00: 13: 32: 23$

0172

$00: 13: 34: 23$

00:13:36:10

0173

00:13:36:12

00:13:38:10

0174

00:13:38:12

00:13:40:22

0175

00:13:40:24

00:13:42:06

0176

00:13:42:08

00:13:45:18

0177

00:13:53:21

00:13:57:07

0178

00:13:57:09

00:13:59:04

0179

00:13:59:08

00:14:00:17

0180

00:14:00:19

00:14:02:11

0181

00:14:03:07

00:14:04:23
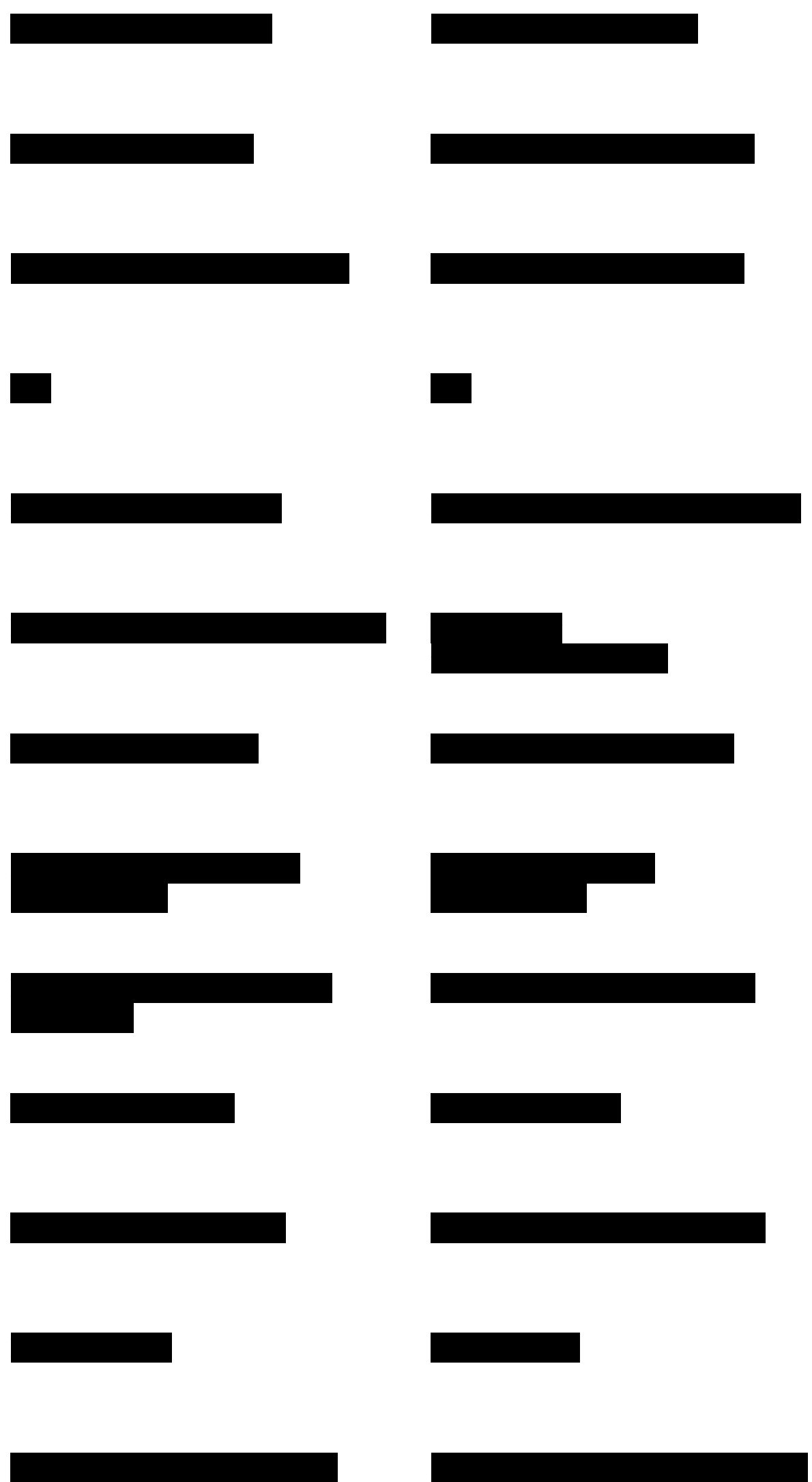

\footnotetext{
${ }^{234}$ As discussed earlier, this is an expression that is repeated across the Fantozzi series and highlights Fantozzi's mistreatment by his colleagues.
} 
0182

00:14:05:00

00:14:08:02

\section{3}

00:14:08:11

00:14:13:11

\section{4}

00:14:13:13

00:14:15:08

0185

00:14:15:11

$00: 14: 17: 12$

\section{6}

00:14:17:15

00:14:20:08

\section{7}

00:14:20:10

00:14:21:11

\section{8}

$00: 14: 21: 13$

$00: 14: 27: 01$

\section{9}

00:14:27:06

$00: 14: 28: 12$

\section{0}

00:14:28:14

00:14:32:06

\section{1}

00:14:32:08

$00: 14: 33: 18$

\section{2}

00:14:33:20

00:14:37:13

\section{3}

00:14:37:15

00:14:39:17
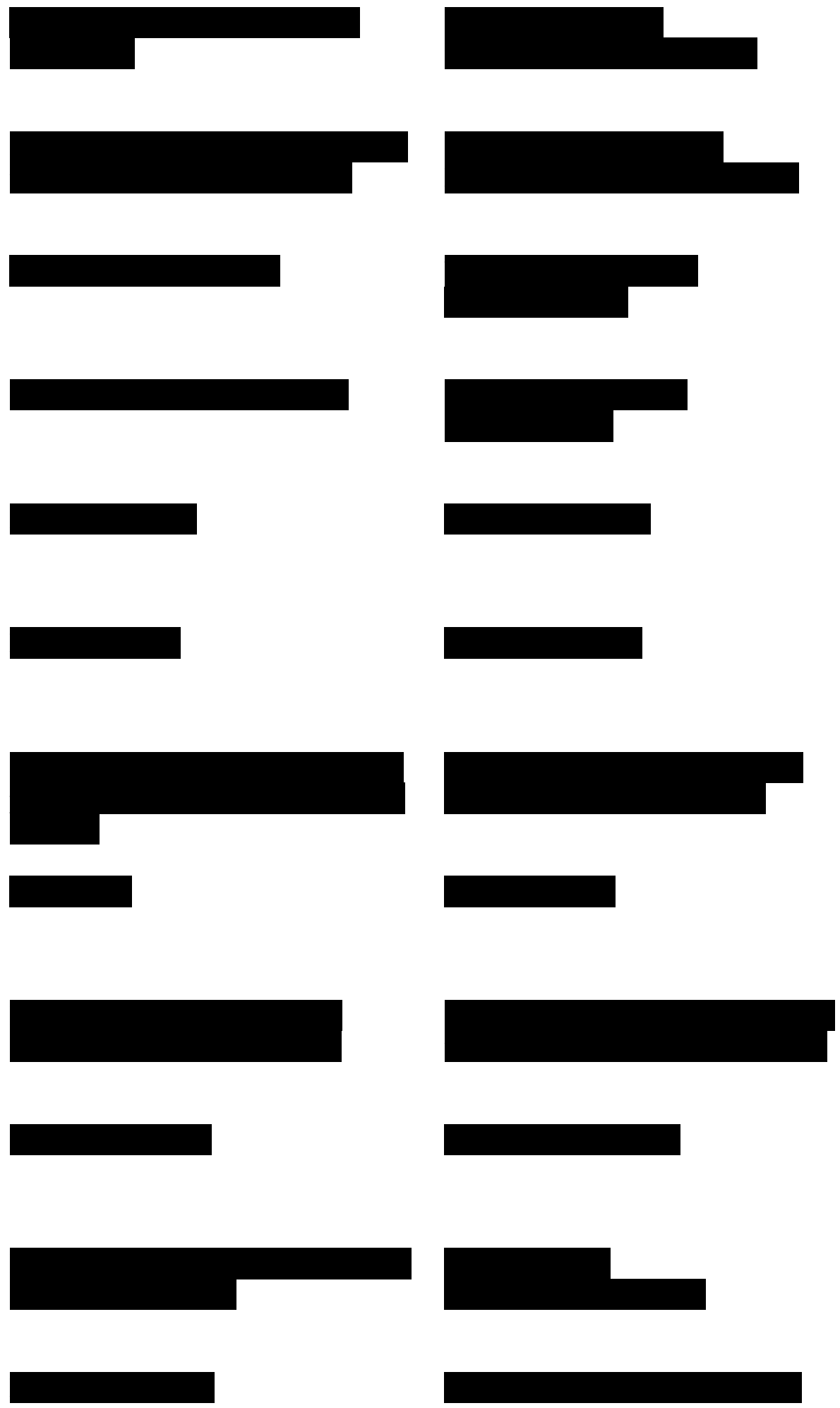

\footnotetext{
${ }^{235}$ This is an extract from Lorenzo de' Medici's Canzone de Bacco (1490). Pre-existing translations exist in English so I have one of these in the subtitles: Christopher DiMatteo, "Translating Lorenzo the Magnificent," http://cdimatteo.com/italian/translating-lorenzo-the-magnificent/. accessed 13th January, 2018.

${ }^{236}$ Literally 'Speak, speak'. 'Keep going' has the same meaning but is more colloquial in English.
} 
0194

00:14:40:17

$00: 14: 42: 19$

\section{5}

00:14:42:23

00:14:44:19

0196

00:15:08:01

00:15:10:02

0197

00:15:32:15

00:15:34:04

0198

00:15:34:06

$00: 15: 37: 15$

0199

00:15:49:23

00:15:51:18

0200

00:15:51:21

00:15:54:06

0201

00:15:59:08

00:16:00:21

0202

00:16:00:23

00:16:02:09

0203

00:16:02:11

00:16:07:21

0204

00:16:09:24

$00: 16: 12: 22$
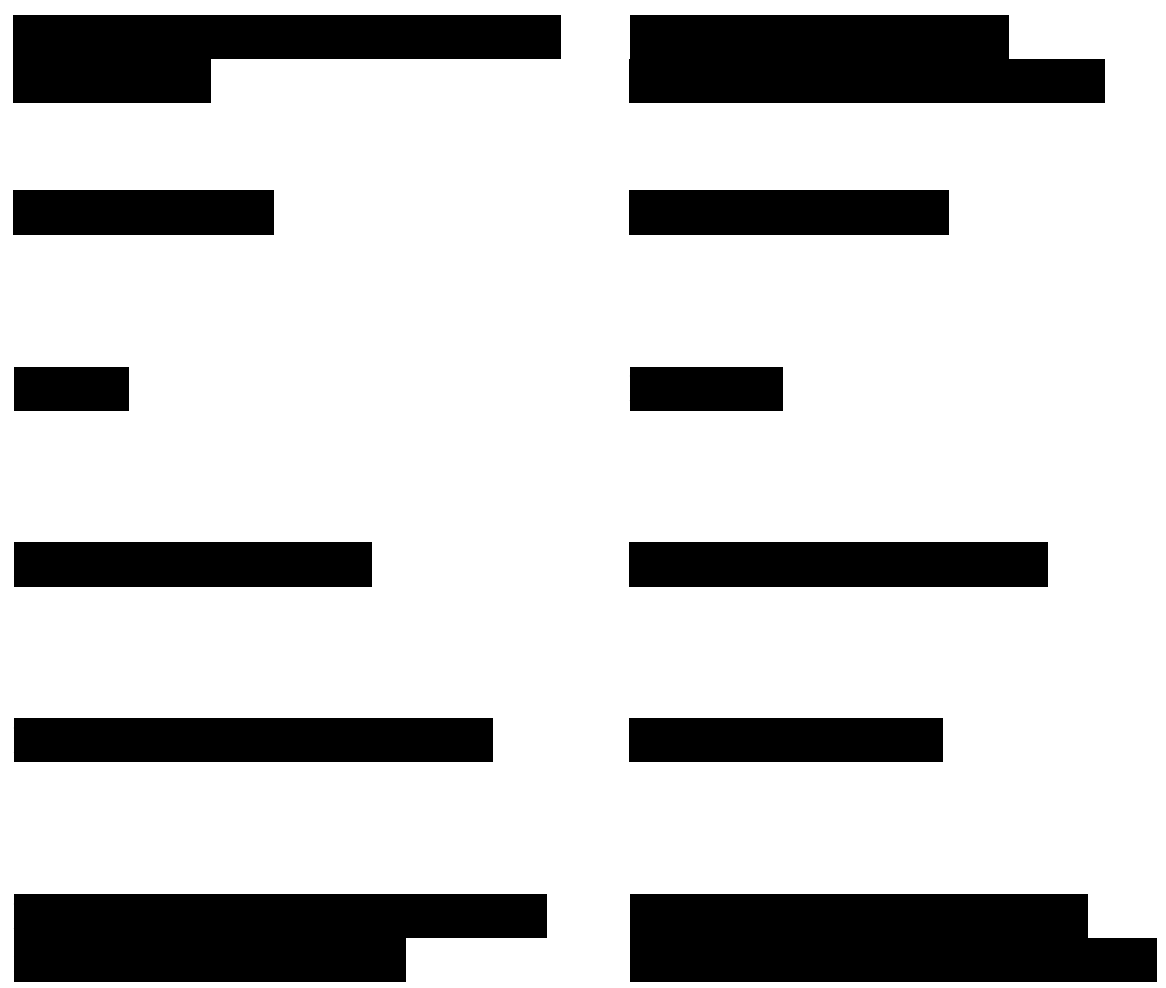

0205

00:16:13:00

00:16:14:13
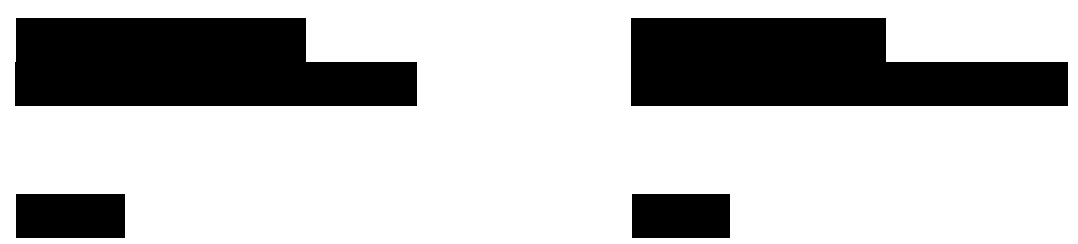

237 The translation could have simply been 'piece of shit' however given that the augmentative has been used in the original, I have decided to retain this.

${ }^{238}$ Literally 'Give him a punch' although this would not be said in English.

239 "If you have the courage" is too long here, given that the subtitle is split across two lines already. Instead the colloquial "if you dare" has been used. 
0206

00:16:16:11

00:16:20:06

0207

00:16:20:09

00:16:22:00

0208

00:16:32:10

$00: 16: 35: 19$

0209

00:16:35:21

$00: 16: 37: 15$

0210

$00: 16: 37: 17$

00:16:39:20

0211

00:16:41:24

$00: 16: 45: 17$

0212

00:16:46:01

00:16:48:20

0213

00:16:48:24

$00: 16: 52: 12$

0214

00:16:52:14

$00: 16: 55: 10$

0215

00:16:57:24

00:17:00:00

0216

00:17:00:02

00:17:02:15

0217

00:17:02:18

00:17:06:18

0218

00:17:06:19

00:17:09:08

0219

00:17:09:09

$00: 17: 11: 10$
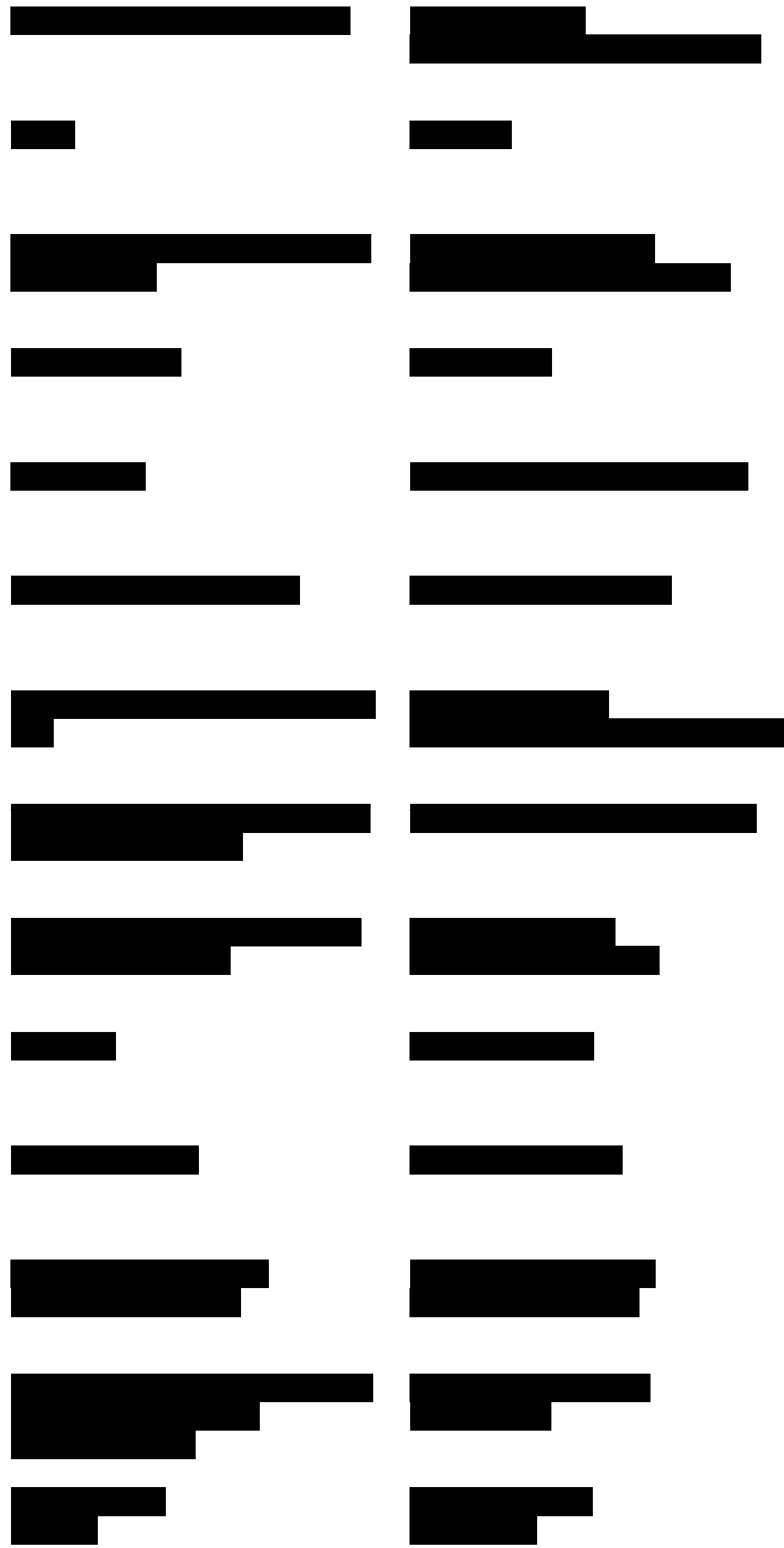
0220

00:17:11:12

$00: 17: 14: 22$

0221

00:17:15:00

00:17:18:09

0222

00:17:18:11

00:17:21:02

0223

00:17:21:04

$00: 17: 22: 17$

0224

00:17:22:21

$00: 17: 25: 23$

0225

00:17:47:15

00:17:48:21

0226

$00: 17: 48: 23$

00:17:51:13

0227

00:17:53:09

$00: 17: 55: 17$

0228

00:17:55:19

00:17:59:06

0229

00:17:59:08

00:18:01:17

0230

00:18:01:20

00:18:03:00

\section{1}

00:18:04:02

00:18:06:12

0232

00:18:06:22

00:18:07:22

\section{3}

00:18:07:24

00:18:09:07
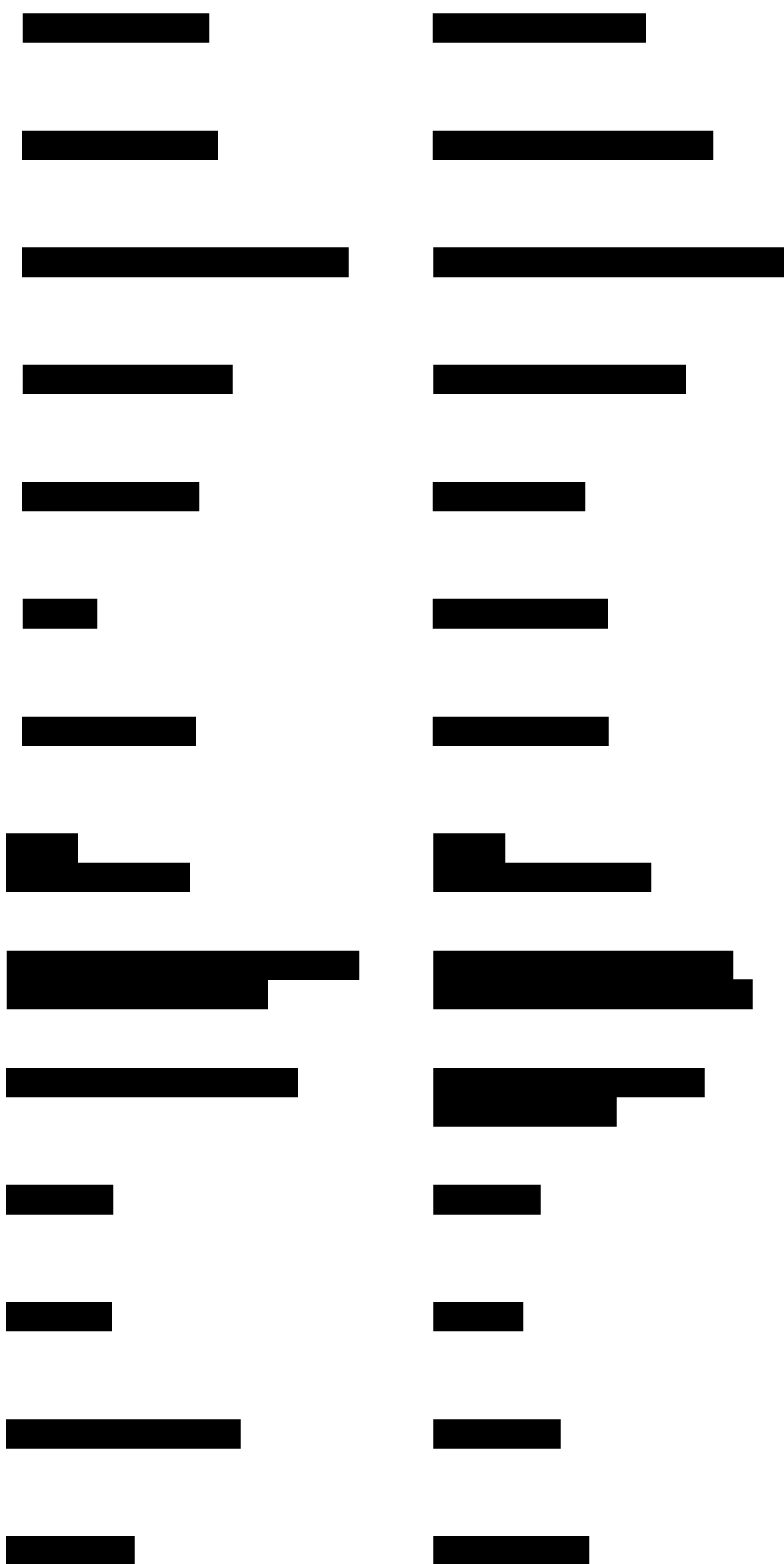
0234

00:18:18:01

00:18:20:07

\section{5}

00:18:33:16

00:18:35:22

\section{6}

00:18:45:03

00:18:47:11

0237

00:18:48:00

00:18:49:08

\section{8}

00:18:49:10

00:18:50:14

0239

00:18:50:16

$00: 18: 53: 15$

0240

00:18:53:19

00:18:56:09

\section{1}

00:18:56:24

00:19:02:01

0242

00:19:15:03

00:19:18:08

0243

00:19:18:10

00:19:21:07

0244

00:19:21:09

00:19:23:20

\section{5}

00:19:23:21

$00: 19: 25: 18$
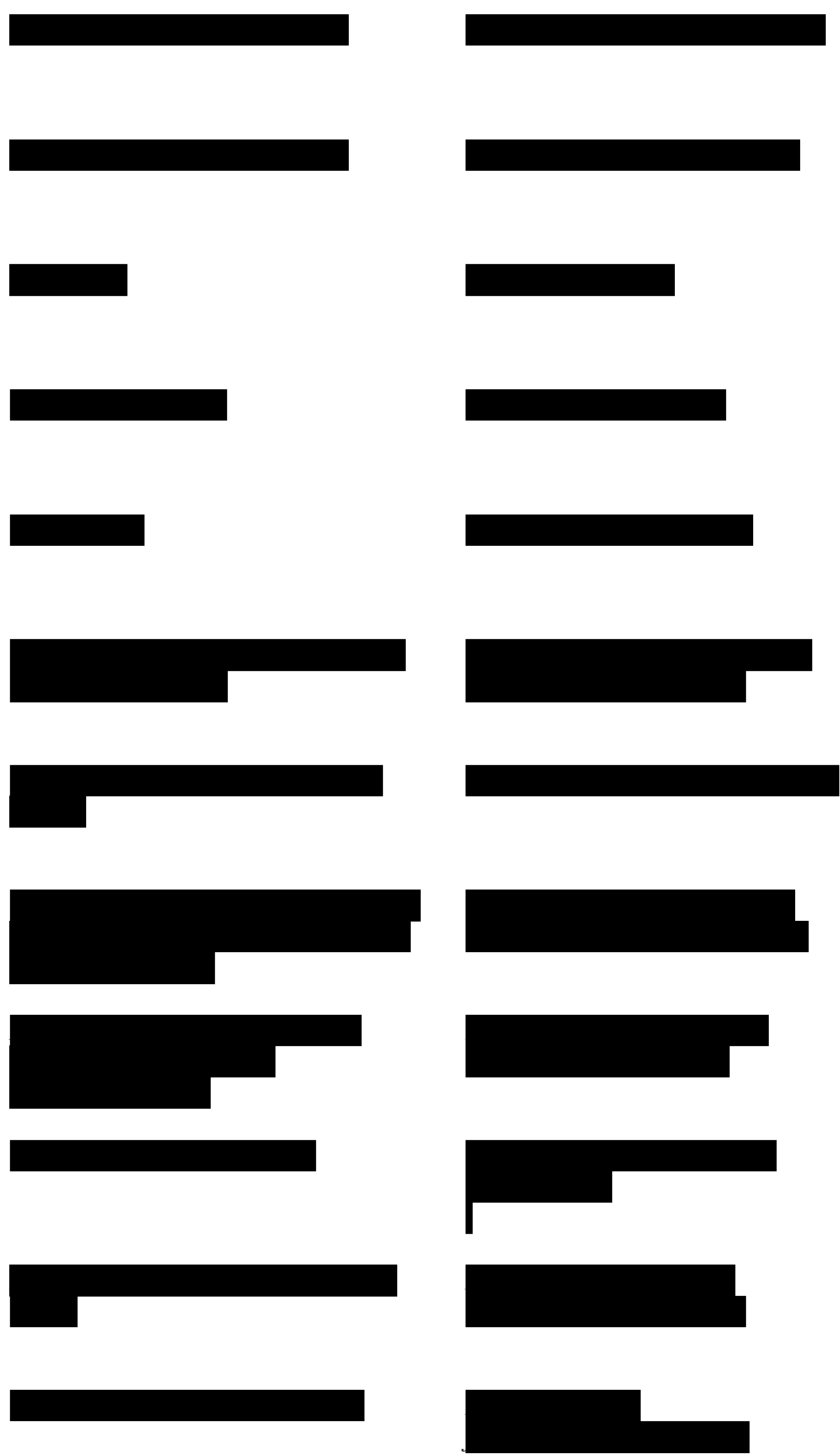

\footnotetext{
${ }^{240}$ Every time text appears on screen it is important to translate this into the TL. Often there are humorous elements, or messages that convey context that would otherwise be lost.

${ }^{241}$ There is no specific reference to the coffee machine apart from the fact that it's a feminine adjective. This reference can be inserted into the English for clarification. 'Maledetta' literally means 'cursed' or 'damned'.

${ }^{242}$ I have simply decided to translate this as coins, as Fantozzi is pulling out a coin on-screen. It also resolves the issue of which currency to use in this particular context, although this problem does occur again later in the film.
} 

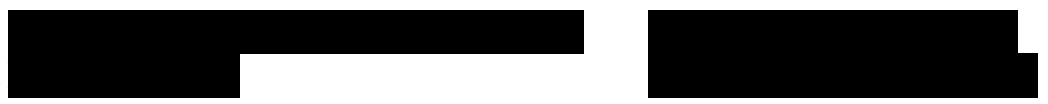

00:19:28:04

0247

00:19:28:05

00:19:29:22

0248

00:19:29:23

00:19:33:10
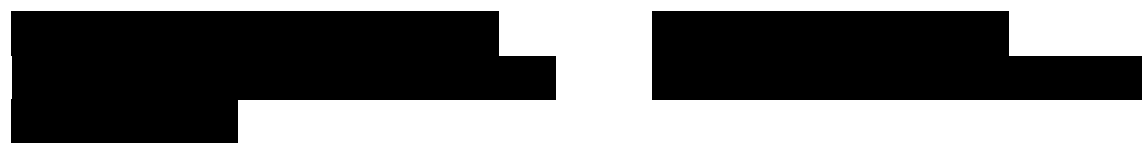

0249

00:19:33:12

00:19:36:13
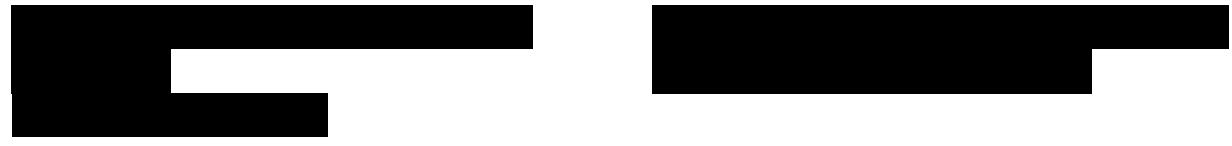

0250

00:19:36:15

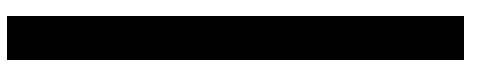

00:19:39:06

0251

00:19:39:08
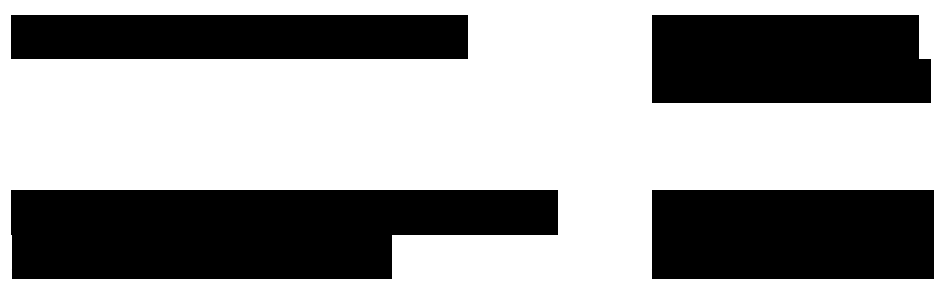

00:19:42:16

0252

00:19:42:18
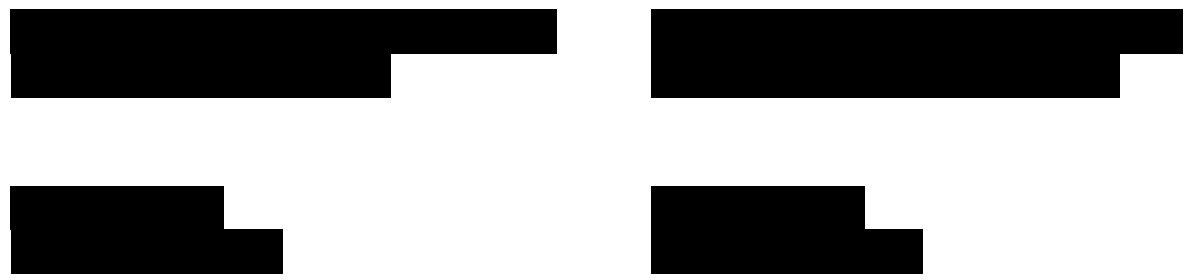

00:19:44:01

0253

00:19:44:03

00:19:46:14
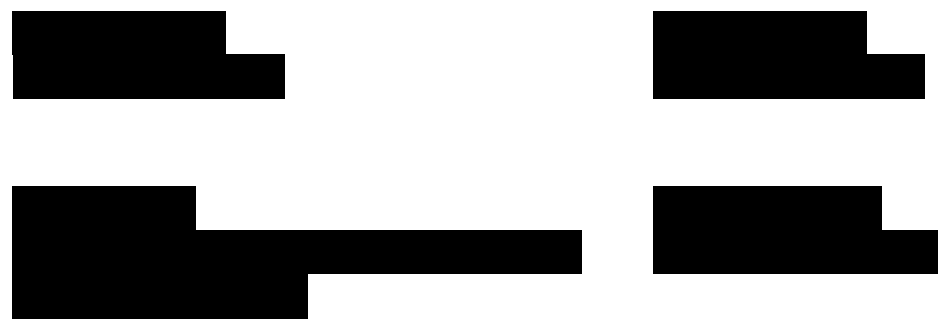

0254

00:19:46:16

00:19:51:05

0255

00:19:51:07

$00: 19: 53: 14$

0256

00:19:53:17

00:19:57:23
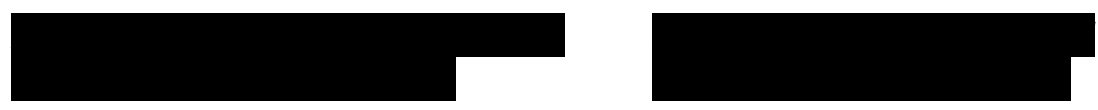

\section{7}

00:19:58:00

00:20:02:00
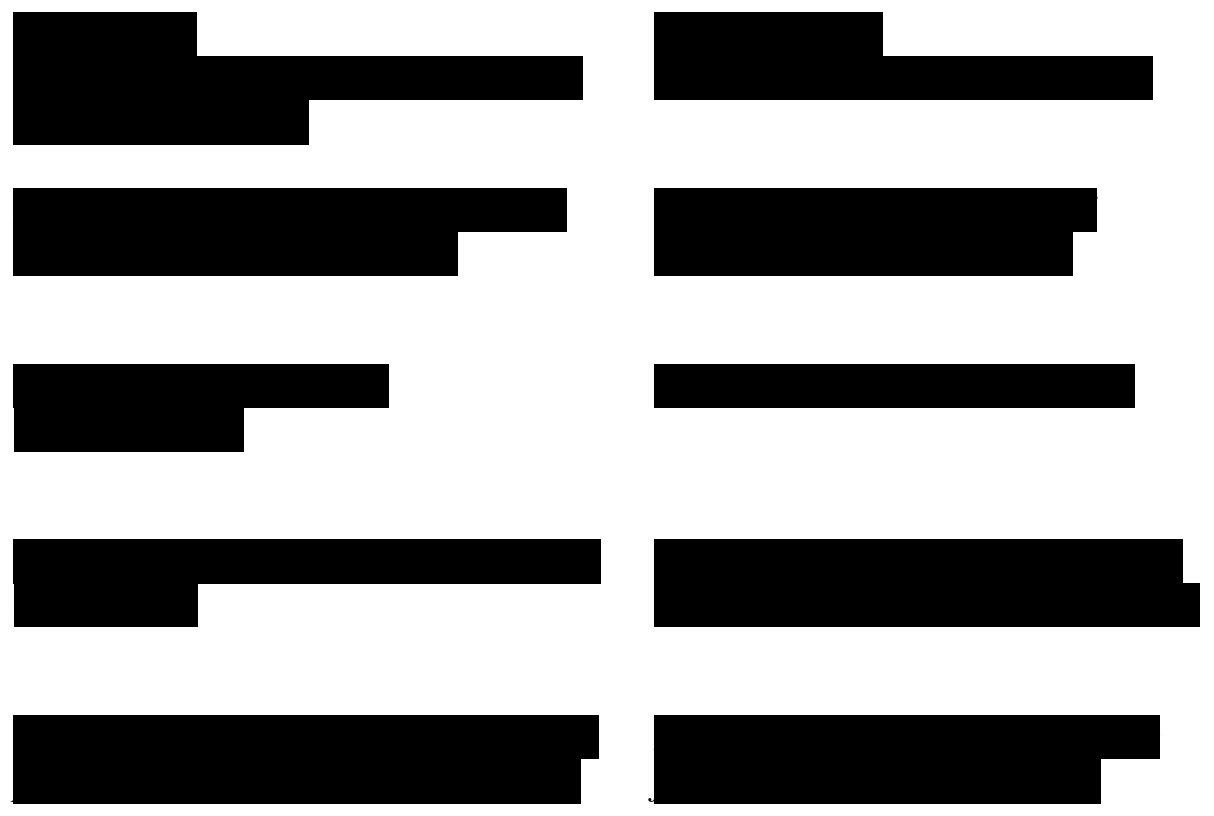

\footnotetext{
${ }^{243}$ The currency of the 'lira' here has been replaced with 'dollars'. This has been done as the currency in the original Italian is no longer in use (the euro has subsequently become the currency of Italy) therefore using 'lira' would mean nothing to the English speaking audience of today. Using 'dollar' resolves this issue, although could theoretically cause problems to an audience that does not use this currency. I have, however, attempted to use similar numbers to the original. For example 5000 lire becomes $\$ 50$ - a rough equivalent in today's currency value. This issue is discussed further in the next chapter. ${ }^{244}$ Another common theme throughout the Fantozzi series is making fun of his daughter Mariangela. Here Filini doesn't remember her name. Because the Italian names are not foreign to an English speaking audience I have decided to retain them. This scene is mirrored in Il secondo tragico Fantozzi (1976).
} 


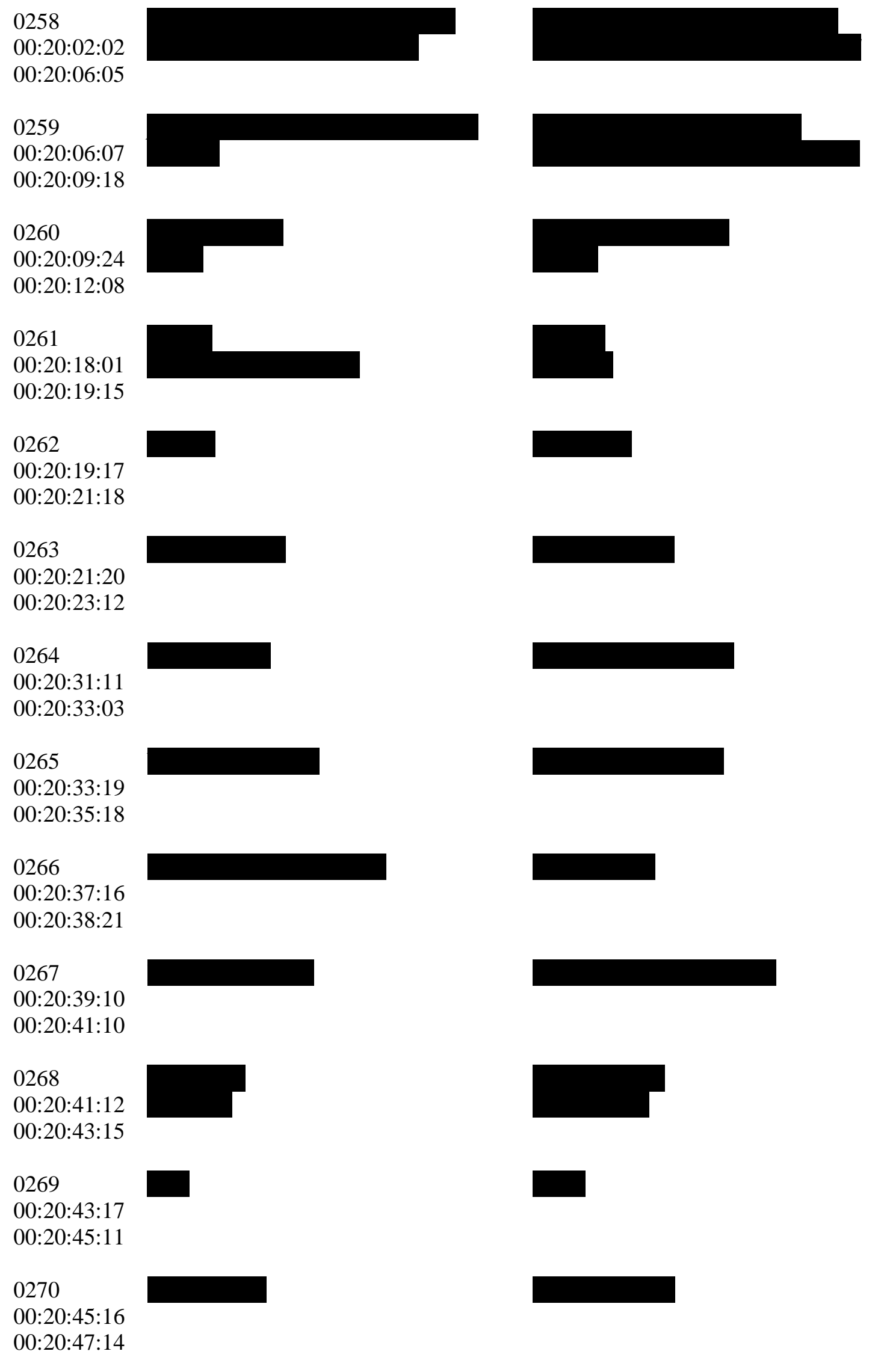

245 'Perito', meaning 'consultant', is another title that has been left out as it would otherwise sound strange in English. 


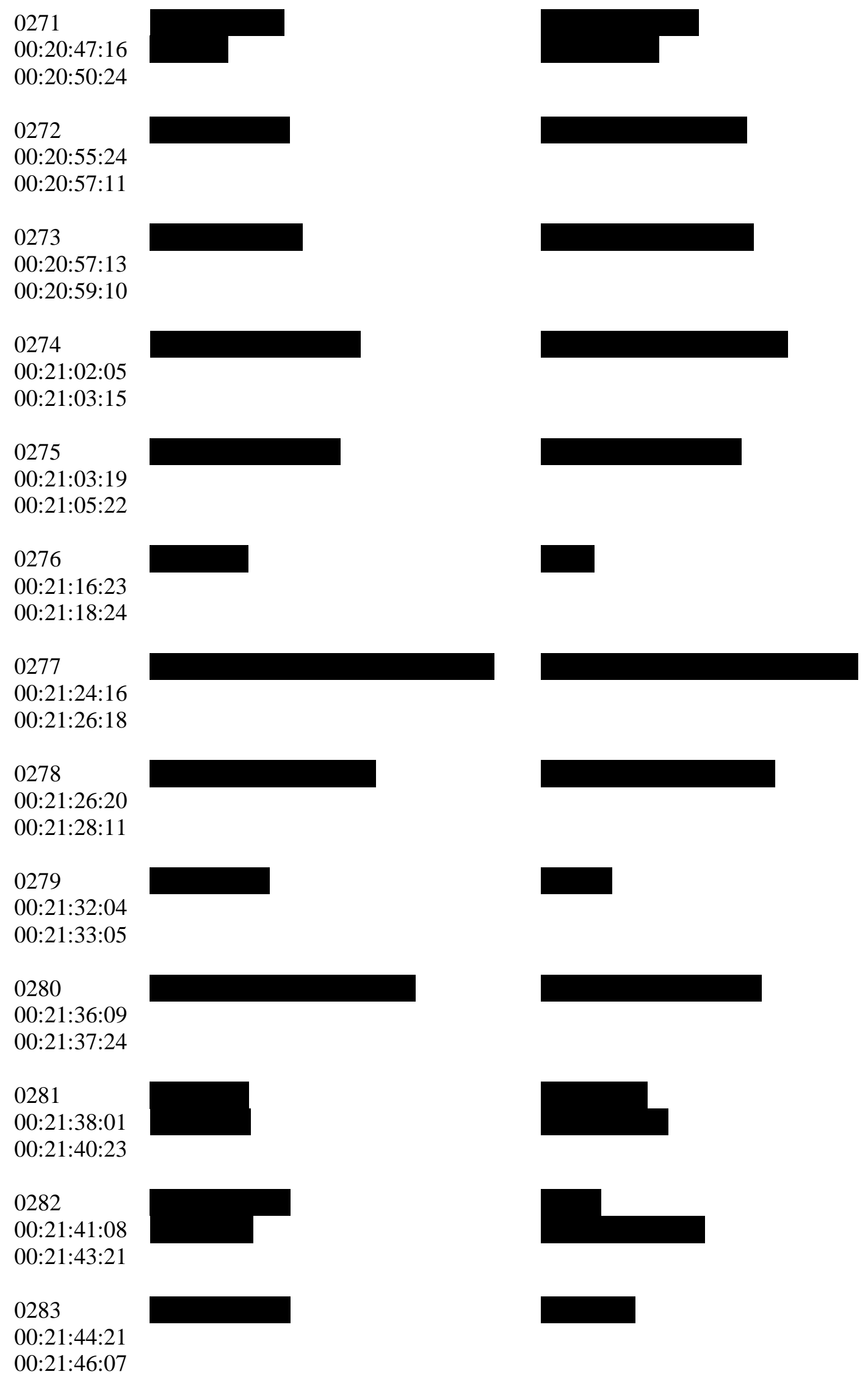

${ }^{246}$ Colloquial term used in football to mean that he pretended to be fouled. Also known as 'simulation'. 
0284

00:21:46:09

00:21:49:11

0285

00:21:53:06

00:21:55:04

0286

00:22:03:18

00:22:05:07

0287

00:22:11:05

$00: 22: 13: 12$

0288

00:22:13:14

$00: 22: 15: 19$

0289

00:22:16:16

00:22:18:17

0290

00:22:18:19

00:22:20:17

0291

00:22:20:18

00:22:22:05

0292

00:22:25:22

00:22:28:04

0293

00:22:28:06

00:22:30:07

0294

00:22:30:09

00:22:32:01

0295

00:22:32:03

00:22:34:02

0296

00:22:35:04

00:22:36:09

0297

00:22:37:17

00:22:38:21
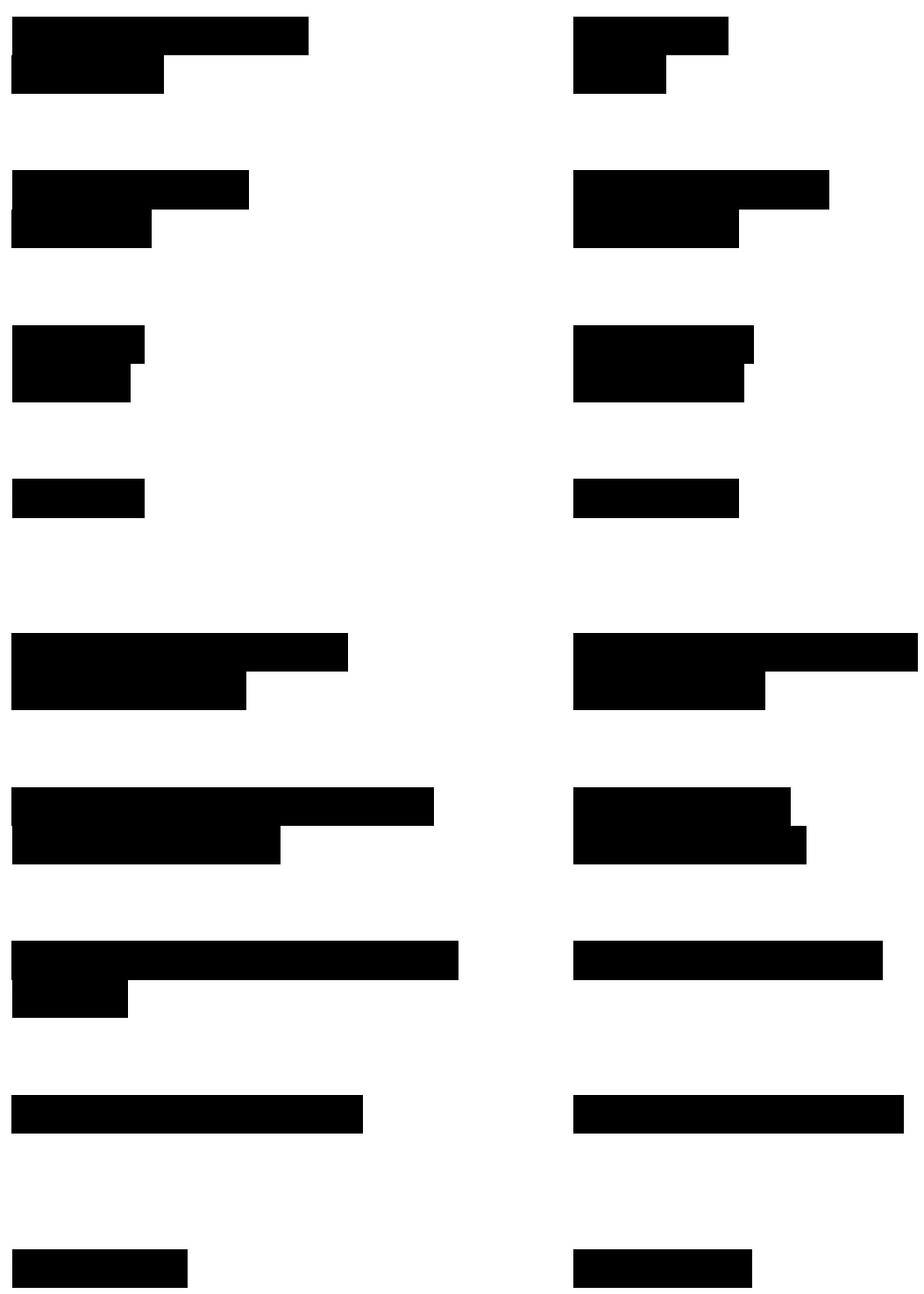
0298

00:22:40:08

00:22:41:11

0299

00:22:41:13

00:22:43:05

0300

00:22:48:21

00:22:52:01

0301

00:22:52:03

00:22:55:08

0302

00:22:55:10

00:22:57:20

0303

00:22:59:04

00:23:00:14

0304

00:23:01:07

00:23:02:19

\section{5}

00:23:04:04

00:23:05:18

0306

00:23:07:20

00:23:13:24

0307

00:23:14:01

00:23:18:20

0308

00:23:19:00

$00: 23: 21: 14$

0309

00:23:21:16

$00: 23: 24: 17$
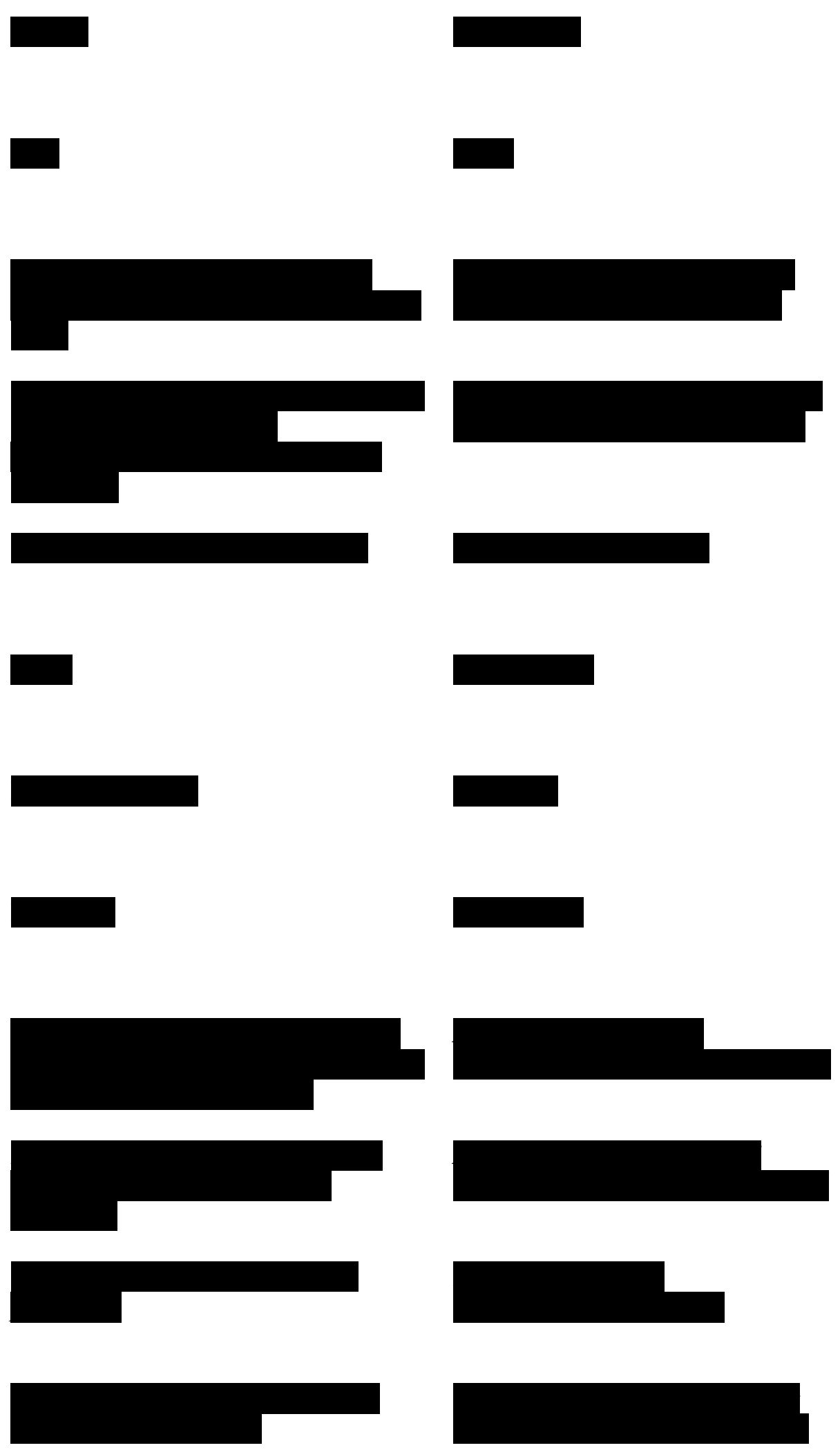

247 'Fantozzi's Cloud' has become a commonly used phrase in contemporary Italian for when it rains while on holiday, going to show how aspects of the Fantozzi saga have become staple references of Italian culture: "Le cose che diciamo per via di Fantozzi," Il Post, http://www.ilpost.it/2017/07/03/fantozzi-paolo-villaggio-lingua/. accessed 13th Februrary, 2018. 
0310

00:23:24:19

$00: 23: 27: 16$

\section{1}

00:23:27:18

00:23:32:09

0312

00:23:39:07

$00: 23: 40: 22$

0313

00:23:45:19

$00: 23: 46: 22$

0314

00:23:47:12

00:23:48:22

0315

00:23:48:24

00:23:51:00

0316

00:23:59:15

00:24:01:14

0317

00:24:06:00

00:24:08:07

0318

00:24:08:09

00:24:10:02

0319

00:24:10:04

00:24:11:13

0320

00:24:12:04

$00: 24: 15: 16$

0321

00:24:15:18

00:24:18:08

0322

00:24:19:13

$00: 24: 23: 10$
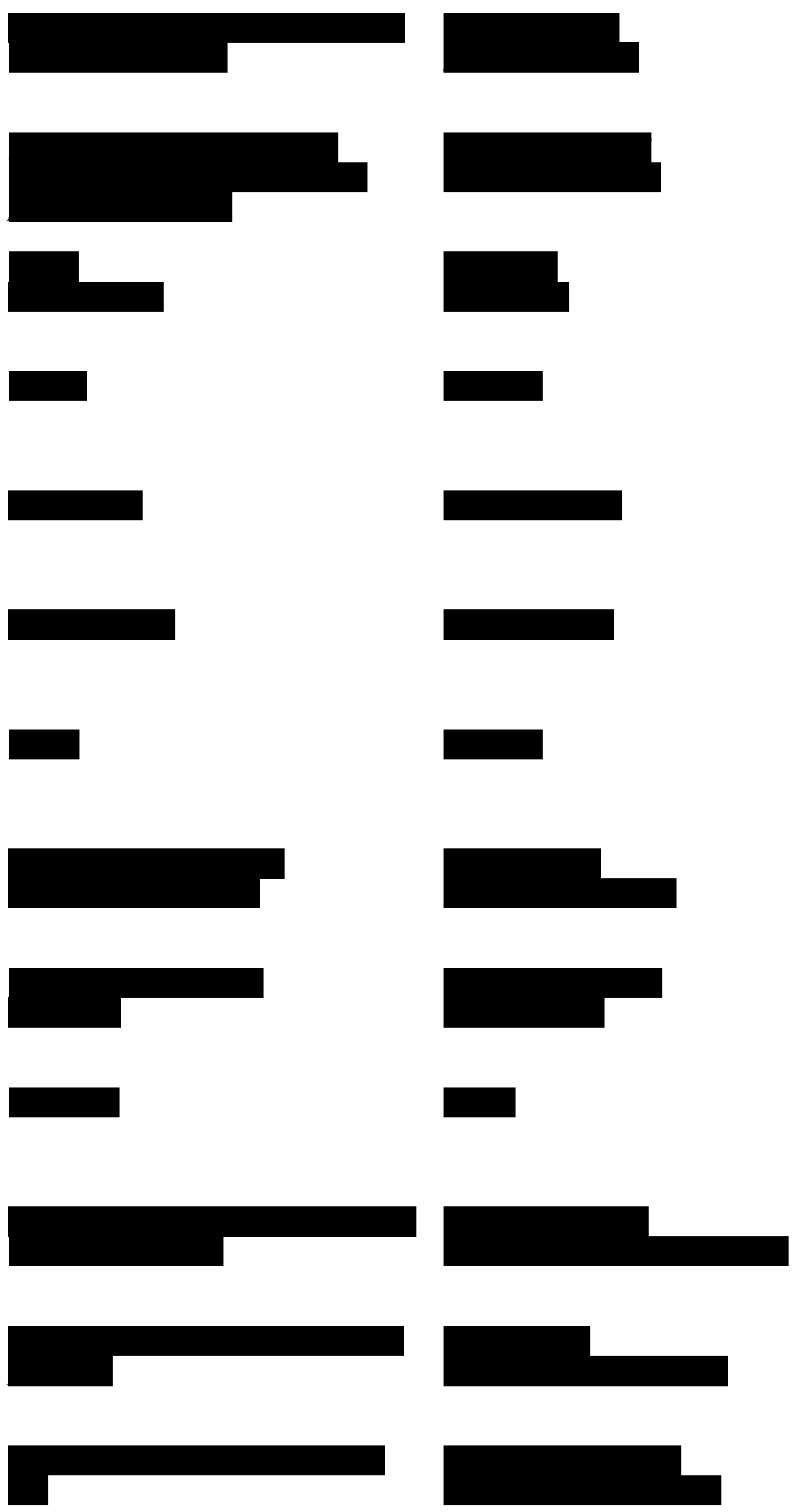

${ }^{248}$ Fantozzi's hallucinations often involve biblical figures. Because these figures have Anglicised names these have been used in translation. 


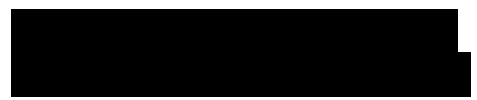

0324

00:24:26:01

00:24:27:20

\section{5}

00:24:27:21

00:24:30:17

\section{6}

00:24:31:15

$00: 24: 33: 24$

\section{7}

00:24:34:00

$00: 24: 35: 19$

\section{8}

00:24:35:20

$00: 24: 38: 16$

0329

00:24:38:19

$00: 24: 42: 23$
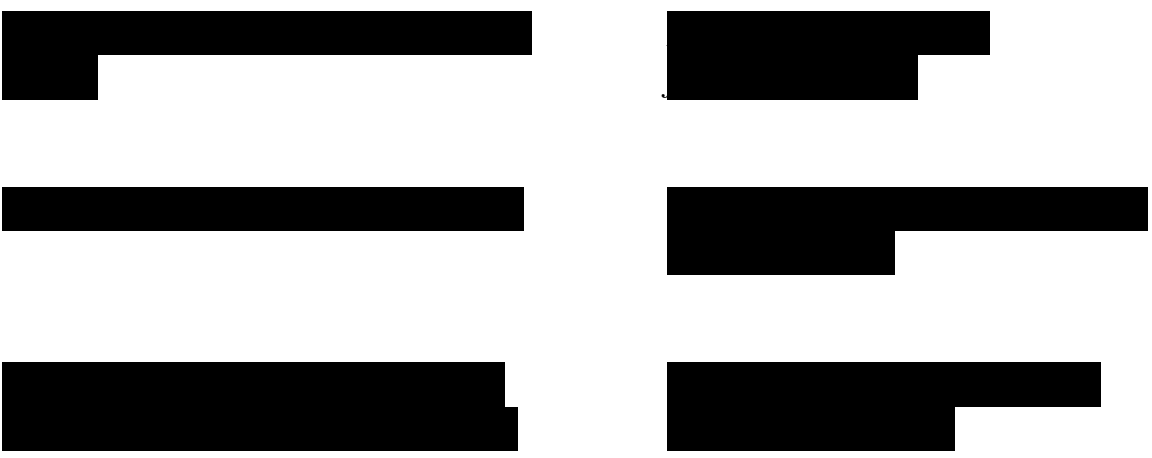

\section{0 \\ 00:24:43:00 \\ $00: 24: 47: 12$}

0331

00:24:55:20

00:24:58:23
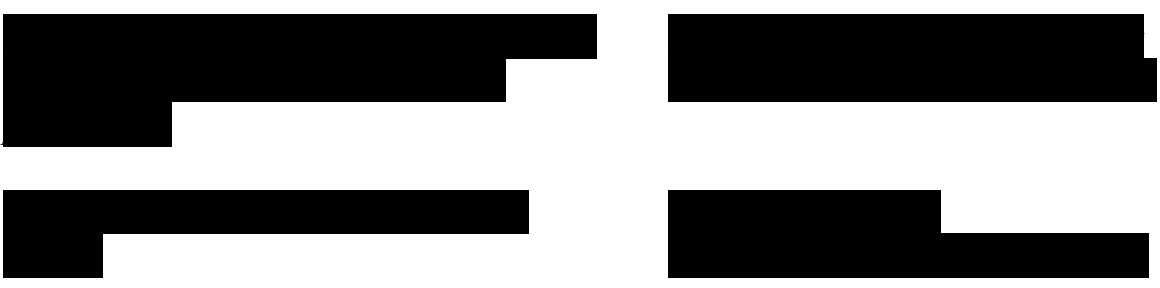

0332

00:24:59:00

00:25:01:17

\section{3}

00:25:02:02

00:25:04:04
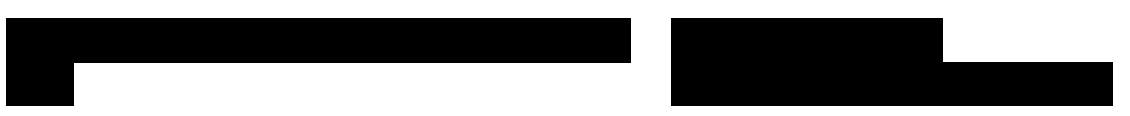

0334

00:25:04:06

00:25:05:23
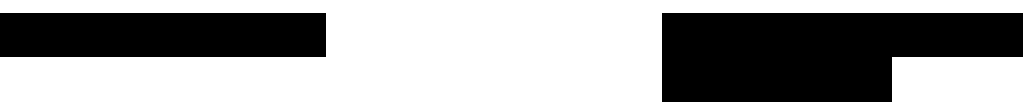

\footnotetext{
249 'Cambiali' is a concept similar to an IOU (I owe you), where a person borrows money and pays it back over time. While it is not exactly the same as a debt, it is the closest alternative that exists in English.

${ }^{250}$ In translating these segments of dialogue I have tried to maintain comprehension to an English audience, given that an Italian audience is, comparatively, more likely to be familiar with French than English-speaking viewers. When there is code-mixing, or when the expressions are well-known, I have translated these. Where the dialogue is meant to be incomprehensible, I have simply subtitled it as [Speaking in $\mathrm{x}$ language]
} 
0335

00:25:06:01

$00: 25: 07: 22$

0336

00:25:09:12

$00: 25: 12: 23$

0337

00:25:13:00

$00: 25: 15: 12$

0338

00:25:17:04

00:25:19:01

0339

00:25:19:06

$00: 25: 21: 12$

0340

00:25:22:02

$00: 25: 25: 20$

0341

$00: 25: 26: 24$

00:25:30:01

0342

00:25:30:03

$00: 25: 33: 11$

0343

00:25:33:20

00:25:35:06

0344

00:25:35:08

$00: 25: 37: 20$

\section{5}

$00: 25: 37: 22$

$00: 25: 39: 24$

0346

00:25:41:19

00:25:45:05

0347

00:25:45:15

00:25:47:20
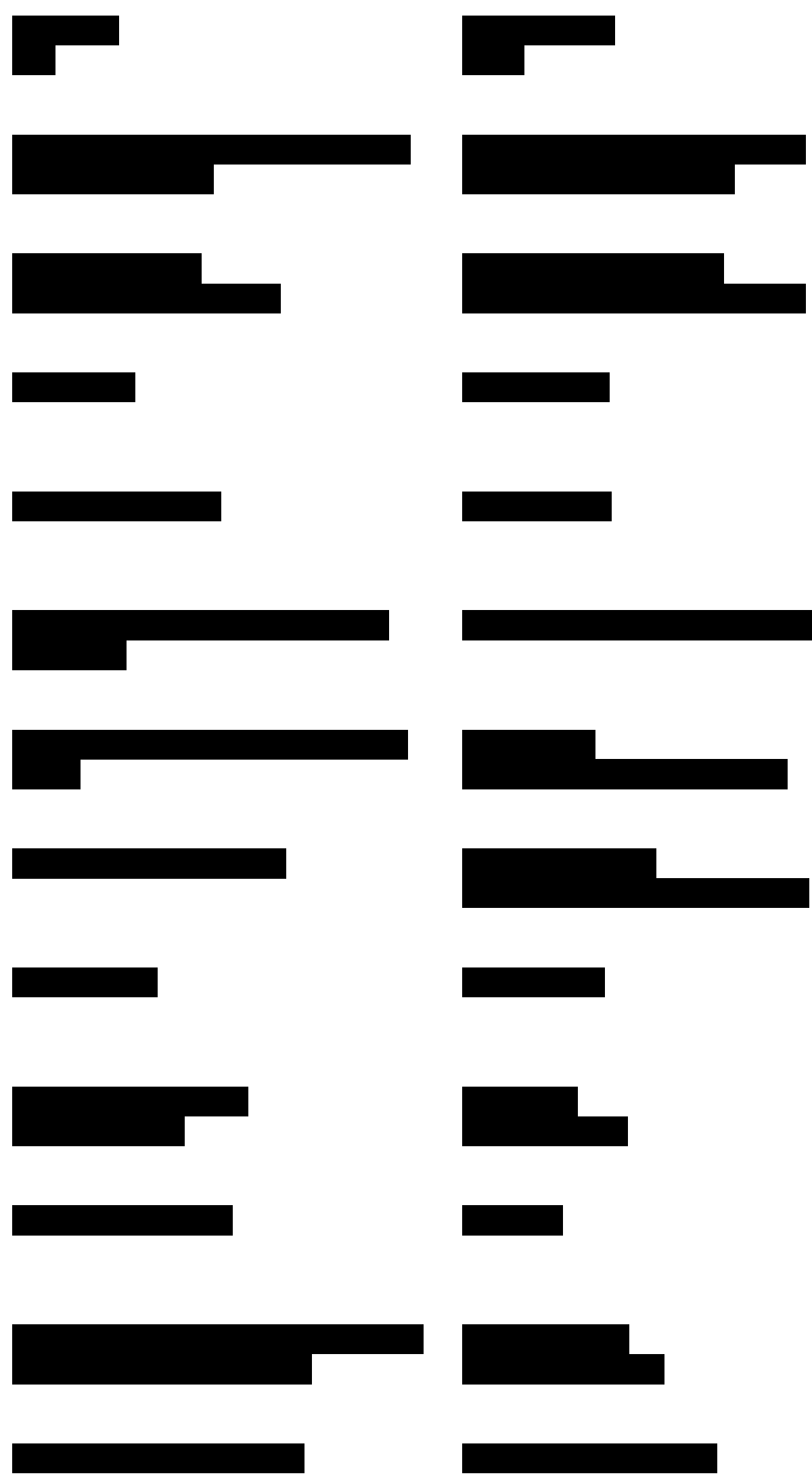

\footnotetext{
${ }^{251}$ Very common expression in French and therefore translated.
} 
0348

00:25:51:01

00:25:53:04

\section{9}

00:25:53:08

$00: 25: 56: 23$

0350
$00: 25: 57: 00$
$00: 26: 02: 17$

0351

00:26:02:19

00:26:07:15

0352

00:26:08:01

00:26:09:14

0353

00:26:09:16

00:26:10:17

0354

00:26:10:19

00:26:12:01

\section{5}

00:26:15:01

$00: 26: 17: 23$

0356
00:26:18:00
00:26:19:06

0357

00:26:19:09

00:26:21:06

\section{8}

00:26:22:21

$00: 26: 25: 14$

\section{9}

$00: 26: 25: 17$

$00: 26: 27: 24$

0360

00:26:28:01

00:26:32:02
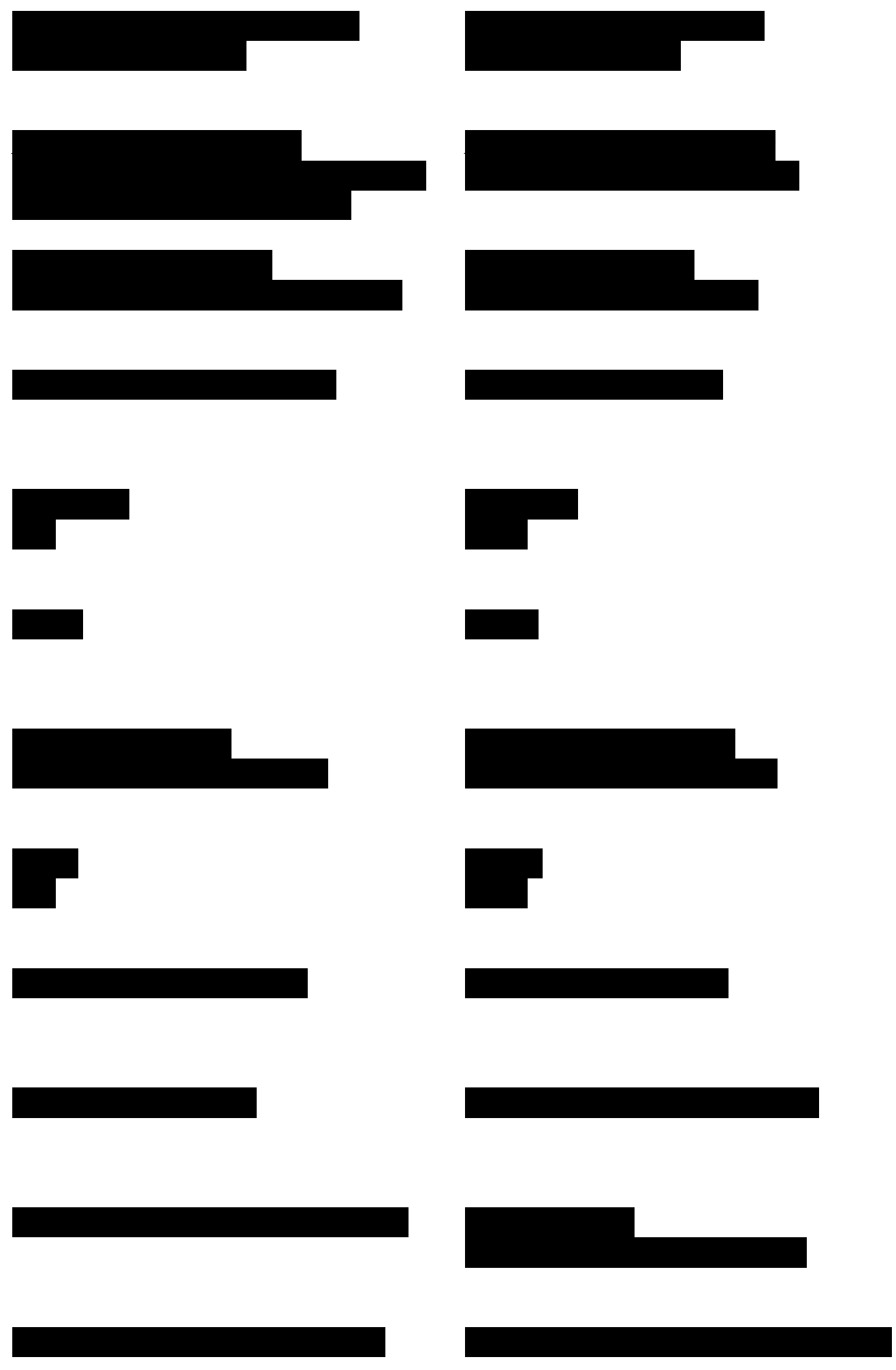

\footnotetext{
${ }^{252}$ This dialogue is mirrored in the hunting scene of Il secondo tragico Fantozzi (1976).
} 
0361

00:26:32:04

00:26:34:05

0362

00:26:34:07

$00: 26: 35: 17$

0363

00:26:35:19

00:26:38:24

\section{4}

00:26:41:02

00:26:44:13

\section{5}

00:26:44:21

00:26:47:03

0366

00:26:55:10

$00: 26: 57: 14$

\section{7}

00:26:58:14

00:27:01:18

0368

00:27:02:20

00:27:04:14

\section{9}

00:27:04:16

00:27:07:21

\section{0}

00:27:07:24

00:27:10:07

0371

00:28:05:06

00:28:08:13

0372

00:28:08:15

00:28:10:19

0373

00:28:10:21

$00: 28: 13: 18$
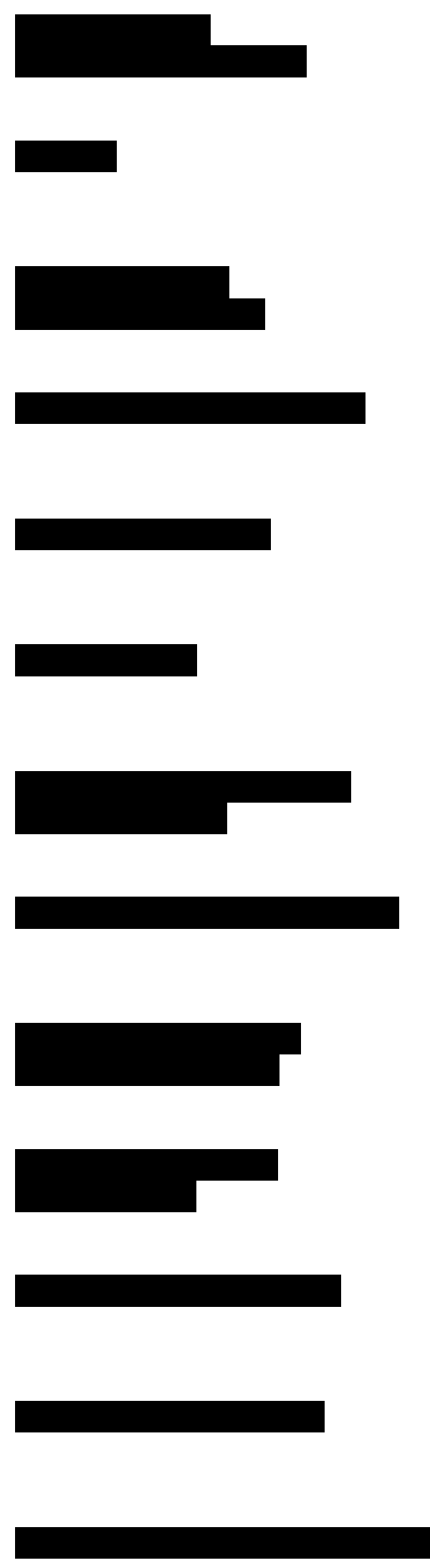

${ }^{253}$ This is a classic example of the visual humour in the film, highlighted in chapter two. Fantozzi's hands or head always tend to be subjected to pain. 
0374

00:29:18:15

00:29:20:11

0375

00:29:22:17

$00: 29: 23: 24$

0376

00:29:27:11

00:29:29:09

\section{7}

00:29:33:15

00:29:36:20

0378

00:29:39:10

00:29:41:21

0379

00:29:42:05

00:29:43:18

\section{0}

00:29:48:19

00:29:51:03

0381

00:29:51:06

$00: 29: 52: 22$

\section{2}

00:29:52:24

00:29:55:06

\section{3}

00:30:00:13

00:30:04:11

\section{4}

00:30:04:15

00:30:06:19

\section{5 \\ 00:30:07:16 \\ 00:30:12:03}

\section{6}

00:30:13:02

00:30:17:06
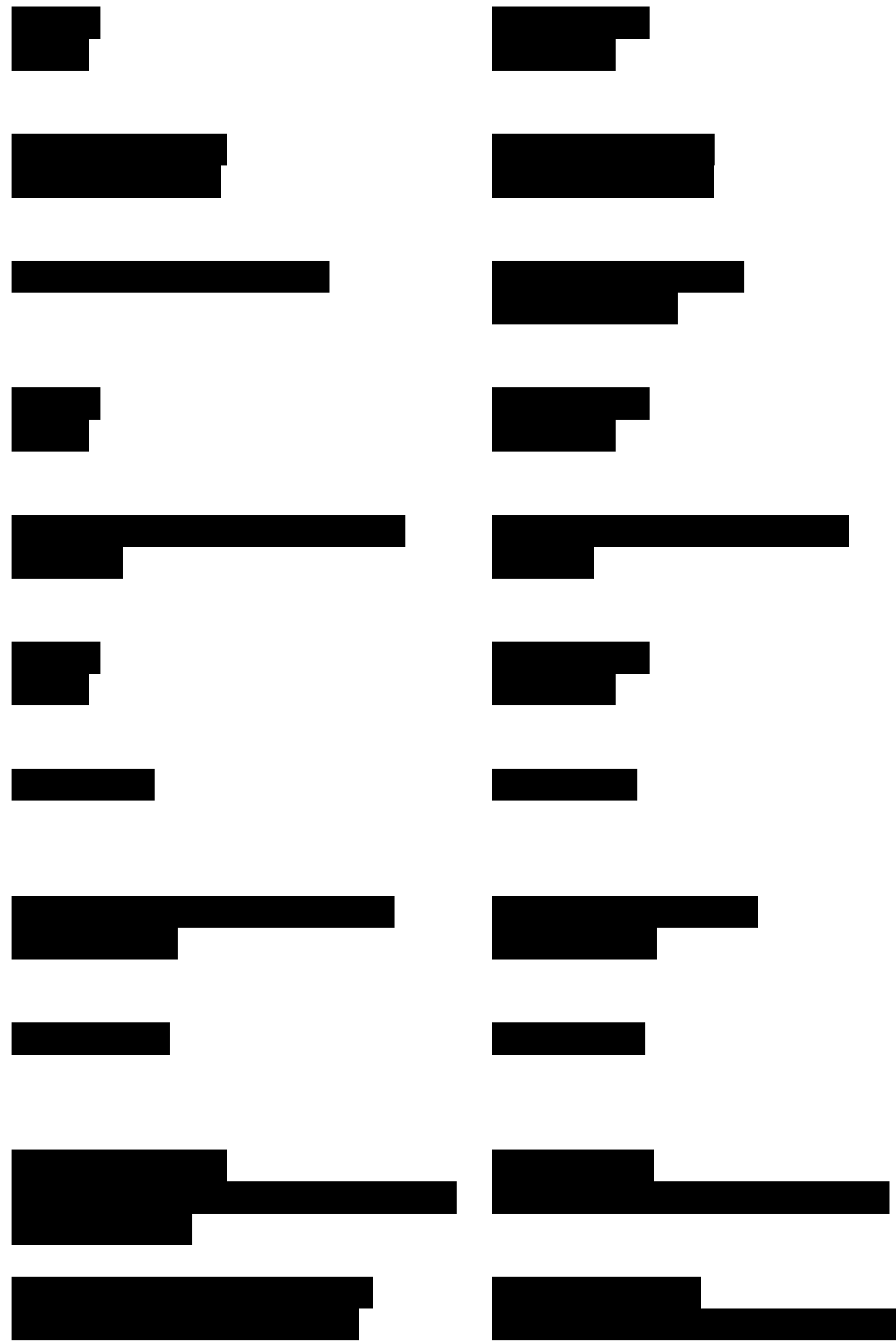

${ }^{254}$ Translates to "You didn't buy one out of stinginess" which is a rather clunky sentence in English, therefore reworked to "you were too cheap" 
0387

00:30:21:00

00:30:24:05

\section{8}

00:30:24:14

00:30:29:23

\section{9}

00:30:32:16

00:30:34:09

\section{0}

00:30:34:12

00:30:41:06

0391

00:30:42:24

00:30:46:01

0392

00:30:46:07

00:30:50:11

0393

00:30:50:18

00:30:52:04

0394

00:30:52:06

00:30:56:07

0395

00:30:56:19

00:30:58:16

0396

00:30:59:22

00:31:03:03

0397

00:31:03:05

00:31:05:04

0398

00:31:06:05

00:31:08:21

0399

00:31:08:24

00:31:13:06
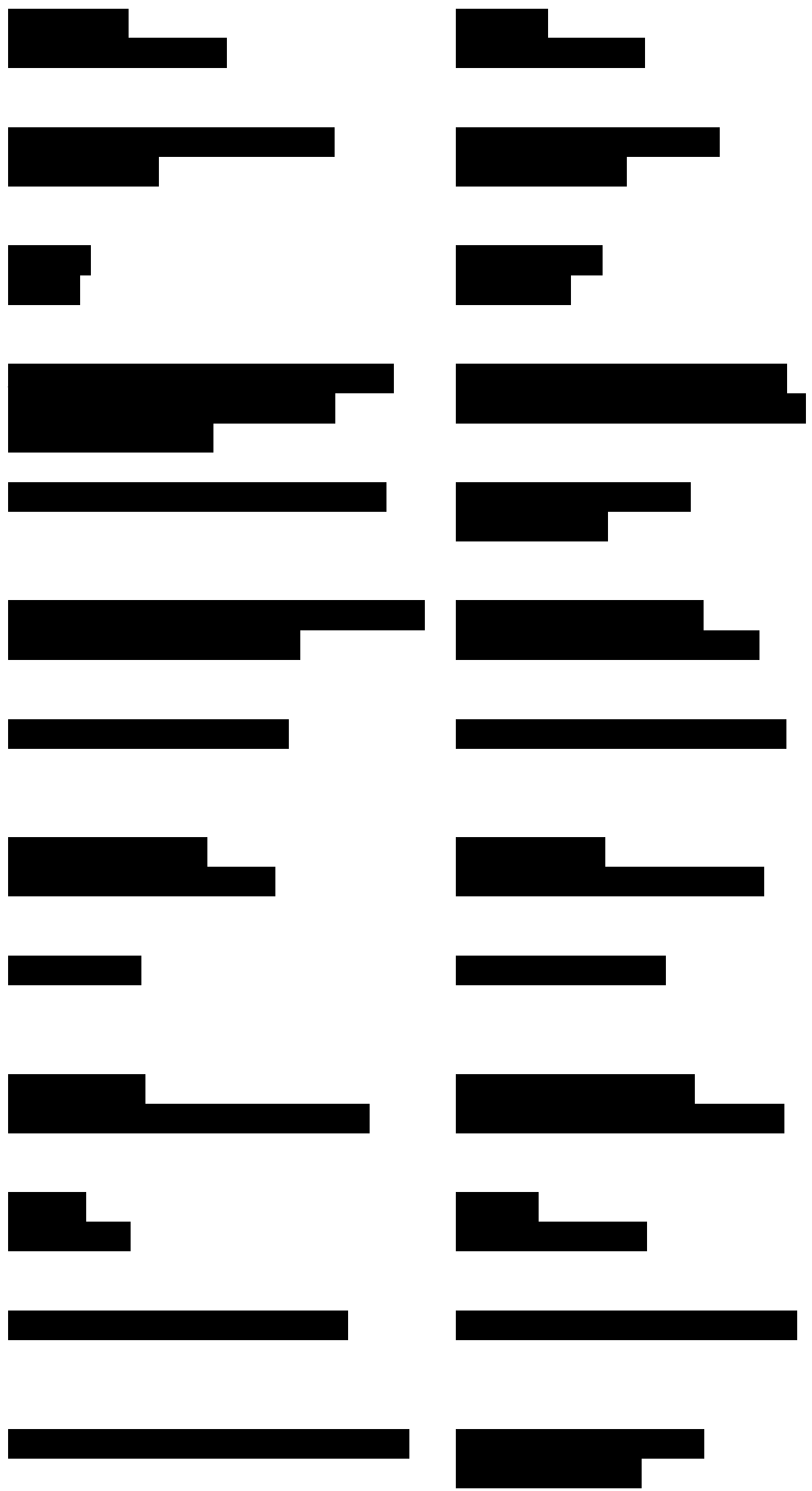

${ }^{255}$ Literally “the wind is coming from the east/west, however 'easterly' and 'westerly' are more common in English 
0400

00:31:14:14

00:31:16:09

0401

00:31:32:23

$00: 31: 35: 13$

0402

$00: 31: 35: 15$

$00: 31: 38: 15$

0403

$00: 31: 38: 21$

00:31:41:18

0404

00:31:41:20

$00: 31: 43: 17$

0405

00:31:43:20

00:31:46:09

\section{6}

00:31:46:12

00:31:48:22

\section{7}

00:31:49:02

00:31:51:06

\section{8}

00:31:52:14

$00: 31: 54: 23$

0409

00:31:55:00

$00: 31: 56: 15$

0410

$00: 31: 56: 17$

00:31:58:21

0411

$00: 31: 58: 24$

00:32:01:24

0412

00:32:02:02

00:32:04:05
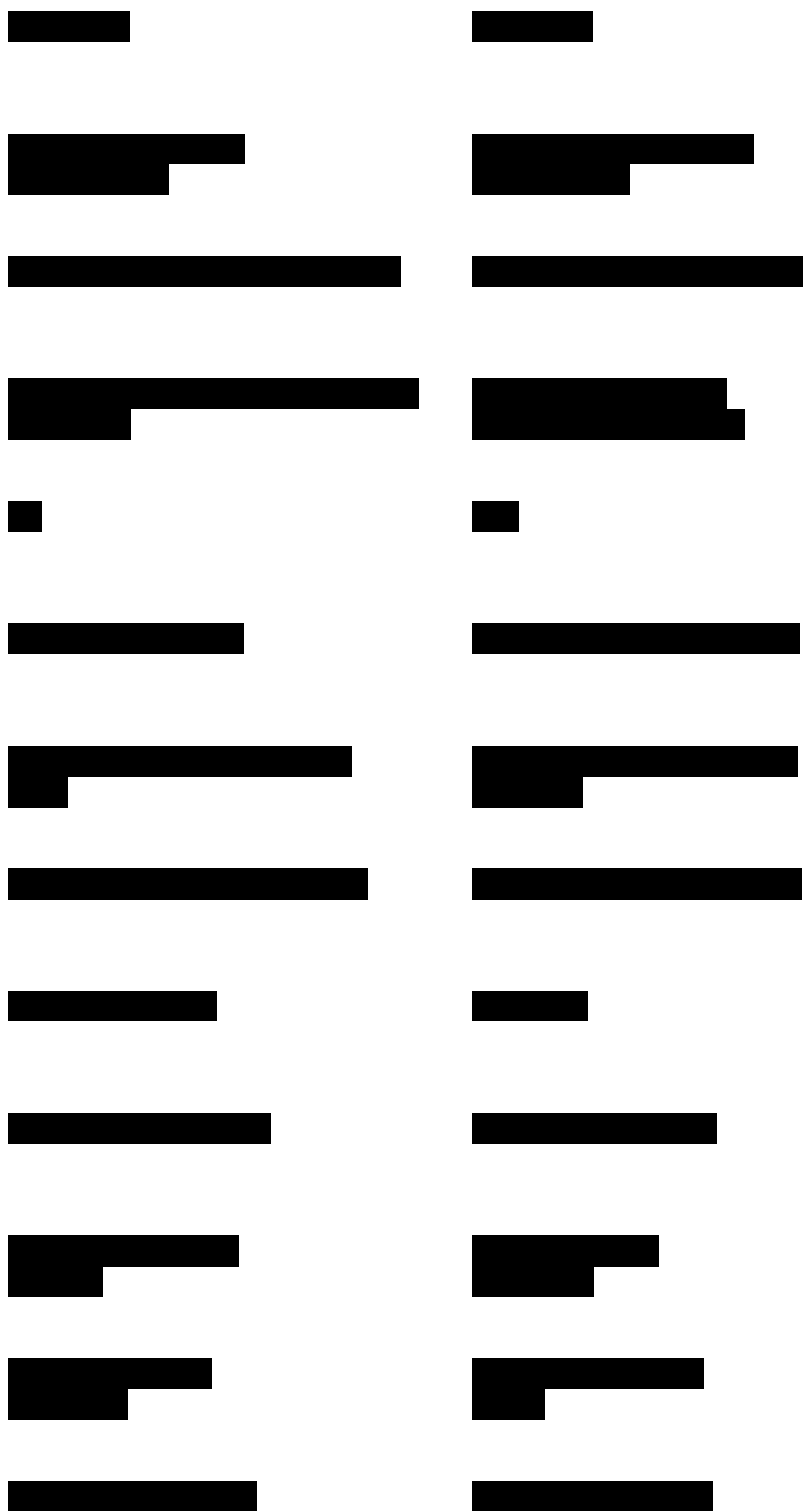

\footnotetext{
${ }^{256}$ Literally "you committed to it". The idiom in English "you gave me your word" works well in this context.
} 
0413

00:32:04:10

00:32:06:24

0414

00:32:09:01

00:32:11:16

\section{5}

00:32:13:17

00:32:17:05

\section{6}

00:32:26:10

$00: 32: 31: 16$

\section{7}

00:32:31:19

00:32:36:07

\section{8}

00:32:37:10

00:32:39:20

\section{9}

00:32:39:22

00:32:42:12

\section{0}

00:32:42:17

$00: 32: 46: 10$

\section{1}

00:32:46:13

00:32:49:16

\section{2}

00:32:49:19

00:32:52:10

\section{3}

00:33:01:24

00:33:04:19

\section{4}

00:33:04:21

00:33:07:06
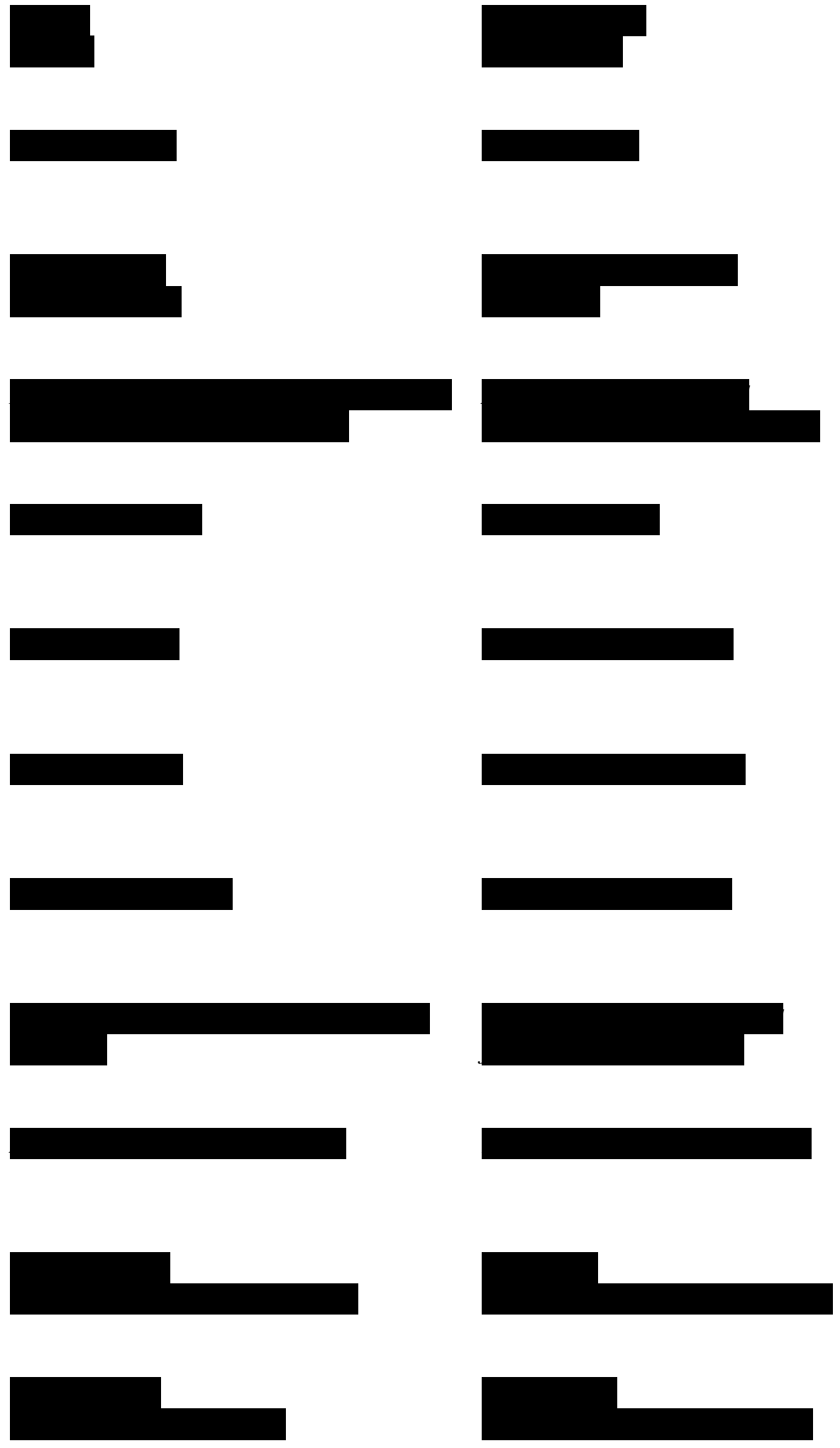

257 'Piovere' is an impersonal verb that the characters are trying to use, incorrectly, as an imperative to encourage the cloud to rain over them. This is therefore reflected in the subtitles.

${ }^{258}$ This is spoken with a heavy accent from the Lazio region, which is very noticeable to the Italian audience. Because of this I wanted to retain a point of difference between the accents of the characters. For a detailed explanation of what I have chosen to do, see the section 4.3.3 "multilingualism and the representation of Jesus" 
0426

00:33:08:14

00:33:11:19

0427

00:33:22:12

$00: 33: 25: 16$

0428

00:33:29:04

$00: 33: 32: 10$

\section{9 \\ 00:33:32:12 \\ 00:33:37:00}
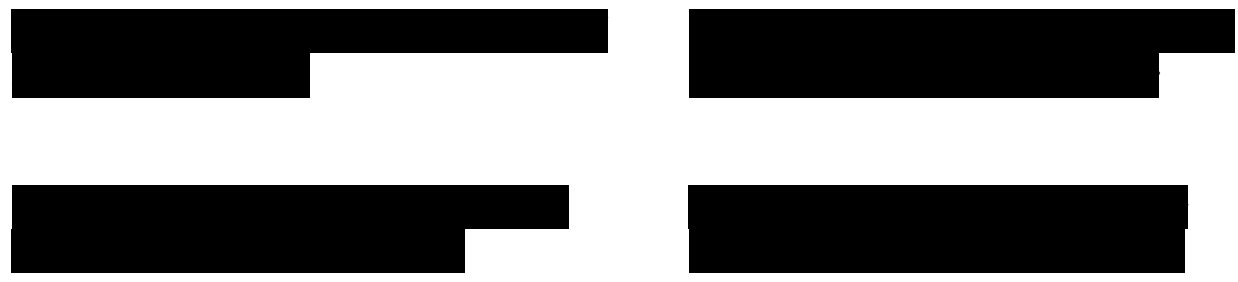

0430

00:33:37:03

00:33:40:17

\section{1}

00:33:40:19

$00: 33: 44: 17$

\section{2 \\ 00:33:44:19 \\ $00: 33: 47: 12$}
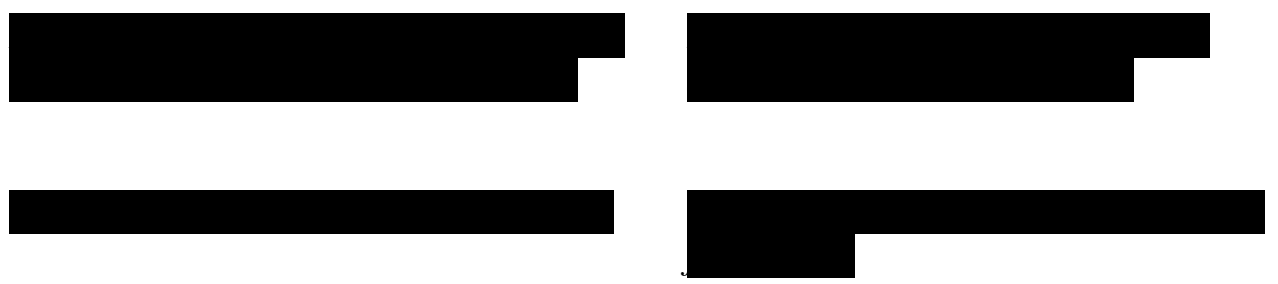

\section{3}

00:33:49:19

00:33:52:07

\section{4 \\ 00:33:52:09 \\ 00:33:54:05}
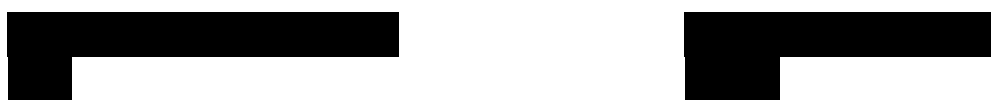

\section{5 \\ 00:33:58:23 \\ 00:34:00:24}

\section{6}

00:34:01:01

00:34:04:00
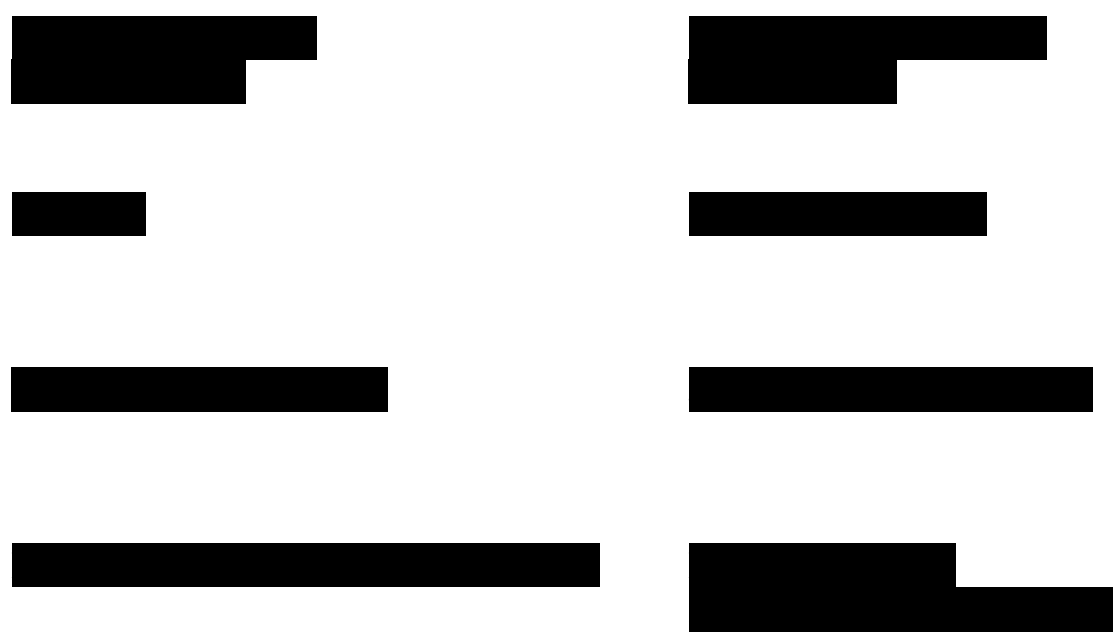

\footnotetext{
${ }^{259}$ Although 'hell' doesn't feature in the original dialogue, in order to keep the dialogue very informal and low register I have decided to include it. This expressions is very common in colloquial speech.

${ }^{260}$ As discussed earlier, often words on-screen are used to provide context. Here, 'Buon Natale' or 'Merry Christmas' appears backwards in the window of Fantozzi's workplace, so I have decided to retain this in translation.
} 
0437

00:34:09:05

00:34:10:12

\section{8}

00:34:10:15

00:34:13:03

\section{9}

00:34:13:13

$00: 34: 17: 15$

0440

00:34:18:05

00:34:21:05

\section{1}

00:34:21:11

$00: 34: 22: 24$

\section{2}

00:34:28:19

00:34:33:23

\section{3}

00:34:34:00

$00: 34: 38: 24$

0444

00:34:39:01

00:34:43:04

\section{5}

00:34:46:10

00:34:49:15

\section{6 \\ 00:34:49:17 \\ 00:34:53:04}

\section{7}

00:34:54:13

00:34:57:05

\section{8}

00:34:57:07

00:35:00:09
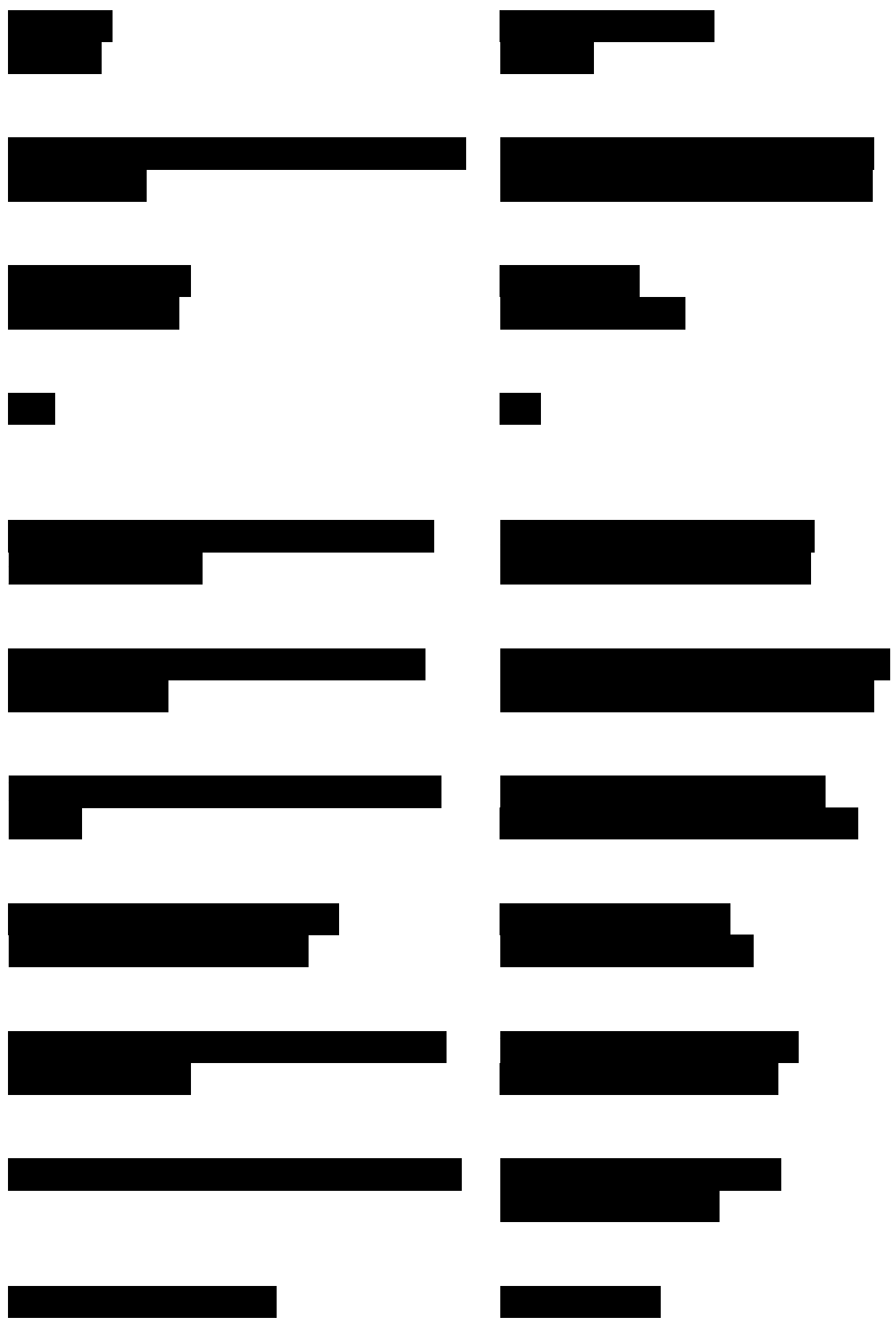

${ }^{261}$ This section required similar thought processes to those needed when translating poetry or music. For a detailed explanation of these translation choices and options see chapter 4.

${ }^{262}$ Literally "thing from the zoo", changing to "are we in a zoo" is more natural in English. 
0449

00:35:01:05

00:35:03:09

\section{0}

00:35:03:11

00:35:05:23

\section{1}

00:35:06:00

00:35:07:08

0452

00:35:09:03

$00: 35: 11: 12$

\section{3}

00:35:29:00

00:35:33:10

\section{4}

00:35:33:13

00:35:36:09

\section{5}

00:35:36:22

00:35:38:21

\section{6 \\ 00:35:42:15 \\ $00: 35: 44: 23$}

\section{7}

00:35:45:01

00:35:46:14

\section{8 \\ 00:35:46:16 \\ 00:35:48:04}

\section{9 \\ 00:35:48:06 \\ 00:35:49:07}

\section{0}

00:35:49:09

$00: 35: 51: 19$

\section{1}

00:35:52:06

00:35:54:09
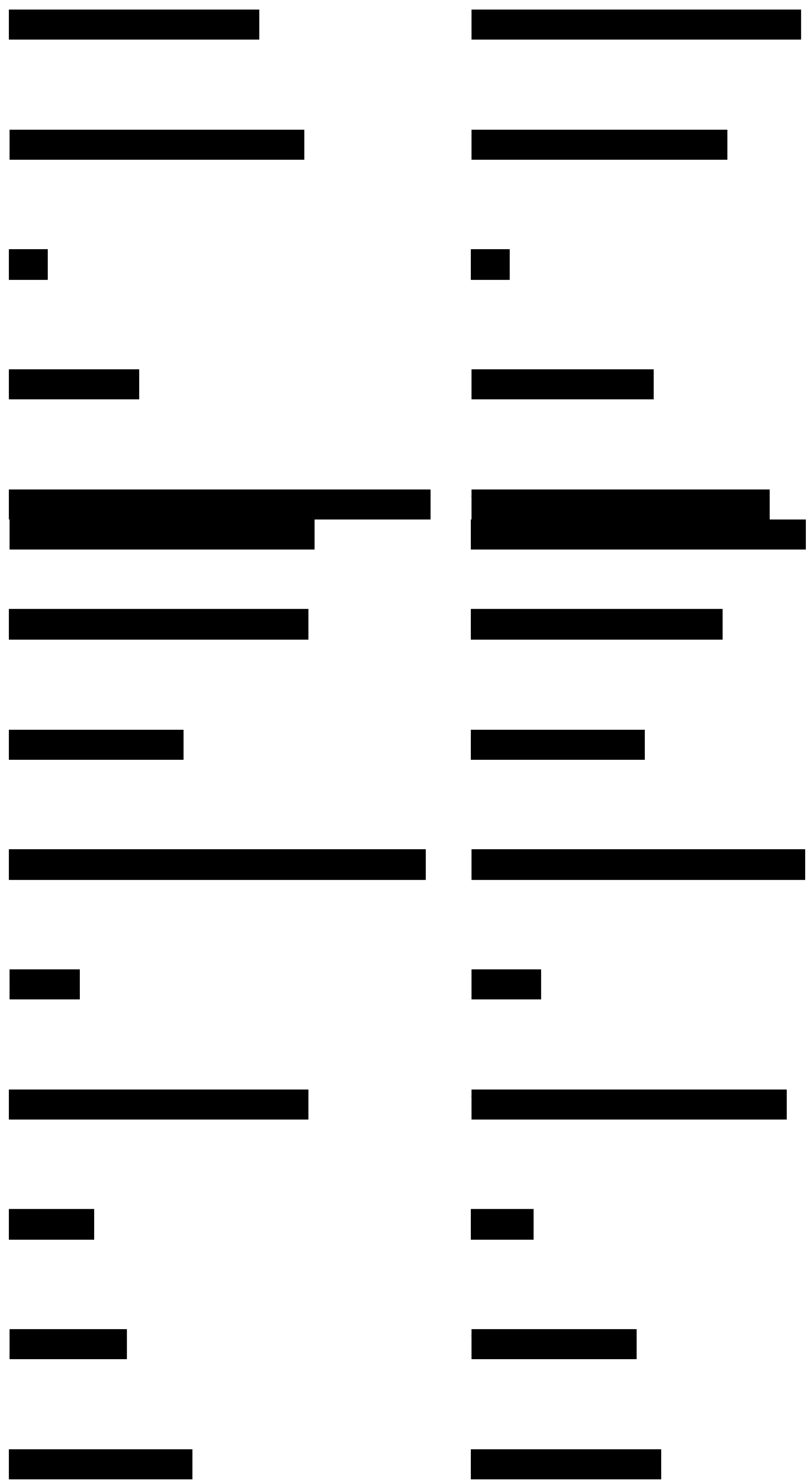

\footnotetext{
${ }^{263}$ This is a reference to Tarzan's monkey, and the comparison between Mariangela and a monkey is prevalent across the entire Fantozzi series. The spelling changes between the English and Italian versions.
} 
0462

00:35:54:11

00:35:56:21

\section{3}

00:35:56:24

00:36:00:19

0464

00:36:00:21

00:36:03:10

\section{5}

00:36:03:14

00:36:07:04

\section{6}

00:36:08:13

00:36:10:12

\section{7}

00:36:44:21

00:36:46:16

\section{8}

00:36:51:05

$00: 36: 52: 23$

\section{9 \\ 00:36:53:02 \\ 00:36:54:17}

\section{0}

00:36:54:19

$00: 36: 56: 24$

\section{1}

00:36:57:01

$00: 36: 59: 12$

0472

00:36:59:14

00:37:01:10

\section{3}

00:37:01:20

00:37:05:16

\section{4}

00:37:06:08

00:37:11:05
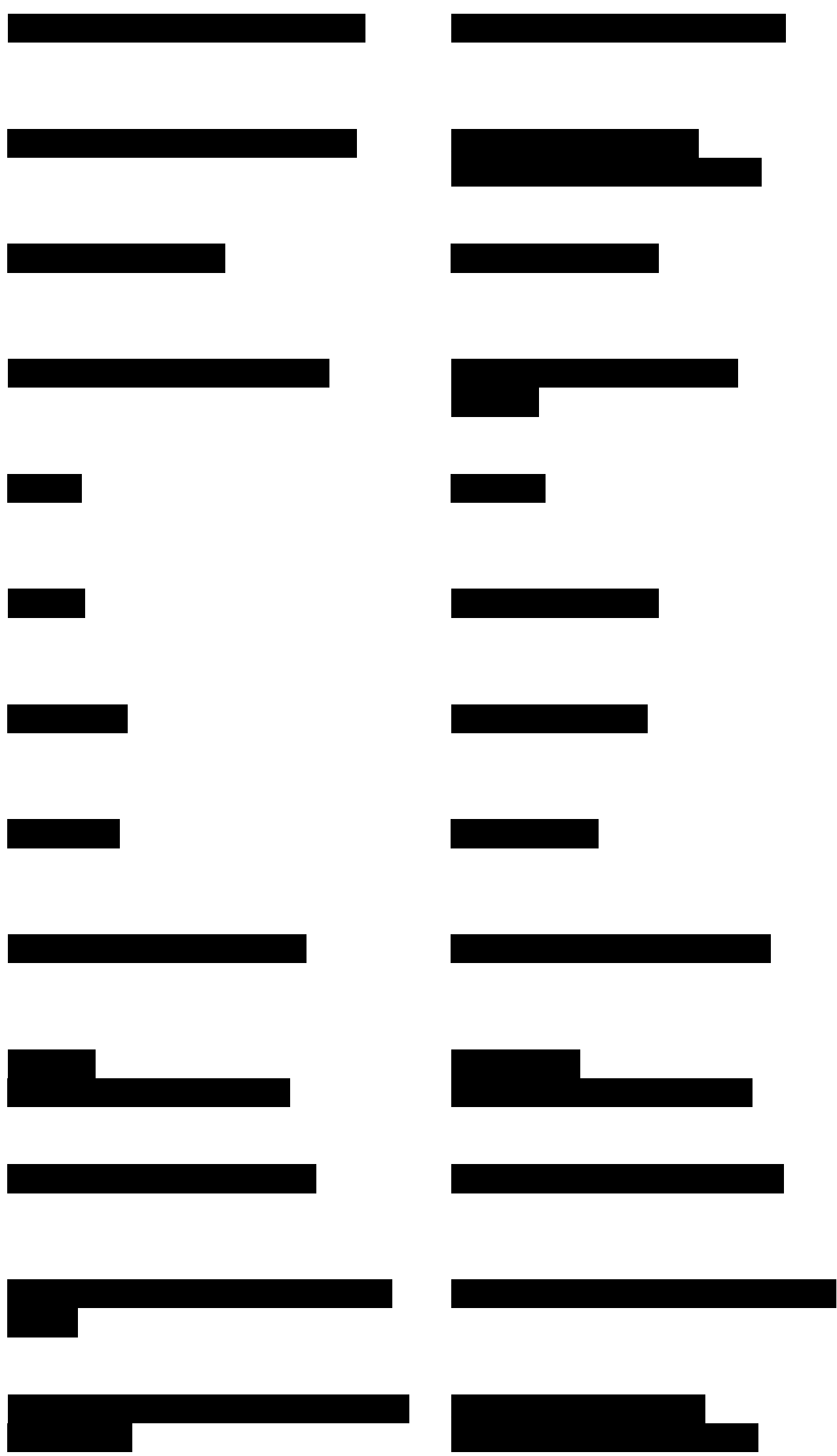

264 'Auguri' literally means 'wishes' and can be used in a variety of different contexts from birthdays to New Years. For clarity, and given the context, I have translated these such instances in this scene as "Happy New Year" 

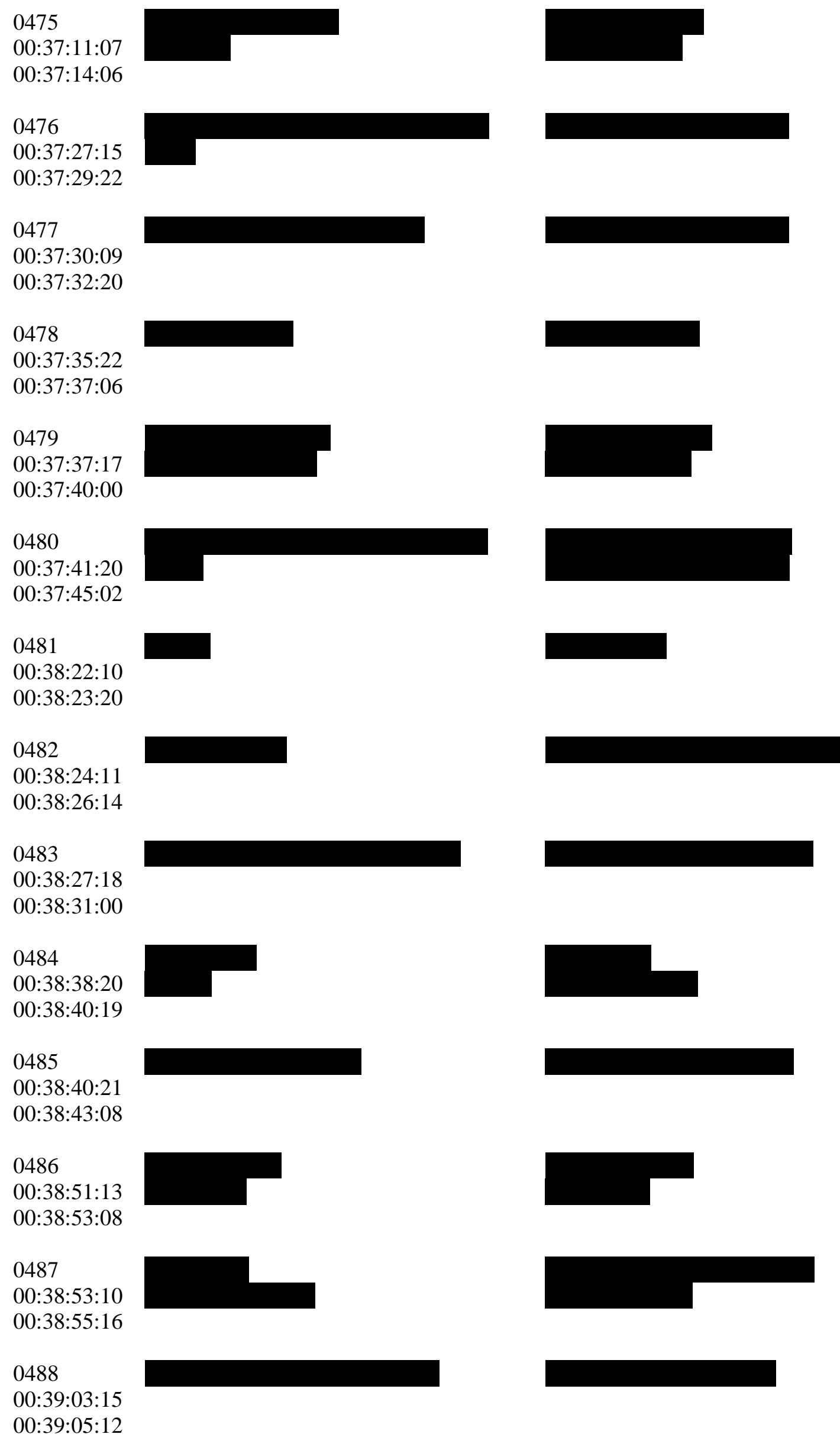


\section{9}

00:39:16:15

00:39:19:24

0490

00:39:28:09

00:39:30:11

0491

00:39:30:19

00:39:33:11

0492

00:39:34:03

$00: 39: 37: 24$

0493

00:39:38:01

00:39:41:13

0494

00:39:49:07

00:39:52:02

\section{5}

00:40:41:13

00:40:45:03

0496
00:40:45:05
00:40:50:06

0497

00:40:50:11

00:40:53:20

0498

00:40:53:23

00:40:56:21

0499

00:40:56:23

00:40:58:09

0500

00:40:58:11

00:41:01:21

0501

00:41:01:23

00:41:05:14
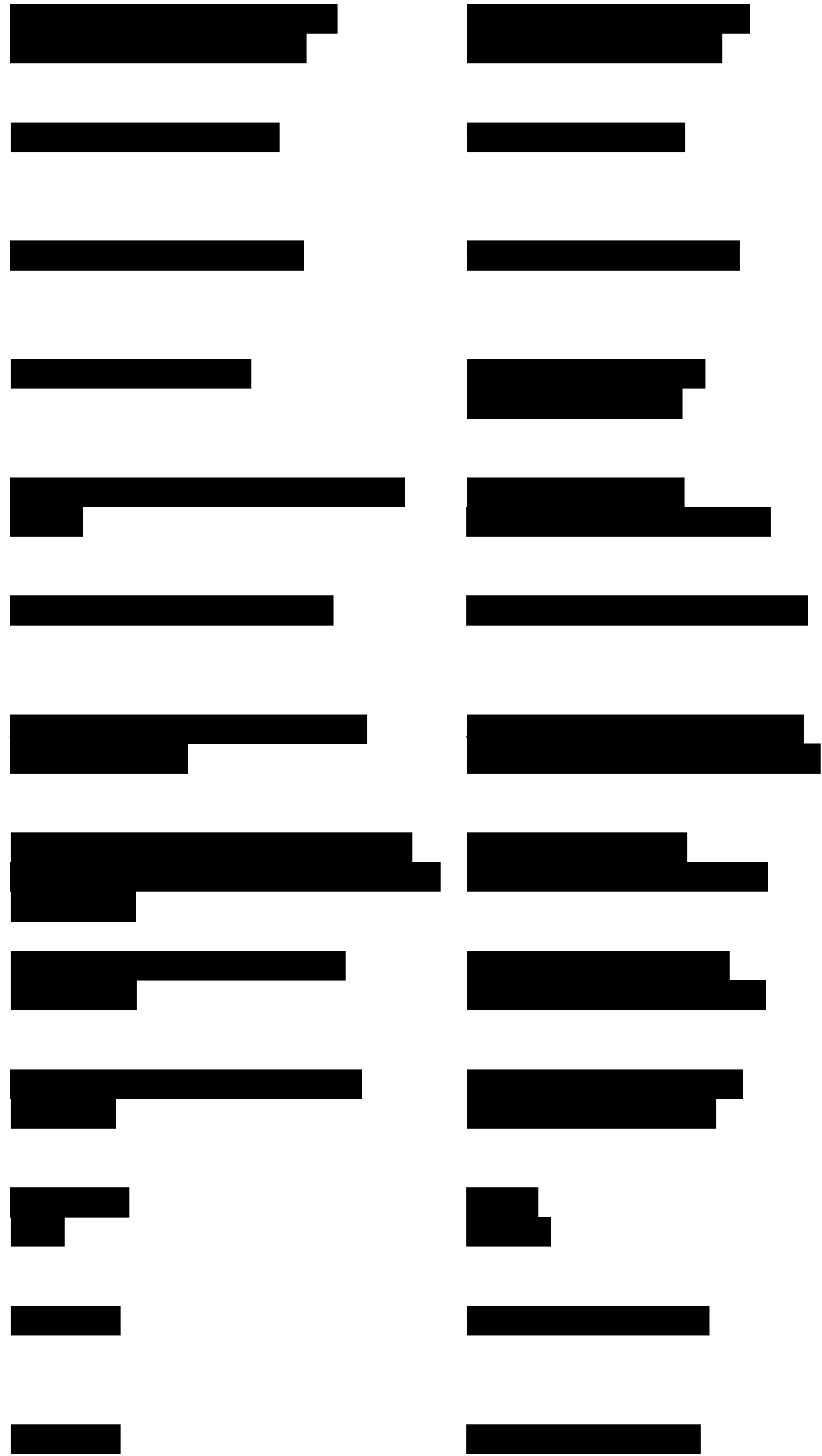

265 'Zampone' has been left in the translation. I have decided to foreignize the text here. This is in keeping with similar scenes in the film where dishes unknown to an English-speaking audience have been retained, where there are equivalents these have been translated. 
0502

00:41:06:07

00:41:09:13

\section{3}

00:41:18:06

00:41:19:17

0504

00:41:19:19

00:41:22:08

0505

00:41:22:11

$00: 41: 24: 17$

0506
$00: 41: 36: 16$
$00: 41: 40: 08$

00:41:40:08

0507

00:41:40:10

00:41:42:14

0508
00:41:51:11

00:41:53:02

0509

00:41:54:04

00:41:55:21

0510

00:42:16:05

$00: 42: 19: 23$

0511

00:42:50:11

00:42:52:08

$00: 42: 50: 11$ 00:42:52:08

\section{2}

$00: 42: 54: 10$

00:42:58:04

0513

00:42:58:06

00:43:00:10

0514

00:43:00:13

00:43:02:19
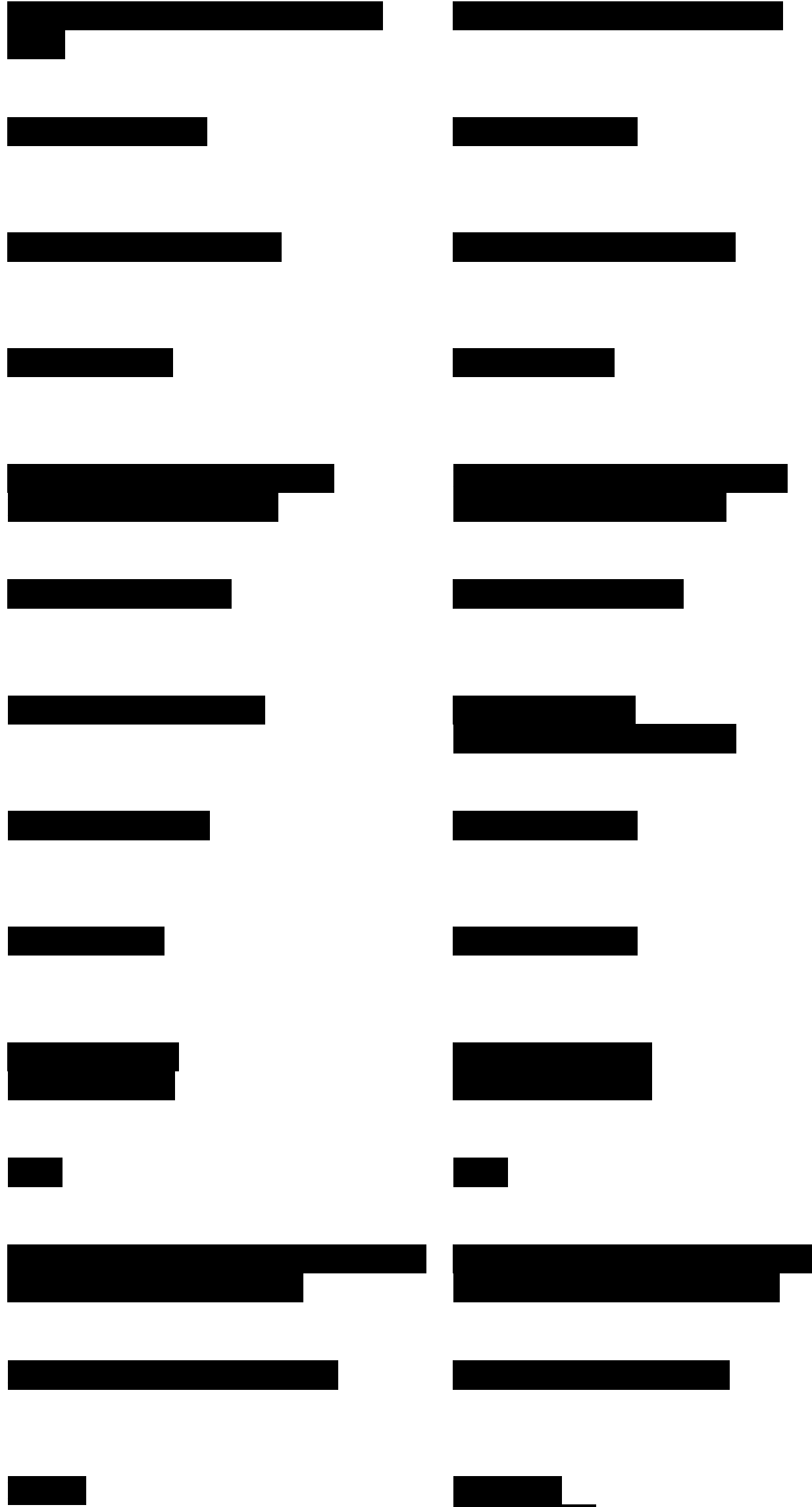
0515

00:43:02:21

00:43:05:11

0516

00:43:06:01

00:43:08:21

0517

00:43:27:15

00:43:32:07

\section{8}

00:43:32:09

00:43:35:10

\section{9}

00:43:39:19

00:43:43:14

0520

00:43:43:16

00:43:46:03

0521

00:43:46:05

00:43:50:07

0522

00:43:50:09

$00: 43: 52: 12$

0523

00:43:52:22

00:43:54:21

0524

00:43:57:01

00:44:00:06

\section{5}

00:44:00:18

00:44:05:04

\section{6}

00:44:05:06

00:44:07:07
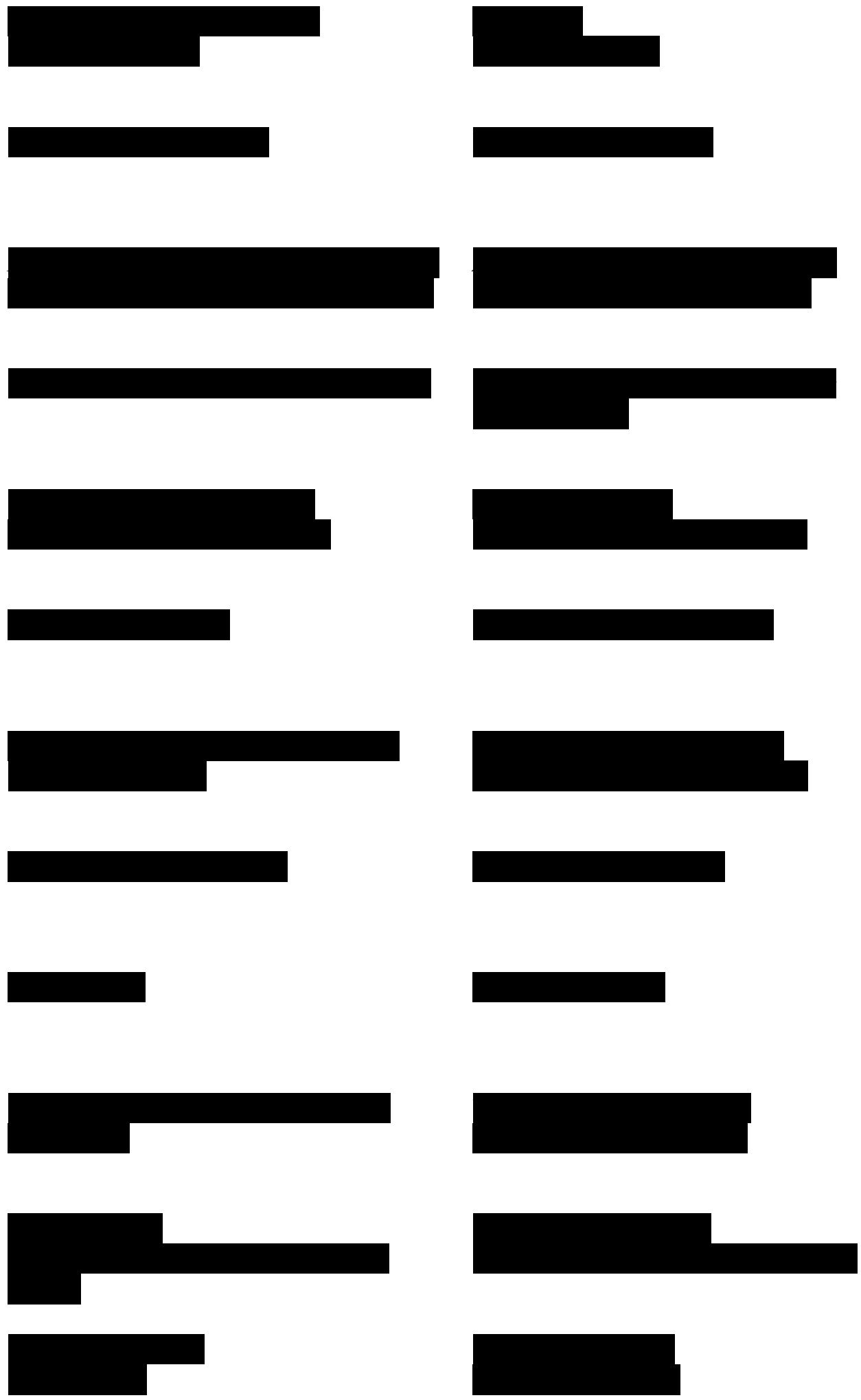

${ }^{266}$ Literally 'the new year exploded in the city'; 'welcomed with a bang' is a commonly used phrase in English which conveys the same meaning.

${ }^{267}$ This is a case of 'cultural humour' as highlighted in chapter two. Throwing items out of the window at New Years a stereotype commonly associated with the south of Italy. To an English-speaking audience this reference is lost, and the humour is associated with the car being crushed, however an Italian audience would pick up this tradition immediately. 
0527

00:44:19:23

00:44:23:10

\section{8}

00:44:26:02

$00: 44: 27: 22$

0529

$00: 44: 27: 24$

00:44:32:20

0530

00:44:32:22

$00: 44: 34: 18$

\section{1}

00:44:35:08

$00: 44: 37: 13$

0532

00:44:37:15

00:44:40:21

\section{3}

00:44:41:08

$00: 44: 44: 15$

0534

00:44:44:17

$00: 44: 46: 12$

\section{5}

00:44:46:14

00:44:51:00

\section{6 \\ 00:44:57:02 \\ 00:44:59:09}

\section{7}

00:45:06:09

00:45:08:20

\section{8}

00:45:08:22

00:45:11:12
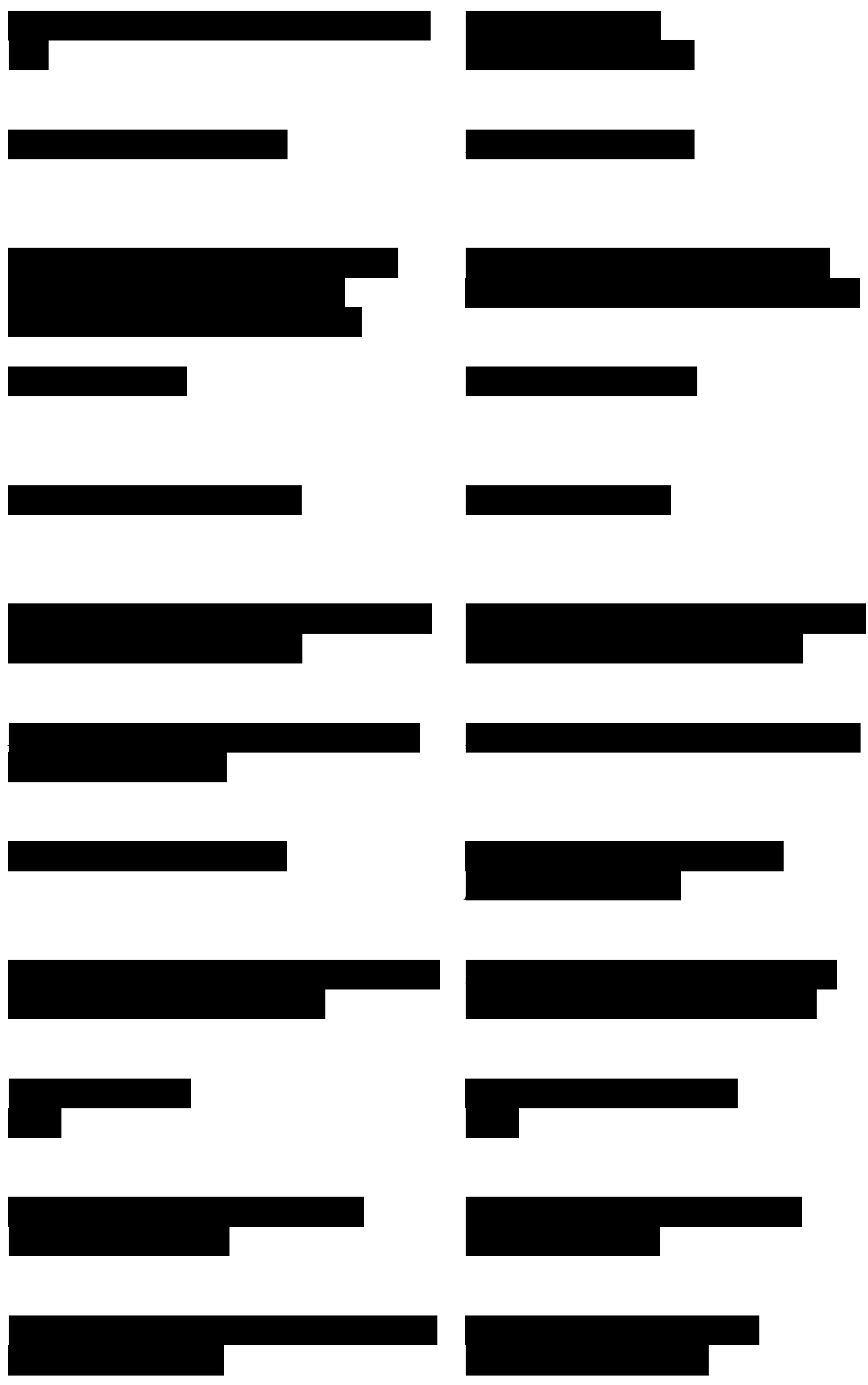

\footnotetext{
${ }^{268}$ Given the context, this literally translates to "Those who don't do things quickly, don't advance their career". The expression "No pain, no gain" while having a slightly different meaning, is the closest expression in English and still conveys that in order to rise through the ranks one must make sacrifices. It also retains the linguistic similarity between 'scatta' and 'scatti', instead as a rhyme in English.
} 

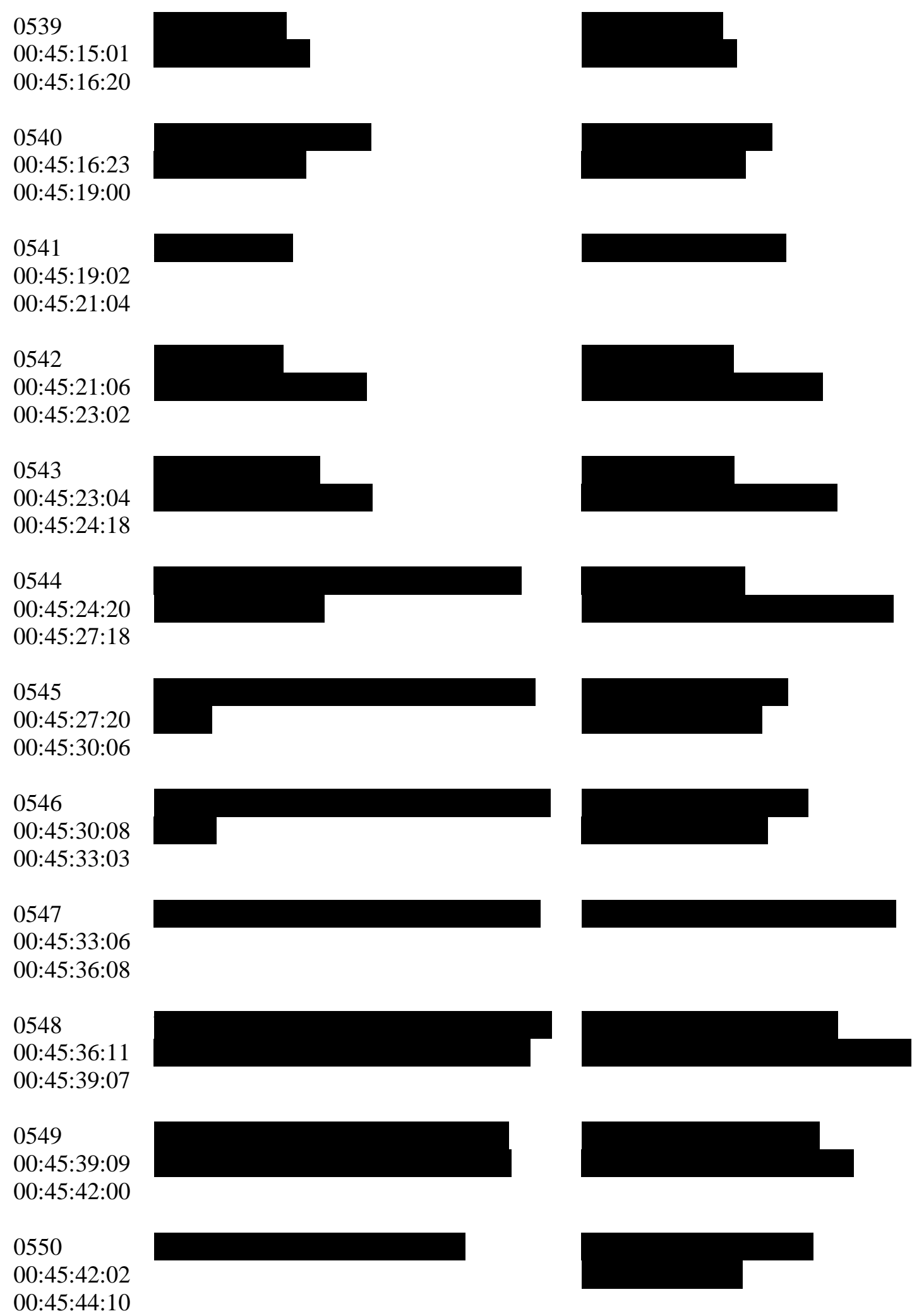

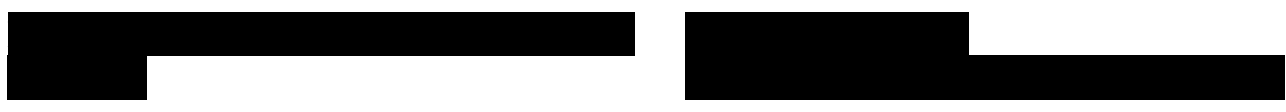

\section{2}

00:45:48:19
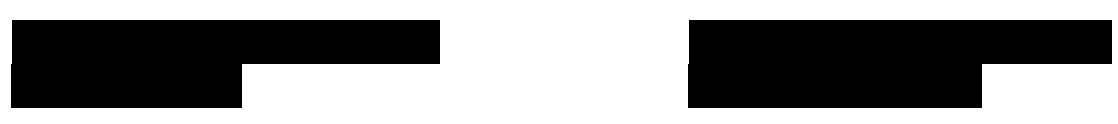

00:45:51:19

\section{3}

00:45:53:15

$00: 45: 54: 22$

\section{4 \\ 00:45:54:24 \\ 00:45:57:00}
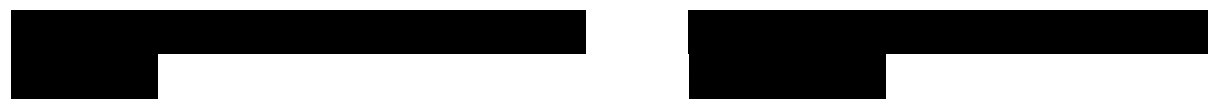

\section{5 \\ 00:45:57:02}

00:45:59:22

\section{6 \\ 00:46:00:00 \\ 00:46:03:21}
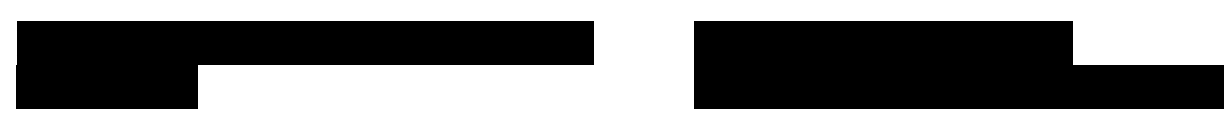

\section{7 \\ 00:46:03:23 \\ 00:46:04:24}

\section{8 \\ 00:46:05:01 \\ 00:46:08:10}

\section{9}

00:46:08:18

00:46:10:12

\section{0}

00:46:12:16
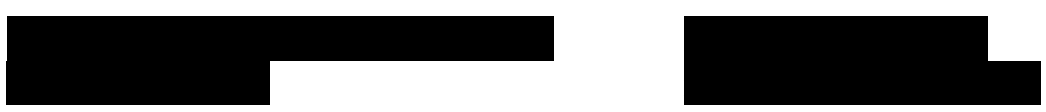

00:46:14:05

\section{1}

00:46:14:21

00:46:16:16

\section{2}

00:46:22:22

$00: 46: 24: 24$
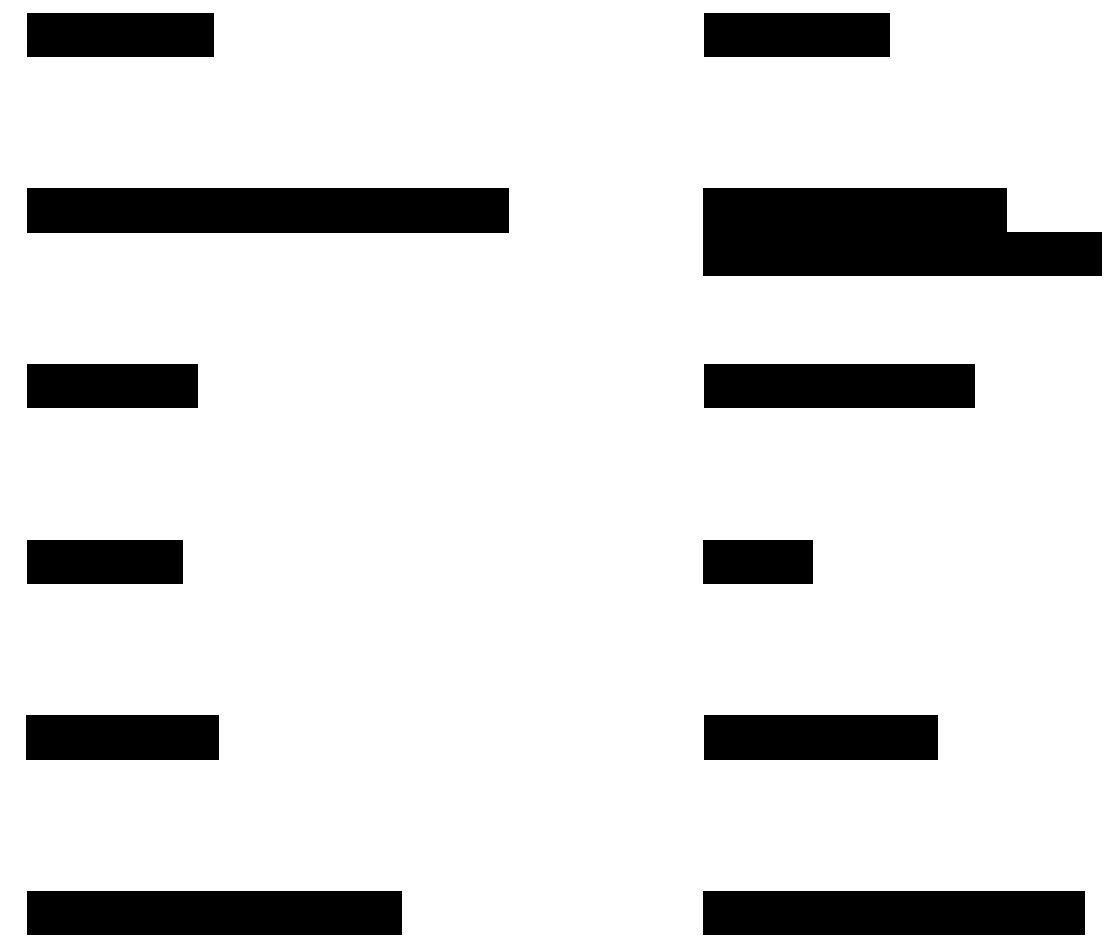

\footnotetext{
${ }^{269}$ The billiards game that Catellani plays is not the well-known form of the game with which the English speaking world will be familiar, it is a particular variety that is popular in Italy with unique terminology. I have decided to retain this terminology in the translation as there are no similar sports with which the one being played could be replaced, despite the inevitability that the audience is unlikely to understand the references.

${ }^{270}$ Although "buon appetit" may be a better translation, here they repeat "buon" so in the translation I attempted to retain this. I also switched out "figli maschi" (male children) for "good health" as it is rather politically incorrect.

${ }^{271}$ Here, 'scatti' is used again. The translation has therefore included 'gain', here in reference to 'No pain, no gain'.
} 
0563

00:46:25:02

00:46:26:17

0564

00:46:34:00

00:46:36:13

\section{5}

00:46:36:15

00:46:39:12

\section{6}

00:46:39:17

00:46:41:10

\section{7}

00:46:44:12

00:46:46:15

\section{8}

00:46:46:17

00:46:48:10

\section{9}

00:46:48:13

00:46:50:09

\section{0 \\ 00:46:50:11 \\ $00: 46: 52: 12$}

\section{1}

00:46:52:14

00:46:54:14

0572

00:46:54:16 00:47:00:07

\section{3}

00:47:00:09

00:47:05:00

\section{4}

00:47:11:17

$00: 47: 14: 18$

\section{5}

00:47:15:02

$00: 47: 18: 17$
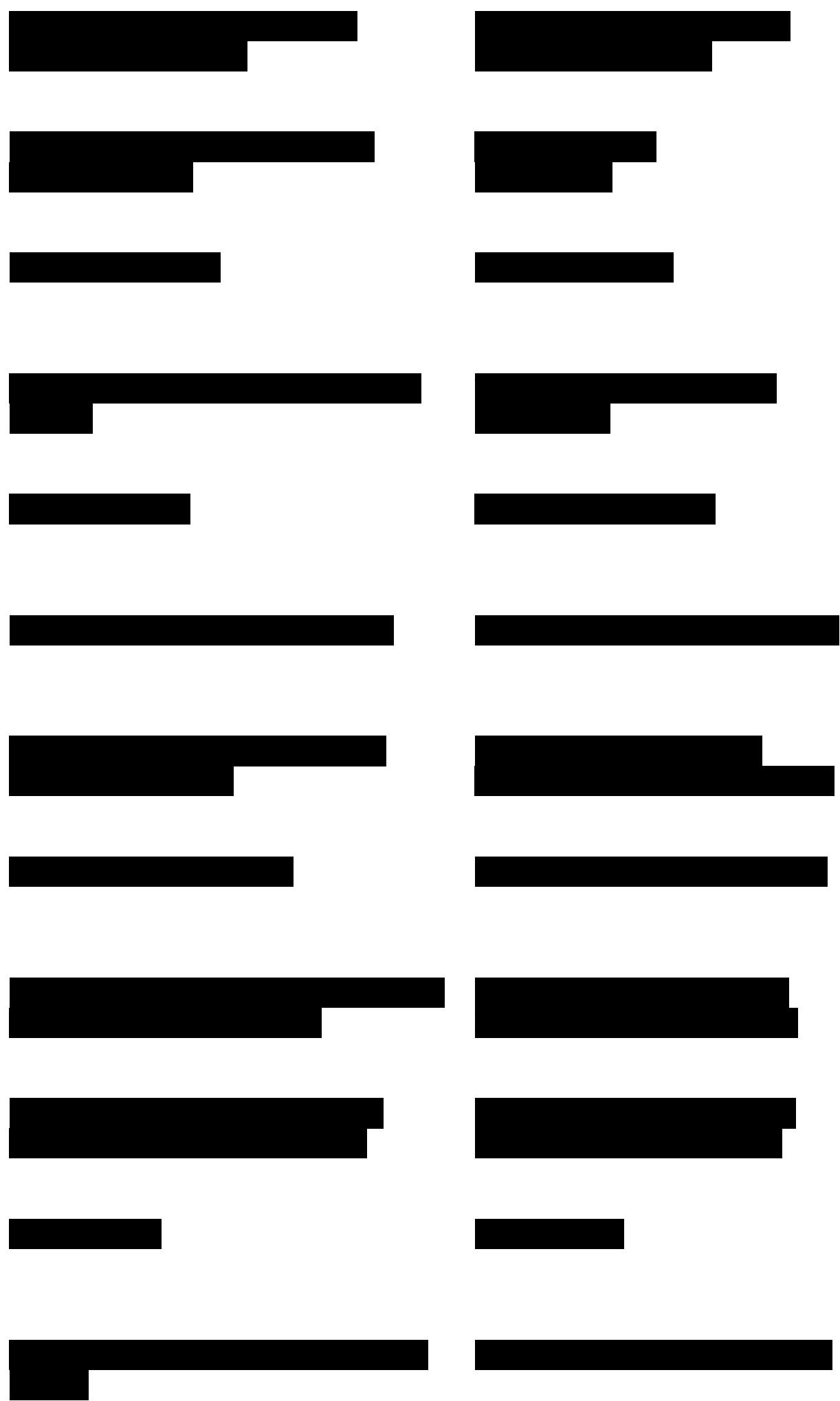

272 'Enel' is an electricity and gas company in Italy. Because this reference would be lost on the English native speaker I have decided to go for a super-ordinate and simply translate it as 'gas company'. 


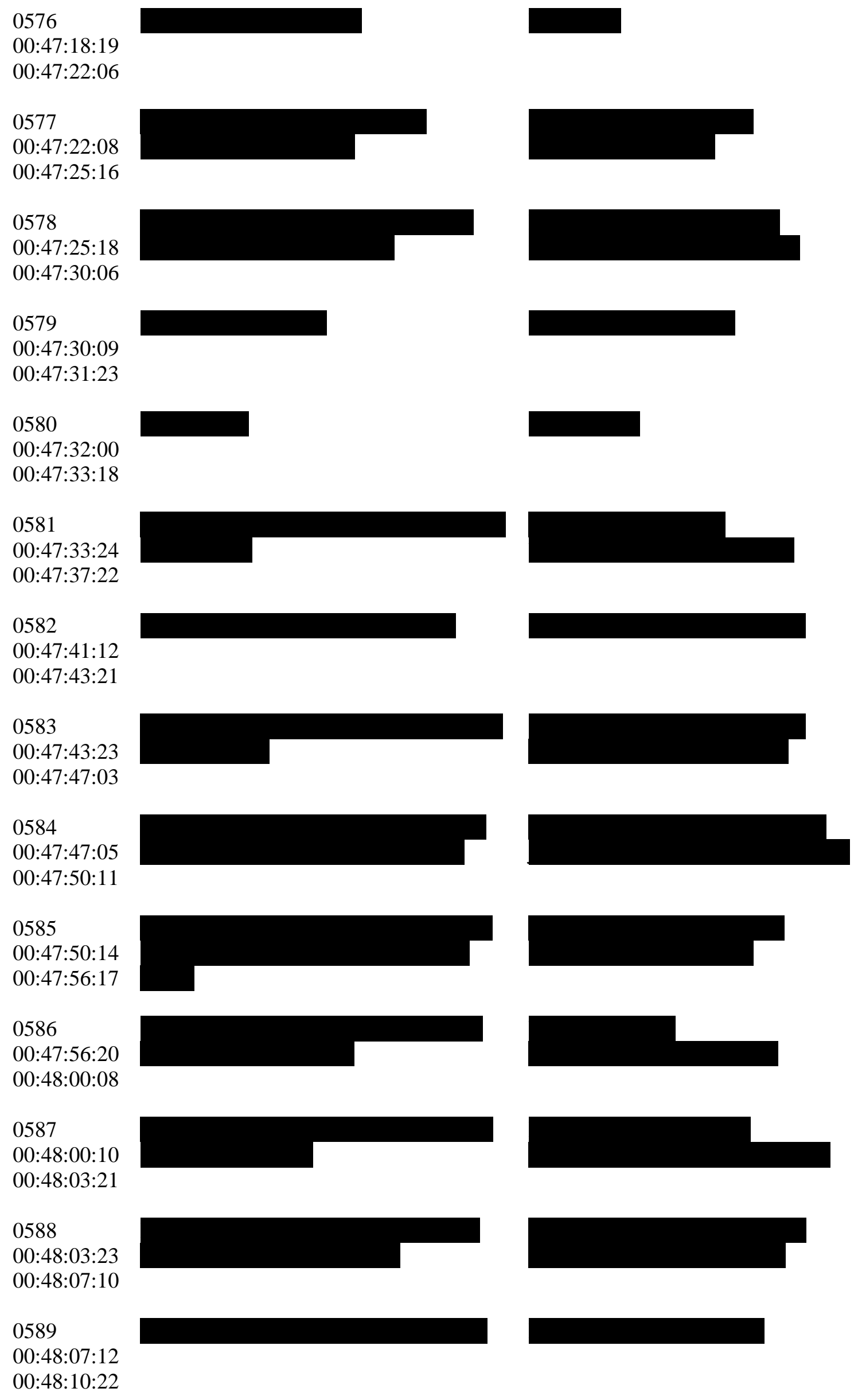


0590

00:48:10:24

00:48:13:09

0591

00:48:13:11

00:48:14:14

0592

00:48:14:16

00:48:16:18

0593

00:48:19:19

00:48:21:13

0594

00:48:22:07

00:48:23:11

\section{5}

00:48:23:13

$00: 48: 24: 20$

0596

$00: 48: 24: 22$

00:48:26:17

\section{7}

00:48:26:19

$00: 48: 28: 16$

\section{8}

00:48:29:03

00:48:31:11

0599

00:48:31:13

00:48:33:02

0600

00:48:33:04

00:48:36:07

\section{1}

00:48:36:09

00:48:39:19

0602

00:48:40:00

$00: 48: 42: 12$
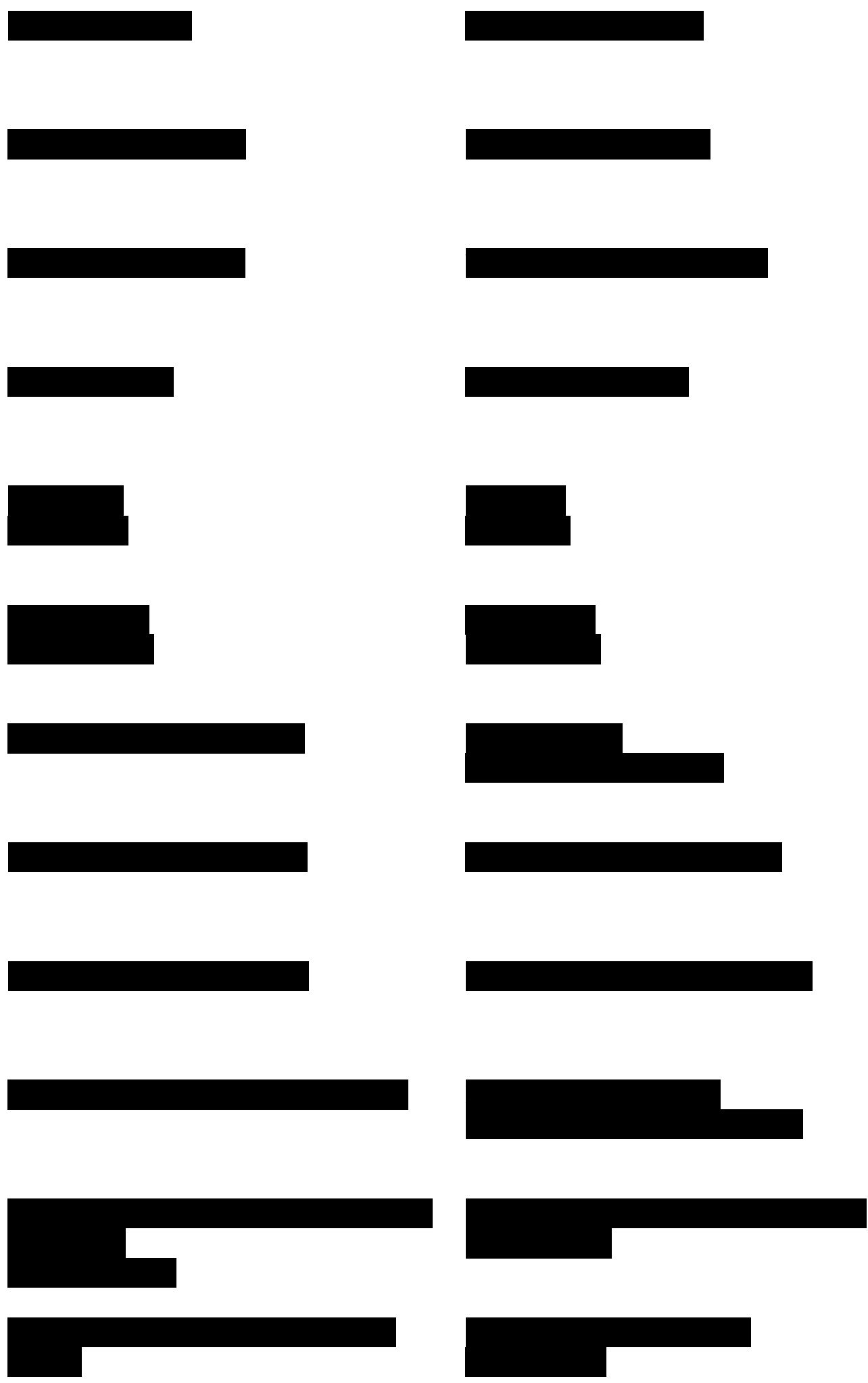

${ }^{273}$ This is an example of the unique terminology used in this form of billiards. I have chosen to translate the terms literally into English. 
0603

00:48:42:14

00:48:44:07

0604

00:48:46:13

00:48:47:24

0605

00:48:48:02

00:48:51:08

0606
$00: 48: 51: 10$

$00: 48: 55: 12$

0607

00:48:56:23

00:48:59:16

0608

00:48:59:18

00:49:01:23

0609

00:49:09:14

$00: 49: 12: 21$

0610

$00: 49: 12: 23$

00:49:13:23

0611

00:49:14:00

$00: 49: 15: 14$

0612

00:49:15:16

00:49:18:04

0613

00:49:18:23

00:49:23:09

0614

00:49:23:11

$00: 49: 25: 19$

0615

00:49:26:00

00:49:28:16

0616

00:49:28:17

00:49:32:04
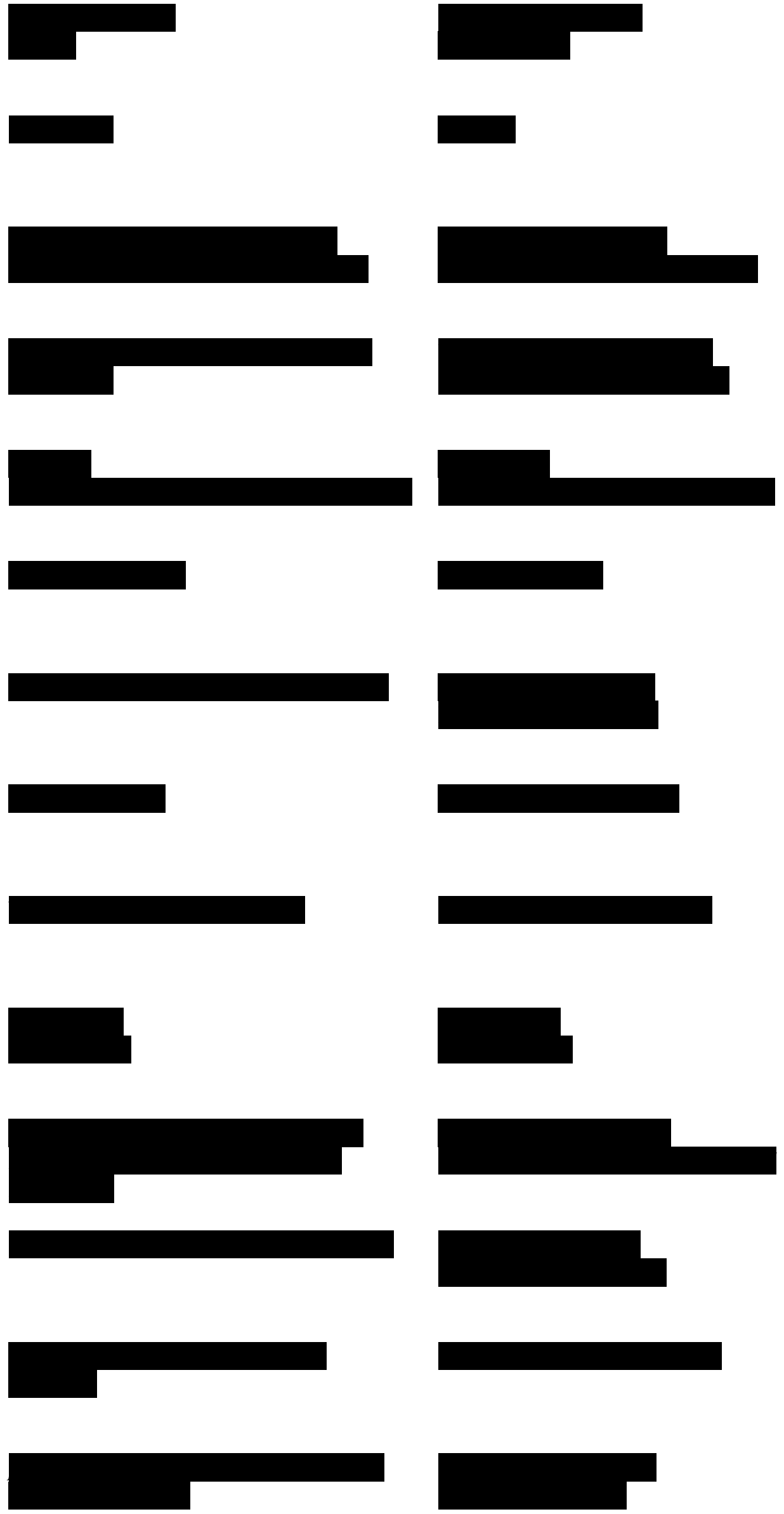
0617

00:49:32:05

00:49:37:12

\section{8}

00:49:40:19

00:49:43:09

0619

00:49:43:10

00:49:47:04

0620

00:49:57:05

00:50:00:02

\section{1}

00:50:00:22

00:50:07:05

0622

00:50:07:07

00:50:14:22

0623

00:50:15:16

00:50:17:01

0624

00:50:17:03

00:50:19:09

0625

00:50:30:06

00:50:32:22

0626

00:50:34:15

00:50:37:00

0627

00:50:42:13

00:50:44:13

\section{8}

00:50:44:17

00:50:49:23
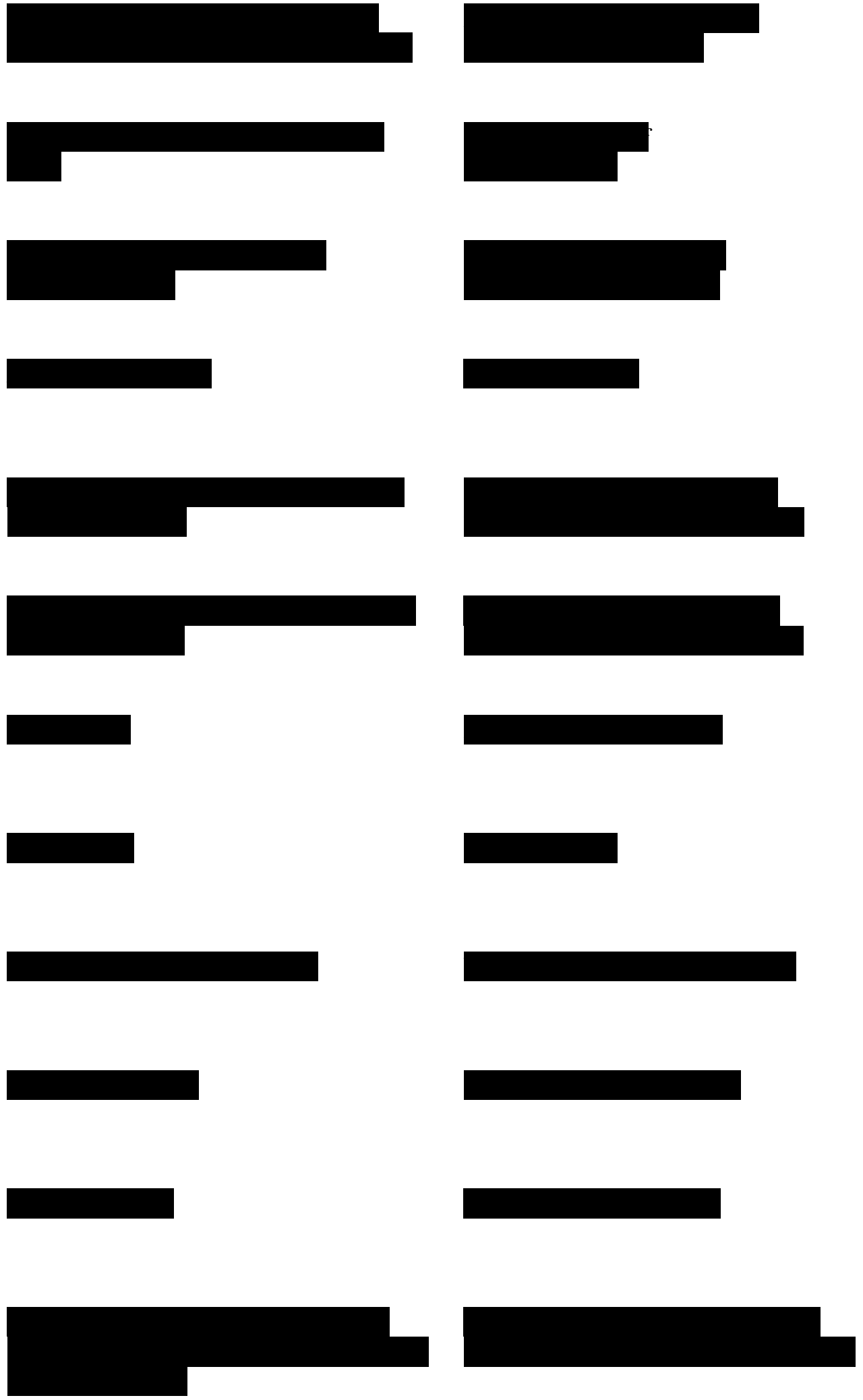

${ }^{274}$ This is a well-known Italian song called Parlami d'amore Mariù written by Ennio Neri and Cesare Andrea Bixio for the voice of Vittorio de Sica. There is a rhyme in the original between 'tu' and 'Mariu', fortunately it still rhymes with the English 'you'.

${ }^{275}$ Again, Fantozzi's head is subject to pain. A common theme throughout the film. 
0629

00:50:50:21

00:50:53:09

0630

00:50:53:11

00:50:55:21

0631

00:50:55:24

00:50:57:13

0632

00:50:57:15

00:50:59:24

0633
$00: 51: 00: 12$
$00: 51: 04: 00$

00:51:04:00

0634

00:51:04:03

00:51:06:15

0635

00:51:20:23

00:51:24:09

0636

00:51:24:11

00:51:26:14

0637

00:51:26:16

00:51:27:21

0638

$00: 51: 27: 23$

00:51:31:00

0639

00:51:31:02

00:51:33:06

0640

00:51:33:08

00:51:35:01

0641

00:51:36:17

00:51:38:07
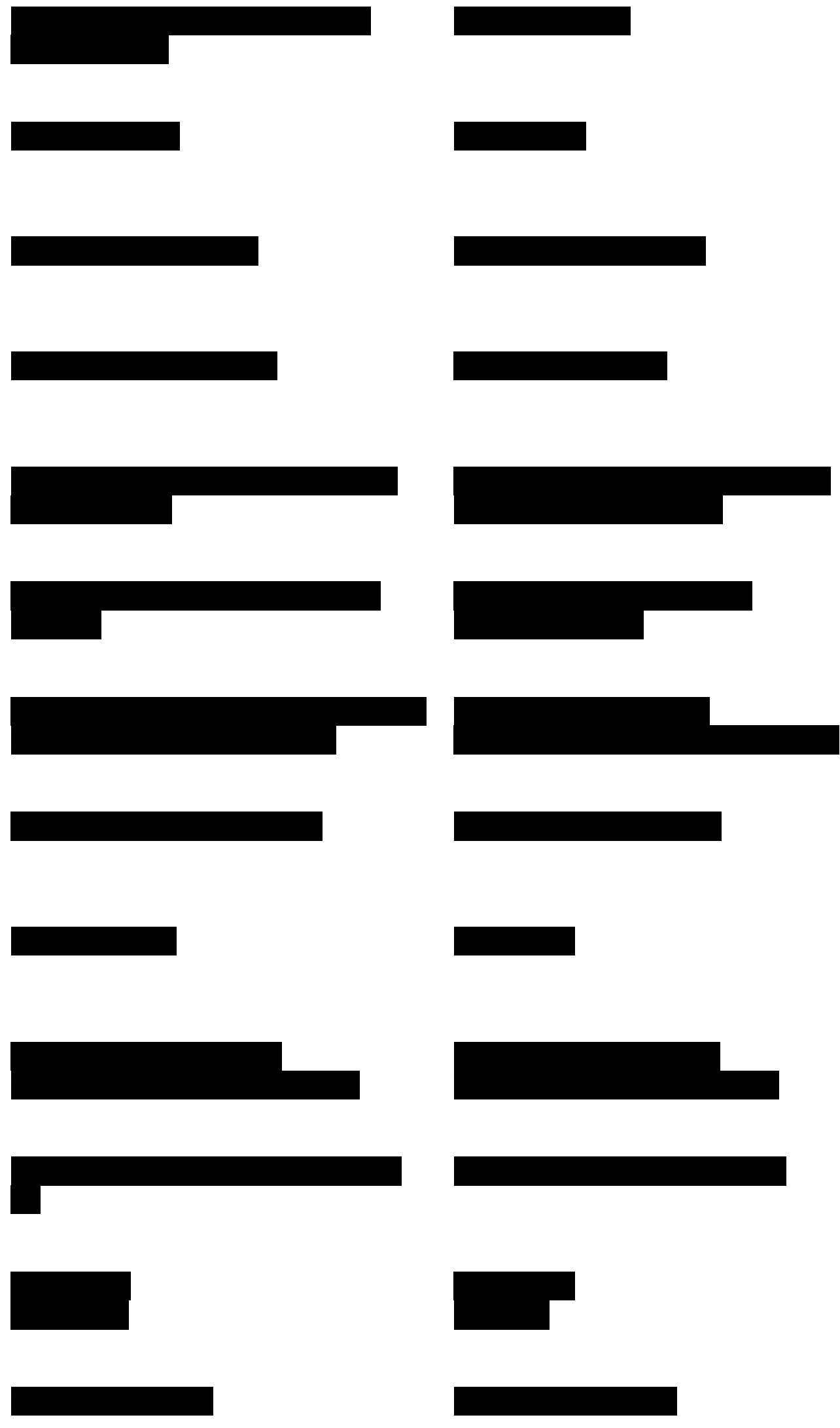

\footnotetext{
${ }^{276}$ Literally "whore" although seems too strong a word given the context.
} 
0642

00:51:38:09

00:51:40:03

\section{3}

00:51:41:08

00:51:43:10

0644

00:51:44:04

00:51:45:05

\section{5}

00:51:45:07

00:51:47:17

0646

00:51:49:22

00:51:51:12

\section{7}

00:51:51:14

00:51:53:07

\section{8}

00:51:53:09

00:51:54:13

0649

00:52:12:00

00:52:14:09

\section{0}

00:52:15:05

00:52:16:11

\section{1}

$00: 52: 16: 22$

00:52:20:00

\section{2}

$00: 52: 21: 19$

00:52:24:04

\section{3}

00:52:24:06

$00: 52: 25: 24$

0654

00:52:26:01

00:52:28:05
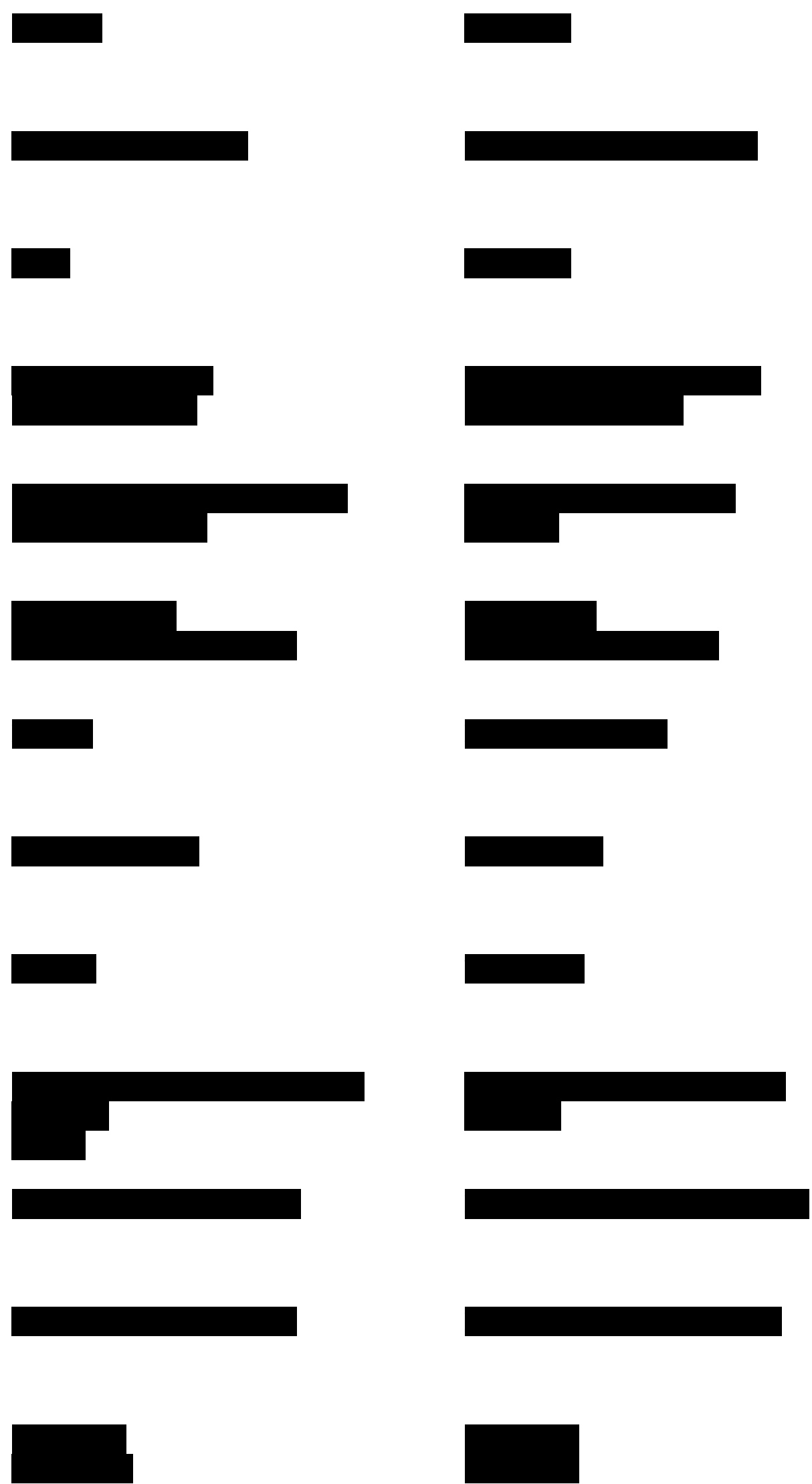

277 A misspelling and mispronunciation of Fantozzi's name. I have kept the original spelling of the Italian despite the 'ch sound that is produced. This shouldn't be too difficult for the English audience to contend with considering words such as 'cappuccino' and 'ciao' exist in English with the same sound and spelling. 


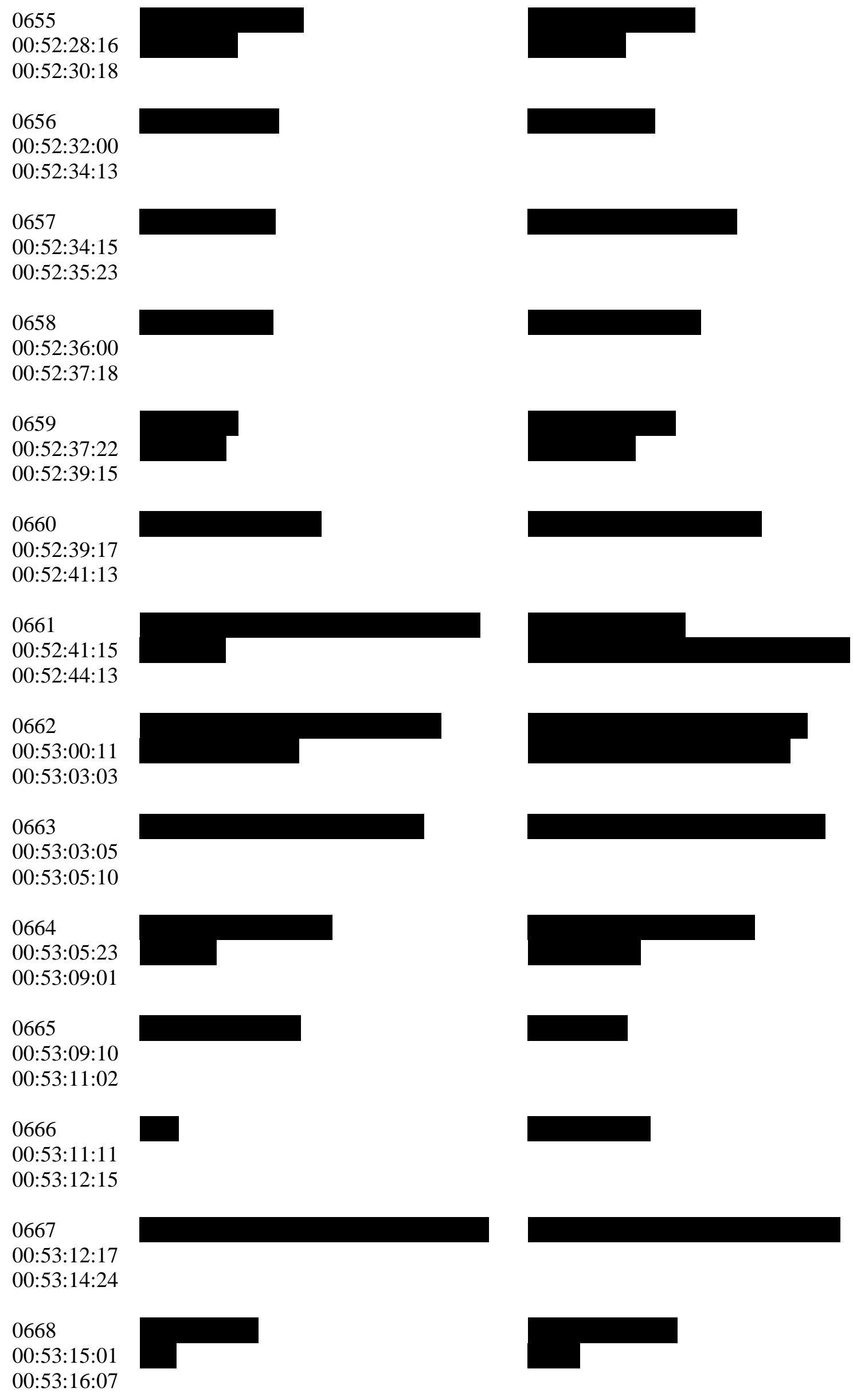



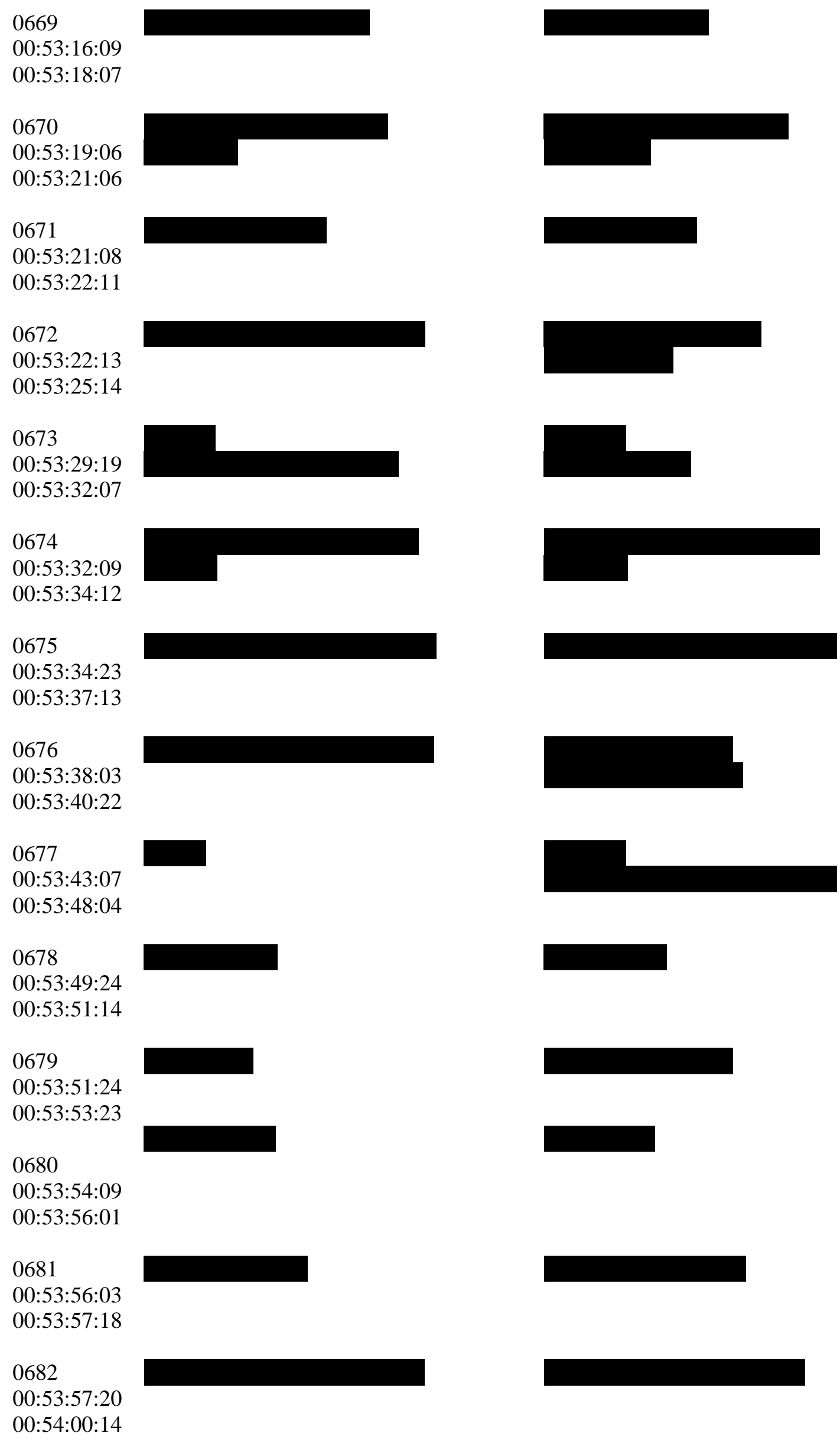

00:53:54:09

0681

00:53:56:03

$00: 53: 57: 20$

00:54:00:14 


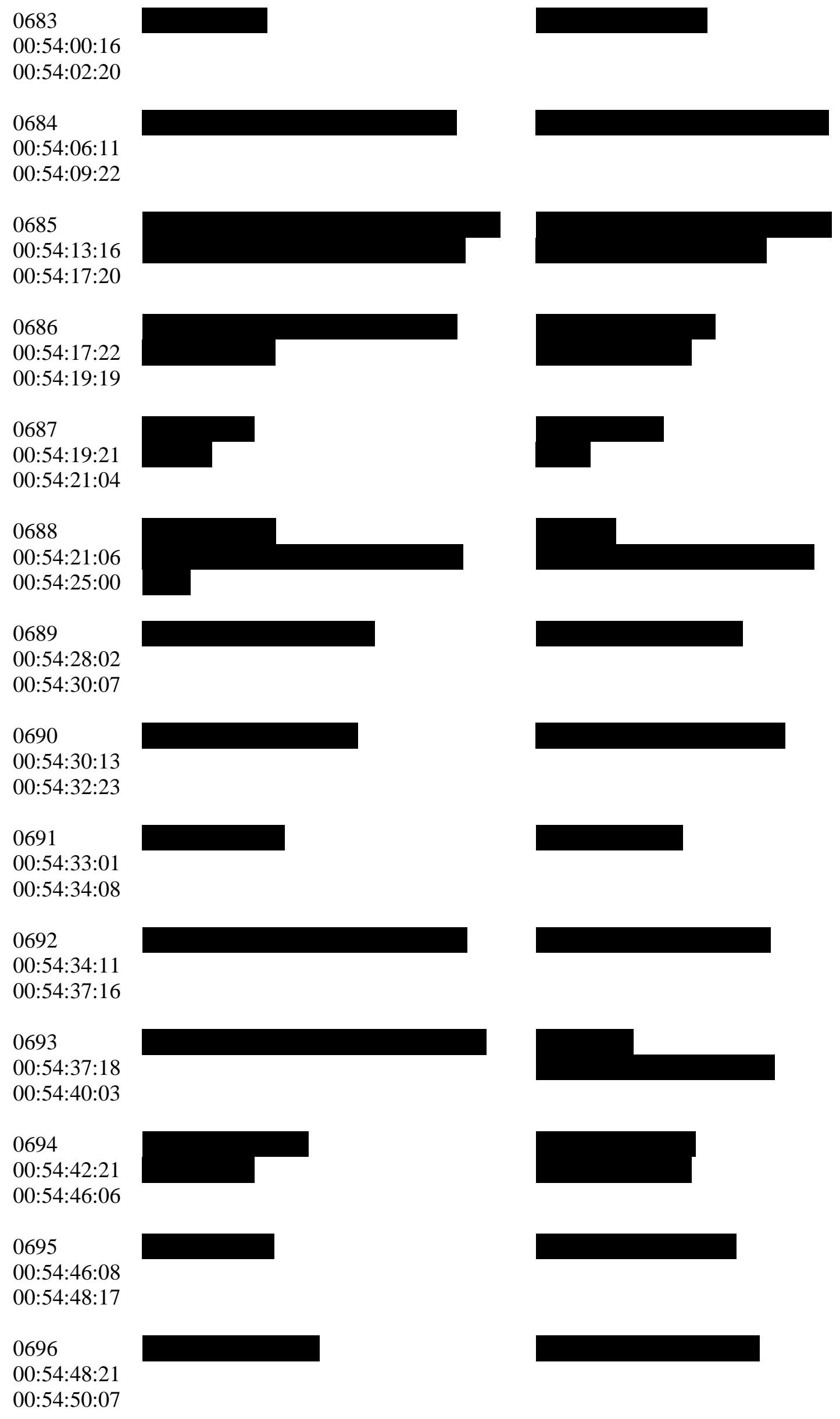




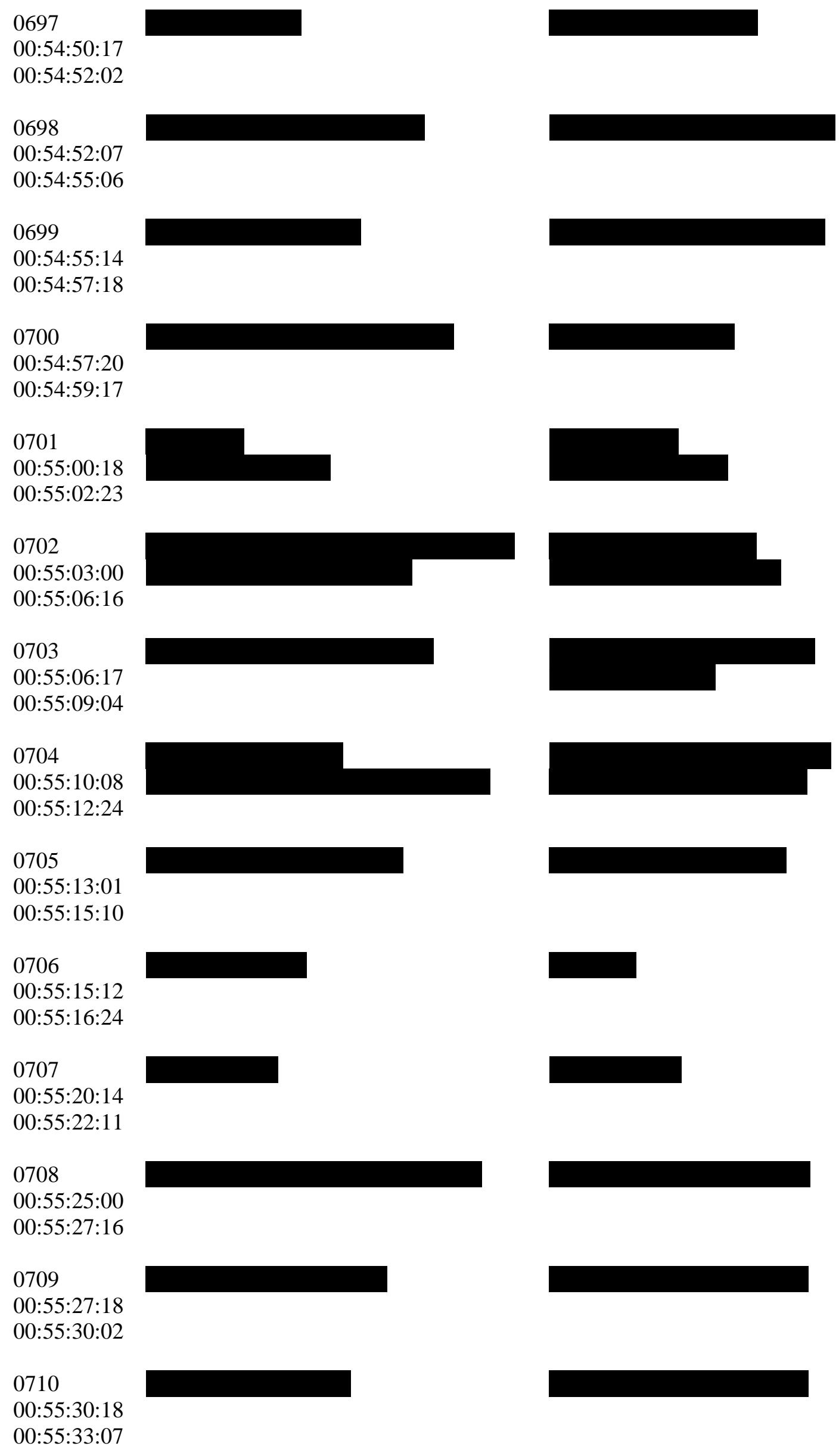


0711

$00: 55: 34: 18$

00:55:36:20

0712

00:55:38:02

00:55:39:08

0713

00:55:42:16

00:55:46:05

\section{4}

00:55:46:23

00:55:49:14

\section{5}

00:55:49:16

00:55:53:10

0716

00:55:57:06

00:56:00:11

0717

00:56:07:22

00:56:11:17

0718

00:56:18:00

00:56:22:02

0719

00:56:22:05

00:56:25:20

0720

00:56:25:22

00:56:29:00

0721

00:56:32:20

00:56:34:24

0722

00:56:37:16

00:56:39:13

0723

00:56:40:19

$00: 56: 42: 20$

0724

00:56:58:06

00:56:59:23
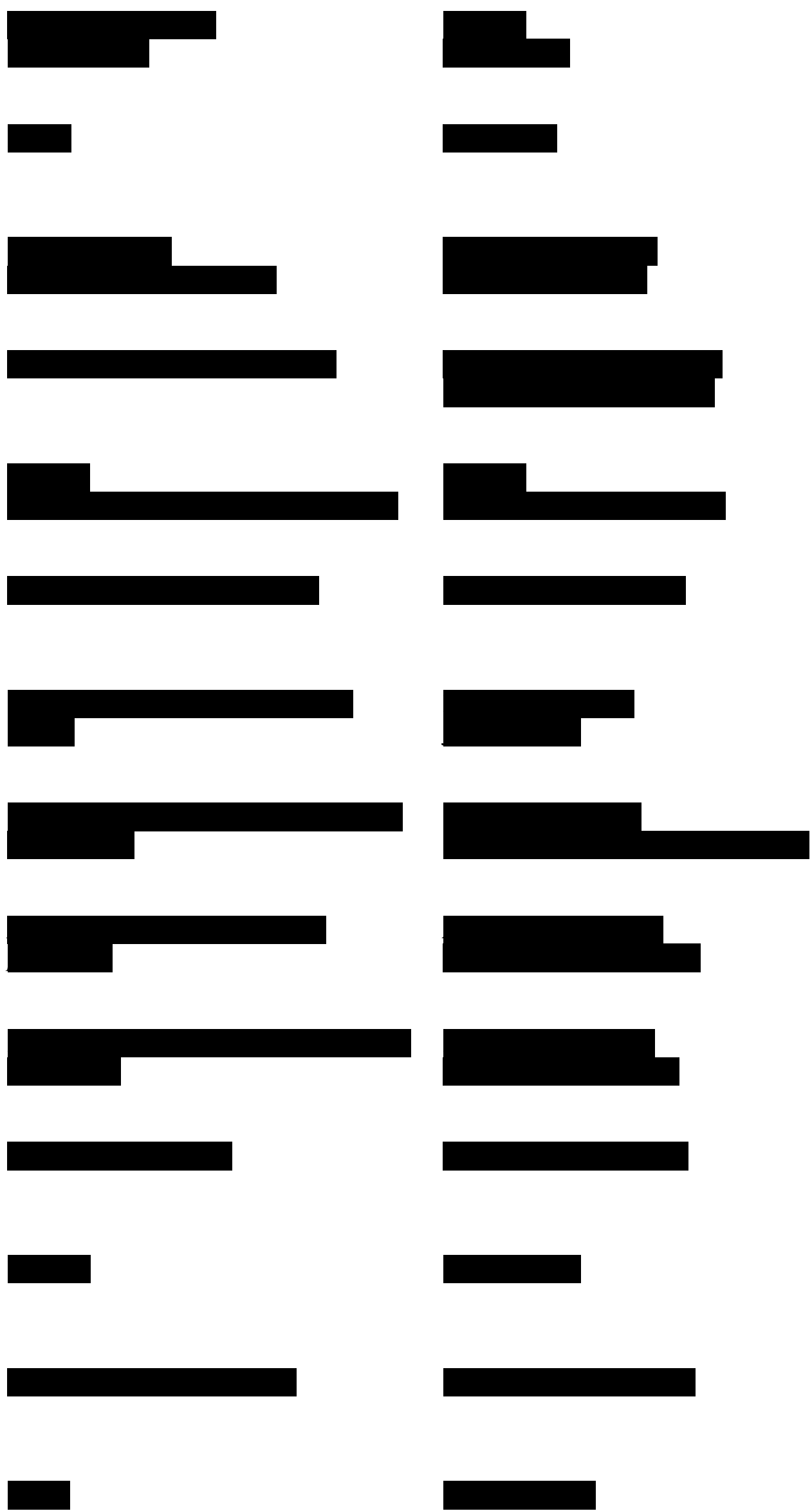

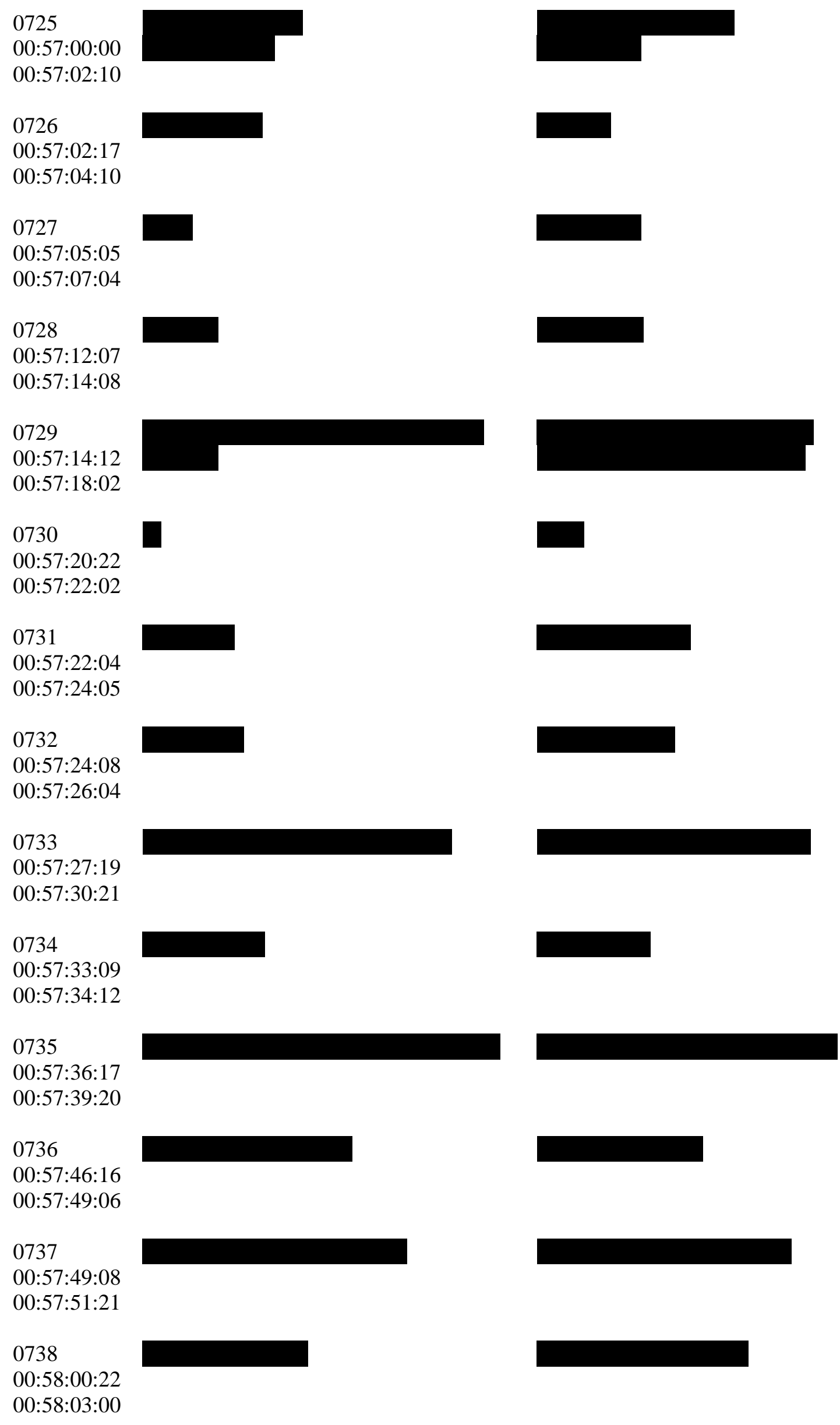
0739

00:58:03:17

00:58:06:00

0740

00:58:06:02

00:58:07:19

0741

00:58:07:22

00:58:09:09

0742

00:58:15:03

00:58:18:03

0743

00:58:18:05

00:58:22:00

0744

00:58:22:02

$00: 58: 25: 19$

0745

00:58:25:21

00:58:30:24

0746

00:58:31:01

00:58:33:15

\section{7}

00:58:34:01

00:58:37:21

0748

00:58:37:23

00:58:40:22

0749

00:58:40:24

00:58:42:13

0750

00:58:42:15

00:58:44:22

0751

00:58:45:00

00:58:47:13

0752

$00: 58: 47: 15$

00:58:50:03
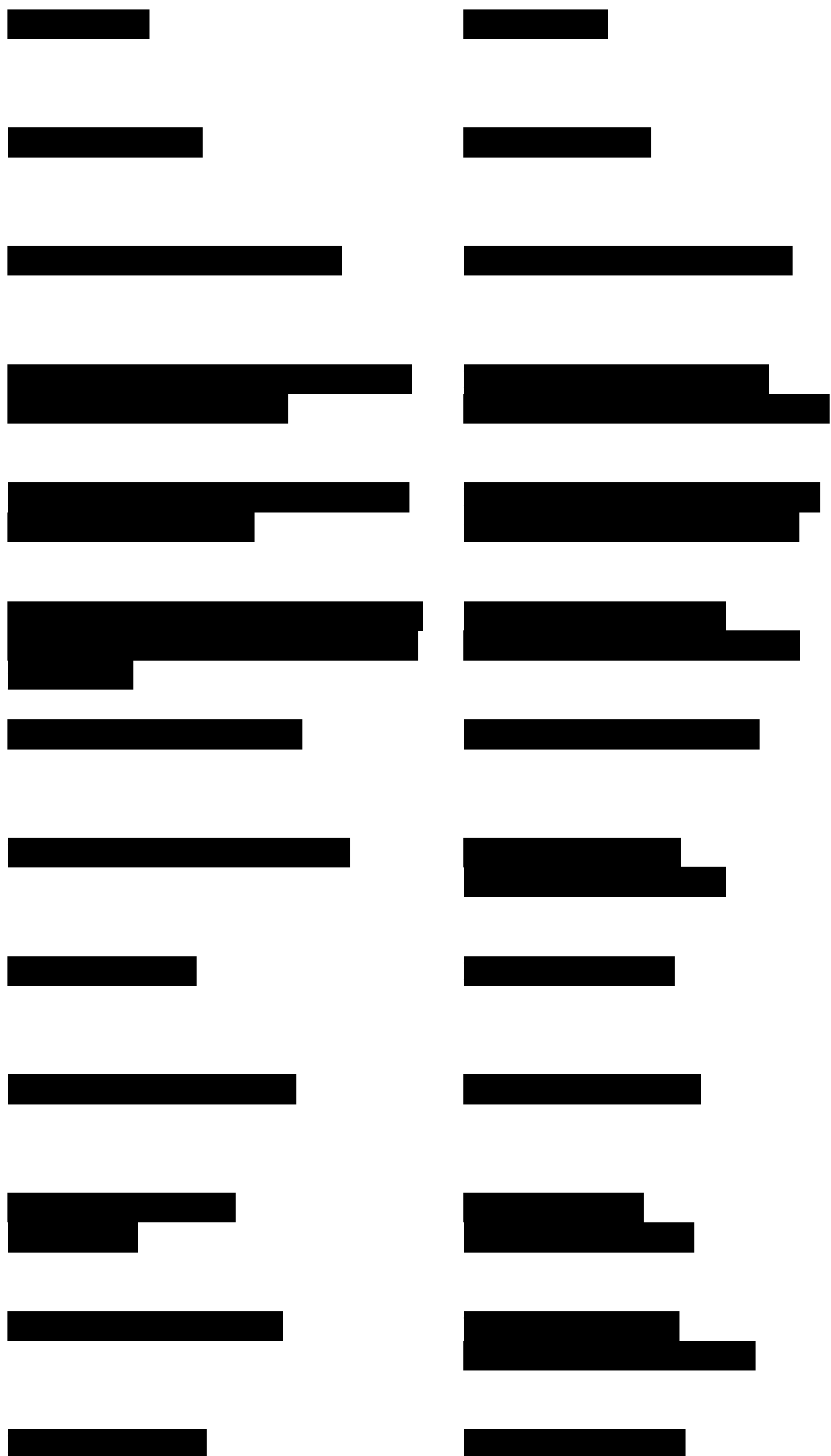
0753

00:58:50:05

00:58:52:01

0754

00:58:52:03

00:58:55:14

0755

00:58:55:16

00:58:56:19

0756

00:58:56:22

00:58:59:24

0757

00:59:01:03

00:59:03:01

0758

00:59:04:15

00:59:09:06

0759

00:59:09:08

00:59:10:21

0760

00:59:10:24

00:59:14:16

0761

00:59:14:19

00:59:18:14

0762

00:59:18:17

00:59:20:05

\section{3}

00:59:20:07

00:59:23:07

\section{4}

00:59:23:10

$00: 59: 25: 21$

\section{5}

00:59:26:24

00:59:28:19
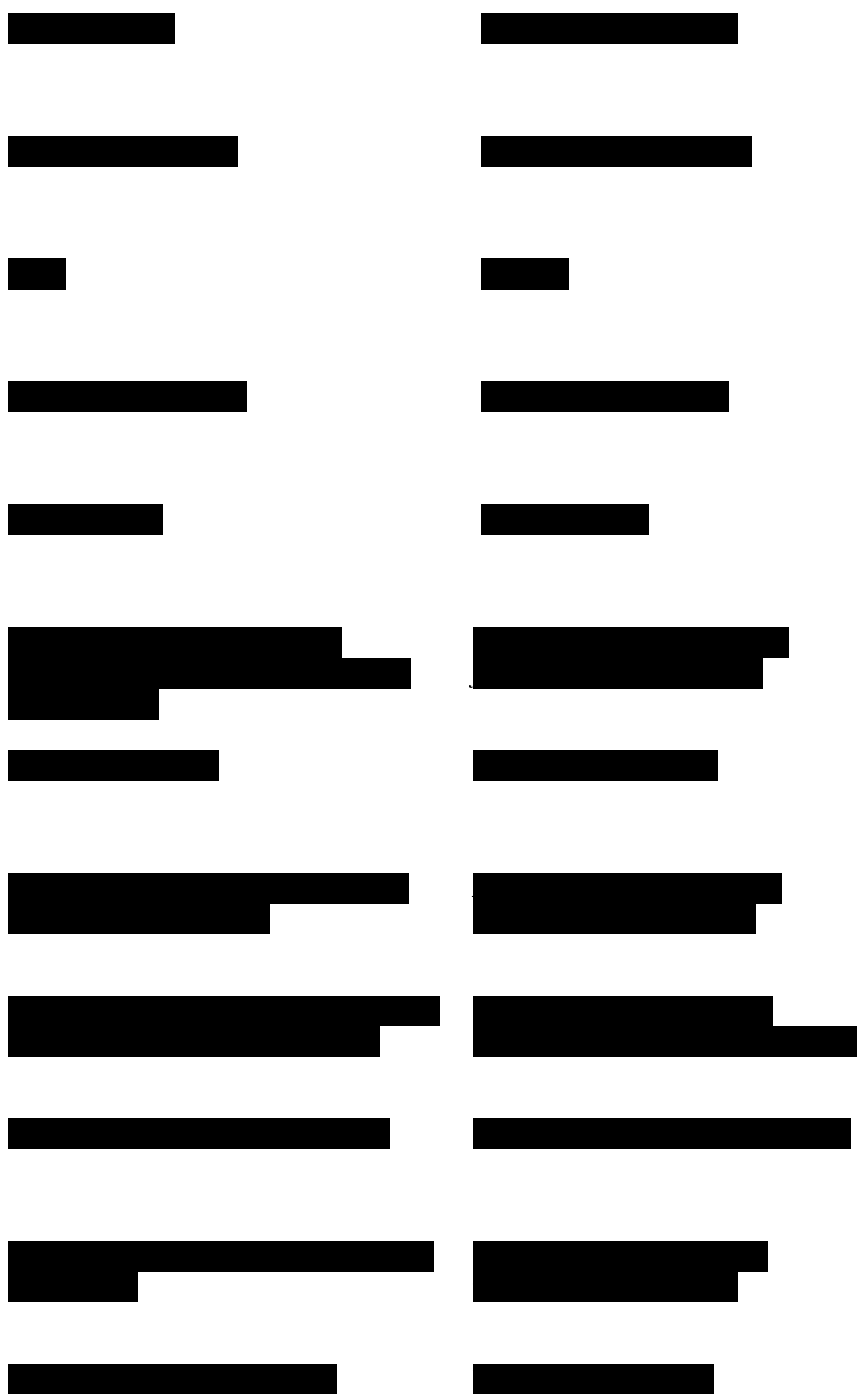

278 The Directors mother is using the 'imperativo formale' incorrectly which, in the context of this film, tends to suggest a low social status. Perhaps this suggests a lower-class status for the mother. It must be noted however that as the Fantozzi series progresses, all characters tend to misuse this verb form not just those of a lower social standing. 


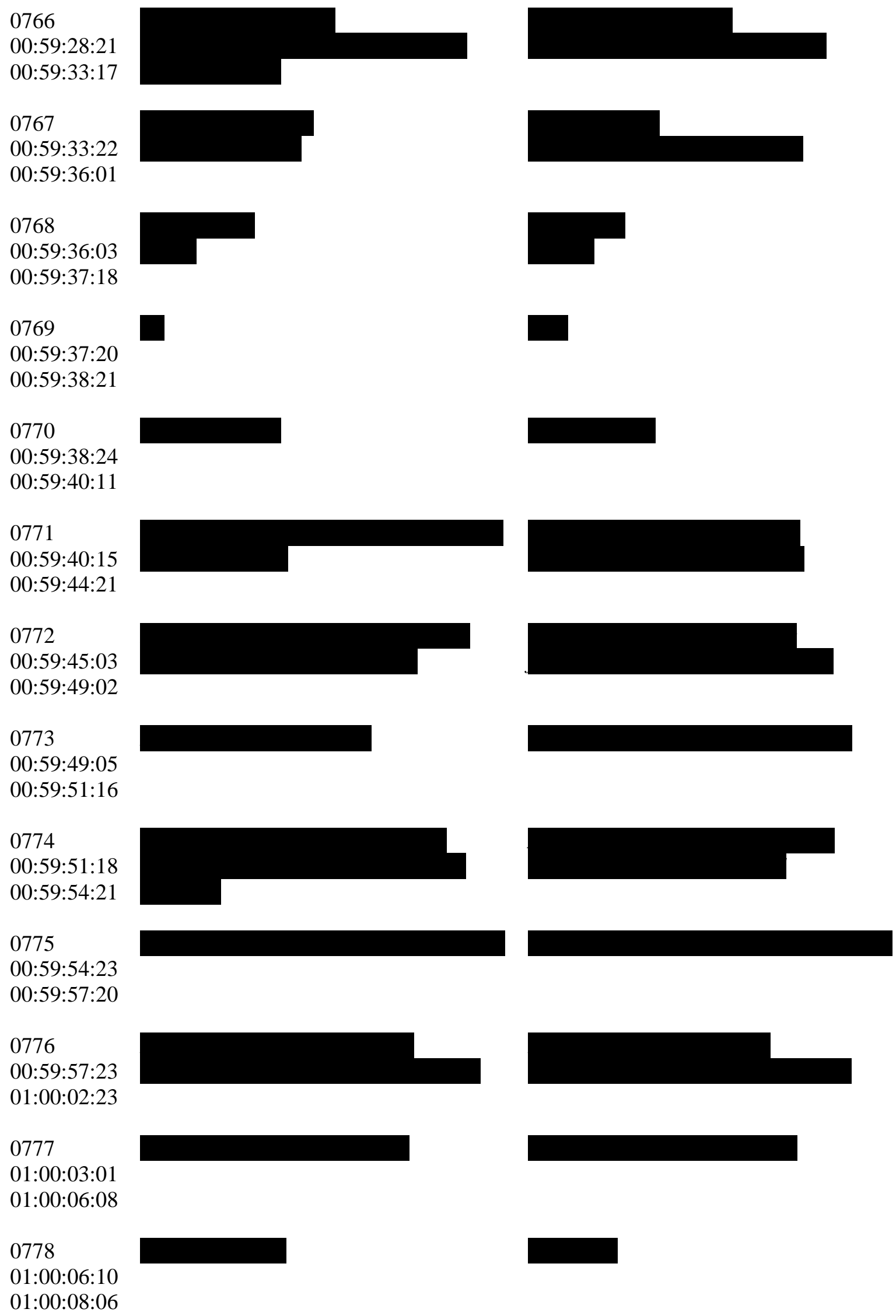


0779

01:00:08:09

01:00:10:18

0780

01:00:12:12

01:00:14:23

0781

01:00:15:16

01:00:18:20

0782

01:00:18:22

01:00:21:16

0783

01:00:21:18

01:00:23:21

0784

01:00:25:22

01:00:27:01

0785

01:00:33:21

01:00:35:12

0786

01:00:35:18

01:00:37:20

0787

01:00:39:24

01:00:44:12

0788

01:00:44:14

01:00:46:09

0789

01:00:46:11

01:00:50:18

0790

01:00:50:20

01:00:53:14
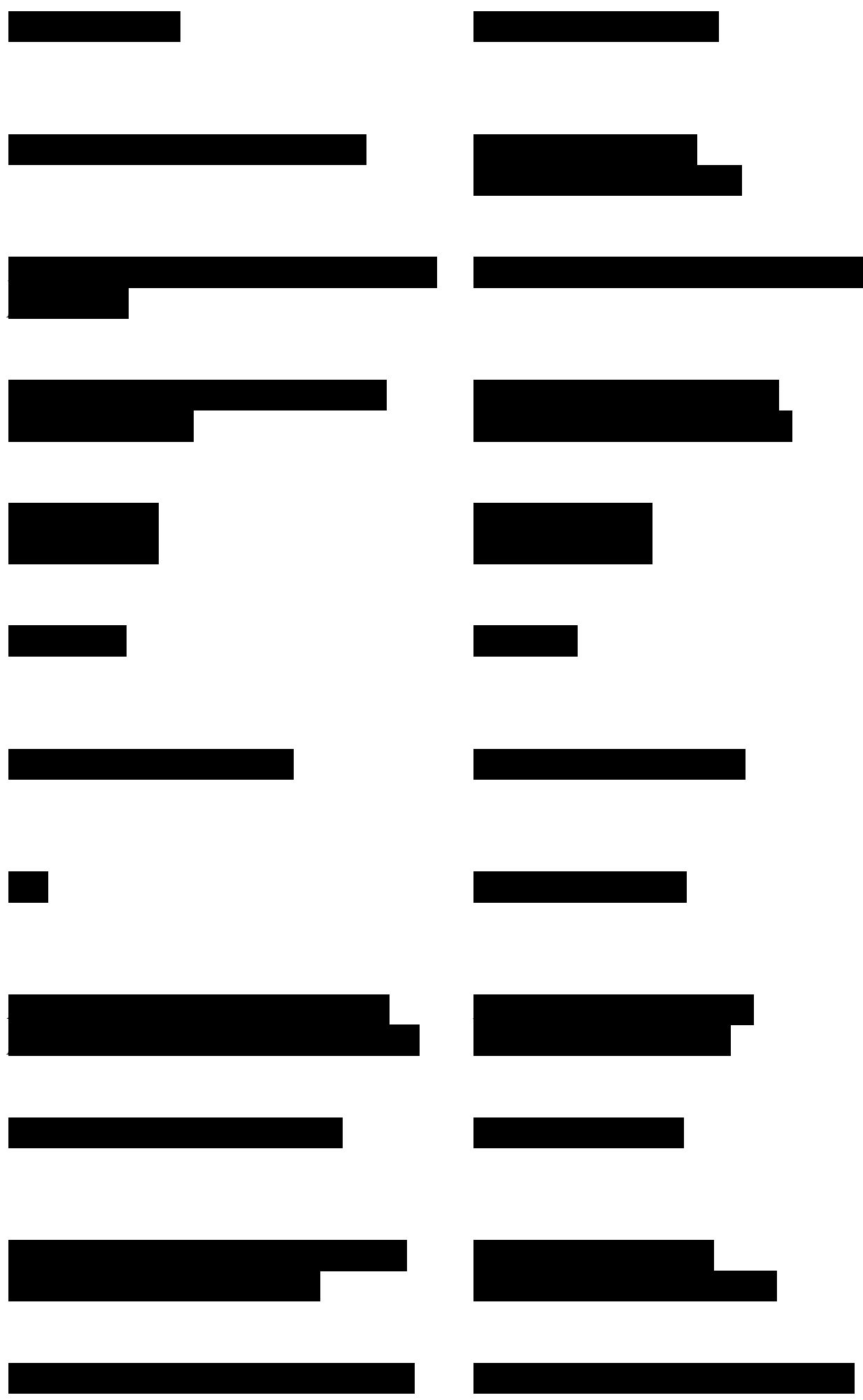

${ }^{279}$ Another instance of incorrect subjunctive/imperative.

${ }^{280}$ The original 'Scottish socks' has been changed to 'tartan' as 'Scottish socks' don't mean anything to an English speaking audience, 'tartan' makes the reference explicit. 
0791

01:00:54:15

01:00:57:08

0792

01:00:57:11

01:01:02:08

0793

01:01:02:10

01:01:04:12

0794

01:01:04:14

01:01:06:15

\section{5}

01:01:06:17

01:01:08:22

0796

01:01:10:22

01:01:13:22

0797

01:01:13:24

01:01:16:20

0798

01:01:30:20

01:01:32:24

0799

01:01:33:01

01:01:34:04

0800

01:01:34:05

01:01:35:21

0801

01:01:35:23

01:01:38:06

0802

01:01:38:08

01:01:41:02
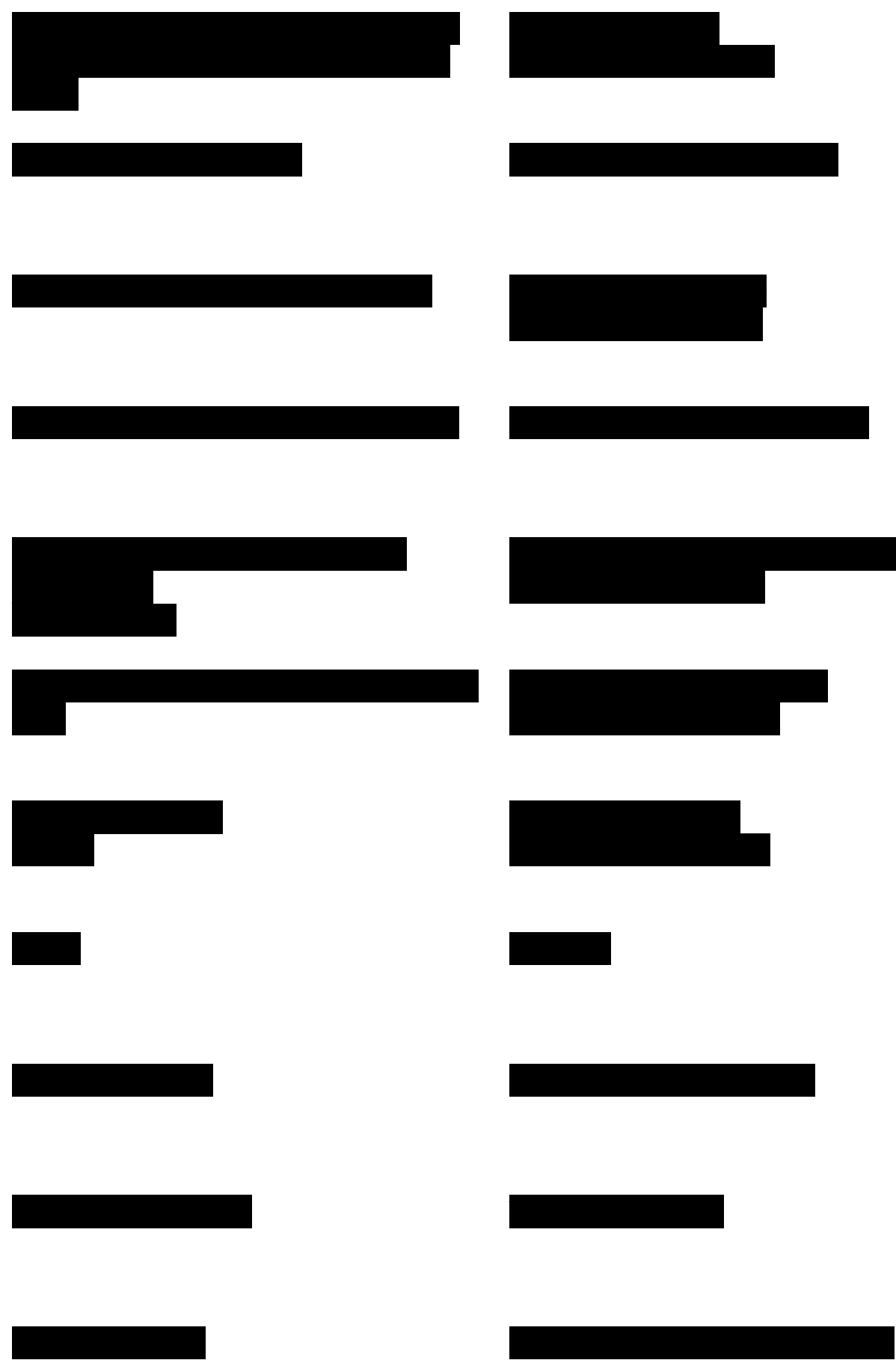

\footnotetext{
${ }^{281}$ A variety of cultural references need to be negotiated in this section of dialogue. A detailed explanation as to the specific translation choices can be found in chapter 4 .

${ }^{282}$ The incorrect use of imperatives is treated differently in this small section of dialogue to maintain the humour. A discussion around this particular translation choice can be found in chapter 4 .
} 


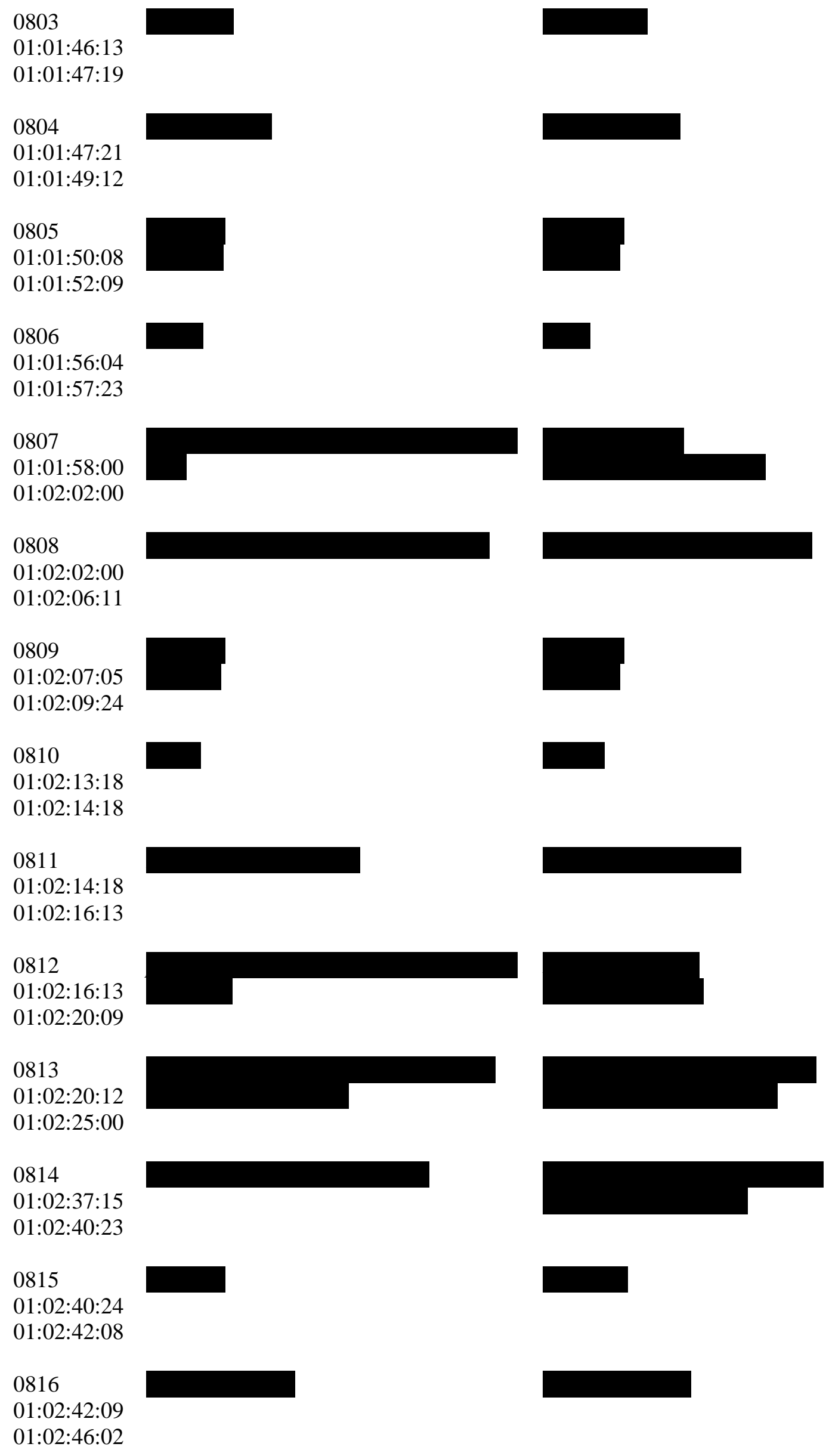




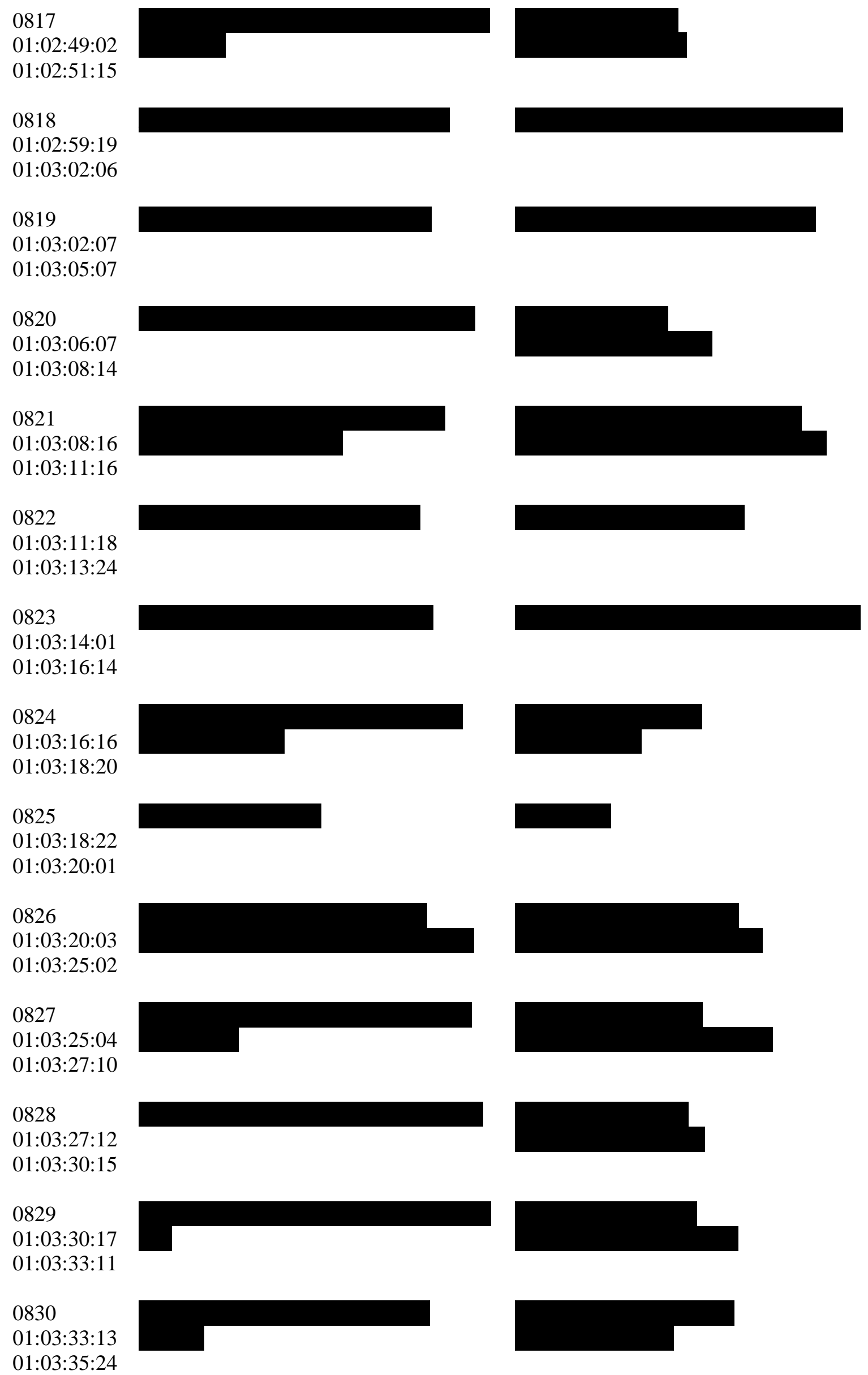


0831

01:03:36:01

01:03:38:13

0832

01:03:38:15

01:03:42:12

0833

01:03:42:14

01:03:47:10

0834

01:03:47:14

01:03:49:13

0835

01:04:13:15

01:04:15:09

0836

01:04:15:11

01:04:18:00

0837

01:04:18:02

01:04:20:04

0838
01:04:20:06

01:04:22:06

0839

01:04:22:08

01:04:24:10

0840

01:04:27:00

01:04:29:18

0841

01:04:40:16

01:04:41:20

0842

01:04:41:22

01:04:43:02

0843

01:04:43:04

01:04:45:06

0844

01:04:54:14

01:04:57:06
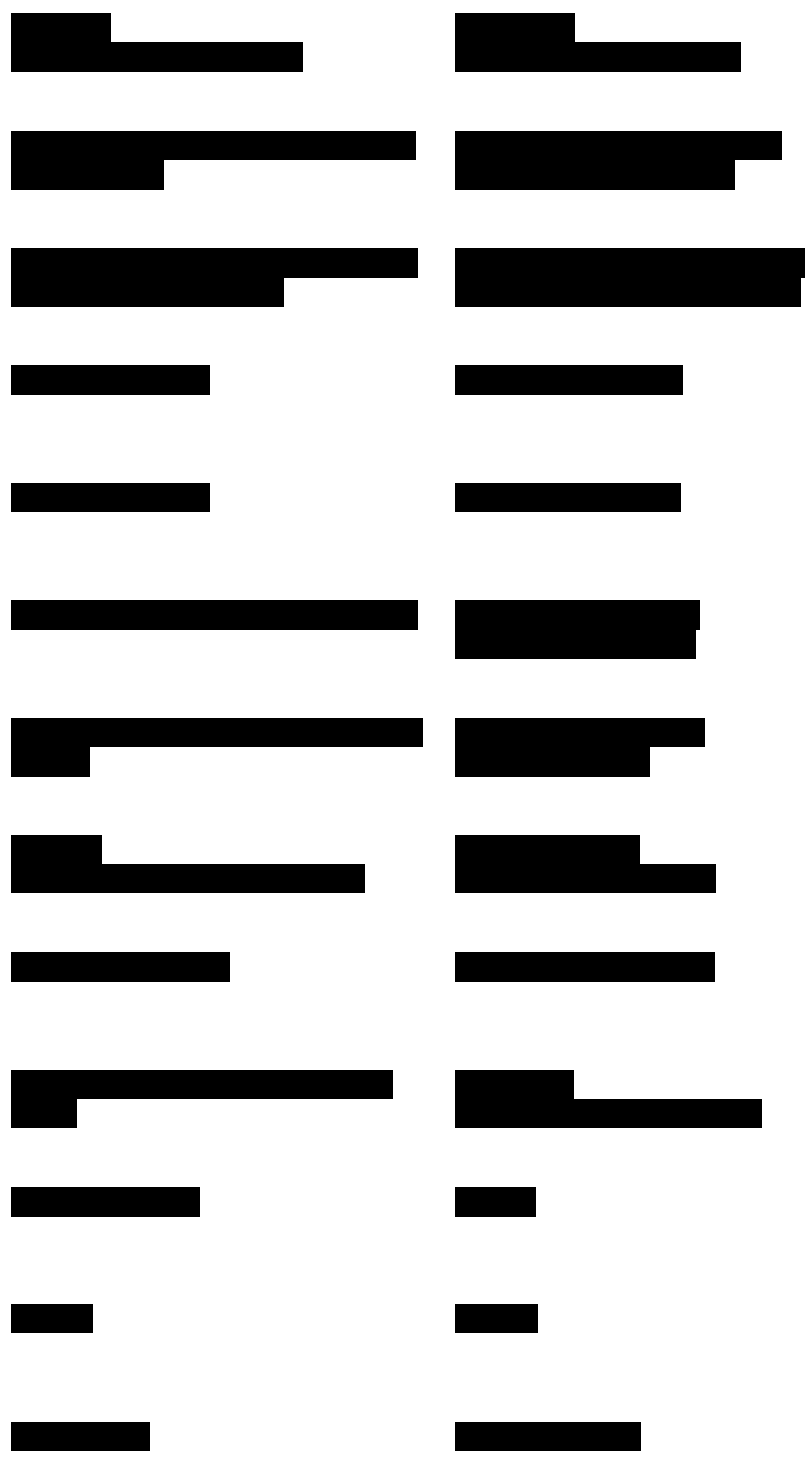
0845

01:04:57:08

01:04:59:01

0846

01:04:59:03

01:05:01:10

0847

01:05:01:12

01:05:06:18

0848

01:05:06:23

01:05:11:02

0849

01:05:11:04

01:05:14:20

0850

01:05:20:19

01:05:22:15

0851

01:05:23:12

01:05:27:23

0852

01:05:27:23

01:05:31:13

0853

01:05:41:14

01:05:44:16

0854

01:05:44:18

01:05:48:21

\section{5}

01:05:49:12

01:05:52:00

0856

01:05:52:07

01:05:54:05

0857

01:05:55:20

01:05:57:10
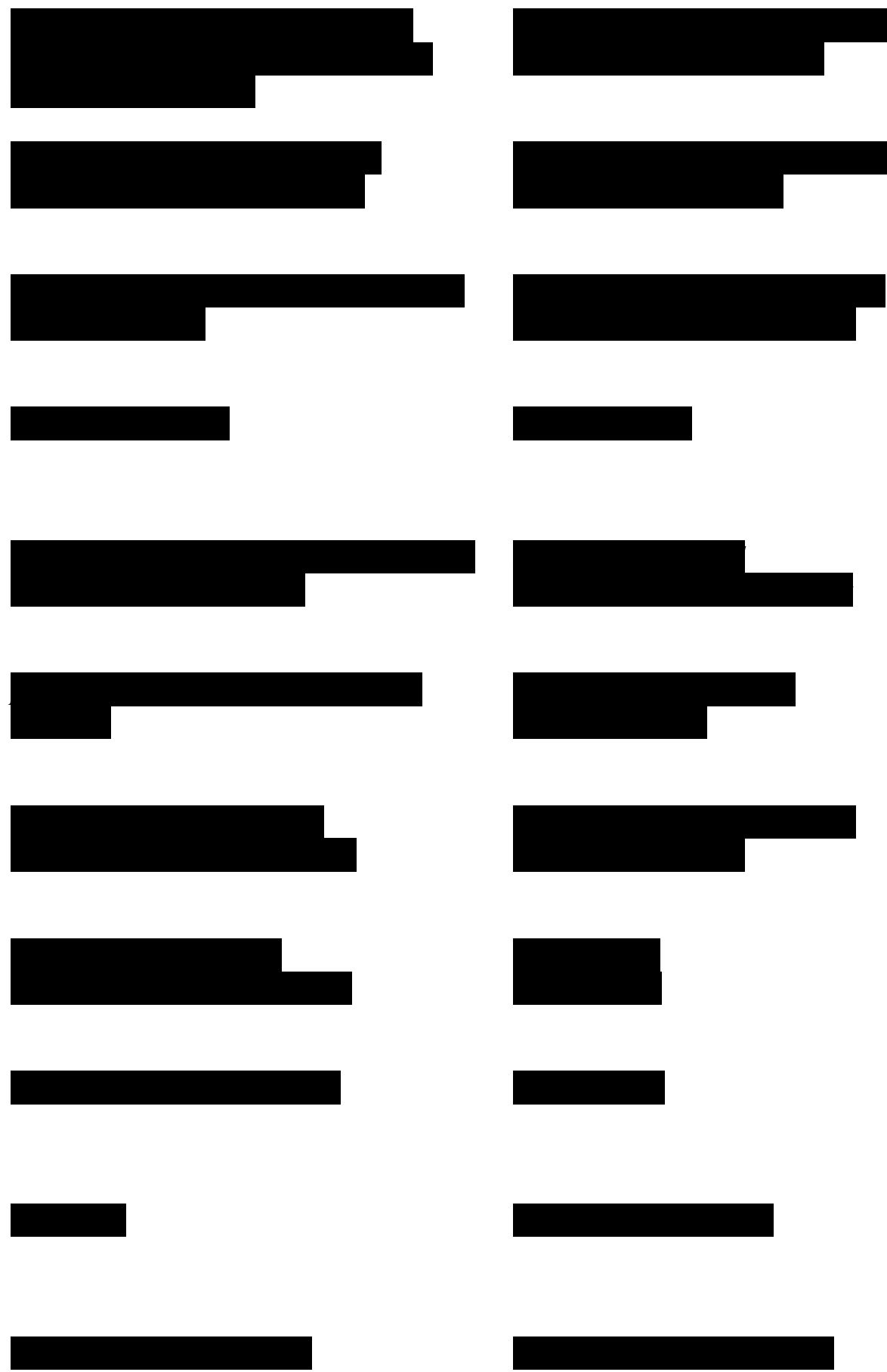

\footnotetext{
${ }^{283}$ A discussion on particular translation choices associated with food can be found in section 4.3 .4 of this chapter.
} 


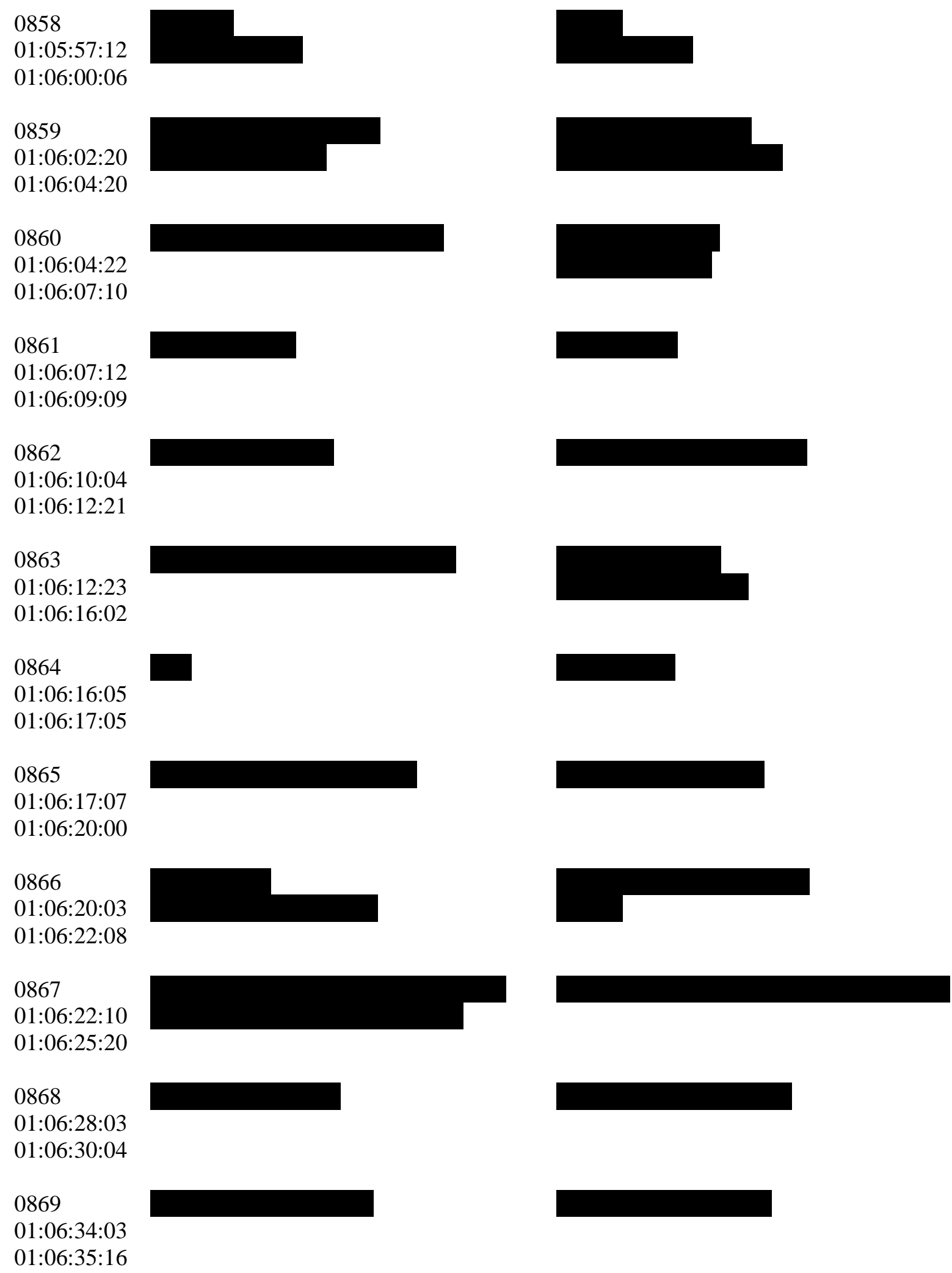

\footnotetext{
${ }^{284}$ This is a particular Roman expression recognised by all Italians meaning "to your dead people", it is used as an insult, particularly on the road, to mean "fuck off" or "go to Hell".

${ }^{285}$ By changing the word order in English we get a reference back to the sir/serve pun that was played on in the previous scene.
} 


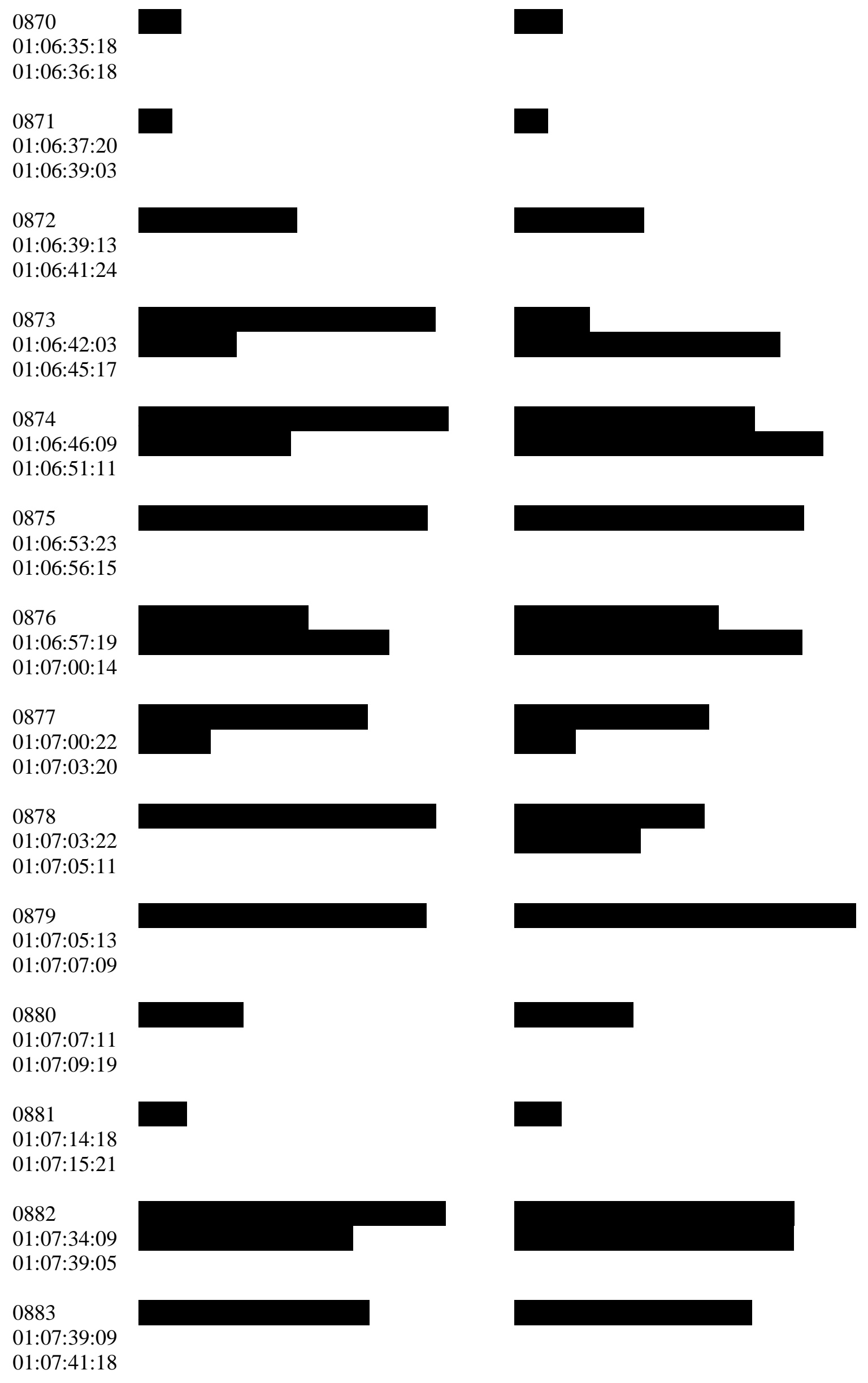




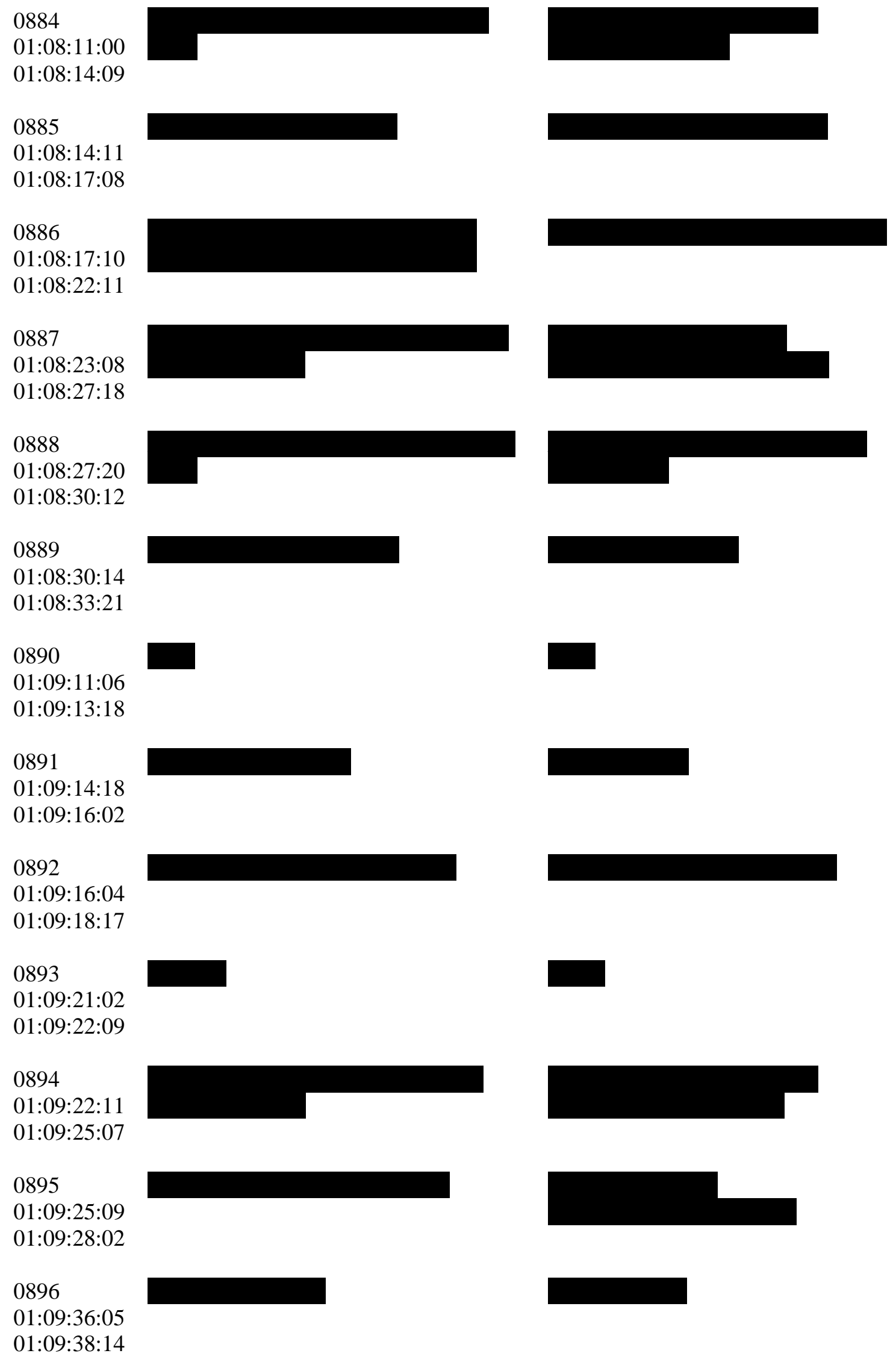

\footnotetext{
${ }^{286}$ A reference to The Count of Montecristo. For a discussion on this scene in particular see section 4.3.4
} 
0897

01:09:38:16

01:09:40:13

\section{8 \\ 01:09:40:15}

01:09:44:10

0899

01:09:45:04

01:09:46:22

0900

01:09:46:24

01:09:48:21

0901

01:09:48:23

01:09:50:19

0902

01:09:50:21

01:09:52:03

0903

01:09:52:05

01:09:53:18

0904

01:09:53:20

01:09:55:17

0905

01:09:55:19

01:09:59:12

0906

01:09:59:14

01:10:02:01

0907

01:10:02:18

01:10:04:10

0908

01:10:06:00

01:10:08:00
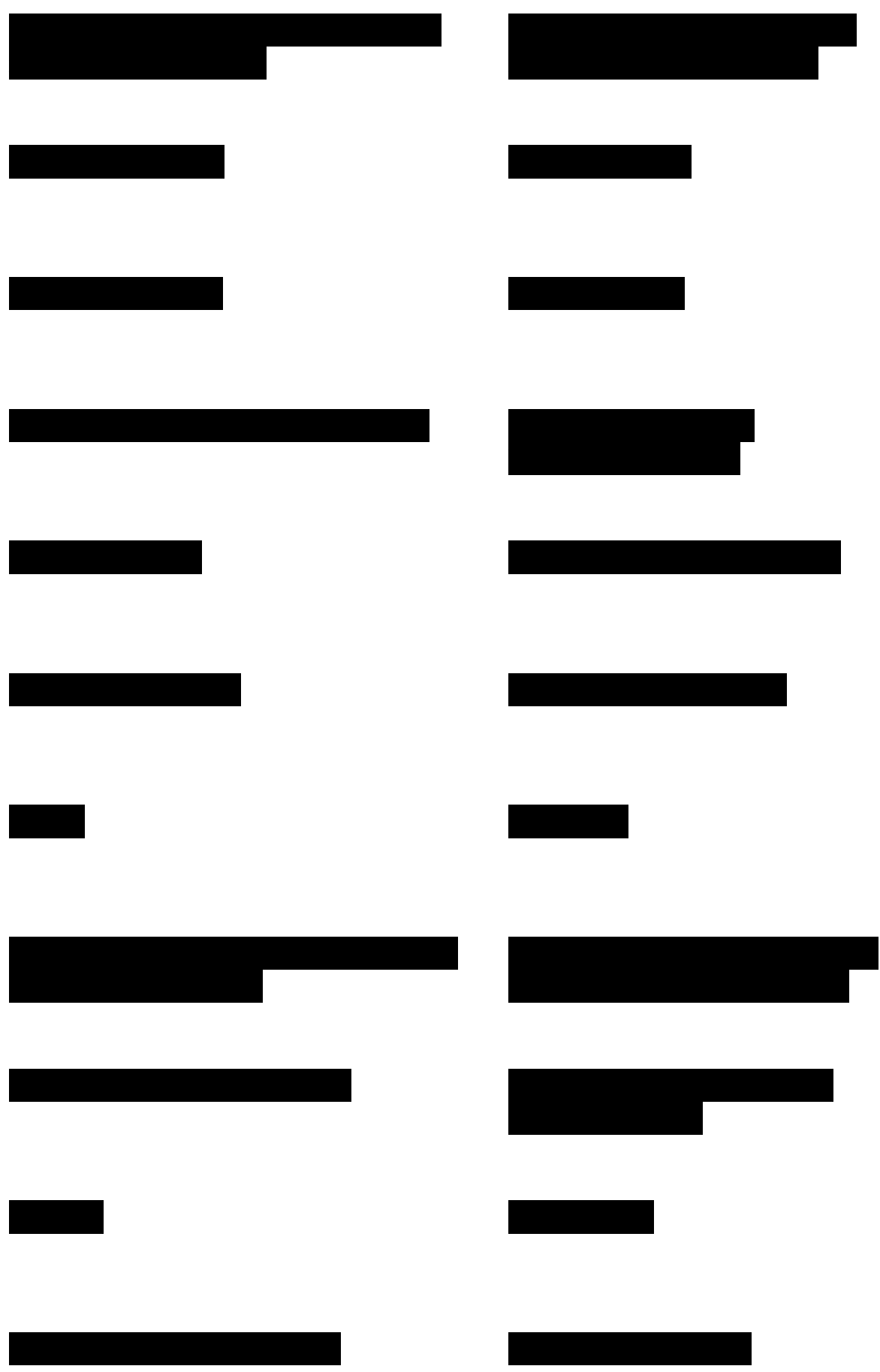

${ }^{287}$ Signorina Silvani is dressed in Japanese clothing, a reference to the 'exotic' used for comedic purposes.

${ }^{288}$ Another interesting reference to Germans. At the end of the film we see Fantozzi say the directors are evil people, only concerned about making money. Salce is perhaps foreshadowing the fact that the directors are perceived as being evil by associating that stereotype with them. 


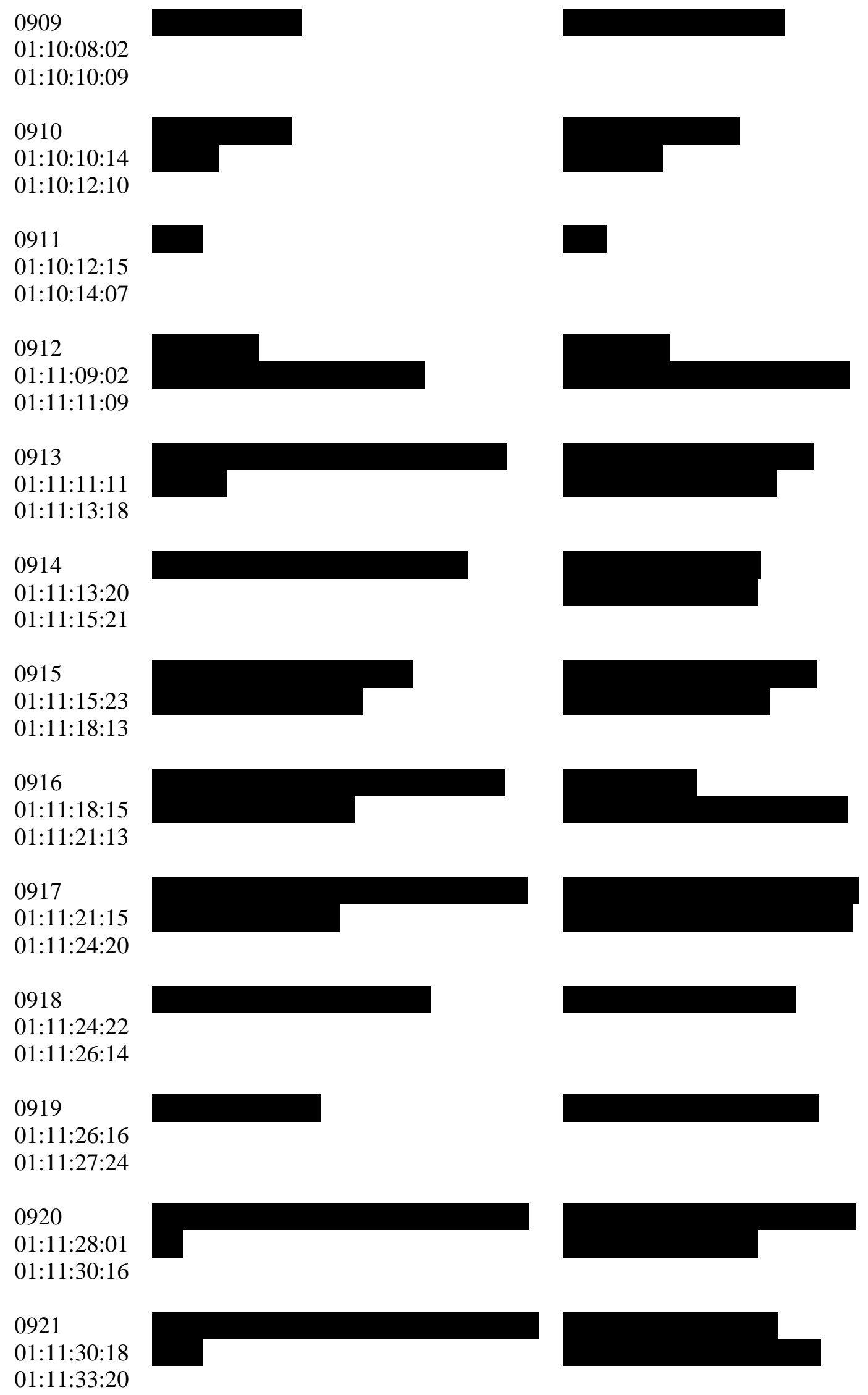
${ }^{289}$ This literally translates to: "He is Pekinese, not far from the Japanese." This is a colloquialism in Italian and therefore has
been translated to one of a similar ilk in English, that is, "to fit right in". 
0922

01:11:39:02

01:11:41:07

0923

01:11:44:12

01:11:48:16

0924

01:11:59:00

01:12:02:08

0925

01:12:02:10

01:12:05:06

0926

01:12:05:08

01:12:09:11

0927

01:12:09:15

01:12:12:21

0928

01:12:12:24

01:12:14:15

0929

01:12:14:17

01:12:17:10

0930

01:12:17:12

01:12:21:01

0931

01:12:21:19

01:12:23:14

0932

01:12:27:06

01:12:28:17

\section{3}

01:12:30:00

01:12:31:11

0934

01:12:32:12

01:12:34:11
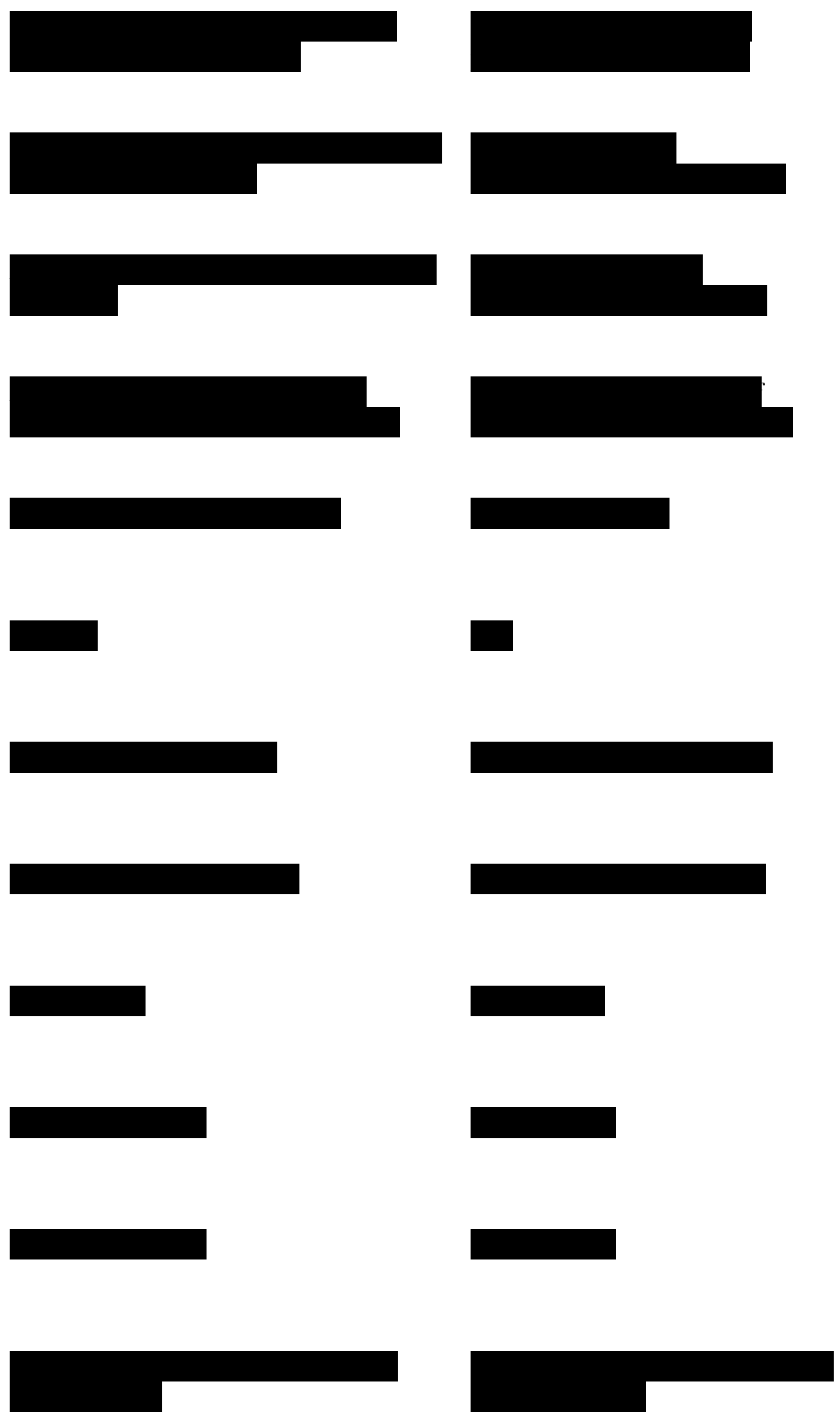

\footnotetext{
290 This translation has a nice ambiguity where we are unsure as to whether Pierugo has been fed, or is going to be fed to
} Fantozzi and Signorina Silvani. 
0935

01:12:34:13

01:12:36:06

0936

01:12:36:08

01:12:38:19

0937

01:12:38:22

01:12:42:03

0938

01:12:44:01

01:12:46:05

0939

01:12:46:08

01:12:49:11

0940

01:12:50:03

01:12:52:10

0941

01:12:53:03

01:12:54:14

0942

01:12:54:17

01:12:56:19

0943

01:12:56:21

01:12:59:16

0944

01:12:59:18

01:13:01:01

\section{5}

01:13:01:03

01:13:02:24

0946

01:13:03:04

01:13:05:22

0947

01:13:05:24

01:13:08:02

0948

01:13:08:04

01:13:09:12
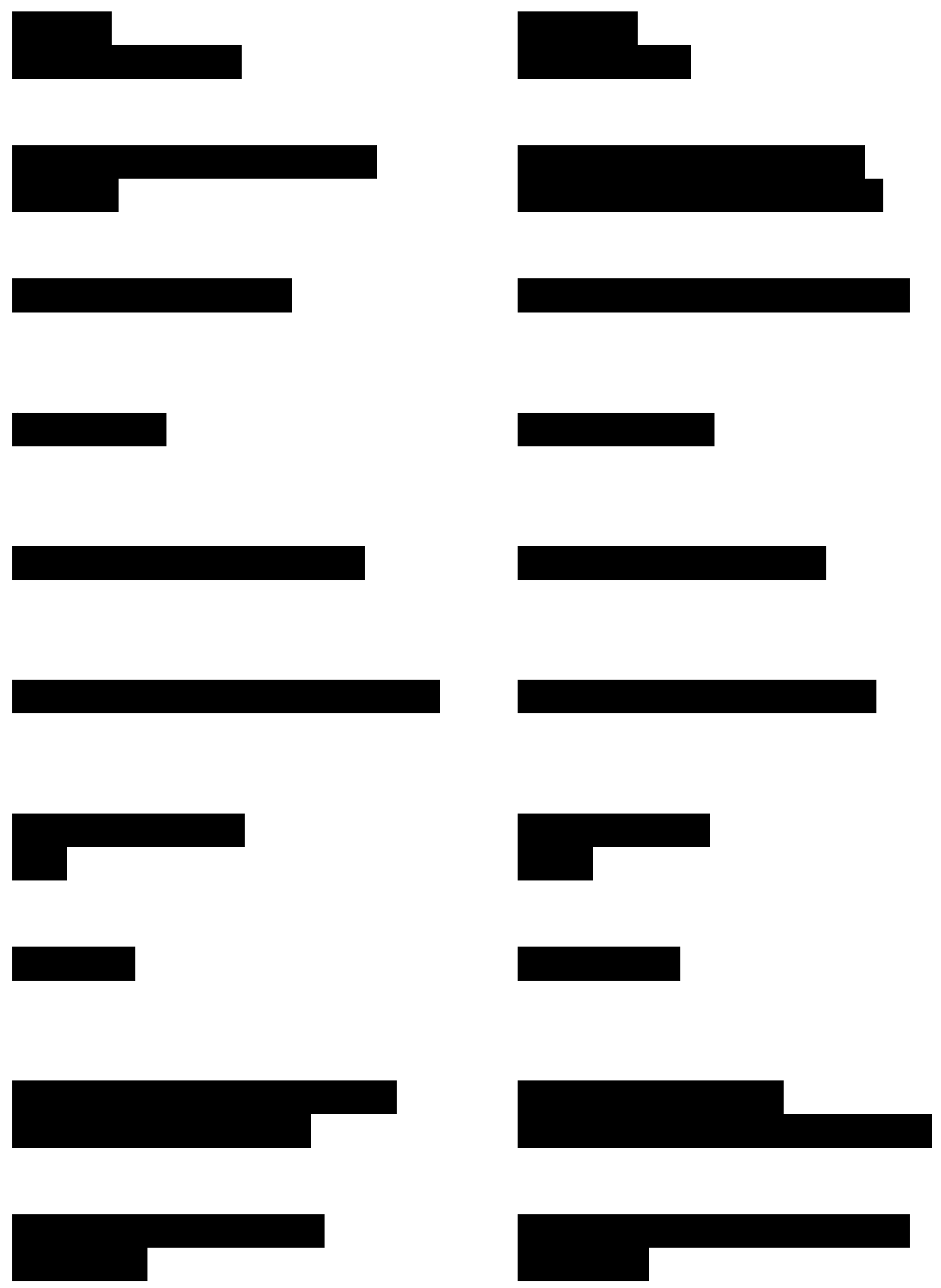
0949

01:13:10:15

01:13:12:03

0950

01:13:12:05

01:13:15:22

01:13:16:08

01:13:18:04

\section{1}

01:13:18:07

01:13:21:18

0952

01:13:21:20

01:13:23:04

0953

01:13:24:16

01:13:26:19

0954

01:13:30:06

01:13:31:18

\section{5}

01:13:31:20

01:13:33:21

0956

01:13:33:23

01:13:35:08

0957

01:13:35:10

01:13:38:19

0958

01:13:39:02

01:13:40:09

0959

01:13:40:12

01:13:41:24

0960

01:13:47:20

01:13:50:06

\section{1}

01:13:56:00

01:13:58:10
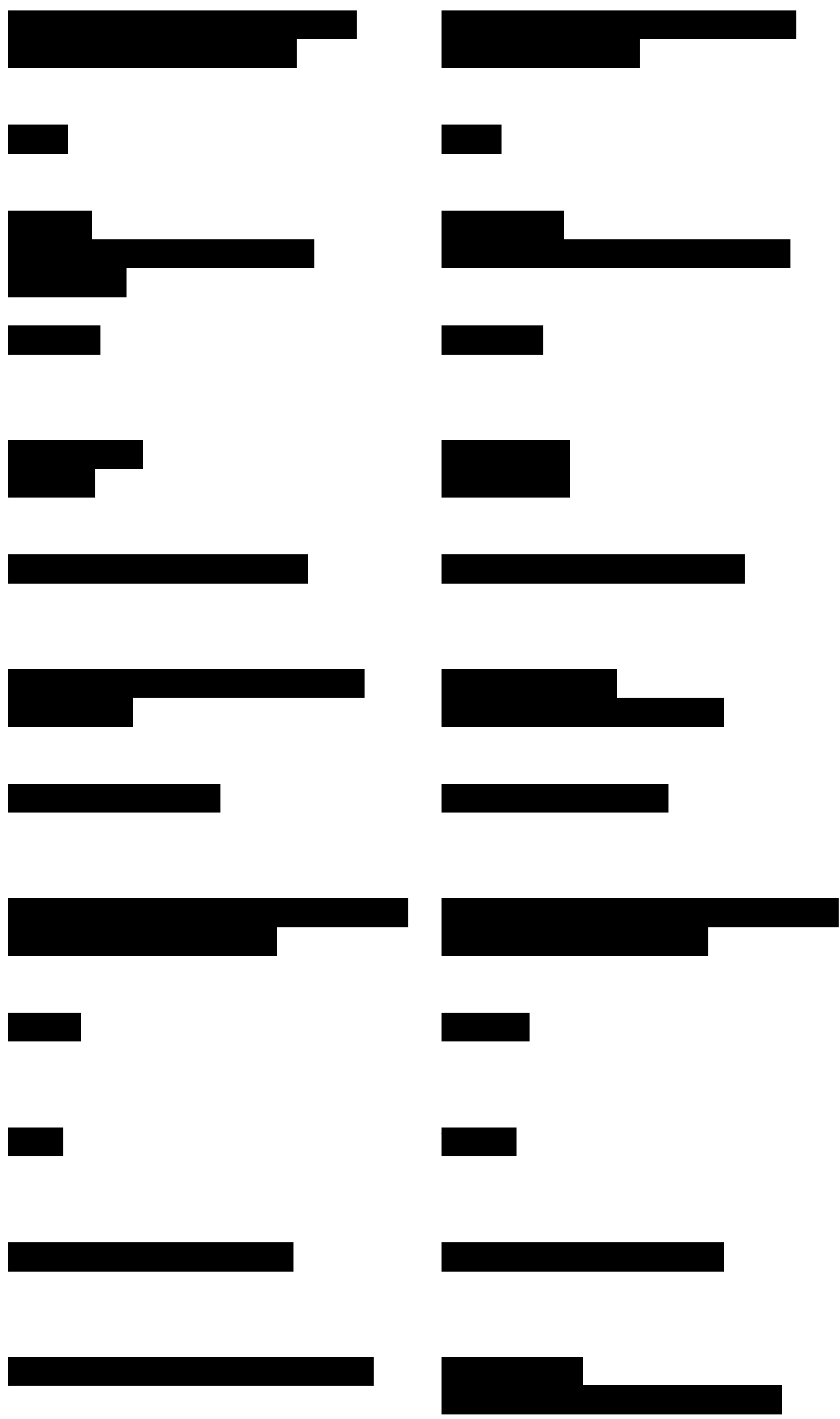


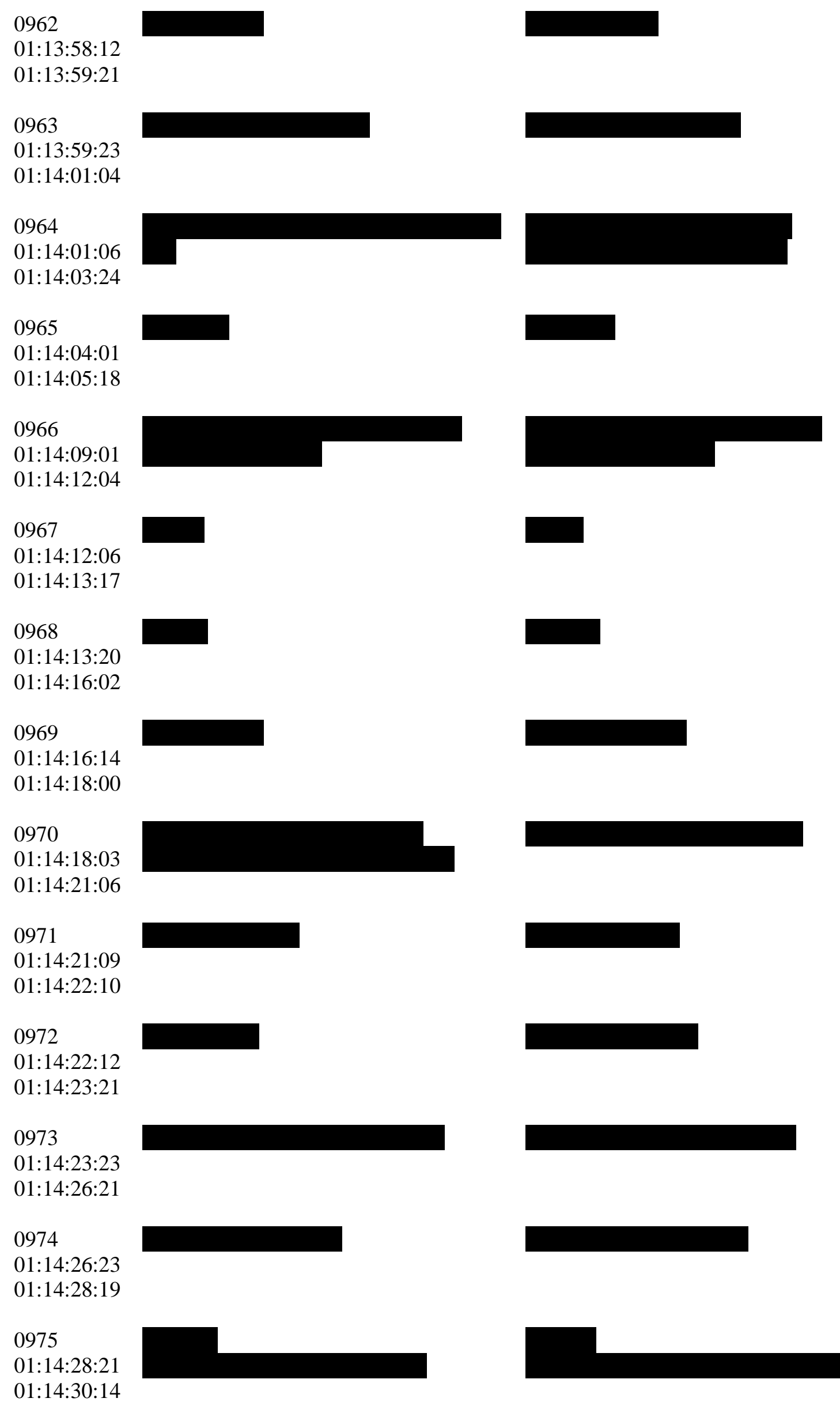




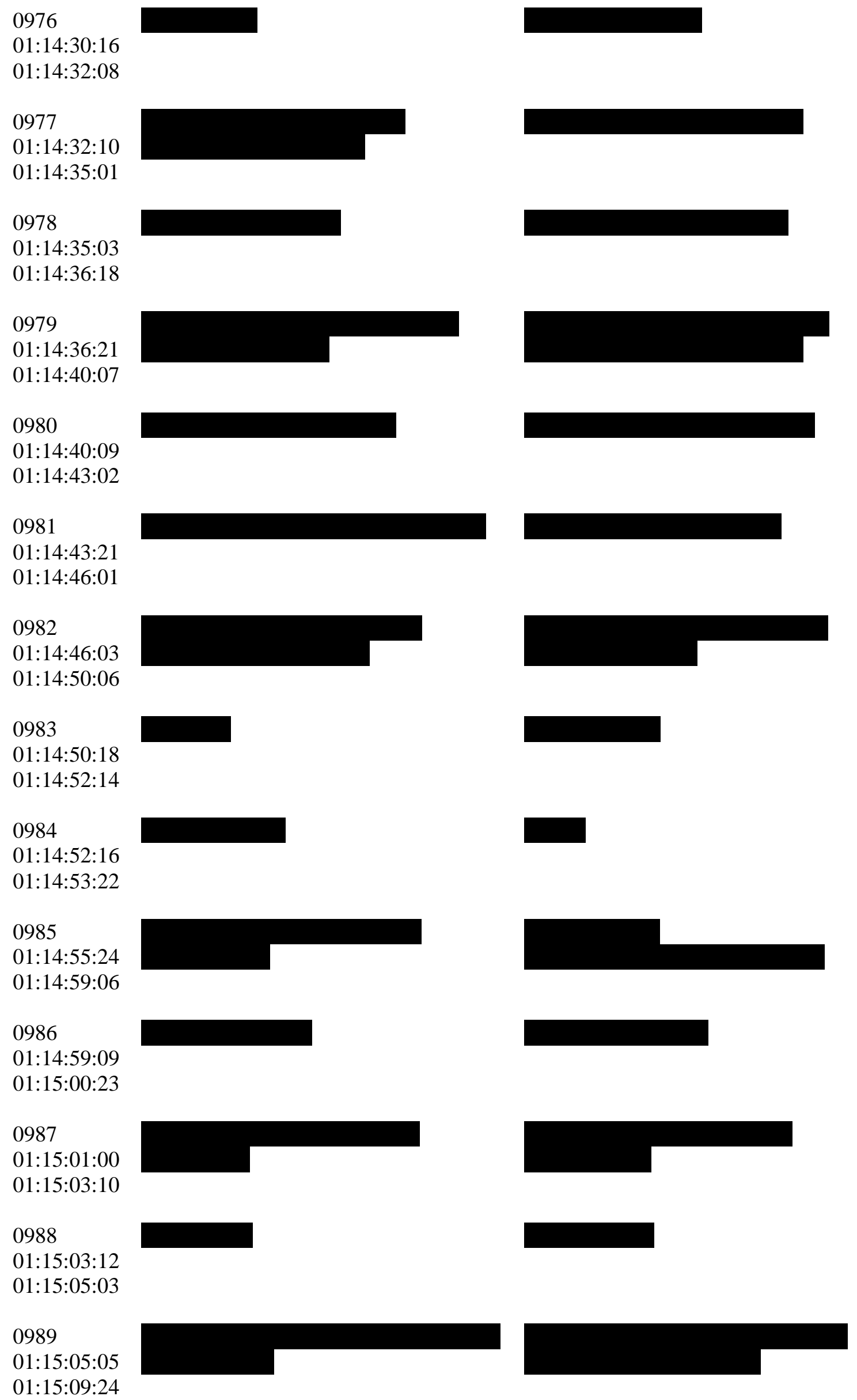




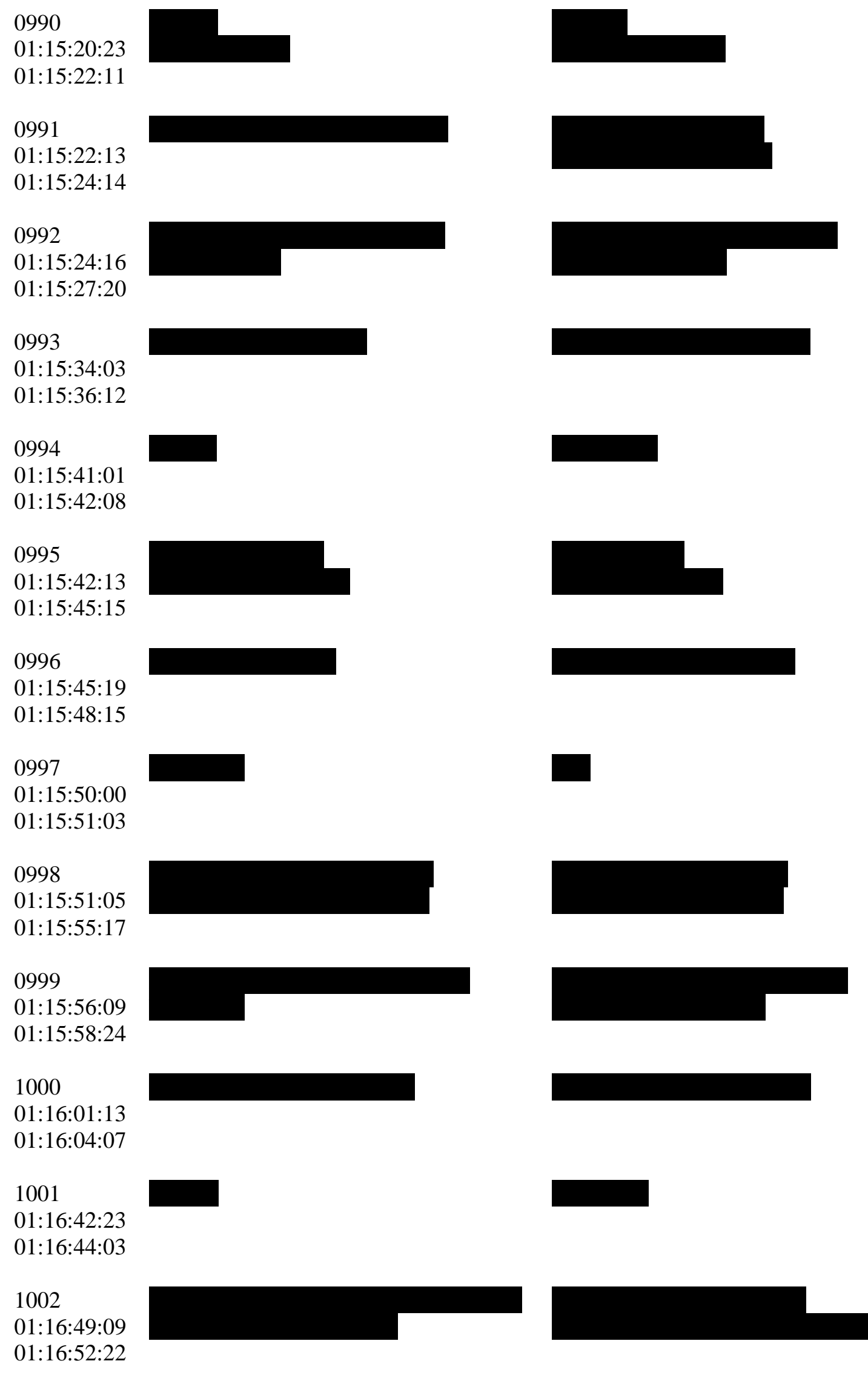

${ }^{291}$ Another reference to the 'exotic' for comedic effect, playing on the idea of different customs in other cultures. 
1003

01:16:53:15

01:16:57:07

1004

01:16:57:09

01:17:00:05

1005

01:17:06:23

01:17:10:02

1006

01:17:10:04

01:17:12:22

1007

$01: 17: 14: 23$

01:17:17:19

1008

01:17:20:01

01:17:23:20

1009

01:17:29:23

01:17:31:19

1010
01:17:31:21
$01: 17: 34: 10$

1011

01:17:34:12

01:17:36:08

1012

01:17:36:10

01:17:38:09

1013

01:17:38:11

01:17:41:00

1014

01:17:41:02

01:17:43:04

1015

01:17:43:06

01:17:45:13
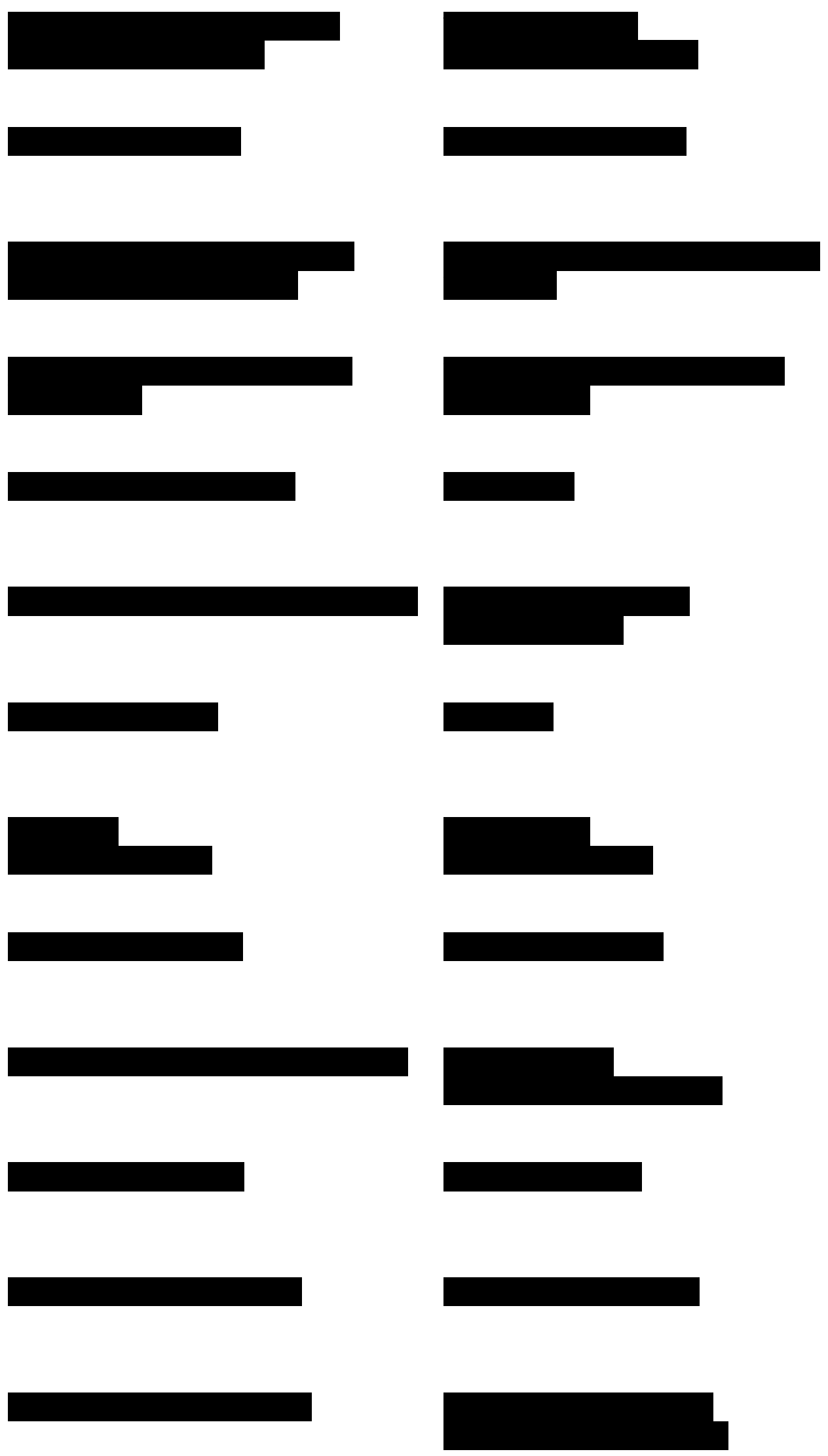
${ }^{292}$ Here, like other references in the Fantozzi series, the humour is based on racist stereotypes that most Italian audiences at
the time would not find objectionable. 
1016

01:17:45:15

01:17:48:17

1017

01:17:48:21

01:17:51:01

\section{8}

01:17:51:04

01:17:54:05

1019

01:17:54:07

01:17:55:13

1020

01:17:55:15

01:17:57:01

1021

01:17:57:03

01:17:59:17

1022

01:17:59:19

01:18:04:14

\section{3}

01:18:11:19

01:18:14:23

1024

01:18:14:23

01:18:18:03

1025

01:18:18:05

01:18:21:04

\section{6}

01:18:21:06

01:18:23:21

\section{7}

01:18:23:23

01:18:28:09

1028

01:18:28:11

01:18:32:03
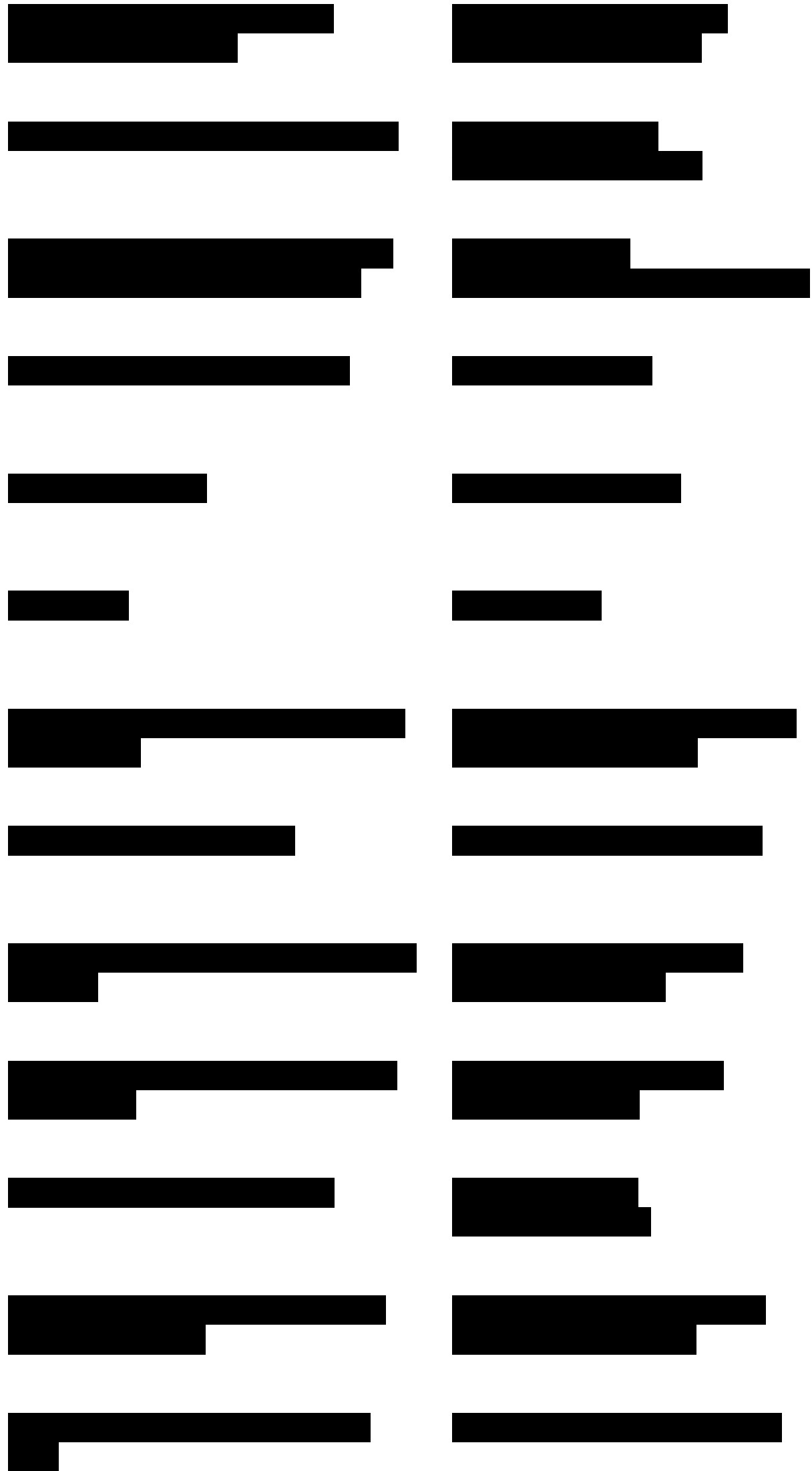

293 "Hara-kiri", otherwise known as "seppuku" is a form of Japanese suicide whereby a person disembowels themselves. I have decided to retain the original Japanese, rather than translating simply as 'disembowel myself' in order to retain the reference to a well-known Japanese practice. 
1029

01:18:34:20

01:18:36:04

1030

01:18:37:11

01:18:40:11

1031

01:18:44:13

01:18:47:21

1032

01:18:47:23

01:18:53:21

1033

01:18:54:17

01:18:56:15

1034

01:18:57:08

01:18:58:23

1035

01:18:59:00

01:19:01:14

1036

01:19:01:23

01:19:04:08

1037

01:19:04:10

01:19:06:20

1038

01:19:06:22

01:19:11:01

1039

01:19:11:03

01:19:12:17

1040

01:19:12:19

01:19:14:21

1041

01:19:14:23

01:19:18:22

1042

01:19:19:10

01:19:23:08
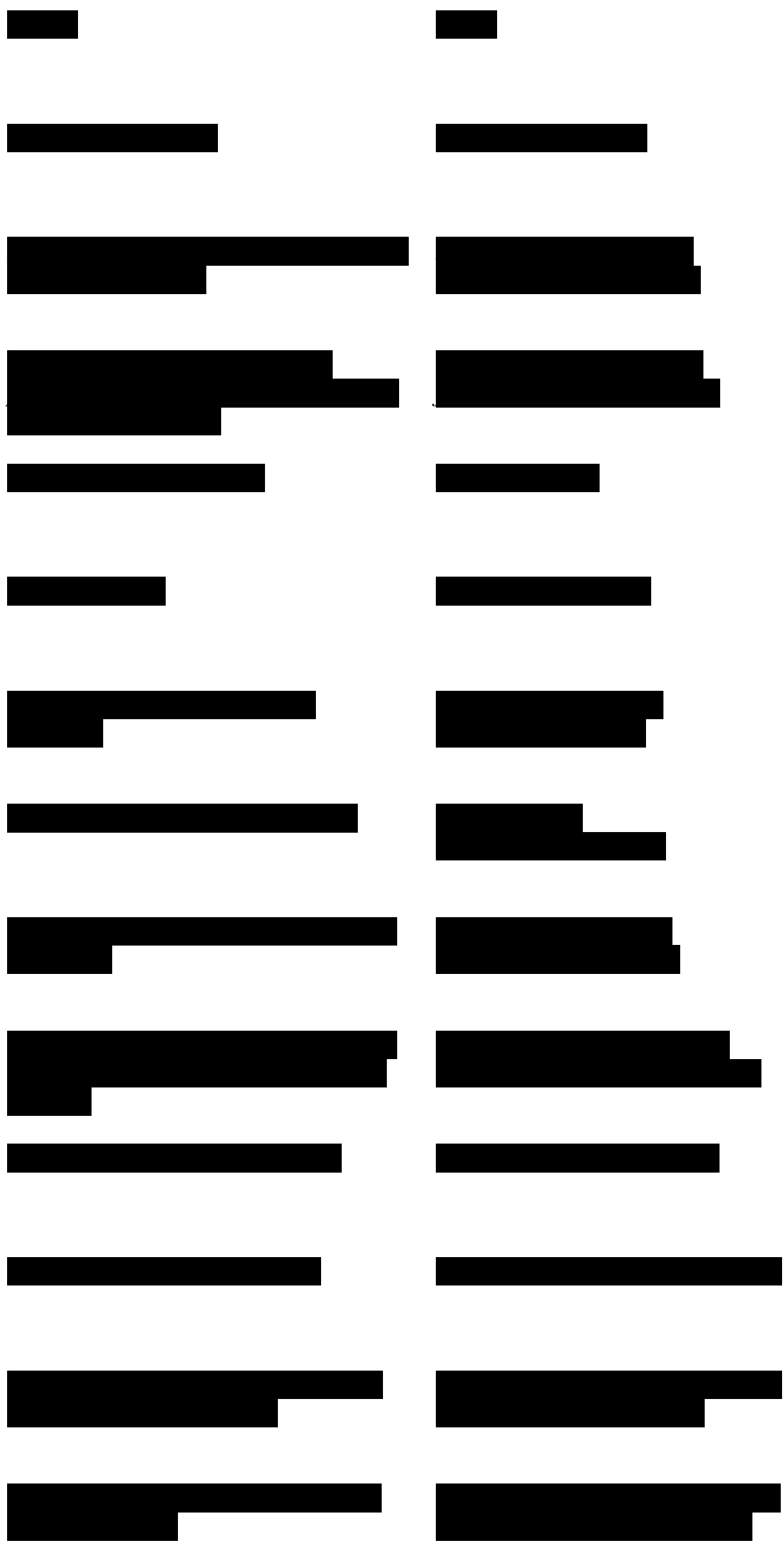
1043

01:19:23:10

01:19:25:17

1044

01:19:25:19

01:19:28:08

1045

01:19:28:10

01:19:29:10

1046

01:19:29:12

01:19:32:10

1047

01:19:32:12

01:19:35:02

1048

01:19:35:04

01:19:36:06

1049

01:19:36:08

$01: 19: 37: 23$

1050

01:19:38:01

01:19:40:17

1051

01:19:40:19

01:19:42:06

1052

01:19:42:08

01:19:45:04

\section{3 \\ 01:19:45:06}

01:19:46:23

\section{4}

01:19:47:00

01:19:52:19

1055

01:20:00:17

01:20:02:09
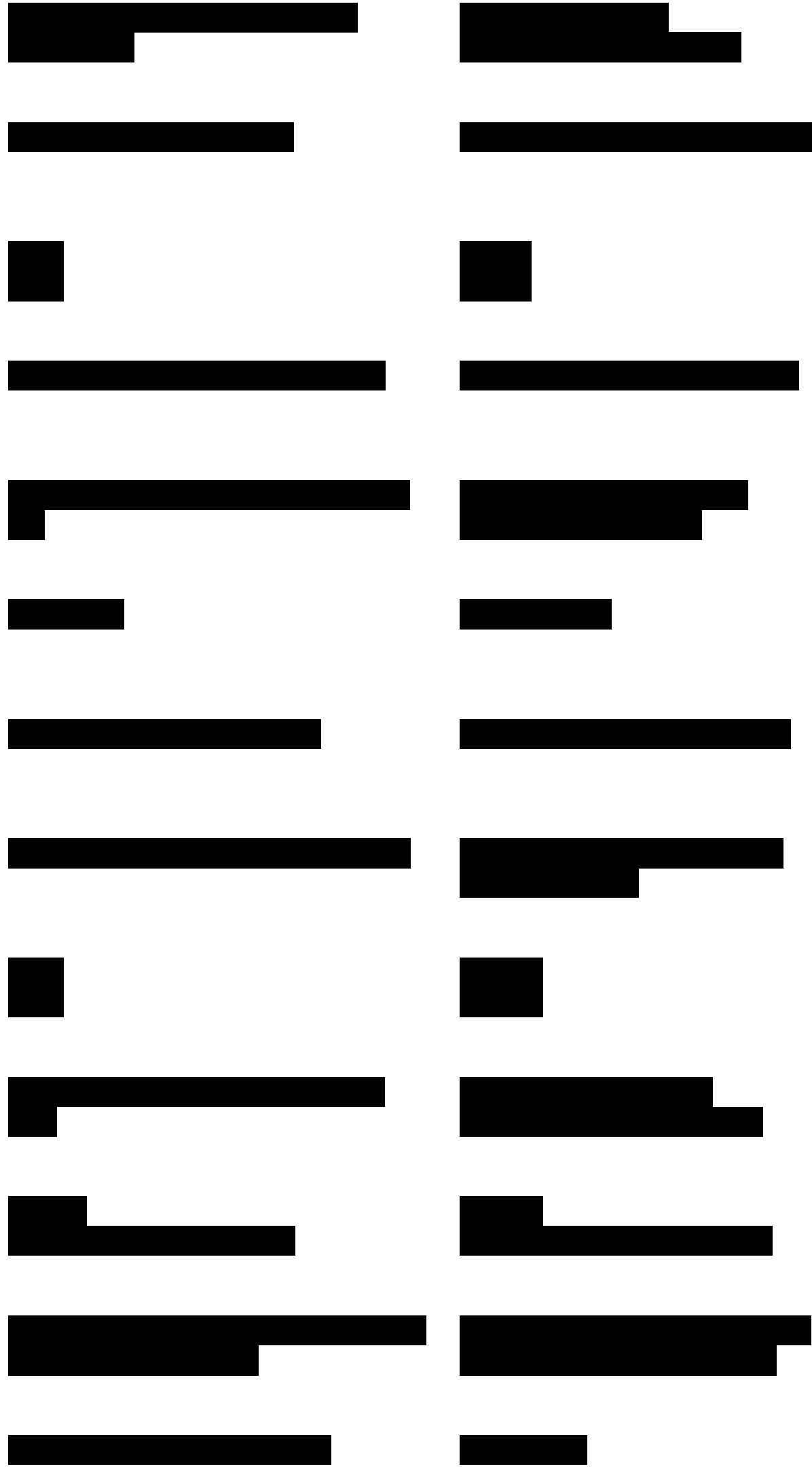
${ }^{294}$ The nickname of the Italian national football team is 'Gli Azzurri' referring to the colour jersey they wear. Here it simply
means that he represented Italy. This is reflected in translation by saying the 'Italian National Team' rather than 'the Blues'. 

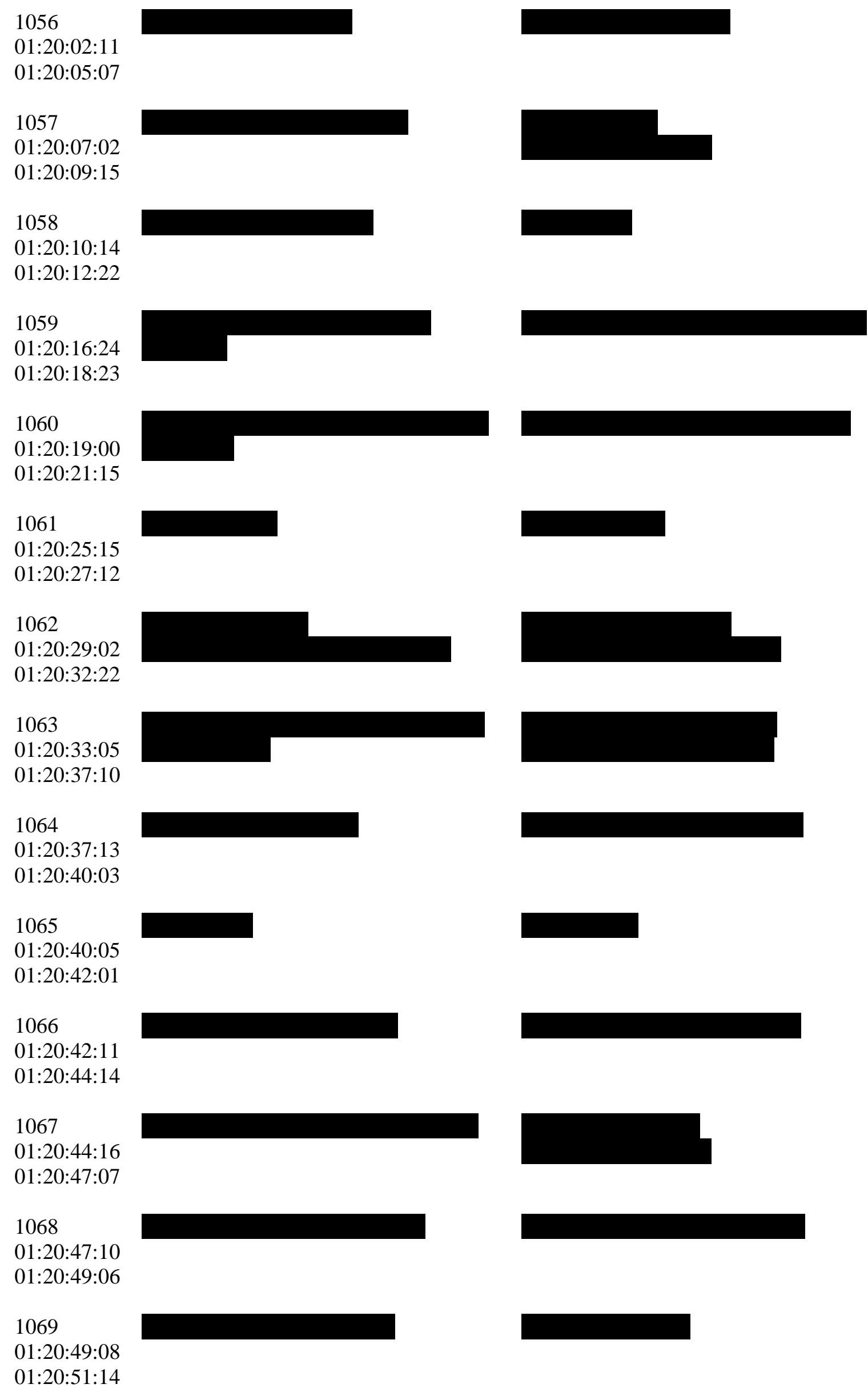


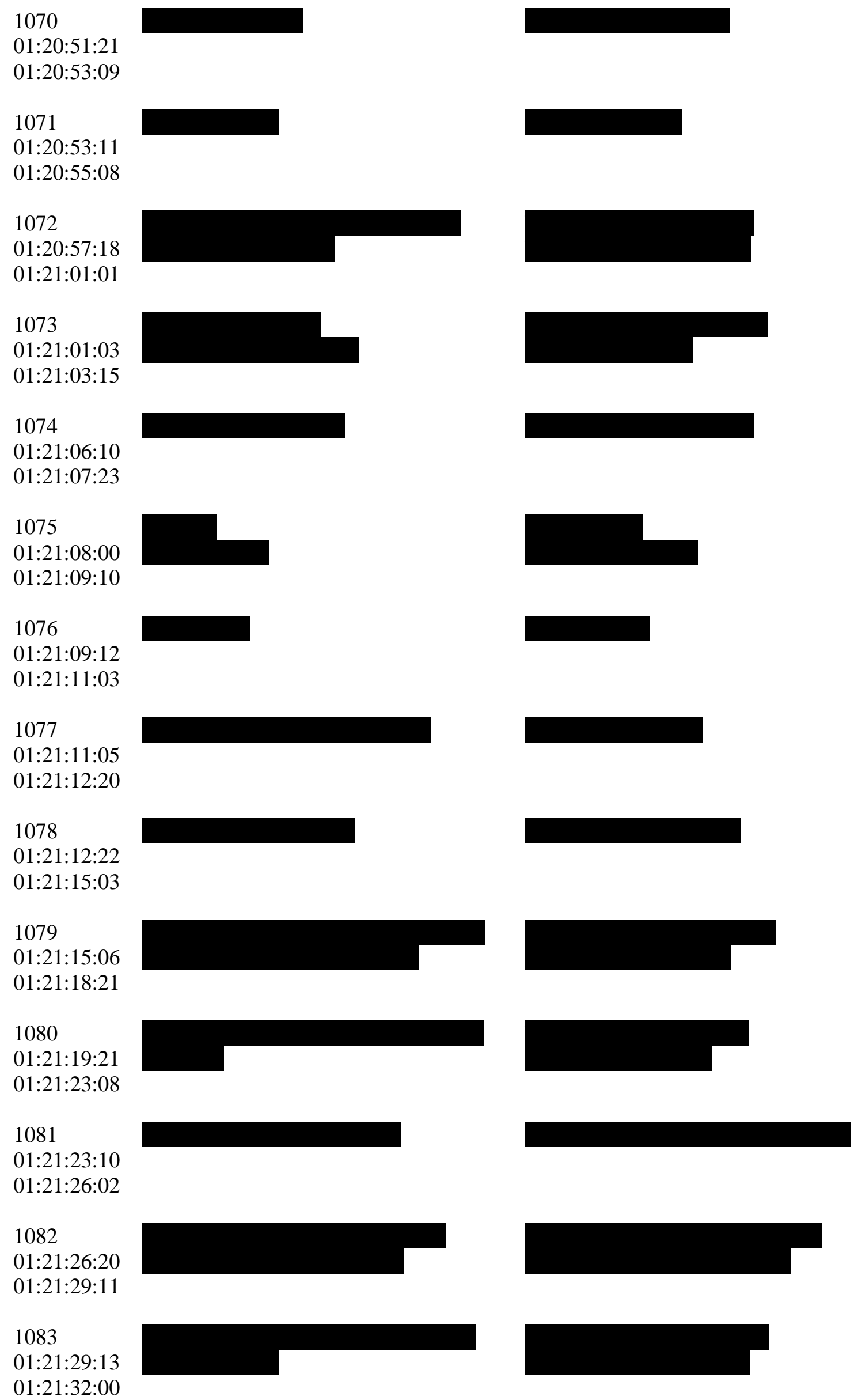


1084

01:21:32:02

01:21:33:22

1085

01:21:33:24

01:21:36:24

1086
01:21:37:01
01:21:42:03

1087

01:21:42:05

01:21:43:22

1088

01:21:44:00

01:21:47:11

1089

01:21:47:13

01:21:50:04

1090

01:21:52:11

01:21:54:21

1091

01:21:54:23

01:21:58:00

1092

01:21:59:02

01:22:01:20

1093

01:22:01:22

01:22:03:03

1094

01:22:03:06

01:22:06:24

1095

01:22:07:13

01:22:09:13

1096

01:22:09:15

01:22:11:17
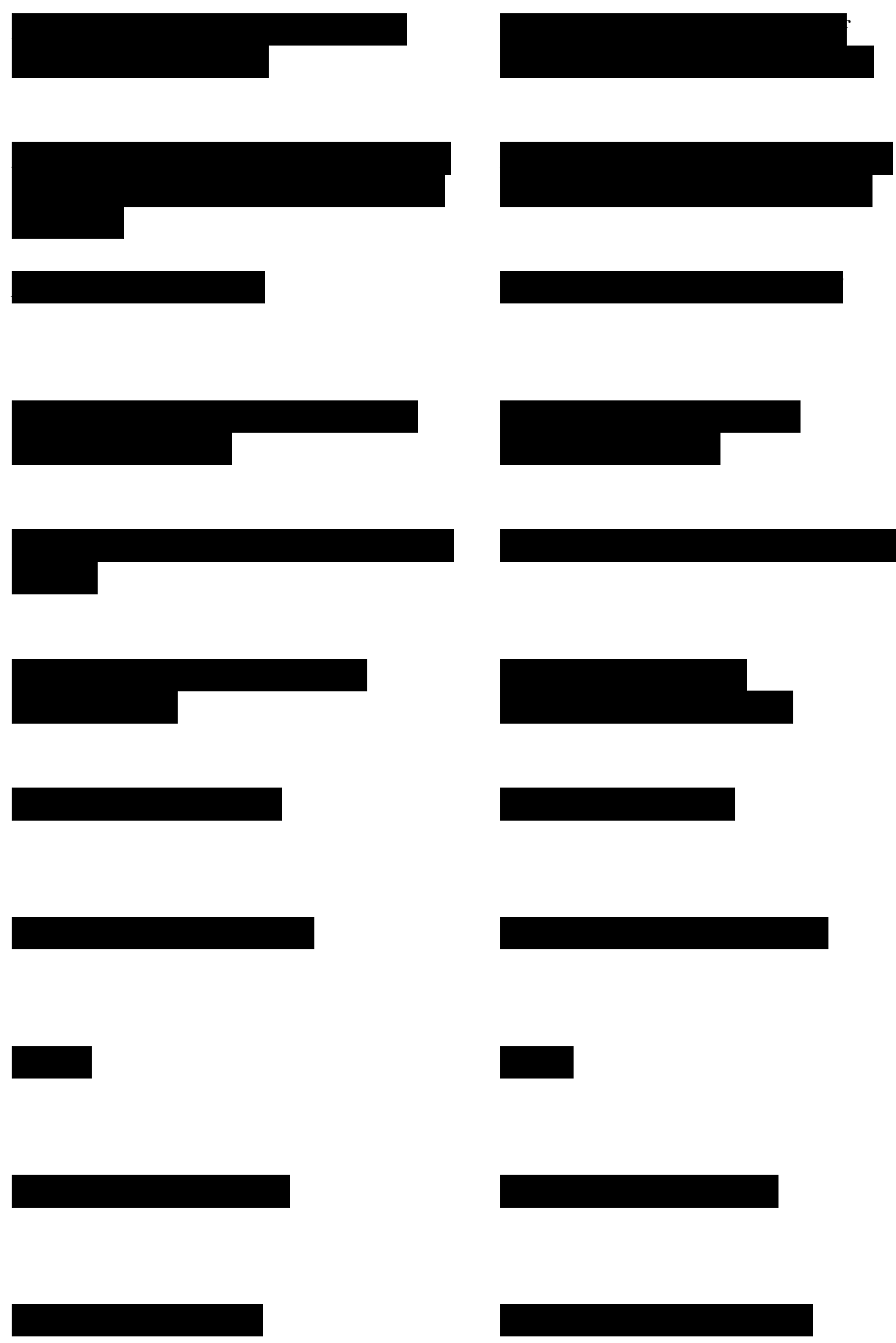
1097

01:22:11:19

01:22:15:12

\section{8}

01:22:15:14

01:22:17:06

1099

01:22:17:09

01:22:21:07

1100

01:22:21:09

01:22:23:15

\section{1}

01:22:23:17

01:22:25:13

1102
$01: 22: 25: 15$
$01: 22: 27: 24$

1103

01:22:28:01

01:22:31:17

1104

01:22:31:19

01:22:33:00

1105
01:22:33:02
$01: 22: 36: 03$

1106

01:22:36:05

01:22:38:13

\section{7}

01:22:38:15

01:22:39:24

\section{8}

01:22:40:01

01:22:41:23

1109

01:22:42:00

01:22:43:18
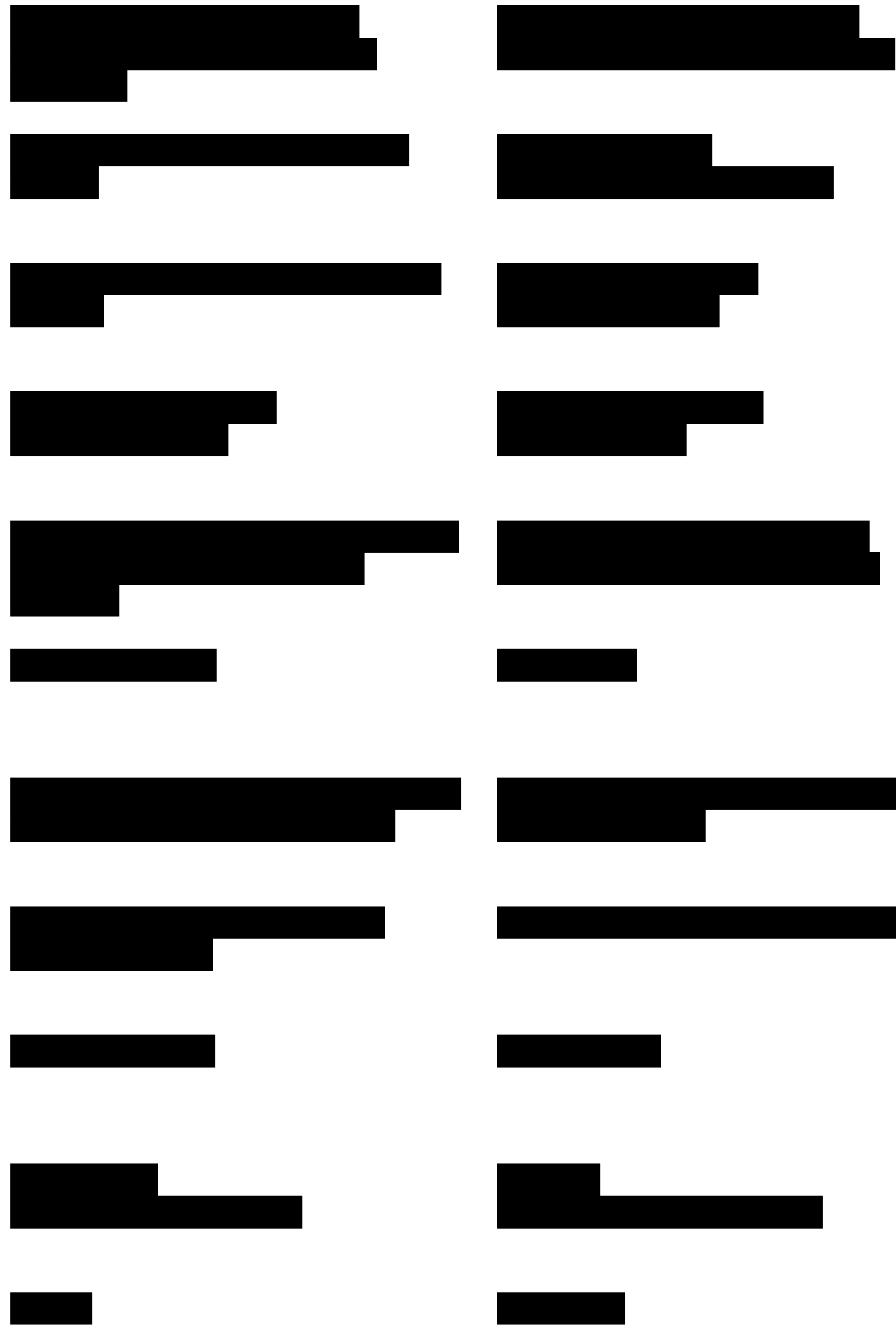

${ }^{295}$ Serbelloni Mazzanti Vien Dal Mare mistakes Fantozzi to be a publisher, rather than someone simply recounting the story to her. This is a commentary on the 'classist' society highlighted in chapter one, where the Countess believes that in order to be there at Courtmayeur he must be of a high standing in society. 
1110

01:22:44:10

01:22:45:20

\section{1}

01:22:45:22

01:22:48:01

\section{2}

01:22:48:03

01:22:50:21

1113

01:22:50:24

01:22:53:24

1114

01:22:54:01

01:22:58:10

\section{5}

01:22:58:12

01:22:59:19

\section{6}

01:22:59:21

01:23:01:02

\section{7 \\ 01:23:01:05 \\ 01:23:03:20}

\section{8}

01:23:03:22

01:23:08:01

1119

01:23:08:03

01:23:10:15

\section{0}

01:23:10:17

01:23:12:17

\section{1}

01:23:12:19

01:23:14:19

\section{2}

01:23:14:21

01:23:16:22
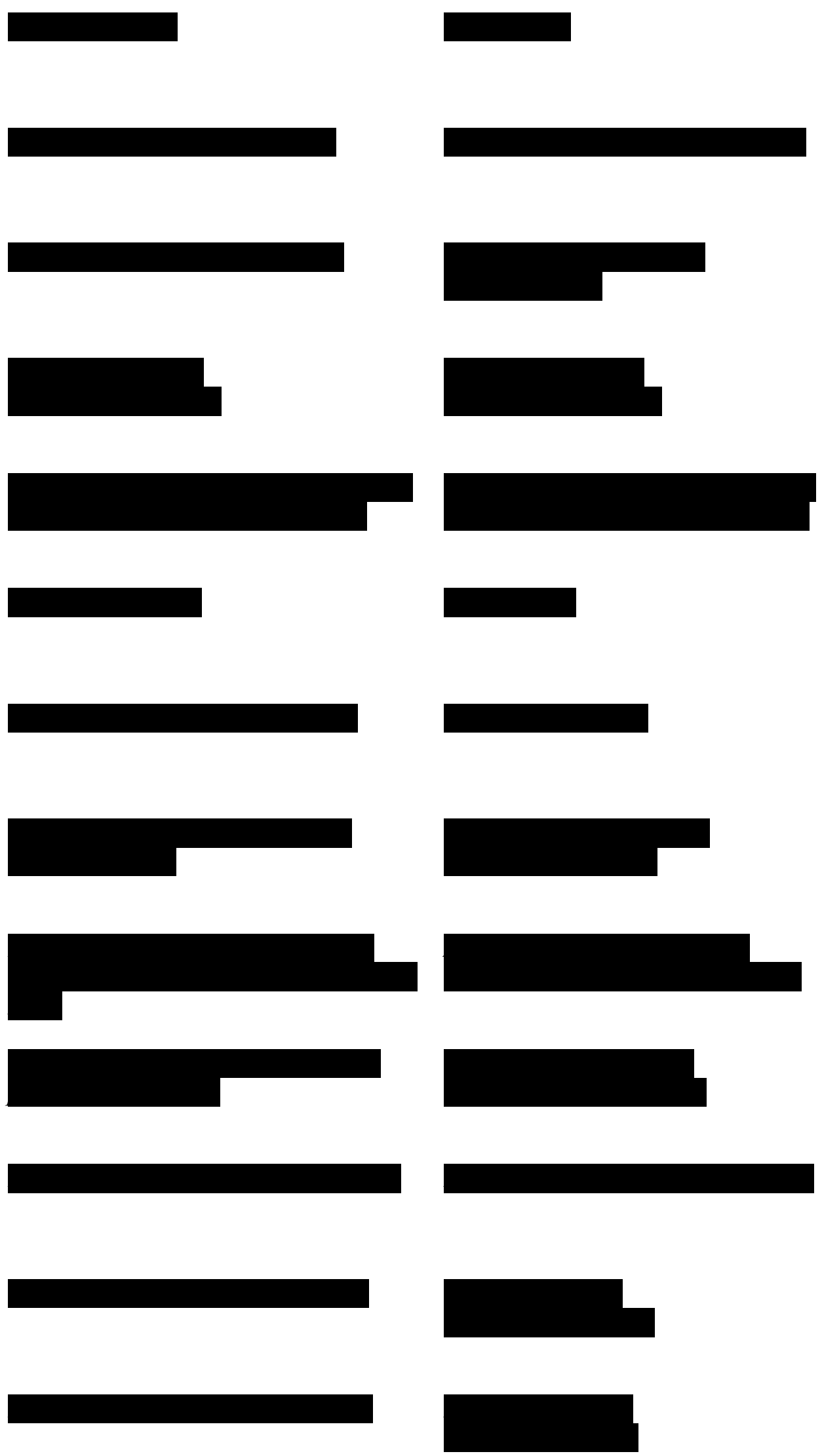

296 These are all references to prestigious members in Italian society, some of whom will not be recognisable to an Englishspeaking audience. I have, however, left the original names as I believe that context of the scene, as well as the few names they are perhaps aware of, allows the audience to understand the associated wealth and prestige. 
1123

01:23:16:24

01:23:18:22

1124

01:23:18:24

01:23:21:20

1125

01:23:21:22

01:23:25:13

1126

01:23:25:15

01:23:27:20

1127

01:23:27:22

01:23:29:18

1128

01:23:29:23

01:23:34:10

1129

01:23:34:19

01:23:38:20

1130

01:23:38:22

01:23:41:08

1131

01:23:43:03

01:23:45:18

1132

01:23:45:20

01:23:47:10

1133

01:23:47:13

01:23:50:19

1134

01:23:50:21

01:23:52:18

1135

01:23:52:20

01:23:55:03

1136

01:23:55:05

01:23:56:15
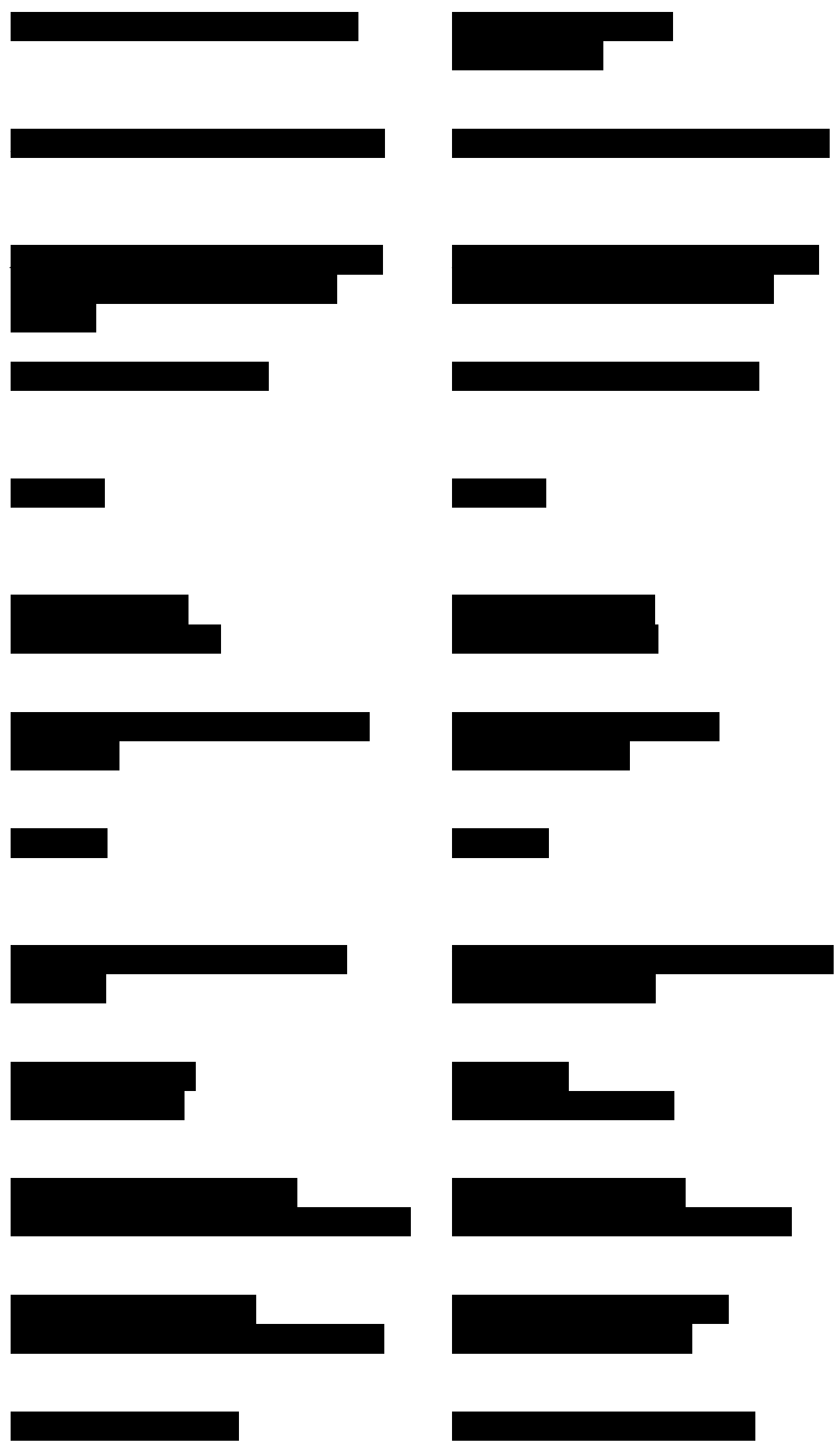


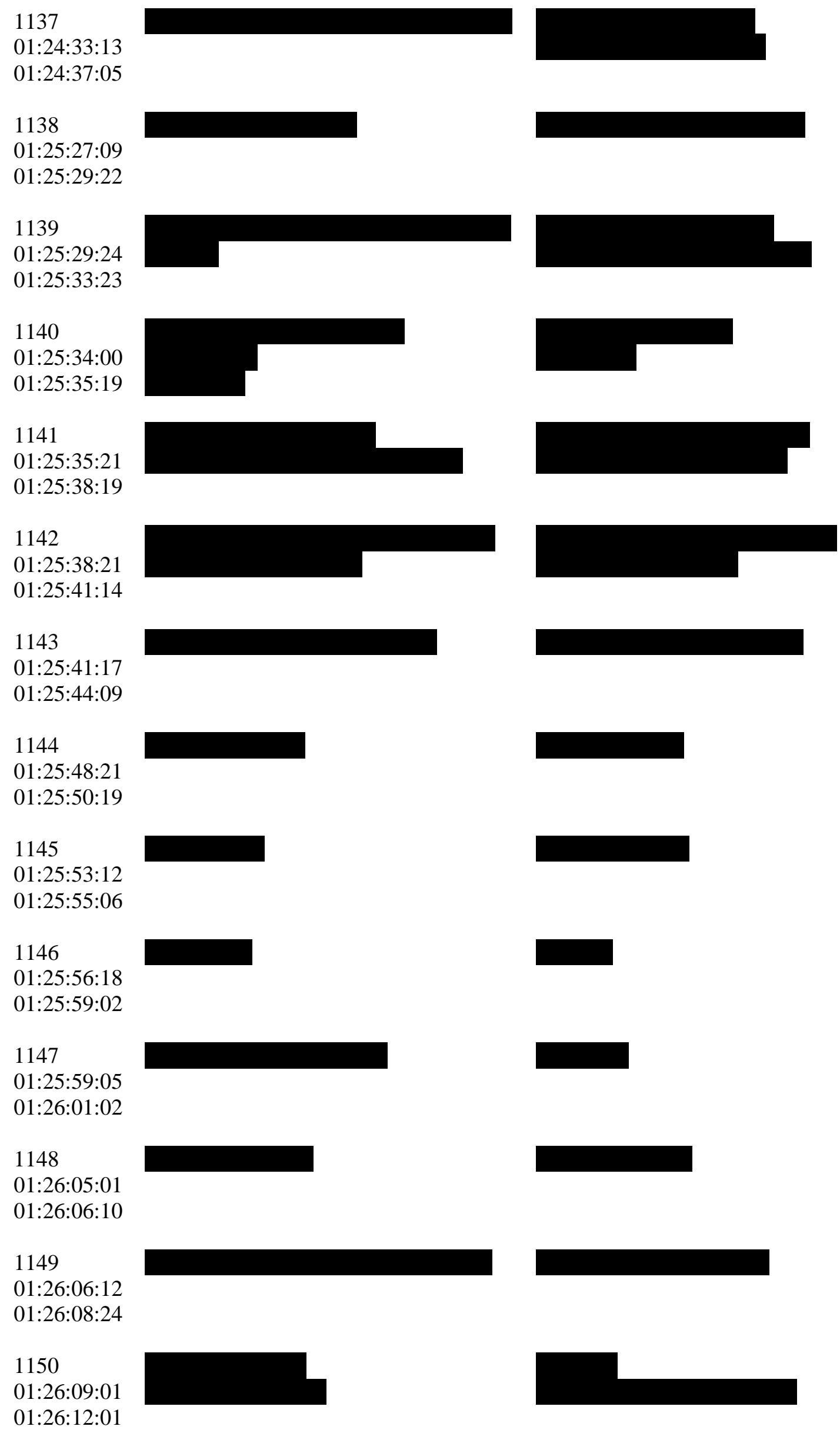


1151

01:26:12:03

01:26:13:13

1152

01:26:13:15

01:26:18:02

1153

01:26:18:04

01:26:20:20

1154

01:26:20:24

01:26:25:13

1155

01:26:25:15

01:26:28:11

1156

01:26:28:14

01:26:32:19

1157

01:26:37:21

01:26:39:10

1158

01:26:40:03

01:26:41:02

1159

01:26:49:18

01:26:51:20

1160

01:26:56:11

01:27:00:12

1161

01:27:01:08

01:27:03:14

1162

01:27:03:16

01:27:07:09

1163

01:27:07:11

01:27:09:13

1164

01:27:09:15

01:27:11:22
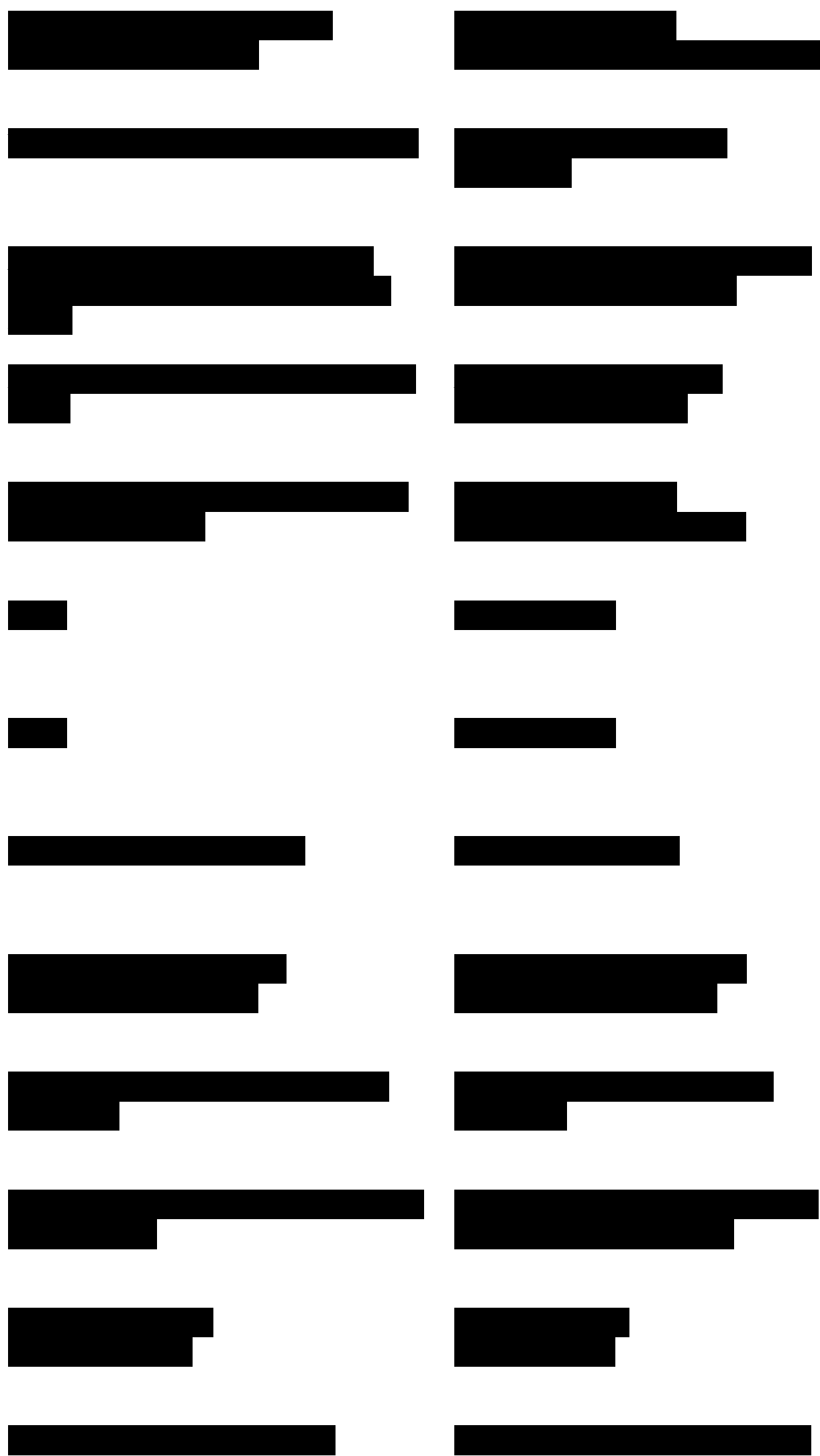


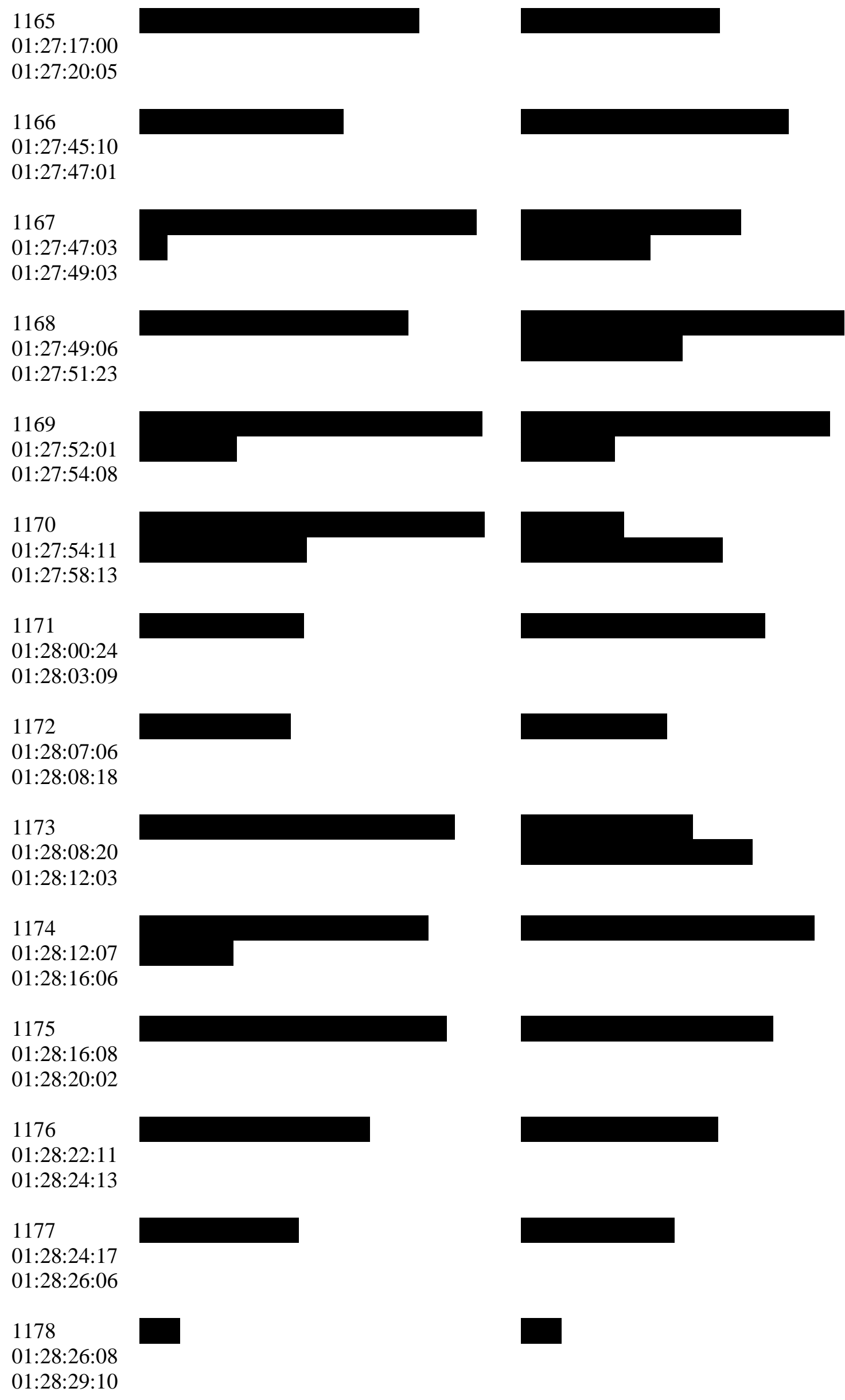




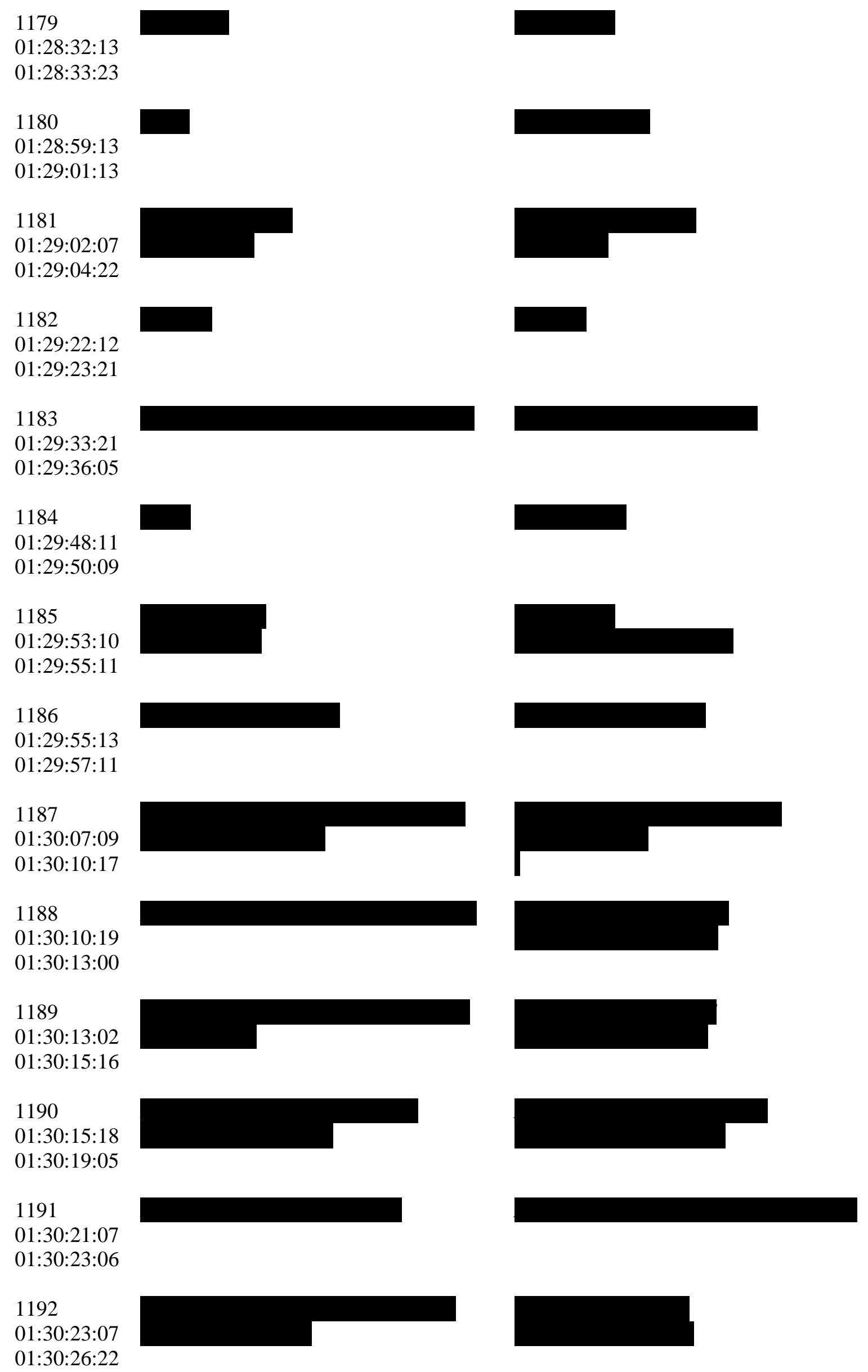




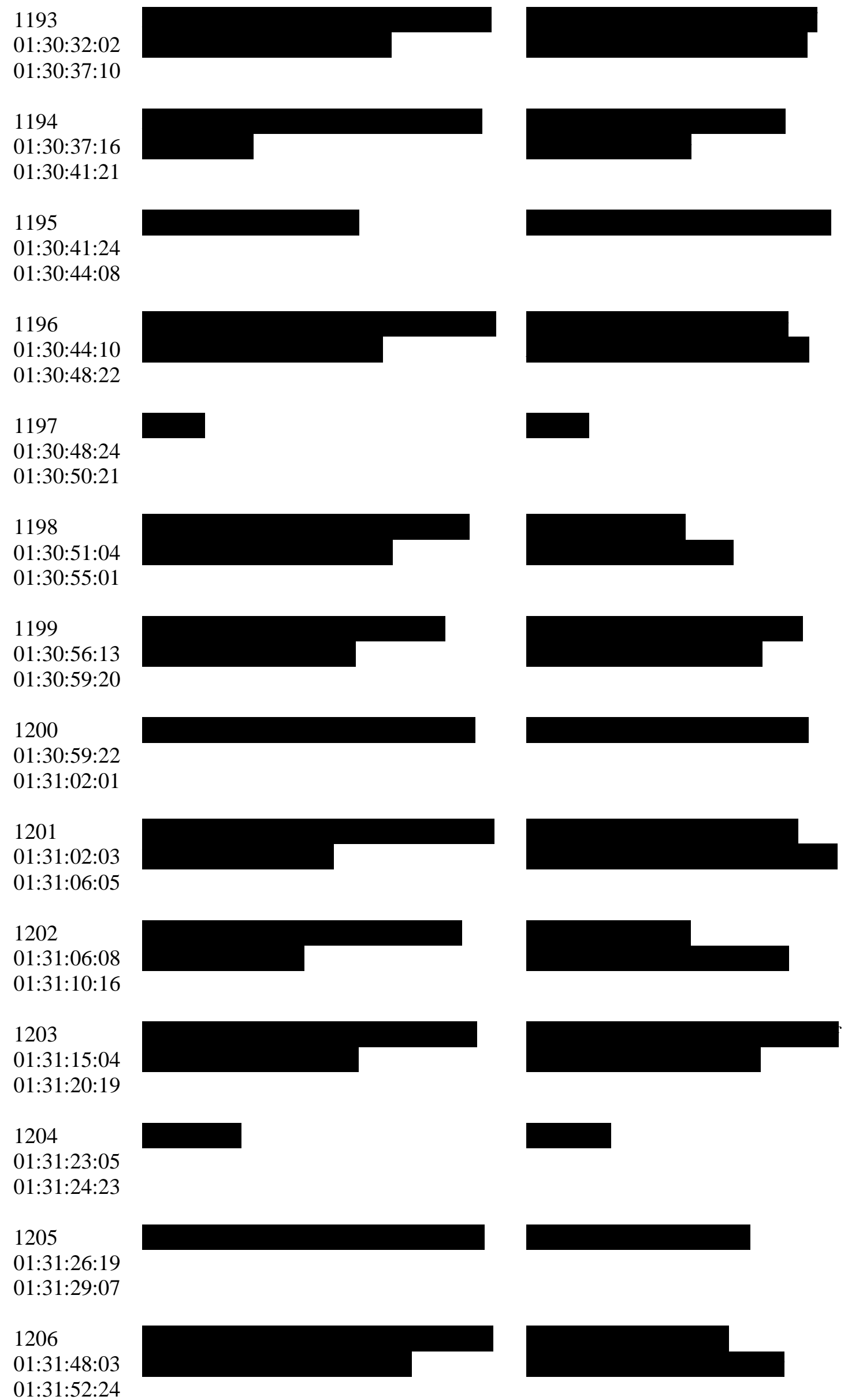


1207

01:31:53:01

01:31:57:12

1208

01:32:00:22

01:32:03:24

1209

01:32:07:05

01:32:09:01

1210

01:32:09:03

01:32:10:19

\section{1}

01:32:11:06

01:32:13:23

\section{2}

01:32:18:13

01:32:22:07

\section{3}

01:32:22:09

01:32:26:01

\section{4 \\ 01:32:26:03 \\ 01:32:29:05}

\section{5}

01:32:29:07

01:32:35:00

\section{6 \\ 01:32:35:02 \\ 01:32:37:14}

\section{7 \\ 01:33:00:00 \\ 01:33:03:05}

\section{8}

01:33:03:07

01:33:08:09

\section{9}

01:33:08:11

01:33:09:22
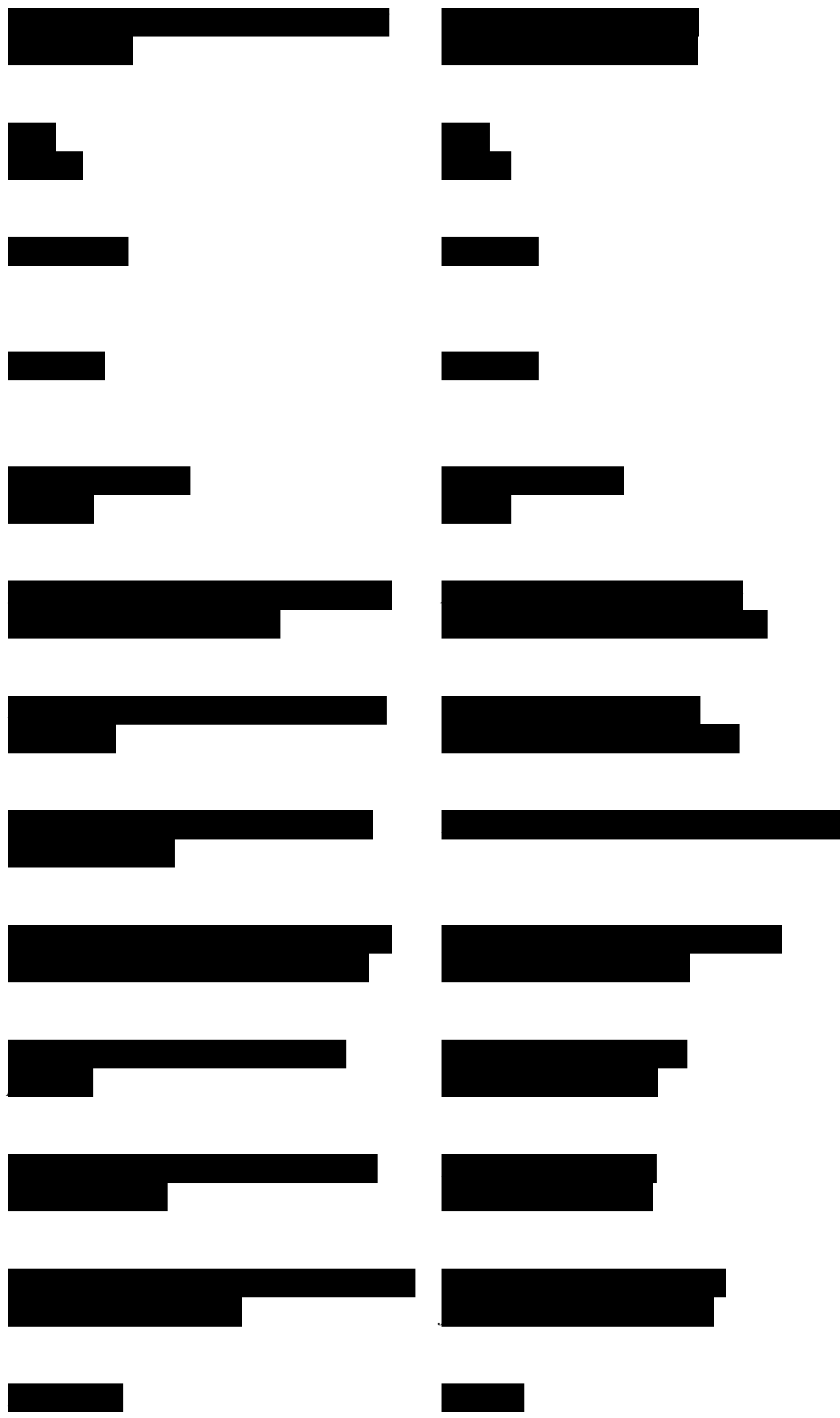

${ }^{297}$ Here we see a rather controversial aspect of Italian culture, politics, be presented as a form of humour. Particularly when this film highlights the importance of wealth, prestige and hierarchy, we see the socialist Folagra go against these themes entirely. 


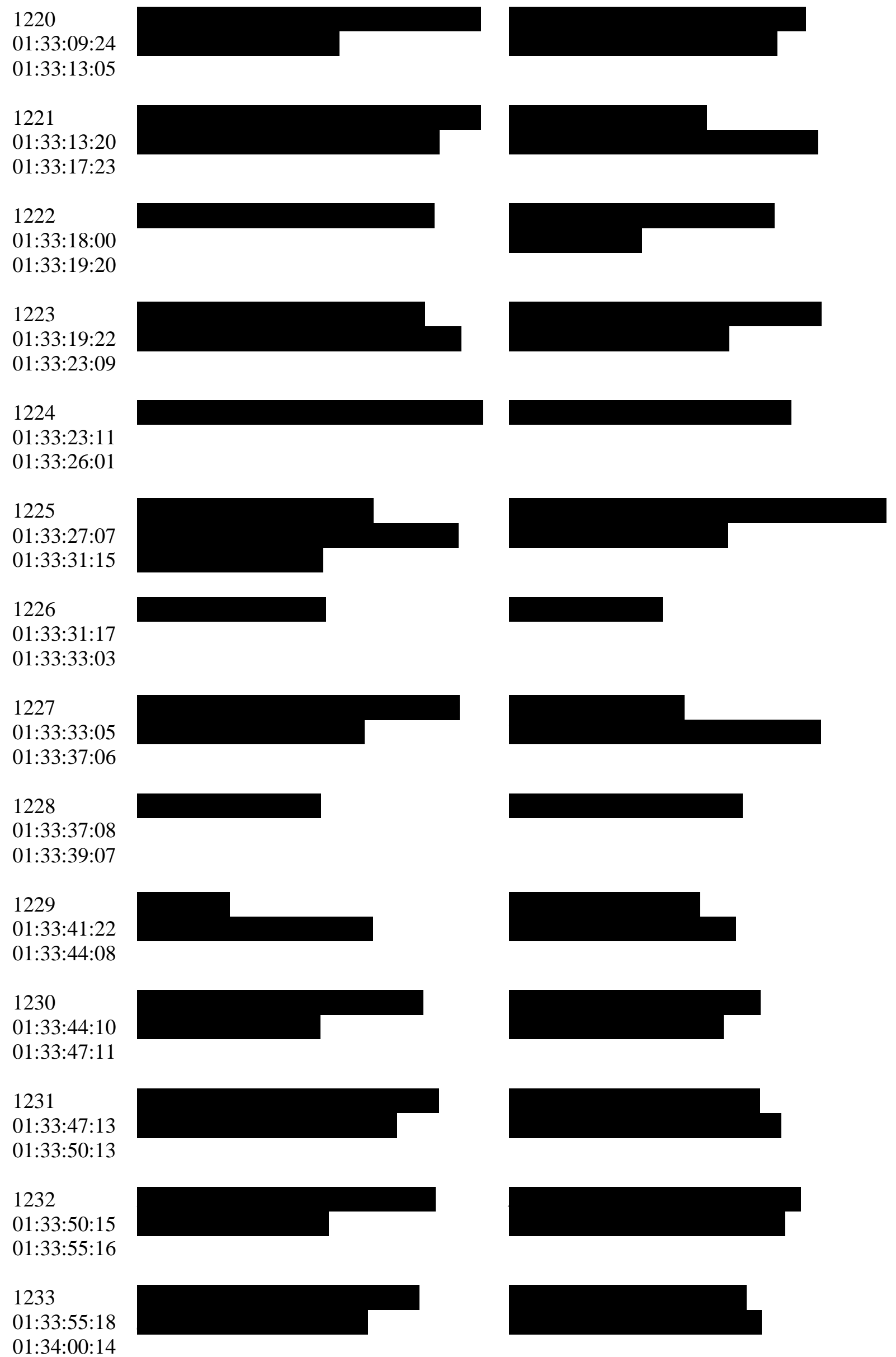


1234

01:34:00:16

01:34:02:21

1235

01:34:02:23

01:34:04:16

1236

01:34:04:18

01:34:08:00

1237

01:34:08:03

01:34:11:11

1238

01:34:11:13

01:34:12:22

1239

01:34:12:24

01:34:14:07

1240

01:34:14:10

01:34:16:08

1241

01:34:17:16

01:34:22:02

1242

01:34:22:04

01:34:24:11

1243

01:34:37:01

01:34:41:01

1244

01:34:41:07

01:34:43:20

1245

01:34:43:23

01:34:46:19

1246

01:34:47:22

01:34:50:18

1247

01:34:57:21

01:34:59:22
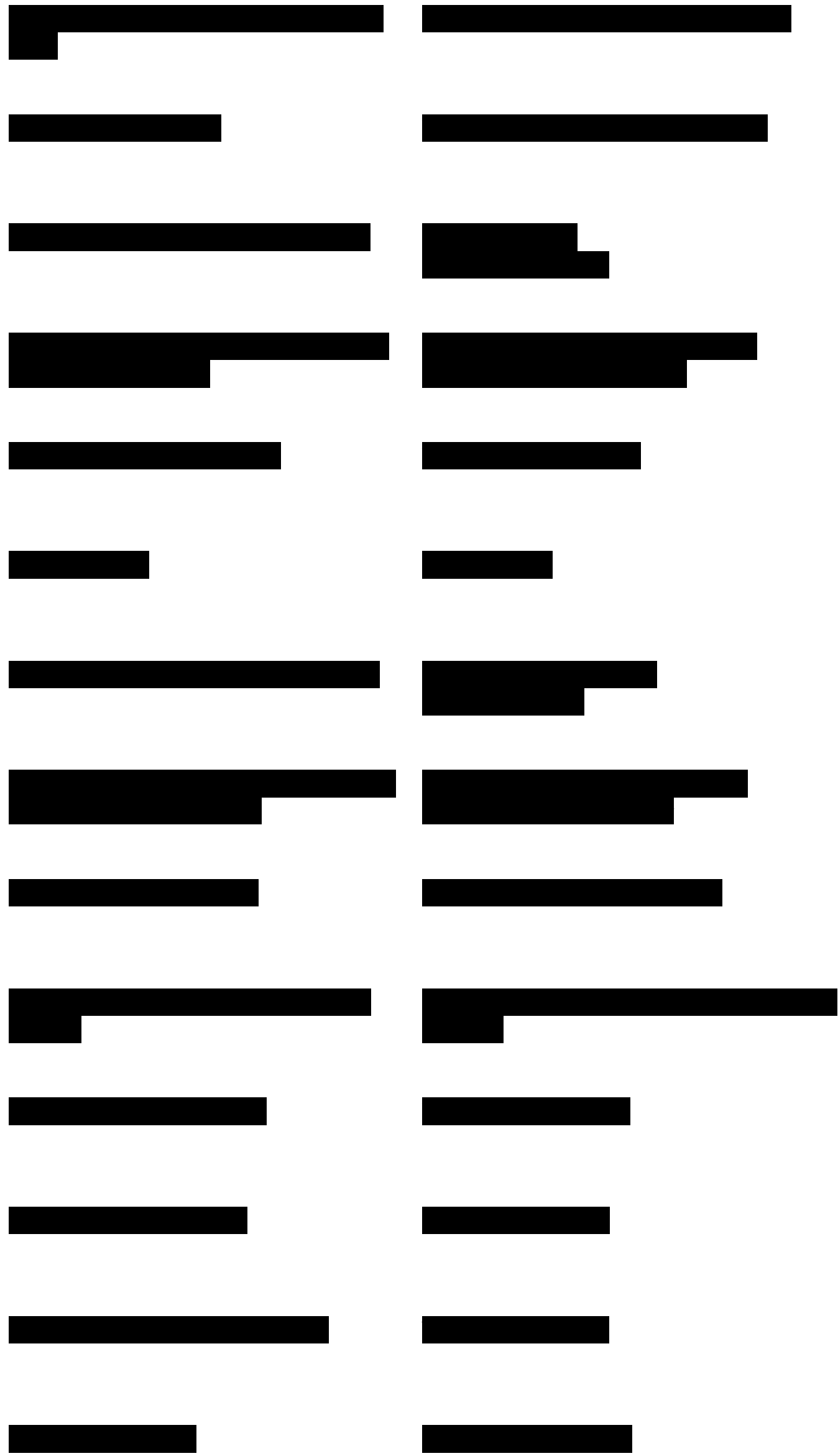


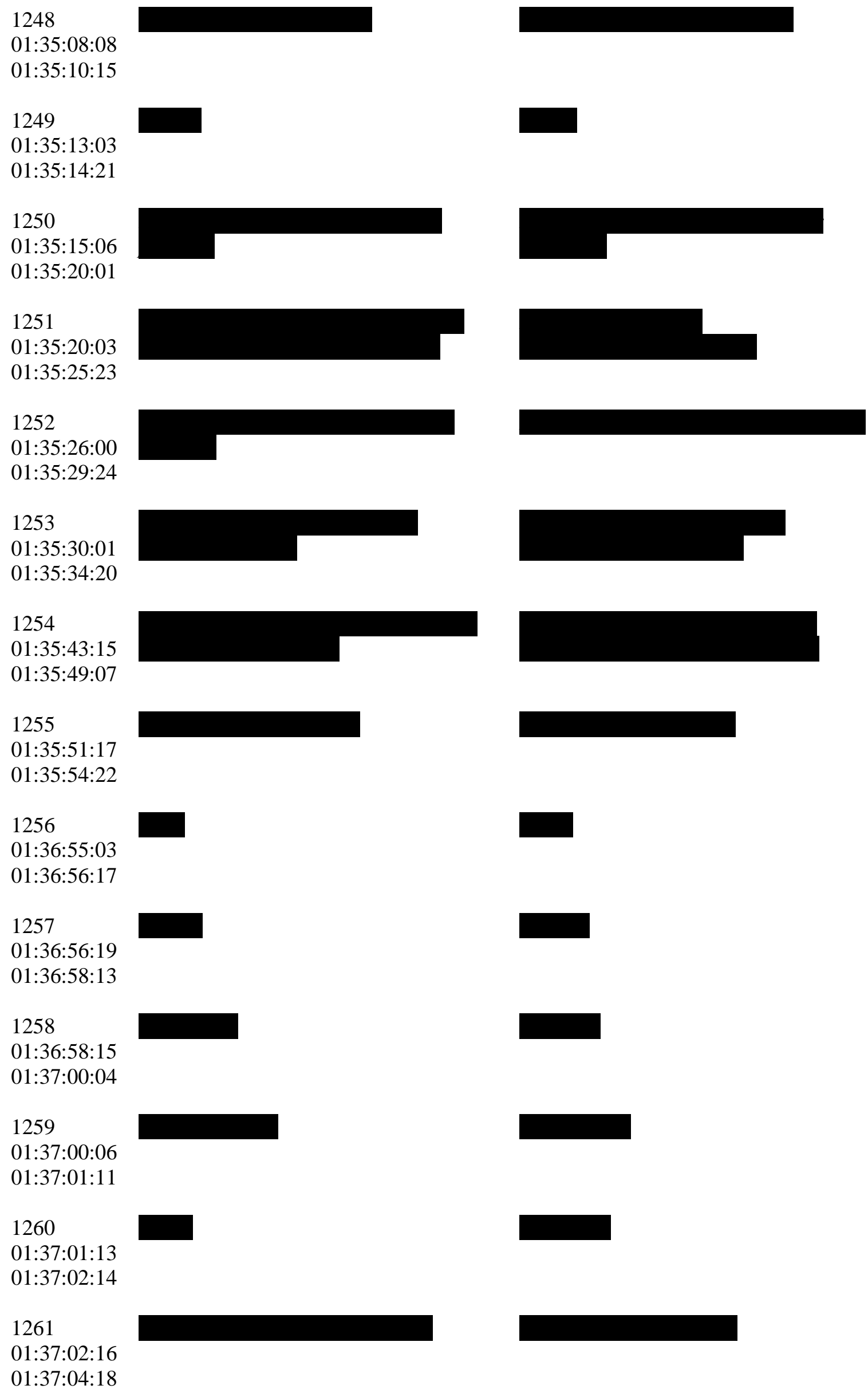


1262

01:37:04:20

01:37:06:24

\section{3}

01:37:07:01

01:37:11:07

1264

01:37:11:17

$01: 37: 16: 20$

1265

01:37:19:01

01:37:21:15

\section{6}

01:37:21:17

01:37:24:04

\section{7}

01:37:24:13

01:37:27:07

1268

01:37:27:09

01:37:29:19

\section{9}

01:37:29:21

01:37:31:10

\section{0}

01:37:31:13

01:37:34:04

1271

01:37:34:18

01:37:36:08

\section{2}

01:37:37:13 01:37:39:24

\section{3}

01:37:40:01

01:37:42:04

1274

01:37:42:07

01:37:44:23
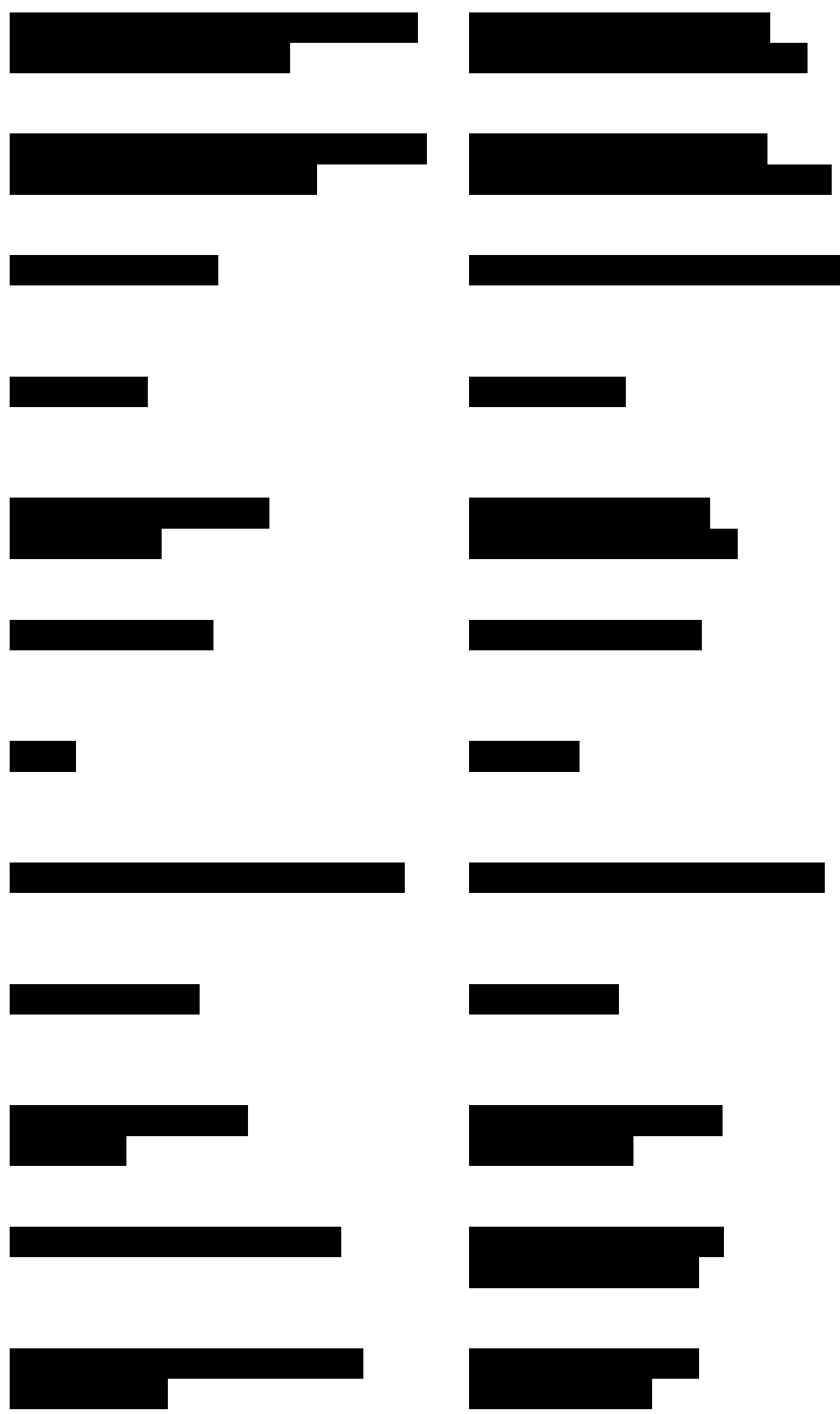
1275

01:37:45:00

01:37:48:00

1276

01:37:48:02

01:37:51:02

1277

01:37:51:04

01:37:53:19

1278
01:37:53:21
01:37:58:22

1279

01:37:59:01

01:38:01:23

1280

01:38:02:00

01:38:06:09

1281

01:38:06:11

01:38:10:03

1282

01:38:10:05

01:38:13:01

1283

01:38:13:04

01:38:14:18

1284

01:38:14:20

01:38:17:06

\section{5}

01:38:17:08

01:38:21:02

1286

01:38:21:04

01:38:24:00

1287

01:38:24:02

01:38:27:12
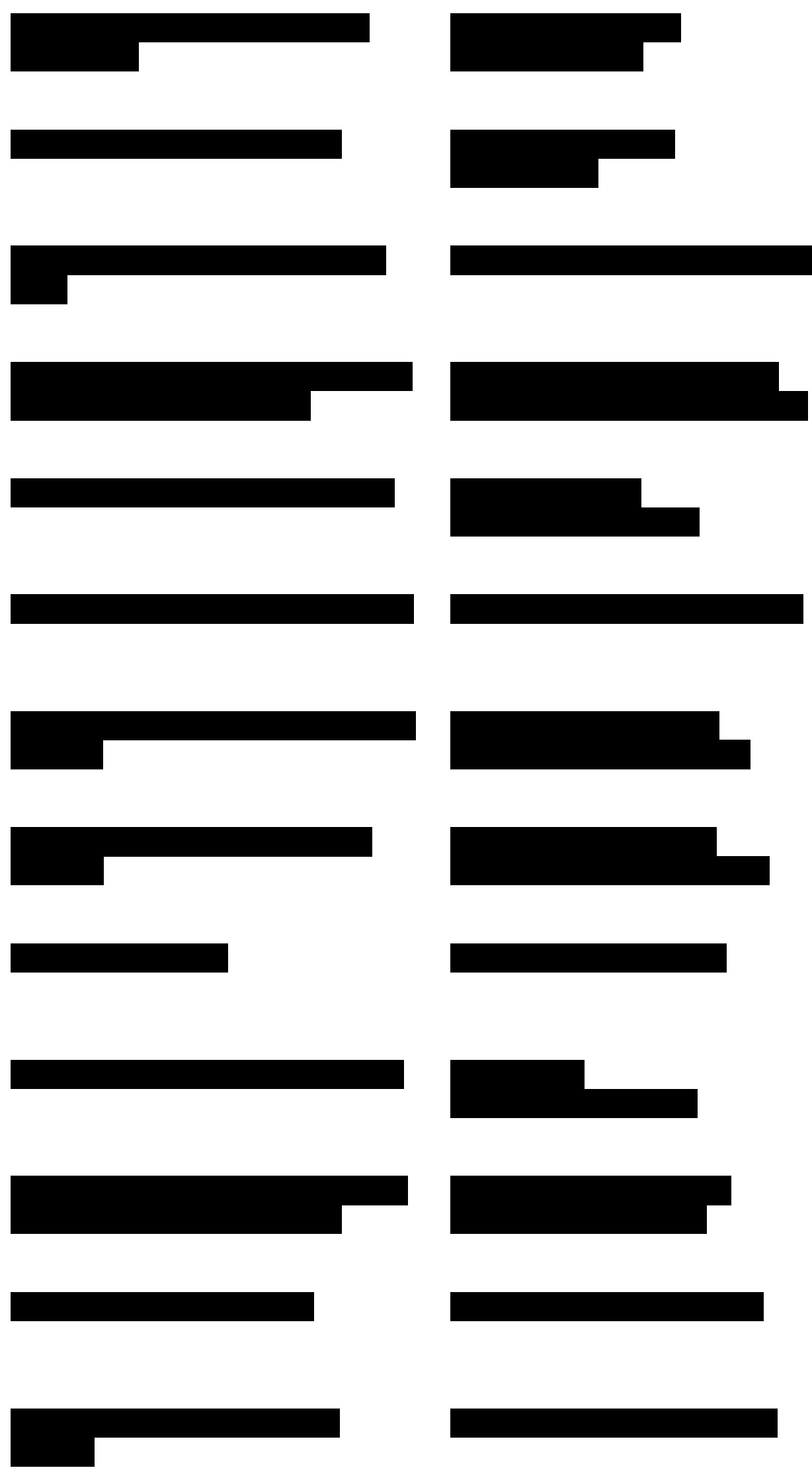

298 The register of the Megadirettore needs to remain very high as he is represented as a God-like figure. For this reason, all words are spelt out in full (no contractions). 


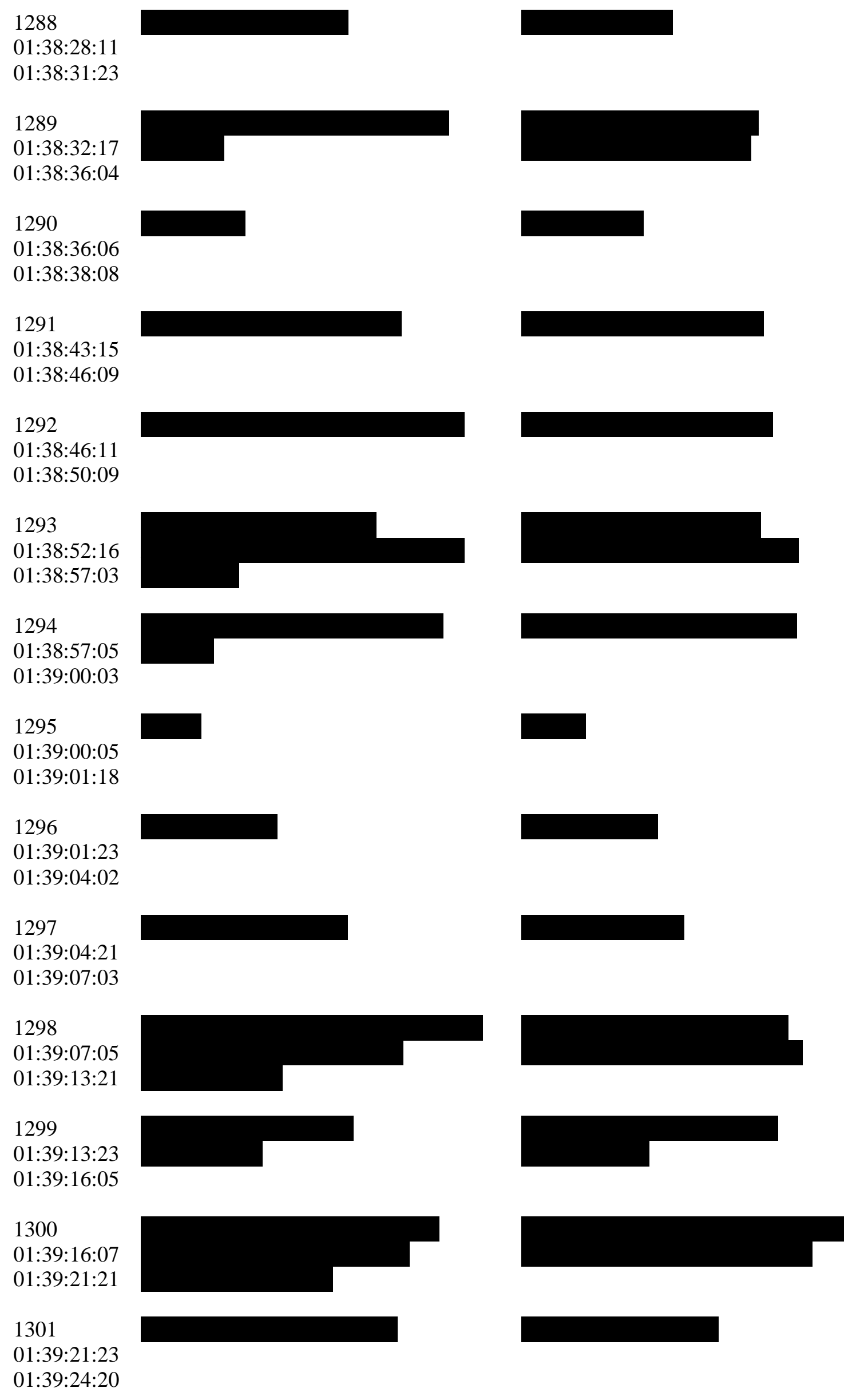


1302

01:39:24:22

01:39:27:06

1303

01:39:27:08

01:39:30:19

1304

01:39:30:21

01:39:32:17

1305

01:39:32:20

01:39:34:04

1306

01:39:35:16

01:39:37:10

1307

01:39:38:05

01:39:39:06

1308

01:39:39:08

01:39:43:14

\section{9}

01:39:47:20

01:39:49:10

1310

01:39:54:00

01:39:55:23

1311

01:39:56:00

01:39:59:18

\section{2}

01:40:01:03

01:40:02:08

\section{3}

01:40:02:10

01:40:07:02

1314

01:40:10:19

01:40:12:16

\section{5}

01:40:12:18

01:40:15:04
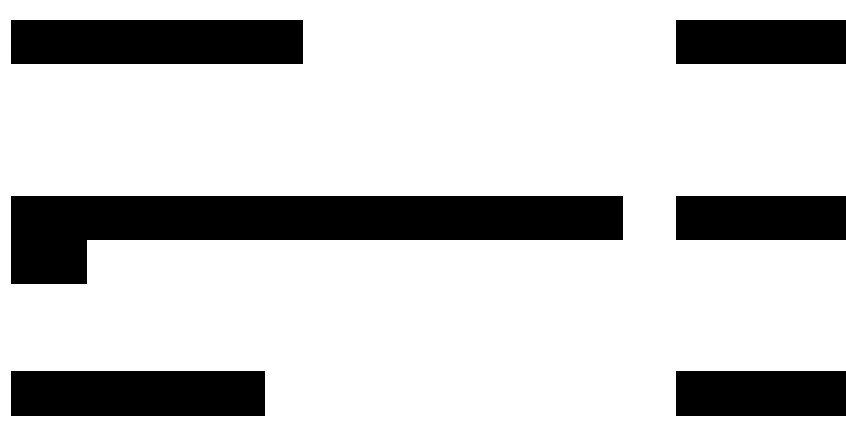

口
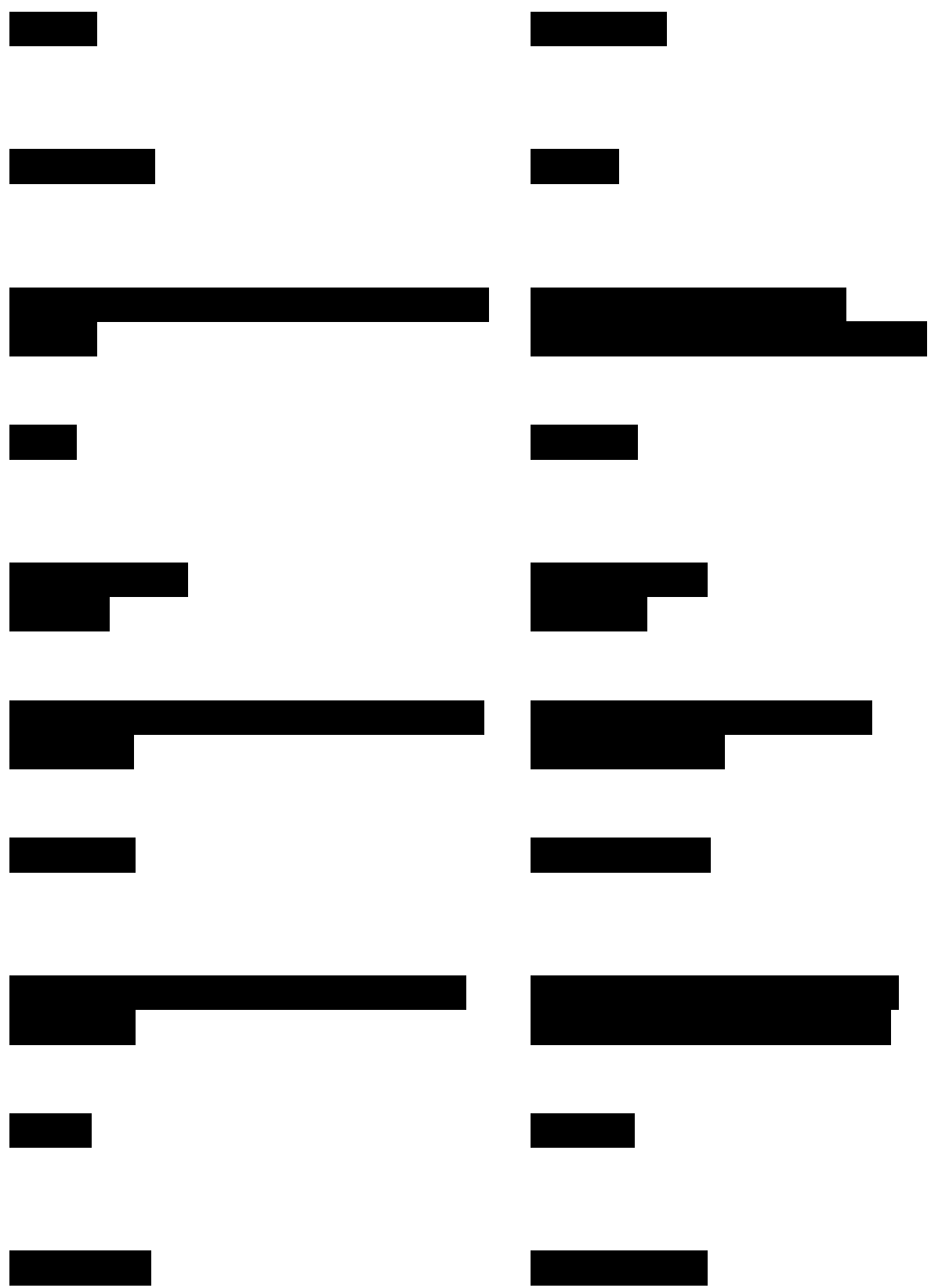


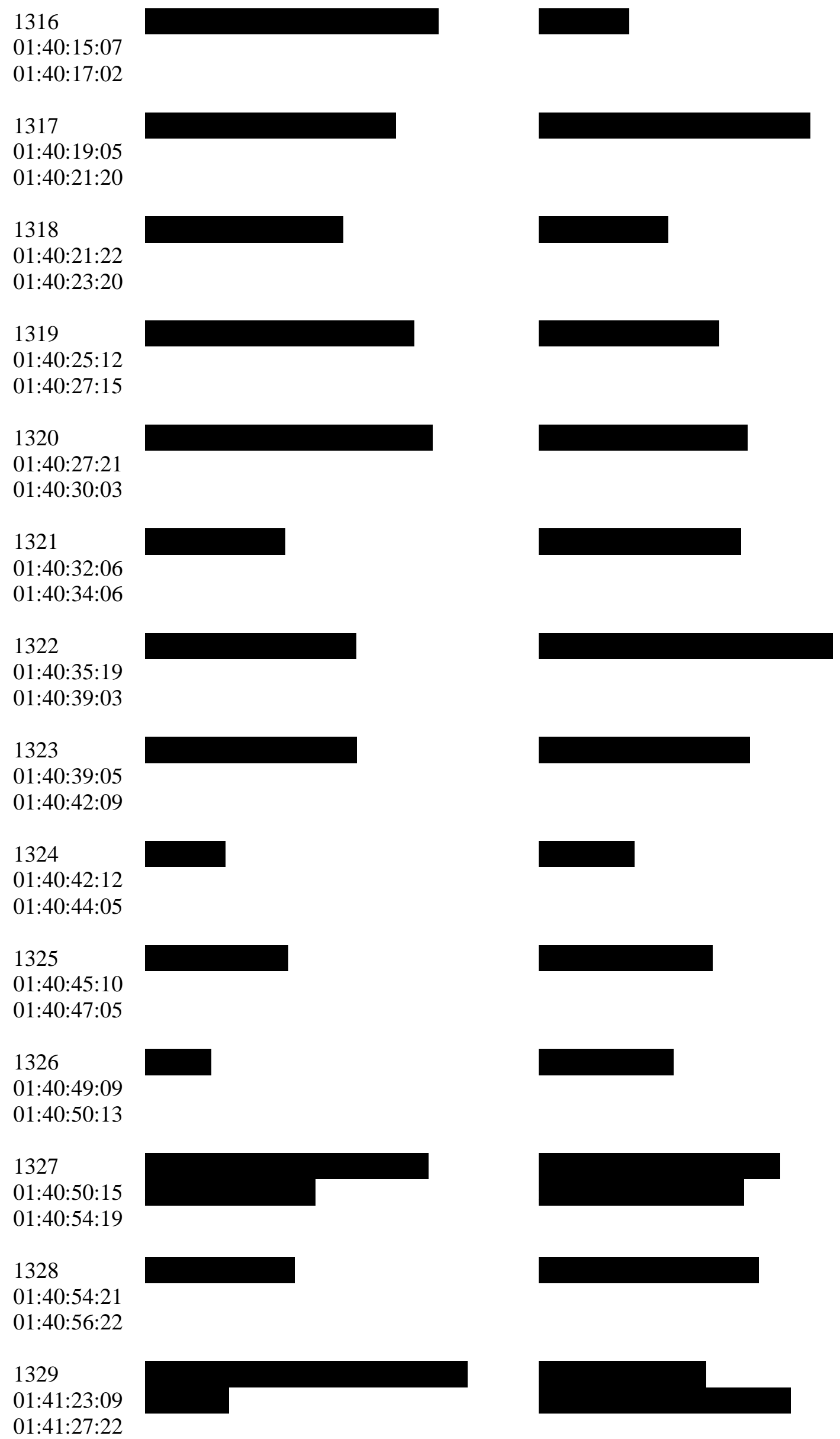


1330

01:41:27:22

01:41:30:04

1331

01:41:31:16

01:41:34:03

1332

01:41:34:06

01:41:38:12

\section{3}

01:41:39:22

01:41:41:24

1334

01:41:42:01

01:41:46:22

\section{5}

01:41:47:00

01:41:50:23

\section{6}

01:41:52:00

01:41:56:12

\section{7 \\ 01:41:56:16} 01:41:58:17

1338
01:41:59:04
01:42:04:23

1339

01:42:05:11

01:42:08:04

1340

01:42:24:24

01:42:29:10

\section{1}

01:42:29:23

01:42:33:10

1342

01:42:35:02

01:42:39:23

\section{3}

01:42:40:00

01:42:44:01
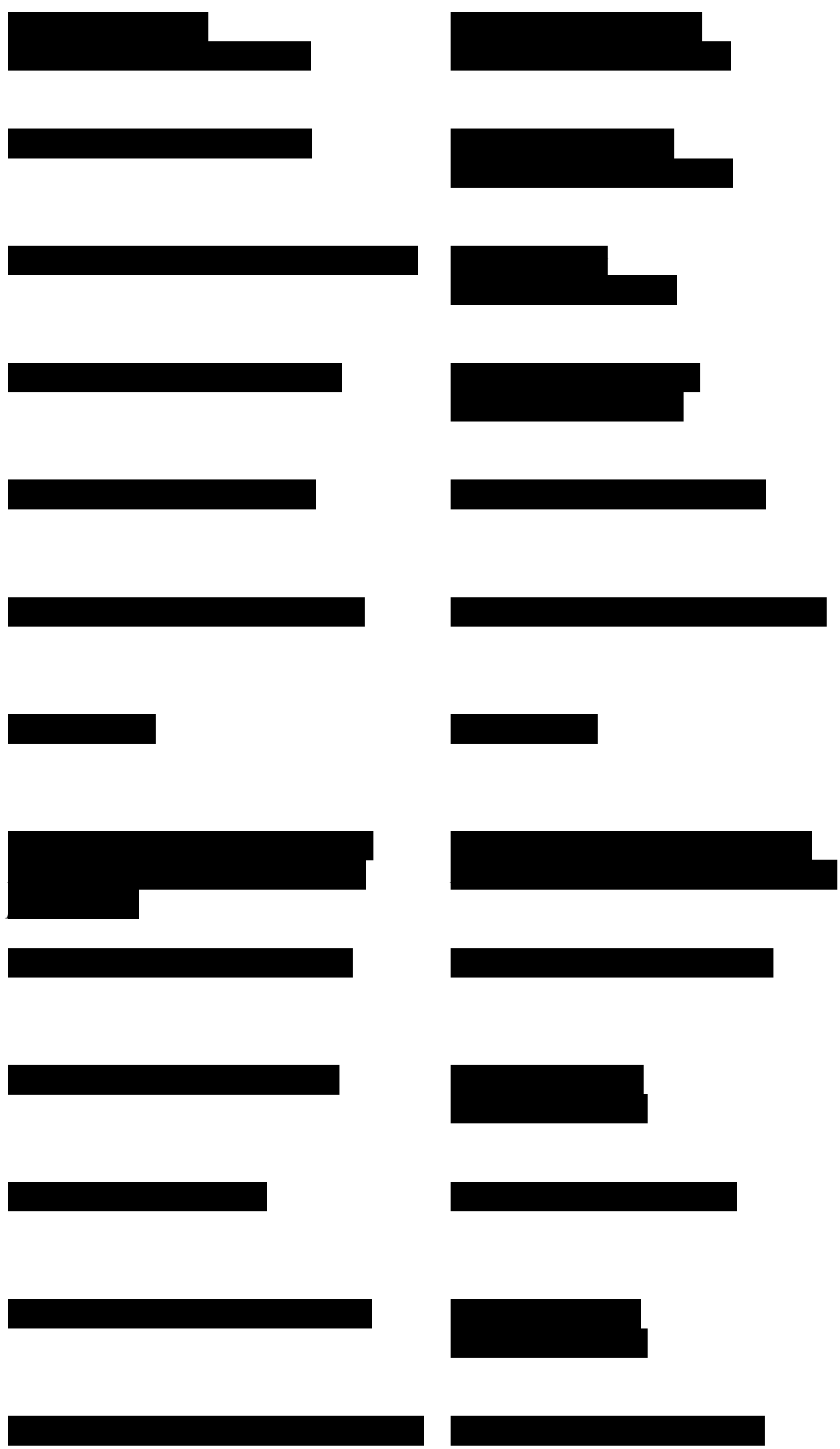


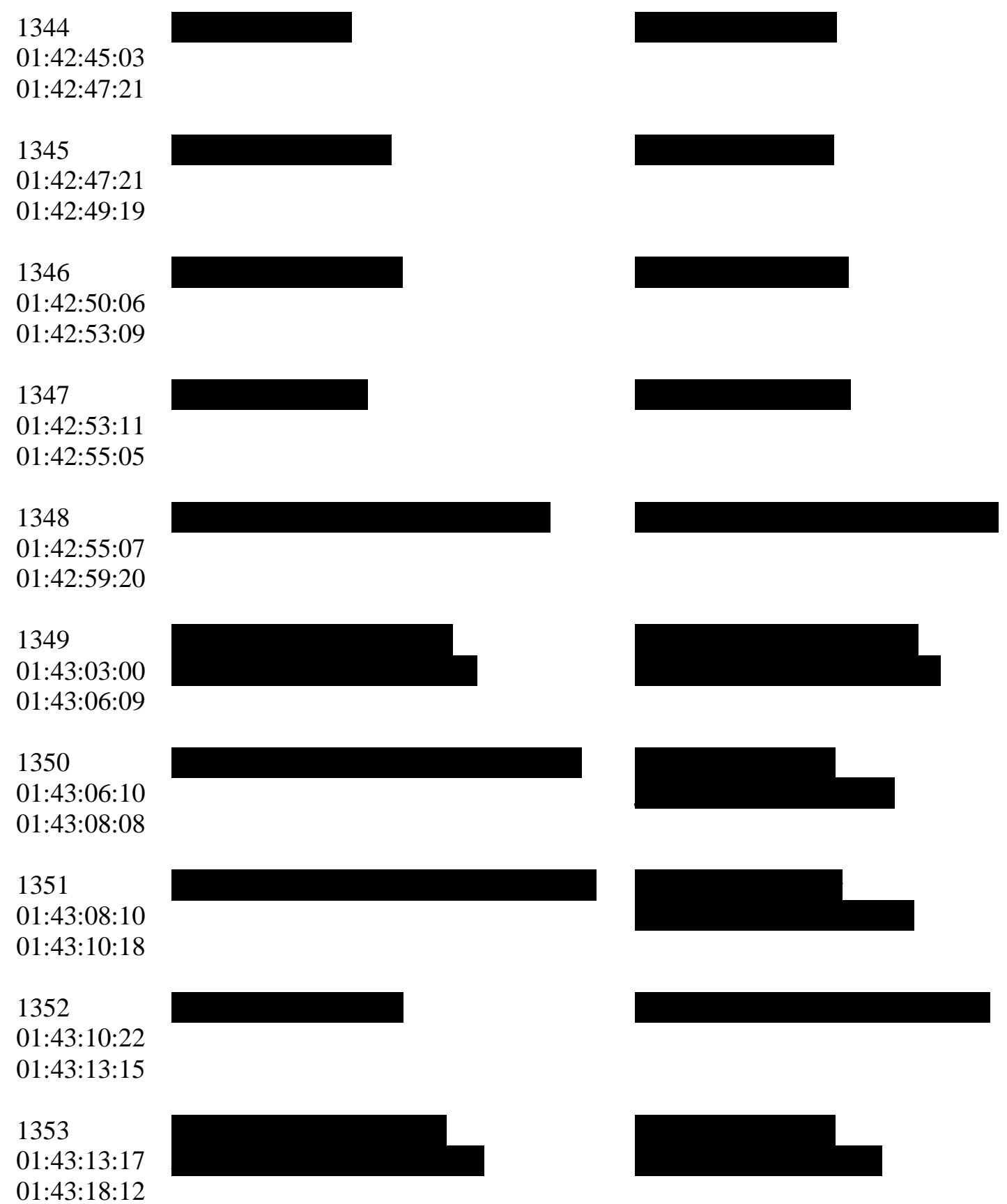




\subsection{Specific difficulties in translation}

Having presented the subtitles of the film in full, I will now analyse some of the specific difficulties encountered when translating Fantozzi from Italian into English. I will endeavour to justify some of my more creative choices in order to convey the original ST humour and cultural content, where a direct translation would not have been as effective. Such difficulties are mainly associated with the difference in grammar between the two languages. A lot of the humour associated with Fantozzi is of a linguistic variety, which pertains specifically to social differences, and is therefore difficult to convey to another culture without knowledge of the social differences of the time. Other aspects of humour are purely culturally specific and have no significance to an English speaking audience unfamiliar with Italian culture and humour. The specific difficulties I will be discussing in depth are those associated with Italian grammar, the translation of rhyme and poetry, representing multilingualism and regional varieties in subtitles as well as translating culturally specific content, including intertextual references.

\subsubsection{The use of the imperativo formale}

The scene in which Fantozzi and Filini play tennis is filled with dialogue and humour which is very difficult to translate into an English context. The two main difficulties in this scene arise from the use of the Italian imperativo formale, or 'formal imperative', and from the use of cultural references that are not applicable or immediately understandable to the English-speaking audience. This specific type of humour is repeated not only throughout the first film, but also across the entire series. It is therefore important to find a translation strategy that can be used in a variety of contexts.

As previously mentioned on multiple occasions, in the Fantozzi series, both Fantozzi and Filini use the imperativo formale in an attempt to sound more educated and intellectual, part of their failed aspirations towards social mobility. This is part of the wider commentary on how complex, and fundamentally useless, formality is to effectively change social status, resulting in the opposite effect. The humour is created by the characters often getting the conjugation of the verbs wrong - in this case the use (or misuse) of formal imperatives, the conjugation of which is very similar (often identical) to the present subjunctive, a form that indicates good education. There are a multitude of examples of this form of linguistic humour in the tennis scene in particular (and throughout the Fantozzi series as a whole). Some of these examples are shown below, where the first line of each example shows what the actual dialogue is, and beneath that is what it should have been if the formal imperative had been used correctly:

Di qua! Ragioniere, sono qua! Da questa parte! Venghi, venghi.

Di qua! Ragioniere, sono qua! Da questa parte! Venga, venga.

Buongiorno. Buongiorno. Vadi, vadi. 
Buongiorno. Buongiorno. Vada, vada.

Lei prefersce di giocare di qua o preferisc...? Oh, facci lei.

Lei preferisce di giocare di qua o preferisc...? Oh, faccia lei

Allora, ragioniere, che fa? Batti?

Allora, ragioniere, che fa? Batte?

The difficulty comes in trying to retain this linguistic humour, where, in a language like English, the subjunctive is very rarely present, nor is there a distinction between a formal and an informal 'you' as there is in Italian. In this particular scene, I have used two different strategies in translation to maintain some of the language play that is occurring.

The first technique was to substitute all of the incorrectly used subjunctive phrases with the incorrect use of the imperative of the verb 'to be' in English, followed by the present participle of the verb. This has both the effects of an incorrect use of imperatives in English, while also giving the sense that the protagonists are using language that is beyond them. Below is an example of the subtitles where this technique was used:

\section{Subtitle}

No. and

Timecode

0778

01:00:06:10

01:00:08:06

0779

01:00:08:09

Venghi, venghi.

Da questa parte!
English Subtitles

Original Italian

This way!

Be coming this way.

01:00:10:18

The other technique that I used to retain the multiple layers of the original wordplay was to insert a pun where one was not originally present, a technique suggested by Delabastita. ${ }^{299}$ Instead of translating the use of the imperative directly, I decided to use the play on words between 'sir' and 'serve'. This changes the connotations of the original, and the formality of the subjunctive is somewhat lost because Fantozzi is confused as to why he is being referred to as 'sir'. However, I figured that retaining the subtle linguistic humour is more important than keeping the formality present in the TT.

\footnotetext{
${ }^{299}$ Delabastita, "Wordplay and Translation."
} 


$\begin{array}{lll}\begin{array}{l}\text { Subtitle } \\ \text { No. and } \\ \text { Timecode }\end{array} & \text { Original Italian } & \text { English Sulbtitles } \\ \text { 0798 } & \text { Allora, ragioniere, } & \\ \text { 01:01:30:20 } & \text { che fa? } & \text { Come on Fantozzi... } \\ \text { 01:01:32:24 } & & \text { What are you doing...? } \\ \text { 0799 } & \text { Batti? } & \text { Serve! } \\ \text { 01:01:33:01 } & & \\ \text { 01:01:34:04 } & & \text { Did you just call me "sir"? } \\ \text { 0800 } & \text { Ma, mi dà del tu? } & \\ 01: 01: 34: 05 & & \\ 01: 01: 35: 21 & & \text { No, I said "serve!" } \\ \text { 0801 } & \text { No, dicevo: batti lei? } & \\ 01: 01: 35: 23 & & \\ 01: 01: 38: 06 & & \text { Ah! My mistake. At your service. } \\ \text { 0802 } & \text { Ah, congiuntivo. } & \\ 01: 01: 38: 08 & & \\ 01: 01: 41: 02 & & \end{array}$

Cultural specific references: 'GIL' and 'Casino Municipale di Saint Vincent'

As well as linguistic humour, the tennis scene also provides several examples of culturally specific references that are only accessible to an Italian audience (and perhaps only an older Italian audience at that); therefore, the jokes are of a cultural nature. The translator can follow one of two approaches, as proposed by Lawrence Venuti: foreignization or domestication. In the former, the translator translates the dialogue directly, so the original message and its connotations in the SL are retained, yet the meaning may be lost. The latter makes the content fully accessible to the target audience, yet the connotations of the original dialogue will change as the references change. In the following example, I have decided to use a domestication strategy for both of the cultural references on show and I have therefore substituted the cultural references:

$\begin{array}{lll}\begin{array}{l}\text { Subtitle } \\ \text { No. and } \\ \text { Timecode }\end{array} & \text { Original Italian } & \text { English Subtitles } \\ \text { 0791 } & \text { Fantozzi: maglietta della "GIL”, } & \text { Fantozzi: 'Fascist Youth" } \\ \text { 01:00:54:15 } & & \text { t-shirt from the 1940s, } \\ \text { 01:00:57:08 } & & \\ \text { 0792 } & \text { mutanda ascellare aperta sul davanti e } & \text { high ridden shorts } \\ \text { 01:00:57:11 } & \text { chiusa pietosamente con uno spillo da } & \text { held up by a safety pin. } \\ \text { 01:01:02:08 } & \text { balia, }\end{array}$


The Gioventù Italiana del Littorio (or GIL) was the consolidated youth movement of the National Fascist Party of Italy that was established in 1937 and disestablished after the fall of the regime in 1943. Such a strong political statement is so out of place in Fantozzi given that, until this point, there are no references to Mussolini, Fasism or Italy during a time of war, therefore the 'out-of-place' nature of the reference needs to be retained at the very least. The reference to GIL is clearly loaded with political connotations; however, it is completely inaccessible to an English speaking audience who are unfamiliar with the details of Italian political history. When using a domestication strategy, it is very important to keep the TL and culture in mind. If translating to purely a New Zealand context then the "Young Nats" group, who are the youth brunch of the centre-right National Party, could be an appropriate translation, although all of the political connotations are lost given that the Young Nats in New Zealand have never been as extreme. The main issue with a domestication strategy is that the audience must suspend their disbelief; that is, they have to be open to the concept that an Italian would, for example, wear a t-shirt that has the "Young Nats" branding on it, posing difficulty for a translator. If the translation were for a global English-speaking audience then using "Fascist Youth" would evoke the type of reaction that is present in the original dialogue. The other aspect of this humour is that Fantozzi has not owned sports gear since the days of GIL's existence, implying he has not exercised since the 1940s. For this reason, to make the humour clear to the audience, I have also added 'from the 1940s' to retain this implication that would be lost on the English-speaking audience otherwise.

The other reference, that to the "Casino Municipale di Saint Vincent" is also likely to be lost to a non-European audience. However, with this reference it is not very important that the audience knows exactly which casino it refers to, as long as they know it is a casino, as the humour is more of a visual nature representing the rather strange fashion choices made by Fantozzi. I have therefore decided to transfer the original term, rather than find a replacement. This is also beneficial as the audience will hear what they are reading. Depending on how far the domestication strategy is taken, the reference could have been changed to something along the lines of "Skycity Casino", the most well-known casino in New Zealand; however, again, the audience would have to be willing to believe that the visor had come from a casino in New Zealand that had yet to be built when the film was released in 1975. The best result seems to be to foreginise the text, and leave it as "The City Casino of Saint-Vincent". 


\subsubsection{Rhyme and Song}

Another scene that caused a lot of difficulty was during Fantozzi's Christmas and New Years celebrations. Each of the children of the employees in the film give recitals for the Mega-Directors in exchange for a gift. This scene proved that subtitling is indeed a multidisciplinary form of translation, presenting the challenge of translating poetry or nursery rhyme.

The scene presented the challenging task of having to translate a made-up nursery rhyme. While the rhyme itself is not intrinsically humorous, it, alongside the visual humour on-screen, are an important allegory of the life of Fantozzi's daughter, Mariangela, as her 'fruit' will supposedly never flower. Mariangela is, in fact, played by a boy actor and is often referred to as a chimpanzee or other species of primate. Because the rhyme of the poem and the images work in tandem to create a certain black-humour, it is important to retain as much of this humour and allegory as possible in the translation.

The translator, in order to deal with rhyme and meaning, needs to use a similar skill set to that used in translating music or poetry. The emphasis is on saving both rhyme and meaning, while also being limited by time and space. There are several ways in which a subtitler can approach this task. The first option is to ignore the rhyming scheme altogether. This allows for the meaning to be passed on to the new audience, yet the intricacies of the original dialogue would be lost. It may also be off-putting to the audience as they will be able to hear the SL rhyme, but no rhyme in the TL. The second option could use intertextuality and replace the rhyme with a recognisable alternative in the TL. For example, retaining the theme of nature, an alternative could be the famous nursery rhyme Mary, Mary, Quite Contrary. While this does provide some familiarity to the target audience, the original rhyme is not a famous one in Italian, so it is, perhaps, a step too far when replacing it with one that is very well known by English speakers. The other issue with this translation is that the allegory of the original is completely lost.

\section{Original Italian:}

Un ciliegio grande e bello disse a un piccolo alberello: "Sì, sei bravo, hai messo i fiori coi lor petali a colori, ma cambiare i fiori in frutti alberello, non saprai."

\section{Original Italian:}

Un ciliegio grande e bello disse a un piccolo alberello: "Sì, sei bravo, hai messo i fiori coi lor petali a colori, ma cambiare i fiori in frutti alberello, non saprai."

\section{Potential English subtitles:}

A tall and beautiful cherry tree said to a little sapling:

"Yes, you are wonderful, starting to bloom with your colourful petals, but how to change the flowers to fruit, Little sapling, you will never know."

\section{Potential English subtitles:}

"Mary, Mary, quite contrary

How does your garden grow?

With silver bells,

And cockle shells,

And pretty maids all in a row." 
The final strategy that a subtitler can adopt, and the one that I have settled on, is to attempt to retain both the rhyming scheme, and the message, although perfect equivalence can rarely be reached. In the translation below, both the rhyming as well as the implied message that being beautiful and adhering to society's aesthetic norms does not, and should not, mean everything, is retained. Slightly different references, or the order in which the references are made, was switched so this meaning could be presented in the English subtitles. For example, instead of a literal translation of "coi lor petali a colori" as "with your colourful petals", translating it to "anything but dour" while changing it from a positive to a negative statement, retains the message of the original verse.

Subtitle

No. and

Timecode

$0442 \quad$ Un cigliegio grande e bello, disse a un

00:34:28:19 piccolo alberello:

00:34:33:23

0443 Sì, sei bravo, hai messo i fiori, coi lor

00:34:34:00 petali a colori.

$00: 34: 38: 24$

0444 Ma cambiarli con i frutti, alberello non

00:34:39:01

00:34:43:04

\section{English Subtitles}

\begin{abstract}
A cherry tree, pretty and tall
told a sapling ever so small:
\end{abstract}

\section{Yes, bravo, you're about to flower. \\ Your petals are anything but dour}

\section{How to get fruit from flower, little sapling, you'll never know.}

Similarly to the translation of poetry, at the end of the film La ballata di Fantozzi or 'Fantozzi's ballad' is the original song that played during the end credits. The rhyming is rather challenging to deal with "caffè" and "bide", "La milletre" with "Frigo e Buffet", and a partial rhyme with "moquette" and "roulotte". The song, written by Paolo Villaggio himself, includes references to his previously published work on Fantozzi, which provided source material for the first and second films directed by Luciano Salce.

As can be seen, to deal with the cases of rhyme, I have tended to go either for a substitution technique, or, bearing in mind Baker's equivalence at both a grammatical level and an above-word level, simply changing the order of the sentence, in conjunction with explicitation where possible, to retain meaning. As a last resort, I went for a simple relative literal translation. For example, "sveglia e caffè, barba e bide" is translated as 'shaves and has coffee, then cleans his body' which retains both the rhyme (at least partial rhyme) in 'coffee' and 'body' as well as the meaning of the line, that being that when Fantozzi wakes up, he has coffee, shaves and washes himself. I decided for a rather loose explicitation as translation of "La mille e tre, frigo e buffet", l'elettro dei frappe, while attempting to retain the rhyme of the original. The words buffet and frappé fortunately already rhyme, and both exist in English. The problem comes with "La milletre", a type of car at the time that acts as an example of 
Fantozzi's middle-class aspirations. I decided to translate it as 'the car of today', thus retaining the rhyme and not detracting from any meaning. While it is not an ideal solution, it does serve its purpose, keeping the content and rhyming scheme of the original.

\section{Subtitle \\ No. and}

Timecode

\section{9}

01:41:23:09

$01: 41: 27: 22$

1330

$01: 41: 27: 22$

01:41:30:04

$1331 \quad-$ J Lei venghi qua!

01:41:31:16 - Sì, Signor Megadirettore! J

$01: 41: 34: 03$

1332

01:41:34:06

$01: 41: 38: 12$

1333

$01: 41: 39: 22$

$01: 41: 41: 24$

1334

01:41:42:01

$01: 41: 46: 22$

1335

01:41:47:00

01:41:50:23

1336

01:41:52:00

01:41:56:12

\section{7}

$01: 41: 56: 16$

01:41:58:17

1338

01:41:59:04

01:42:04:23

1339

01:42:05:11

01:42:08:04

\section{Original Italian}

1001/bis.

Dell’Ufficio Sinistri.

J No vadi là!....E si muovi! J



(1)

J Presto che perdo il tram! $\mathrm{J}$

(n)

J Fantozzi! J

pubblica...J
Fantozzi, ragioniere Ugo, matricola

J Ah! Hanno messo la parete a vetri! J

J Sveglia e caffè barba e bidé $\boldsymbol{J}$

J Se il cartellino non timbrerò.... J

- J You be coming here!

- Yes, Mr Mega-Director Jo

J No, be going there!

....and get out of the way! J

Fantozzi, Mr Ugo,
employee number 1001/bis.

From the Accidents Office.

English Subtitles

(.

J Ah! They put
the glass walls up... J

Jo Shaves and has coffee

then cleans his body $\mathrm{d}$

J Quickly! I'll miss the bus! J

J If I do not punch my timecard... J

J Fantozzi! J

I0 No! Not crucified in the canteen!

I'm ashamed... What humiliation... 8 J

J No! Crocefisso in sala mensa no.

Mi vergogno... ma che umiliazione

J E poi mi si intrecciano i diti! ס

J My hands start to tremble! $\mathrm{J}$ 
1340

01:42:24:24

01:42:29:10

1341

01:42:29:23

01:42:33:10

1342

01:42:35:02

01:42:39:23

1343
$01: 42: 40: 00$

01:42:44:01

1344

01:42:45:03

01:42:47:21

1345

01:42:47:21

01:42:49:19

1346
01:42:50:06

01:42:53:09

1347

01:42:53:11

01:42:55:05

1348
$01: 42: 55: 07$

01:42:59:20

1349

01:43:03:00

01:43:06:09

1350

01:43:06:10

01:43:08:08

1351

01:43:08:10

01:43:10:18

1352

01:43:10:22

01:43:13:15

1353 J Ma non sempre la mia!

01:43:13:17 Ma perché sempre la mia? ס

01:43:18:12

J L'elettro dei Frappé $\delta$

J Sì Direttore $\delta$

J Conte Ingegner $\mathrm{J}$

J Sire sua Santità! J

J In ginocchio! J

J Sottoscrizione regalo al megapresidente galattico! J

Jo Bella! Bell'idea! J
J La mille e tre, frigo e buffet $\mathrm{d}$

Jo Mixes up a cold frappé $\mathbb{\delta}$

J Più la moquette poi la roulotte $\int$

Jo First the carpet, then the caravan $\mathbb{J}$

J Le rate... 16 chili e 700 di cambiali! J

J An enormous debt to pay! J

J Yes, Director 8

J Mr Count, sir J

JJ Your Holiness! J

J On your knees! J

J Faccia di me quello che vuol....J

J Make of me what you want... $\mathcal{J}$

Jo Gift subscription for the

Galactic Mega-President! J

J Ma che gli facciamo quest'anno?

J But what are we

getting him this year? J

JUna bella poltrona in pelle umana! J

J A beautiful chair

made from human skin! Jo

d Wonderful! Wonderful idea! J

J But not my skin!

Why is it always me! J 


\subsubsection{Multilingualism and the representation of Jesus}

The next scene that I would like to draw specific attention to is one where Fantozzi and Filini go on a camping and fishing trip. This scene is particularly challenging as the dialogue is not solely in Standard Italian, but also includes French, German and also a strong and identifiable Roman accent. I would like to draw specific attention to how dialects and accents can be translated, as an Italian audience would have no difficulty in distinguishing between standard language and dialect, yet an English audience, unfamiliar with Italian and the complex linguistic system that exists within Italy, would undoubtedly struggle in this regard. Multiple languages and dialects are also used across the entire Fantozzi series so again, translation strategies must have the flexibility to be replicated across different contexts.

As I have mentioned earlier on the constraints of subtitling, a character's tone of voice, their accent, or any other distinguishing features, such as lisping, pitch, or stuttering, for example, can be used by an audience to determine who is speaking. Subtitles, being a written form of communication, cannot account for these features. This is where it can be difficult to convey that different languages or dialects are being used on-screen, particularly if the audience is unfamiliar with the ST language. For example, in the film Benvenuti al Sud, described in chapter 2, both the dialect of the Castellabate region, and Standard Italian are used, yet in the subtitles the distinction between the standard language and dialect is often lost and as a result, the film becomes less comedic to an English-speaking audience.

The same problem occurs in Fantozzi. At the end of the film's fifth chapter we see Fantozzi and Filini hallucinating, while Fantozzi has a vision of Jesus walking across the water towards their stranded boat. This representation of Jesus speaks with a Roman accent, which is recognisable to an Italian audience, but it is not of a particularly high register - in fact the character almost comes across as a hippy. Why Jesus speaks with this Roman accent here is open to interpretation, perhaps because the Vatican City is located in Rome, however it is a stylistic choice made by director Luciano Salce and therefore it needs to be signalled in the TL subtitles. It is interesting to note that on the DVD of the film, the Italian captions for the deaf and hard of hearing do not provide a distinction between Italian and Roman.

Several options were available when I translated this piece of dialogue, following Perteghella's discussion on translating dialect highlighted in Chapter 2. One option could be a variant of the paralleldialect translation technique, where a similar geographical dialect or a dialect with similar connotations is used. However, instead of loading the subtitles with new cultural connotations, the subtitler can simply adjust the English subtitles so they read as if an Italian speaker was reading it. That is, the subtitles are a phonetic representation of a stereotypical Italian accent. As mentioned, this will not add regional connotations to the dialogue, and should not cause the viewer's discomfort, as they presumably know that the original language of the film is Italian. While it does not necessarily allow the audience to understand that the original is using a dialect rather than Standard Italian, it will at least be an 
indication to the audience that it is a different form of language, which is represented in the subtitles.

Such a translation could be as follows:

\section{Original Italian:}

\section{Avete pesce?}

Non abbiamo pescato, dottore.

Avete pane?

No, doveva portarlo lui.

\section{Potential English subtitles:}

Do you av' anya fesh?

We haven't been fishing, sir.

Do you av' anya bread?

No, he was meant to bring it.

Another option is a parallel dialect translation where similar connotations are associated with the source and TT. I have therefore decided to use Bible speech for the Jesus character. This works for a variety of reasons, the most obvious being that Jesus himself is a biblical character. However, there is also the fact that the Vatican, home of the Catholic Church, is situated in Rome. Roman being replaced with biblical speech is therefore not too far of a stretch, and means that the audience will have complete comprehension of the scene without being distracted from the content:

\section{Original Italian:}

\section{Avete pesce?}

Non abbiamo pescato, dottore.

Avete pane?

No, doveva portarlo lui.

\section{Potential English subtitles:}

Dost thou have fish?

We haven't been fishing, sir.

Dost thou have bread?

No, he was meant to bring it.

The final option that I have produced is to translate the dialogue using the implied register, that is, signalling a change is speech not through bibilical language as above, but to over-correct the translation and standardize it so the character speaks with very normal language, in a way achieving what Salce hoped to achieve by demistifying religion, showing that this Jesus figure could really be anyone. The language is therefore simple, if not at a lower register than Fantozzi and Filini. I also decided to add the expletive "What the hell" as this only adds to the informality and shock that this accents brings:

\section{Subtitle No. Original Italian \\ and}

Timecode

0423

00:33:01:24 - Non abbiamo pescato, dottore.

00:33:04:19

0424

00:33:04:21

00:33:07:06

\section{English Subtitles}

Got fish?

- We haven't been fishing, sir.

- Got bread?

- No, he was meant to bring it. 
0425

00:33:07:09

00:33:08:12

0426

00:33:08:14

$00: 33: 11: 19$
No, lui.

Allora, che vi moltiplico io?
No, he was!

Then what the hell am I meant to multiply?

\subsubsection{Culturally specific references}

The final scene that I would like to discuss in detail is the scene where Fantozzi admits himself to the diet therapy clinic Le Magnolie. This is a particularly challenging scene for a variety of reasons: not only is the language challenging in and of itself, but there are particular cultural references to food and money as well as intertextual references from outside litterature that require a lot of thought on the translator's part. Moreover the scene carries visual resemblances to Nazi internment camps, the diet therapy effectively consisting of starving the patients, and as discussed in chapter 1, the fact that the doctors are all German is surely not by coincidence. The translator must therefore be very mindful of this and tread with extreme caution when translating this particular scene.

The first challenge, and one that, just like humour, is both culturally specific yet universal, is the translation of particular traditional Italian dishes. Food evokes emotion, it creates particular images in the audience's mind and is something to which everyone around the world can relate. This scene plays on the idea of greed through both the consumption of food and the cruelty of the directors in charge of the centre who both withhold food from the patients, as well as exploiting them when they are at the peak of their hunger. The audience is confronted by the idea of starvation, and then presented with particular traditional Italian dishes that are meaningful to an Italian audience. Trying to capture the emotion associated with the food in a translation becomes difficult. The context itself, fortunately, means the translator does not need to worry too much about the first factor, that of a starving patient, as the images and dialogue are enough to convey this message.

However, the difficulty comes in retaining the emotion associated with the traditional Italian dishes. The translator cannot simply replace the meals with traditional English equivalents like "steak and chips" as the meals are being shown on-screen; in other words, the subtitler is restricted by the images present in the film. For some meals, such as "Risotto ai funghi" or 'mushroom risotto' a simple translation can take place as the meals exist across languages and cultures. For other meals, such as "Pasta alla boscaiola" (literally 'Woodsman's Pasta') an equivalent does not exist in English and therefore it would mean nothing to the English viewer. For the meals where an equivalent does exist, I have translated these as such, or at least made the original more recognisable to the viewer, for example "Rigatoni alla bella Bologna" becomes the very recognisable dish 'Bolognese', "Penne alla carbonara" becomes 'Carbonara', and "Paglia e fieno ai quattro formaggi" simply becomes 'Four cheese pasta'. However, for the meals for which no equivalent exists, I have decided to foreignise the text, leaving 
them untranslated in the hope that their presence on-screen provides familiar enough reference to adequately convey the intended sentiment, as can be seen below.

Furthermore, later in the scene just before Fantozzi breaks out of the clinic-prison, he orders the "Pasta alla Montecristo", which comes with an iron file that Fantozzi then uses to saw the window bars of the cell and escape - which, as discussed in chapter 1, is a reference to the escape of Edmond Dantes in Alexandre Dumas' adventure novel, The Count of Monte Cristo (1844), of which multiple film adaptions were made from 1901 to 2012, one of them in 1975, the same year as Fantozzi was released. Yet 'Pasta alla Montecristo' does exist as an Italian dish: it is a type of pasta with seafood. Retaining both references is simply impossible in translation. For this issue, I settled on the creative translation of 'Filefish pasta', obviously referring to the fact that Fantozzi is sawing his way out using a file, and that 'Filefish' is indeed a type of fish that has some edible species. I have translated it as such also because the original reference to Montecristo is unlikely to be understood by today's Englishspeaking audiences.

$\begin{array}{lll}\begin{array}{l}\text { Subtitle } \\ \text { No. and } \\ \text { Timecode }\end{array} & \text { Original Italian } & \text { English Subtitles } \\ & & \\ \text { 0853 } & \text { - Tagliatelle alla boscaiola. } & \text { - Tagliatelle alla boscaiola! } \\ \text { 01:05:41:14 } & \text { - Paglia e fieno ai 4 formaggi. } & \text { - Four cheese pasta! } \\ \text { 01:05:44:16 } & & \\ & & \\ 0854 & \text { - Penne alla Carbonara. } & \text { - Carbonara! } \\ 01: 05: 44: 18 & \text { - Rigatoni alla bella Bologna. } & \text { - Bolognese! } \\ 01: 05: 48: 21 & & \end{array}$

\section{Subtitle \\ No. and Timecode}

\section{7}

01:08:23:08

01:08:27:18

\section{8}

01:08:27:20

01:08:30:12

0889

01:08:30:14

Original Italian

Quella stessa notte, Fantozzi firmò due

cambiali giganti.

E si pagò la specialità più costosa della casa:

01:08:33:21

Spaghetti alla Montecristo.
English Subtitles

That same night, Fantozzi

signed two enormous cheques.

He bought the most expensive dish

on the menu:

Filefish Spaghetti. 


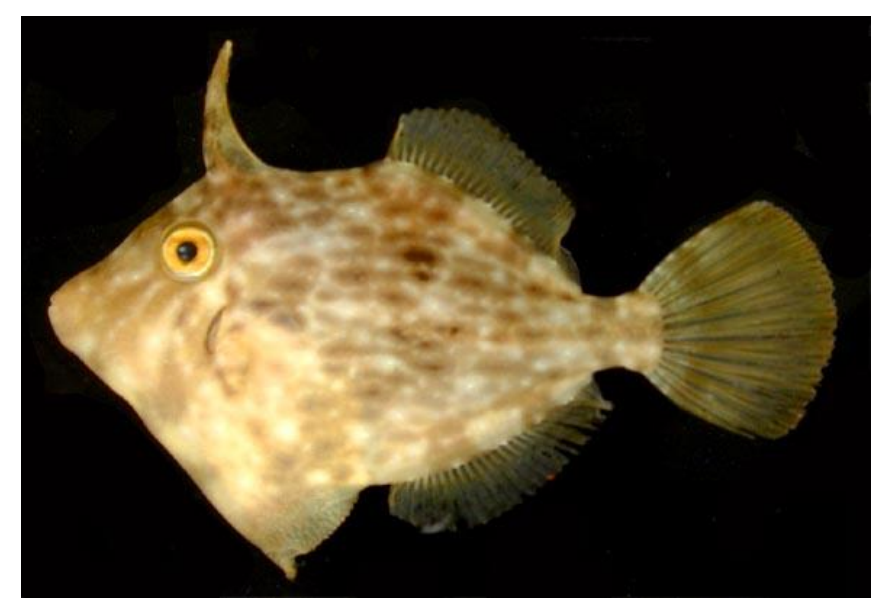

Figure 23: The Filefish, used in the translation of Pasta alla Montecristo ${ }^{300}$

The next difficulty in this particular scene (and subsequently throughout the Fantozzi series) is the translation of currency. Part of the humour is associated with the absurdity of the prices, yet the currency used is the lira, Italy's currency prior to the Euro. This is difficult because a foreign audience has no concept of the lira, including younger generations of Italians. I decided to change it into dollars for the English-speaking audience; this does come with its own barriers: the Australian, New Zealand, American, Canadian and any other country that uses the dollar would be able to understand, but other audiences may not, although the dollar is considered a global currency. Despite the change in currency, I have tried to keep the numbers similar: 10,000 lira as $\$ 100,28,000$ lira as $\$ 280$ as can be seen in the example below. Other alternatives could involve leaving the currency out completely, although this then places tangible value on the amount, removing the humour entirely, or simply leaving it in 'lira', knowing that the audience may not be able to fully access the content.

$\begin{array}{lll}\begin{array}{l}\text { Subtitle } \\ \text { No. and } \\ \text { Timecode }\end{array} & \text { Original Italian } & \text { English Subtitles } \\ & & \\ 0877 & - \text { Me ne dia due porzioni... } & - \text { Give me } 2 \text { portions... } \\ 01: 07: 00: 22 & -56,000 & -\$ 560 . \\ 01: 07: 03: 20 & & \\ 0878 & \text { Non ho più contanti, come faccio? } & \text { I don't have any cash, } \\ 01: 07: 03: 22 & & \text { what can I do? } \\ 01: 07: 05: 11 & & \end{array}$

There is a precedent for translating the currency 'lira' to 'dollars' in contemporary Italian film. The English subtitler of Nani Moretti's Caro Diario (1993) chose to do exactly this in the scene where

\footnotetext{
${ }^{300}$ Image found on: https://en.wikipedia.org/wiki/Filefish. Accessed 5 ${ }^{\text {th }}$ January, 2019.
} 
Nanni Moretti talks about the prices of penthouses in Rome. In the original, the price of the penthouse is "dieci milioni [di lire] al metro quadro" or "ten million lire per square metre". In the English subtitles, however, the subtitler not only changed the currency but also the numbers used. In 1993, the year the film was released, ten million lira would have been worth approximately five thousand euro. As can be seen in the subtitles in Figure 25, the translator changed this to seven hundred dollars thus drastically altering the content and value of the penthouses. While I disagree with this second practise, the domestication strategy to change lira to dollars is certainly a viable technique in an Italian to English translation of a contemporary film. In fact, Anthony Pym, when talking about equivalence in translation, highlights the fact that currency in particular is not about exact values. He cites the example of the British game show Who Wants to Be a Millionaire? The translation, therefore, for an American audience should realistically be Who Wants to Win $\$ 1,516,590$ ? Obviously here, the sentiment is more important than the exact figures, which is exactly what I tried to achieve in my translation of 'lira' to 'dollars'. ${ }^{301}$

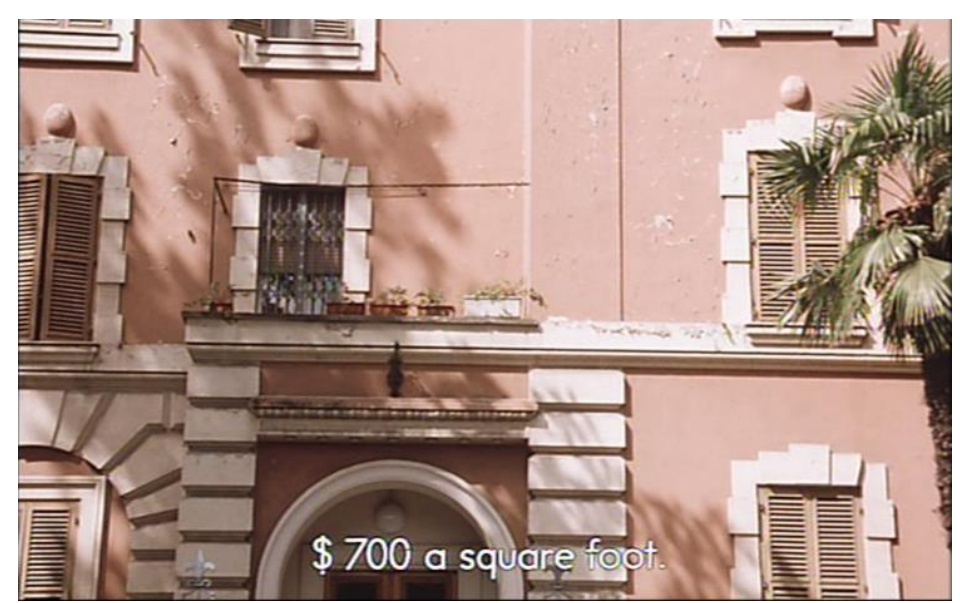

Figure 24: The use of "dollars" in translation, rather than the retention of "lira" in Nanni Moretti's Caro Diario.

\subsection{Conclusion}

Subtitling is certainly not a straightforward translation from SL to TL. The subtitler must be well versed in a variety of different areas of translation studies. In the context of Fantozzi these include, but are not limited to: the translation of humour, cultural specific content, rhyme, dialect and songs. The variety of content that exists within one film alone undoubtedly makes the translators job exponentially harder.

The translation decisions made during the subtitling of Fantozzi attempted to resolve such difficulties with creative solutions. However it must be said that such strategies may not be adopted by commercial subtitling agencies given that their main purpose tends to be communicating the content,

\footnotetext{
${ }^{301}$ Anthony Pym, Exploring Translation Theories (New York: Routledge, 2014), 8.
} 
rather than incorporating the subtleties that exist in the orignal language. It is my belief that these could work well in commercial subtitles as well should they be adopted, as while they do require some thought on the translator's part, they have, in my experience so far, been met positively by audiences. At the screenings of the film with these English subtitles that have taken place in Wellington, the subtitles have been been well received; however further testing with wider audiences would be required to determine their true success or failure. 


\section{Conclusion}

Fantozzi (1975) proved to be a very interesting case study of Italian culture in the 1970s and a complex example of a humorous text, filled with rich linguistic, cultural, visual and historical elements that required careful attention. Recognised and enjoyed by different generations of Italians, Fantozzi is viewed as one of the most iconic figures in Italian comedy: not only when the first film was released in 1975 (and earlier appeared in the form of short stories), but also in today's society. Recently, the books have been re-published and, shortly before Paolo Villaggio's death, the books were released in an audioformat read by Villaggio himself.

Given that there has been very little academic attention given to Fantozzi, I hope to have provided a research-based understanding to an often dismissed film and series as a whole that have been the object of very little academic study, even in the context of constantly developing research in Italian comedy. I aimed to do this by presenting Fantozzi through the eyes of theories of humour, theories of translating humour, theories of subtitling, as well as the need for cross-cultural competency to adequately render content across linguistic systems. I set out to give the film, and as a result the books, an ounce of the academic attention that they warrant also through both contextualising the character in Italian culture, introducing some of the important social, cultural and historical themes that run through the series, and also by presenting some of the clever uses of humour made by Villaggio and Salce through my translations. Both Fantozzi as a character and the series as a whole have been largely left unexplored: the way the films portray cultural aspects, of which I have only just scratched the surface, such as the position of women, religion, the representation of other cultures, politics, history, food and intertextual references could each be the subject of academic research in their own right.

This wealth of possible approaches and the interdisciplinary nature of the thesis meant that I had to prioritise the varying aspects that contributed to my research while trying to negotiate the different theoretical, creative and practical components that feed into it. I tried to bear in mind at all times that this was a thesis in Literary Translation Studies, so that remained my focus throughout. There are certainly concepts and examples that warrant expansion, but given the scope of this thesis they were not possible in the current project. The most obvious output, and indeed ambition, of this project was to provide an English-speaking audience with the opportunity to learn about and enjoy an important staple of Italian comedy by ensuring that as a subtitler and as a linguistic and cultural mediator, I could convey the film's textual and contextual implications, its subtlety, and most importantly its humour. The theoretical analysis of both subtitling and the translation of humour, as well as the introduction of some of my own ideas and techniques to cope with specific trends in the Fantozzi series or difficulties in the translation, allowed me to provide well-thought out, justifiable translations in an attempt to do the original content justice through its translation into English.

Another one of the main purposes of this project was to shed further academic light on the process of subtitling and translating humour, each fraught with their own challenges and complexities 
that are as interesting as they are arduous to resolve. By looking into the role of the subtitler, the history of subtitles, the different types of practices that exist and how they are projected on-screen, as well as the future of the industry, I hope to have provided a comprehensive and in-depth analysis of this very unique form of inter-semiotic, multi-modal translation. The theoretical and practical analysis of subtitling brought to the forefront exactly what these challenges and complexities are, and then provided creative and useful techniques that could be used to alleviate some of the unavoidable associated challenges. Introduced by a discussion on translating humour, I sought to determine specific techniques that could be used in conjunction with those proposed for subtitling to aid me when undertaking the translation component of this thesis: the creation of the English subtitles for Fantozzi, a case study that has never been translated into English, despite its success in Italian cinema and Italian culture as a whole. Moreover, the language pairing of Italian and English has received very limited academic study to date since the primary form of audiovisual translation between the two has traditionally been dubbing. This project therefore offers further insight into an area that scholars have largely neglected.

While I do not intend to claim that my translations are perfect, I am overall satisfied that they convey important aspects of the original humour and source culture content and that they are justifiable in their own right. Ultimately, as is the case in any form of translation, the translator must let go, something that I certainly struggled with throughout this project. However it is precisely on this point, on the need to choose one solution over another, that translating and subtitling present the greatest challenge, and indeed reward: it is very difficult to find a perfect answer to questions of practical translation.

The are several directions in which this research could develop. One would be to test my translation on a selected target audience of English native speakers to determine whether it has been a success in terms of the humour of the ST being retained. This is, arguably, a difficult task given the subjectivity of humour that has been discussed in the previous pages. However, I could follow the methodology proposed by Martin et al. or by Schauffler. ${ }^{302}$ It would certainly be an interesting and enlightening analysis to undertake, particularly if comparing the English speaking audience's reaction to the film with a native Italian speaking audience, thus determining whether the humour was indeed transferred or lost in the translation. It would also be an interesting task to compare the similarities in senses of humour between culturally and linguistically distinct audiences. Even from the relatively small sample size from the few screenings of the film with these subtitles, it seemed like the English speaking audience laughed alongside the Italian audience, therefore a preliminary hypothesis is that the retention of humour appears to have been successful. However, these general observations would need to be tested in a systematic and scientific way.

\footnotetext{
${ }^{302}$ Neil Martin et al., "Measuring responses to humor: How the testing context affects individuals reaction to comedy," Humor 21, no. 2 (2008).; Schauffler, Subtitling Wordplay - An Audience Study: Approaches to the Translation of Linguistic Humour, 180-90.
} 
Another direction would be to use the translation strategies that I have adopted for Fantozzi and apply them to the whole Fantozzi series (ten films in total). I have stressed throughout this project that the techniques used would need to be able to be replicated across a variety of different texts and contexts. Applying these techniques to the other films in the series would allow me to measure the success of my choices and strategies. For certain translation decisions - for example the use of 'be coming', 'be going' etc. to render the improper use of the imperativo formale, or the use of "dollars" instead of "lira"- I would argue that they are indeed suitable across contexts. However, there may be other examples that cannot simply be replicated, something which could only be found out if properly tested. Perhaps in discovering whether or not these translations are viable in the context of Fantozzi, we could provide an example to other texts as to how this type of humour may be retained, and how the cultural content can be expressed into English. The success of similar comedic characters like Reginald Perrin and Homer Simpson means that there is potential demand for a character like Fantozzi to grace English-speaking audiences' televisions.

Another aspect of this research that warrants pursuing is the use of subtitling as a pedagogical tool in second language learning and teaching. As studies have proven (some of which were mentioned in this project) subtitling and subtitles can be useful tools to aid second language education, in particular for children. As a tutor of Italian at Victoria University of Wellington, I incorporated subtitling into both tutorial classes and assessments, something which received overwhelmingly positive responses from students, particularly when the students were allowed to work on small subtitling projects involving materials of their own choosing. I would be very interested to learn if this project, and others like it, could have a positive effect on language progression as well as enjoyment.

An aspect of subtitling that I did not explore from a practical sense was the idea of trying to integrate more of the recent technological advancements and research associated with the industry into my project. For example, I considered following in Fox's footsteps and subtitling the film using integrated subtitles, and then comparing the results of an English-speaking audience watching integrated subtitles with her German audience, to see if language background and cultural norms around subtitling played any role in the effectiveness of the integrated titles on comprehension or ease of viewing. Unfortunately, the technology available to me was not sufficient to undertake this research reliably and successfully, but it leaves ample room for further experimentation.

I hope that this study has gone some way in building on the very strong research of my predecessors in this field, showing how subtitling is undoubtedly a rich and creative form of translation that warrants greater academic attention. This is particularly the case given recent trends in fan-subbing, the globalisation of the film industry and developments in technology, which demonstrate that the need for audiovisual translation is greater than it has ever been and will only continue to increase. As a case study, Fantozzi has also shown that translating humour, particularly in the case of subtitling, requires a deep cultural knowledge, a high level of linguistic competency in both the SL and TLs as well as a combination of scholarly understanding and creative freedom. 


\section{Bibliography and Filmography}

\section{Publications by Paolo Villaggio}

Villaggio, Paolo. Fantozzi. Milano: Rizzoli, 1971.

- Come farsi una cultura mostruosa. Milano: Bompiani, 1972.

—. Il secondo tragico libro di Fantozzi. Milano: Rizzoli, 1974.

—. Le lettere di Fantozzi. Milano: Rizzoli, 1976.

_. Fantozzi contro tutti. Milano: Rizzoli, 1979.

—. Fantozzi subisce ancora. Milano: Rizzoli, 1983.

—. "Caro direttore ci scrivo... ”: Lettere del tragico ragioniere, raccolte da Paolo Villaggio. Milano: Mondadori, 1993.

_. Fantozzi salute se ne va. Le ultime lettere del rag. Ugo Fantozzi. Milano: Mondadori, 1994.

—. Vita, morte e miracoli di un pezzo di merda. Milano: Mondadori, 2002.

—. 7 grammi in 70 anni, L'odissea di un povero obeso. Milano: Mondadori, 2003.

- Sono incazzato come una belva. Milano: Mondadori, 2004.

—. Gli fantasmi. Milano: Rizzoli, 2006.

—. Storia della libertà di pensiero. Milano: Feltrinelli, 2008.

—. Storie di donne straordinarie. Milano: Mondadori, 2009.

_. Crociera Lo Cost. Milano: Mondadori, 2010.

- Fantozzi totale. Torino: Einaudi, 2010.

—. Mi dichi. Prontuario comico della lingua italiana. Milano: Mondadori, 2011.

- Giudizio universale. Milano: Feltrinelli, 2011.

—. Non mi fido dei santi. Reggio Emilia: Aliberti, 2011.

—. La fortezza tra le nuvole. Pezzan di Carbonera: Morganti, 2011.

_. La vera storia di Carlo Martello. Milano: Dalai, 2011.

—. Tragica vita del ragionier. Fantozzi. Milano: Mondadori, 2012.

—. Siamo nella merda. Pillole di sagezza di una vecchia carogna. Milano: Mondadori, 2013.

—. Fantozzi, rag. Ugo. La tragica e definitiva trilogia. Milano: Rizzoli, 2013.

—. Lei non sa chi eravamo noi. Milano: Mondadori, 2014.

—. Come farsi una cultura mostruosa OGGI. Milano: Cairo, 2014.

_. Italiani brava gente... ma non è vero. Milano: La nave di Teseo, 2018.

Villaggio, Paolo and F. Schietroma. Fantozzi Forever. Milano: Cairo, 2014. 


\section{Fantozzi Films}

Salce, Luciano, dir. Fantozzi. 1975; Roma: Warner Bros. Entertainment Italia, 2015. DVD.

_. Il secondo tragico Fantozzi. 1976; Florence: CG Home Video, 2013. DVD.

Parenti, Neri. , dir. Fantozzi contro tutti. 1980; Roma: Rai Cinema, 2017. DVD.

_. Fantozzi subisce ancora. 1983 ; Roma: Rai Cinema, 2016. DVD.

—. Superfantozzi. 1986; Florence: CG Home Video, 2013. DVD.

—. Fantozzi va in pensione. 1988; Florence: CG Home Video, 2004. DVD.

_. Fantozzi alla riscossa. 1990; Florence: CG Home Video, 2003. DVD.

_. Fantozzi in paradiso. 1993; Florence: CG Home Video, 2004. DVD.

- Fantozzi - il ritorno. 1996; Roma: 01 Distribution, 2005. DVD.

Saverni, Domenico, dir. Fantozzi - La clonazione. 1999; Florence: CG Home Video, 2007. DVD.

\section{Other films/series referenced}

Collett-Serra, Jaume, dir. Non-Stop. 2014; London: Studio Canal, 2014. DVD

Gattis, Mark, Moffat, Steven, Thompson, Stephen. Sherlock.. 2010-Present; London: BBC Worldwide.

Groening, Matt. The Simpsons. 1989-Present; California: Twentieth Television.

Harlin, Renny, dir. Skiptrace. 2016; Los Angeles: Saban Films, 2016. DVD.

Miniero, Luca, dir. Benvenuti al Sud. 2010; Roma: Medusa Film, 2010. DVD.

Moretti, Nanni, dir. Caro Diario. 1993; Hertfordshire: Arrow, 2009. DVD.

Nobbs, David. The Fall and Rise of Reginald Perrin. 1976-1979; London: BBC.

Stahelski, Chad, dir. John Wick. 2015; California: Warner Home Video, 2015. DVD. 


\section{Referenced texts}

Ayonghe, Suzanne. "Translation of Emotions in Subtitling of the Film Paris àtout prix." International Journal of English Language \& Translation Studies 4, no. 2 (2016): 177-91.

Baker, Mona. In Other Words: A Coursebook on Translation. Oxon: Routledge, 2018.

Balirano, Giuseppe. "The strange case of The Big Bang Theory and its extra-ordinary Italian audiovisual translation: a multimodal corpus-based analysis." Perspectives: Studies in Translatology 21, no. 4 (2013): 563-76.

Baresi, Giacomo. "Fantozzi e lo sport: il ciclismo e la coppa Cobram." http://www.giacomobaresi.com/category/fantozzi-e-lo-sport/.

Benecke, Bernd. "Audio-description." Meta: Translators' Journal 49, no. 1 (2004): 78-80.

Bergen, Lori, Tom Grimes, and Deborah Potter. "How Attention Partitions Itself During Simultaneous Message Presentations." Human Communications Research 31 (2005): 311-36.

Bittner, Hansjörg. "The Quality of Translation in Subtitling ". trans-kom 4, no. 1 (2011): 76-87.

Bogucki, Łukasz. "The Constraint of Relevance in Subtitling." The Journal of Specialised Translation, no. 1 (2004): 71-88.

Borghetti, Claudia. "Intercultural Learning Through Subtitling: The Cultural Studies Approach." In Audiovisual Translation - Subtitles and Subtitling, edited by Laura Incalcaterra-McLoughlin, Marie Biscio and Máire Aine Ní Mhainnín. Bern: Peter Lang, 2011.

Brant, Rosemary. "The History and Practise of French Subtitling." The University of Texas, 1986.

Buratto, Fabrizio. Fantozzi: Una maschera italiana. Torino: Lindau, 2003.

Calvino, Italo. Marcovaldo. Torino: Giulio Einaudi editore, 1963.

Carroll, Noël. Humour: A Very Short Introduction. New York: Oxford University Press, 2014.

Castelli, Diego. "Hold the Door: ecco il doppiaggio nelle varie lingue." http://www.serialminds.com/2016/05/31/hold-the-door-doppiaggio-lingue/.

Catania, Alessandra. "La traduzione audiovisiva: techniche, strategie e difficoltà: proposta di traduzione di quattro articoli tecnico-informativi." Università Ca' Foscari Venezia, 2013.

Celli, Carlo, and Marga Cottino-Jones. A New Guide to Italian Cinema. New York: Palgrave Macmillan, 2007.

Cerón, Clara. "Punctuating Subtitles: Typographical Conventions and their Evolution." In (Multi) Media Translation: Concepts, Practises and Research, edited by Yves Gambier and Henrik Gottlieb. Philadelphia: John Benjamins Publishing Company, 2001.

Chai, Judy, and Rosemary Erlam. "The Effect and the Influence of the use of Video and Captions on Second Language Learning." New Zealand Studies in Applied Linguistics 14, no. 2 (2008): $25-44$.

Chaume, Frederic. Audiovisual Translation: Dubbing. New York: Routledge, 2012.

Chiaro, Delia. The Language of Jokes in the Digital Age. New York: Routledge, 2018. 
_. The Language of Jokes: Analysing Verbal Play. London: Routledge, 1992.

, ed. Translation, Humour and Literature Vol. 1, Continuum Advances in Translation.

London: Continuum International Publishing Group, 2010.

. "Verbally expressed humor and translation: an overview of a neglected field." International Journal of Humour Research 18, no. 2 (2005): 135-46.

Corrizzato, Sara. Spike Lee's Bamboozled: A Contrastive Analysis of Compliments and Insults from English into Italian. Cambridge: Cambridge Scholars Publishing, 2015.

Cronin, Michael. Translation Goes to the Movies. New York: Routledge, 2009.

D'Agostini, Paolo. Romanzo popolare: Il cinema di Age e Scarpelli. Napoli: Edizioni Scientifiche Italiane, 1991.

D'Amora, Paolo. "Cinematic Representations of Italian Office Workers From the Death of the Travet to Fantozzi, 1952-1983." The University of Texas at Austin, 2017.

d'Ydewalle, Géry, and Ingrid Gielen. "Attention Allocation with Overlapping Sound, Image, and Text." In Eye Movements and Visual Cognition, edited by Keith Rayner, 415-27. New York: Springer New York, 1992.

d'Ydewalle, Géry, and J. Van Rensbergen. "Developmental Studies of Text-Picture Interactions in the Perception of Animated Cartoons with Text." In Knowledge Acquisition from Text and Pictures, edited by Heinz Mendl and Joel R. Levin, 233-48. Amsterdam: Elselvier Science, 1989.

Dan, and Dav. "Simpson Tribute to Paolo Villaggio (Fantozzi)." 9GAG, https://9gag.com/gag/agYvjV6/simpson-tribute-to-paolo-villaggio-fantozzi.

Dannenberg, Hilary. "Marketing the British Situation Comedy." Journal for the Study of British Cultures 11, no. 2 (2004): 169-81.

Delabastita, Dirk. "Wordplay and Translation." Special Issue of The Translator 2, no. 2 (1996).

Design, Centre for Inclusive. "The Hunger Games with audio description: Katniss hunting." https://www.youtube.com/watch?v=B8BD9txkGL4.

Díaz-Cintas, Jorge. "Back to the Future in Subtitling." Paper presented at the MuTra Conference: Challenges of Multidimensional Translation, Saarbrücken, 2-6 May 2005.

. "Dealing with Multilingual Films in Audiovisual Translation." In Translation Sprachvariation - Mehrsprachigkeit: Festschrift für Lew Zybatow zum 60. Geburtstag (English, German and Russian Edition), edited by Wolfgang Pöckl, Ingeborg Ohnheiser and Peter Sandrini, 215-33, 2011.

. "Dubbing or Subtitling: The Eternal Dilemma." Perspectives 7, no. 1 (1999): 31-40.

. "'Subtitling's a carnival': New practices in cyberspace." The Journal of Specialised Translation, no. 30 (2018): 127-49. 
—. "Teaching and learning to subtitle in an academic environment." In The Didactics of Audiovisual Translation, edited by Jorge Diaz-Cintas, 89-103. Amsterdam: John Benjamins, 2008.

. "The value of the semiotic dimension in the subtitling of humour." In Aspects of specialised translation, edited by Lucile Desblache, 181-90. Paris: La Maison du Dictionnaire, 2001.

Díaz-Cintas, Jorge, and Aline Remael. Audiovisual Translation: Subtitling. Translation Practises Explained. New York: Routledge, 2007.

DiMatteo, Christopher. "Translating Lorenzo the Magnificent." http://cdimatteo.com/italian/translating-lorenzo-the-magnificent/.

Dwyer, Tessa. Speaking in Subtitles: Revaluing Screen Translation. Edinburgh: Edinburgh University Press, 2017.

Ellender, Claire. Dealing with Difference in Audiovisual Translation: Subtitling Linguistic Variation in Films. New Trends in Translation Studies. Edited by Jorge Diaz-Cintas Vol. 14, Bern: peter Lang, 2015.

Fantozzi subtitles - English. OpenSubtitles, 2010.

"fantozziano." Trecanni, http://www.treccani.it/vocabolario/fantozziano/.

"'fantozziano, adj."." La Repubblica, https://dizionari.repubblica.it/Italiano/F/fantozziano.html.

Fawcett, Peter. "The Manipulation of Language and Culture in Film Translation." In Apropos of Ideology: Translation Studies on Ideology-ideologies in Translation Studies, edited by Maria Calzada Perez, 145-64. London: Routledge, 2014.

. "Translating film." In Translating French Literature and Film, edited by Geoffrey Harris, 6586. Amsterdam: Rodopi, 1996.

Foundation, Blind. "Latest stats at a glance." https://blindfoundation.org.nz/eye-info/latest-statistics/. Fox, Wendy. "Integrated titles: An improved viewing experience?". In Eye-tracking and Applied Linguistics, edited by Silvia Hansen-Schirra and Sambor Grucza, 5-30. Berlin: Language Science Press, 2016.

Franco, Eliana, Anna Matamala, and Pilar Orero. Voice-Over Translation: An Overview. Bern: Peter Lang, 2013.

Gambier, Yves. "Challenges in research on audiovisual translation." In Translation Research Projects 2, edited by Anthony Pym and Alexander Perekrestenko, 17-25. Tarragona: Intercultural Studies Group, 2009.

—. "Introduction. Screen Transadaptation: Perception and Reception." The Translator 9, no. 2 (2003): 171-89.

Gilbert, Morris. "PICTORIAL ENTERTAINMENT IN PARIS.; "Broadway Melody" Attracts Huge Crowds, the Dialogue Being Translated by the Now Despised Subtitle A Study in Contrasts. Royalty at Film Opening." New York Times, 1st December 1929. 
Giovanni, Elena Di, and Yves Gambier, eds. Reception Studies and Audiovisual Translation,

Benjamins Translation Library. Amsterdam: John Benjamins Publishing Company, 2018.

Gottlieb, Henrik. Screen Translation: Six Studies in Subtitling, Dubbing and Voice-over. Cph. :

Centre for Translation Studies, Department of English, University of Copenhagen, 2001.

. "Subtitling - A New University Discipline." In Teaching Translation and Interpreting:

Training Talent and Experience. Papers from the First Language International Conference,

Elsinore, Denmark, 1991, edited by Cay Dollerup and Anne Loddegaard. Amsterdam: John

Benjamins Publishing Company, 1992.

. "Subtitling: Diagonal Translation." Perspectives: Studies in Translatology 2, no. 1 (1994):

$101-21$.

. "Texts, Translation and Subtitling - In Theory, and in Denmark." Screen Translation (2001):

$149-92$.

. "You Got the Picture? On the Polysemiotics of Subtitling Wordplay." In Traductio: Essays

on Punning and Translation, edited by Dirk Delabastita, 207-32. New York: Routledge, 1992.

Guardini, Paola. "Decision-Making in Subtitling." Perspectives: Studies in Translatology 6, no. 1 (1998): 91-112.

Hanczakowski, Allira. "Translating Emotion from Italian to English: A Lexical-Semantic Analysis."

The AALITRA Review 13 (2018): 21-34.

"Hard Facts." The Subtitler's Association,

http://www.subtitlers.org.uk/ajax.php?modulo=paginas\&accion=sitio_ver\&idpaginas=16.

Henry, Matthew. The Simpsons, Satire, and American Culture. New York: Palgrave Macmillan,

2012.

Herbst, Georg-Michael Luyken

Thomas. Overcoming Language Barriers in Television: Dubbing and Subtitling for the European

Audience. Manchester: The European Institute for the Media, 1991.

Hutcheson, Frances. Reflections Upon Laughter, and Remarks Upon the Fable of the Bees. Glasgow1750.

"Il "Fantozzi" neozelandese: nasconde la pistola nei pantaloni e si spara." Blitz Quotidiano, https://www.blitzquotidiano.it/cronaca-mondo/fantozzi-nuova-zelanda-pistola-pene-986897/.

Ina, Lekkai. "Incidental Foreign-Language Acquisition by Children Watching Subtitled Television Programs." TOJET 13, no. 4 (2014): 81-87.

Ivarsson, Jan, and Mary Carroll. Subtitling. Simrishamn: TransEdit HB 1998.

Jakobson, Roman. "On Linguistic Aspects of Translation." On Translation (1959): 232-39.

Kaihao, Wang. "Poor subtitles mar prospects of Chinese cinema abroad." China Daily, 24 March 2016. 
Karamitroglou, Fotios. "A Proposed Set of Subtitling Standards in Europe." European Association for Studies in Screen Translation,

http://www.sub2learn.ie/downloads/karamitroglou fotiosa proposed set of subtitling stand ards in europe.pdf.

Koolstra, Cees M., and Johannes W.J. Beentjes. "Children's Vocabulary Acquisition in a Foreign Language through Watching Subtitled Television Programs at Home.". Educational Technology Research and Development 47, no. 1 (1999): 51-60.

Koolstra, Cees, Allerd Peeters, and Herman Spinhof. "The Pros and Cons of Dubbing and Subtitling." European Journal of Communication 17, no. 3 (2002): 325-54.

Kuo, Szu-Yu. "Quality in Subtitling: Theory and Professional Reality." Imperial College London, 2014.

LaBerge, David, and S. Jay Samuels. "Toward a Theory of Automatic Information Processing in Reading." Cognitive Psychology 6, no. 2 (1974): 293-323.

Lambourne, Andrew. "Future Trends in Subtitling." In 9th Languages in the Media Conference. Berlin, 2006.

Lang, Annie. "Defining Audio/Video Redundancy from a Limited Capacity Information Processing Perspective." Communication Research 22, no. 1 (1995): 86-115.

"Le cose che diciamo per via di Fantozzi." Il Post, http://www.ilpost.it/2017/07/03/fantozzi-paolovillaggio-lingua/.

Leach, Ben. "Susan Boyle of Britain's Got Talent gets subtitles on Oprah Winfrey." The Daily Mail, 2009.

Linde, Zoe De, and Neil Kay. "Processing Subtitles and Film Images." The Translator 5, no. 1 (1999): 45-60.

. The Semiotics of Subtitling. Manchester: St Jerome Publishing, 1999.

Lomheim, Sylfest. "L'écriture sur l'écran: stratégies de sous-titrage à NRK, une étude de cas." Translatio, Nouvelles de la FIT/FIT Newsletter 15, no. 3-4 (1995): 288-93.

Maher, Brigid. Recreation and Style: Translating humorous literature in Italian and English. John Benjamin's Translation Library. Amsterdam: John Benjamins Publishing Company, 2011.

Maori, Andrea. Luciano Salce prigioniero n. 120842. Storia di un intellettuale internato 1943-1945. Perugia: Tozzuolo, 2018.

Martin, Neil, Sharon Sadler, Clare Barrett, and Alison Beaven. "Measuring responses to humor: How the testing context affects individuals reaction to comedy." Humor 21, no. 2 (2008): 143-55.

Maszerowska, Anna, Anna Matamala, Pilar Orero, and Nina Reviers. "From source text to target text: The art of audio description." In Audio Description : New perspectives, edited by Anna Maszerowska, Anna Matamala and Pilar Orero. Amsterdam: John Benjamins Publishing Company, 2014. 
McDonough, Frank. "Class and Politics." In British Cultural Identities, edited by Mike Storry and Peter Childs, 177-206. London: Routledge, 2007.

McKenzie, Rory. "A Dark Horse in Translation Studies: Subtitling Kiwi Culture." Journal of Italian Translation 11, no. 2 (2016): 30-49.

. "Subtitling in the Classroom: Il secondo tragico Fantozzi (1976)." Neke: The New Zealand Journal of Translation 1, no. 1 (2018): 1-14.

Mereghetti, Paolo. Dizionario dei film 2011. Il Mereghetti. Milano: B.G Dalai editore, 2010.

Morreall, John. "Philosophy of Humor." In Stanford Encyclopedia of Philosophy, edited by Edward Zalta. California: Metaphysics Research Lab, Stanford University, 2012.

Mubenga, Kajingulu Somwe. "Investigating Norms in Interlingual Subtitling: A Systematic Functional Perspective." Perspectives 18, no. 4 (2010): 251-74.

Neuman, Susan B., and Patricia Koskinen. "Captioned Television as Comprehensible Input: Effects of Incidental Word Learning from Context for Language Minority Students." Reading Research Quarterly 27, no. 1 (1992): 94-106.

Nilsen, Alleen, and Don Nilsen. The Language of Humor: An Introduction. Cambridge: Cambridge University Press, 2018.

Nornes, Mark Abé. Cinema Babel: Translating Global Cinema. Minneapolis: University of Minnesota Press, 2007.

. "For an Abusive Subtitling." Film Quarterly 52, no. 3 (1999): 17-34.

O'Sullivan, Carol. "'A splendid innovation, these English titles!' The invention of Subtitling in the USA and the UK." Proceedings of the British Academy 218 (2018): 267-90.

. Translating Popular Film. Basingstoke: Palgrave MacMillan, 2011.

Pedersen, Jan. Subtitling Norms for Television: An Exploration Focusing on Extralinguistic Cultural References. Amsterdam: John Benjamins, 2011.

Pelsmaekers, Katja, and Fred Van Eesien. "Subtitling Irony." The Translator 8, no. 2 (2002): 241-66.

Perego, Elisa, Fabio Del Missier, Marco Porta, and Maoro Mosconi. "The Cognitive Effectiveness of Subtitle Processing." Media Psychology 13, no. 3 (2010): 243-72.

Pérez-González, Luis. Audiovisual Translation: Theories, Methods and Issues. New York: Taylor and Francis, 2014.

Pérez, Francisco Javier Díaz. "Worldplay in film titles: Translating English puns into Spanish." Babel 54, no. 1 (2008): 36-58.

Pergolari, Andrea, and Emanuele Salce. Luciano Salce: Una vita spettacolare. Roma: Edilazio, 2009.

Perteghella, Manuela. "Language and Politics on Stage: Strategies for Translating Dialect and Slang with References to Shaw's Pygmalion and Bond's Saved." Translation Review 64, no. 1 (2002): 45-53.

Pirandello, Luigi. L'umorismo. Milano: Garzanti, 1908.

Pittman, Robert. "We're talking the wrong language to 'TV Babies.'." New York Times, 1990. 
Pym, Anthony. Exploring Translation Theories. New York: Routledge, 2014.

Rhodes, Joe. "The Making of The Simpsons." Entertainment Weekly, http://ew.com/article/1990/05/18/making-simpsons/.

Ricatti, Francesco. "Humiliation and Love: Villaggio, Benigni and the cultural politics of emotions." Incontri: Rivista europea di studi italiani 29, no. 2 (2014): 8-18.

Rigoletto, Sergio. "The Italian Comedy of the Economic Miracle." In Italy On Screen: National Identity and Italian Imaginary, edited by Lucy Bolton and Christina Siggers Manson, 31-47. Bern: Peter Lang, 2010.

Robertson, James Napier. "The Dark Horse." Transmission Films, 2014.

Romualdi, Massimiliano. "41 anni di Fantozzi: com'è cambiata l'Italia?" Artspecialday, http://www.artspecialday.com/9art/2016/03/27/41-anni-di-fantozzi-come-cambiata-litalia/.

Rota, Alessandra. "Paolo Villaggio - Il ragionier Ugo è un vendicatore." La Repubblica, 5 Luglio 1999.

Salway, Andrew. "A corpus-based analysis of audio description." In Media for all: Subtitling for the deaf, audio description and sign language, edited by Jorge Diaz-Cintas, Pilar Orero and Aline Remael, 151-74. Amsterdam: Rodopi, 2007.

Savransky, Rebecca. "Gary Johnson: Trump watching Olympics to see how high Mexican pole vaulters go." The Hill, http://thehill.com/blogs/ballot-box/presidential-races/290625-garyjohnson-trump-watching-olympics-to-see-how-high.

Schauffler, Svea. Subtitling Wordplay - An Audience Study: Approaches to the Translation of Linguistic Humour. Saarbrücken: Scholars' Press, 2012.

Schröter, Thorsten. "Language-play, Translation and Quality - with Examples from Dubbing and Subtitling." In Translation, Humour and The Media, edited by Delia Chiaro. London: Continuum International Publishing Group, 2010.

. "Quantity and quality in screen translation." Perspectives: Studies in Translatology 11, no. 2 (2003): 105-24.

_. "Shun the Pun, Rescue the Rhyme? The Dubbing and Subtitling of Language-Play in Film." Karlstad University, 2005.

Scrivano, Paolo. "Signs of Americanization in Italian Domestic Life: Italy's Postwar Conversion to

Consumerism." Journal of Contemporary History 40, no. 2 (2005): 317-40.

Simone, Marco De. Memorie dal sottoscala. Rieti: Amarganta, 2015.

Spinazzola, Vittorio. Cinema e pubblico: Lo spettacolo filmico in Italia 1954-1965. Rome: Bulzoni, 1985.

"There's going to be a Te reo Maori version of Moana." Stuff, http://www.stuff.co.nz/entertainment/film/93512377/theres-going-to-be-a-te-reo-maoriversion-of-moana. 
"Timed Text Style Guide: General Requirements ". Netflix,

https://partnerhelp.netflixstudios.com/hc/en-us/articles/215758617-Timed-Text-Style-Guide-

General-Requirements.

Treisman, Anne. "Strategies and Models of Selective Attention." Psychological Review 76, no. 3 (1969): 282-99.

Valle, Valeria Della, and Giuseppe Patota. Viva il congiuntivo! : Sperling \& Kupfer, 2011.

Venuti, Lawrence. The Translator's Invisibility: A History of Translation. 2nd ed. New York: Routledge, 2008.

Villaggio, Paolo. Fantozzi. Milano: Rizzoli, 1971.

—. Fantozzi, rag. Ugo. Milano: RCS Libri S.p.A, 2013.

. Fantozzi: L'audiolibro inedito. Volume S.R.L., 2016.

Villaggio, Paolo, and Luca Sommi. Non mi fido dei santi: autobiografia bugiarda. 2011.

Wescott, Sarah. "When words fail BBC's subtitles: Ironic mess up on Breakfast show." In Express. London: Express, 2015.

Whitehead, Jill. "What is audio description." International Congress Series 1282 (2005): 960-63.

Whorf, Benjamin Lee. "A Linguistic Consideration of Thinking in Primative Communities." In Language, Thought and Reality: Selected Writings of Benjamin Lee Whorf, edited by John Bissell Carroll. Massachusetts: MIT Press, 1956.

Williams, Gareth Ford. "Subtitling Guidelines v1.0." BBC, https://www.bbc.co.uk/guidelines/futuremedia/accessibility/subtitling_guides/online_sub_edit orial guidelines vs1 1.pdf.

Wissmath, Bartholomäus, David Weibel, and Rudolf Groner. "Dubbing or subtitling?: Effects on spatial presence, transportation, flow, and enjoyment." Journal of Media Psychology: Theories, Methods, and Applications 21, no. 3 (2009): 114-25.

Zabalbeascoa, Patrick. "Humor and translation - an interdiscipline." International Journal of Humor Research 18, no. 2 (2005): 185-207.

_ "The nature of the audiovisual text and its parameters." In The Didactics of Audiovisual Translation, edited by Jorge Diaz-Cintas, 21-37. Amsterdam: John Benjamins, 2008. "Translating Jokes for Dubbed Television." The Translator 2, no. 2 (1996): 235-57. 


\section{Appendices}

1. The original Fantozzi short stories from $L^{\prime}$ Europeo (25 ${ }^{\text {th }}$ July 1968 - 10 April 1969$)$

2. A selection of Fantozzi comics from Il Corrierino (June 1993-August 1993) 

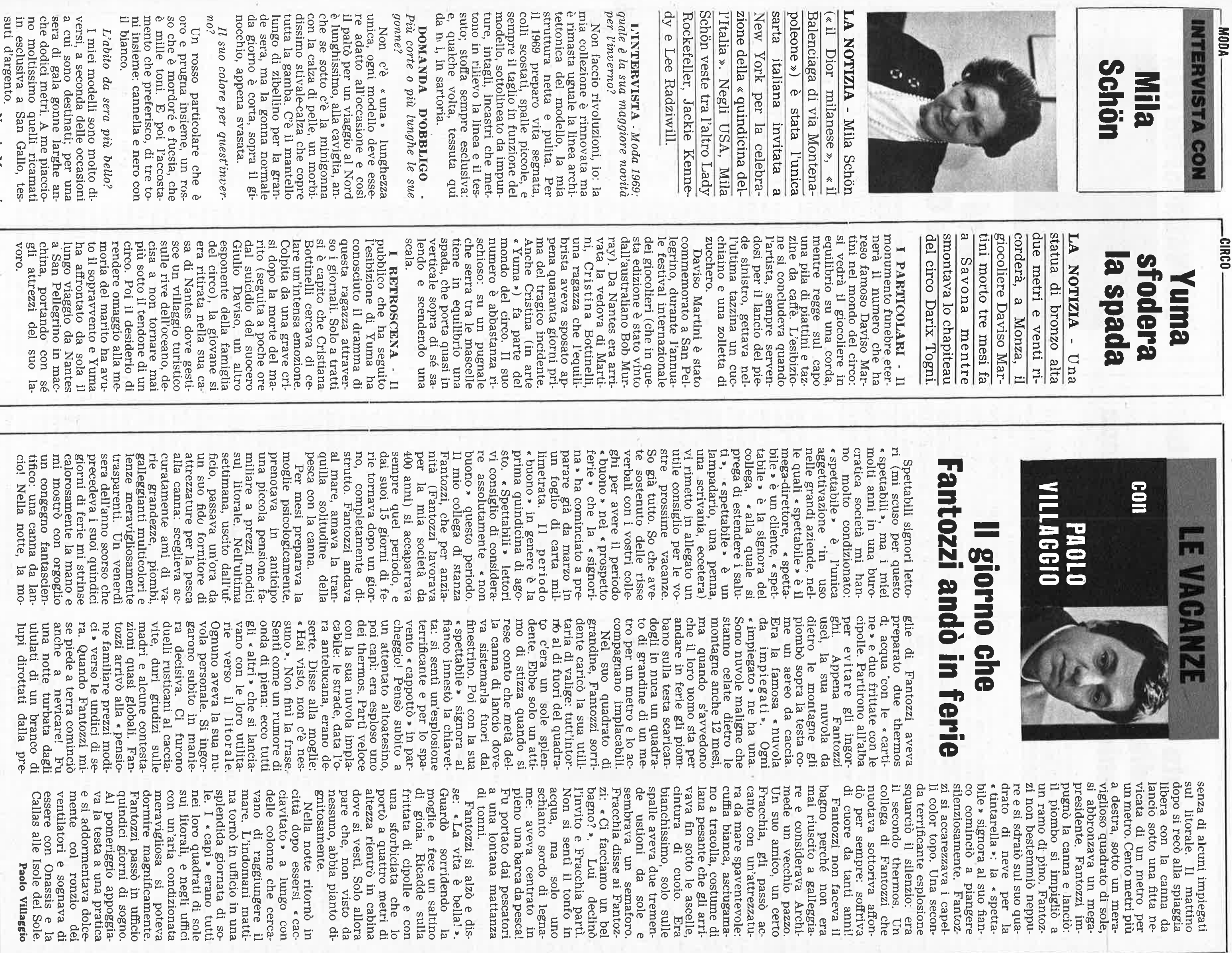

$r$
0
3
0
$N$
0
0
0
0
0
0
$N$
0
0 


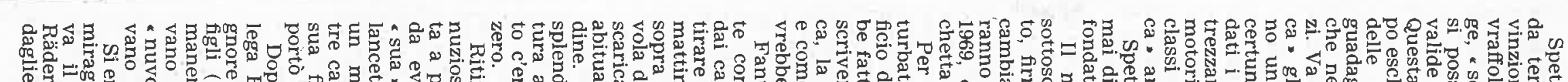

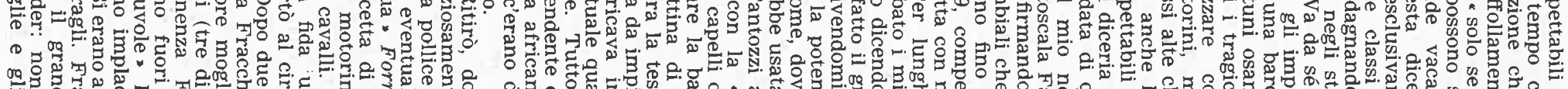

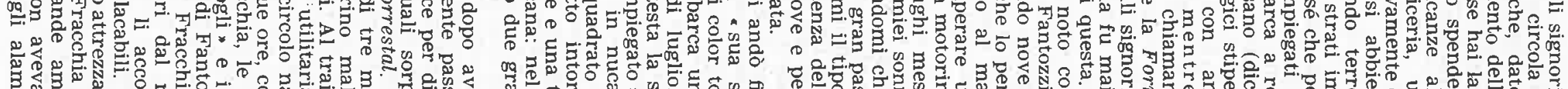

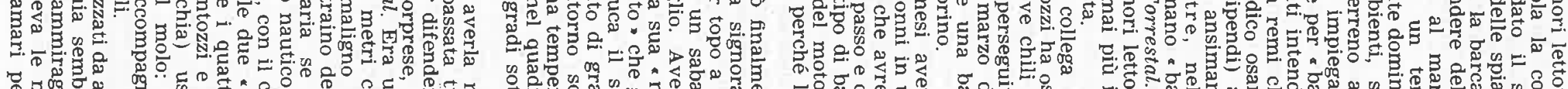

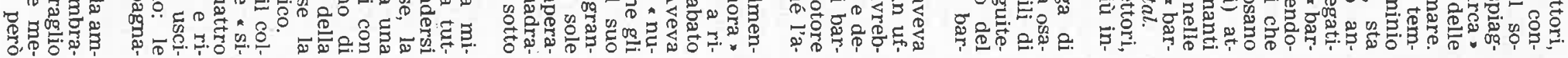

\begin{tabular}{|c|c|c|}
\hline 溜( & (1) & 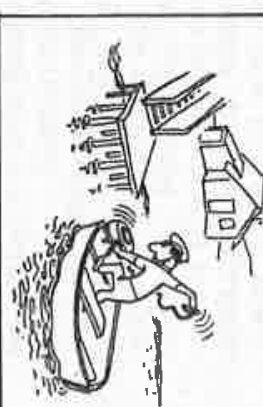 \\
\hline 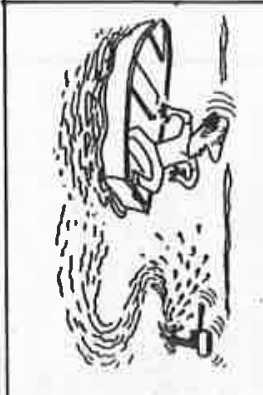 & 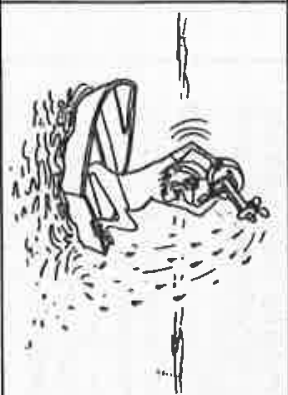 & 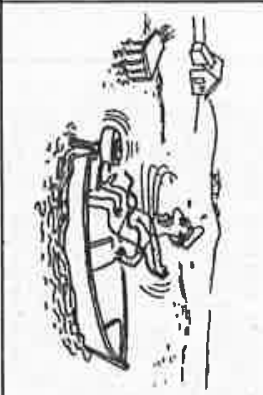 \\
\hline
\end{tabular}
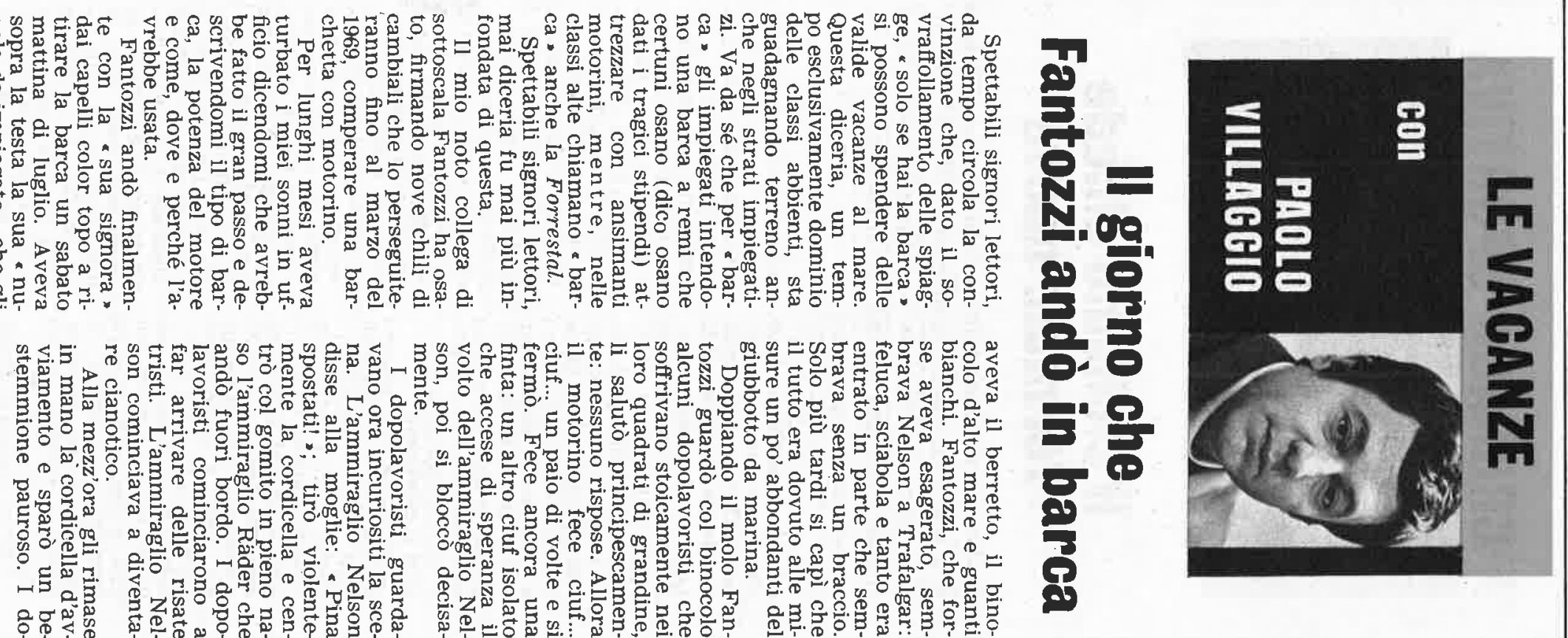

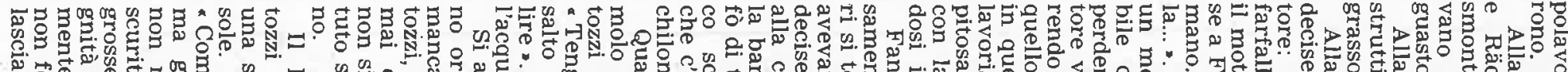

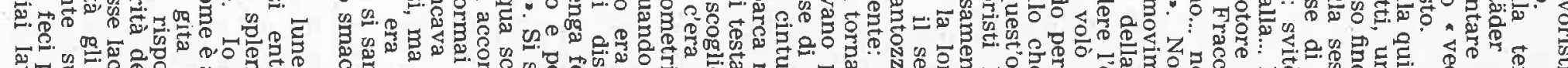

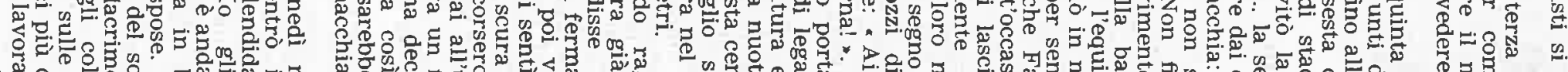

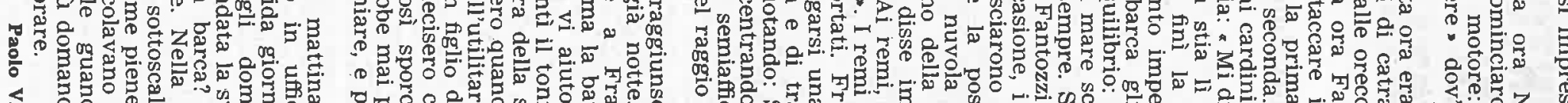

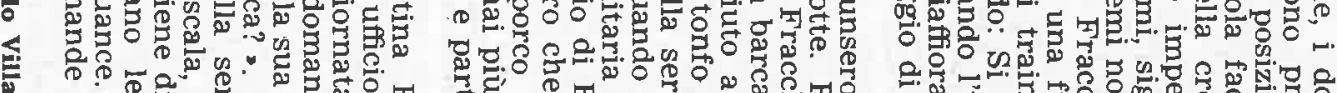

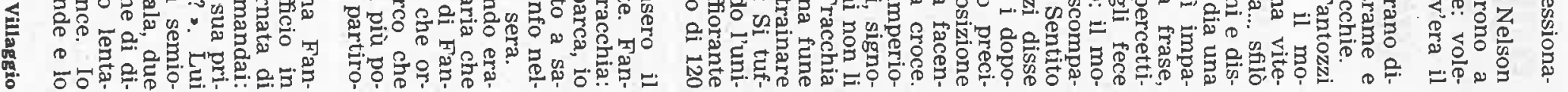

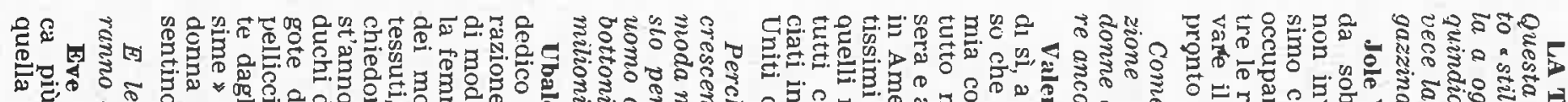

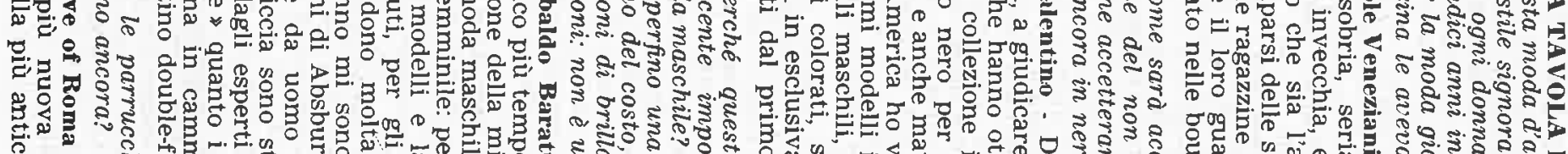

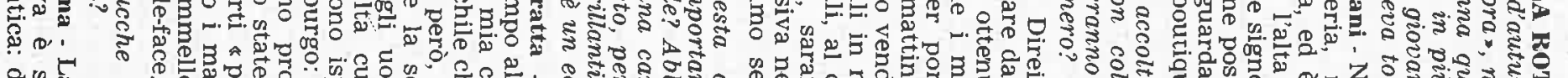
\&W

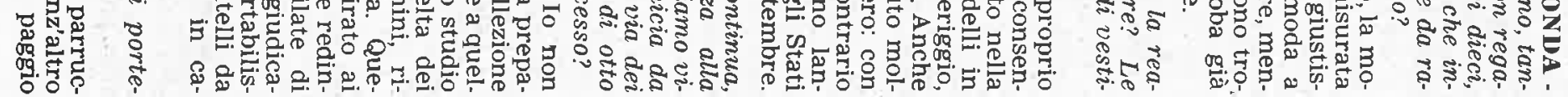

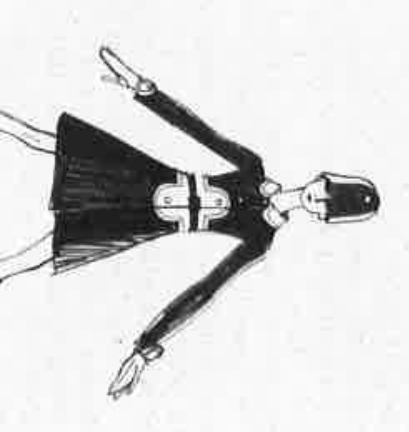

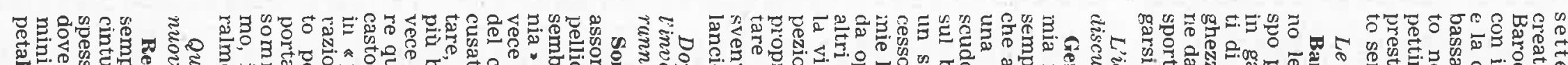

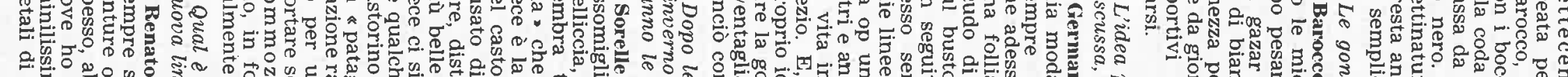

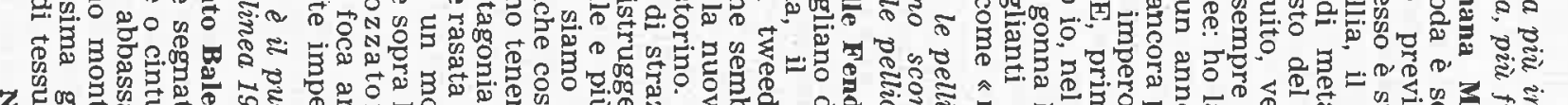

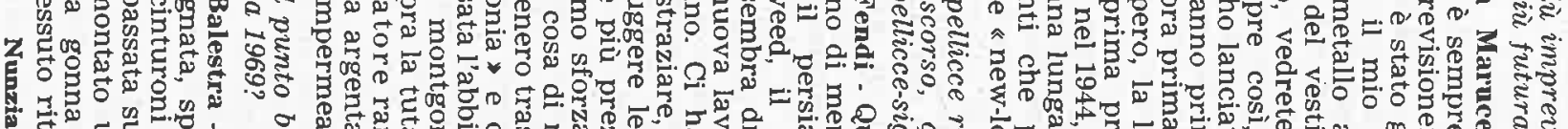

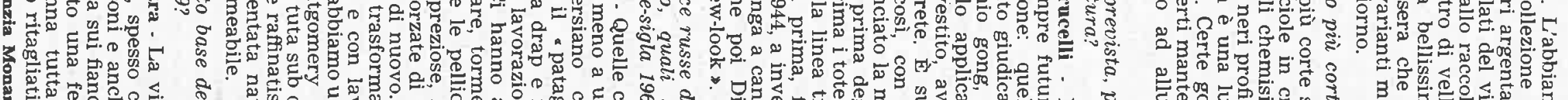

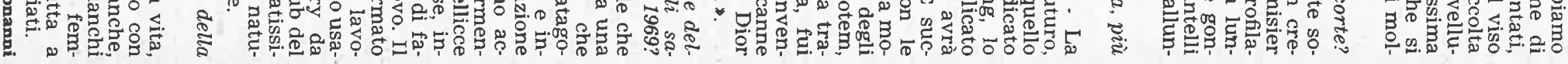
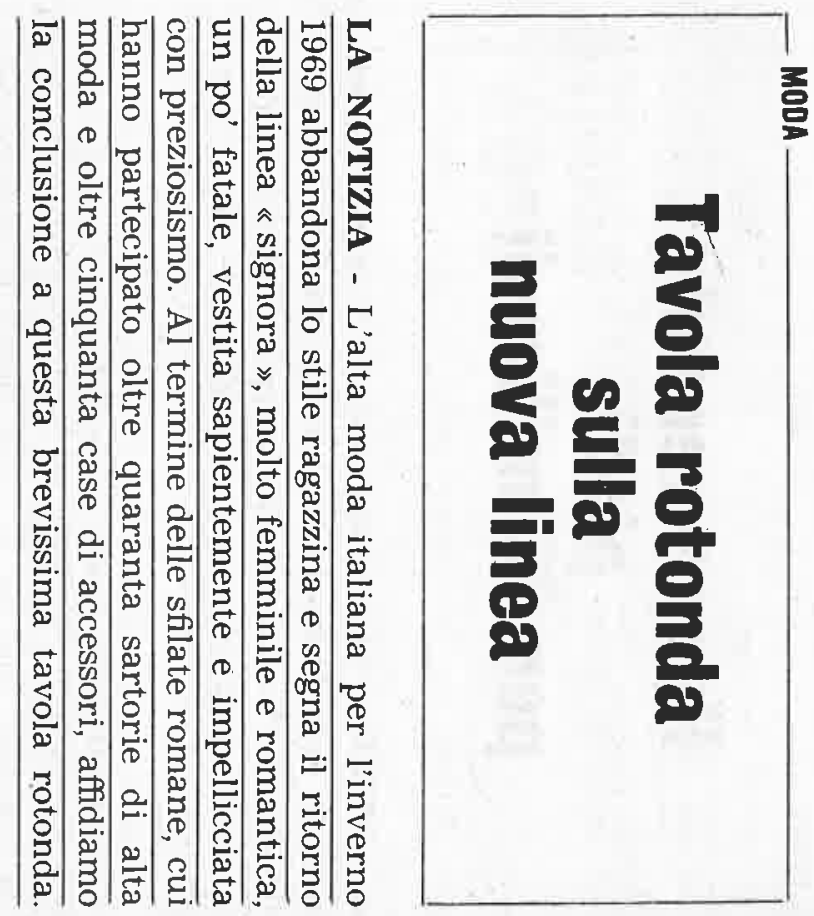

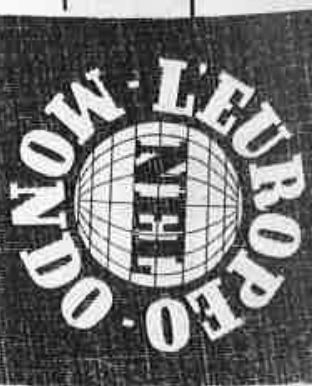

1
2
N
0
0
0
0
0
0
0 


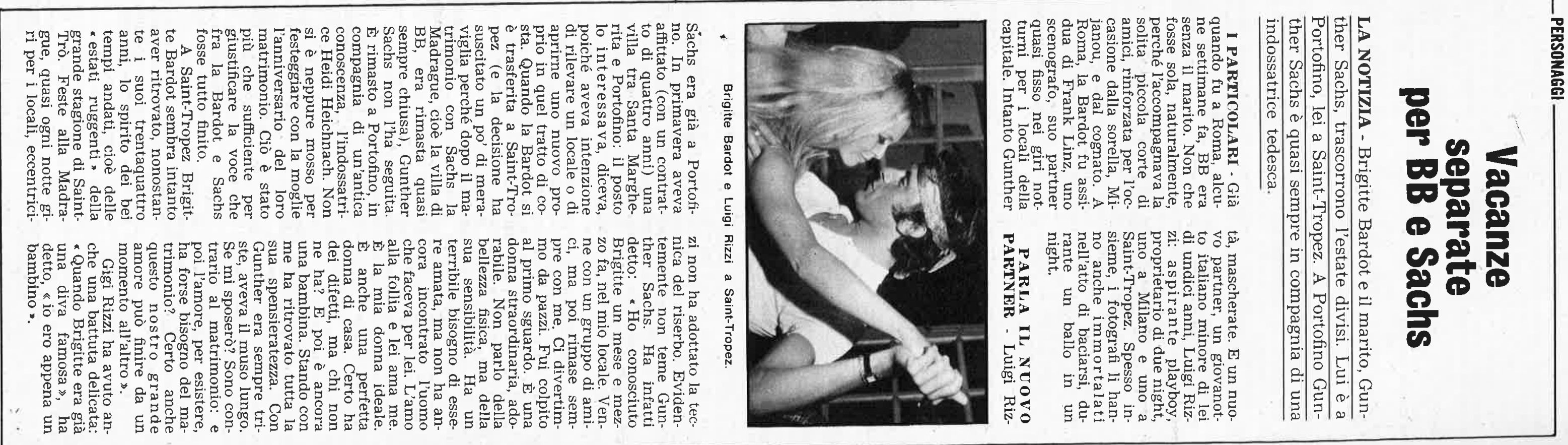




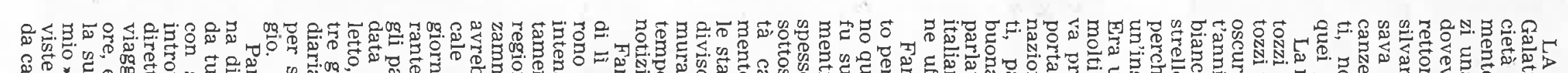

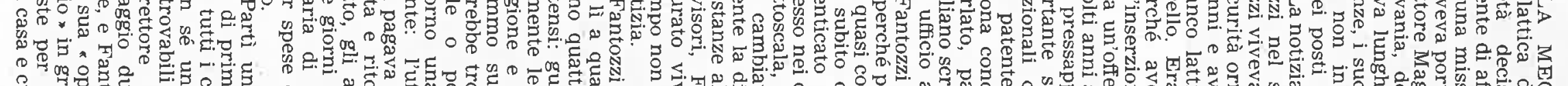

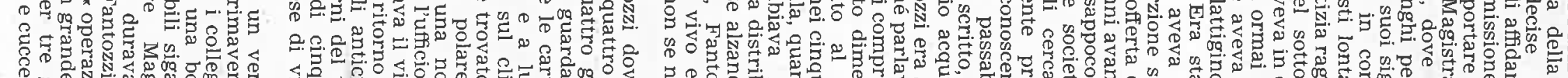

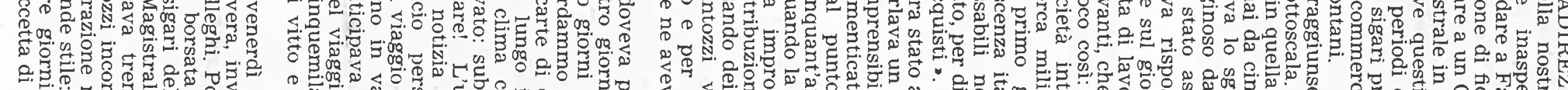

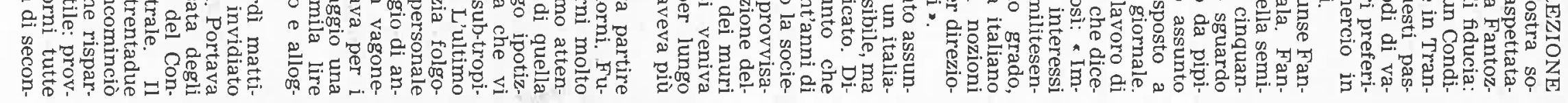

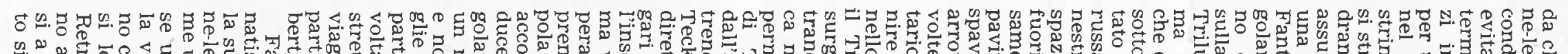
w. F,

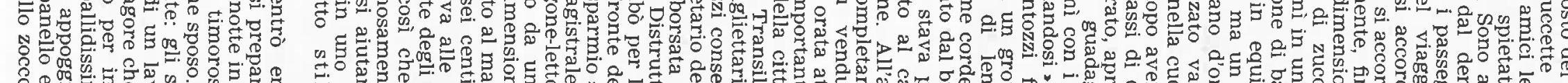

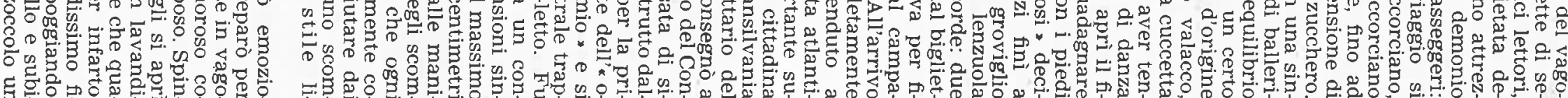

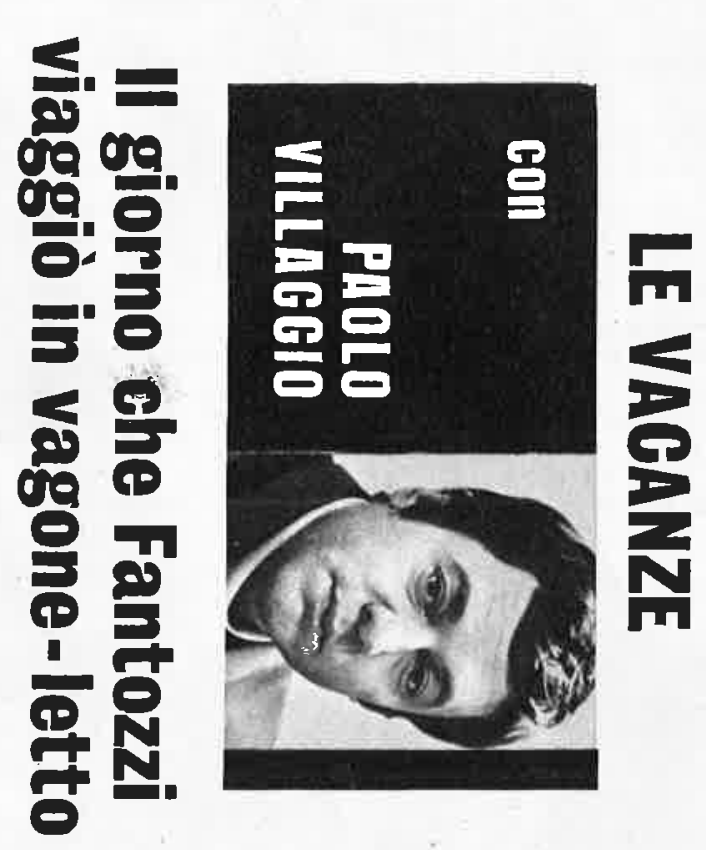

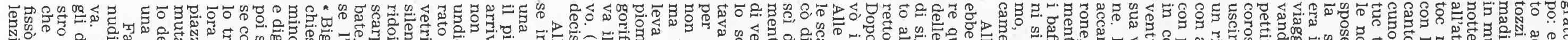

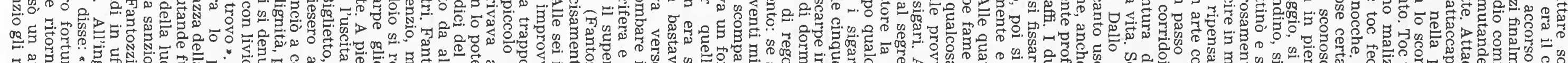

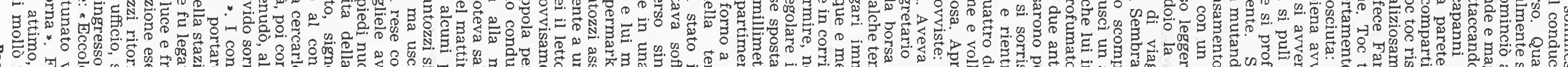

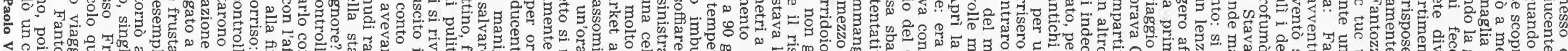

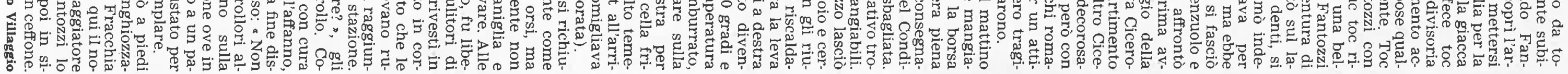

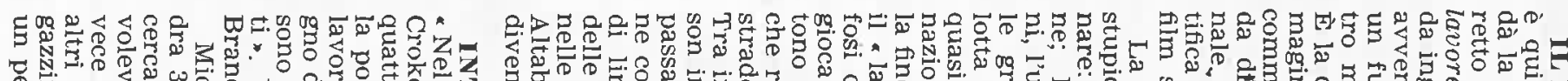

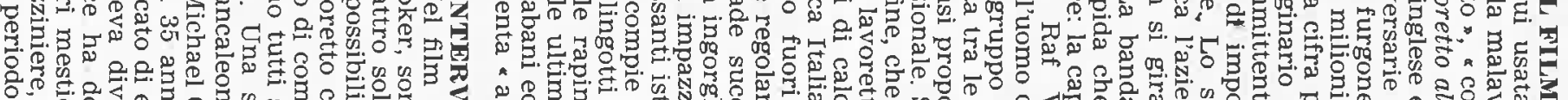

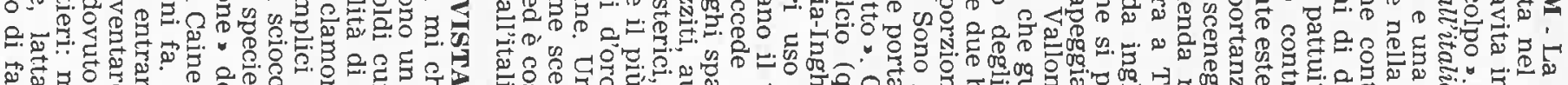

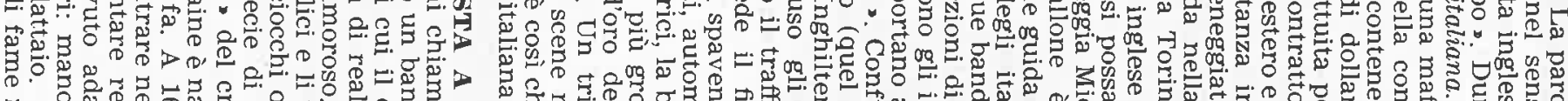

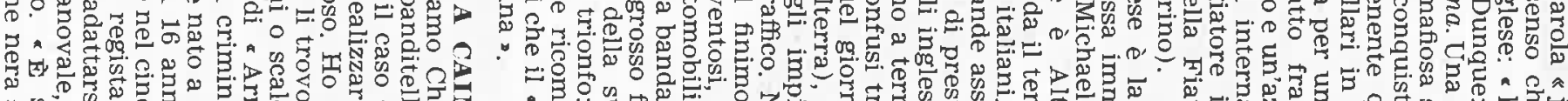

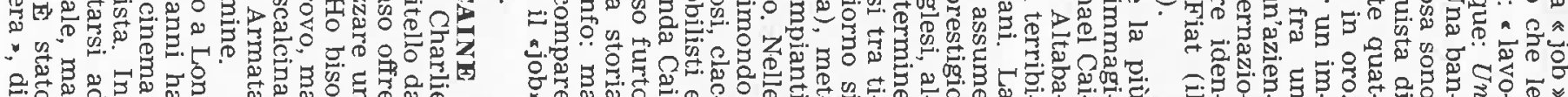

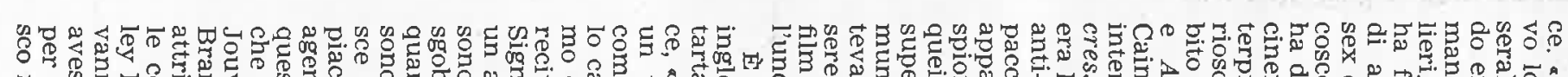

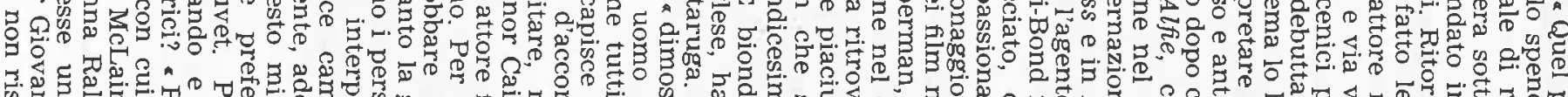

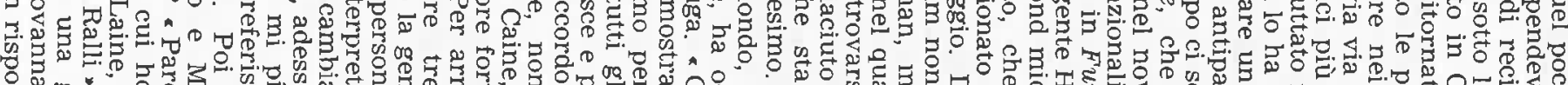

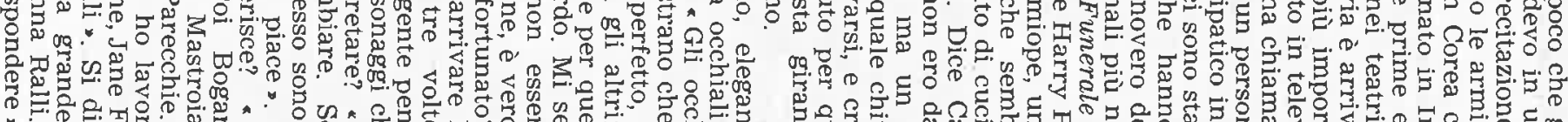

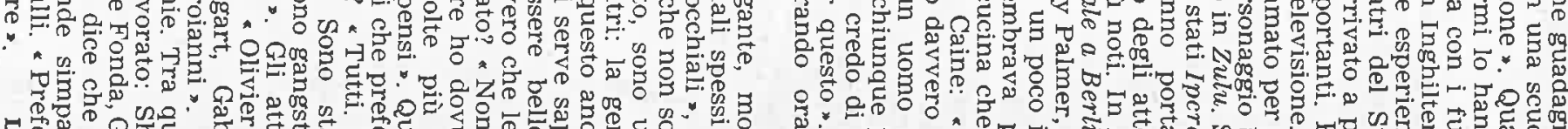

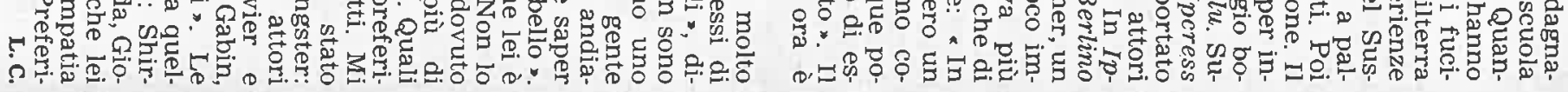
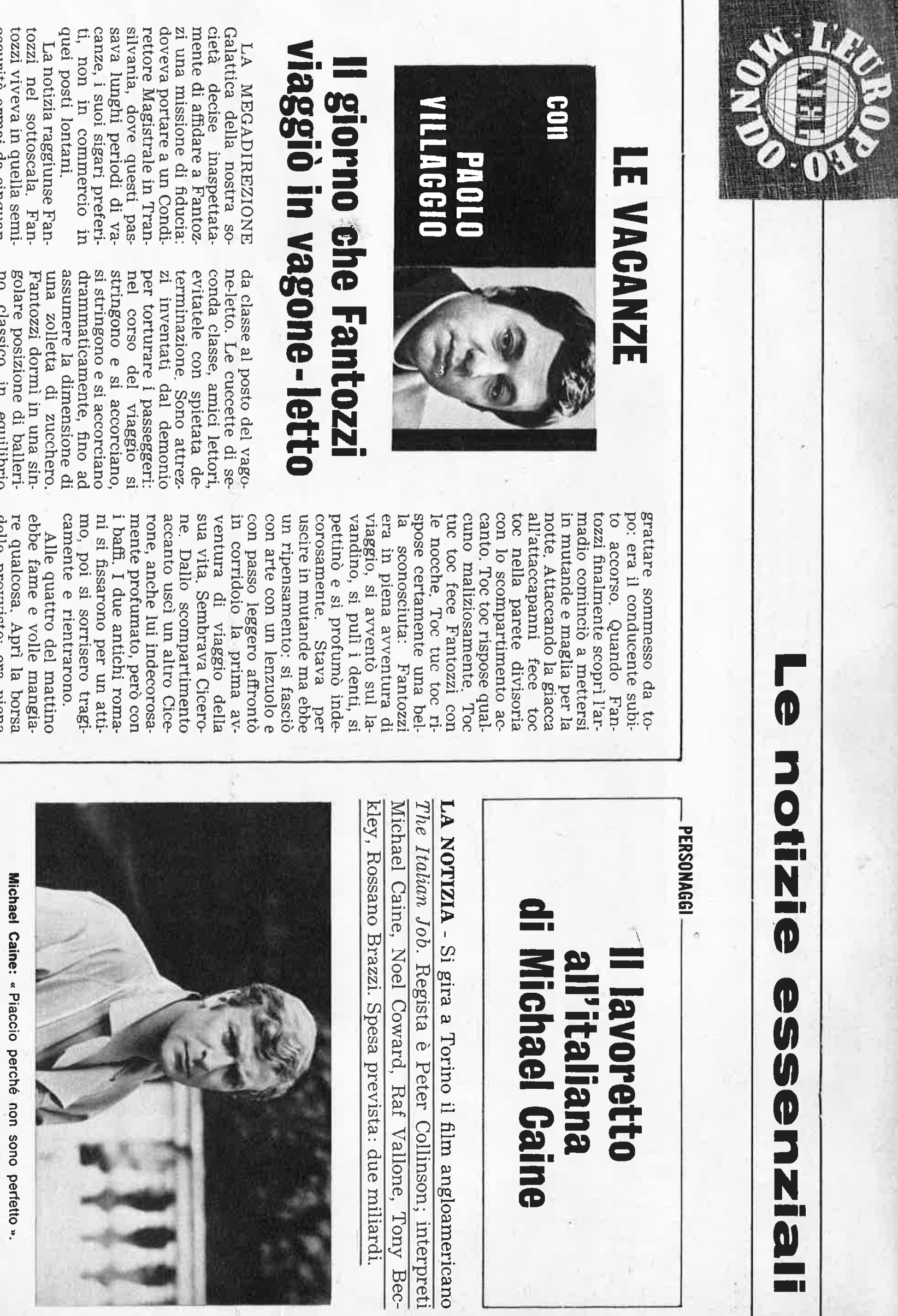


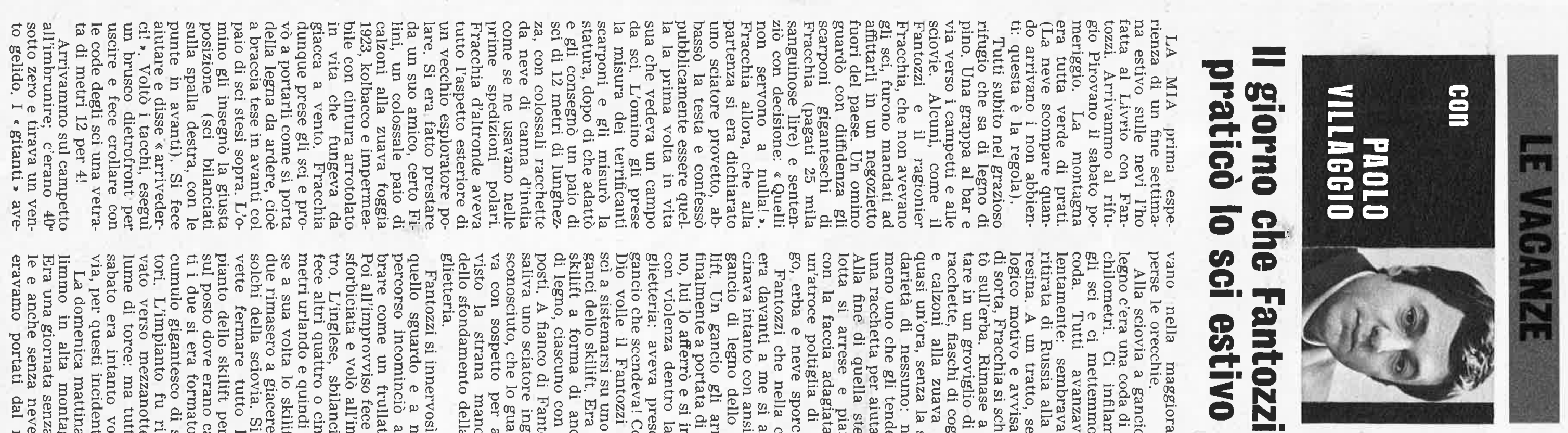

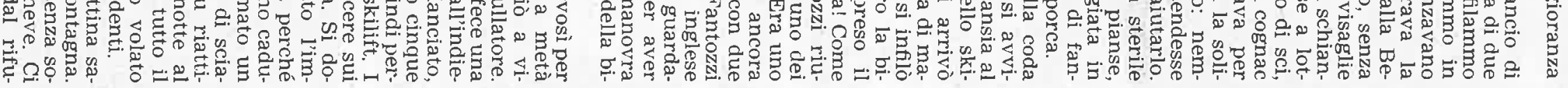

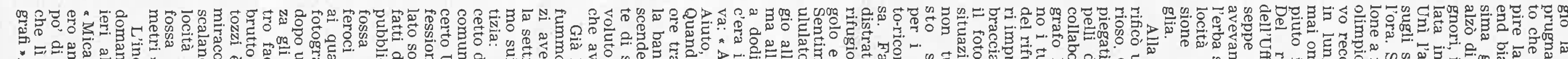

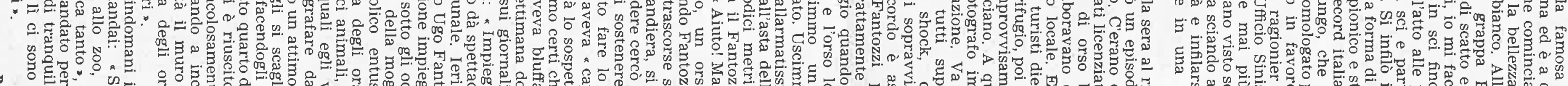
J

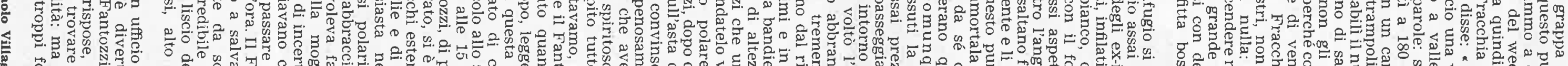
要

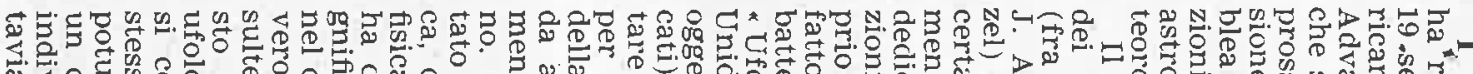

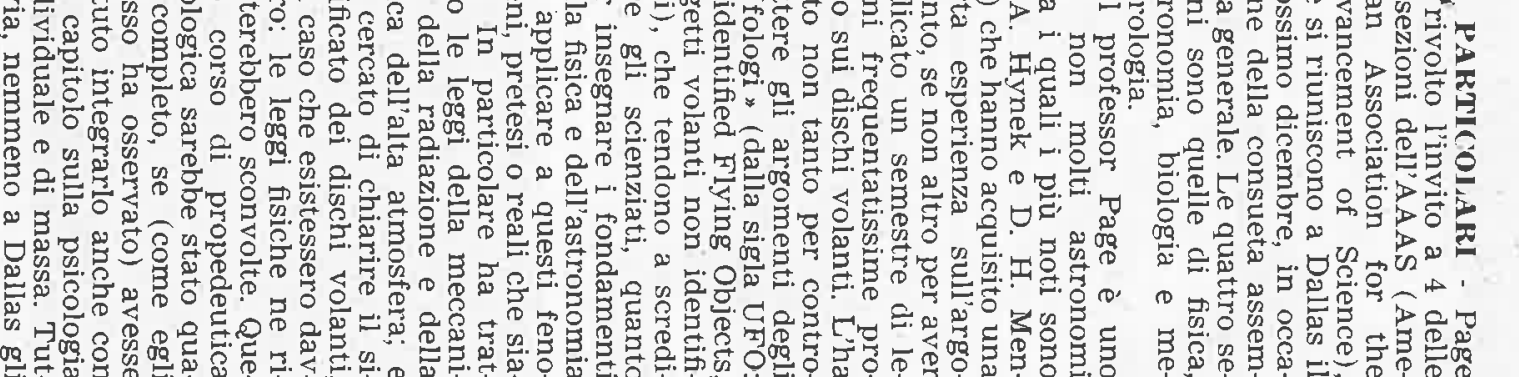

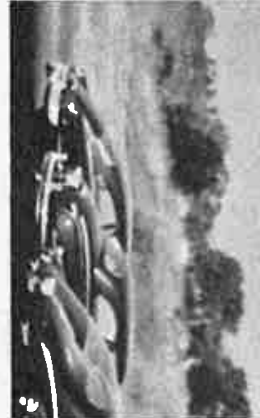

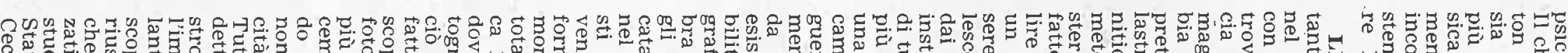

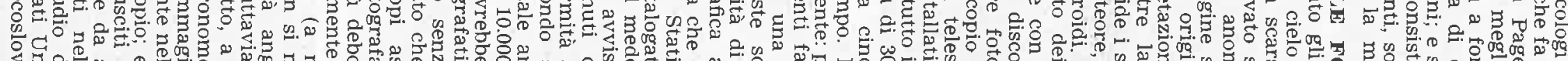
政

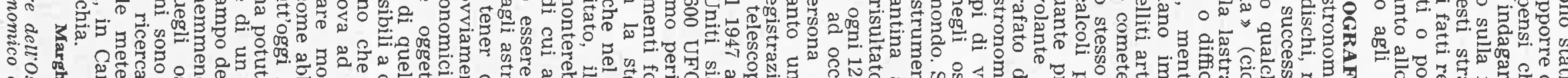

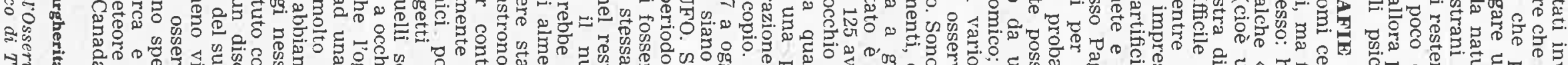

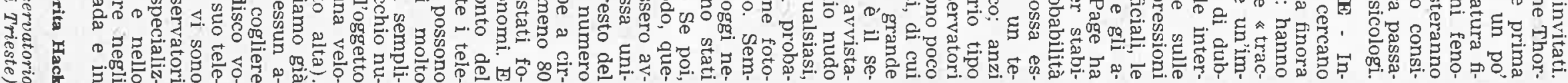
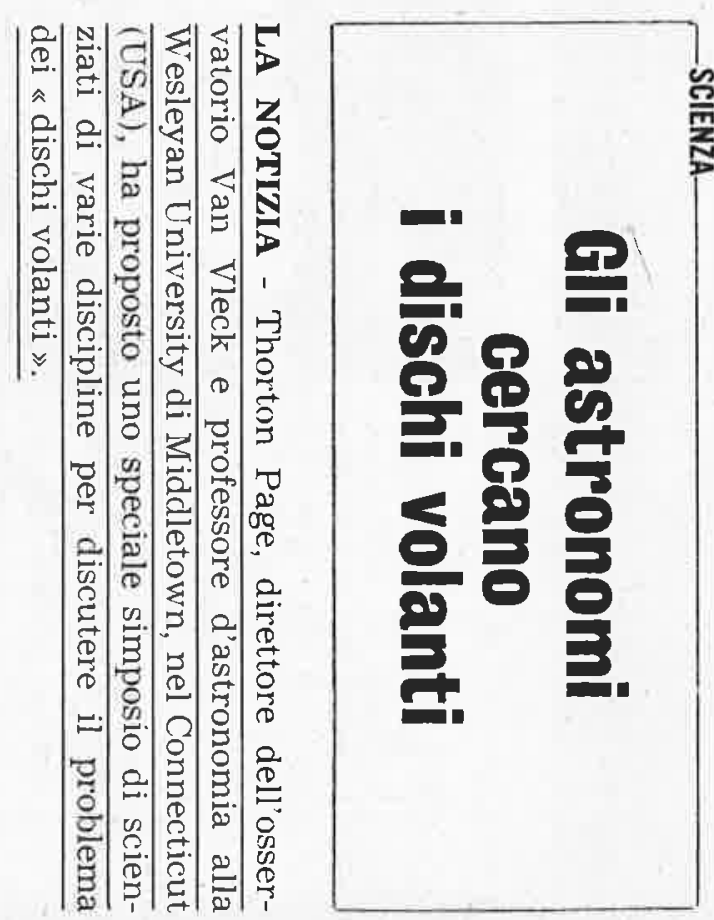

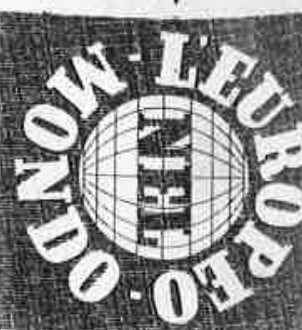

1
0
0
5
$N$
0
0
0
0
0
2
$N$
0
0 

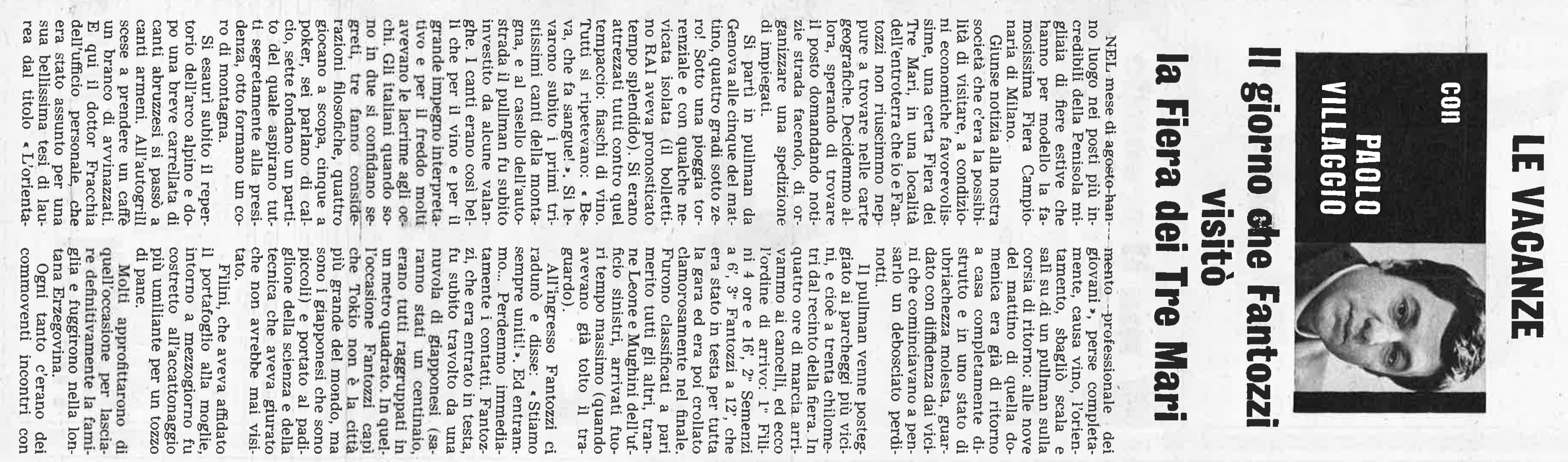

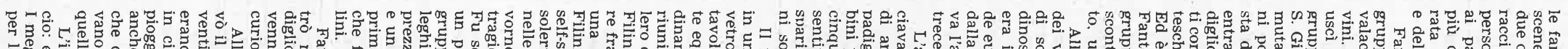
w.

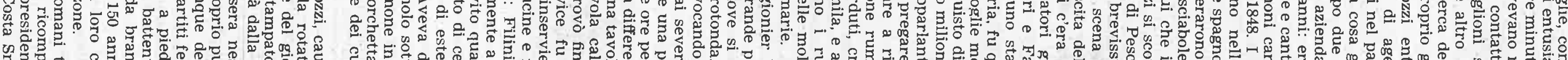

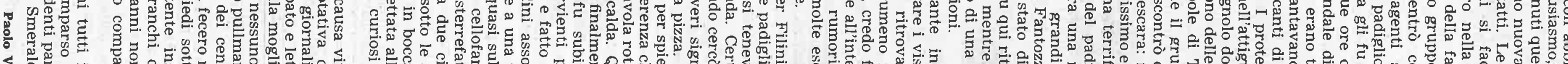

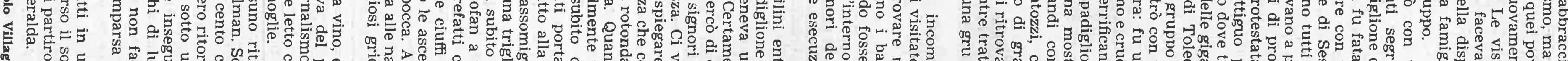

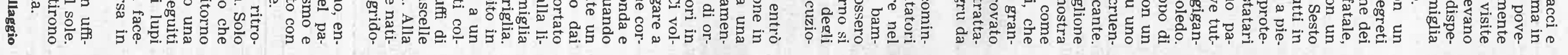

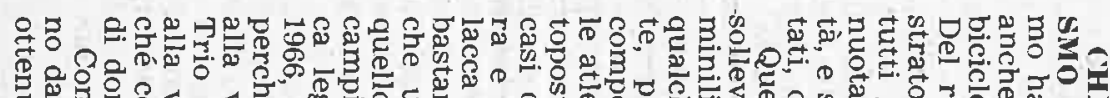

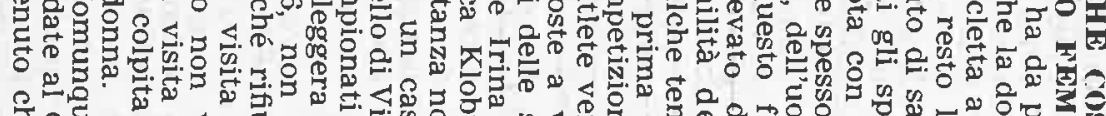
等. 和 它。

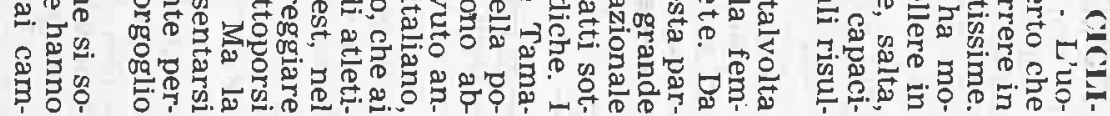

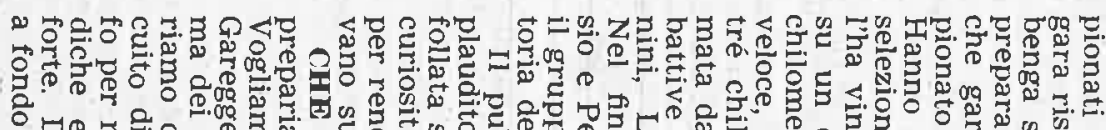

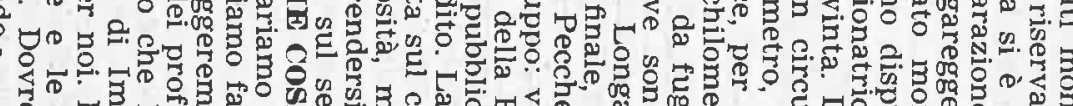

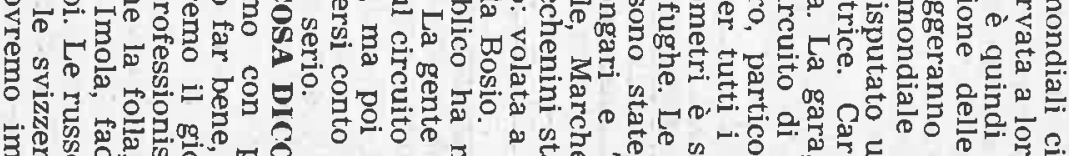

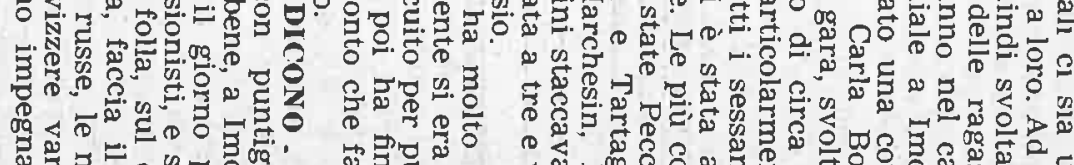

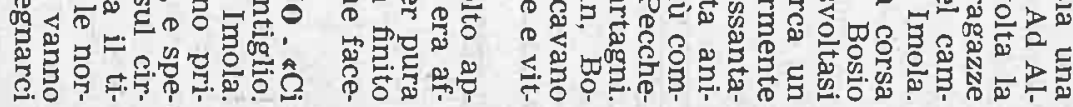
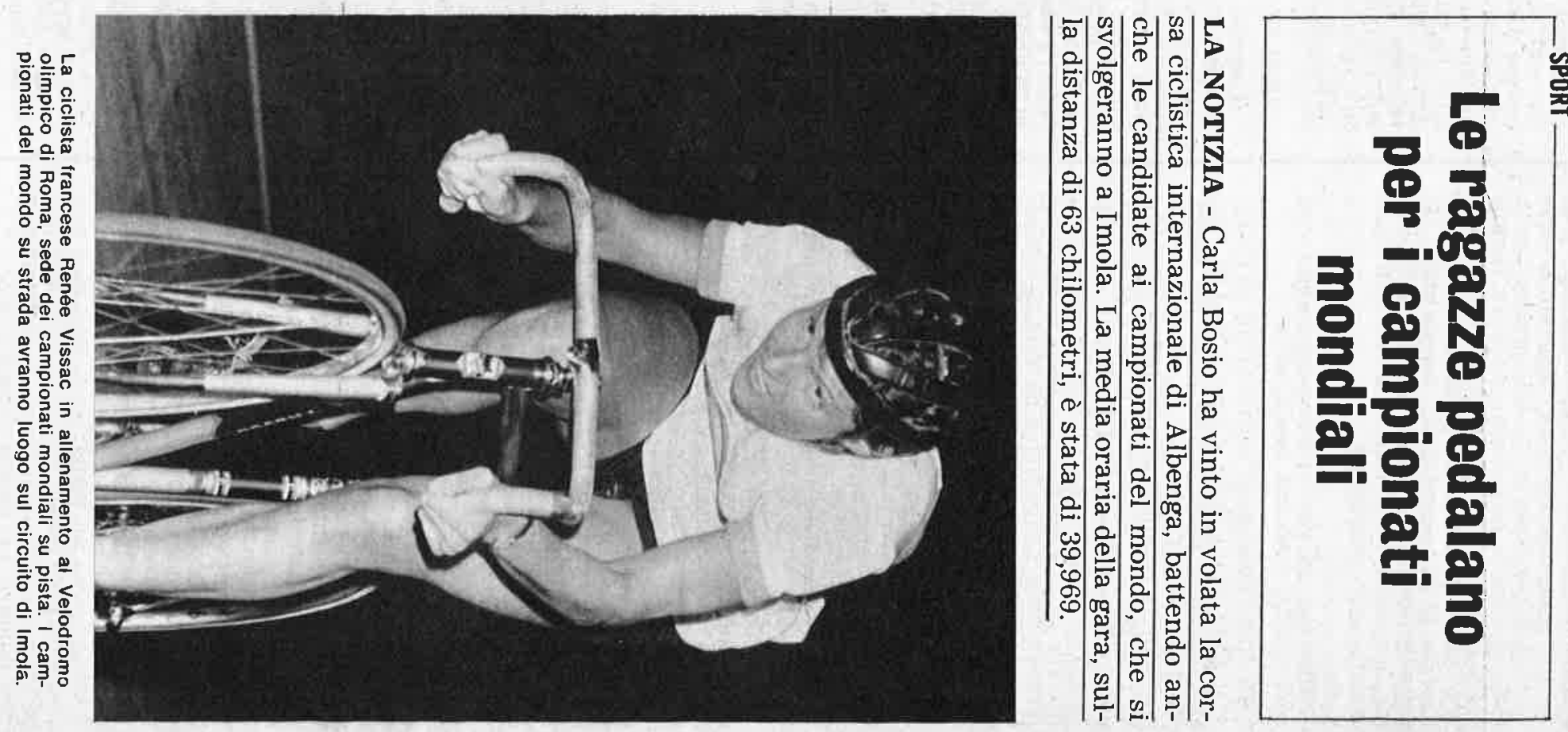


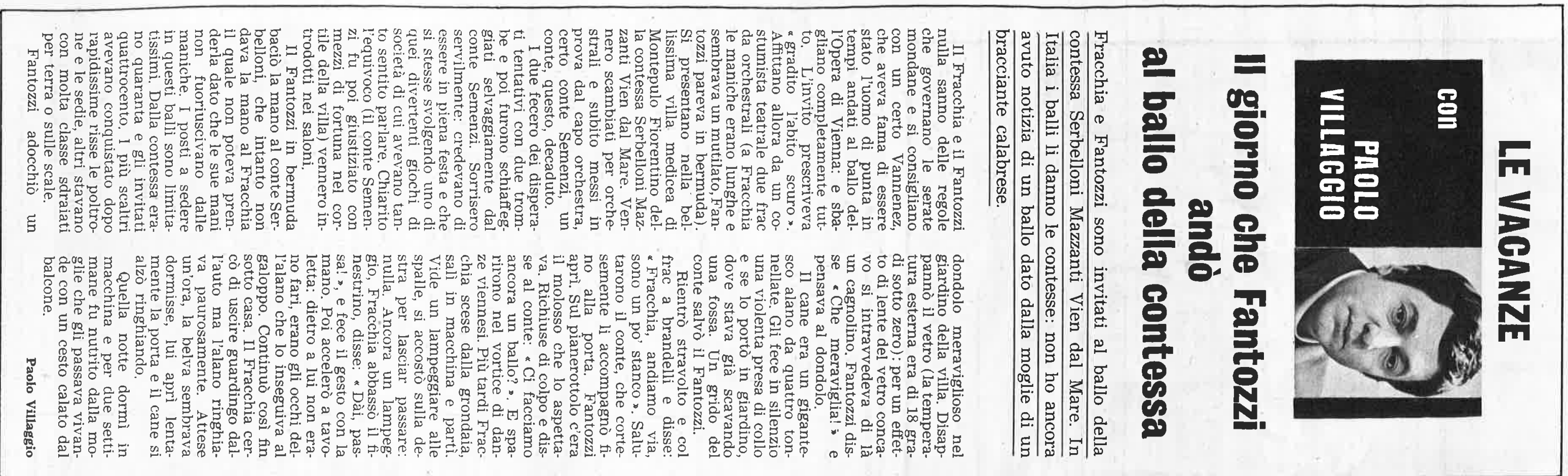

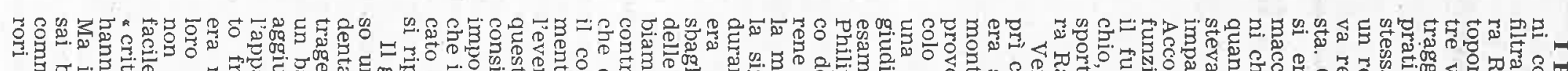

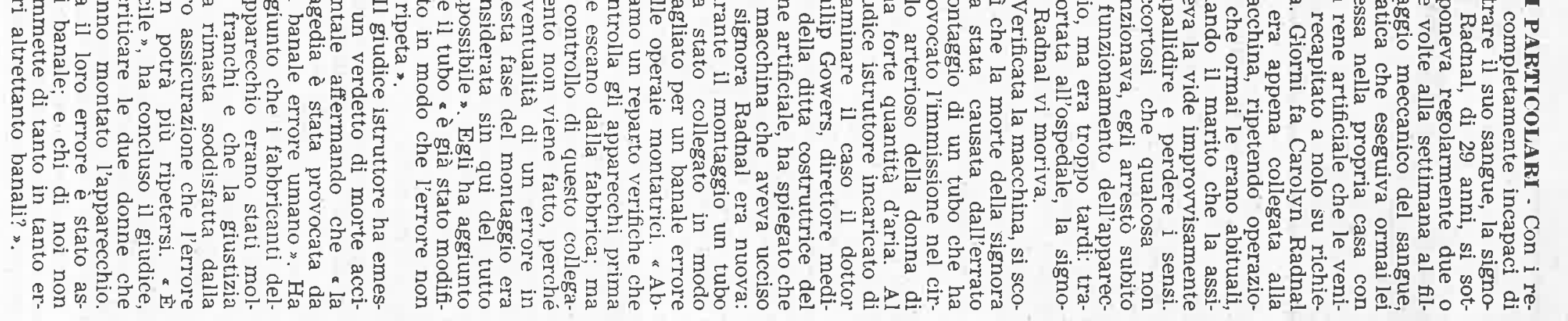
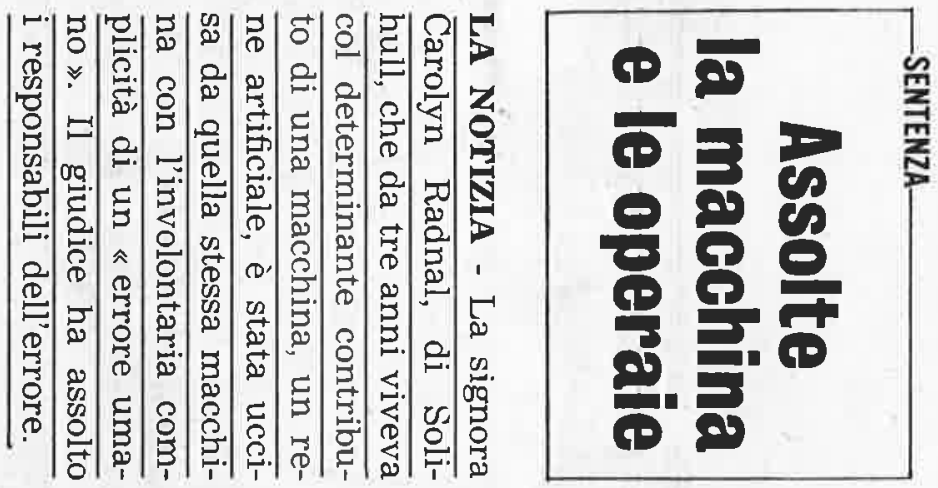

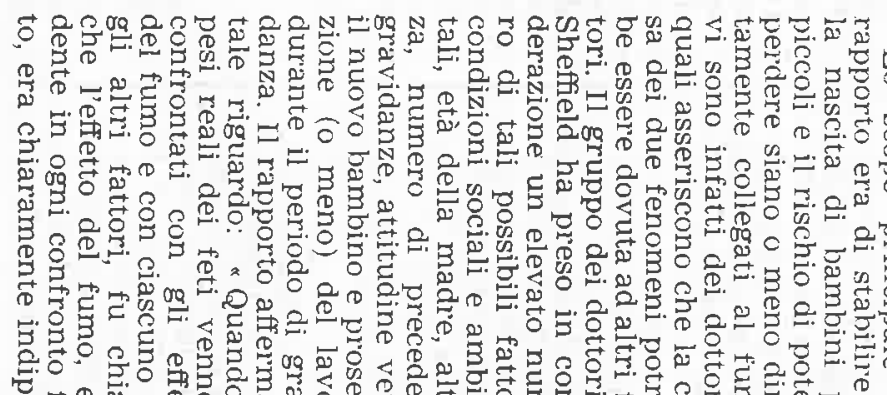

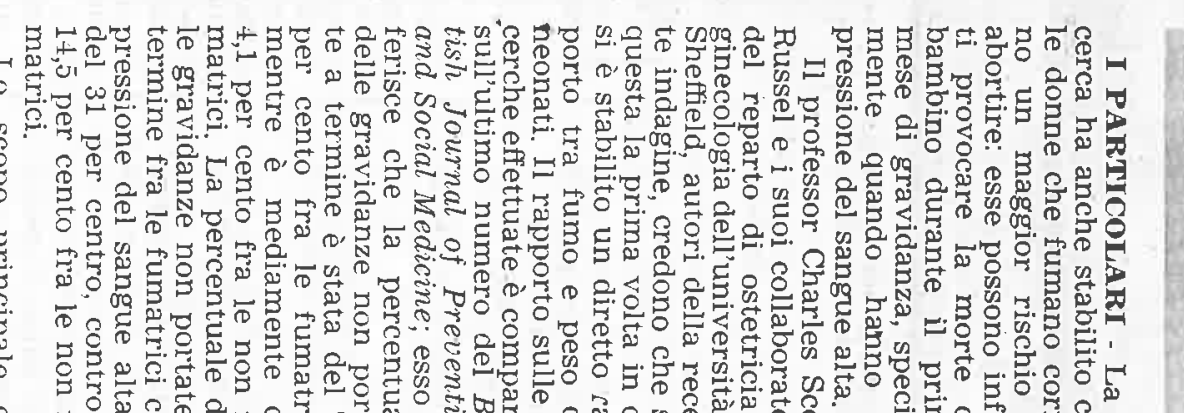

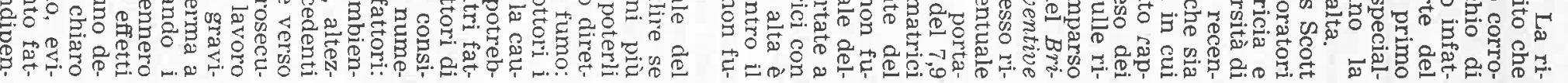

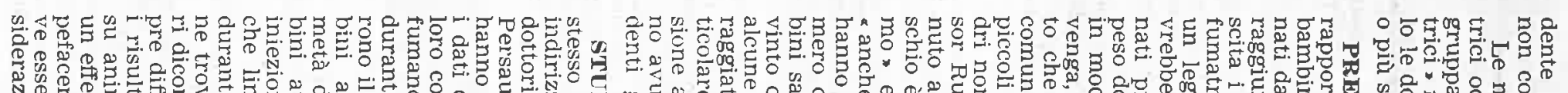

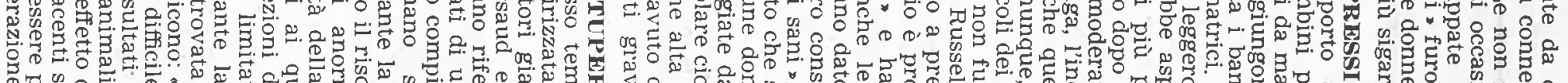

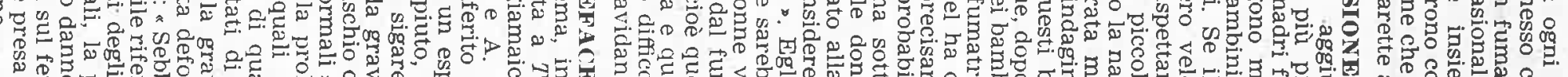

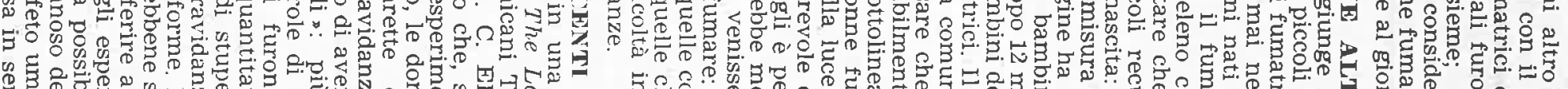

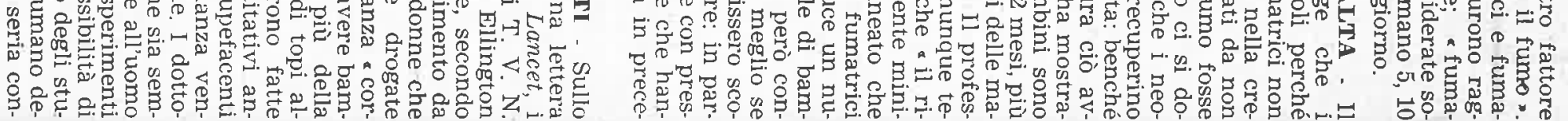
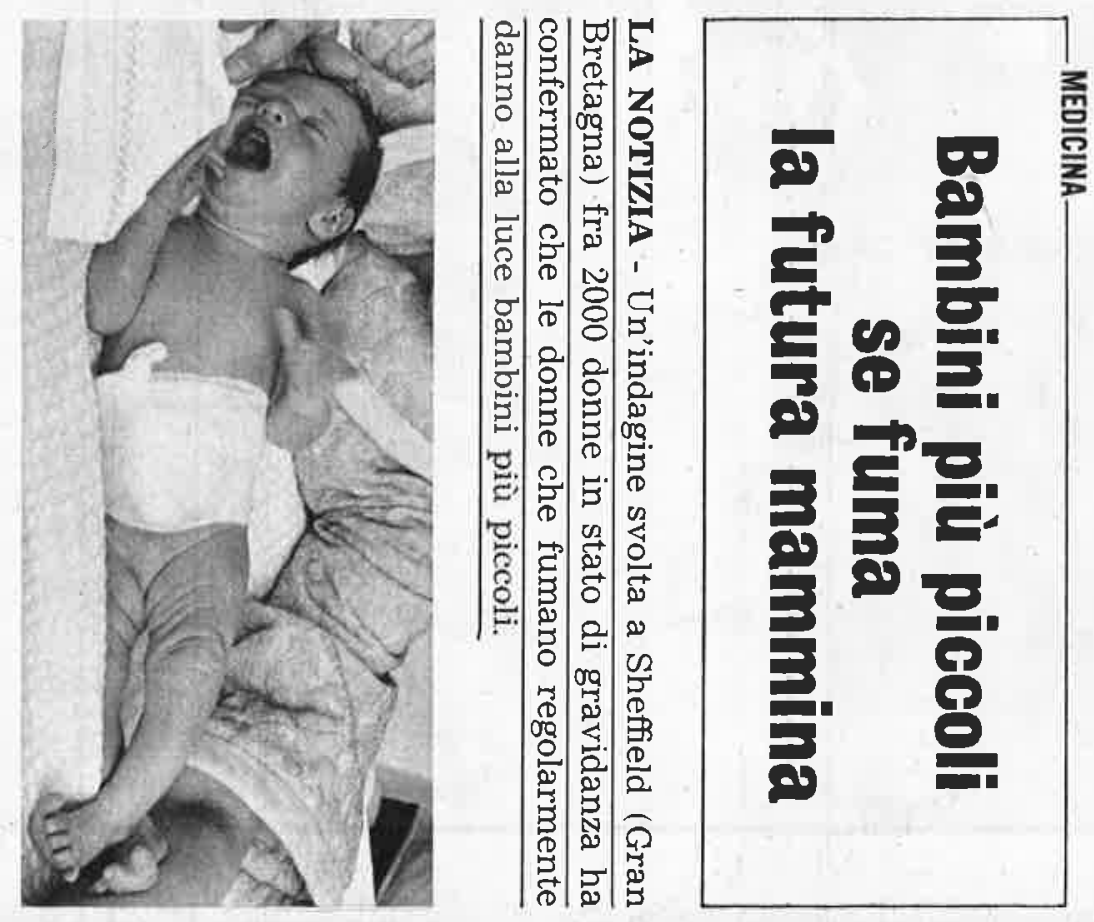


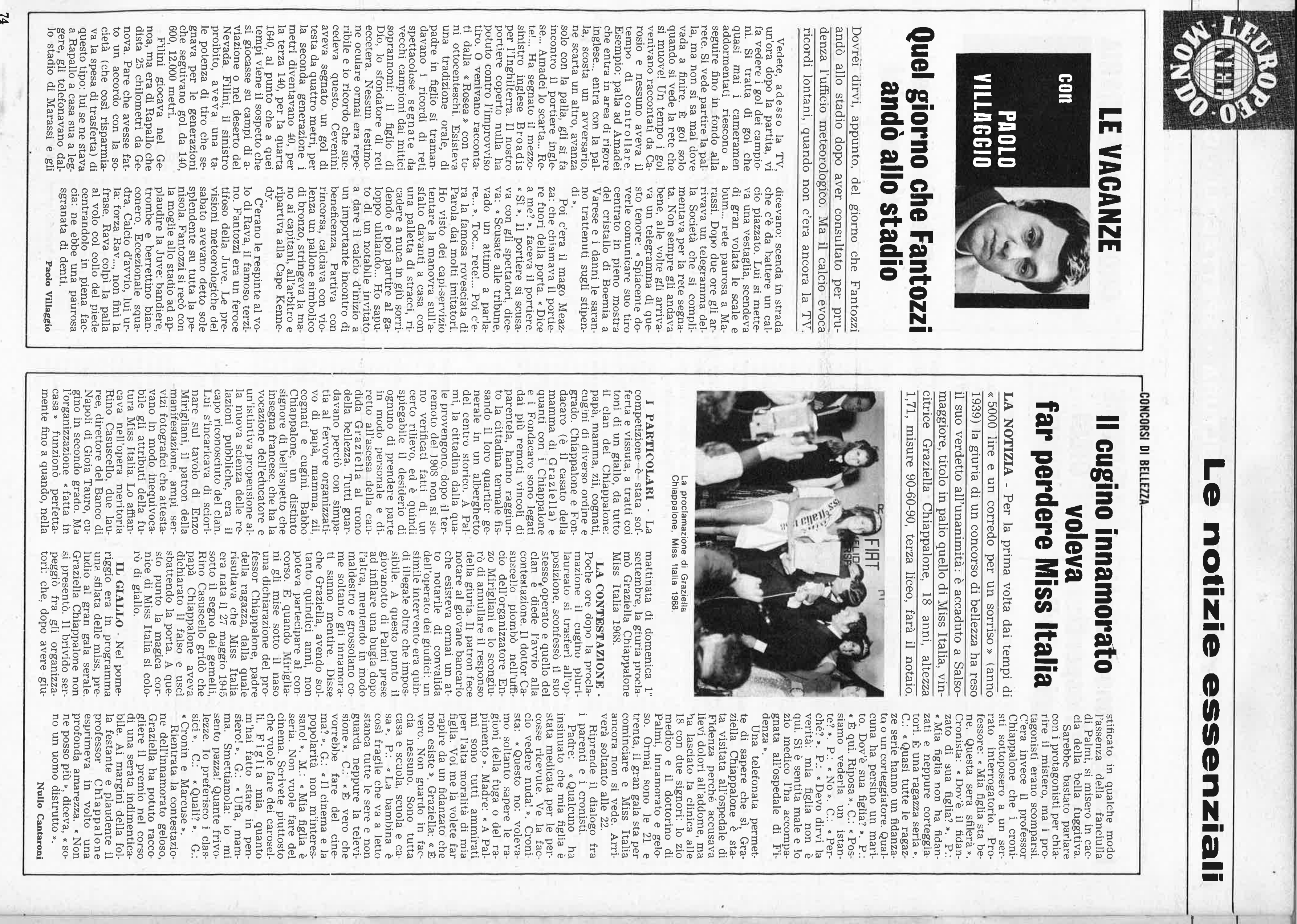



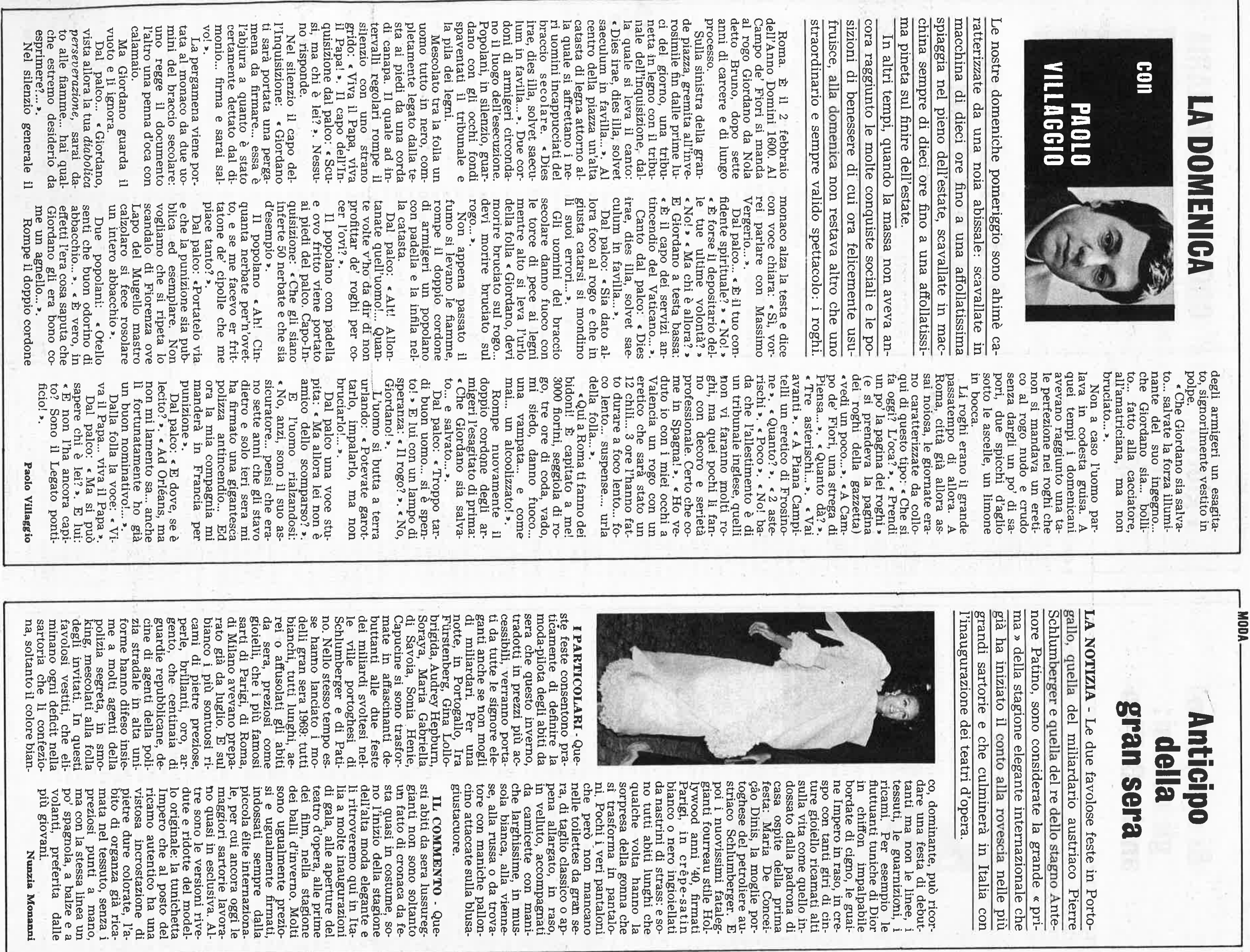


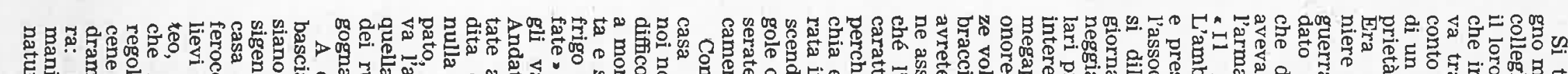

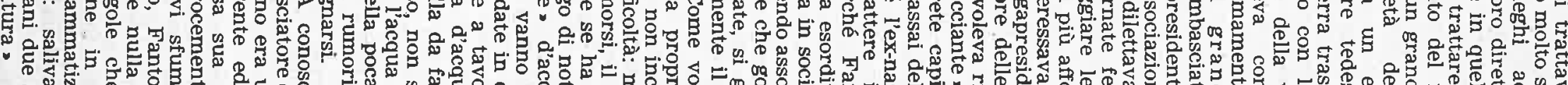

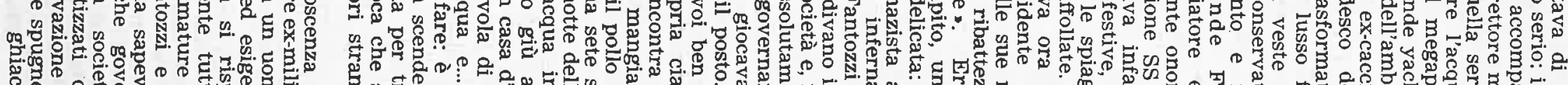

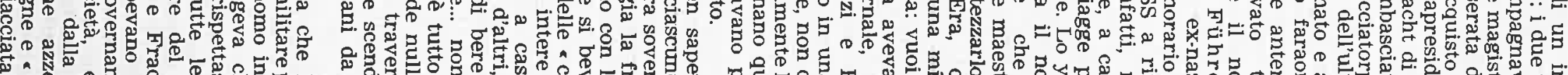

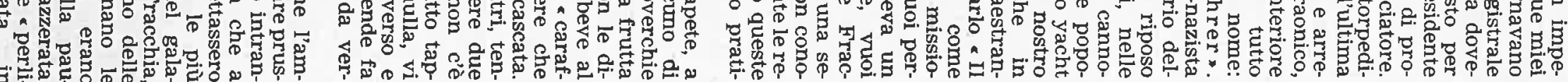

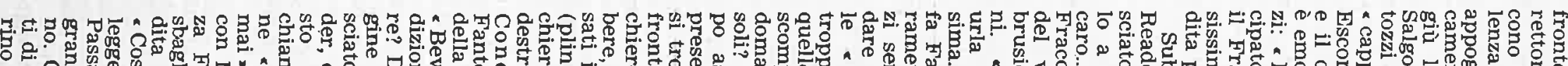
\&7.

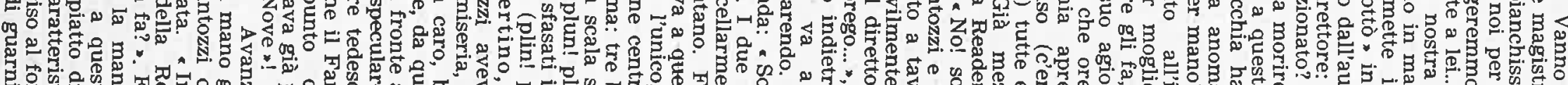

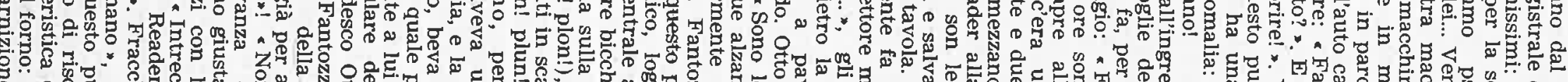

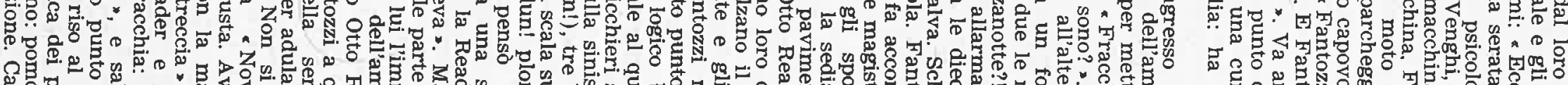

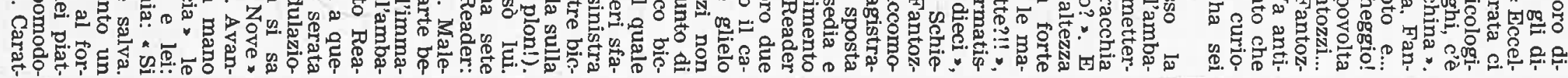
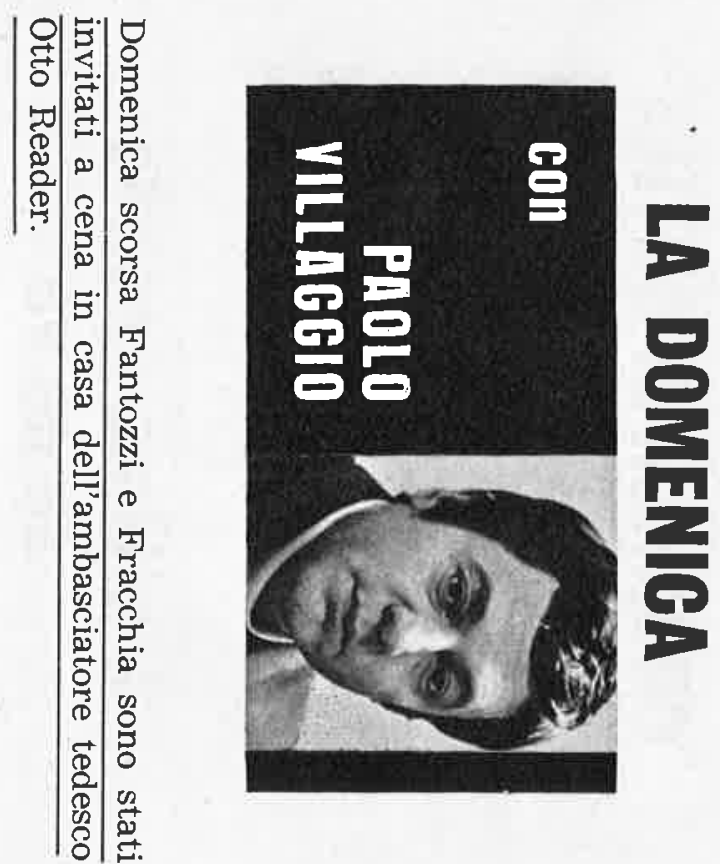

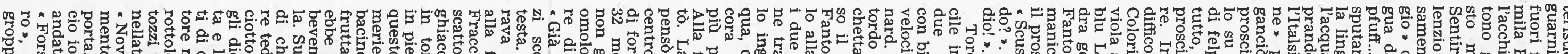
70

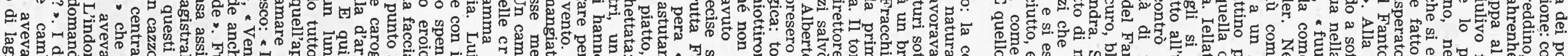

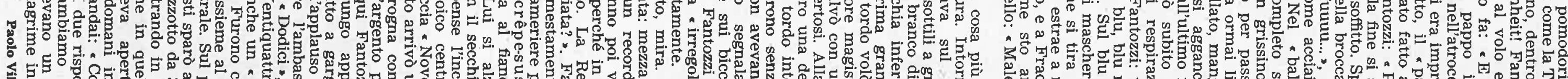

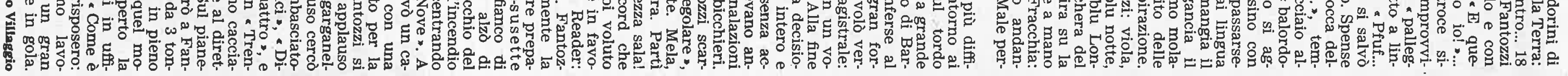

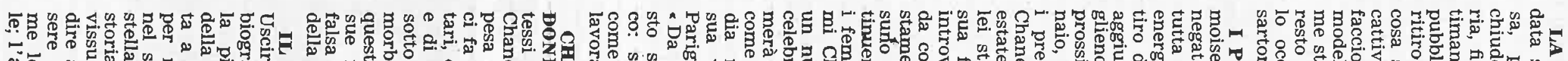

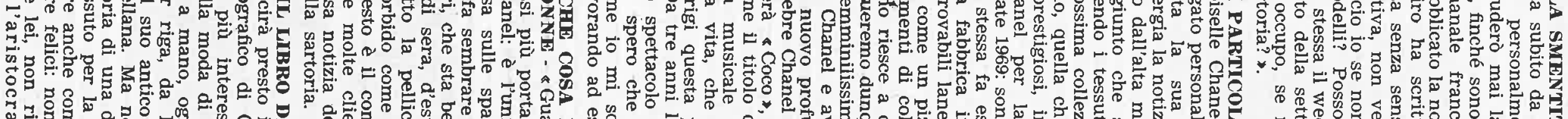

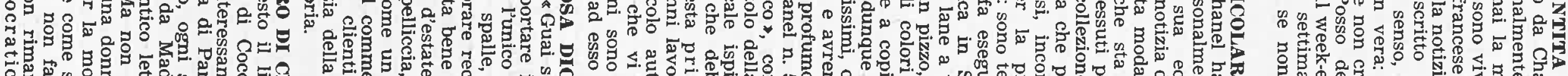

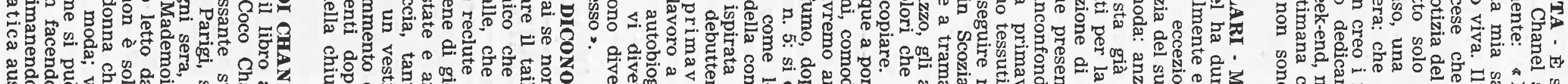

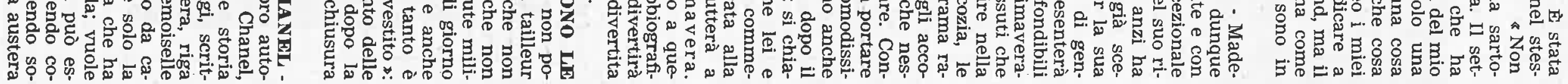

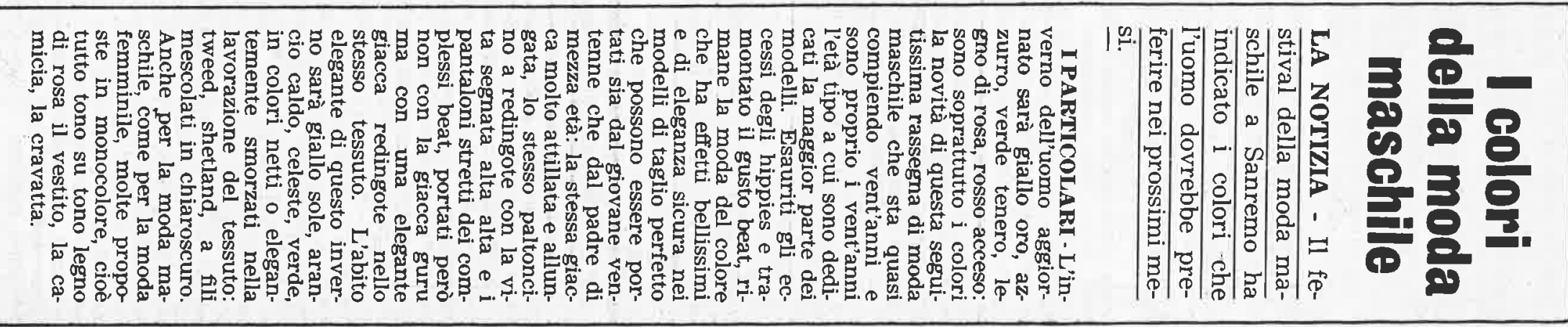

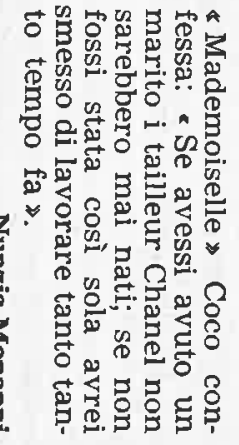
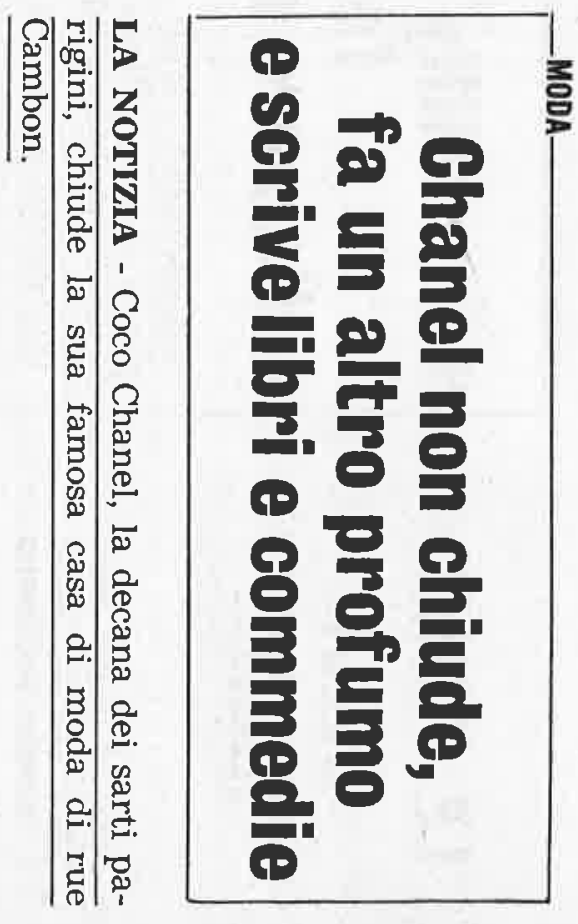

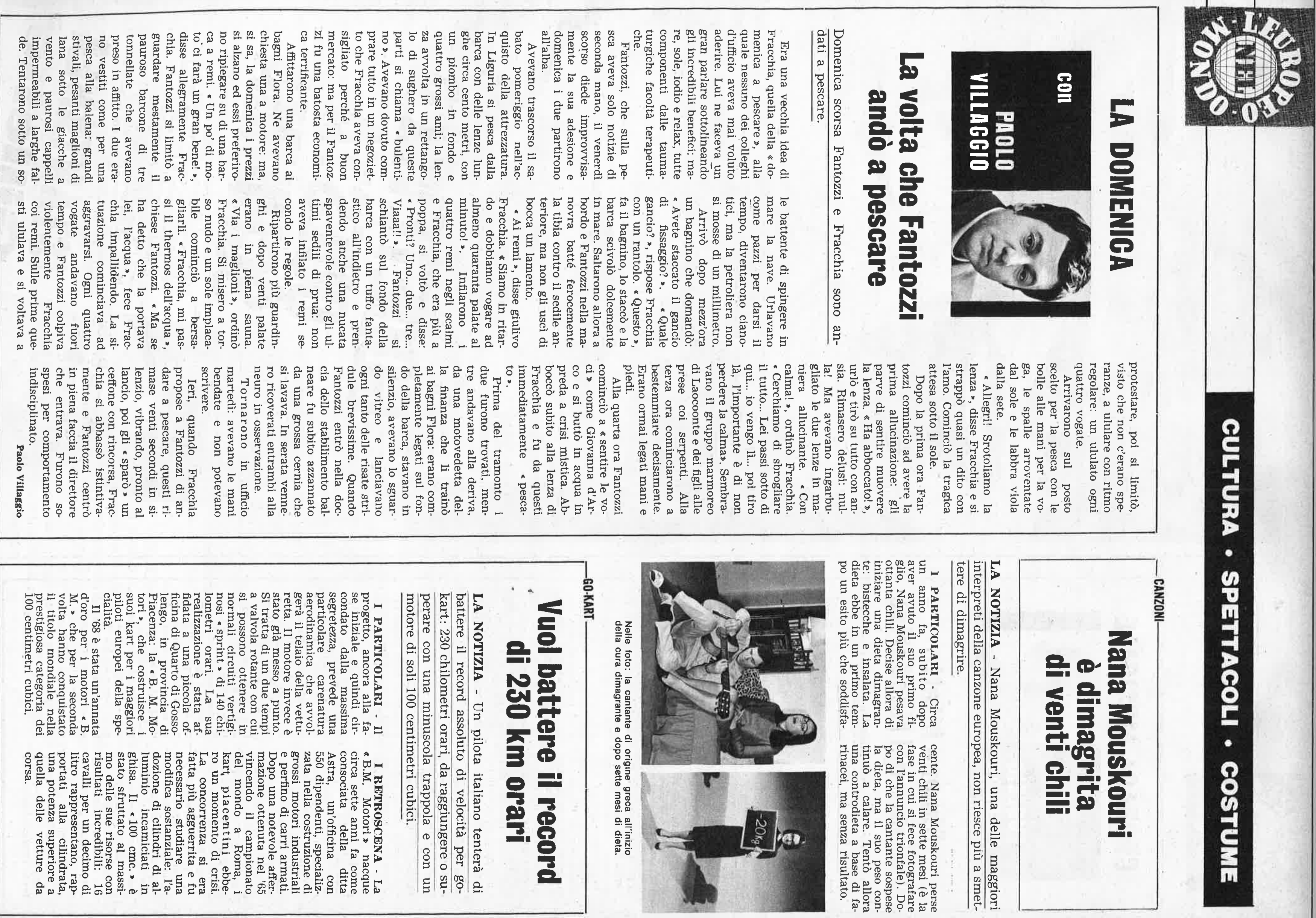


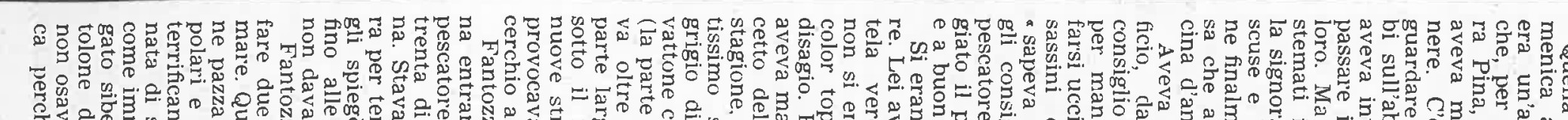
等

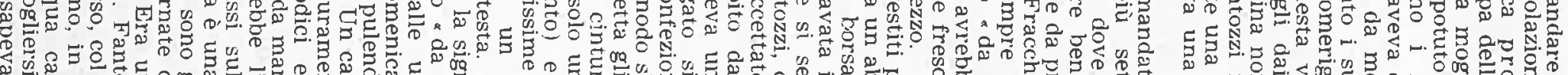

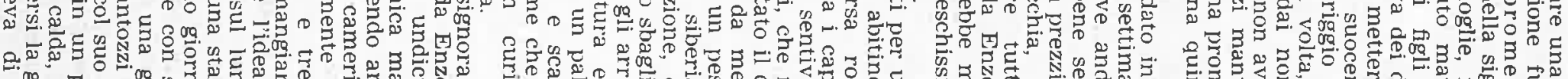

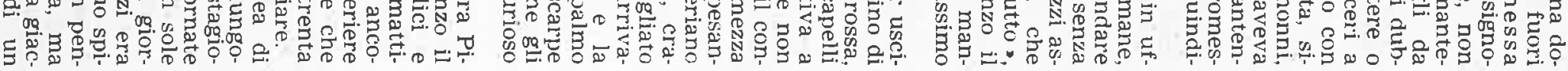

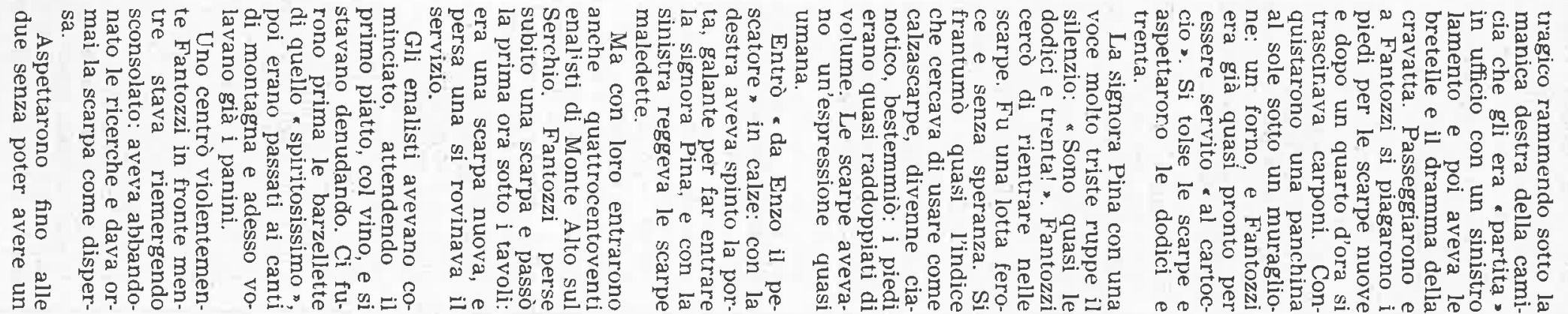

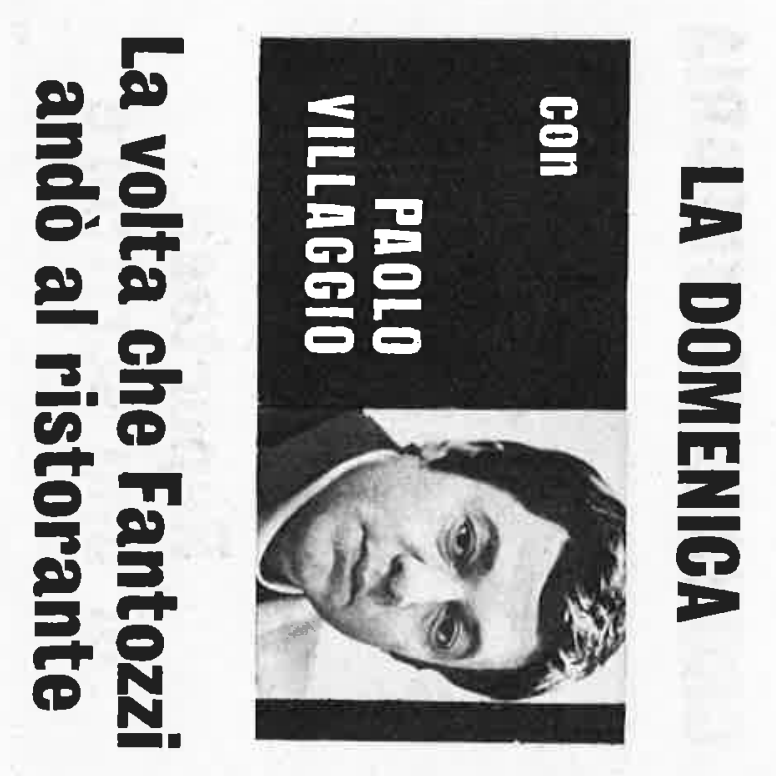

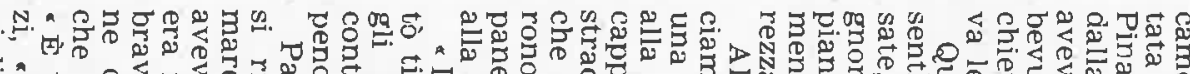

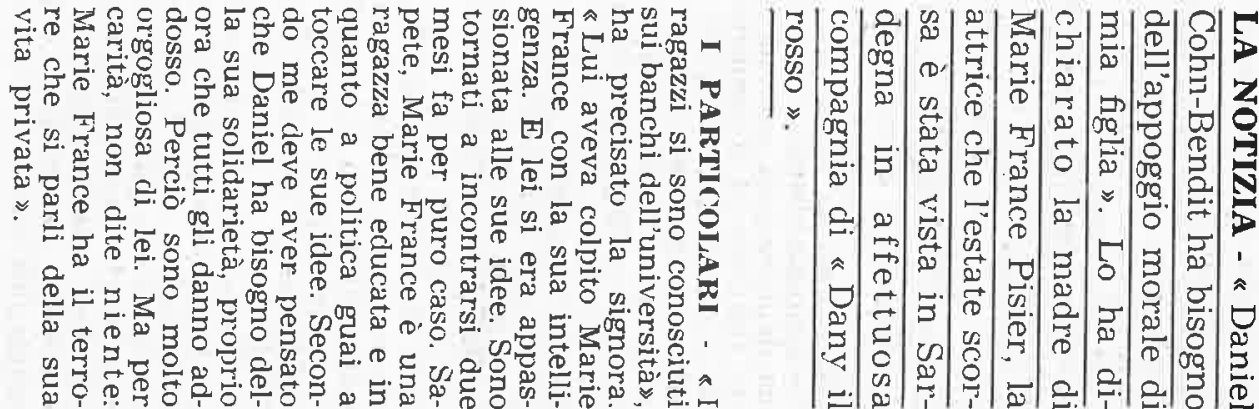

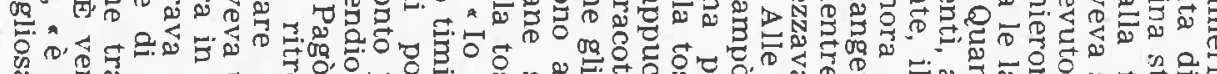

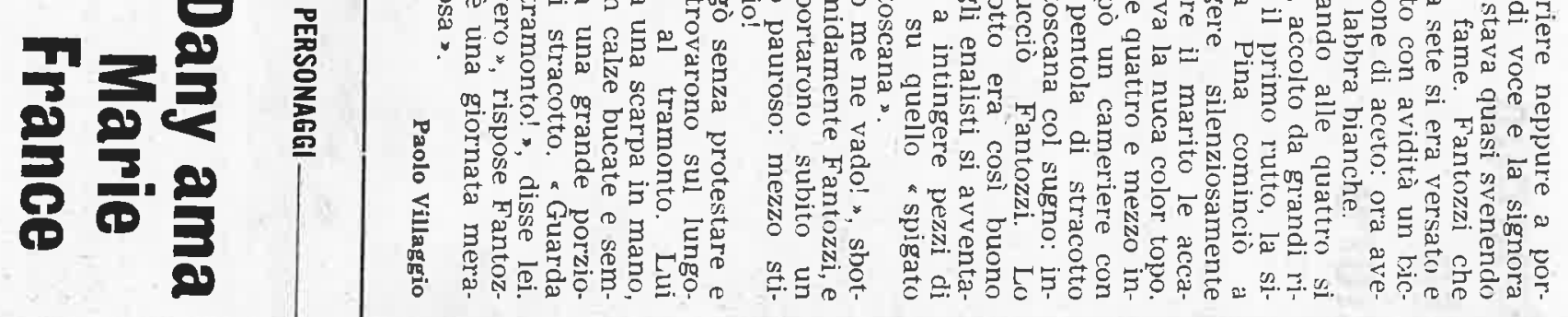

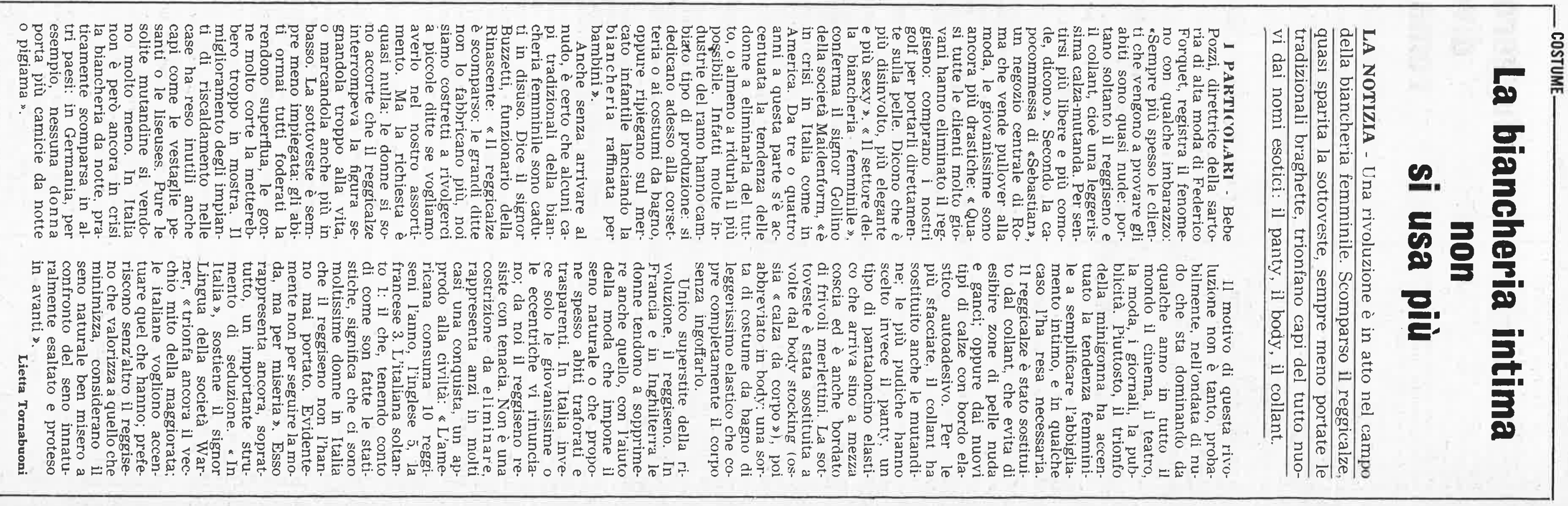



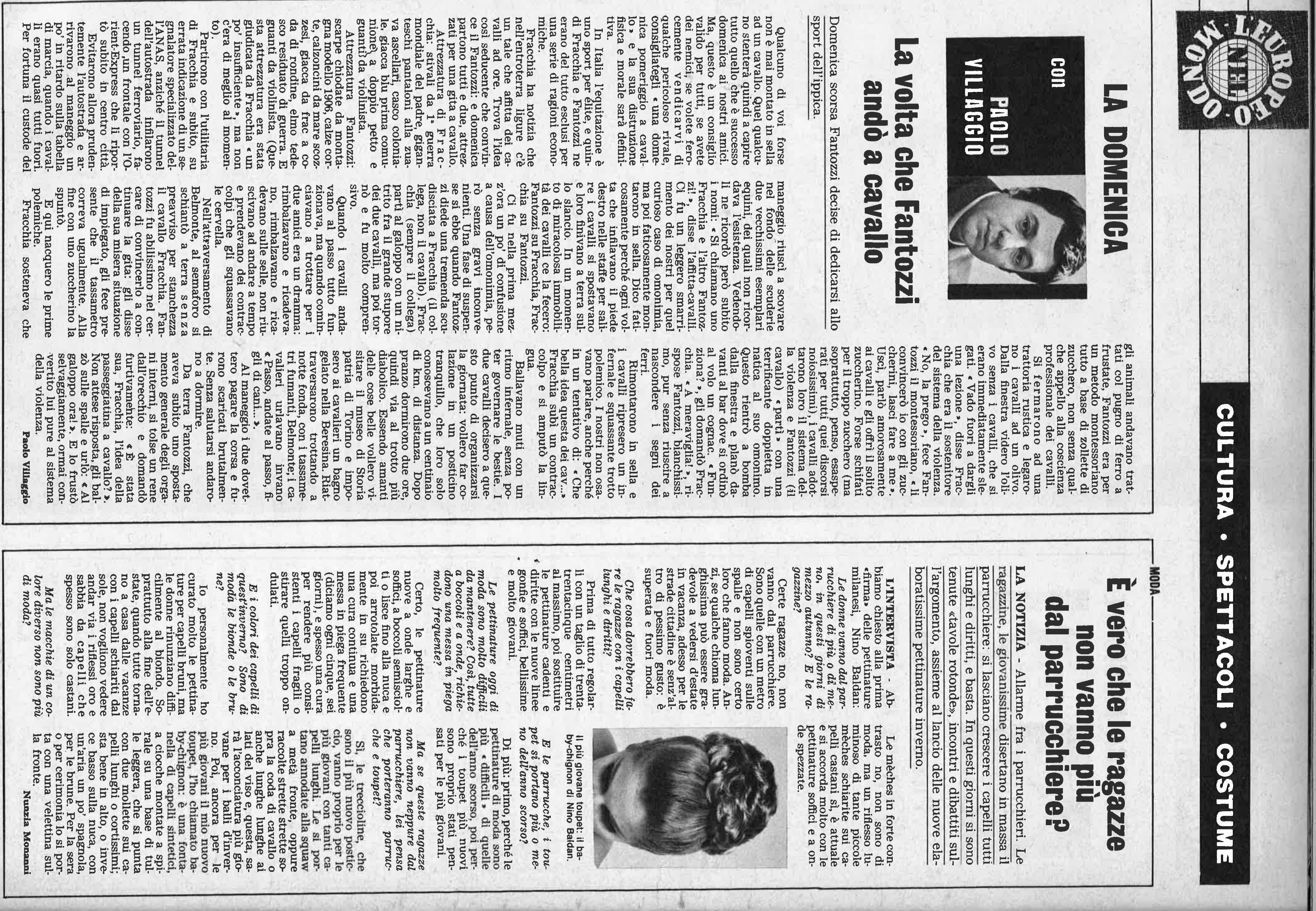


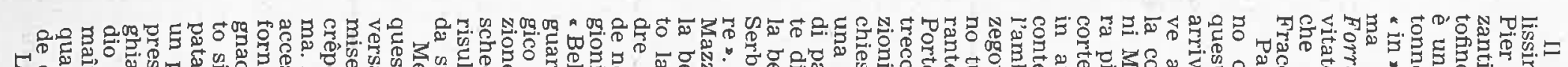

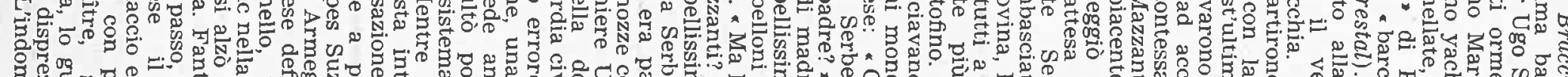

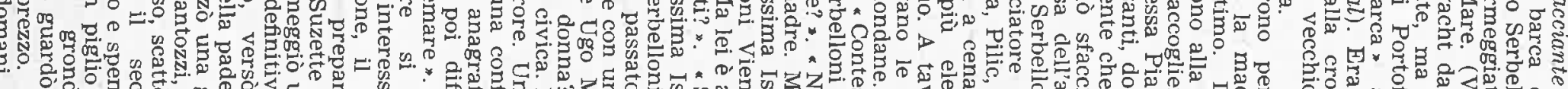

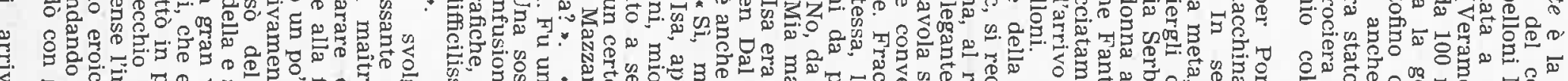

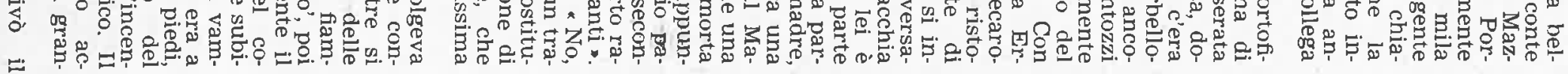

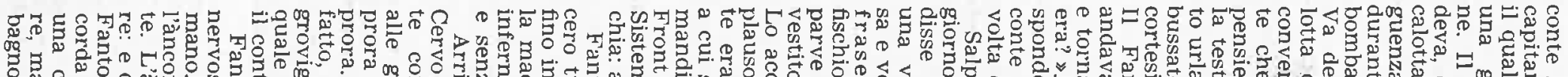

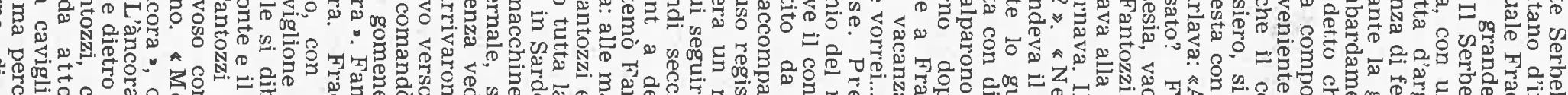

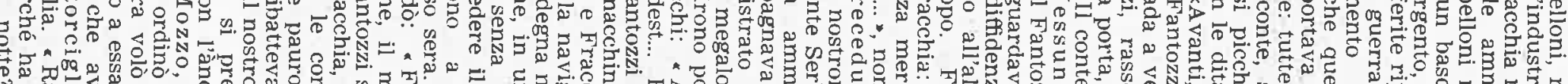

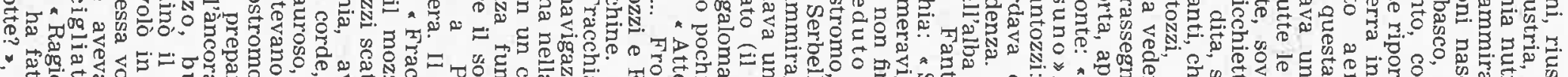

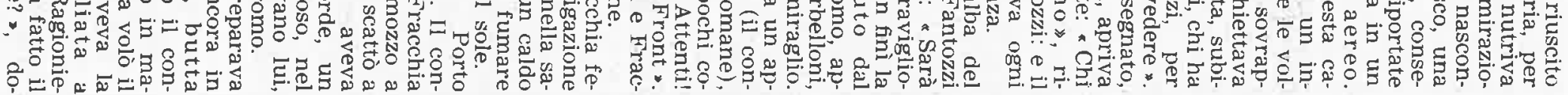
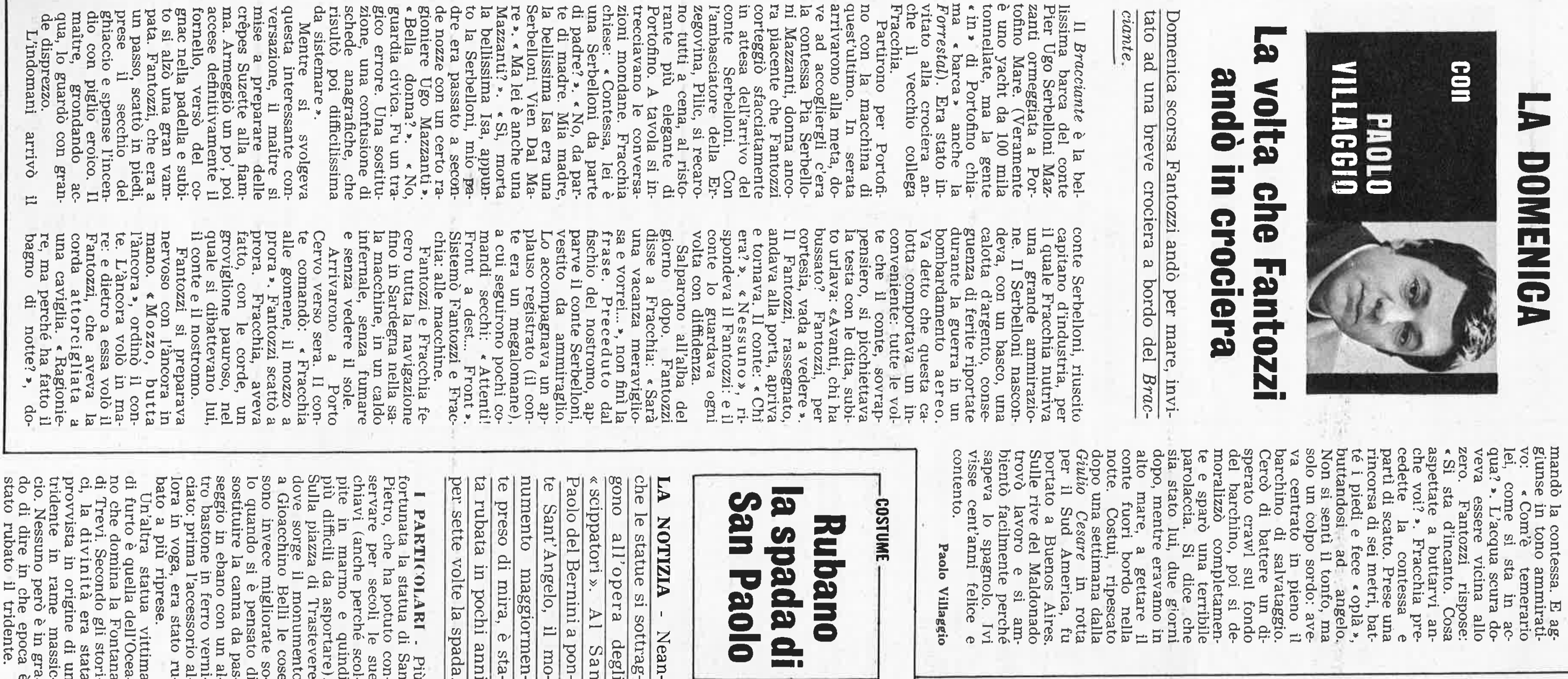

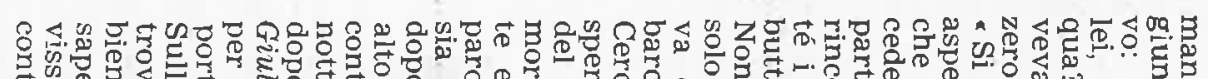
等

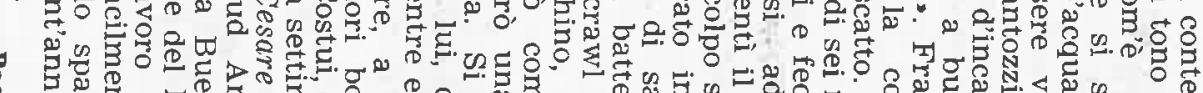

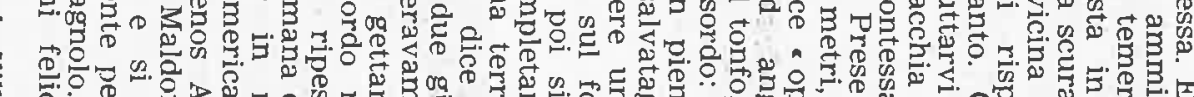

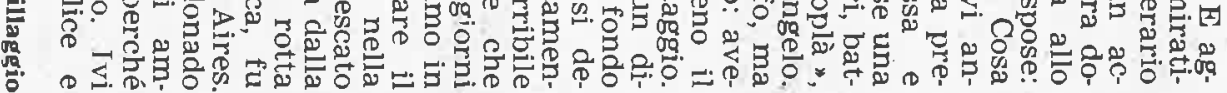

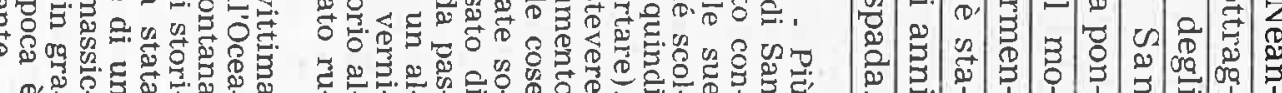

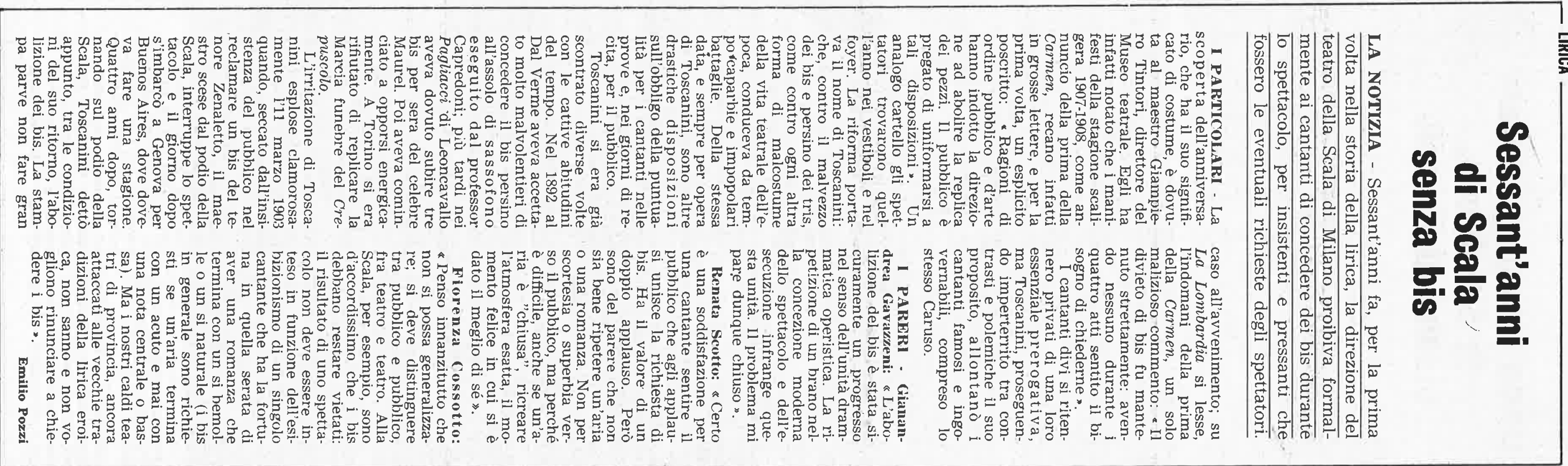




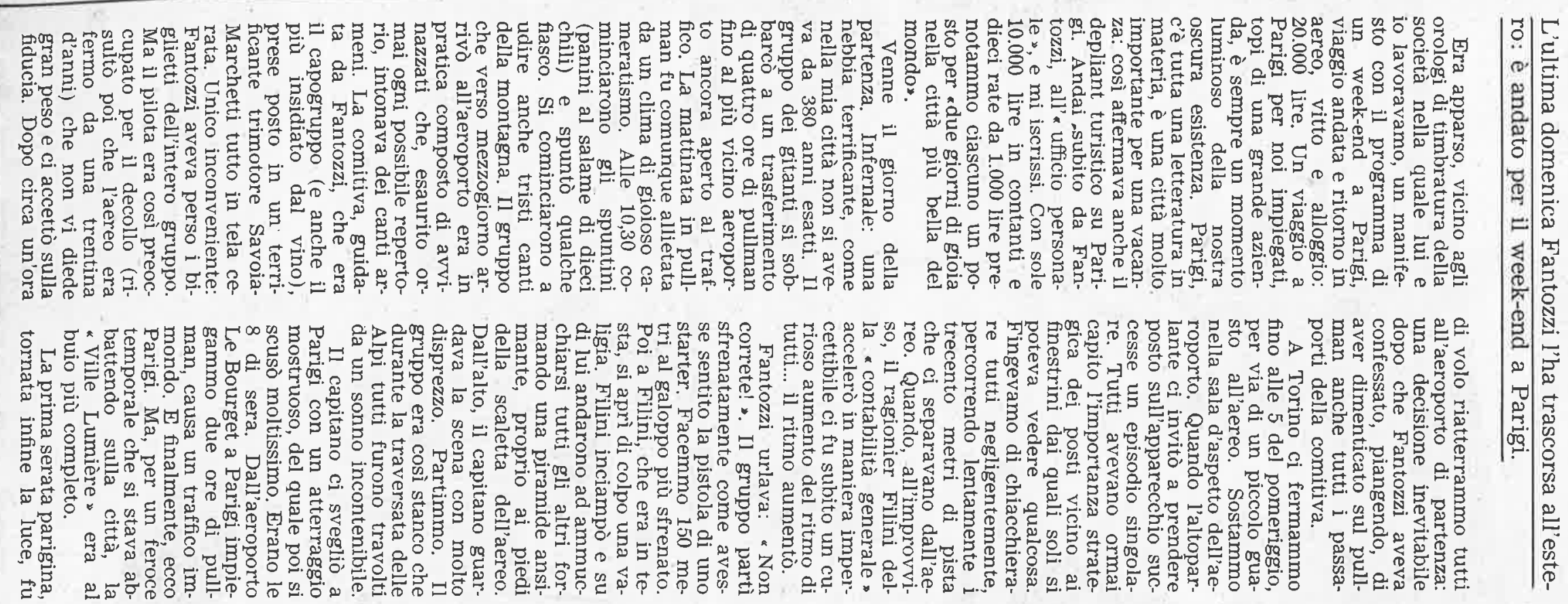

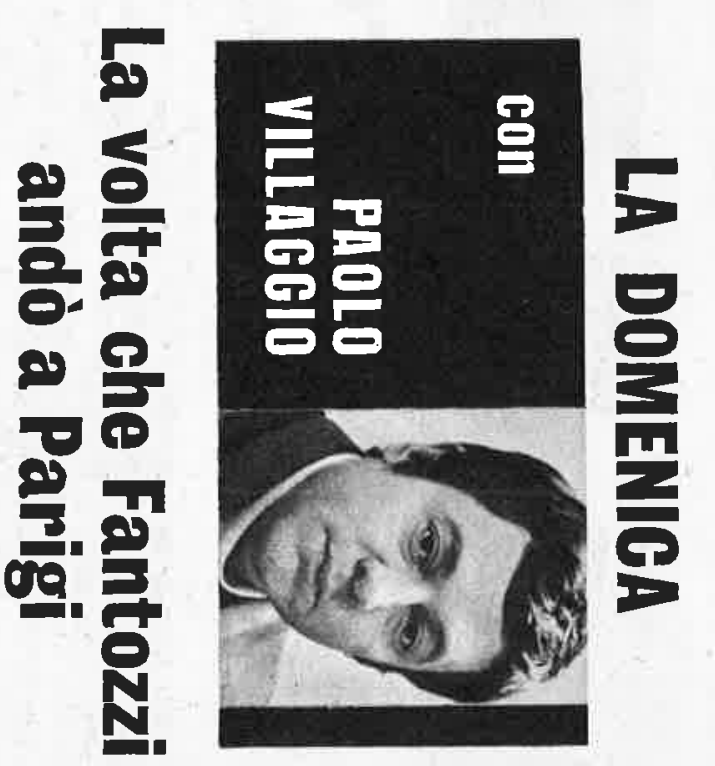

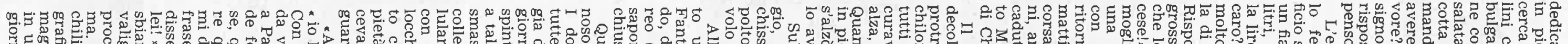

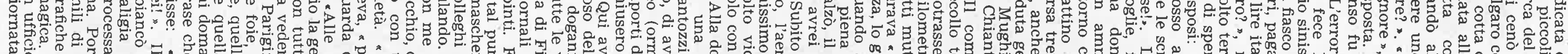

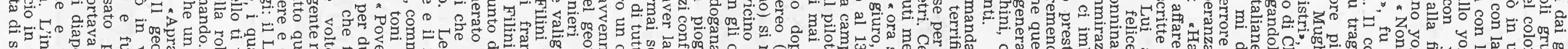

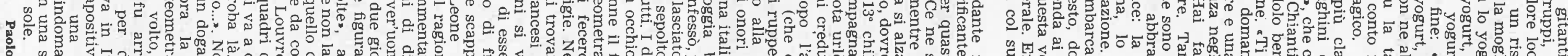

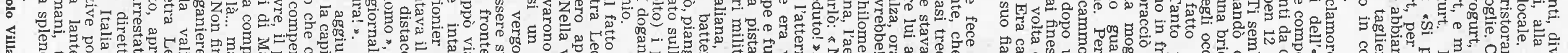

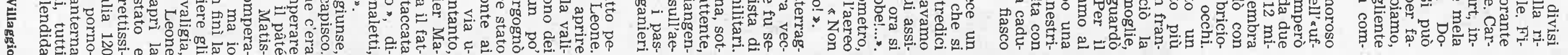

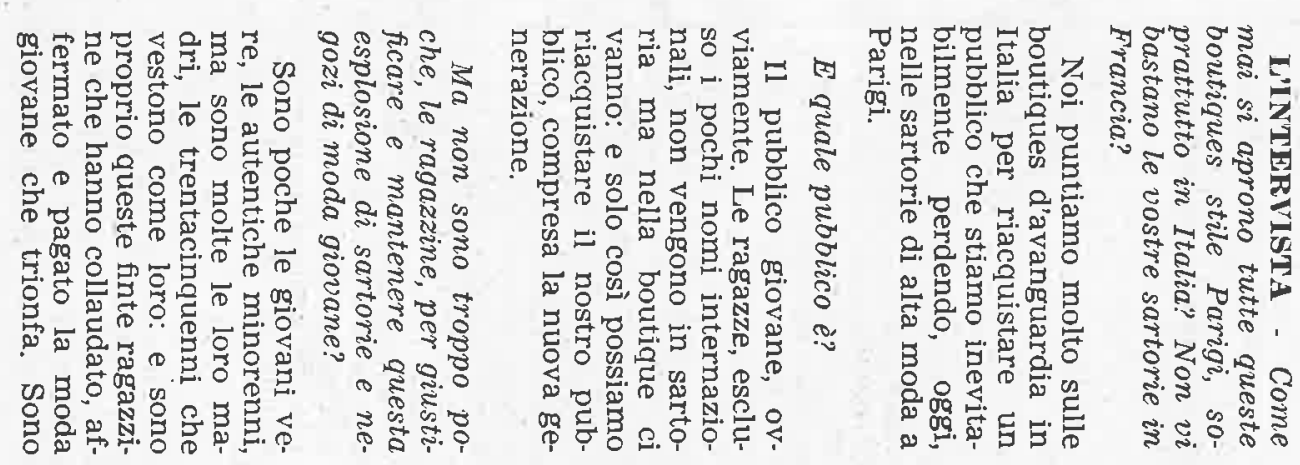
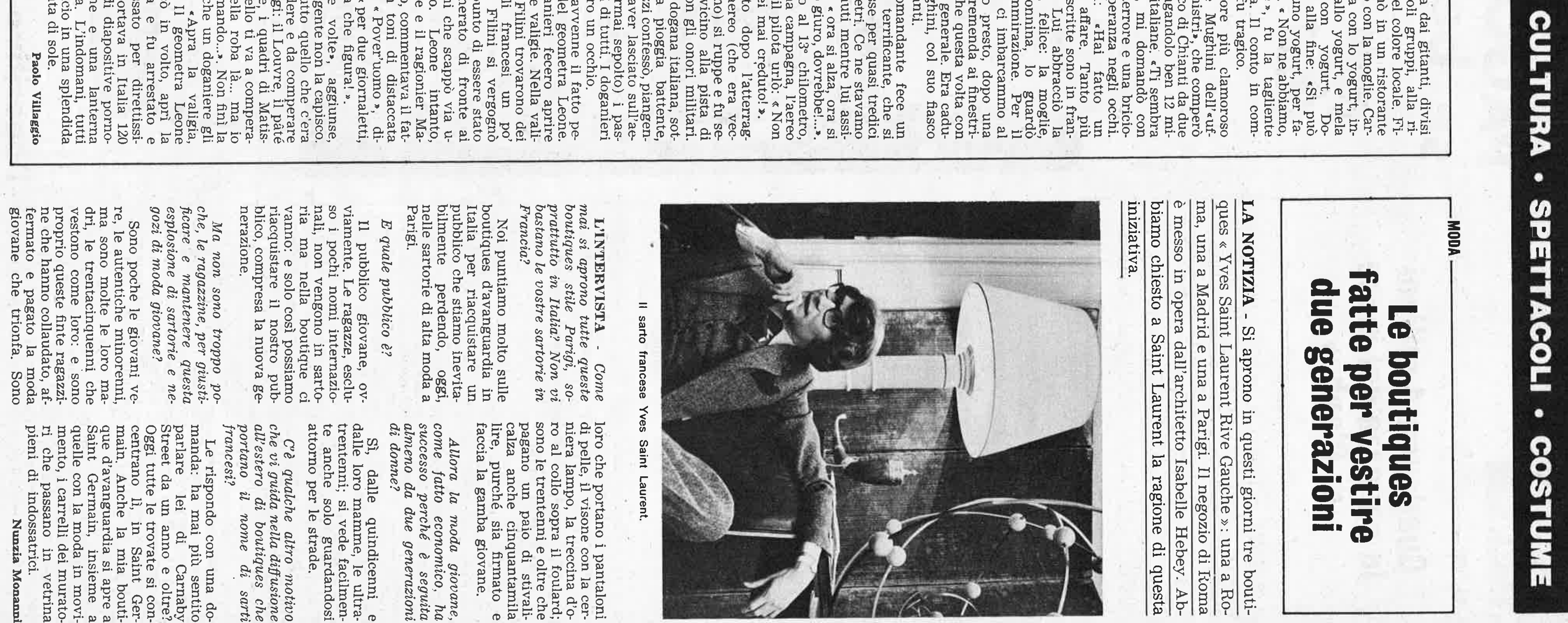


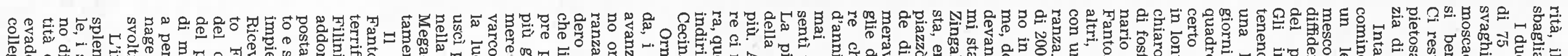

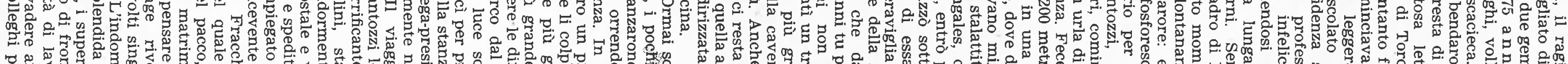

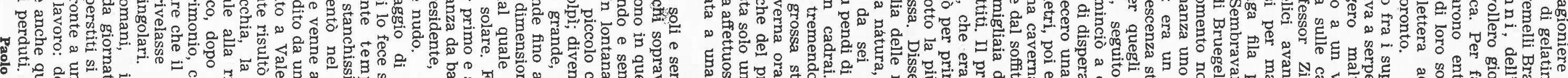

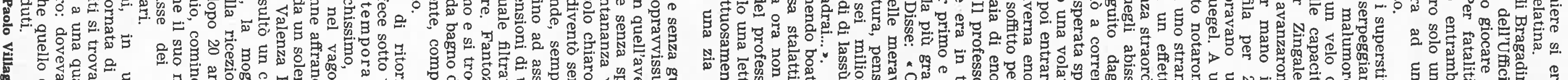

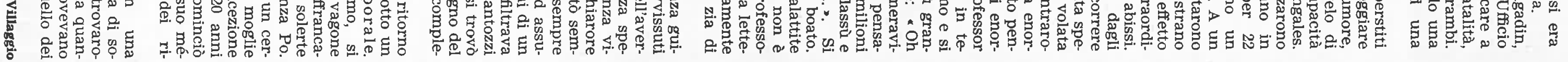
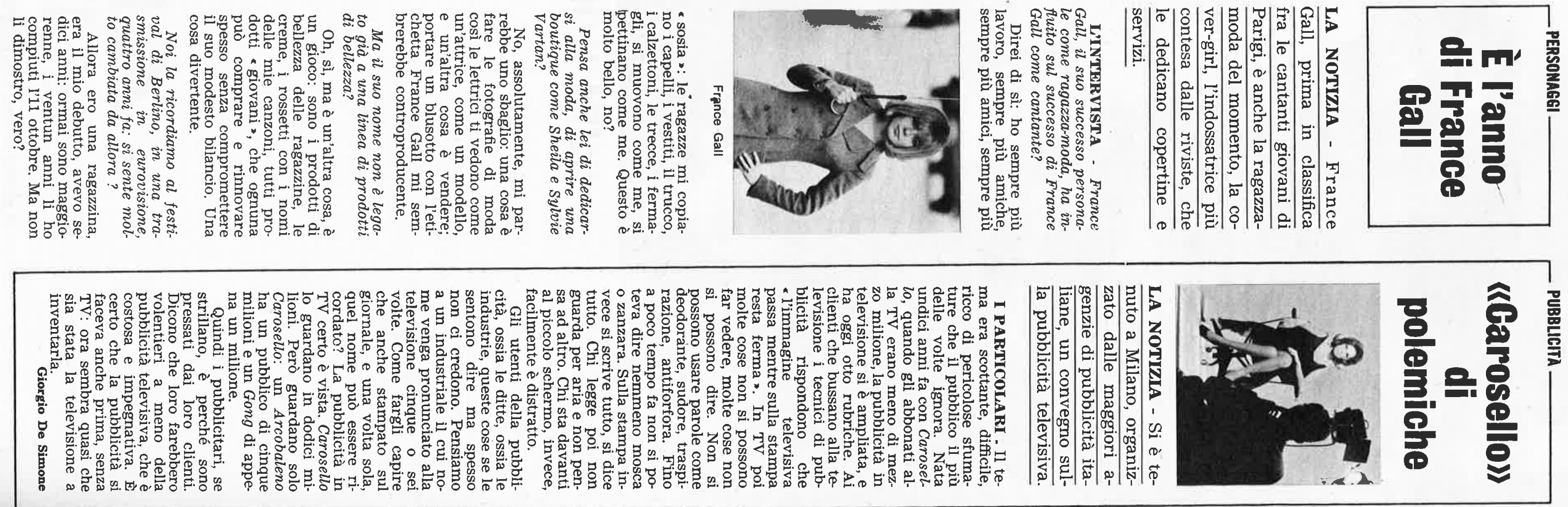


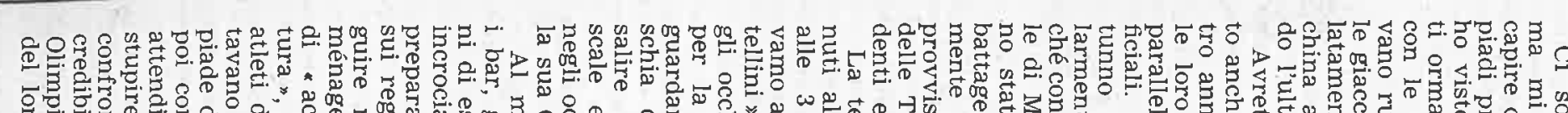

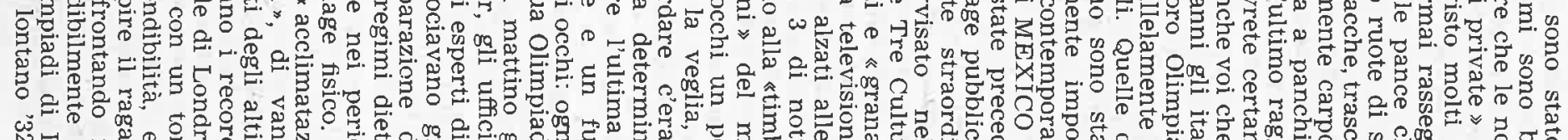

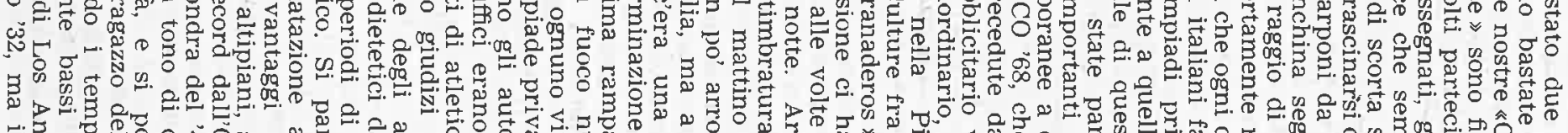

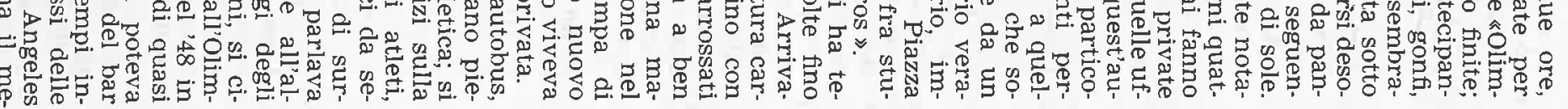

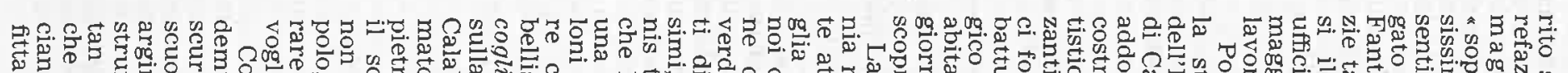

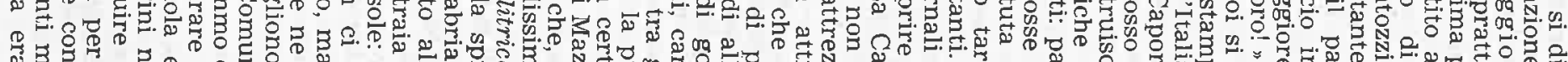

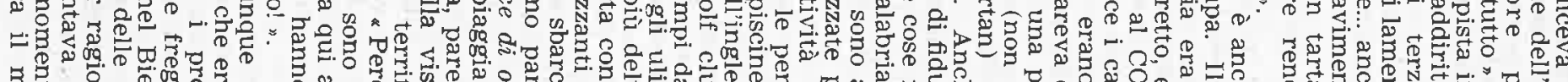

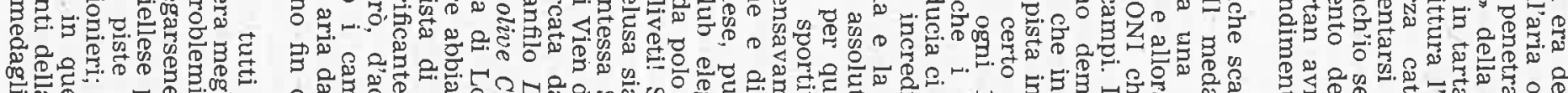

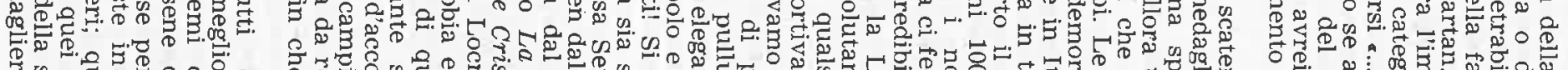

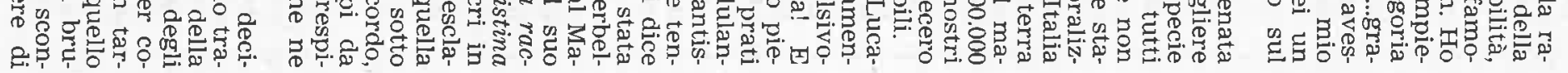
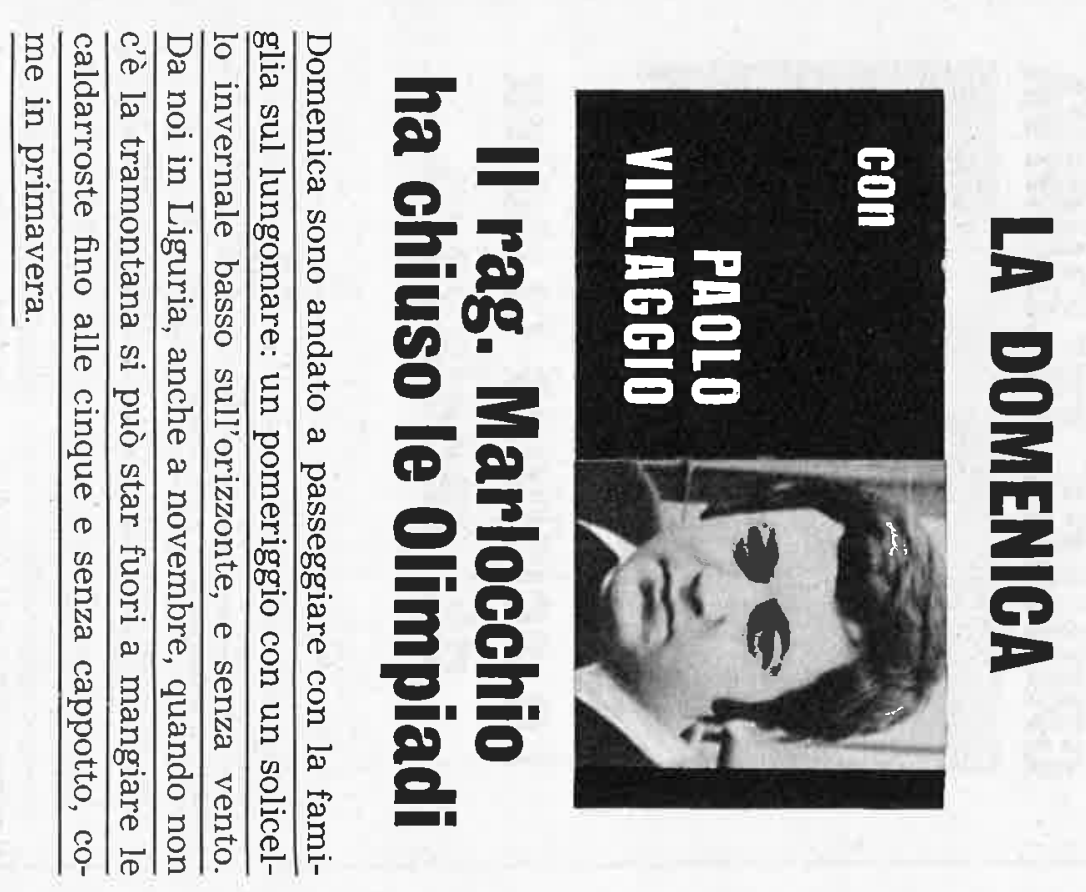

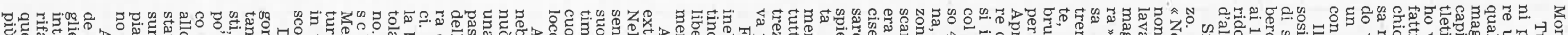

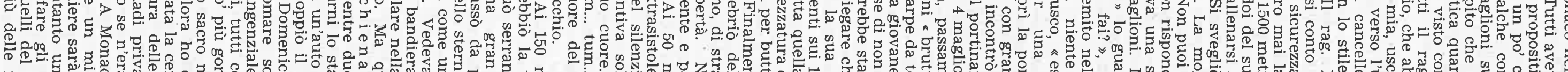

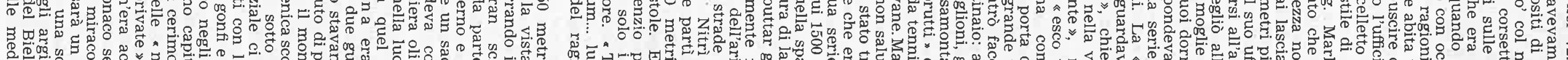

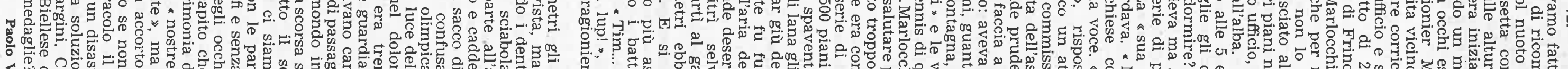

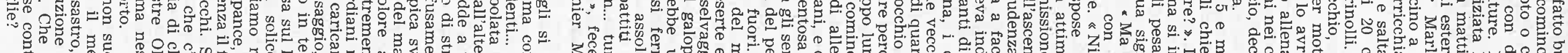

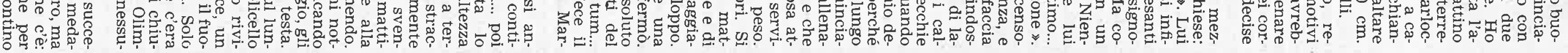

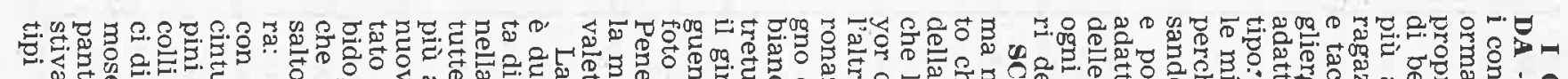

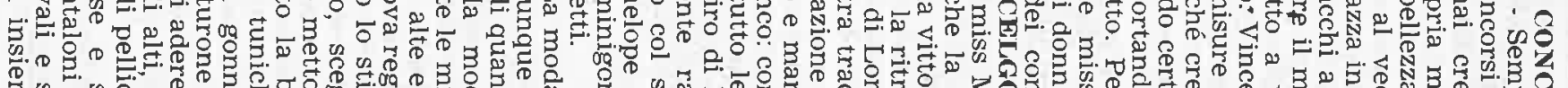

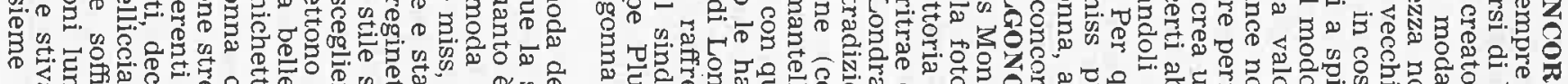
w

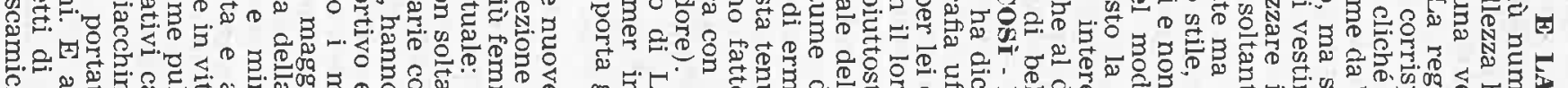

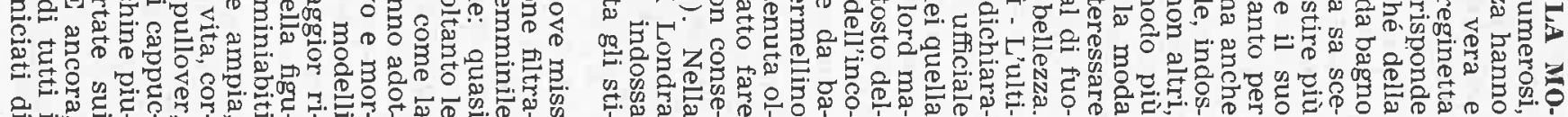

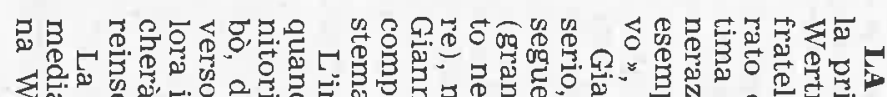

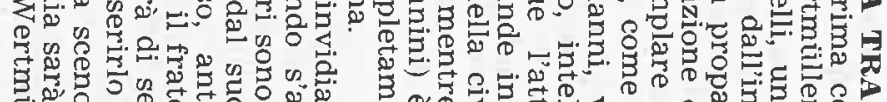

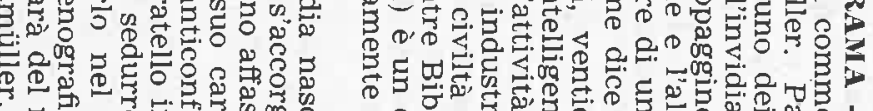

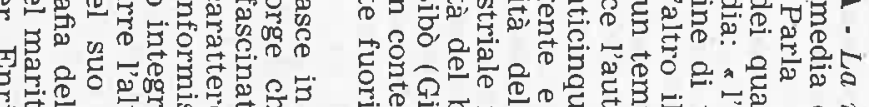

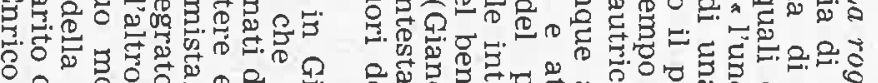

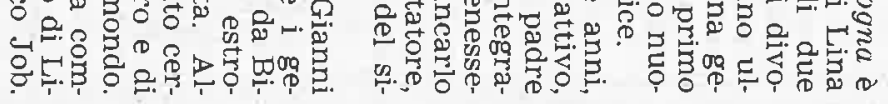

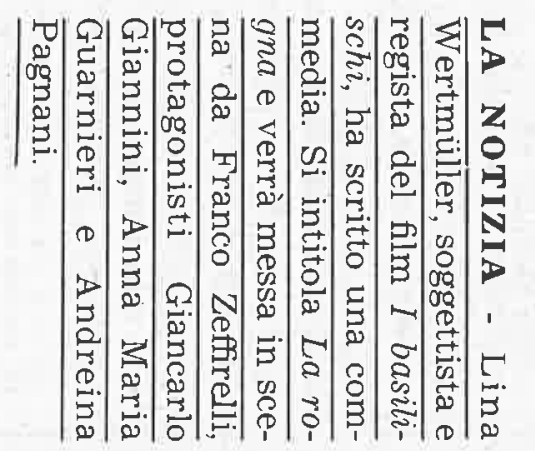

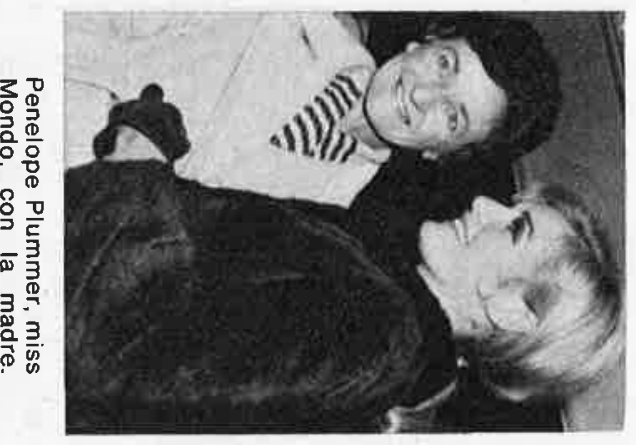

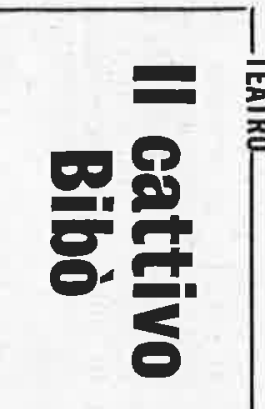

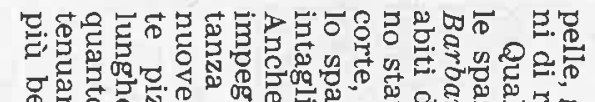

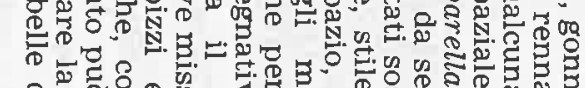

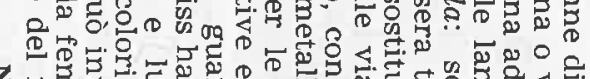

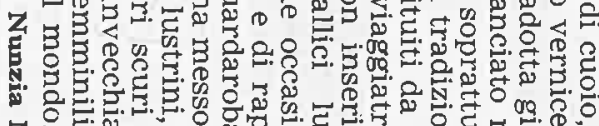

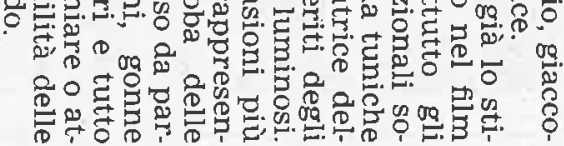
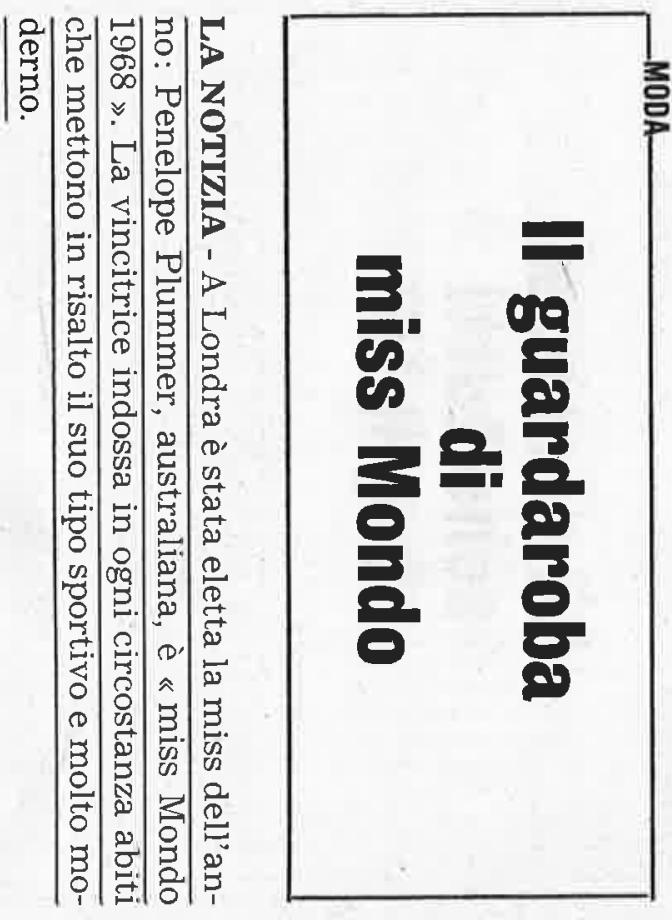

0
3
0
$N$
0
0
0
0
0
$\mathbf{N}$
0
0 


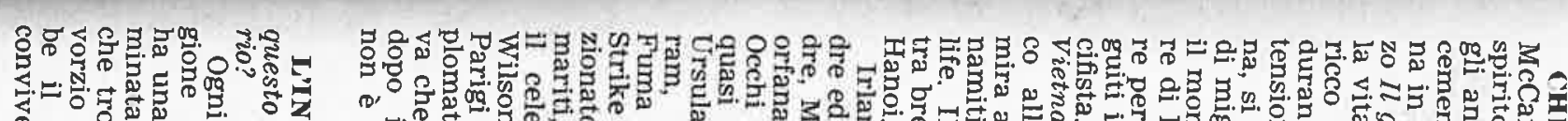

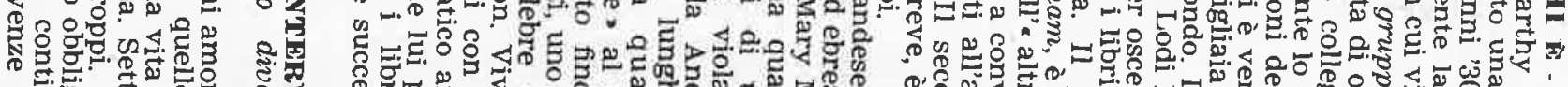

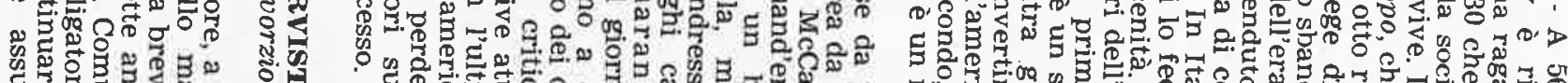

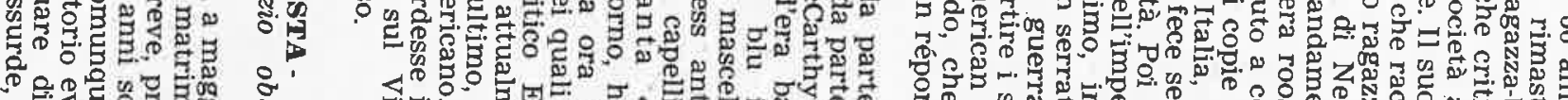

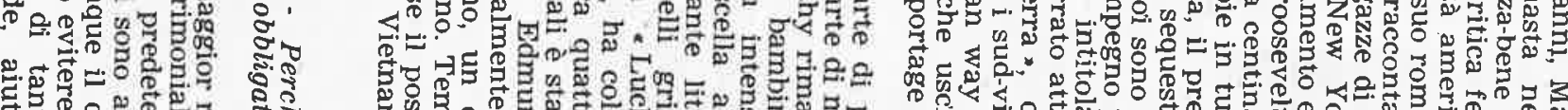

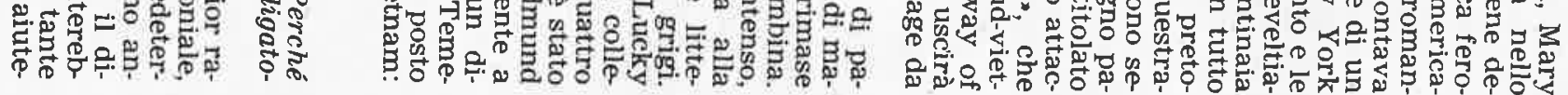
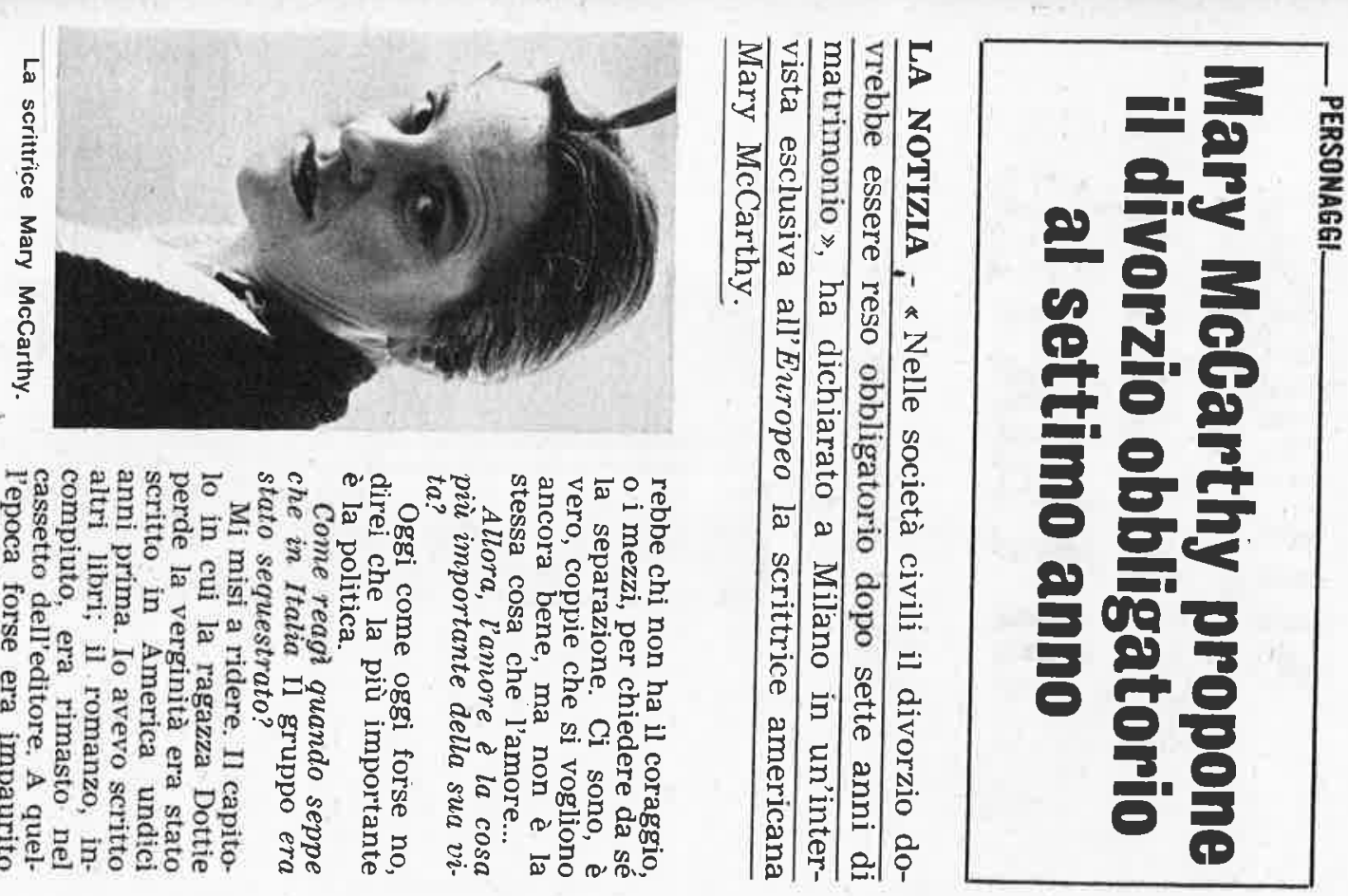

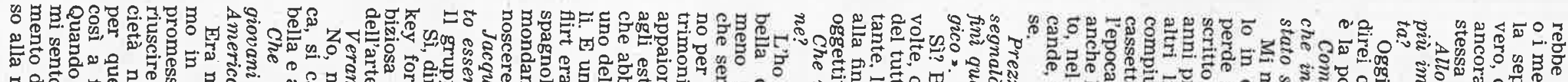

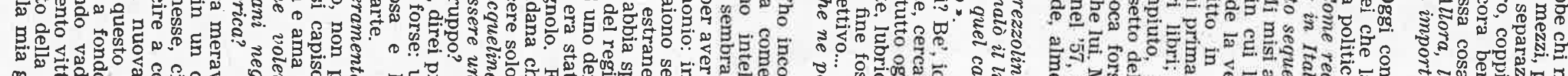

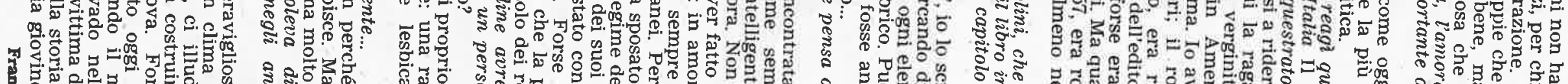
8 4.

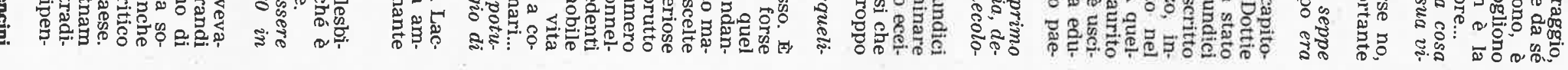

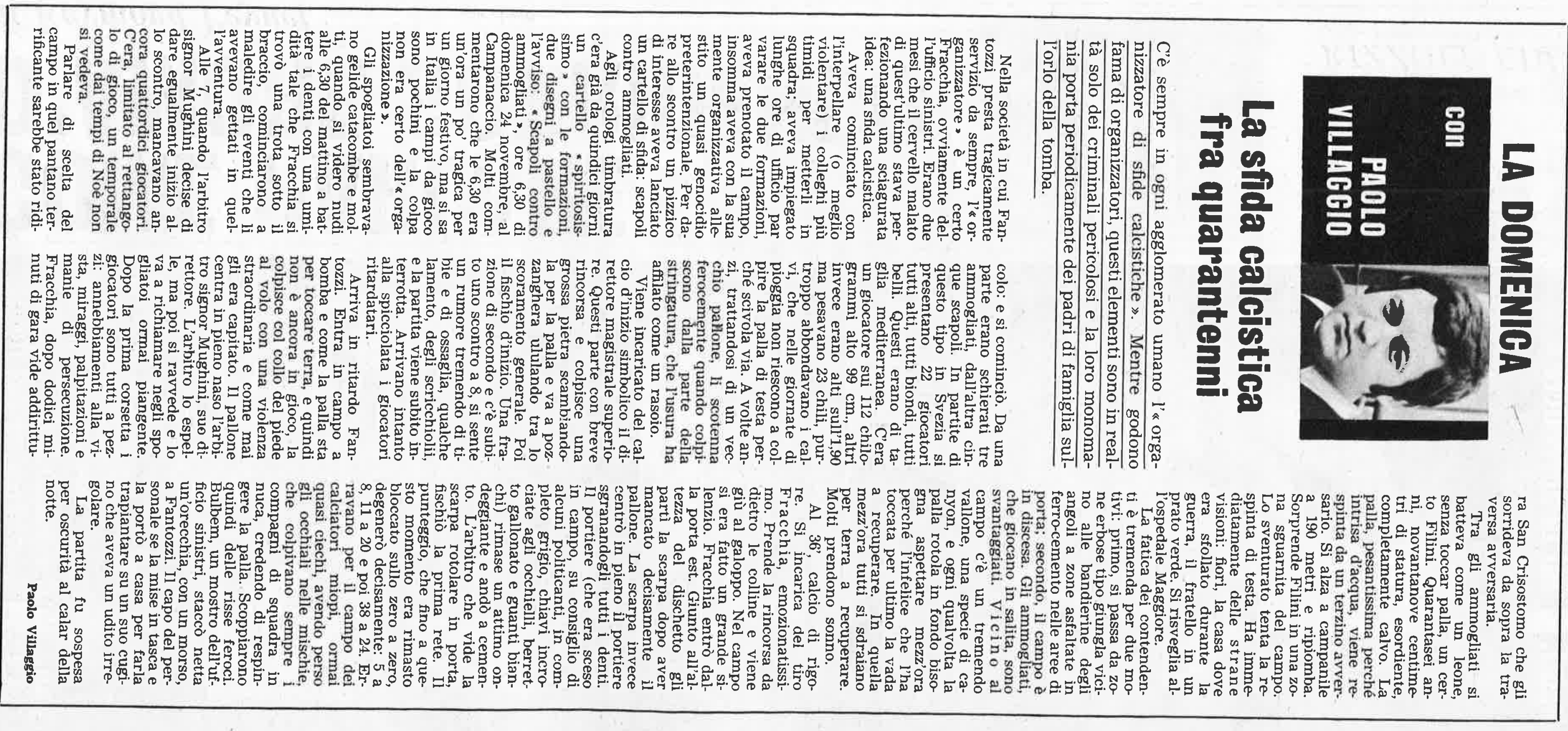



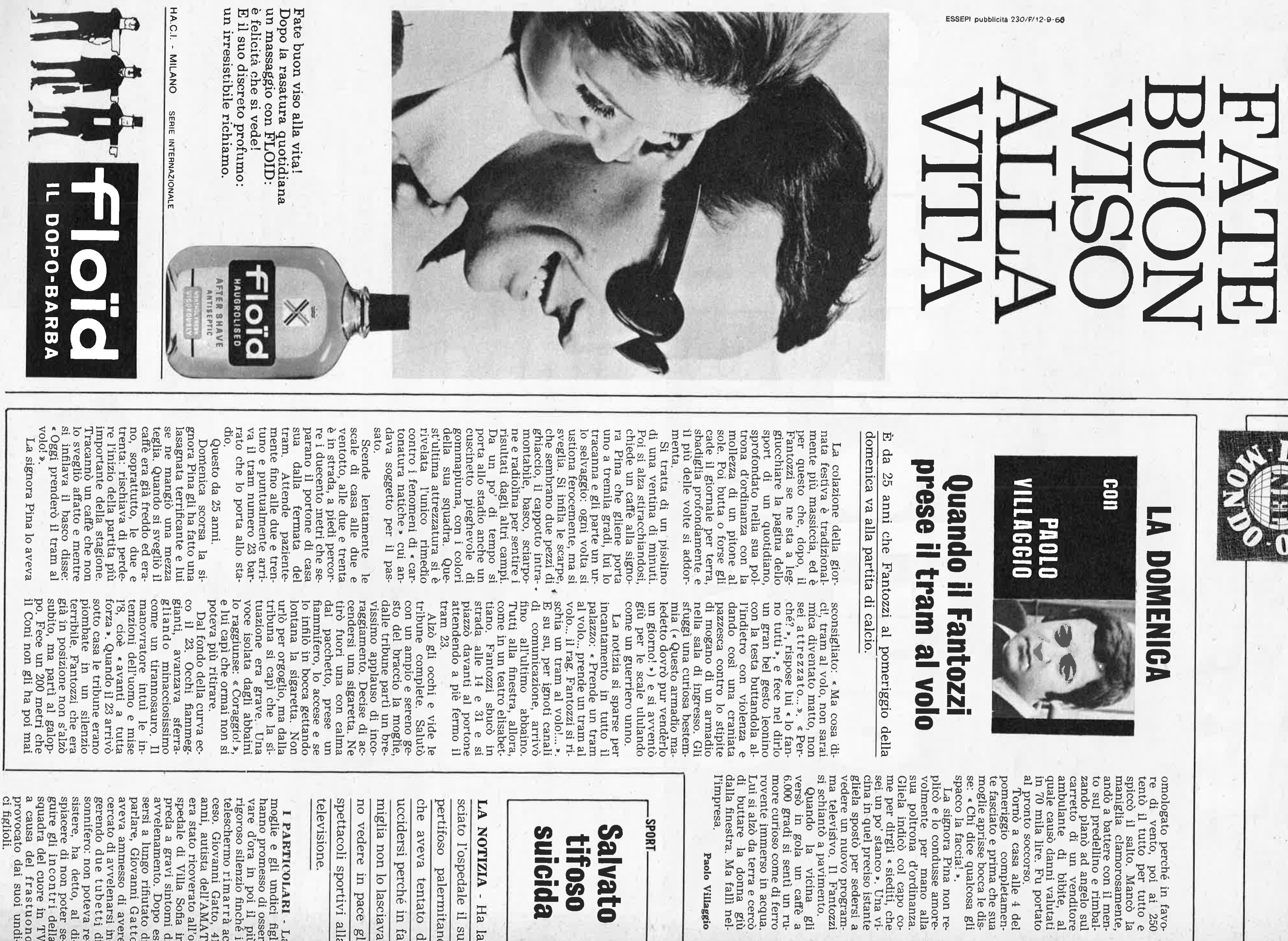

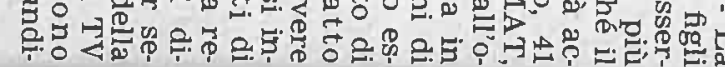
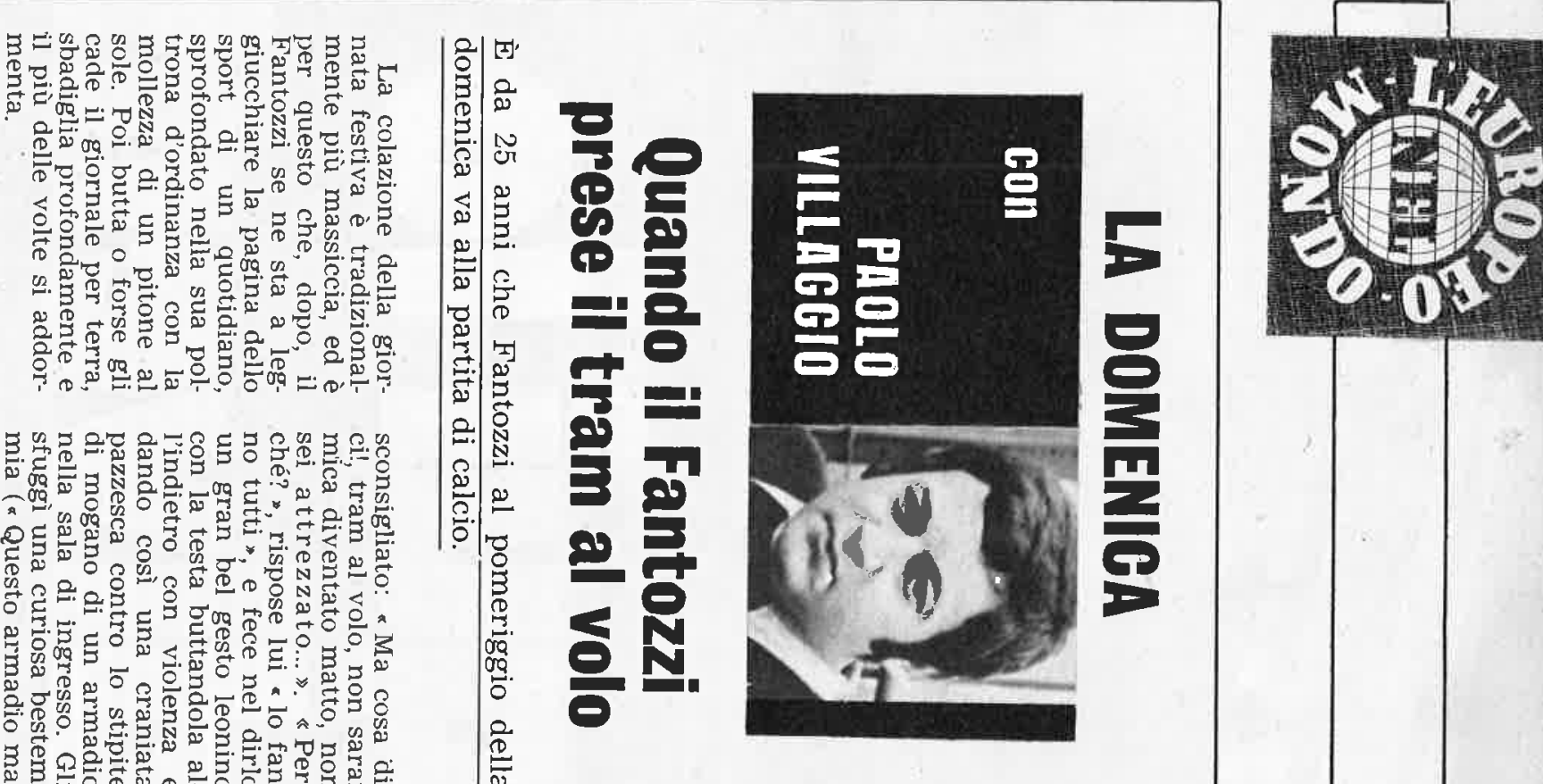

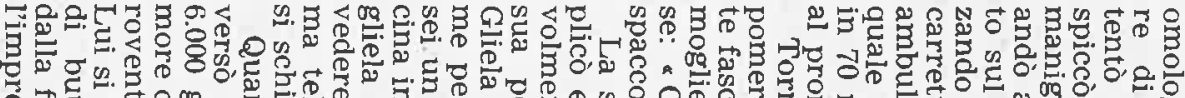

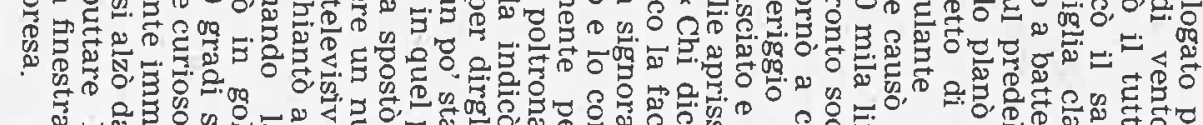

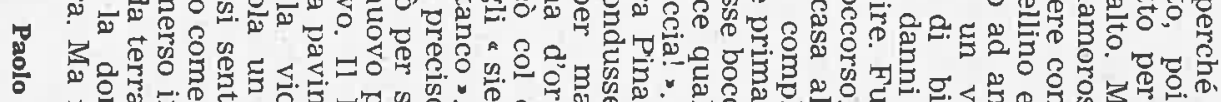

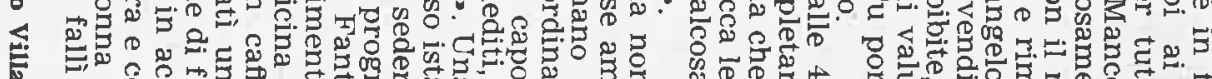

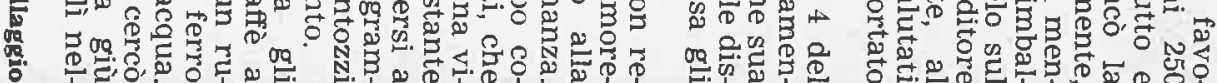




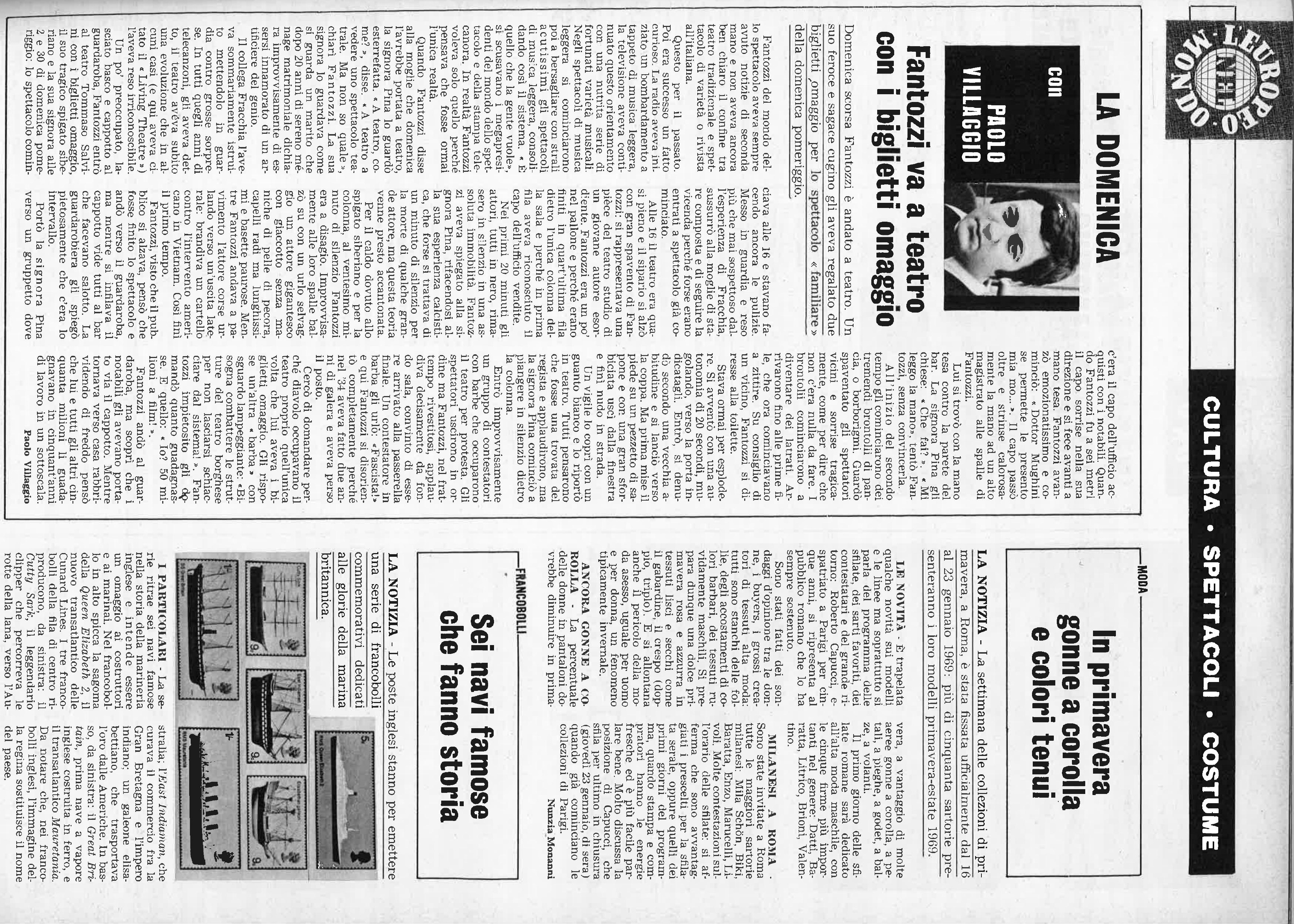




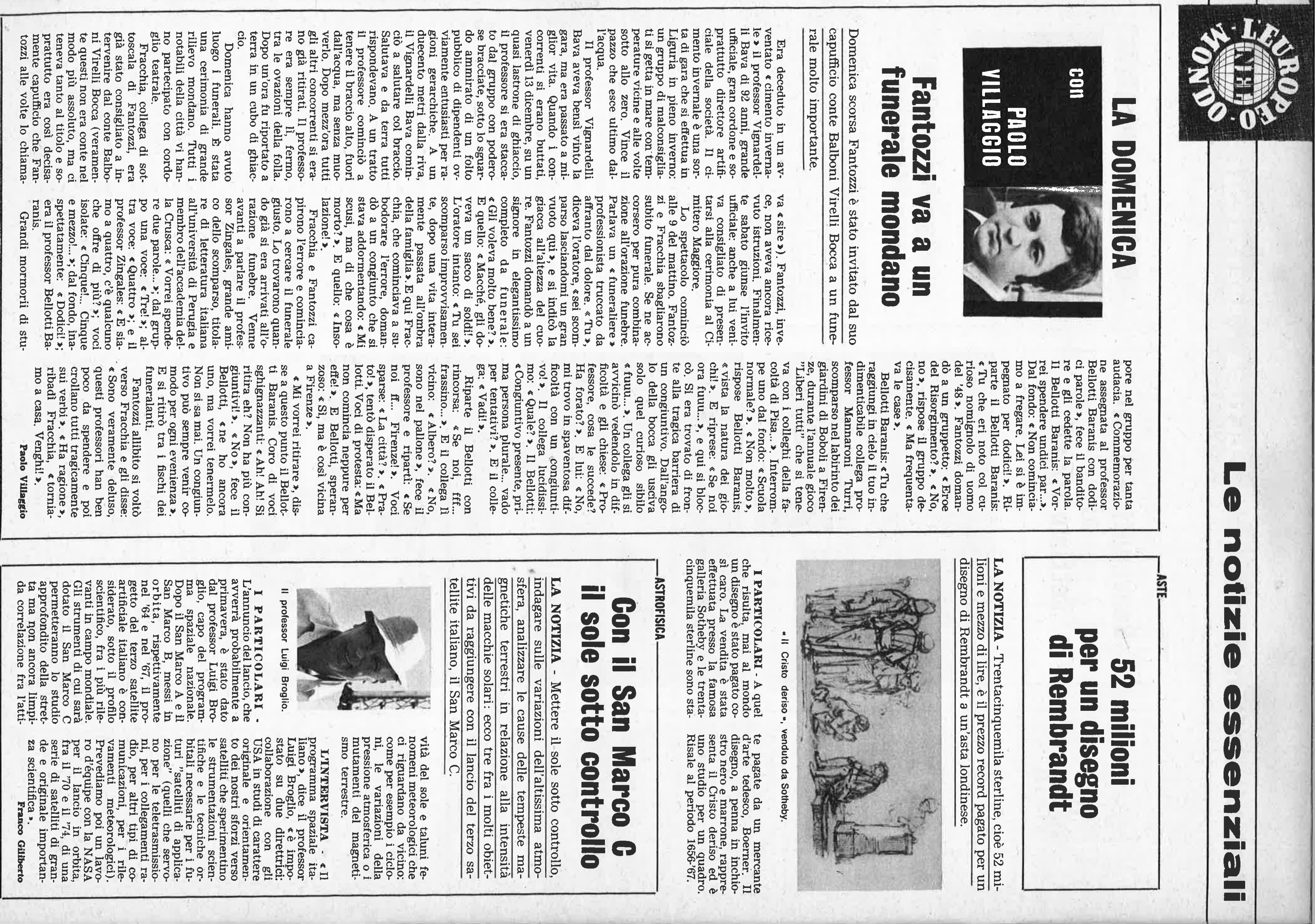




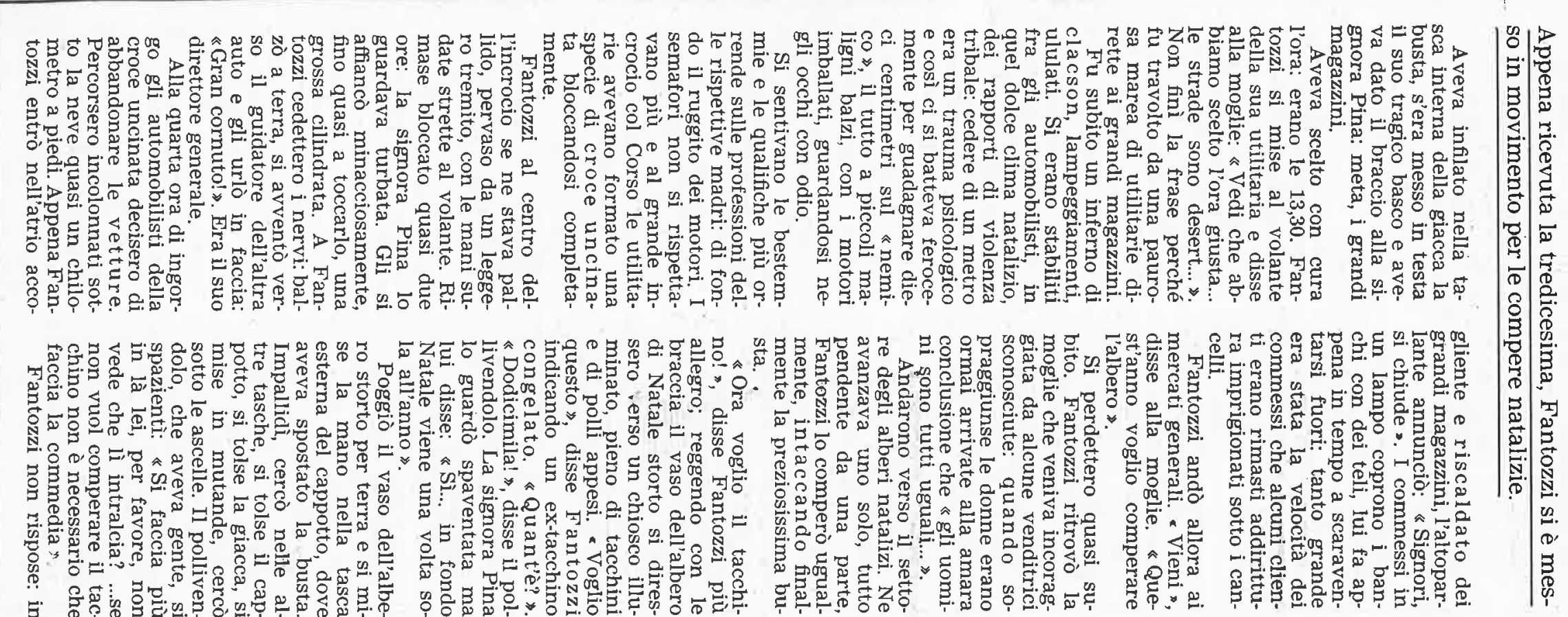
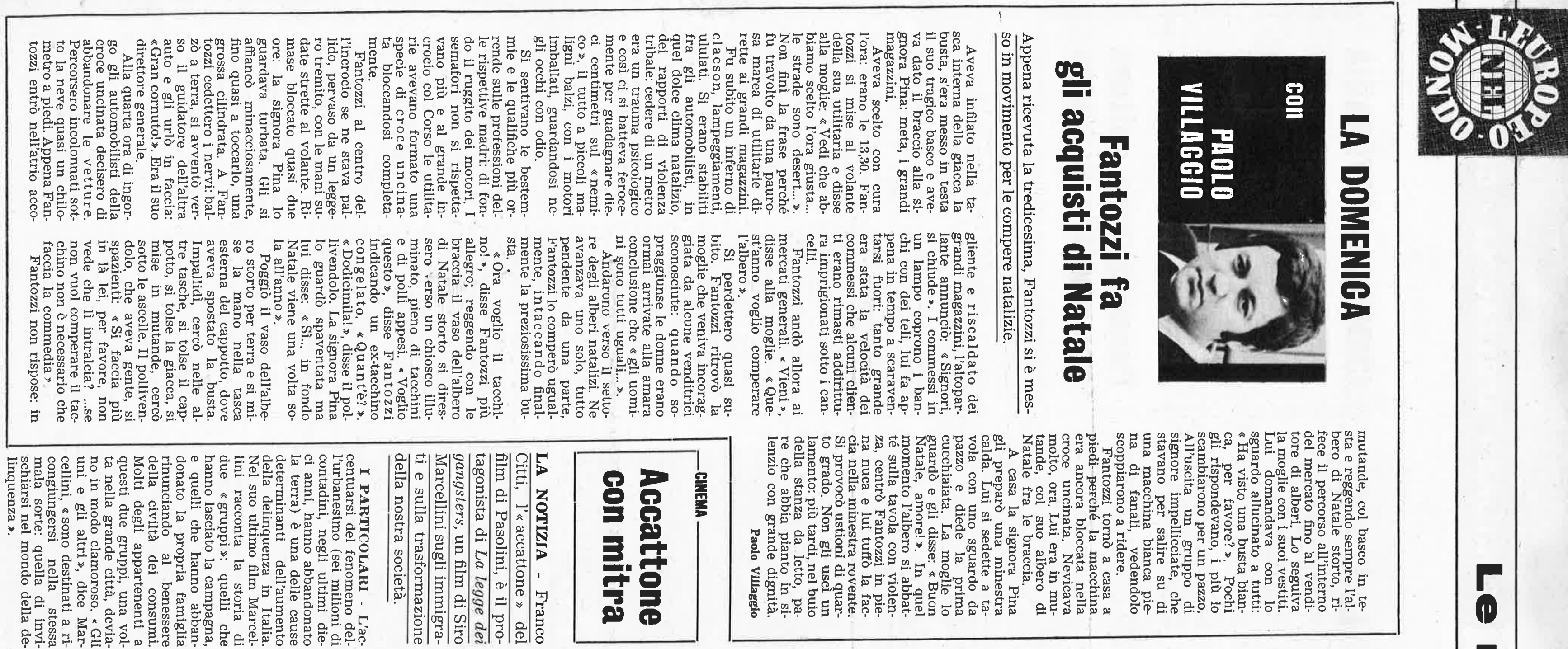

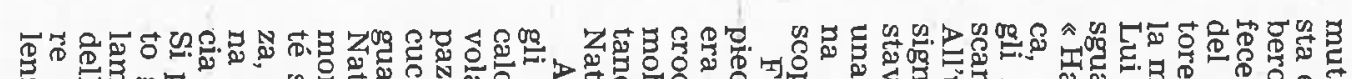

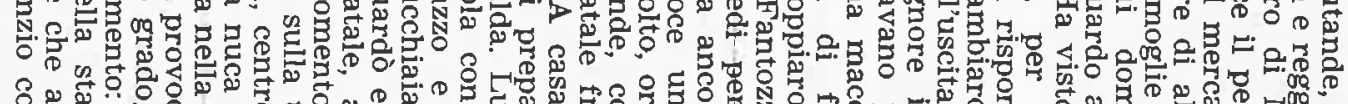

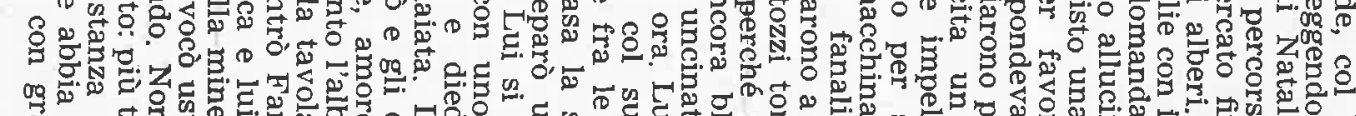

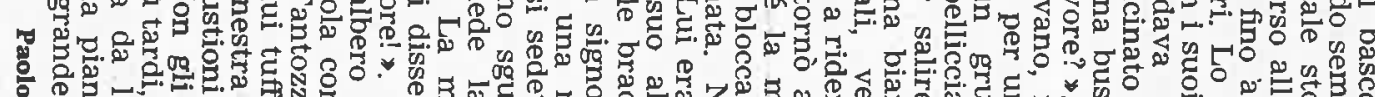

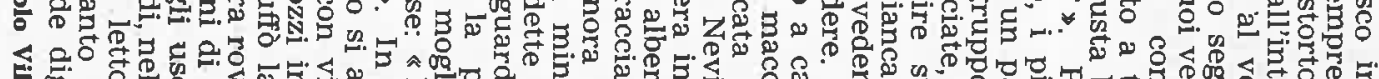

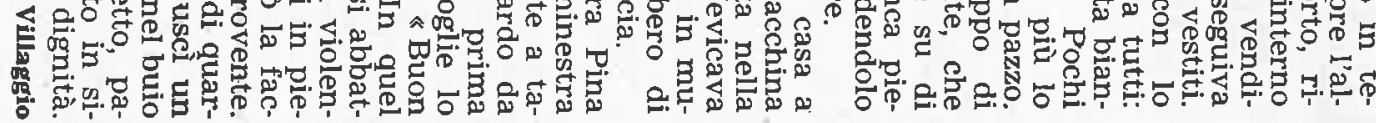

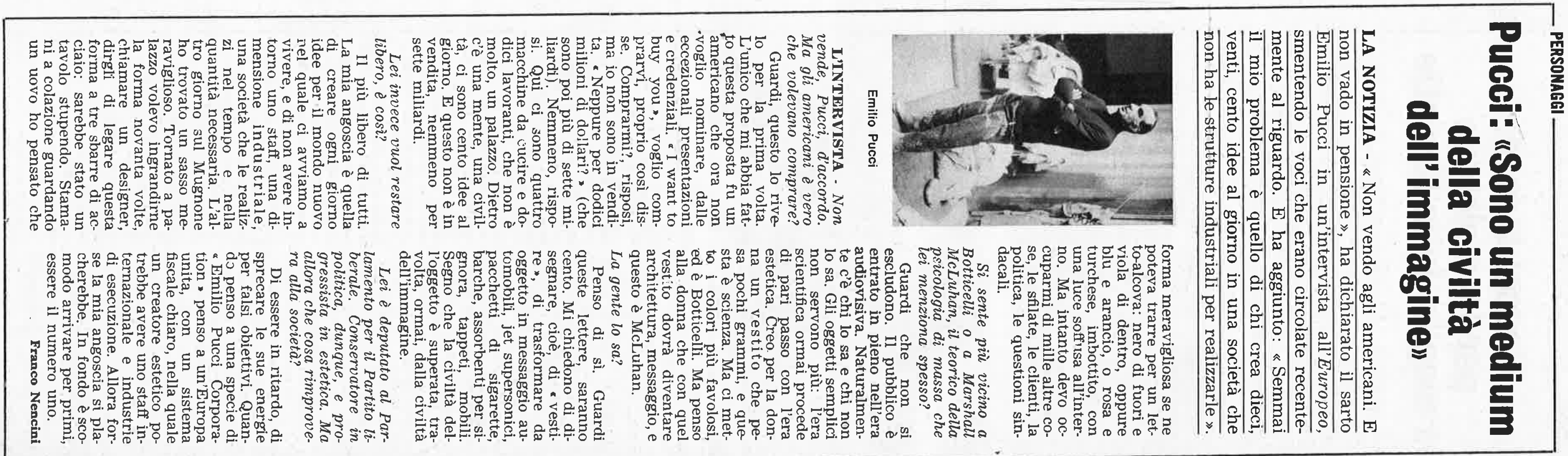




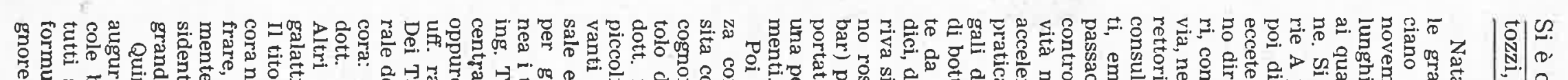

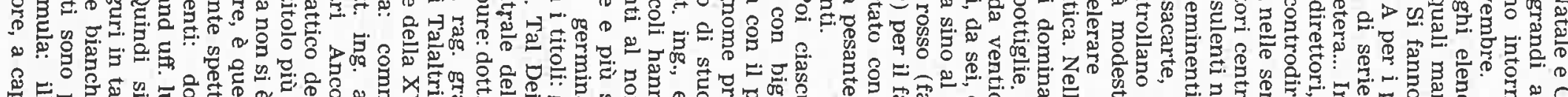

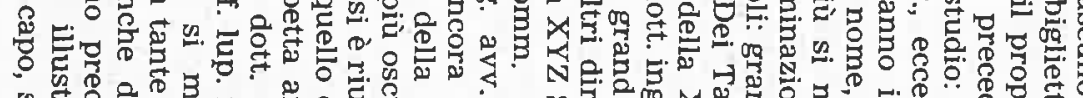

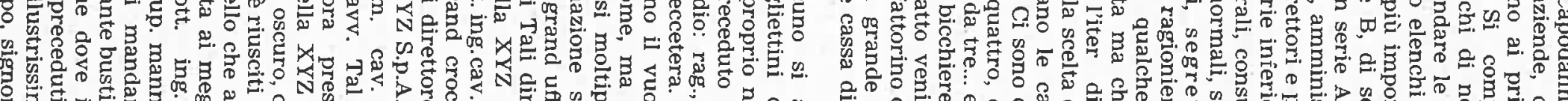

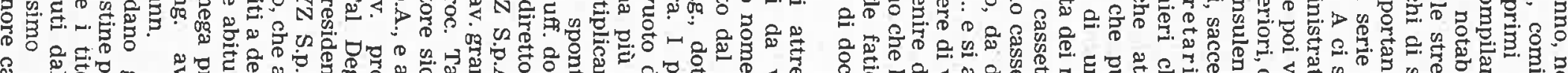

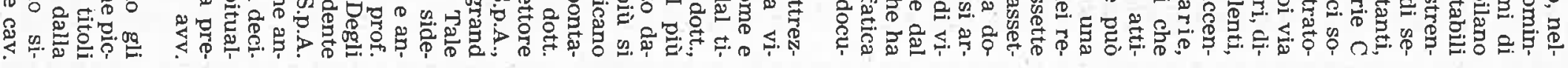

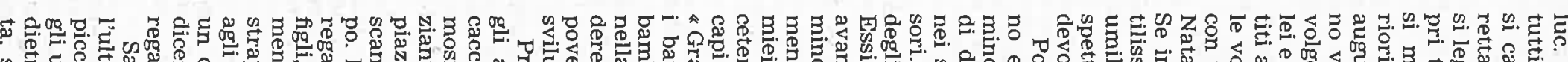
w.

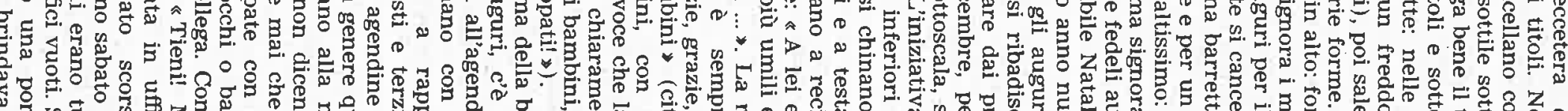

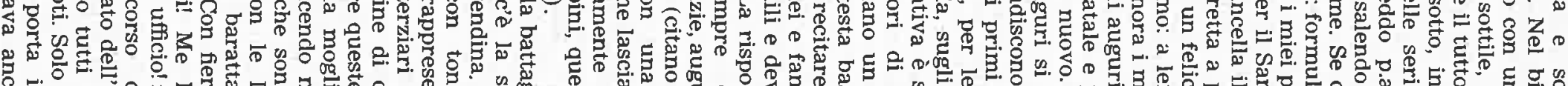

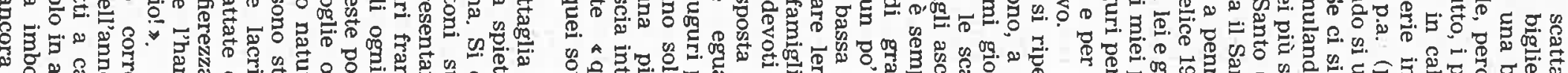

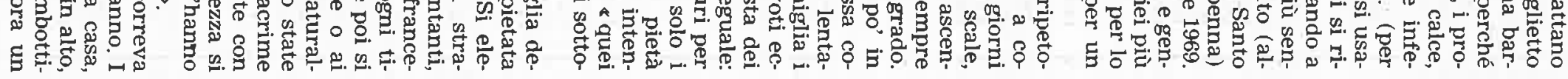

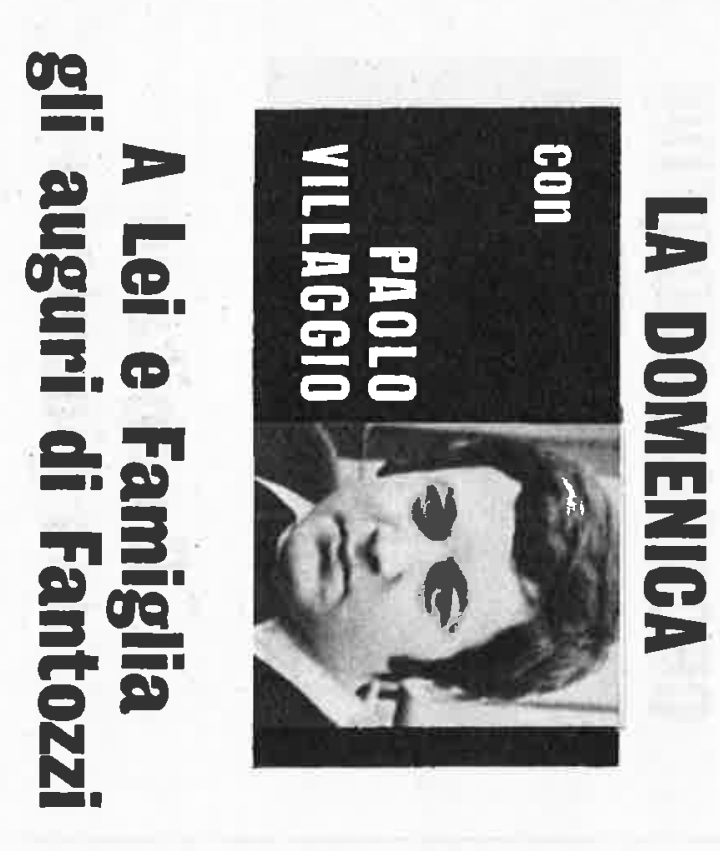

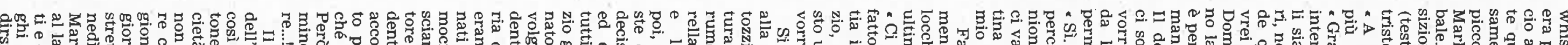

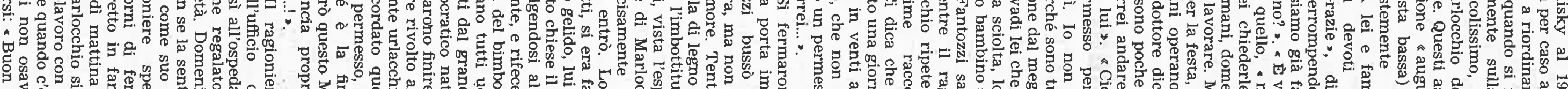

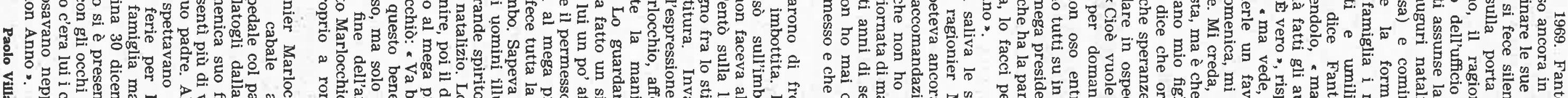

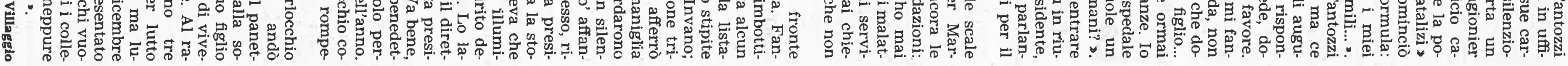

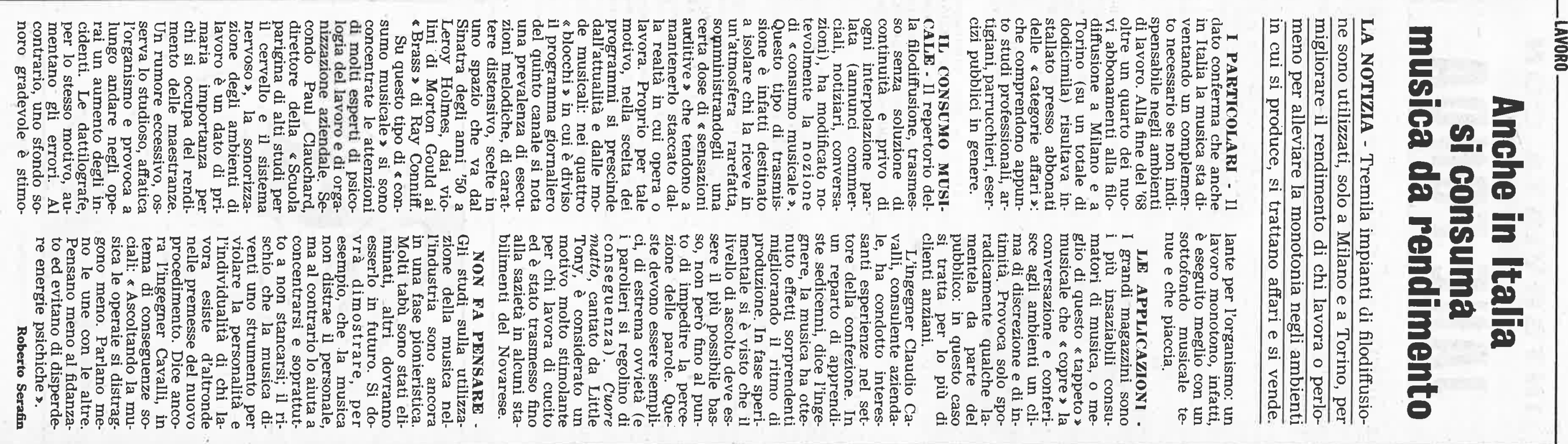




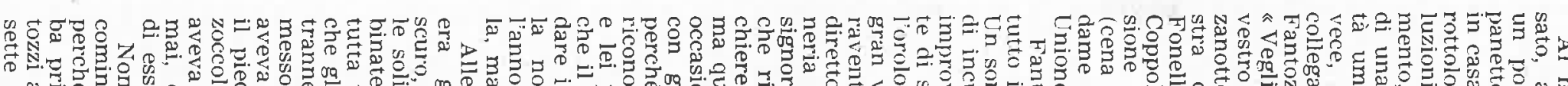

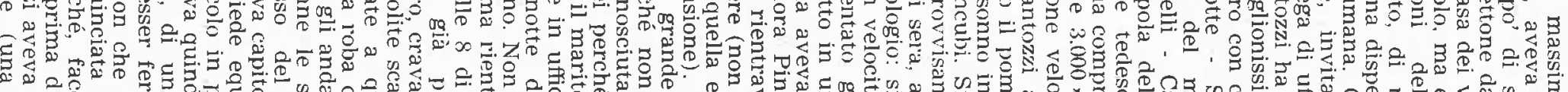

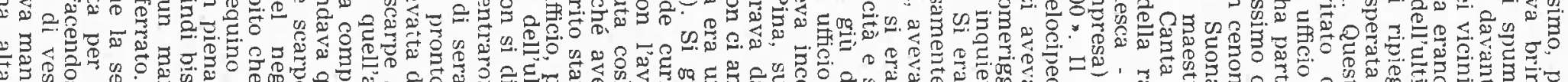

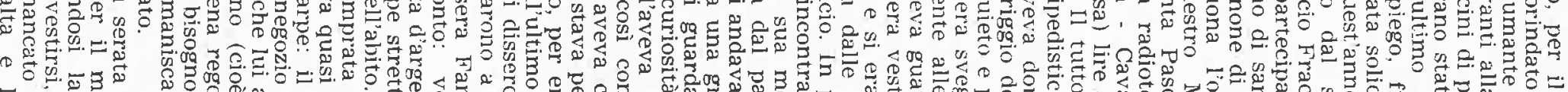

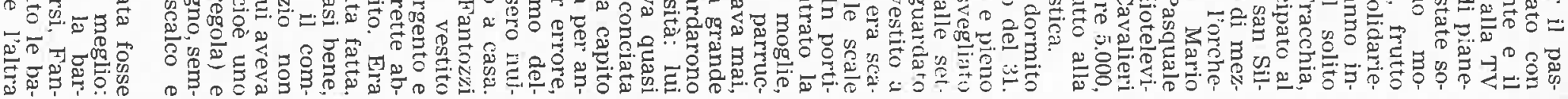

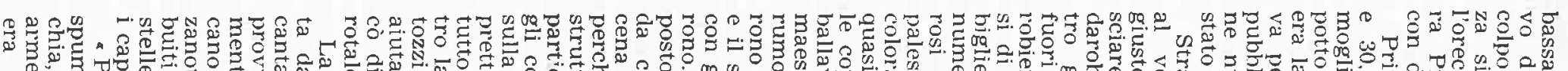

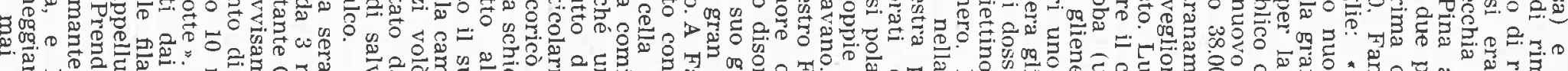

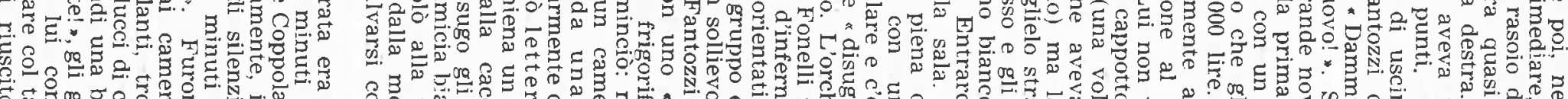

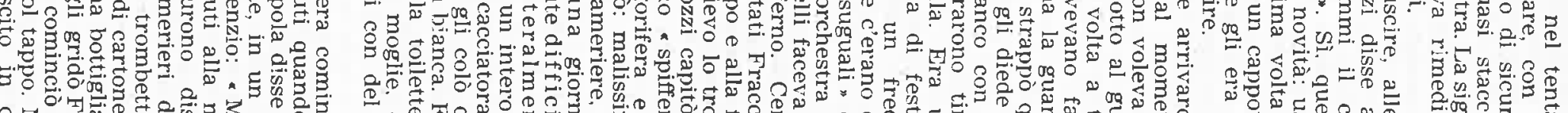

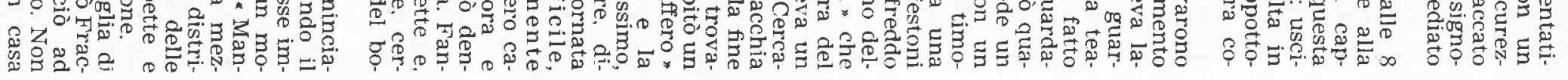
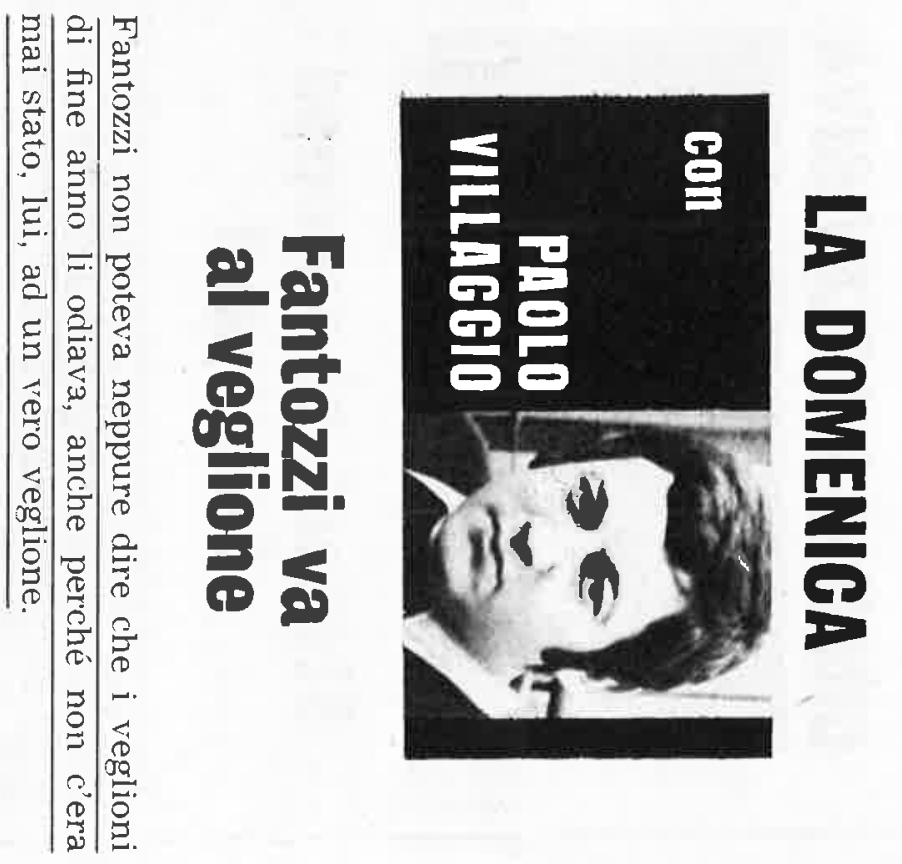

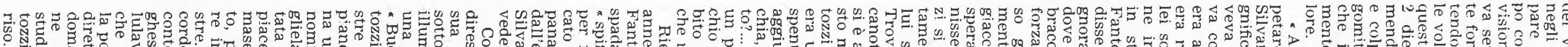

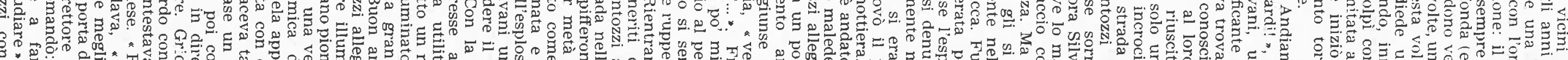

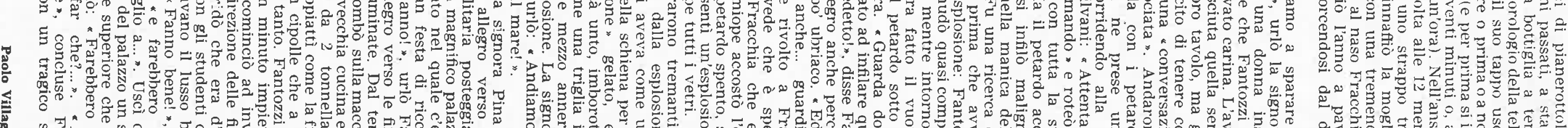

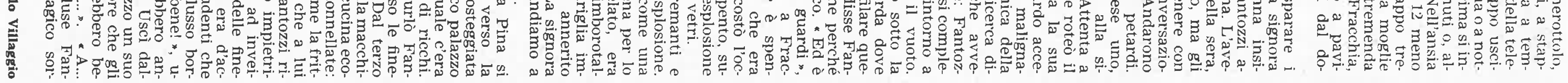
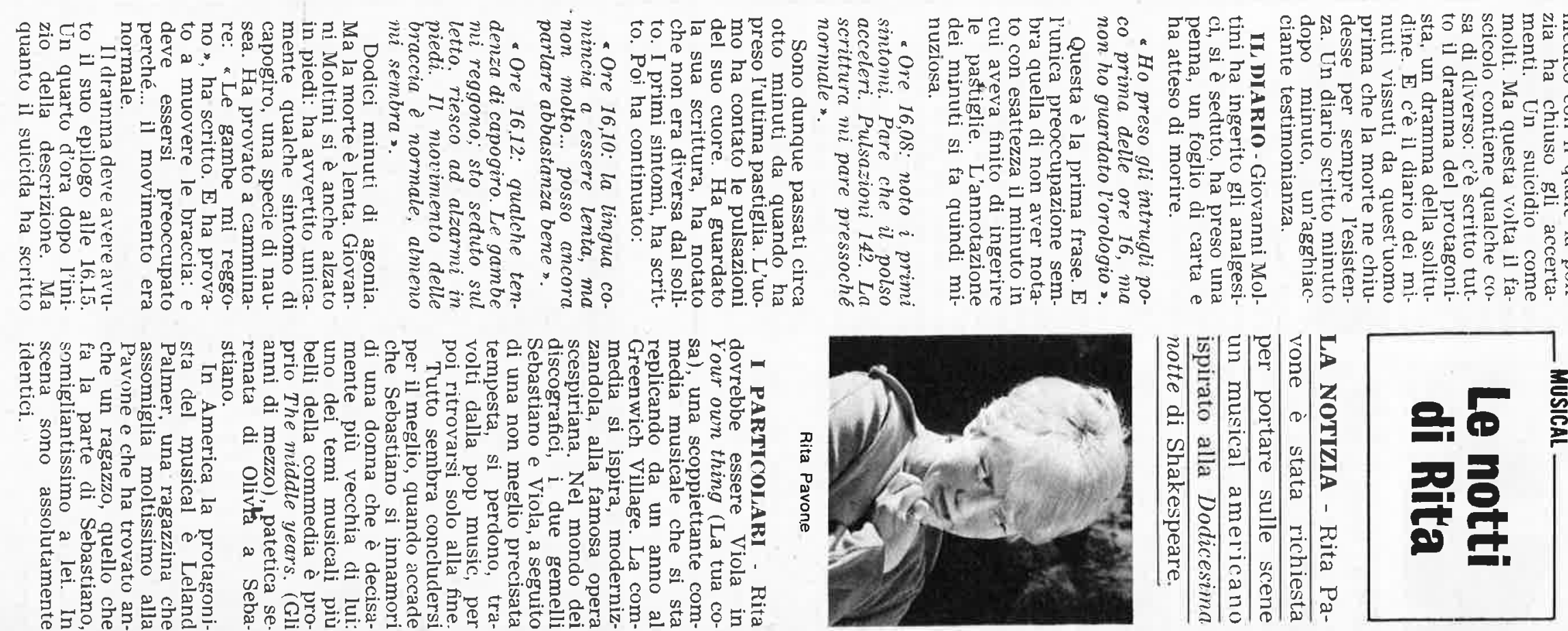

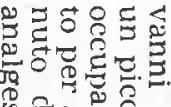
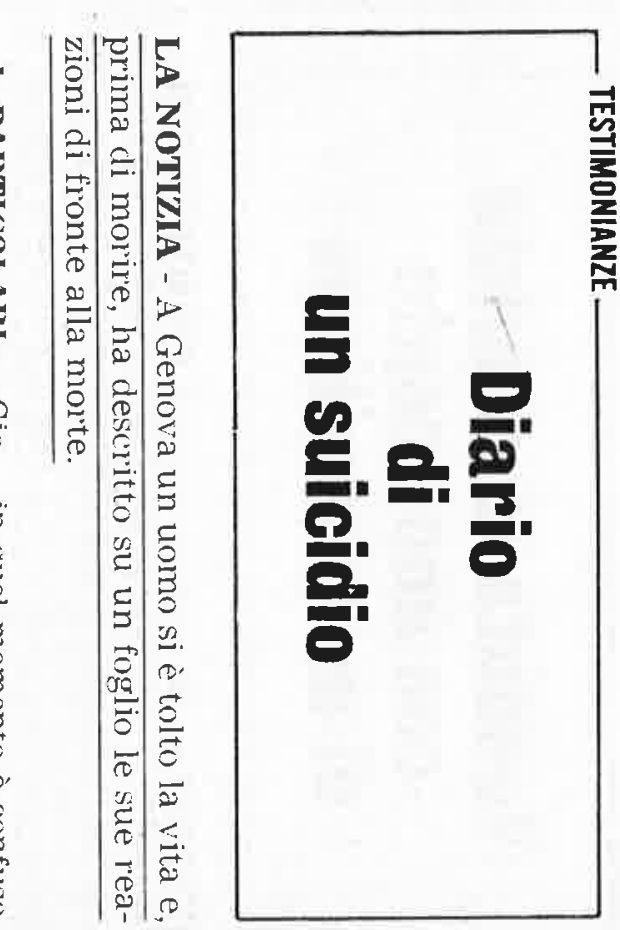


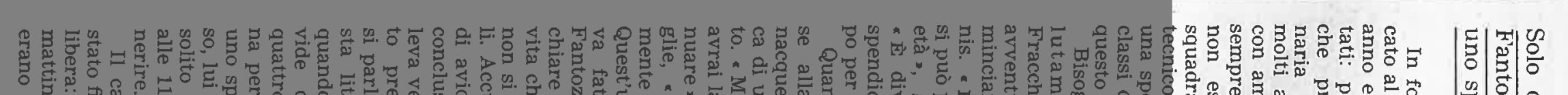

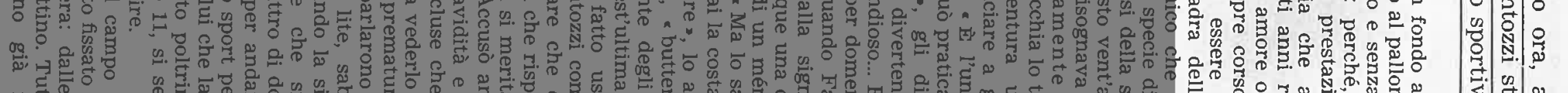

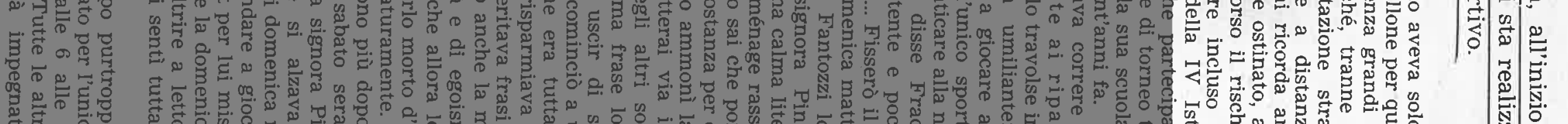

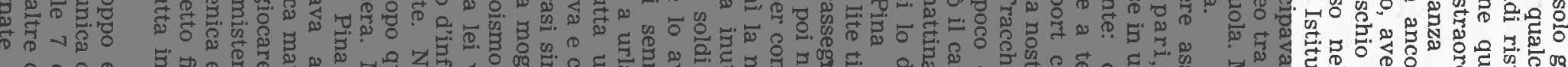

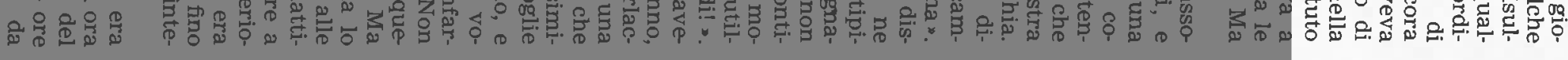

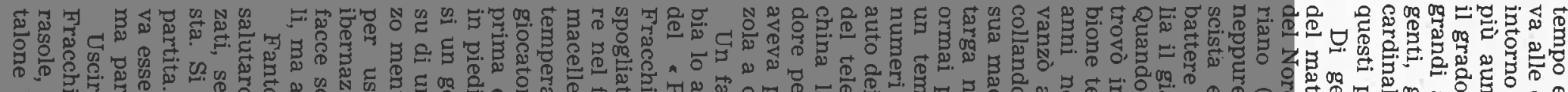

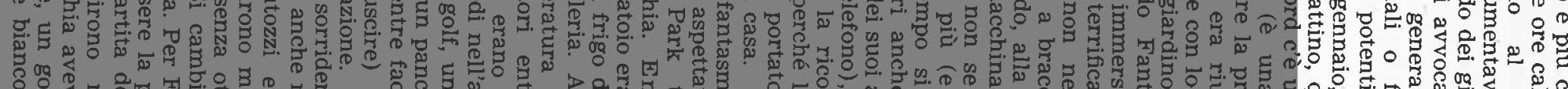

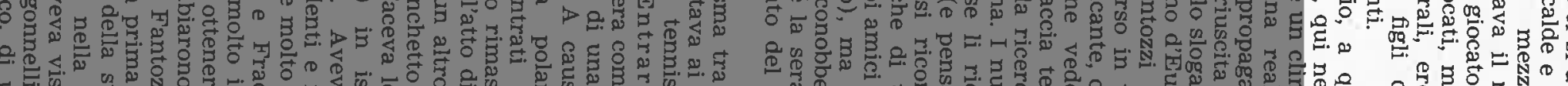

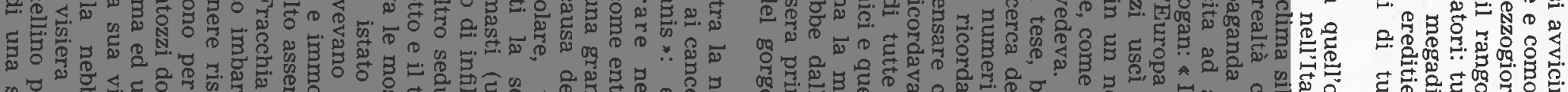

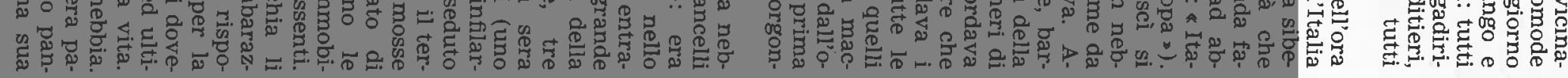

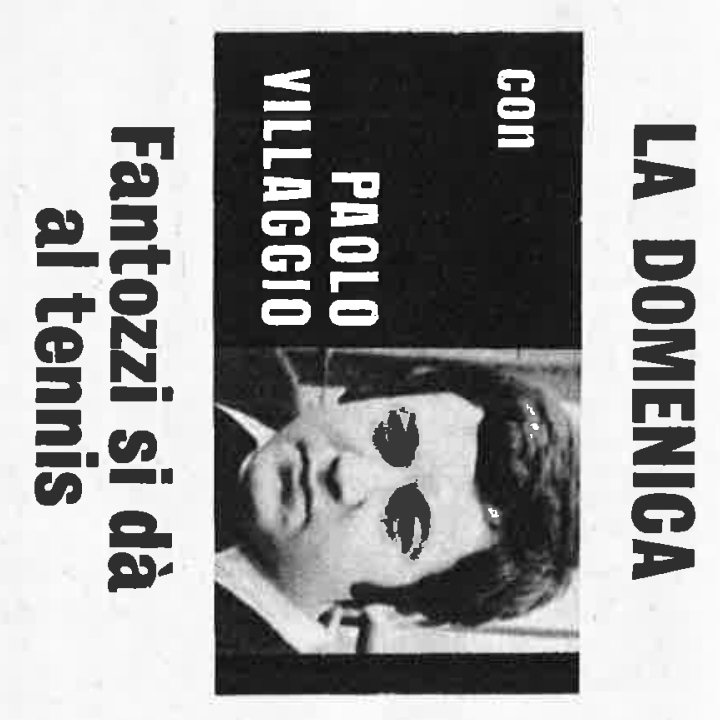

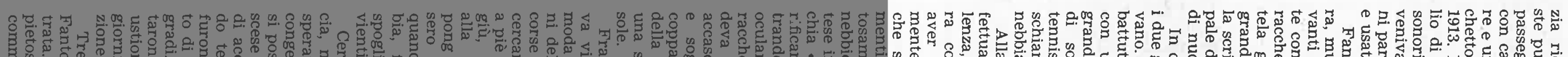

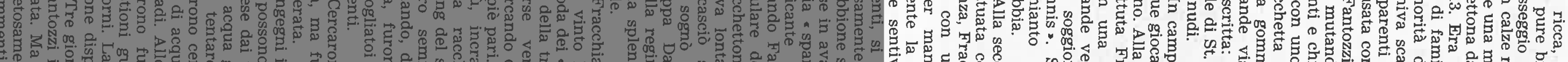

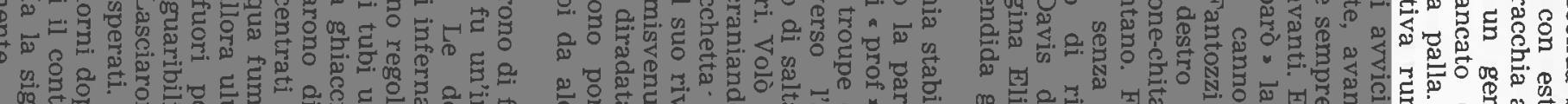

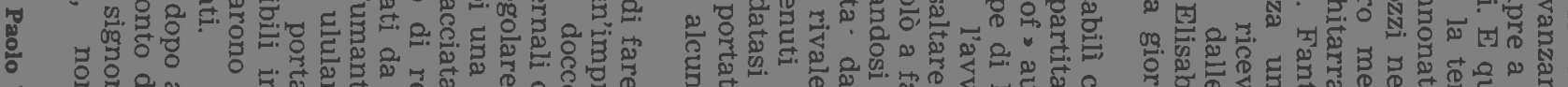

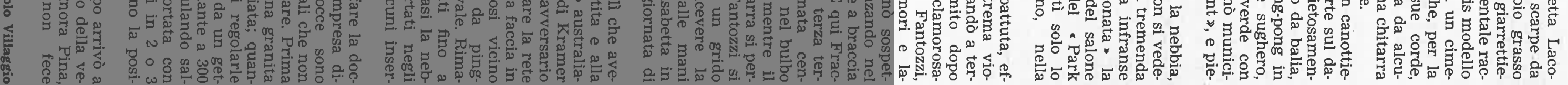
等

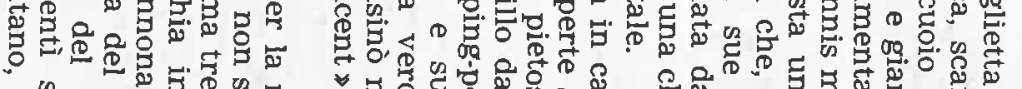

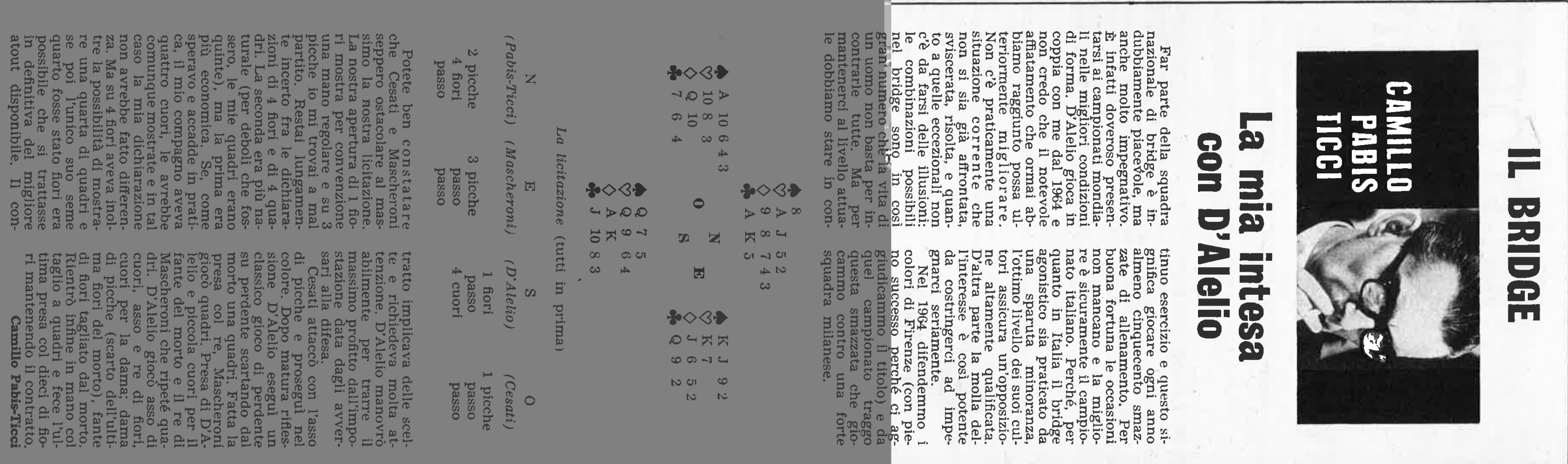




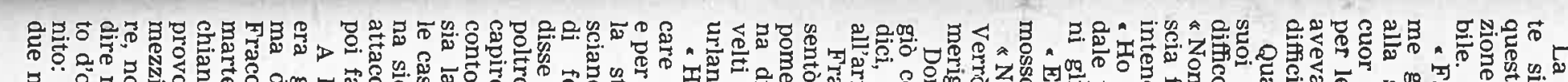

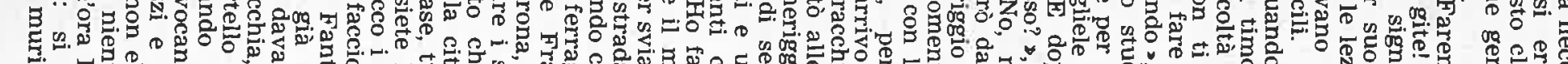

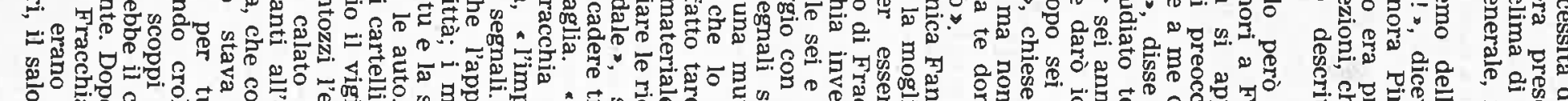

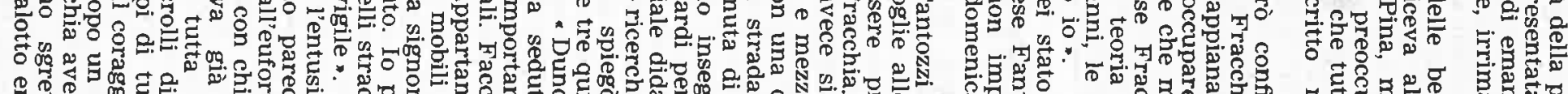

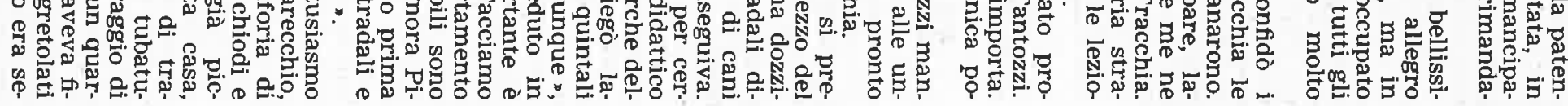
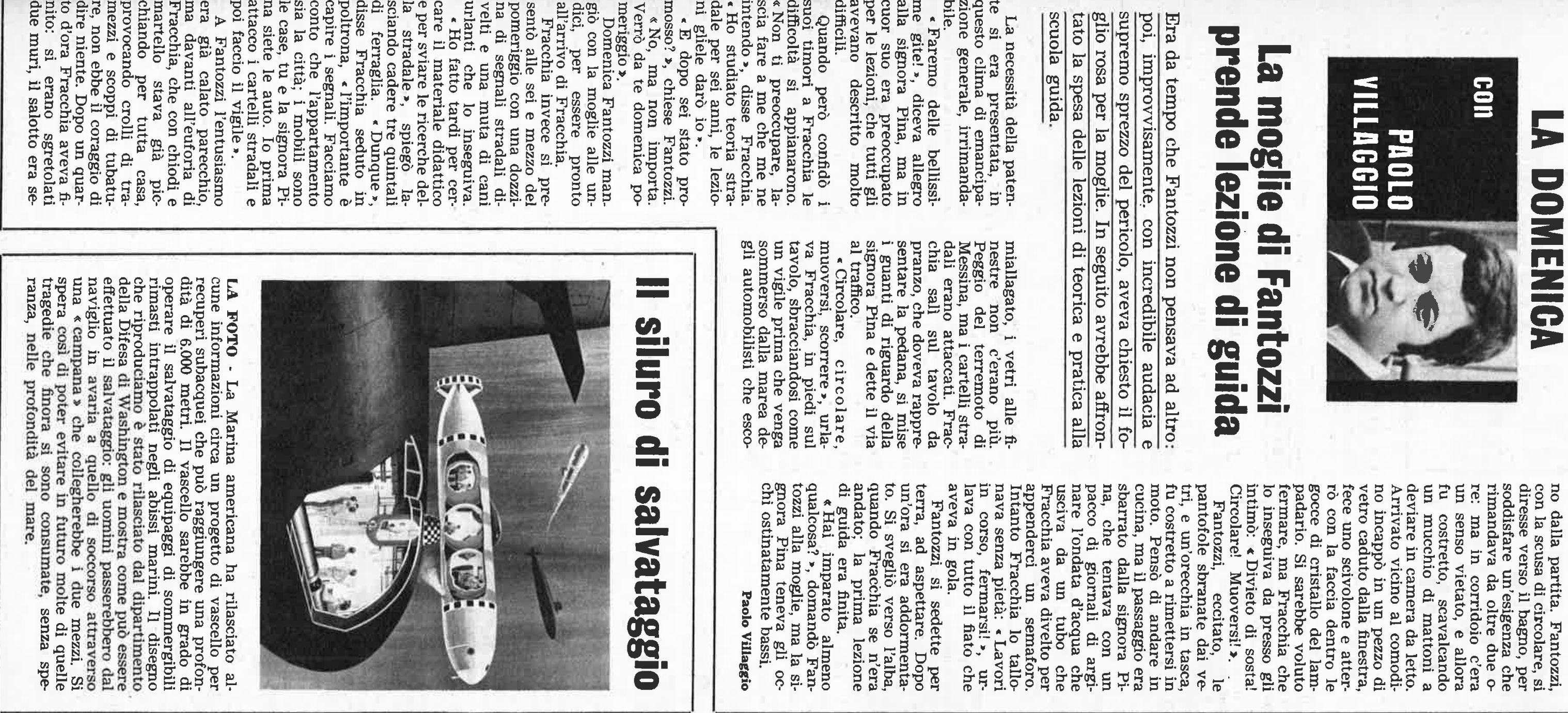

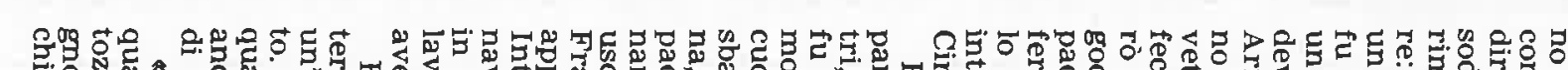

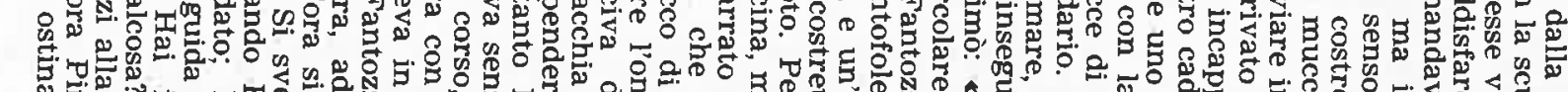

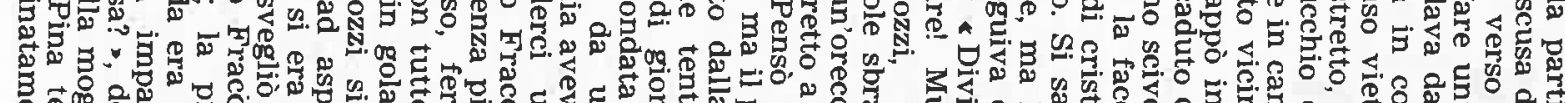

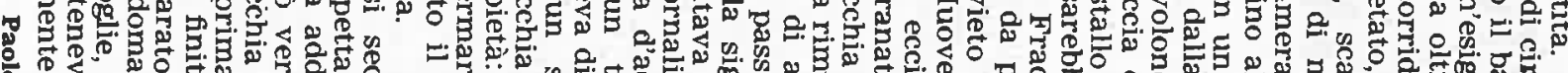

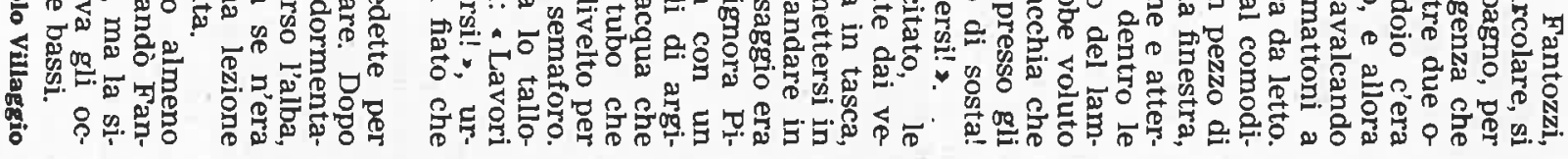
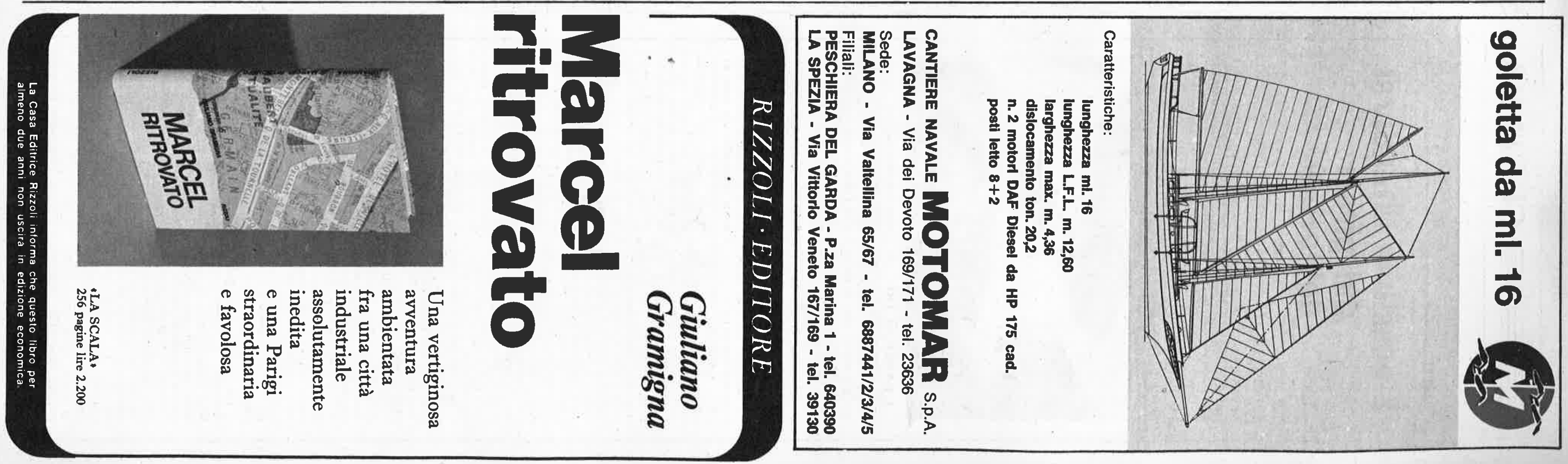


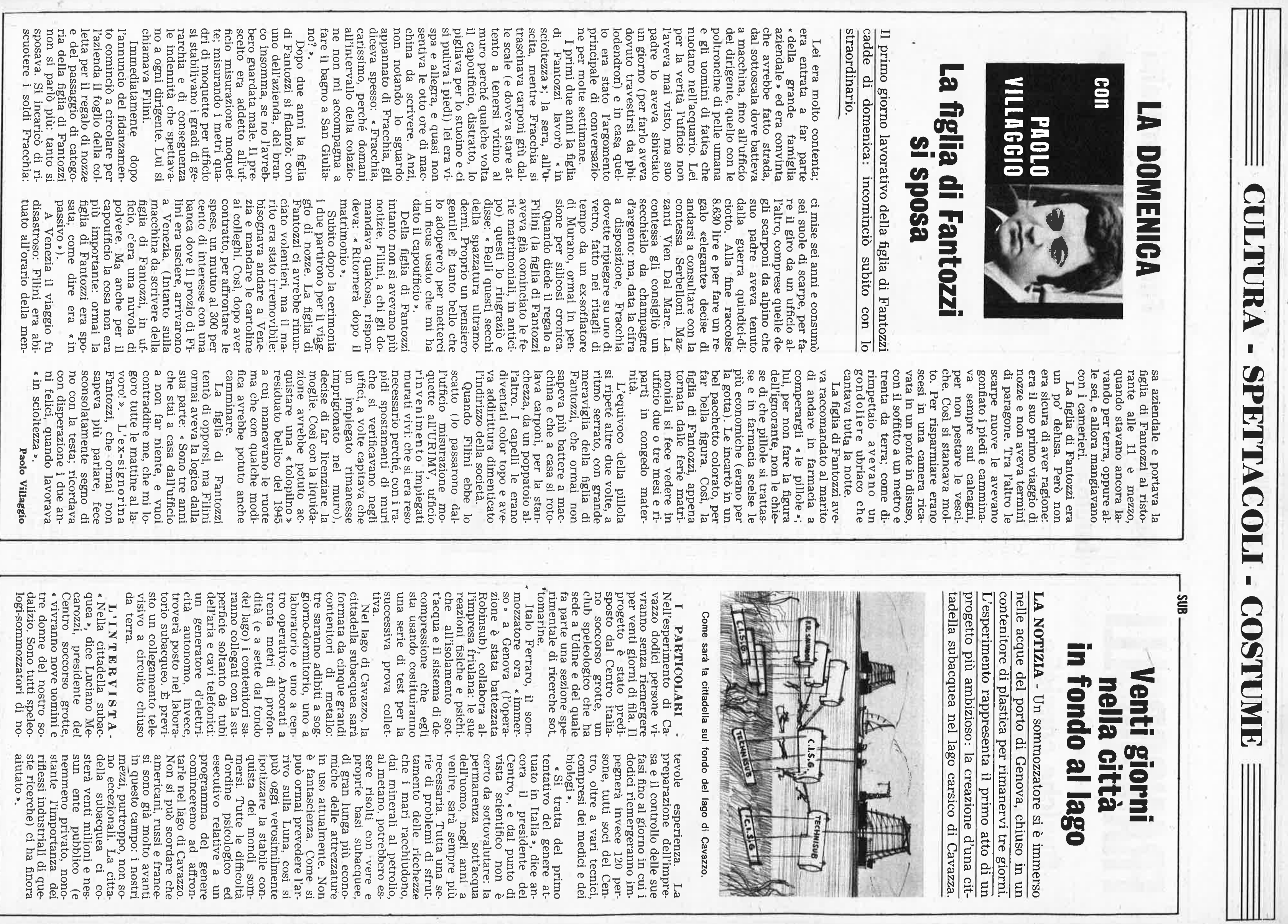



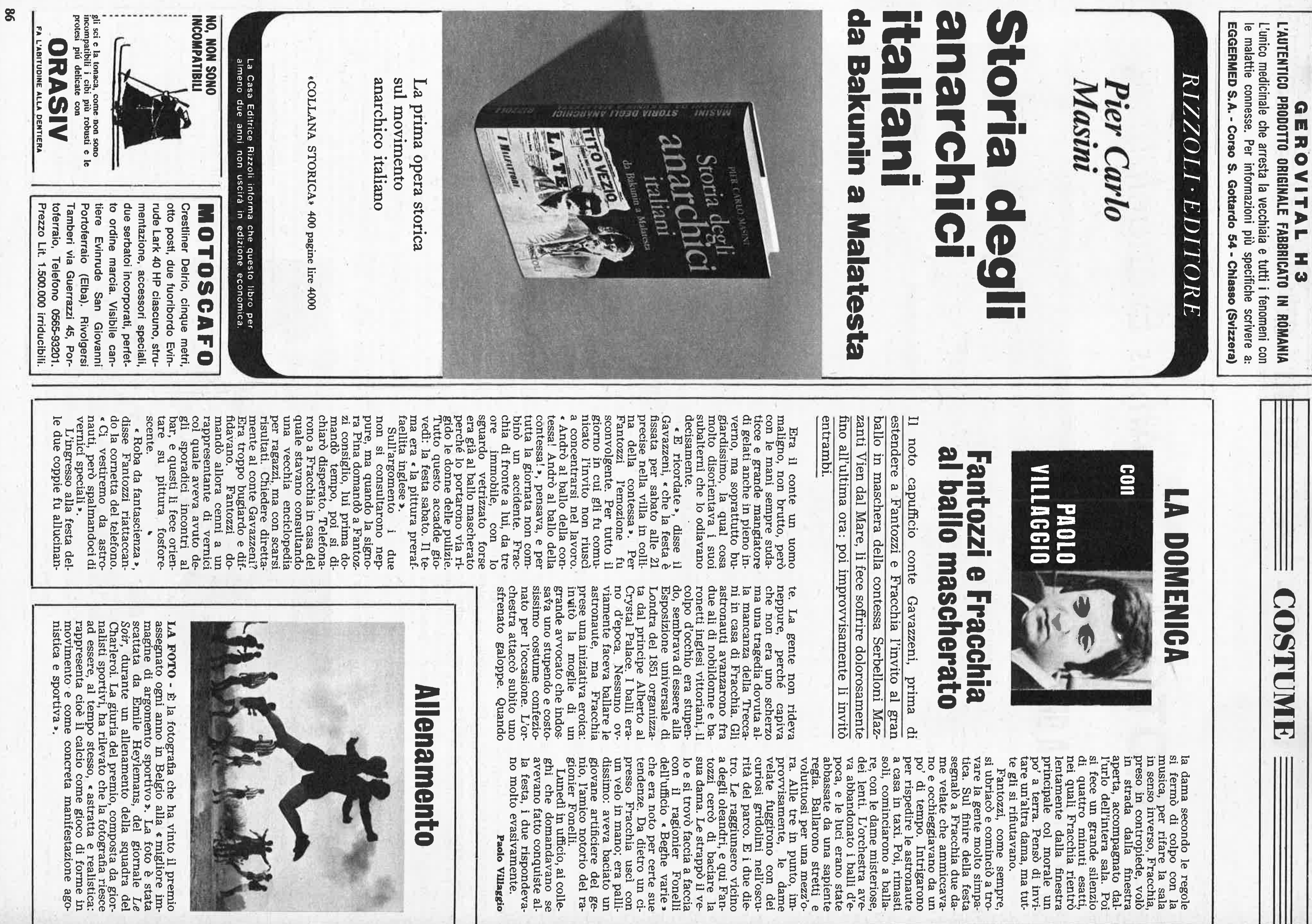

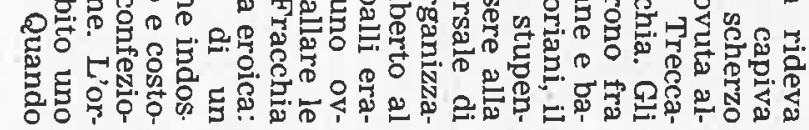

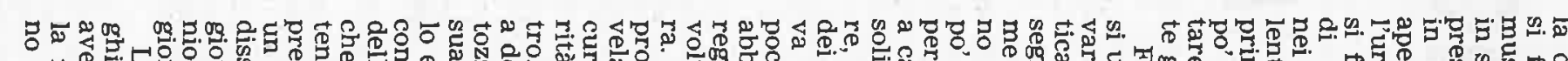

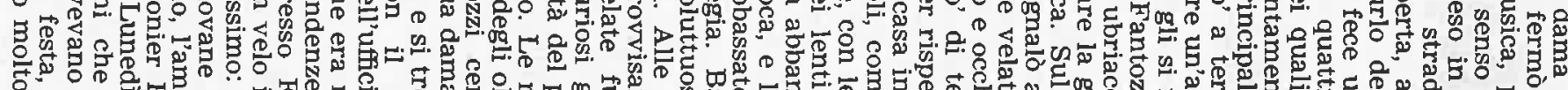

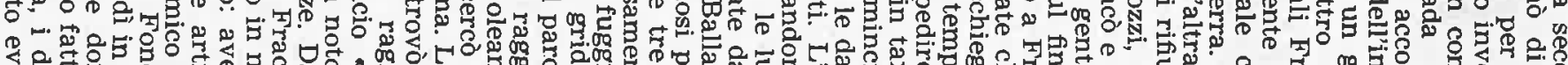

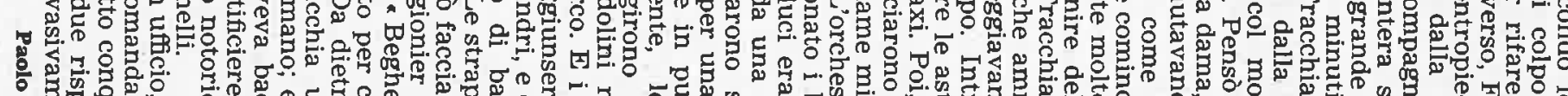

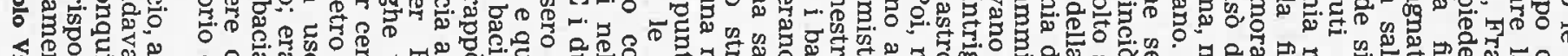

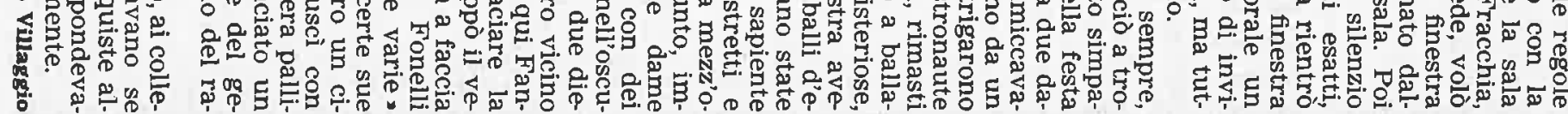

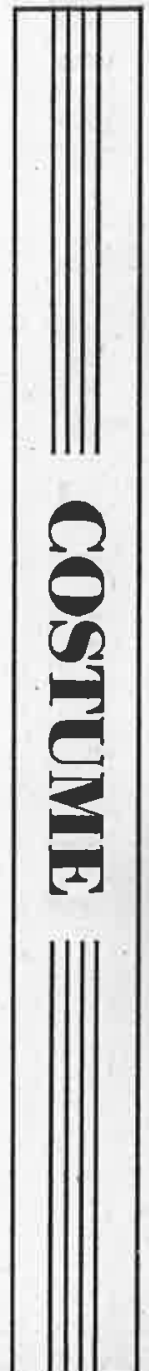



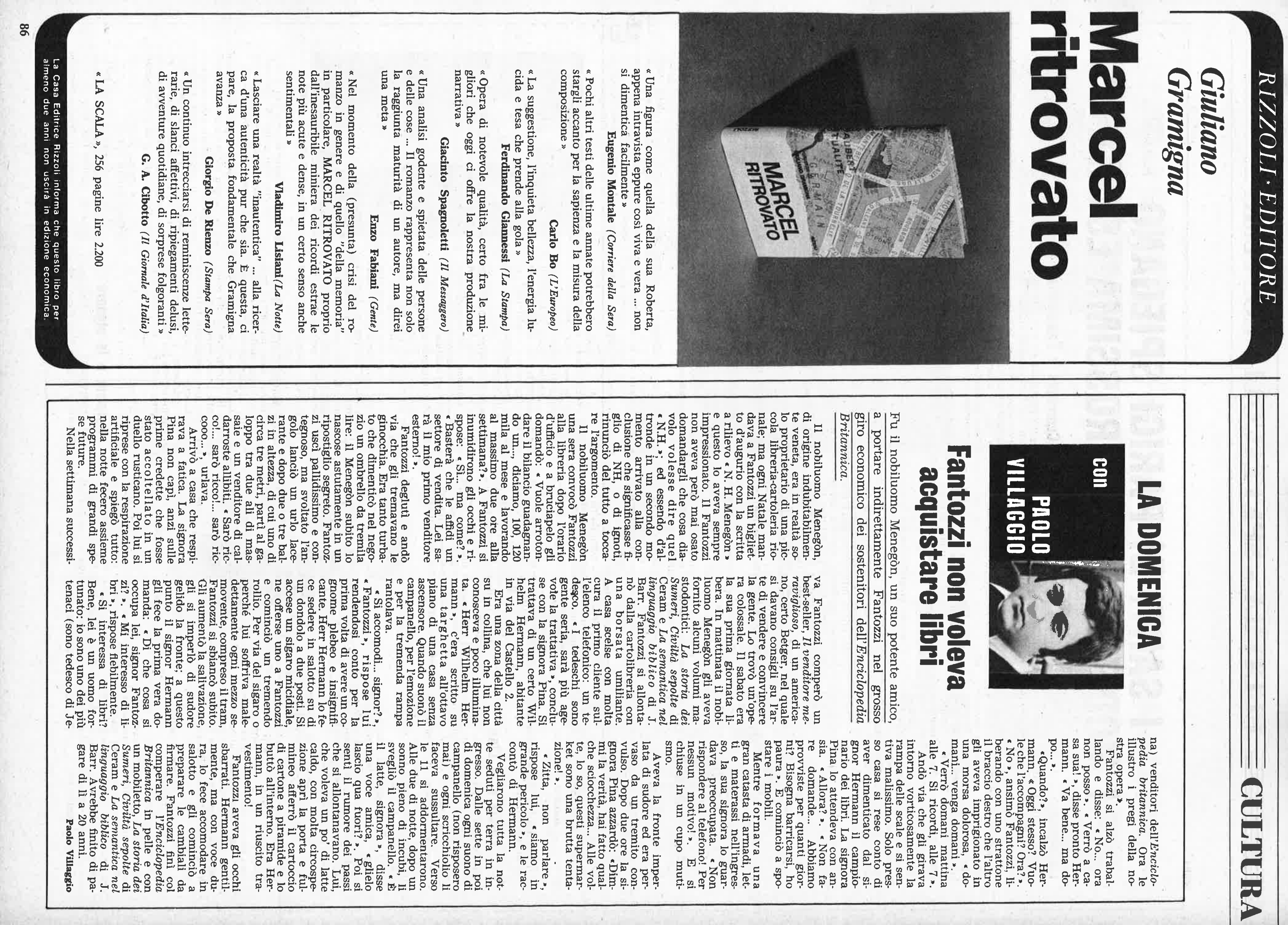

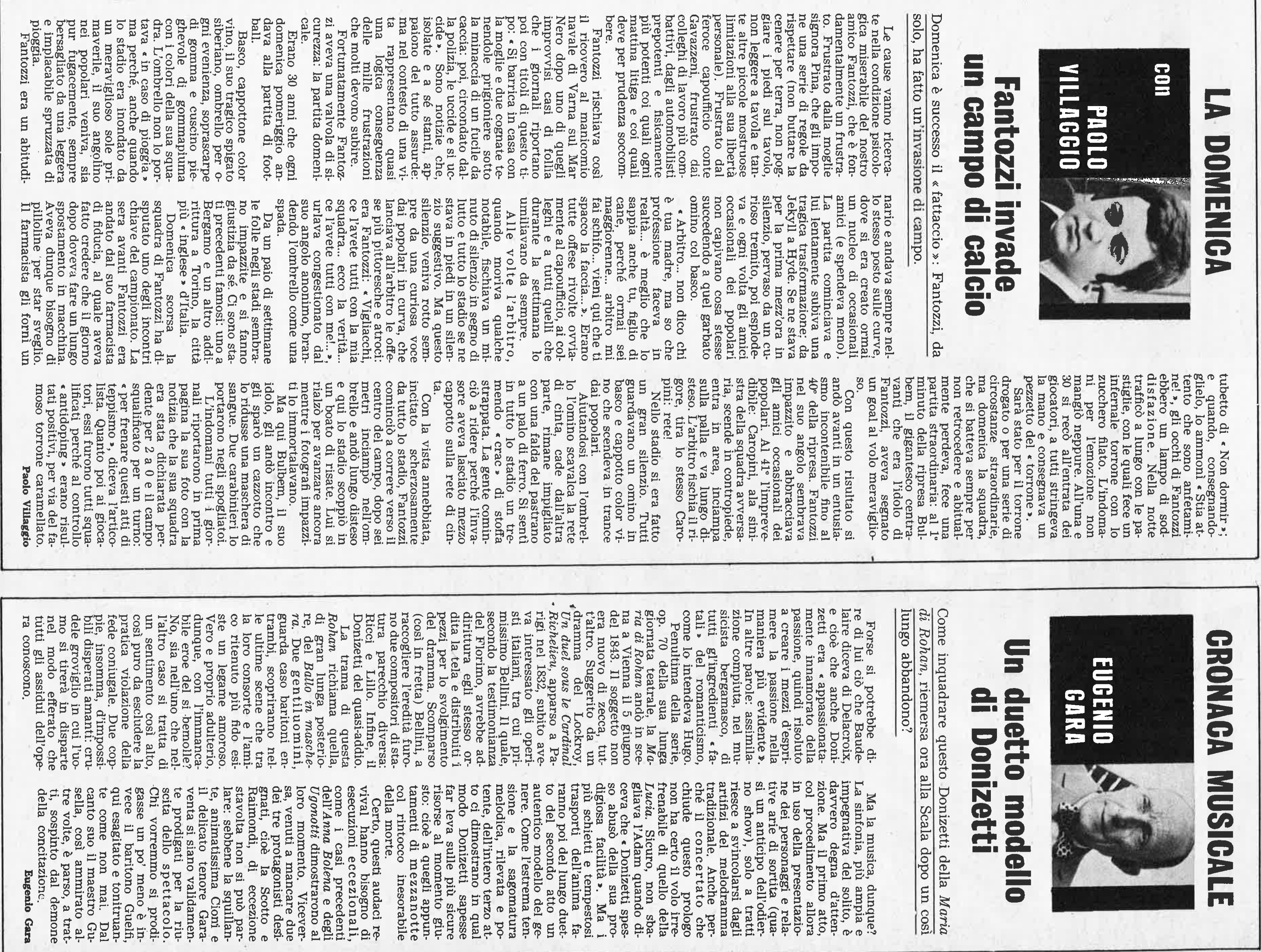

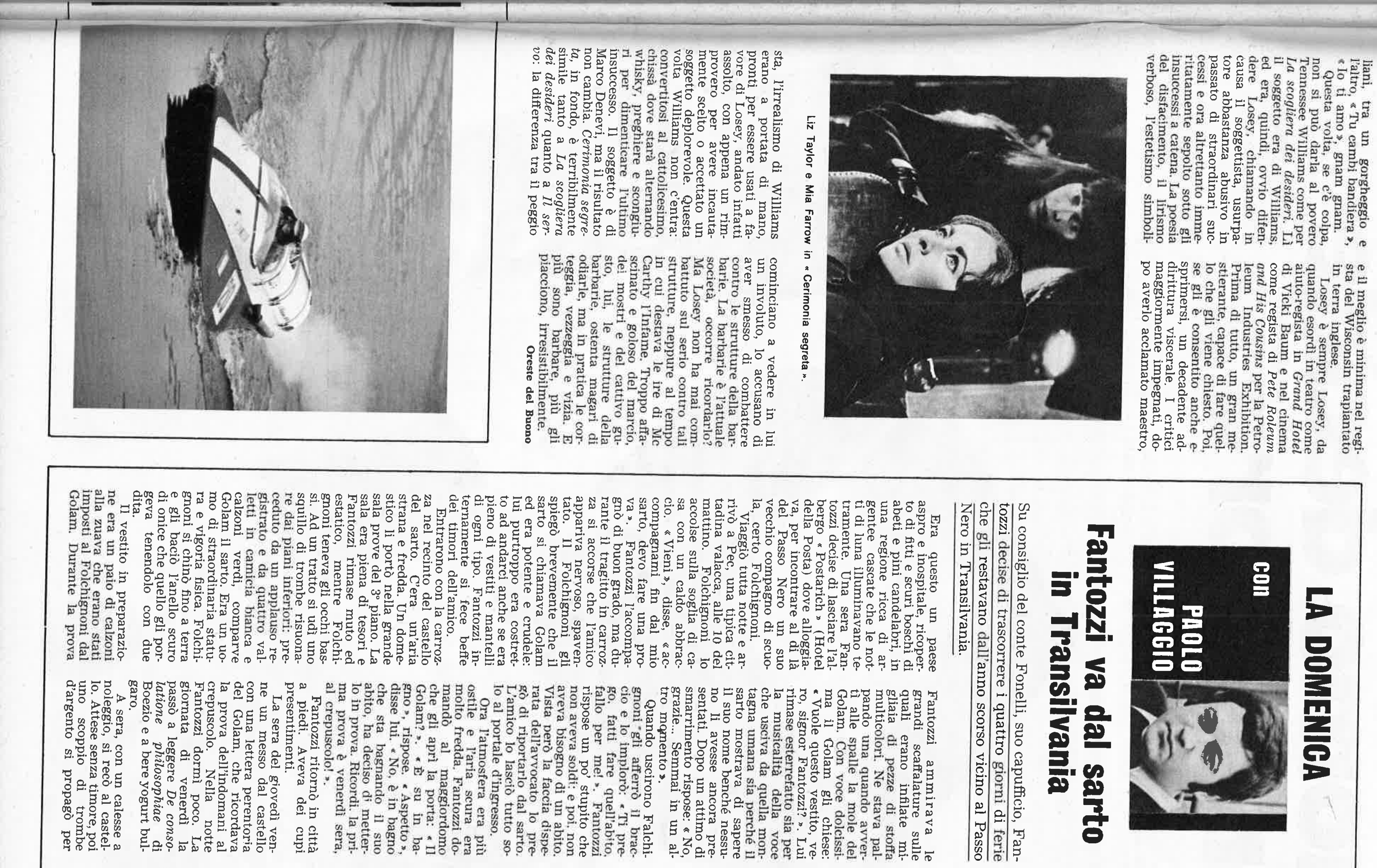

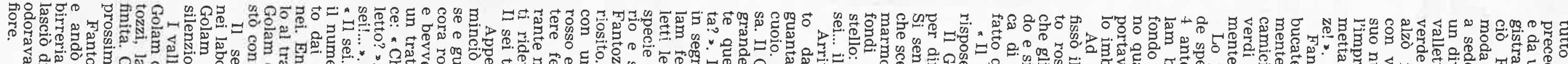
世.

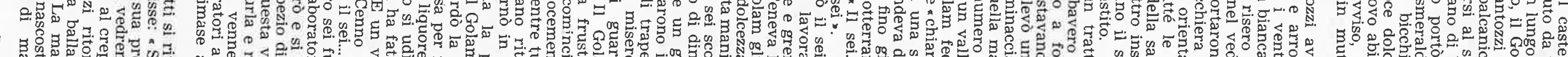

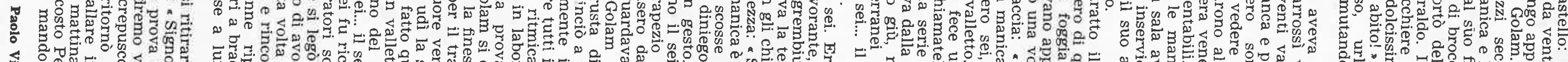

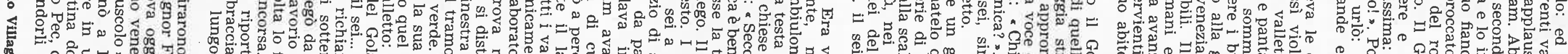

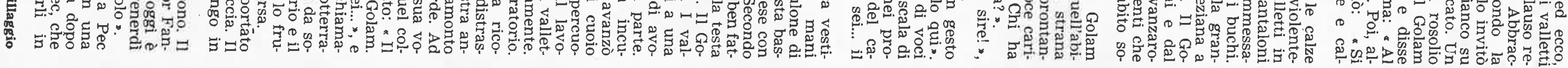



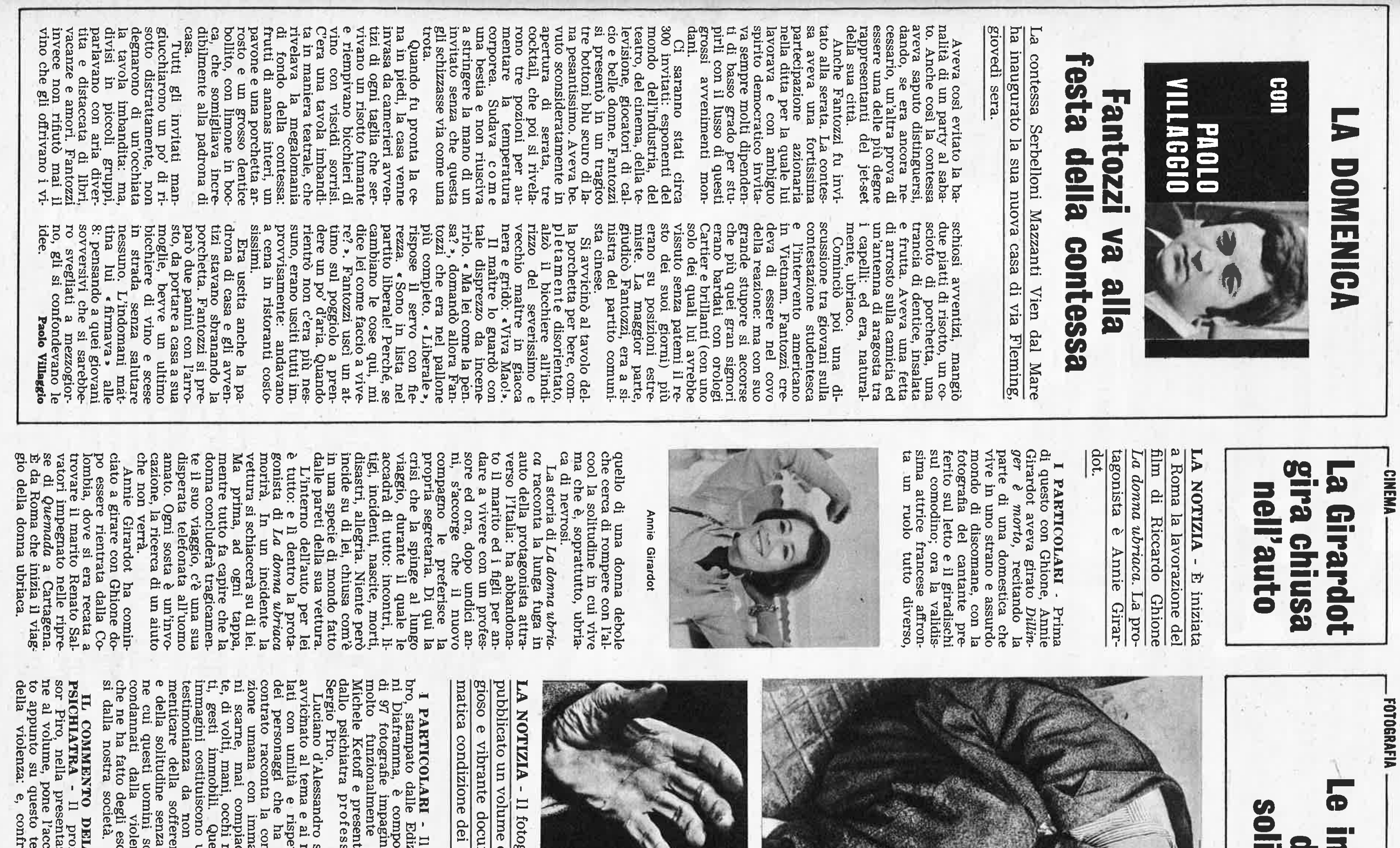

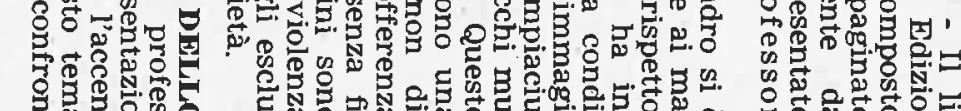

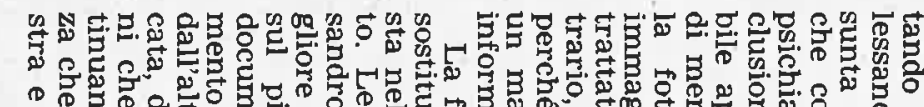

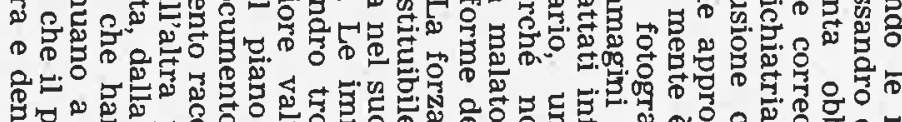

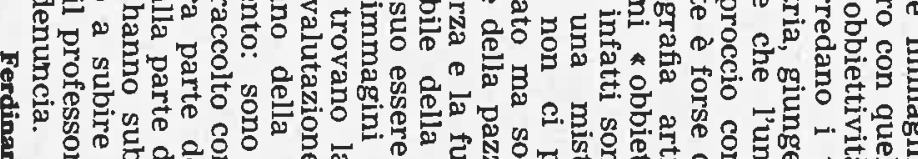

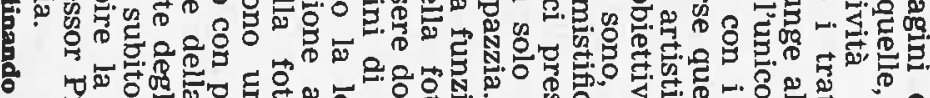

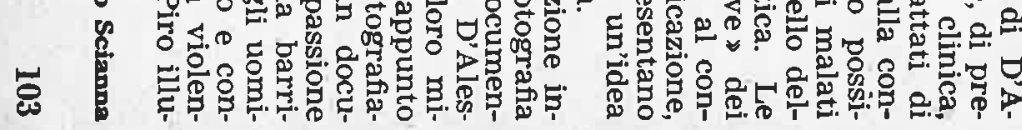
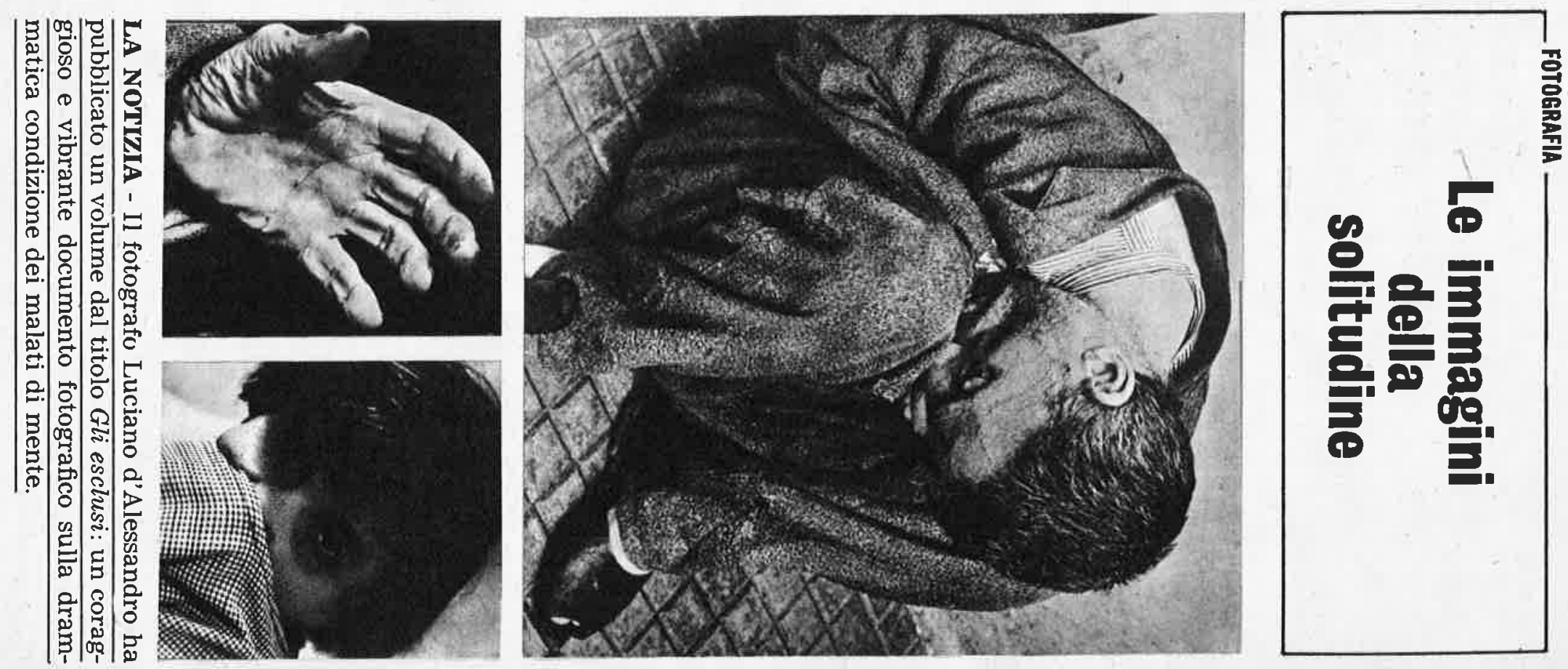


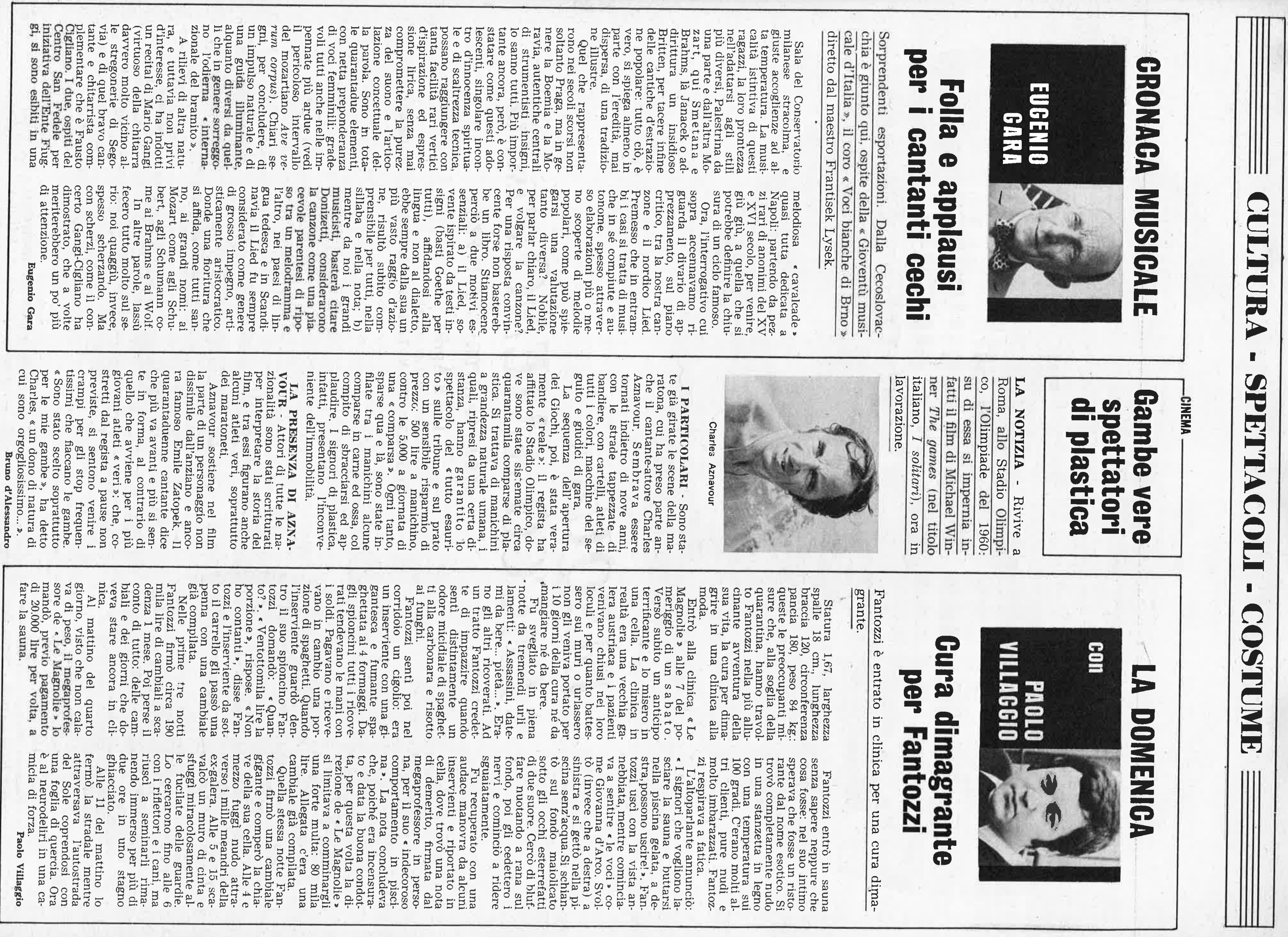



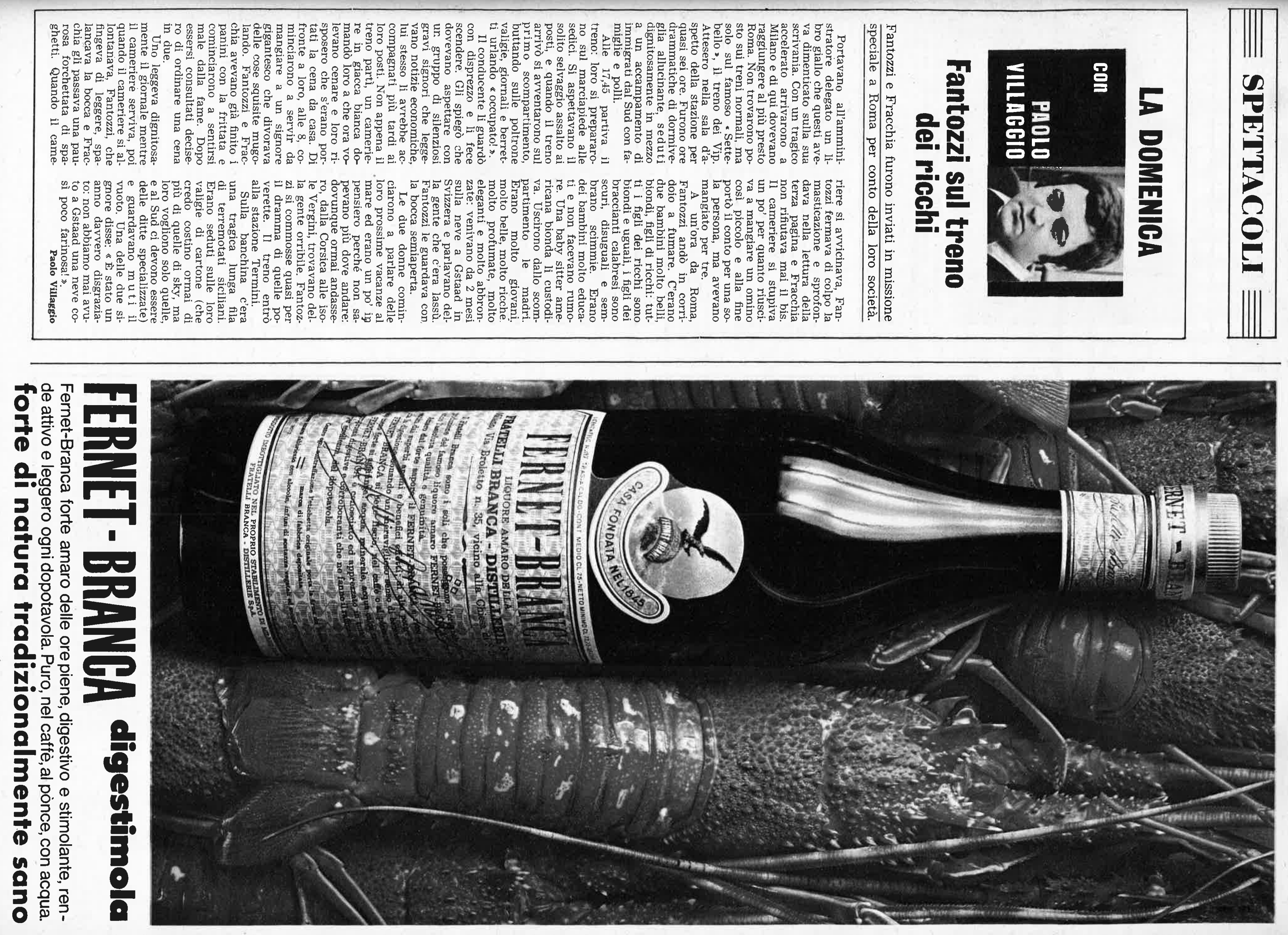


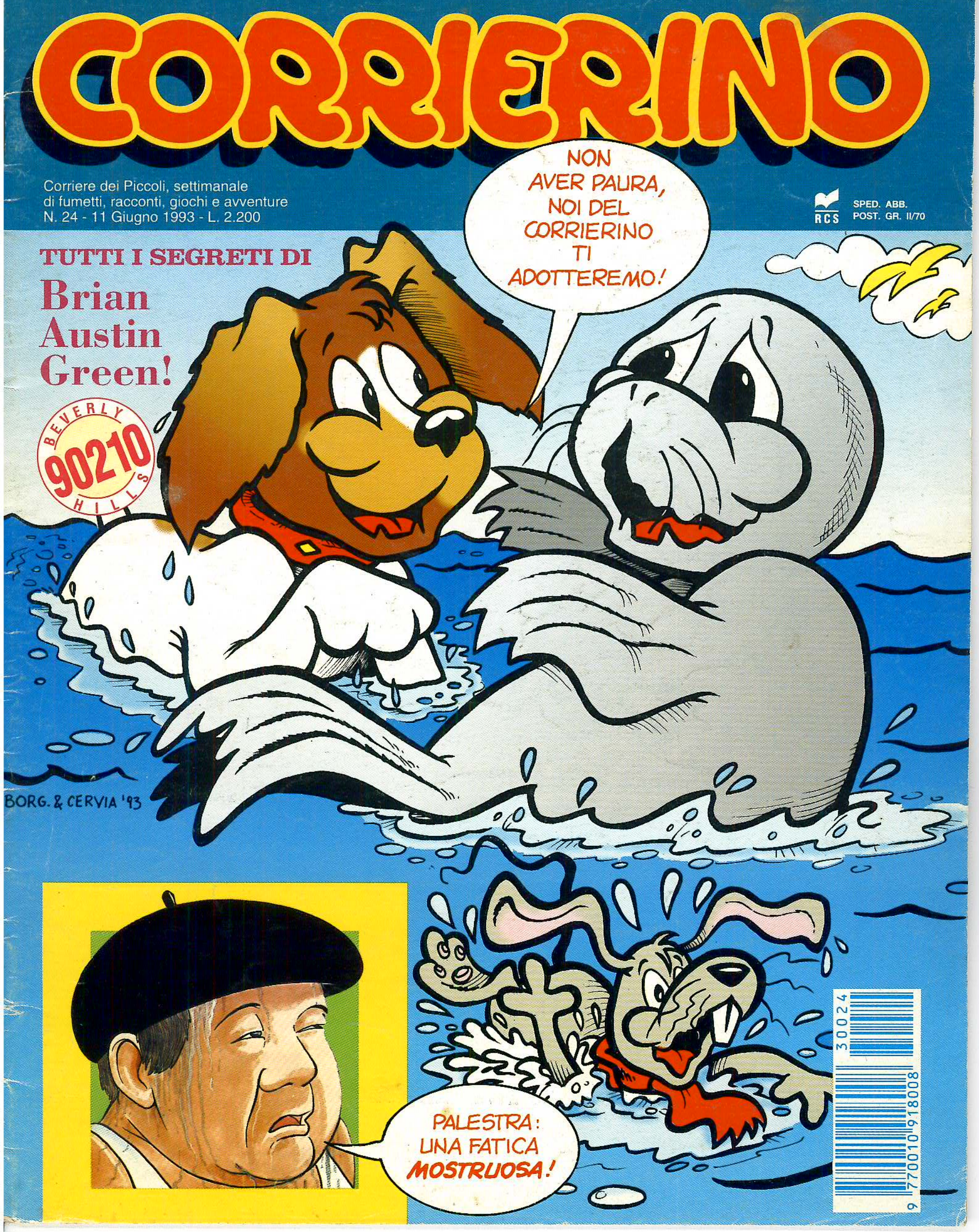




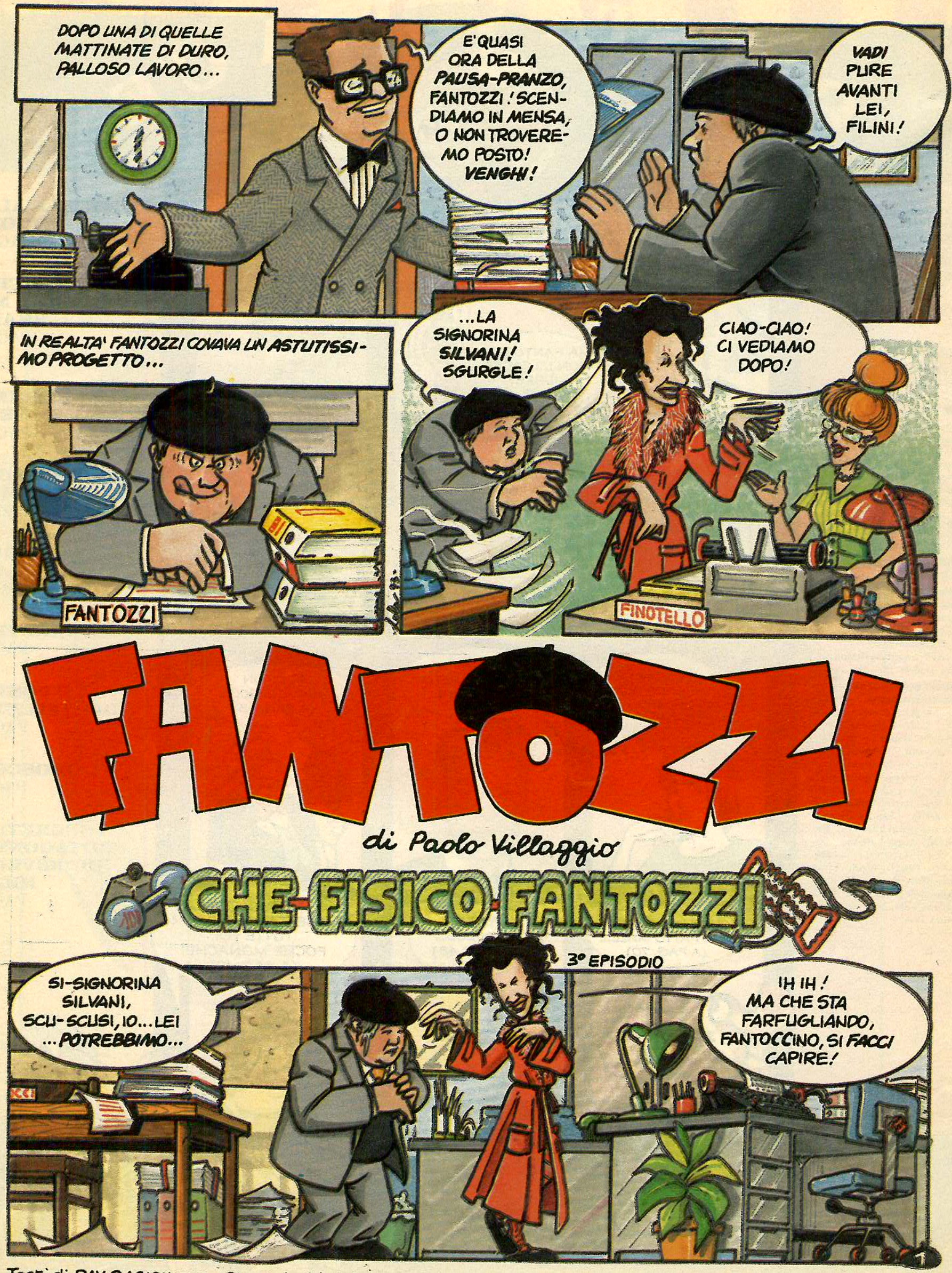

Testi di RAY RAGIONETTI Disegni di LOLA AIRAGHI 


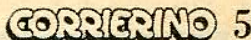

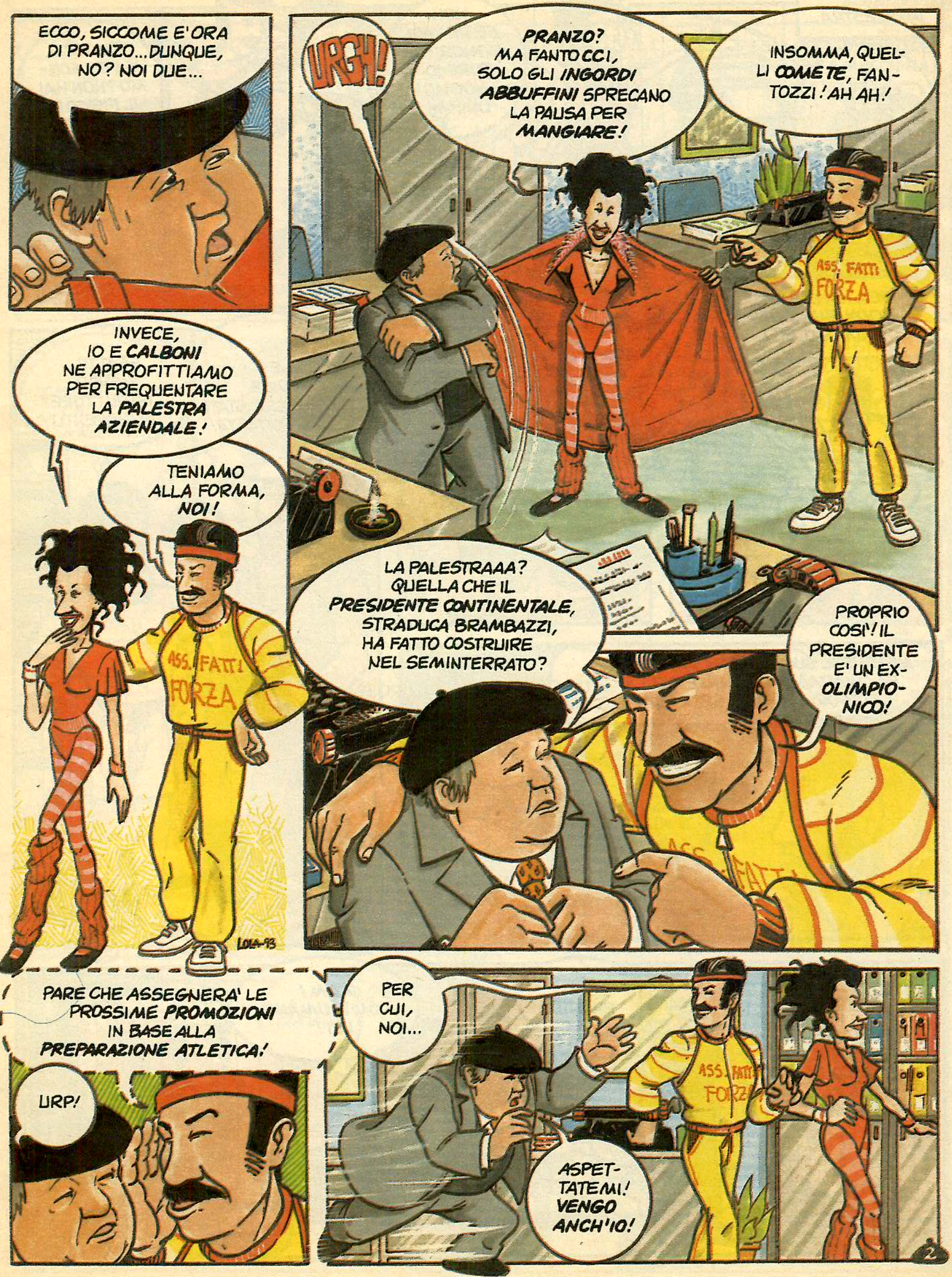




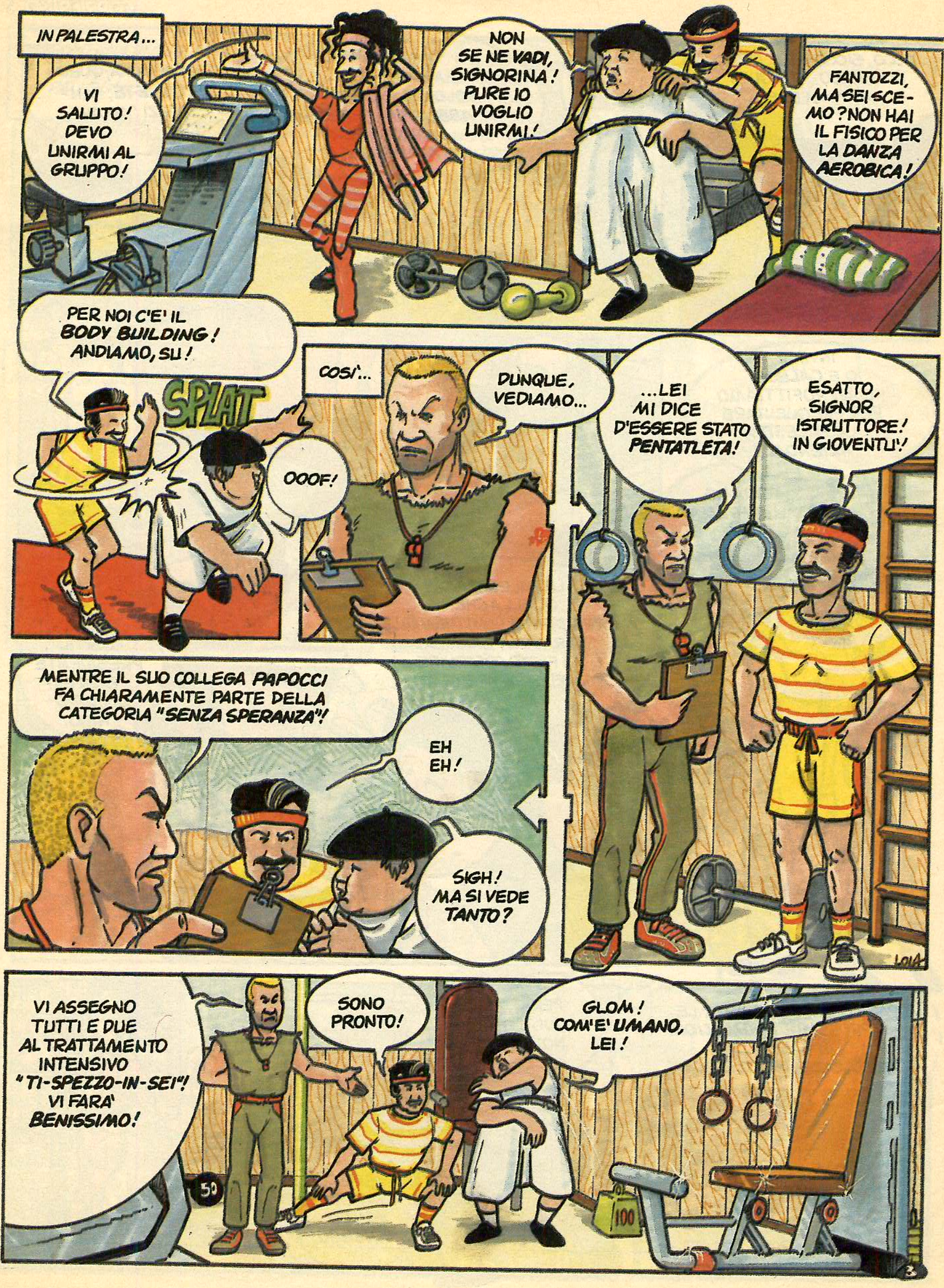




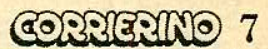

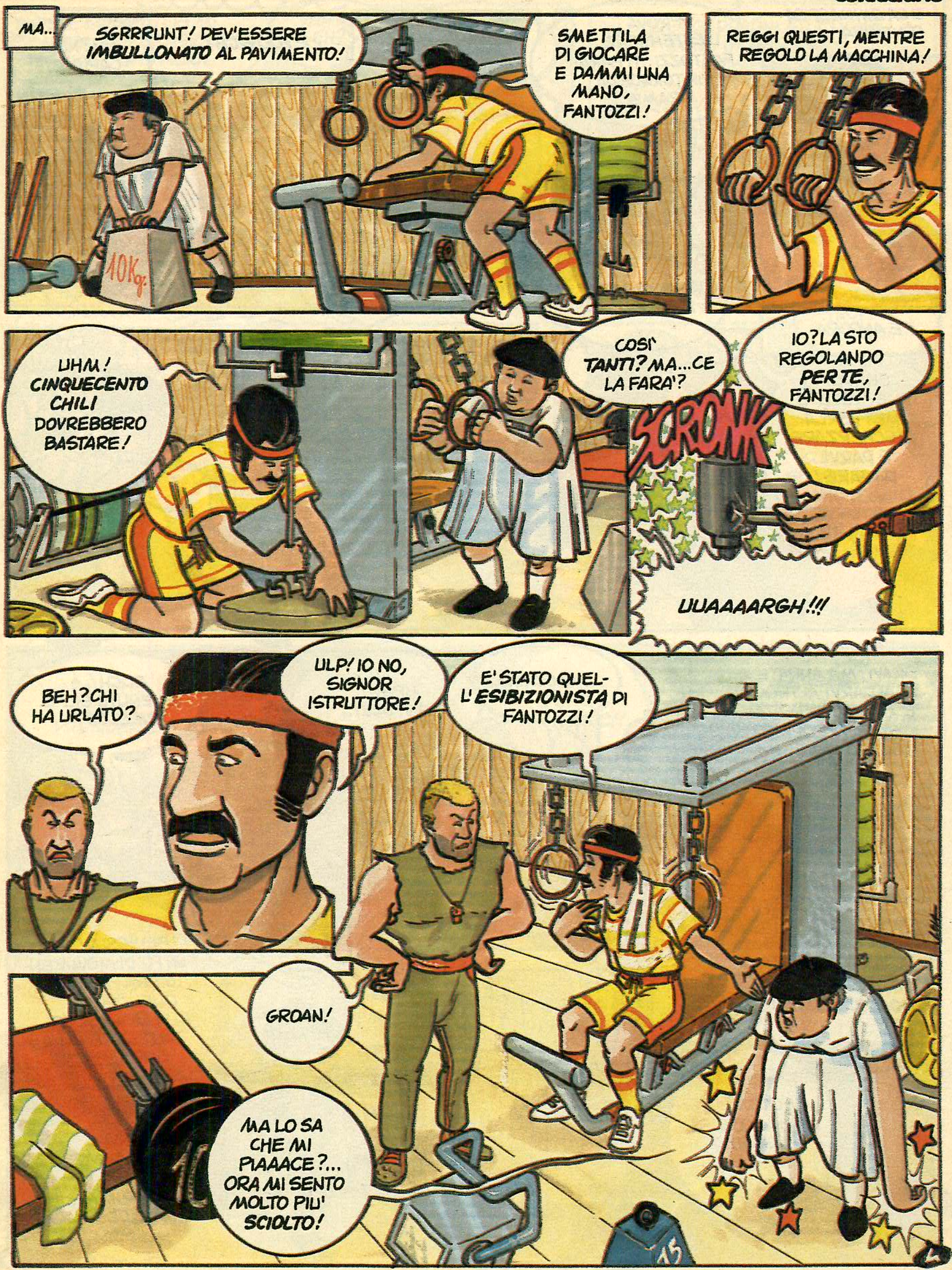




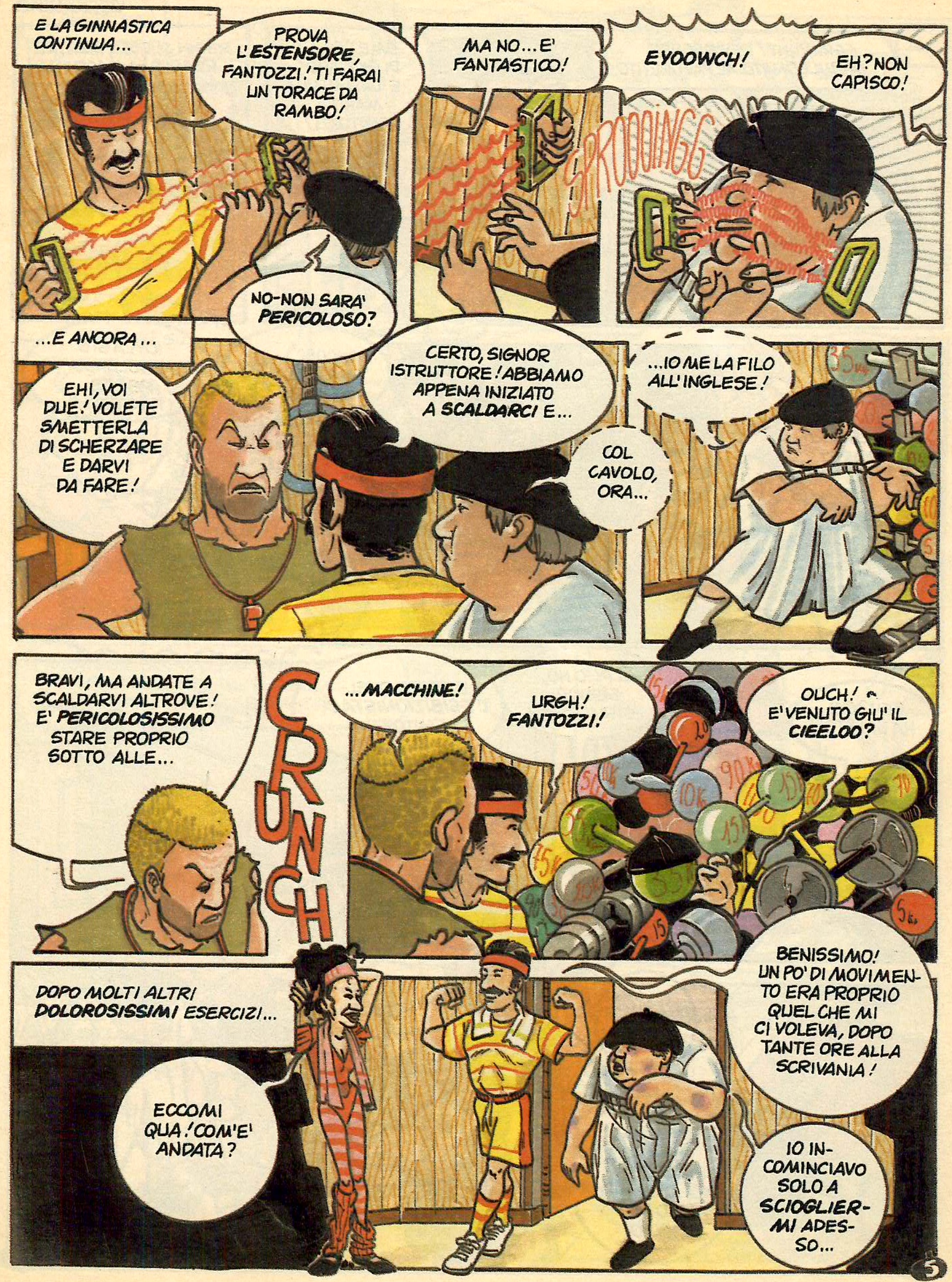




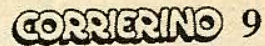

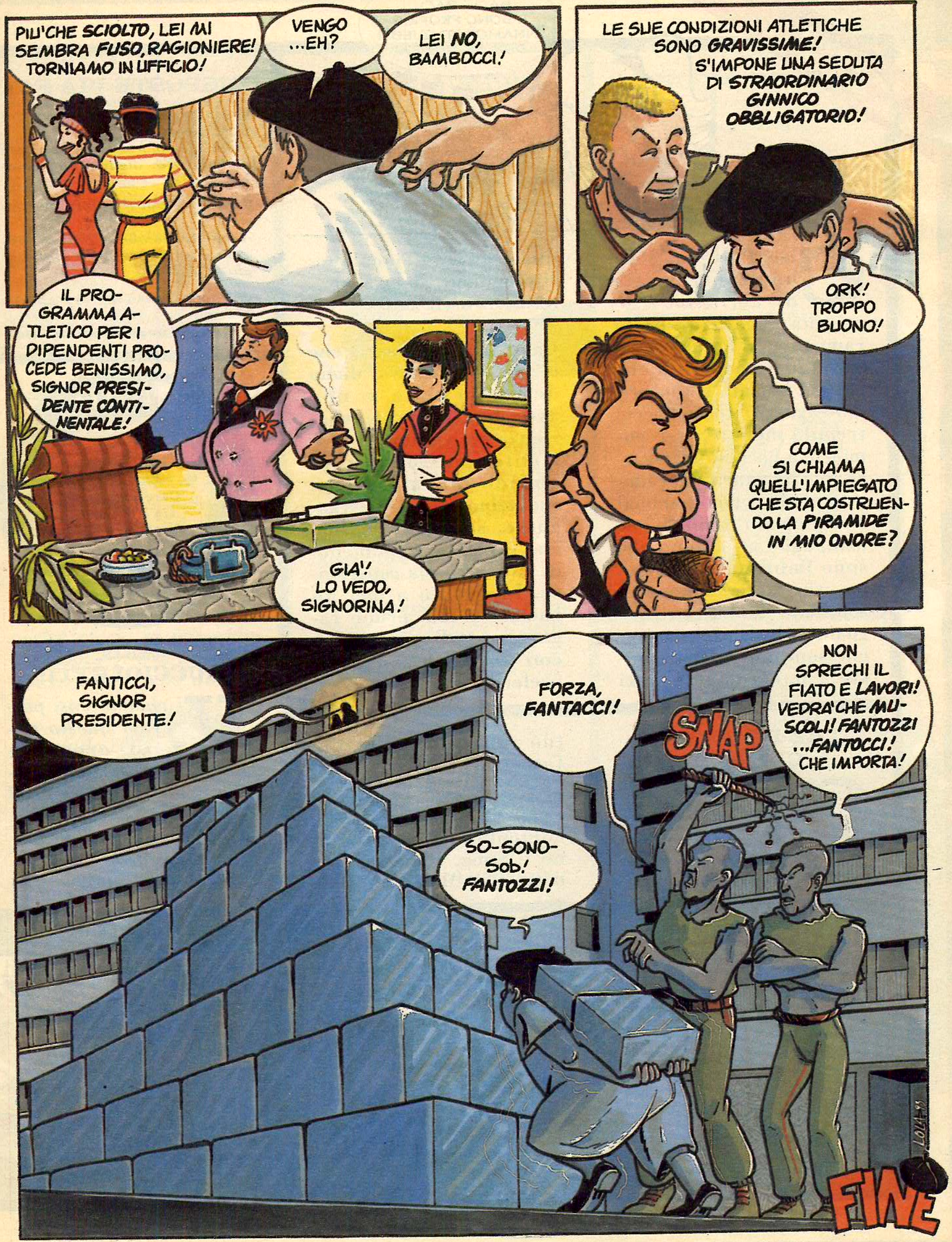




\section{(20)is $3: 3$}

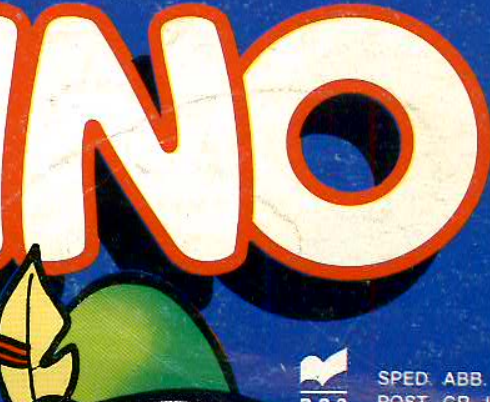

Corriere dei Piccoli, settimanale

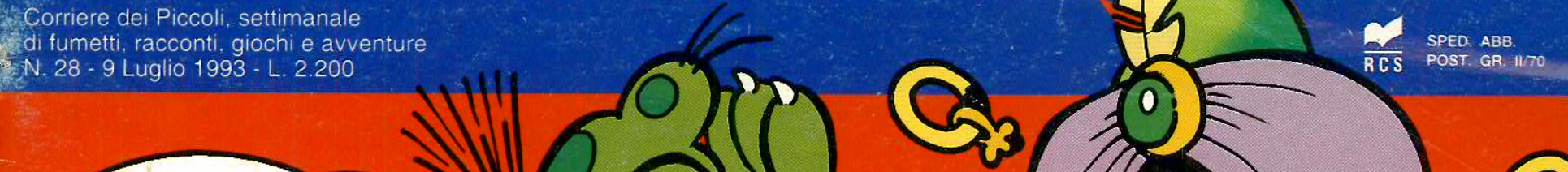

EHI, MA COSA $\because$ VEDI NEL FUTURO?

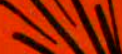
EUTURO?

Una
dieta

MISSIONE

BALENE

SALVATE!

mostruosa!

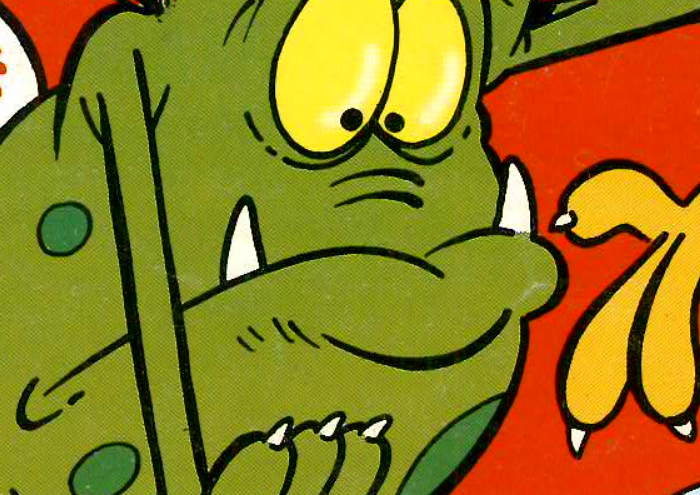




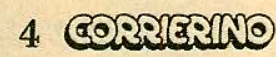

"FINO A QUEL MOMENTO ERA SEMBRATA LINA MALEDETTA ALBA LAVORATIVA COME TANTE ALTRE. IL CAFFELATTE GLI AVEVA USTIONATO IL PALATO. L'ACQUAA DI COLONIA GLI BRLCIAVA ANCORA SOTTO LE ASCELLE E, COME SEMPRE, ERA IN TERRIFICANTE RITARDO! MA IL PEGGIO ERA IN AGGLATO..."

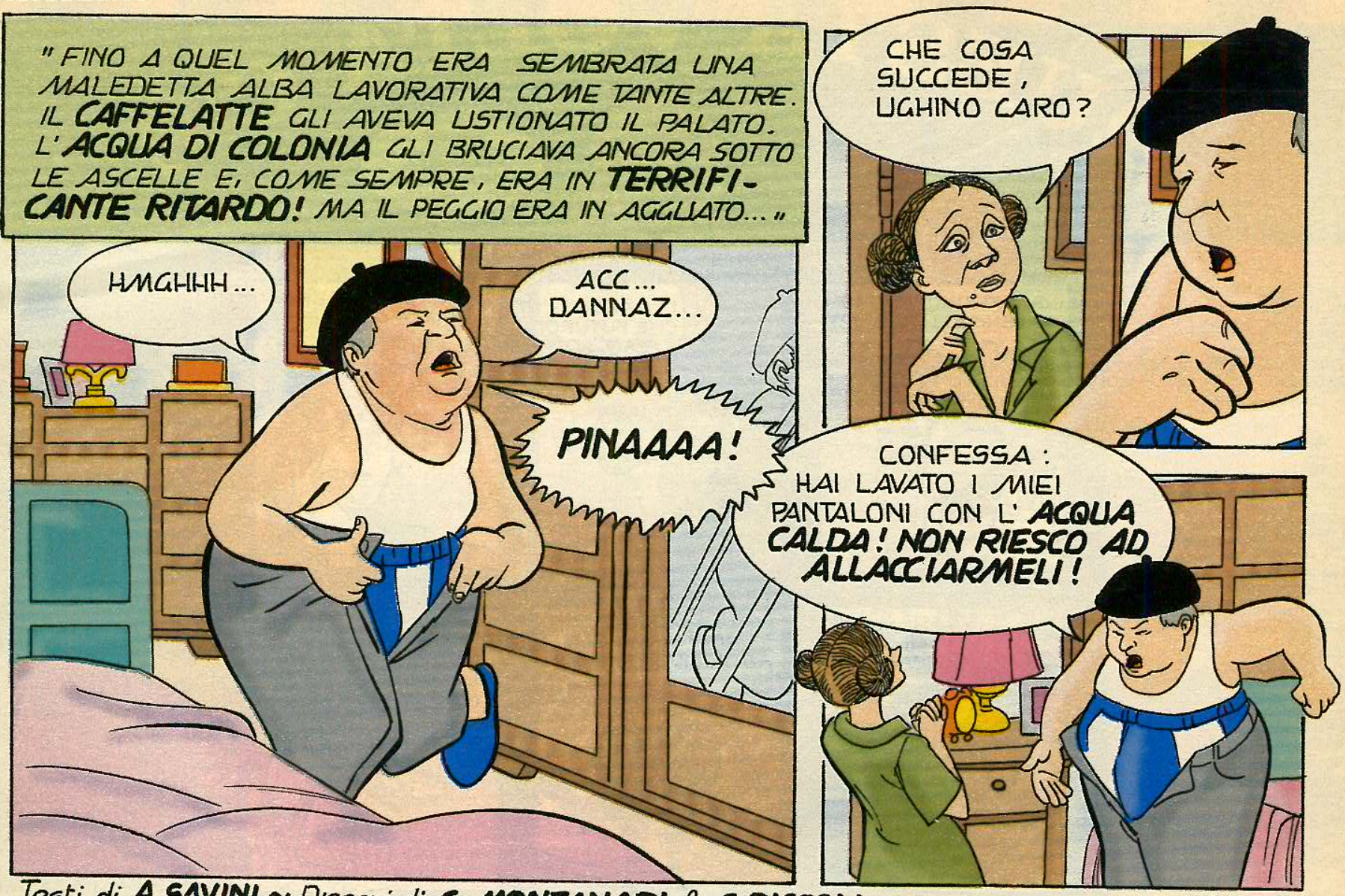

Testi di A.SAVINI DIsegni di G. MONTANARI \& C.PICCOLI

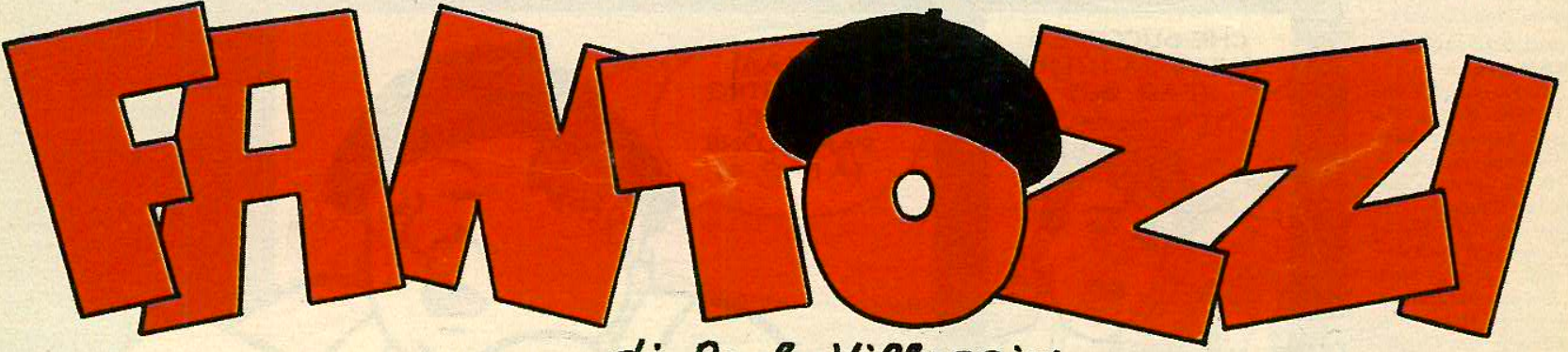

di Pado Villaggio

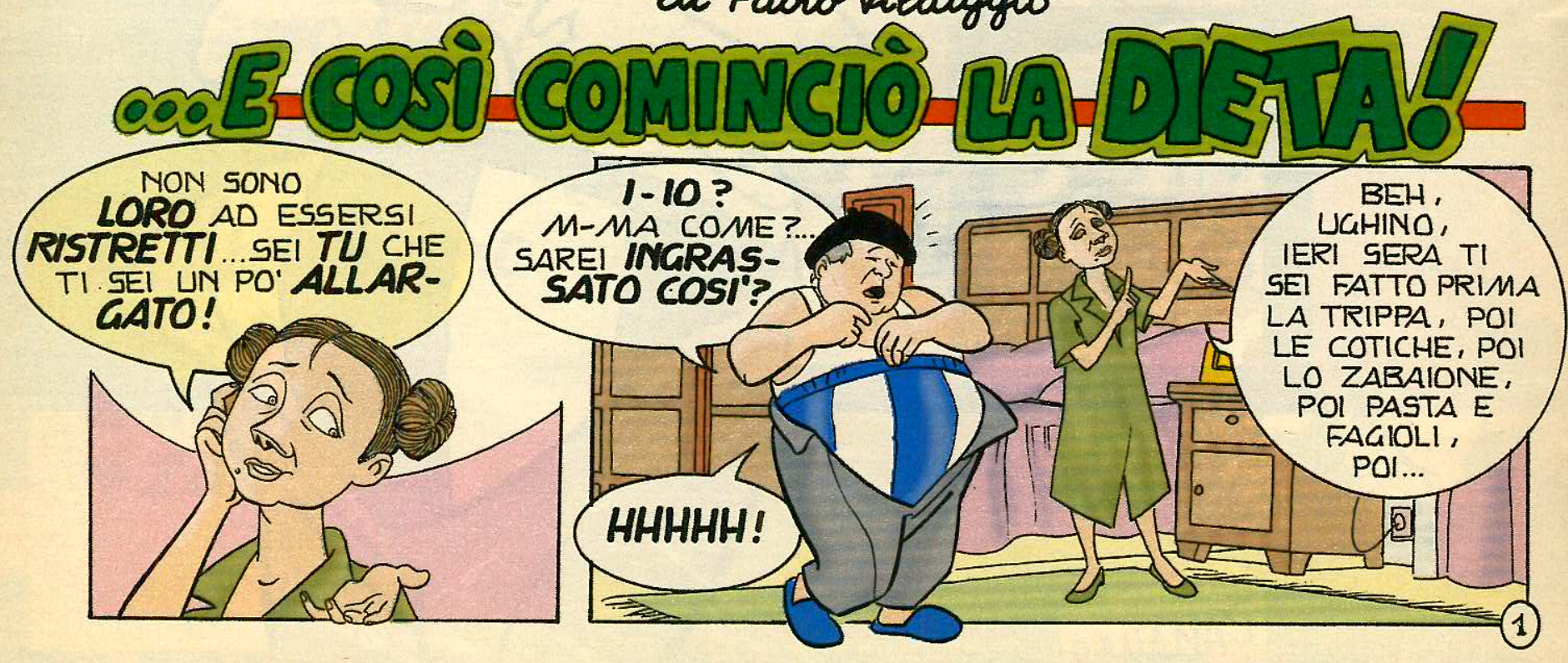




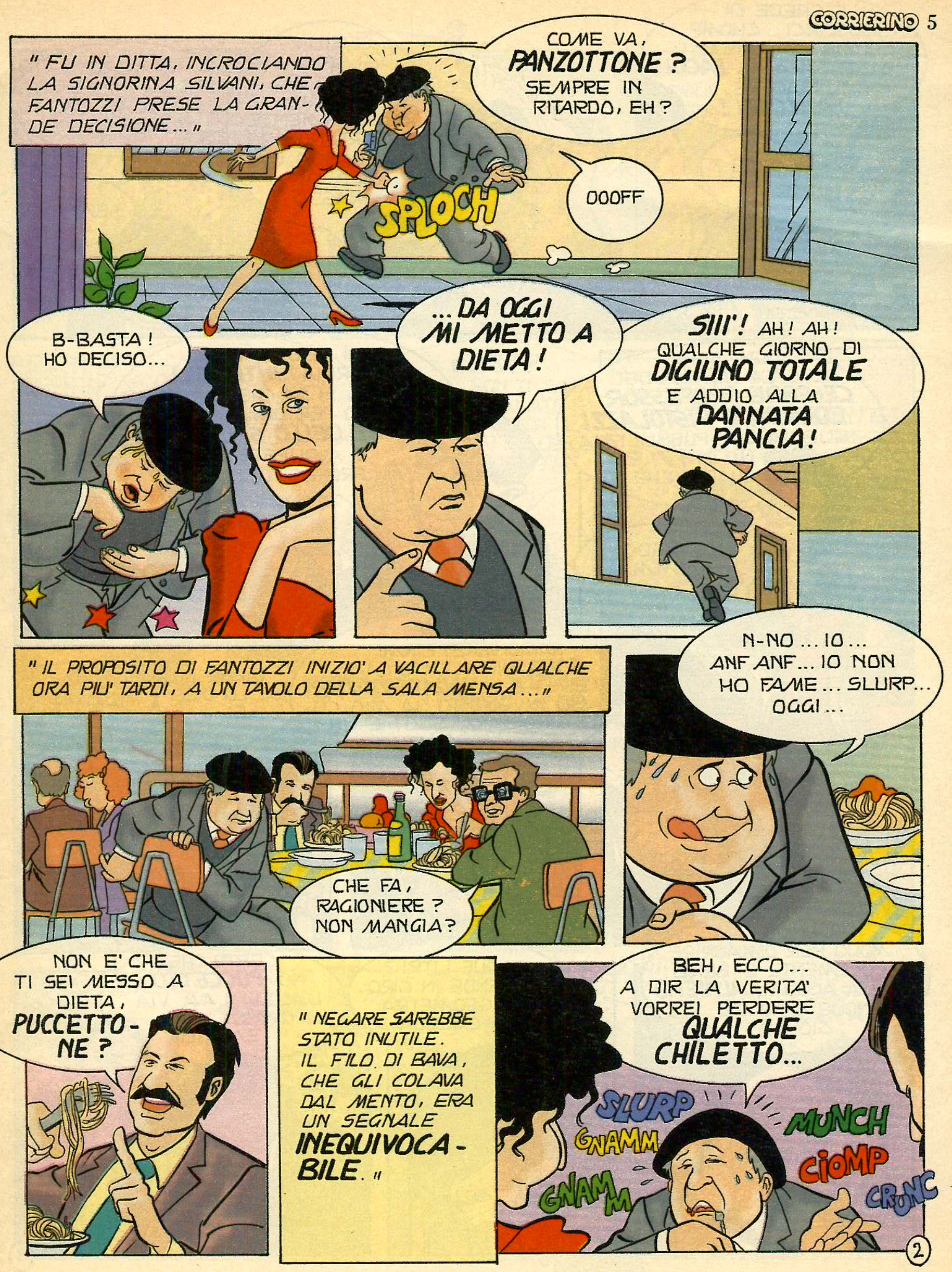




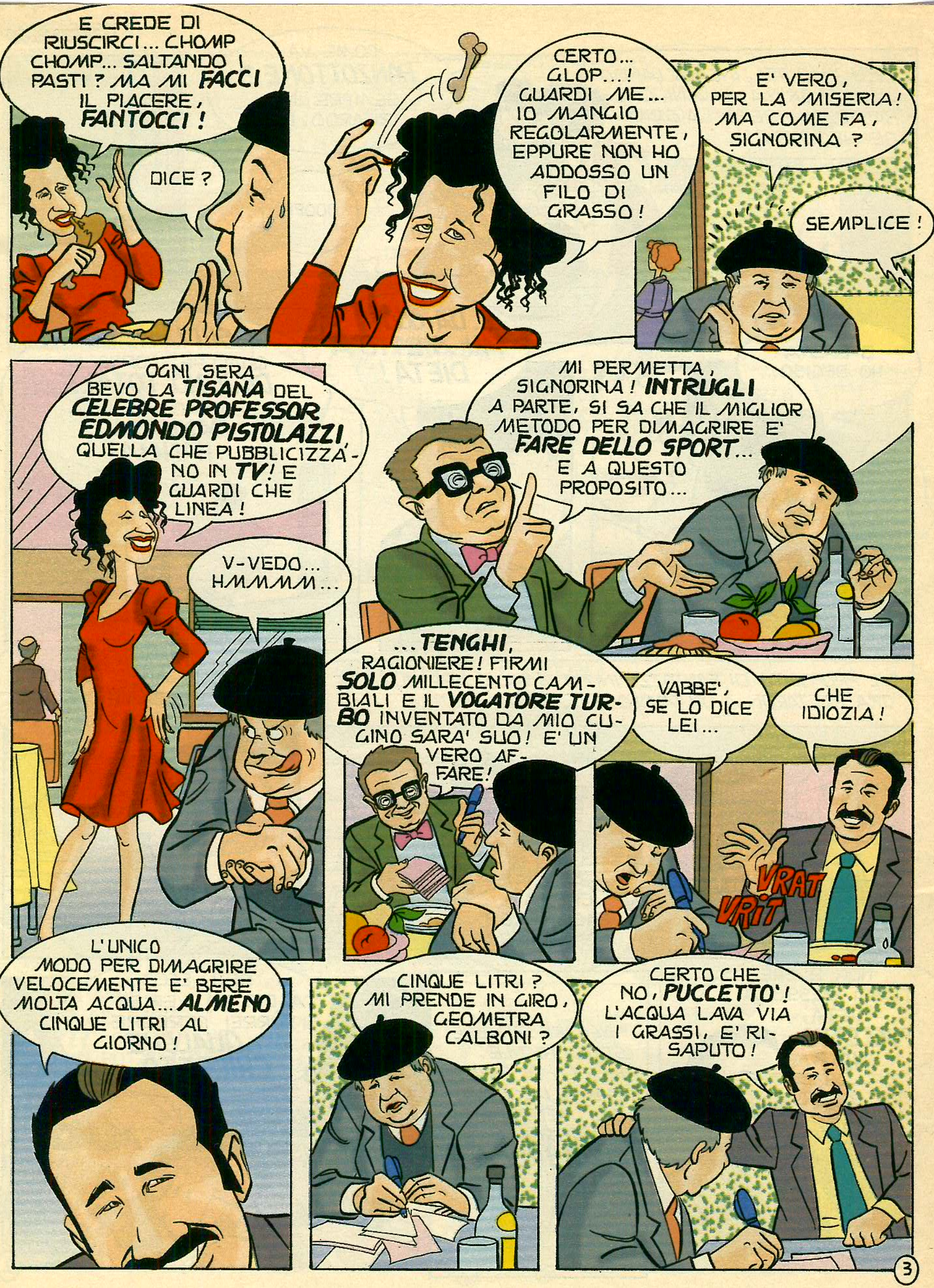



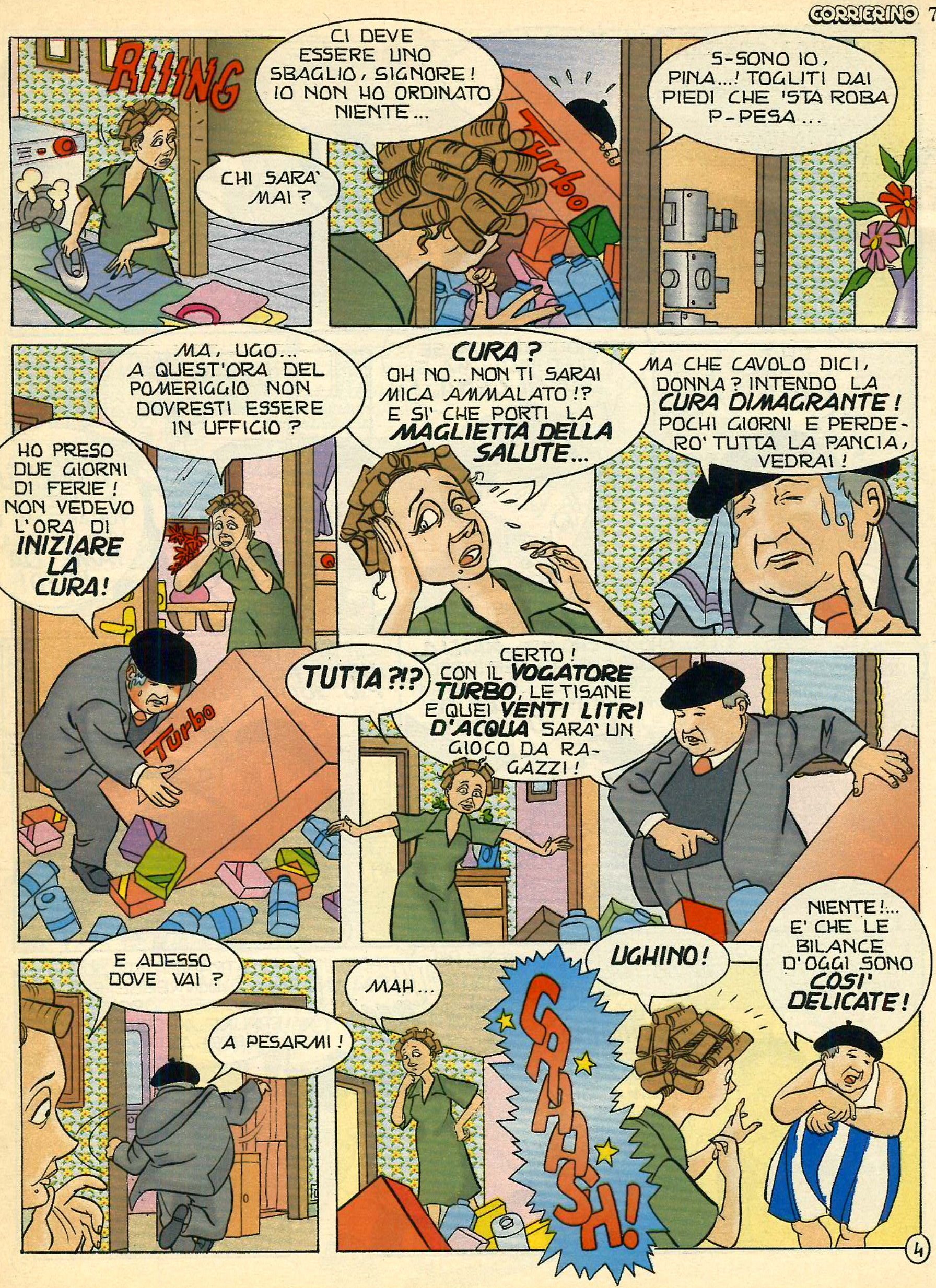
8 बூअ3

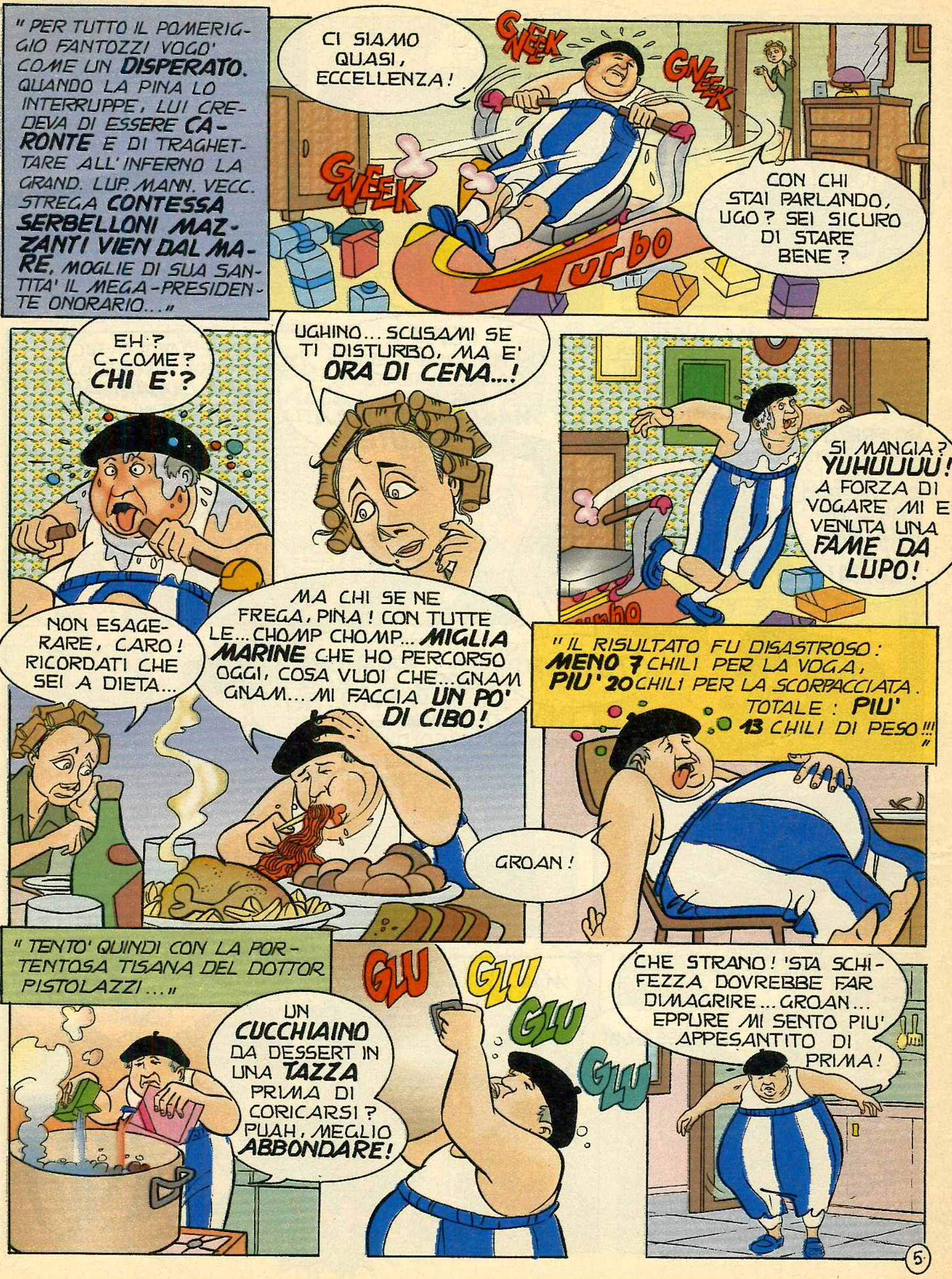




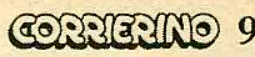
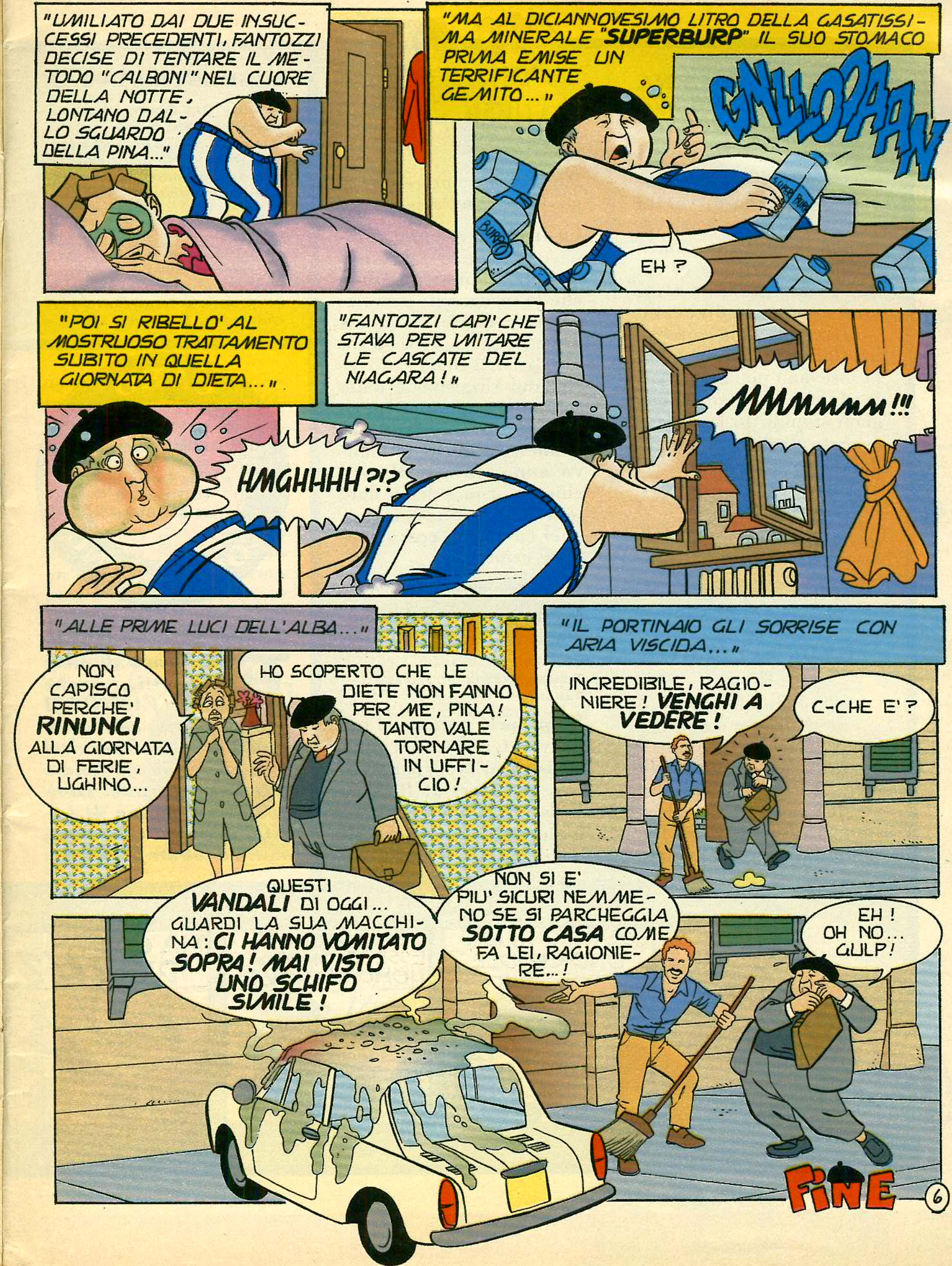


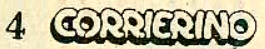

"ERA UNA GIORNATA PARTICOLARE: L'UFFKCIO SINISTRI AVEVA INFATTI DIFFUSO UN'INCREDIBILE NOTIZIA ..."

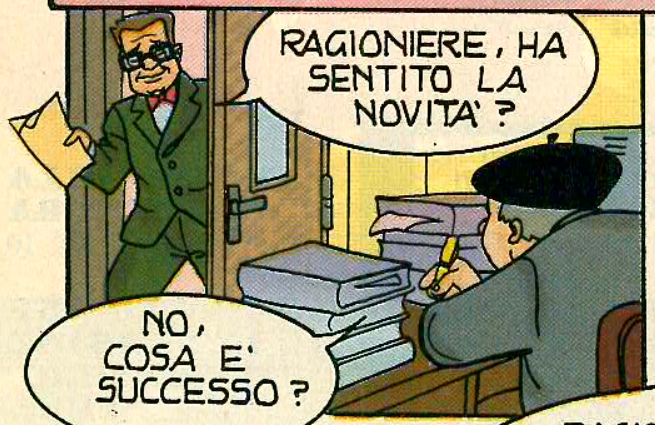

\section{LEGCHI OUI:}

IL MEGADIRETTORE ARCIM-. BOLDO DE LEYVA, COLTO DA LN ATTACCO DI DEMOCRA. ZLA, HA ORGANIZZATO UNA LOTTERIA 5TS AZIENDALE!

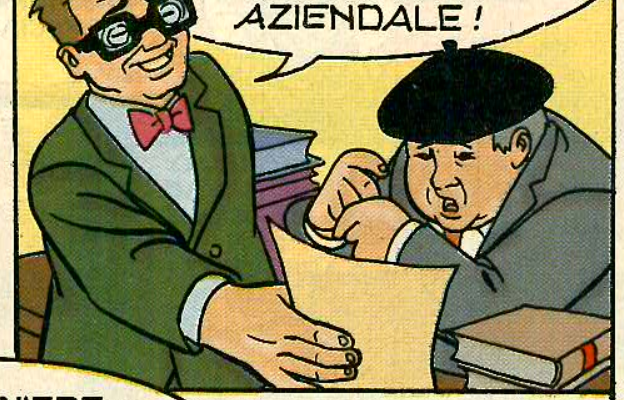

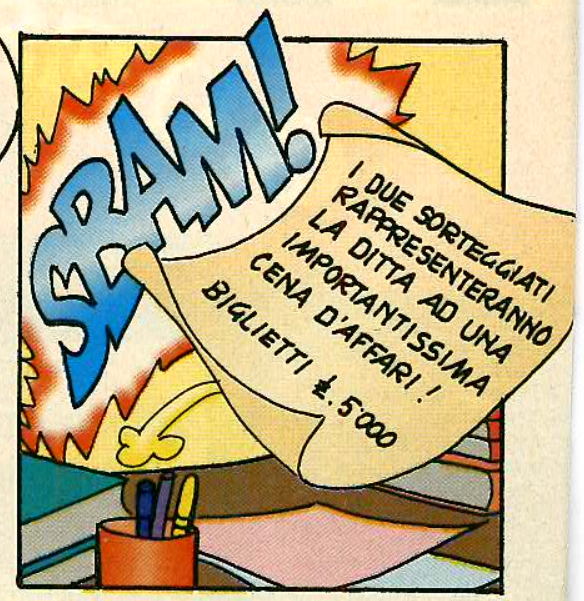

RAGIONIERE, DOV'E'? CHE FA,

\section{GIOCA A}

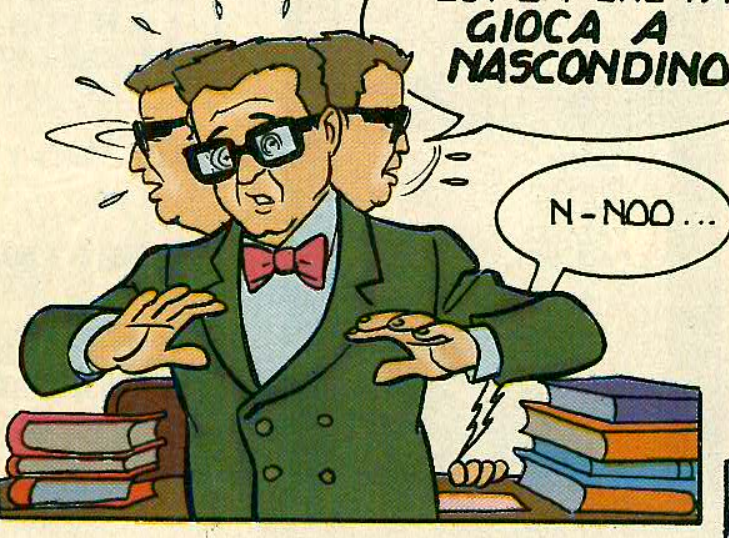

E'L'EMOZIONE!.. UN' OCCASIONE COSI NON CAPITAVA

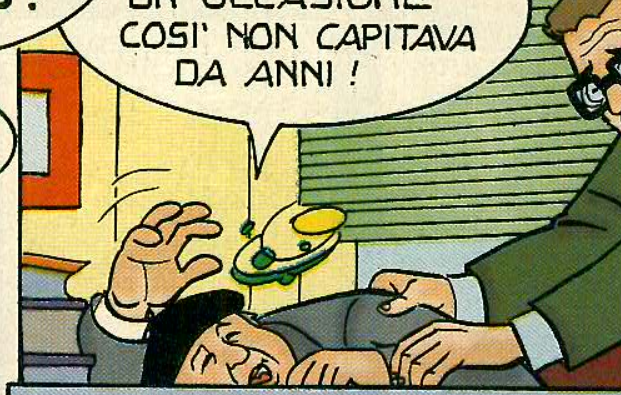

" QUELLA CENA ERA LN' OPPORTUMITA' STORICA PER FARE CAREIERA. "

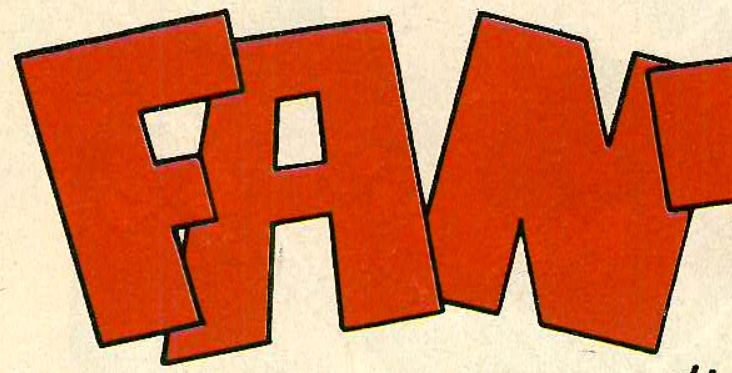

di Pado Villaggio

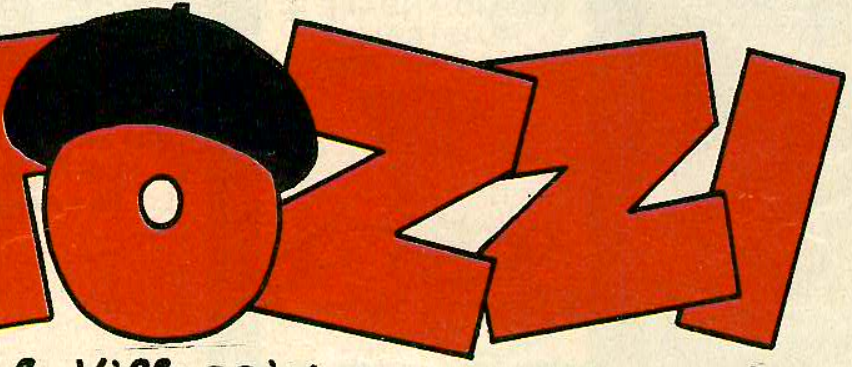

G4] [U] I]

CAPISCI, PINA? POTREI DIVENTARE LA "MASCOTTE PERSONALE DA ACCO MPAGNA MENTO", E CIOE' AVERE UN UFFICIO CON SEDUA IN PEDLA IN
PELLE
LUAMA:

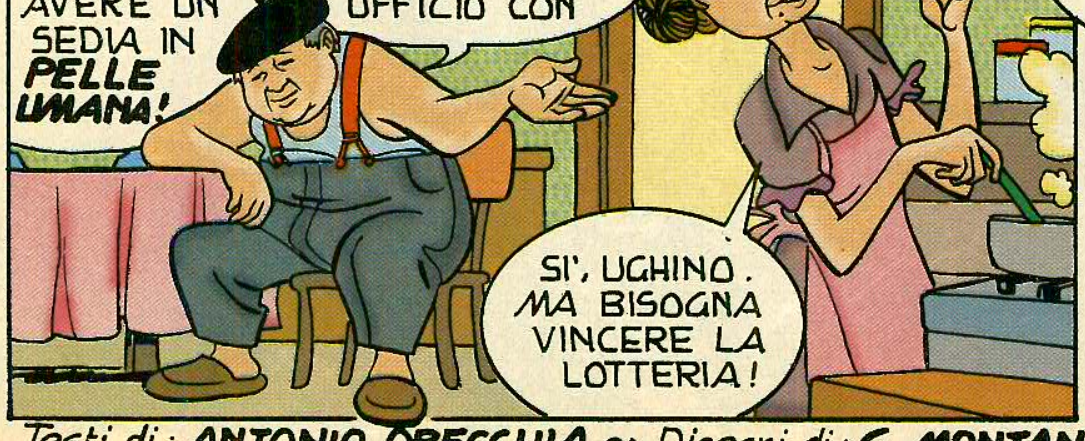

C) 0

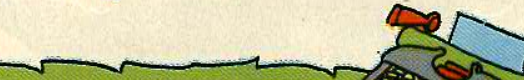

APPUNTO.

ค) ORGANIZZARCI!
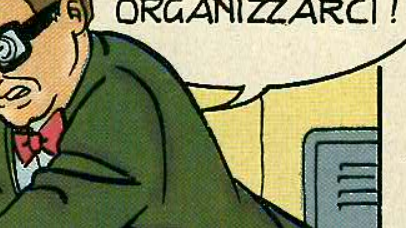
" PER PRIMA COSA BISOGNAVA TROVARE I FOMDI NECESSARI PER COMPRARE PIU' BIGLIETTI POSSIBILE: FANTOZZI SI LASCIO' LEGGERISSIMAMENTE PRENDERE LA MANO..."
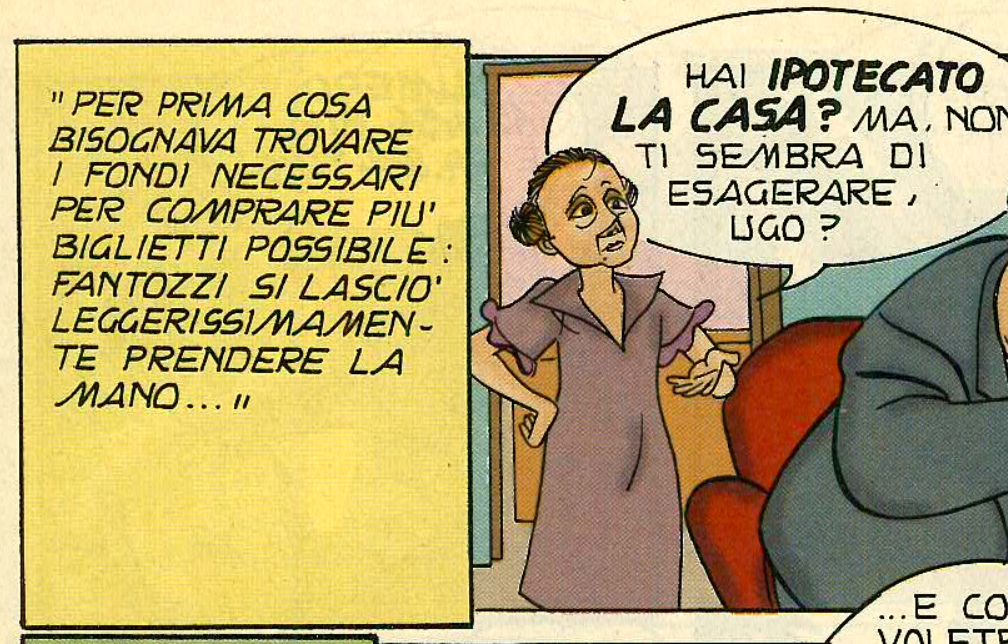

MA NO,
DONNA! NELLA

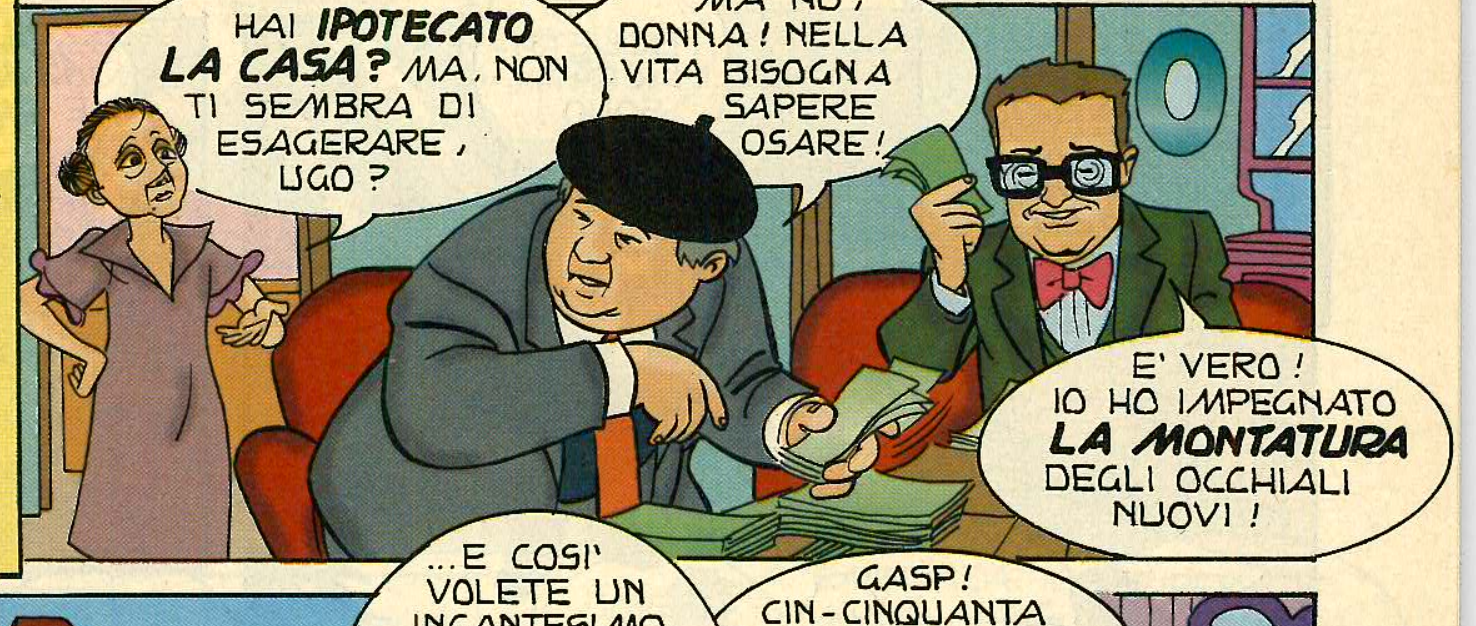

INCANTESIMO

CIN-CINOUANTA

"POI SCATTO"

$\angle A$ SECONDA

PARTE DEL

PIANO, CHE

FILINI $\triangle V E V A$

SOPRANNOMI-

NATO "FASE

PARANORMALE"...

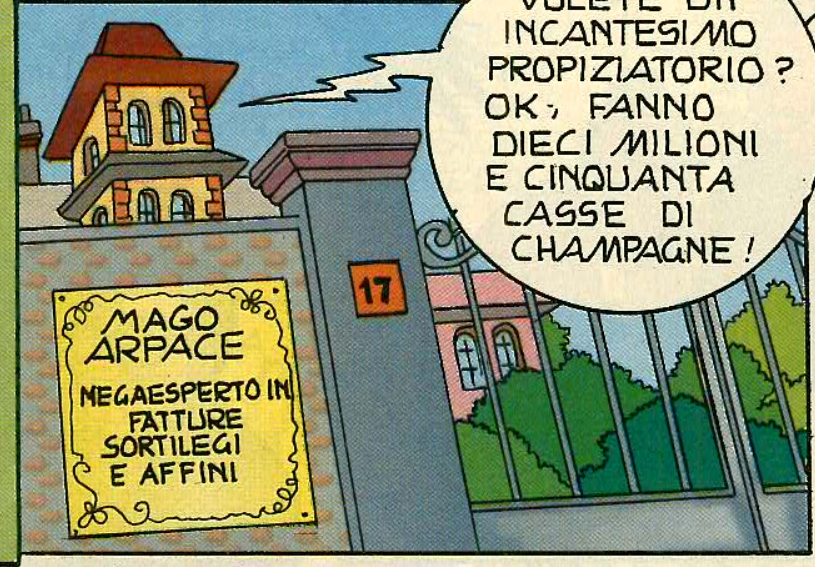

CASSE DI CHAMPA-

GNE E DIECI

MILIONI?

IO HO IMPEGNATO
LA MONTATURA
DEGLI OCCHIALI

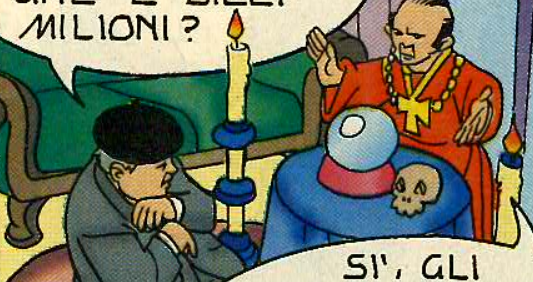

SPIRITI SONO PIU POSITIVI SE BEVONO CHAMPAGNE... E I MILIONI SONO IL MIO ONORARIO.

"E, PER FINIRE, FANTOZZI FECE ANCHE VOTO DI DIETA FERREA..."

"FINAL MENTE ARRIVD' IL GRANDE GIORNO

GLI IMPIEGATI DEBOLI DI CUORE ARRIVARONO IN AZIENDA CON L'AMBLLANZA, MENTRE FANTOZZI....,

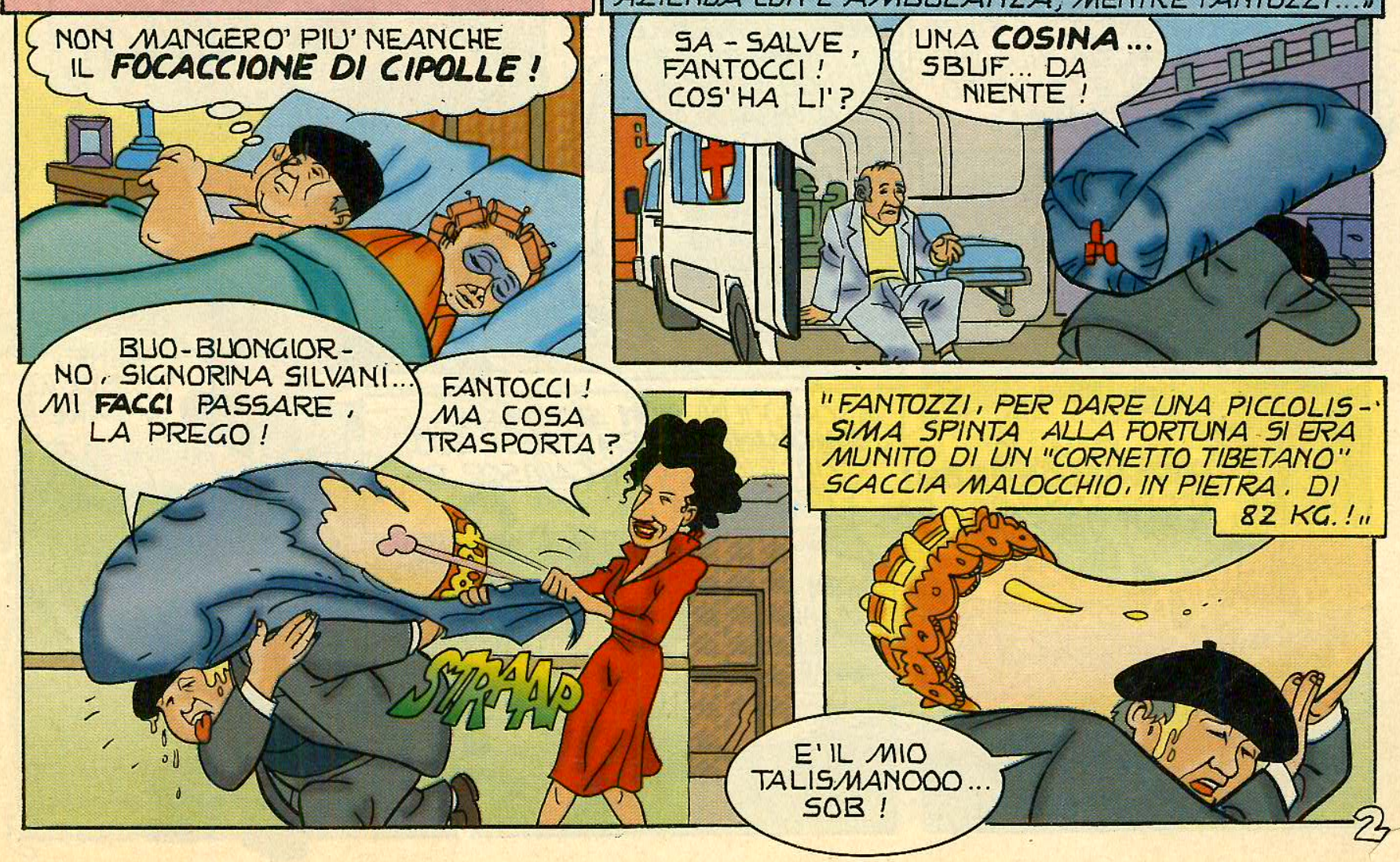




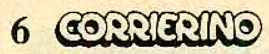

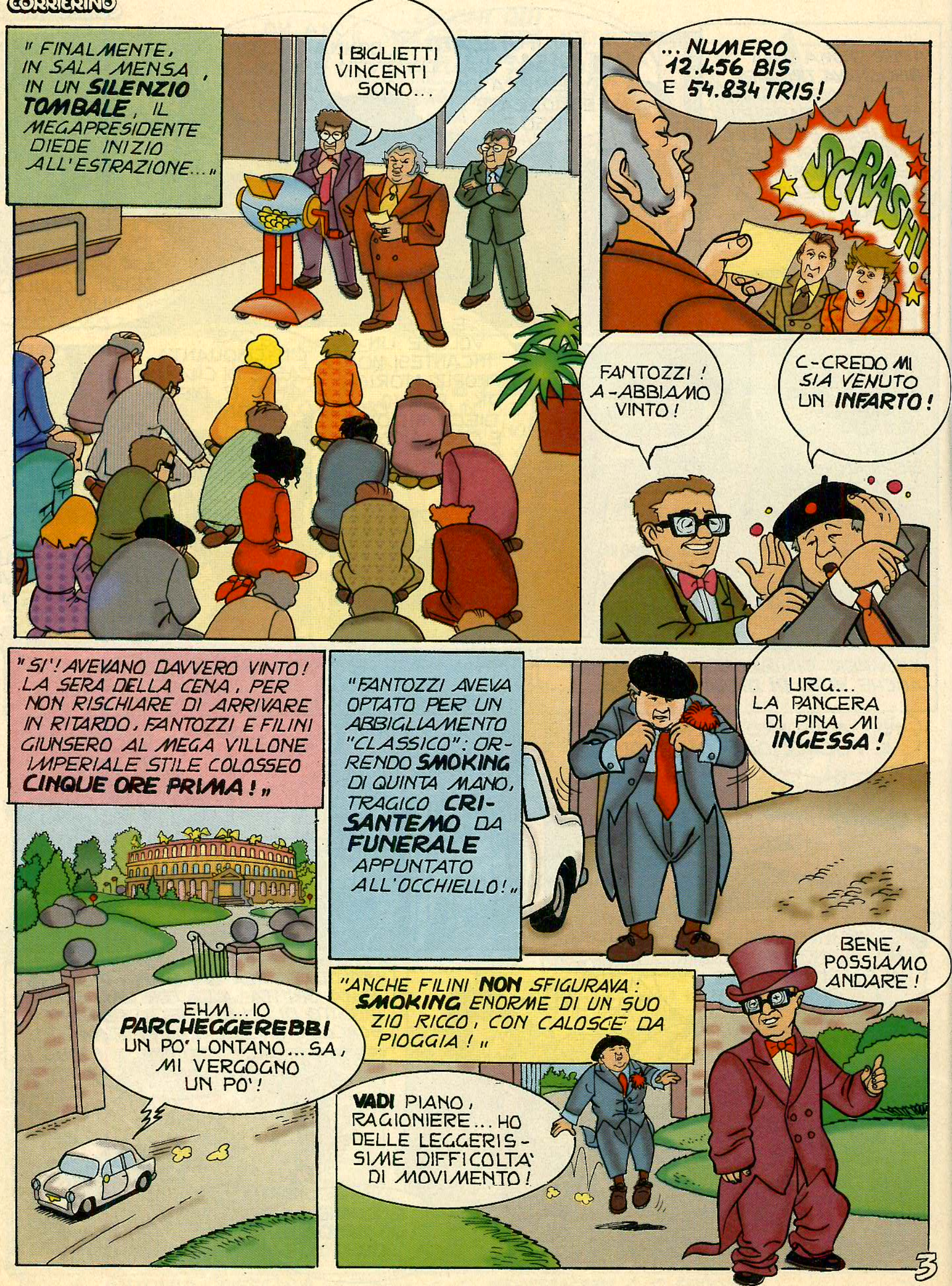




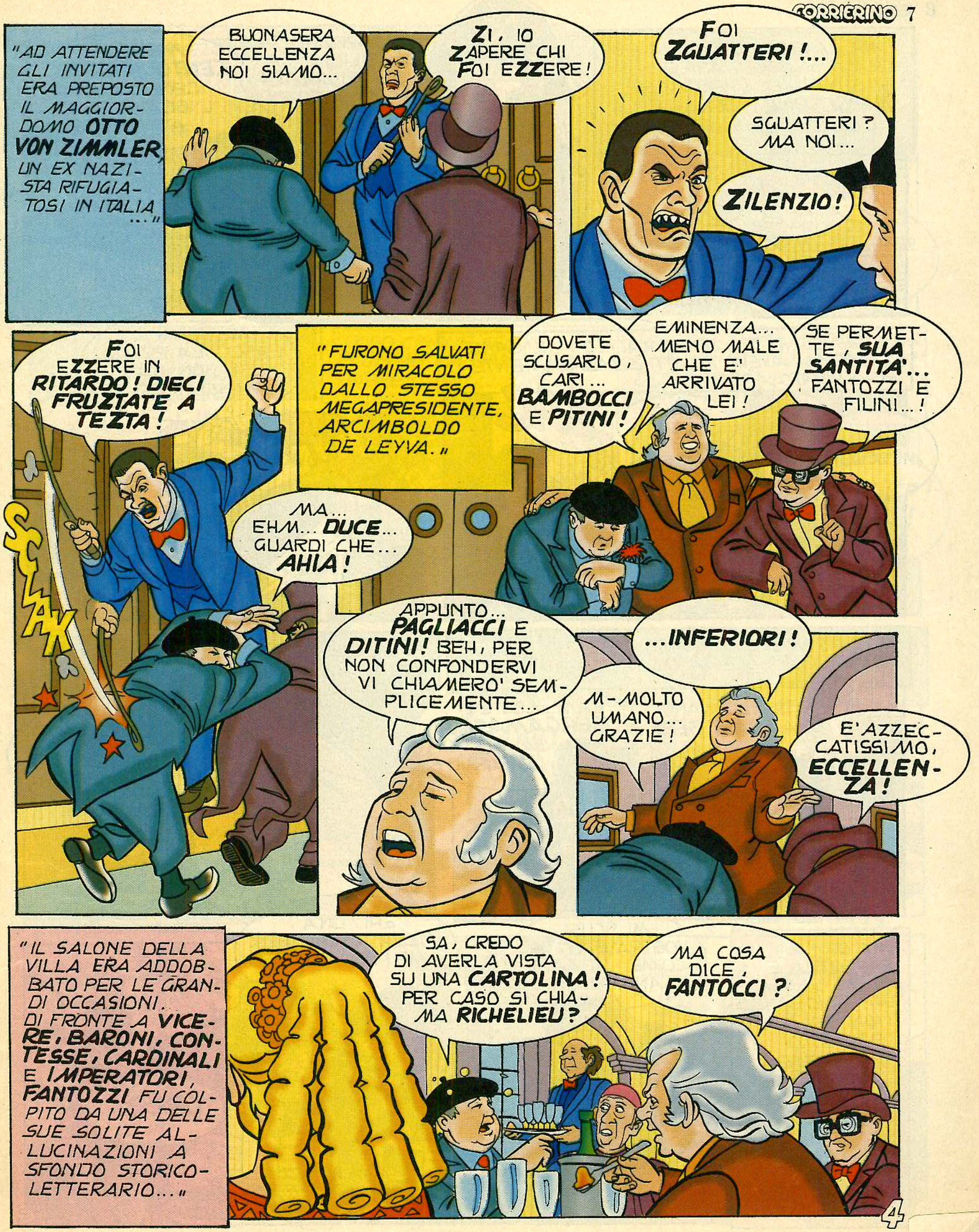




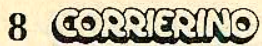

"MA LA CRISI VISIONARIA ERA ORMAI INARRESTABILE SI SENTI' ALL' IMPROVVISO NAPOLEONE, DURANTE LA CAMPAGNA DI RUSSIA..."
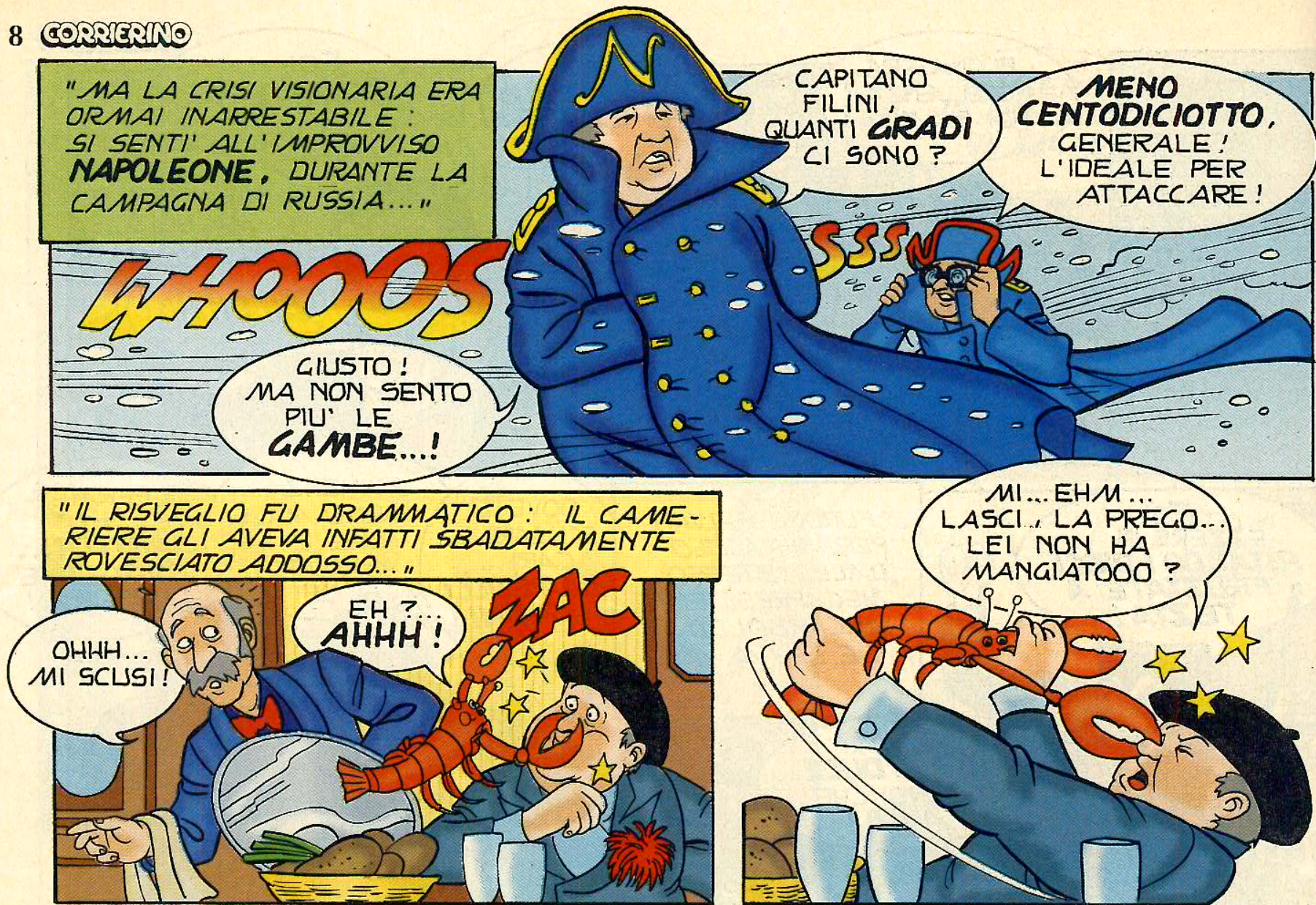

"IL CAMERIERE CE LAVEVA PROPRIO CON
$\angle U I:$ PER LA SCELTA DELLA TERZA PORTATA..."
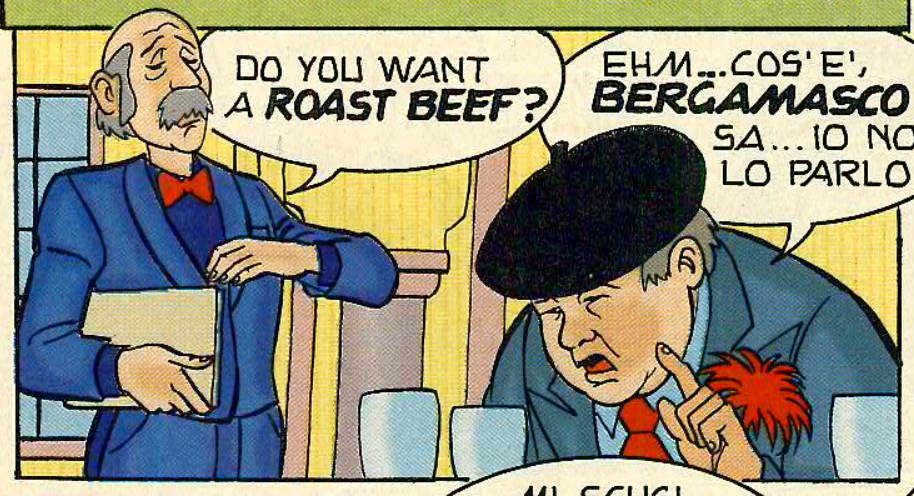

"PER USCIRE DA QUELLA IMBARAZZANTE SITUAZIONE CONTRATTACCO'!"

"MA ORMAI FANTOZZI E FILINI SI STA VANO GIOCAMDD NON SOLO IL POSTO DI MASCOTTE PEESONALE $\triangle A$ ACCOMPA. GNAMENTO, MA. IL LICENZIAMENTO IN TRONCO..."
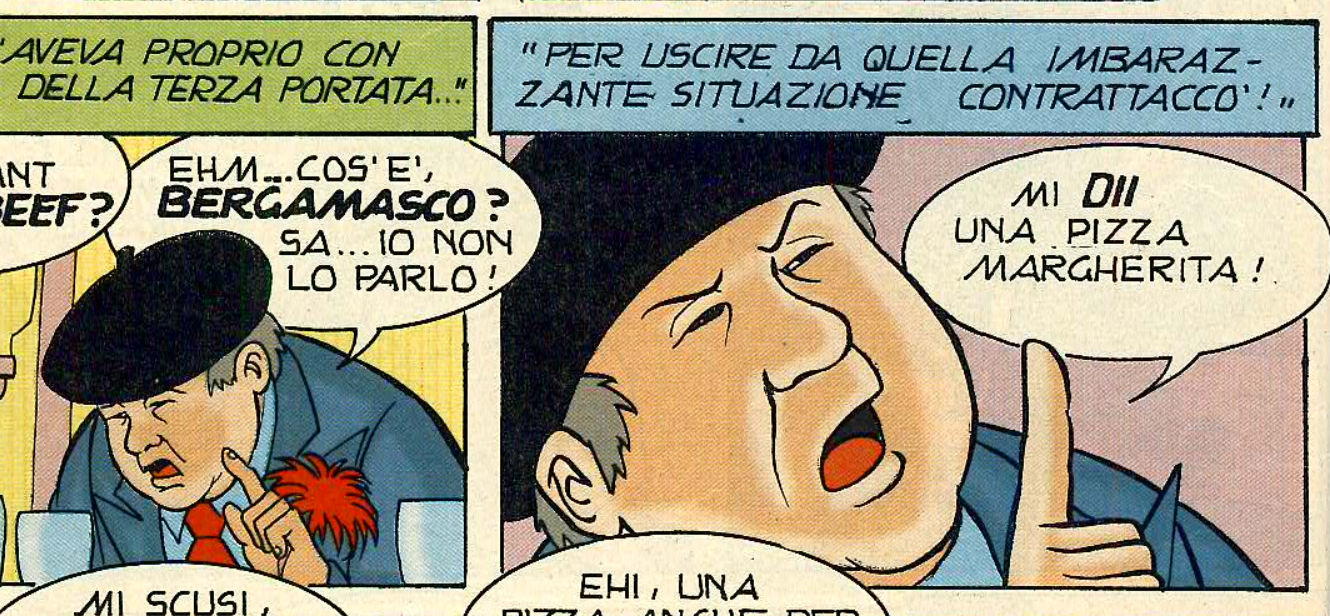

EHI, UNA MI...EHM...
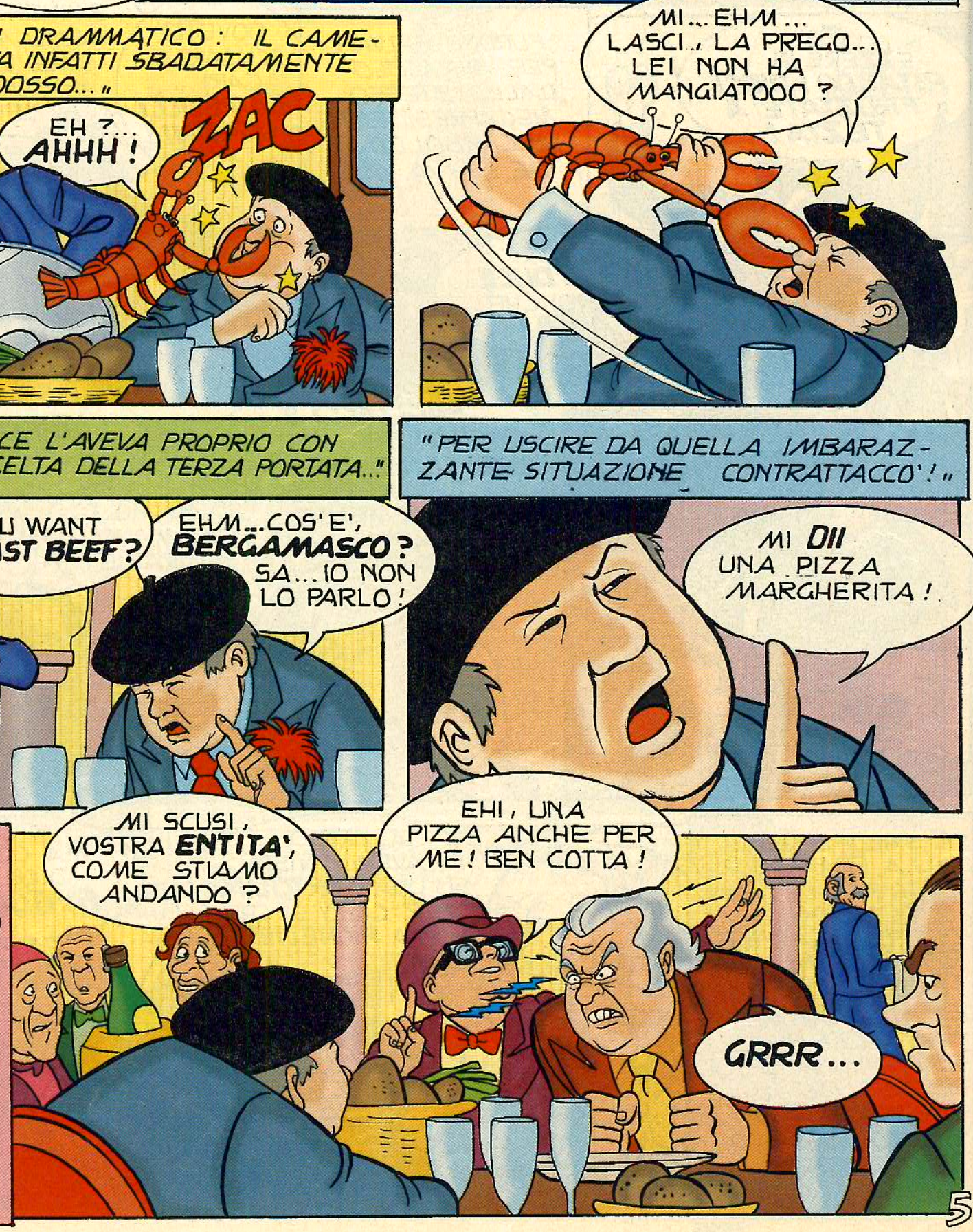

MEI ANCHE PER 
"AVREBBERO POTU. TO SPERARE DI RIABILITARSI ALLA FRUTTA QULANDO VENNE SERVITO UN TRE MENDO LIQUORE SPAGNOLO, DETTO PURGATORIO DE FUEGO..."

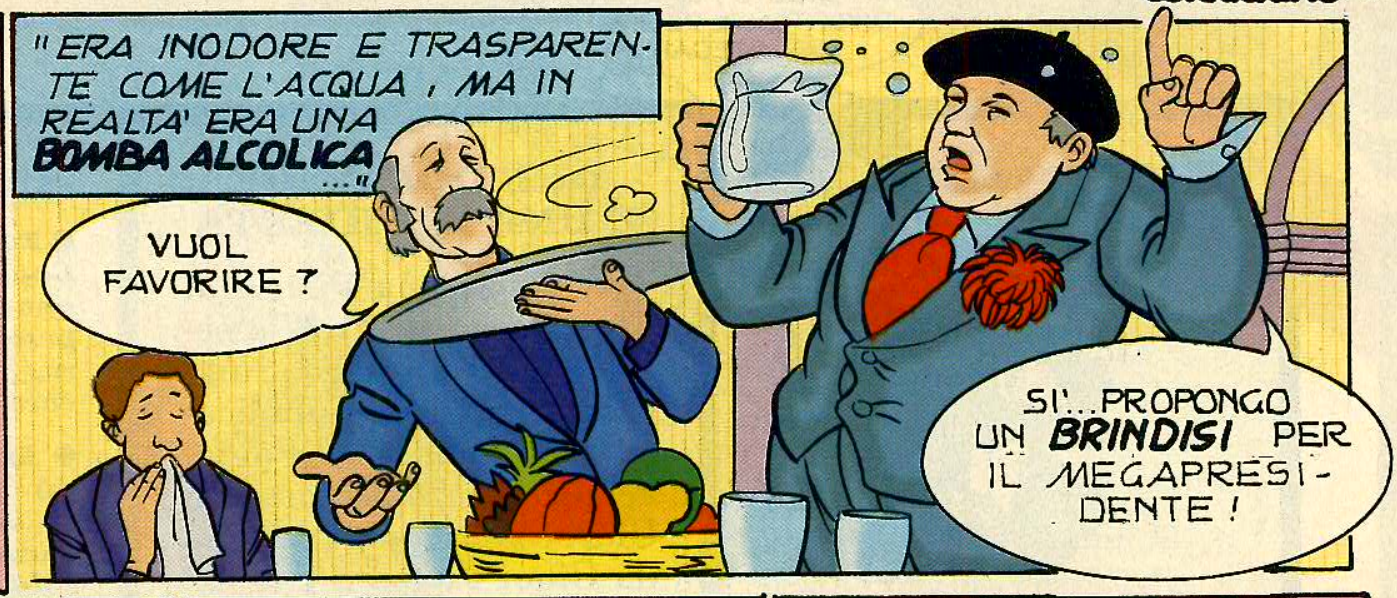

"FANTOZZI SI TRACANMO AVID AMENTE LA CARAF-

"DOPO CINQUE SECONDI GLI SCOPPIO' LA PANCERA IMPRESTATAGLI DALLA

FA IN UN SORSO!"

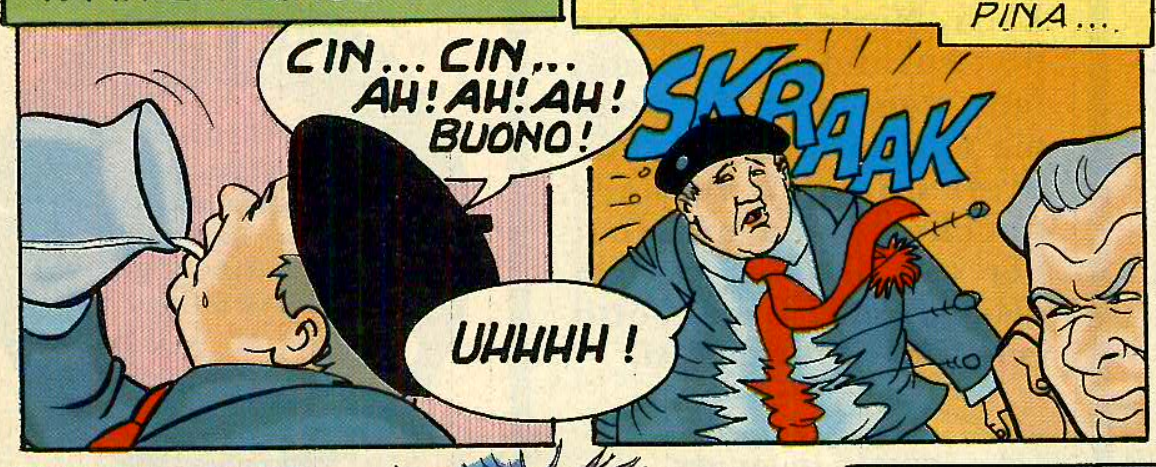

"POI GLI SI USTIONO'LA LINGUA, GLI SCHIZZARONO GLI OCCHI OLTRE IL NASO EI CONATI DI VOMITO DIVENTARONO REGOL ARI..."

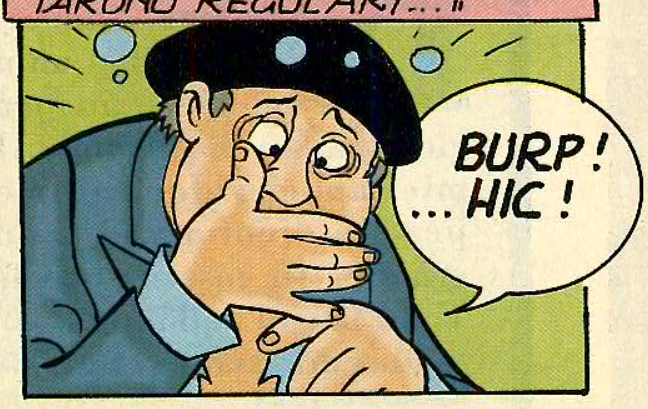

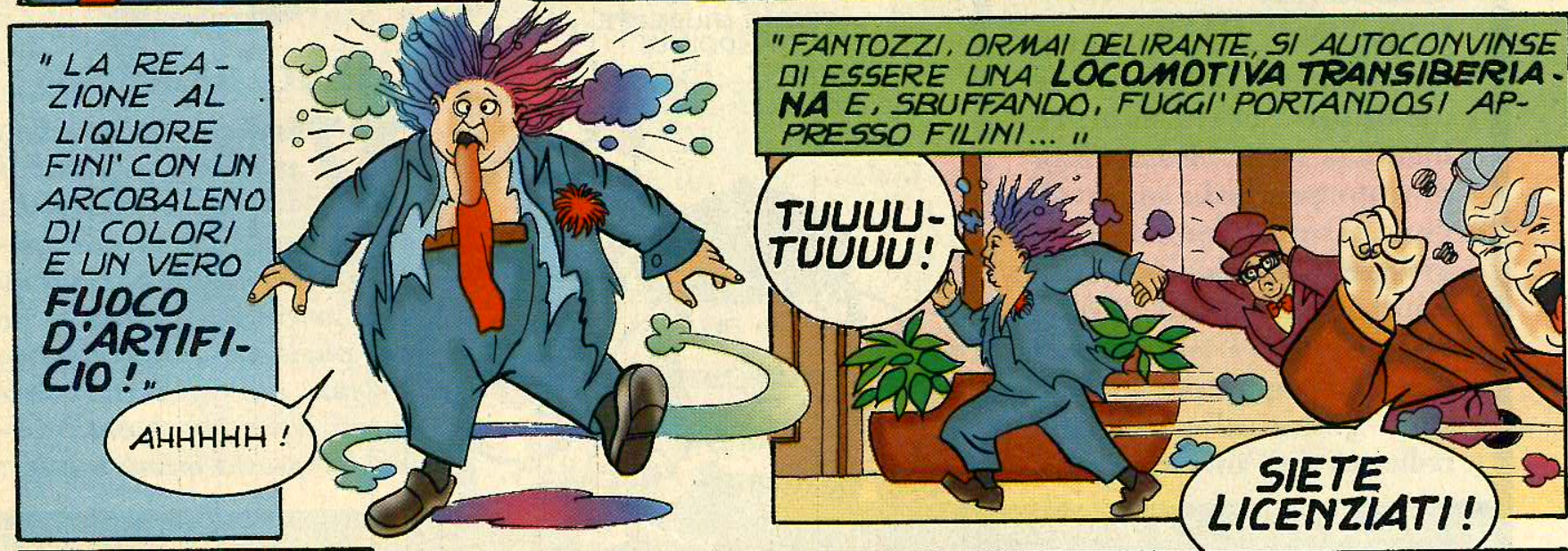

"DOPO DUE SETTIMANE, GRAZIE AL PORTAFORTUNA TIEETANO,

FAMTOZZI E FILINI FURONO RIASSUN TI... UNO COME POGGIAPIEDI DEL MEGAPRE SIDENTE, L'AL-

TRO COME ZERBINO DEL. L'UFFICIO..."

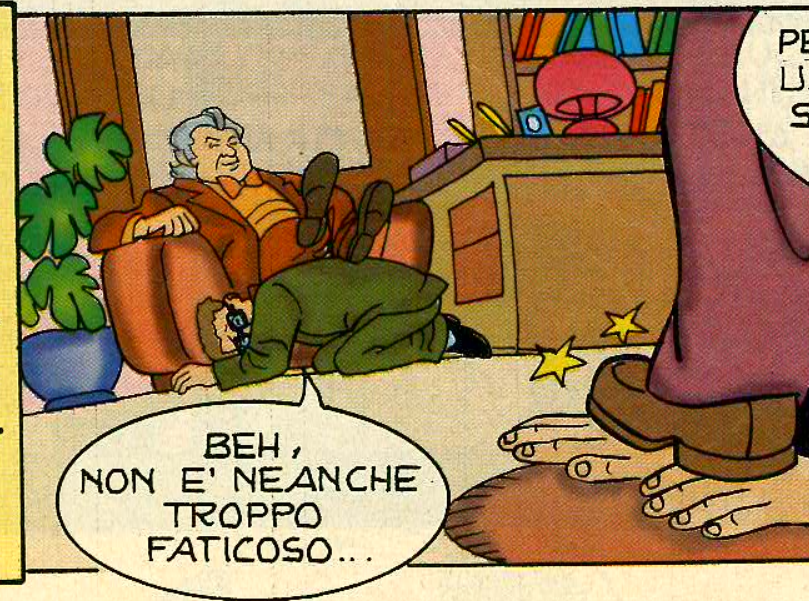

PERO 'E' LEGGER MENTE IMILIANTE! AHHH.. S-SCUS1... MI STA LIEVIS. SIMAMENTE FRANTUMANDO IL POLLICE

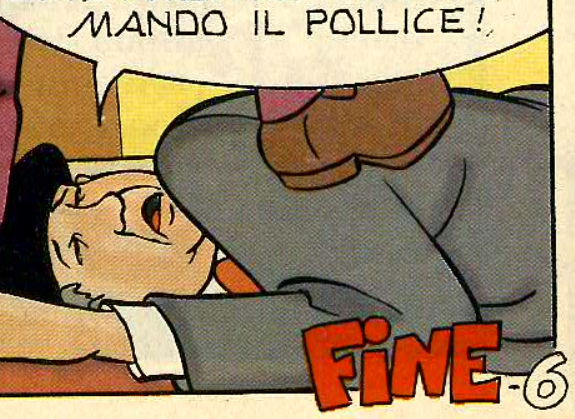




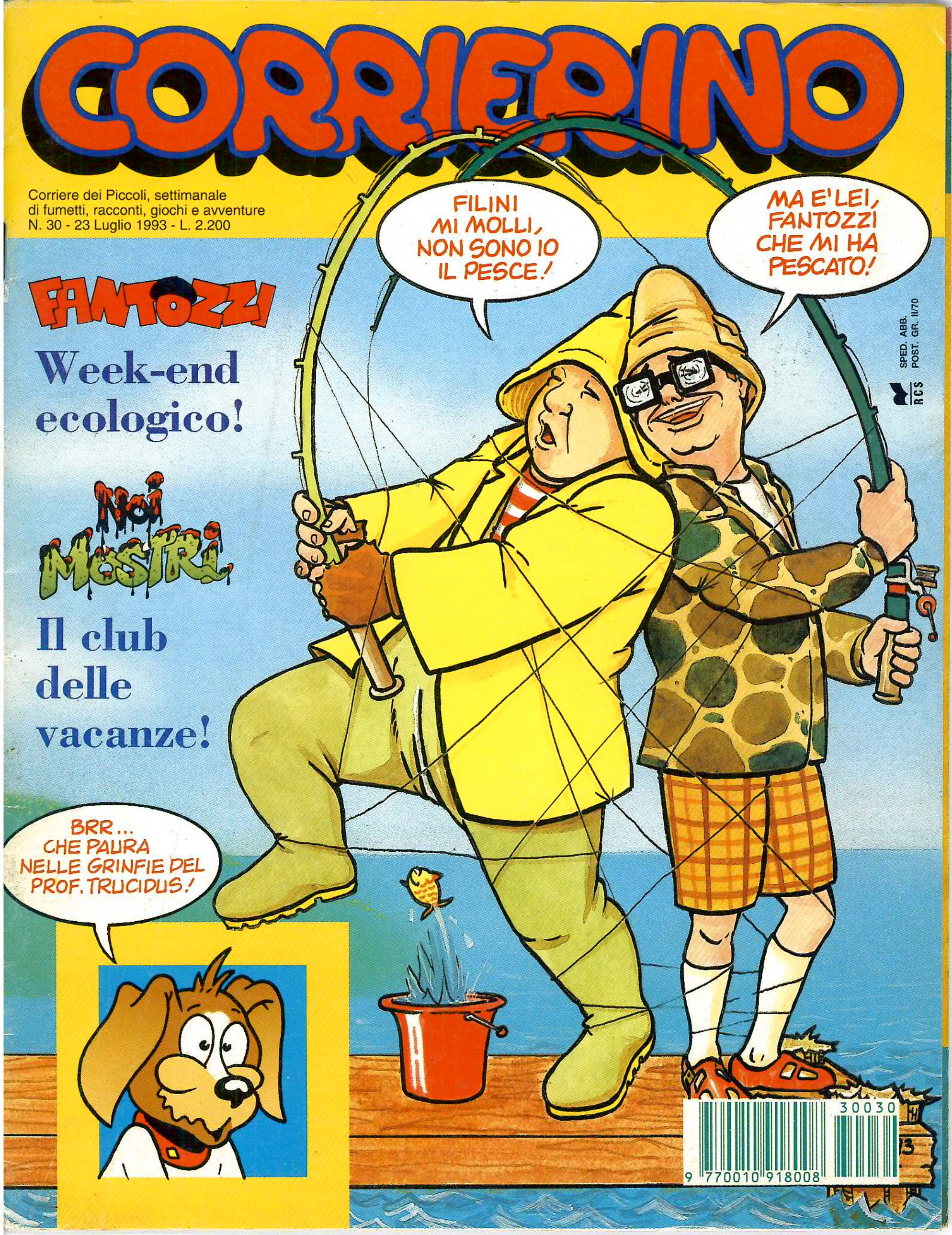




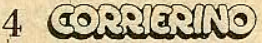
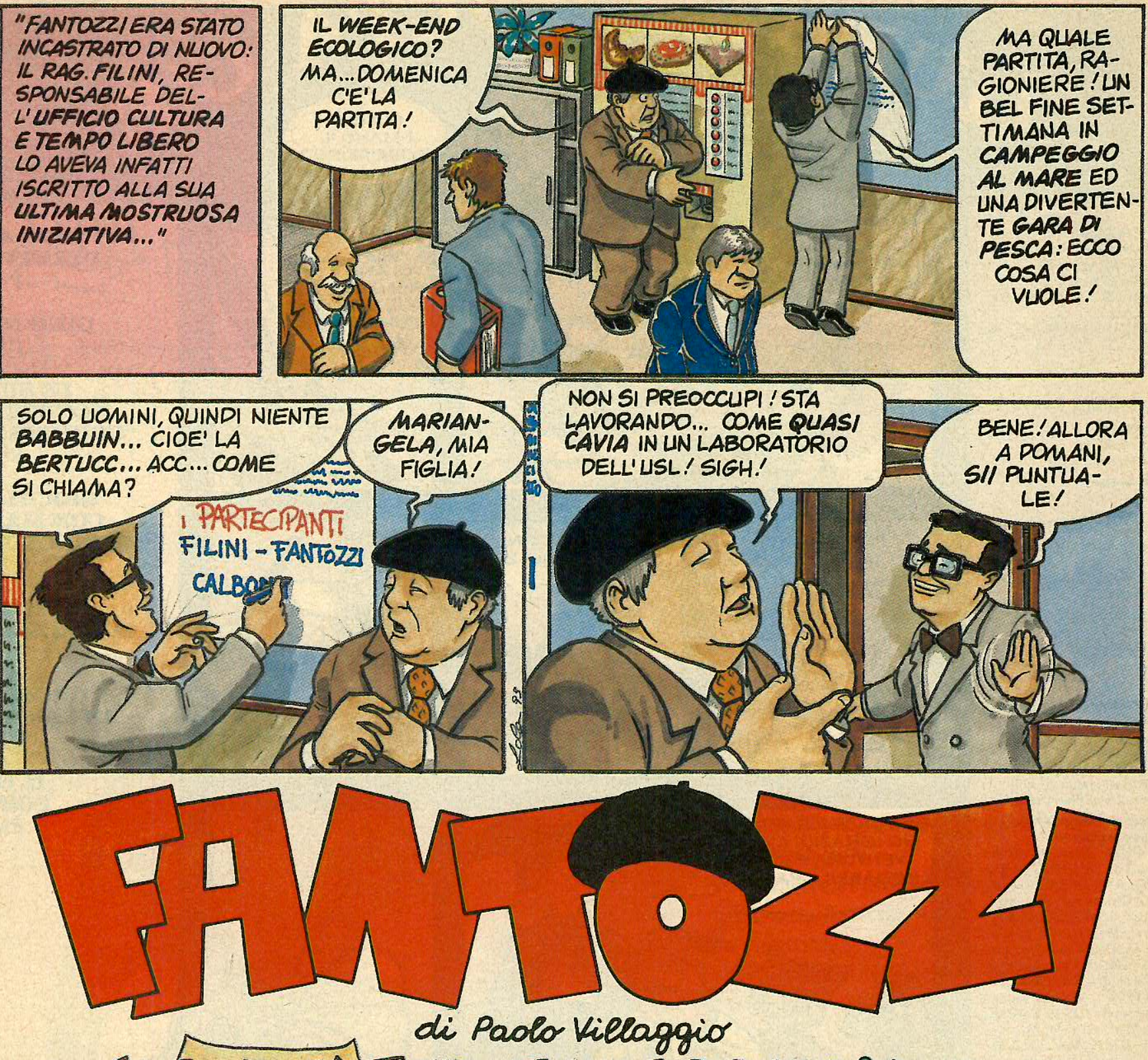

(a)

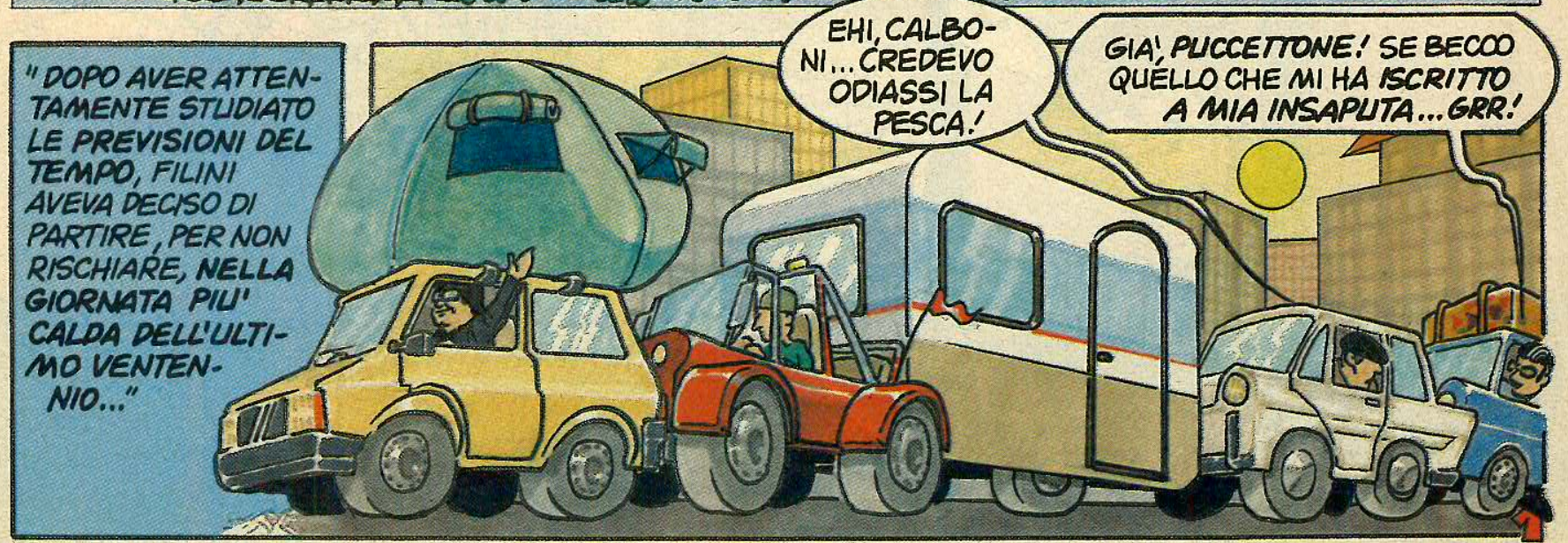

TESTO dI ANTONIO ORECCHIA DISEgni di LOLA AIRAGHI 


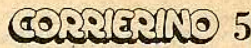

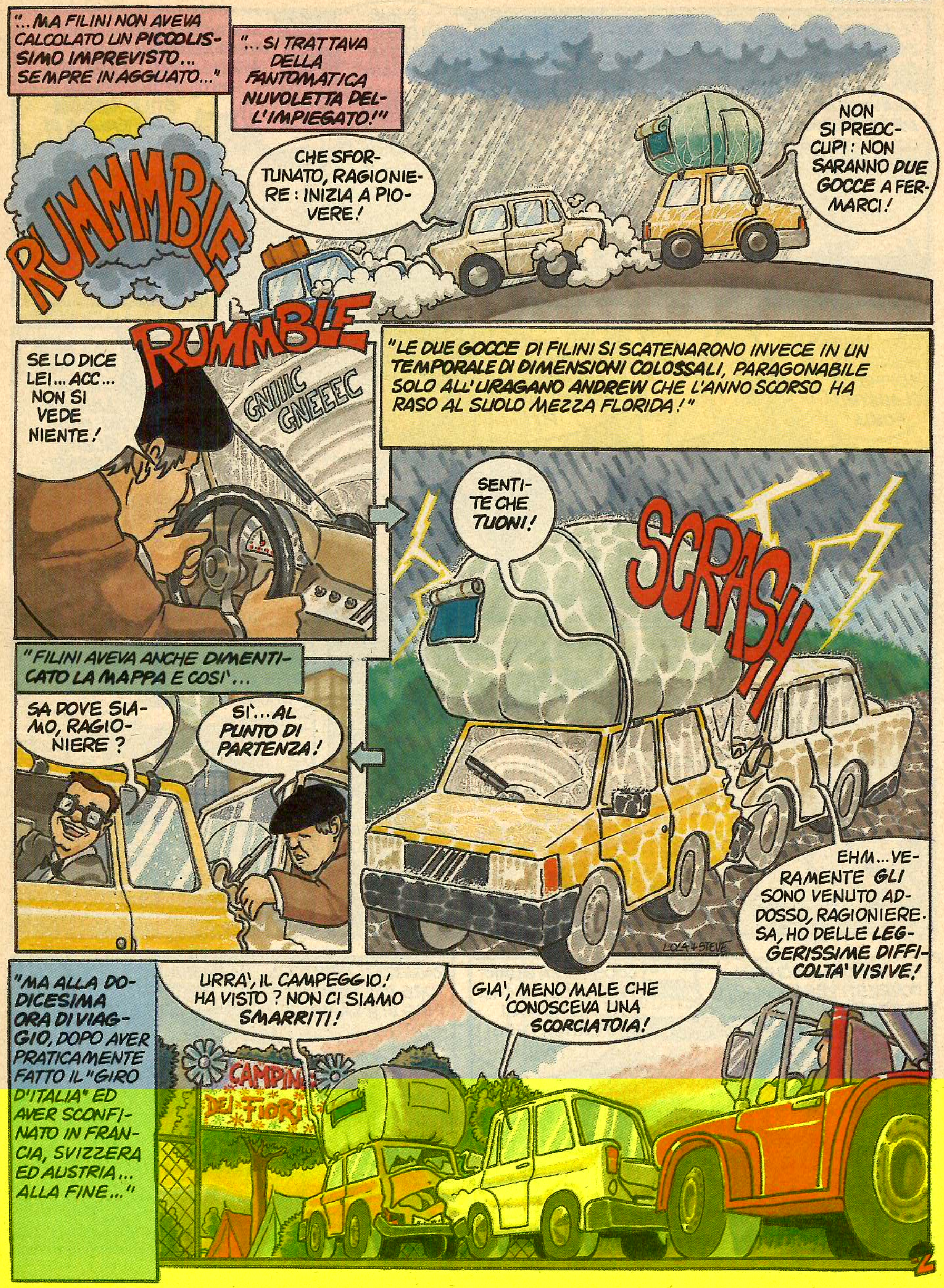


6 ๔อभiฺn:
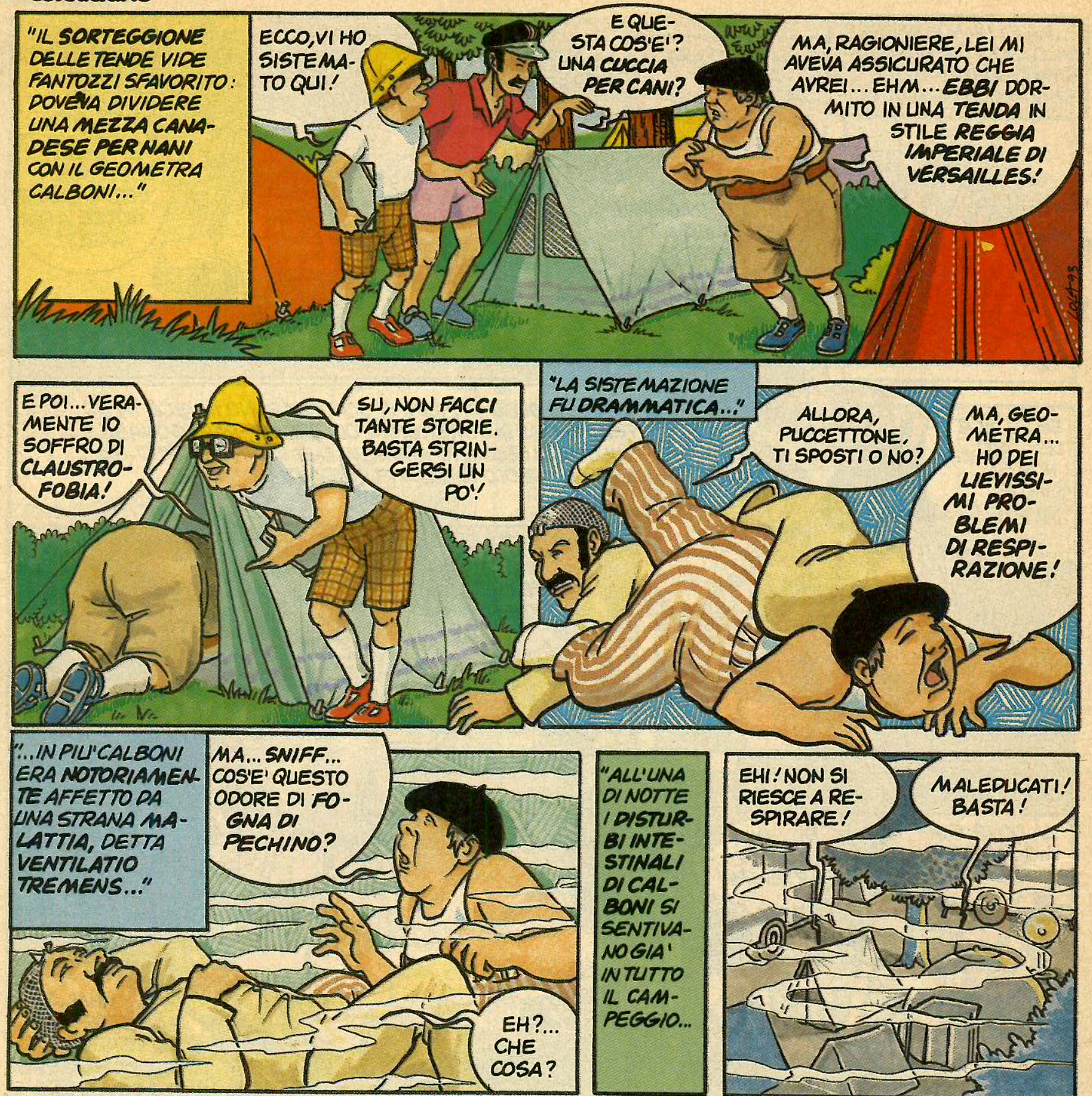

"LA SISTEMAZIONE

FUDRAMMATICA..." ALLORA, MA, GEOPUCCETTONE. TISPOSTIONO? HODEI LIEVISS/MI PRO-

BLEMI OIRESPIRAZIONE!
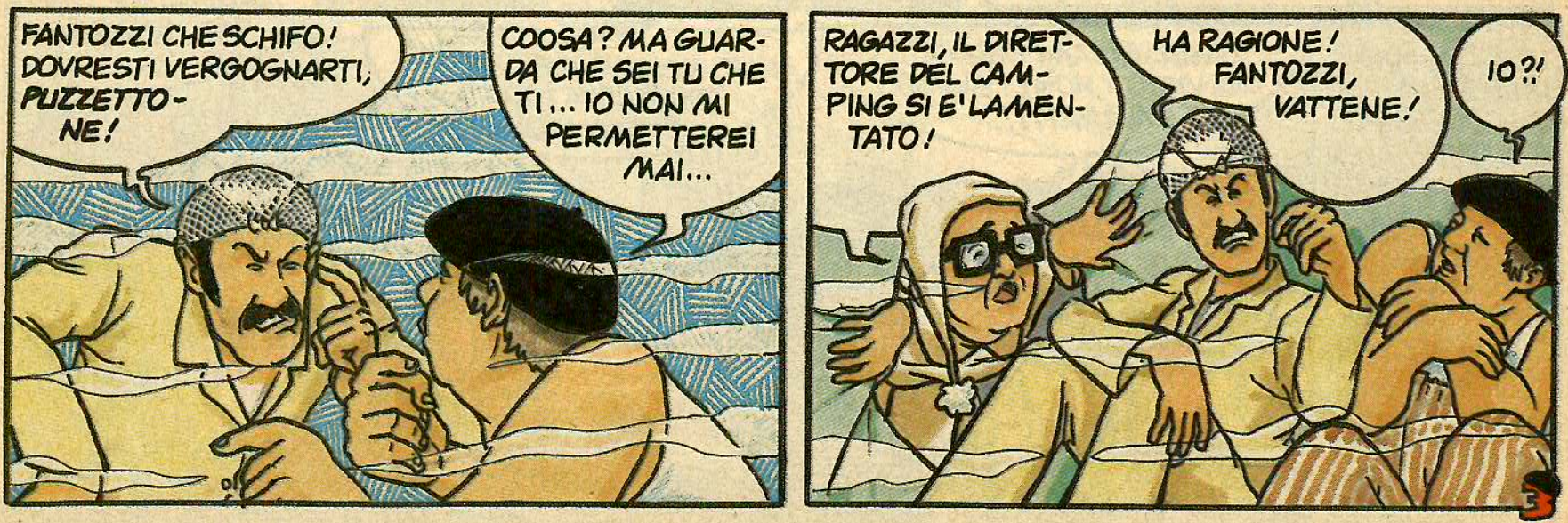
eosingina 7

"FANTOZZI VENNE ESPLILSO PAL CAMPEGGIO PER INDECNITA: E COSTRETTO A DORMIRE IN GINOCCHIO SUICECI, PER PUNIZIONE!"
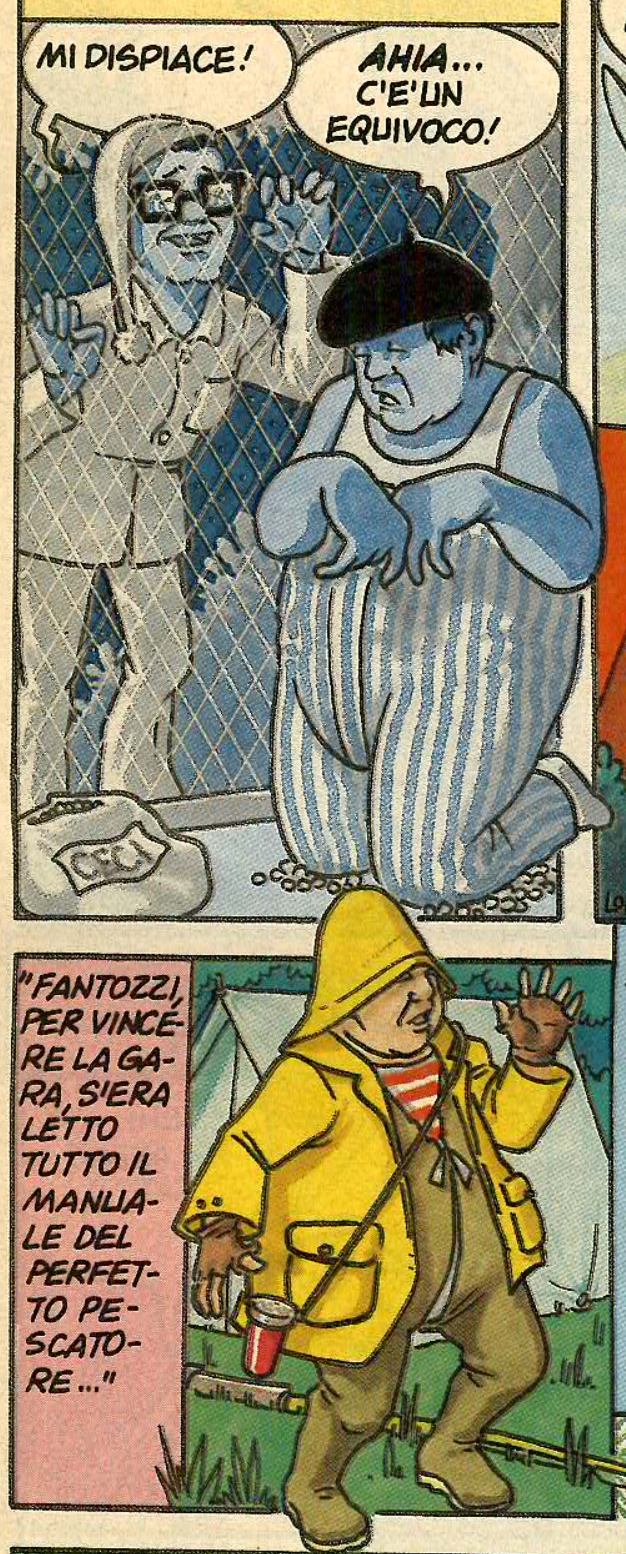

\section{"PER UN BUUN LANCIO: PIEGARE LEG- GERMENTE IL BUSTO, ROTEARE CON FORZA LE ERACCIA..."}

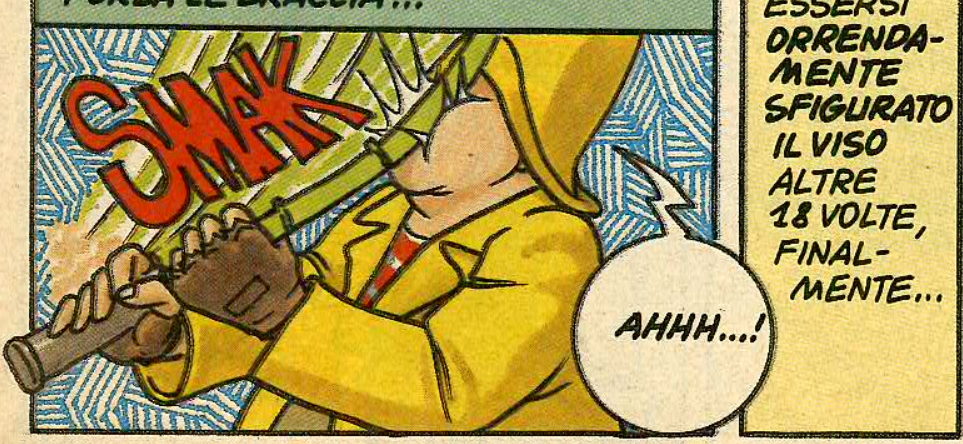

"LA NOTTE PROVOCO' ALLE GINOCCHIA DI FANTOZZI ESCORIAZIONI MULTIPLE DI QUAPTO GRADO, CON CONSEGUENTE SEMI-PARALISI ALLE GAMBE. COSI:

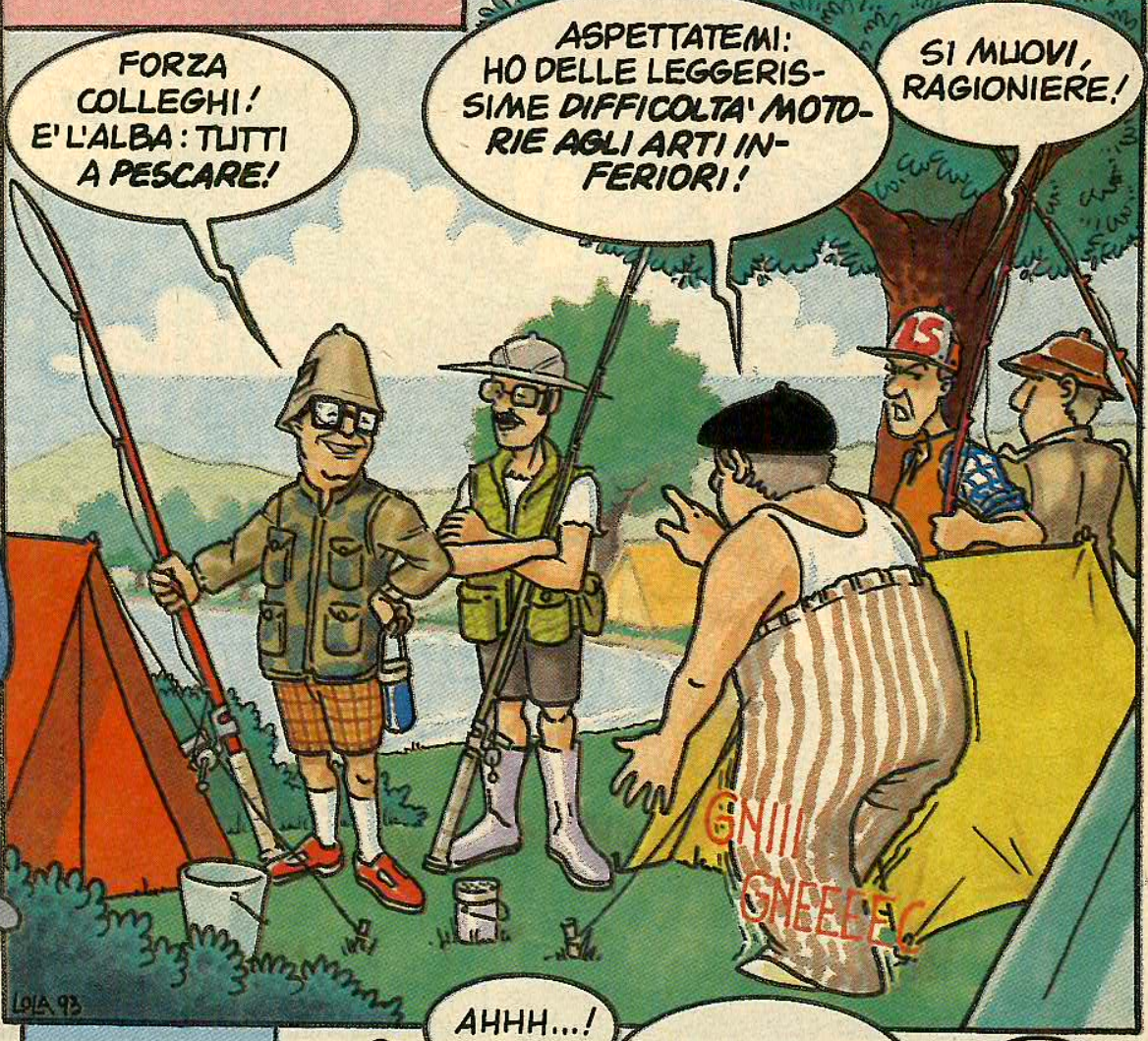

"LA SUA CANNA DA PESCA ERA DEL PERIODO PRE. BELLICO. $150 \mathrm{Ko}$. DI PESO!

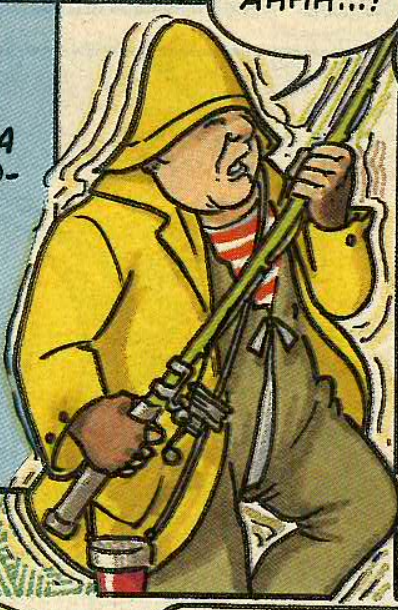

INCOMINCIAMO RAGIONIERE?
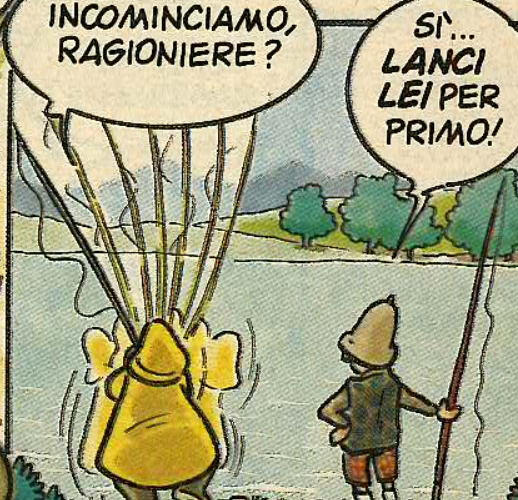

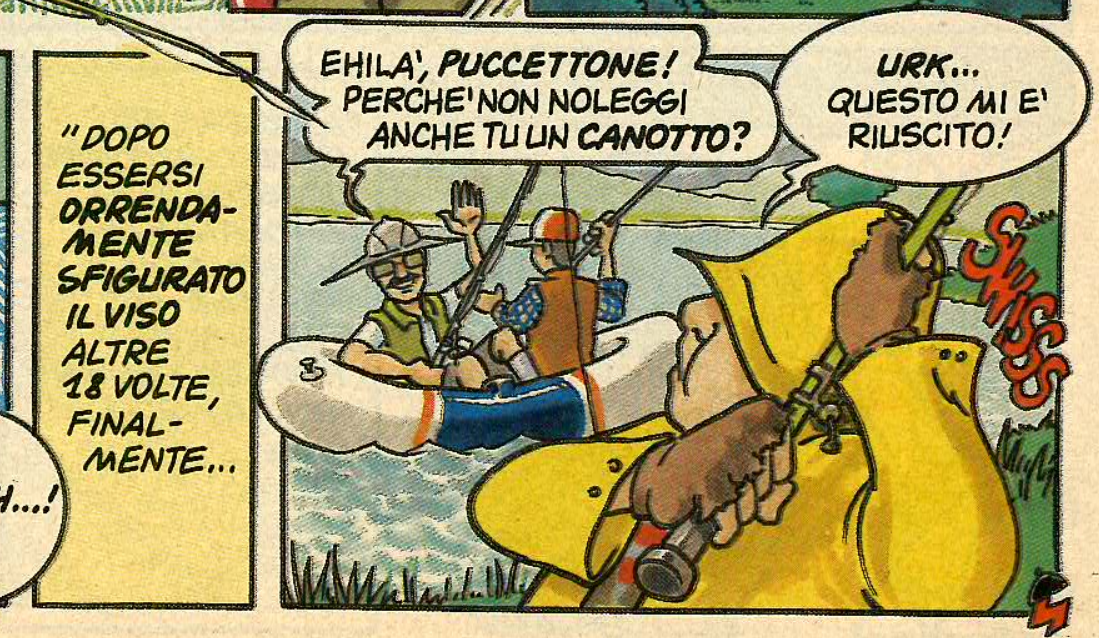


8 Coisiant
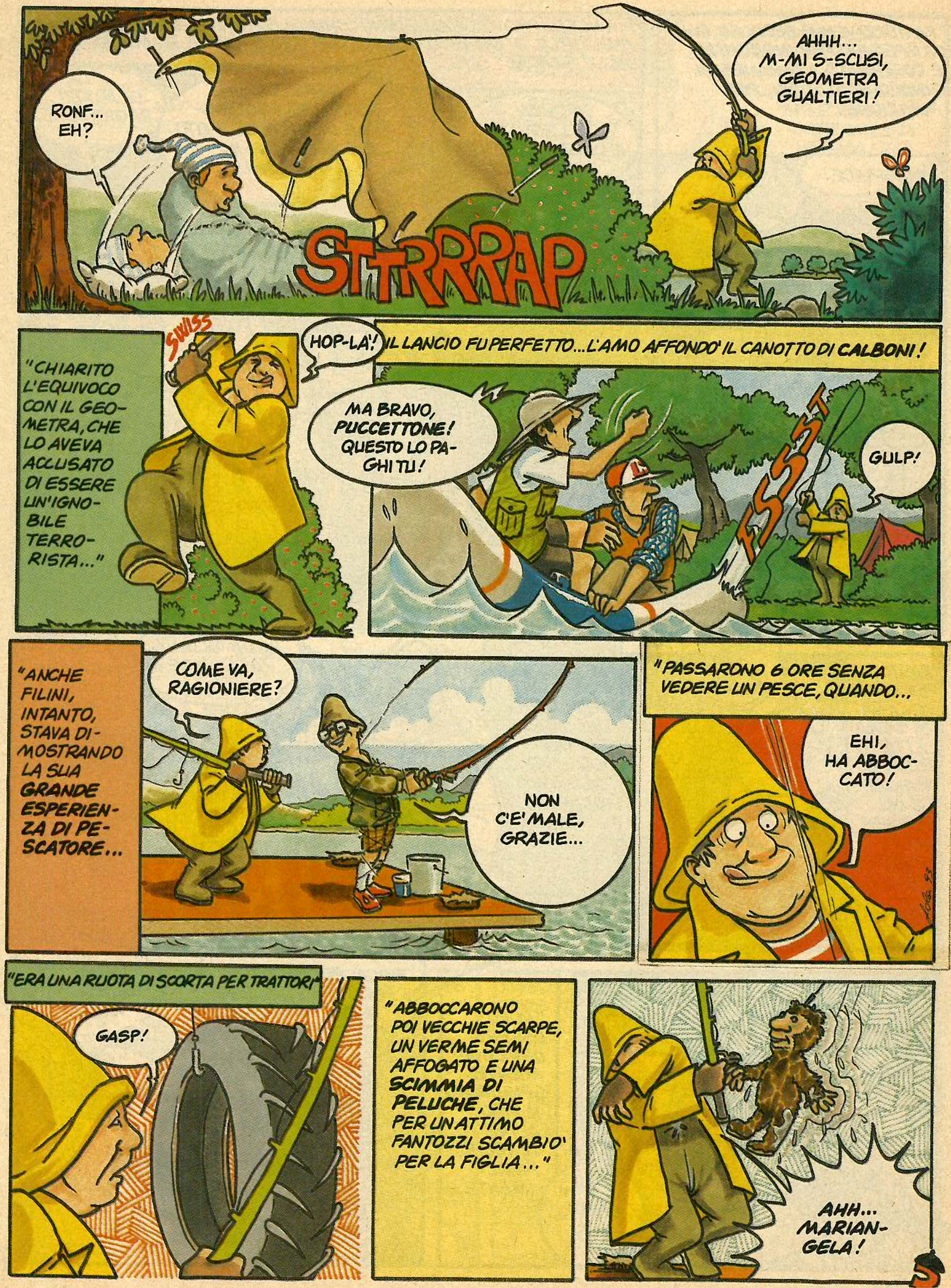


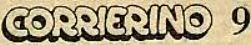

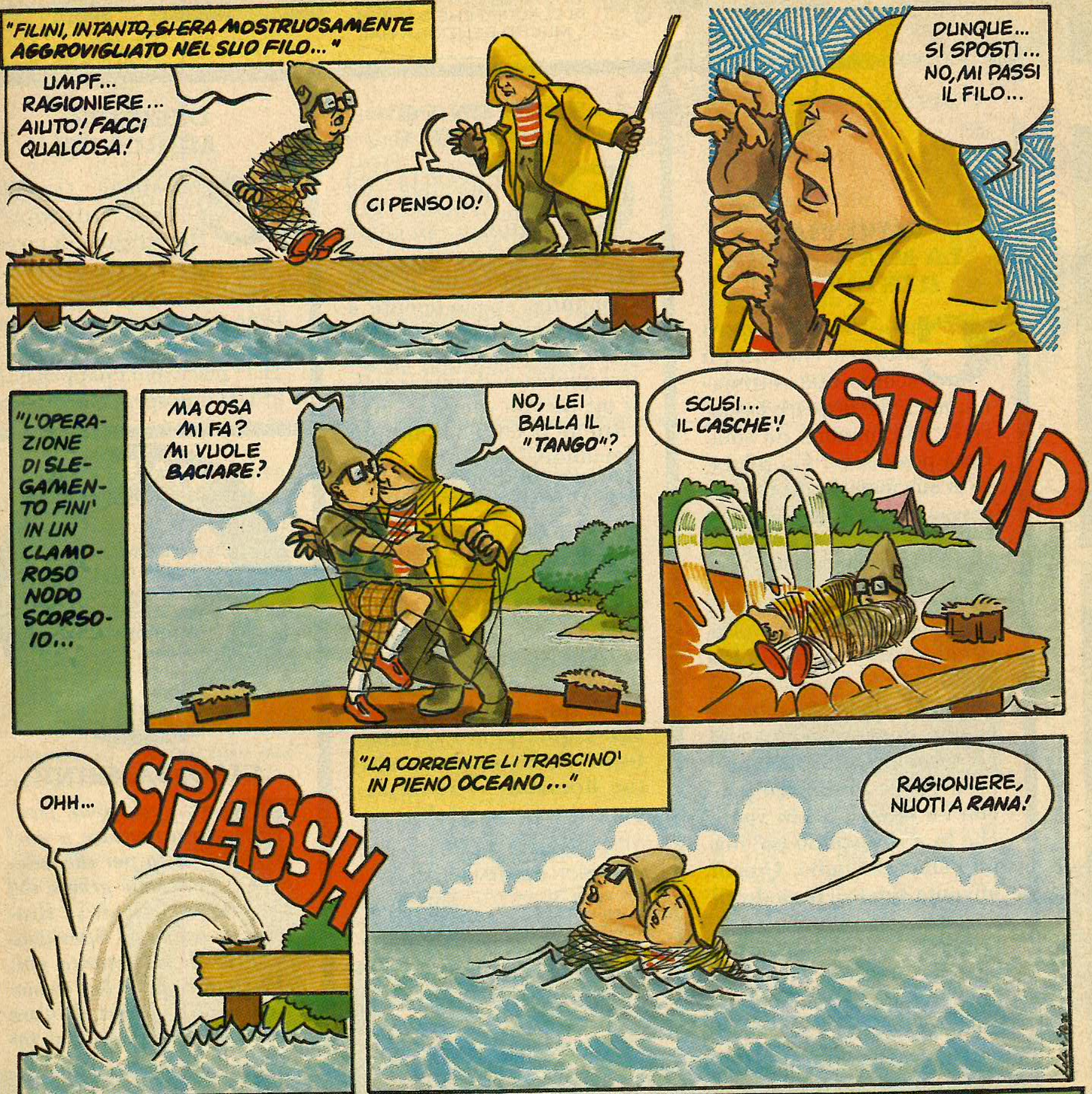

"FANTOZZIE FILINI FURONO RIPESCATI DA UN PESCHERECCIO TUROO BATTENTE

BANDIERA PANAMENSE EASSUNTI UNO COME ANCORA LALTRO COME ZAVORRA!"

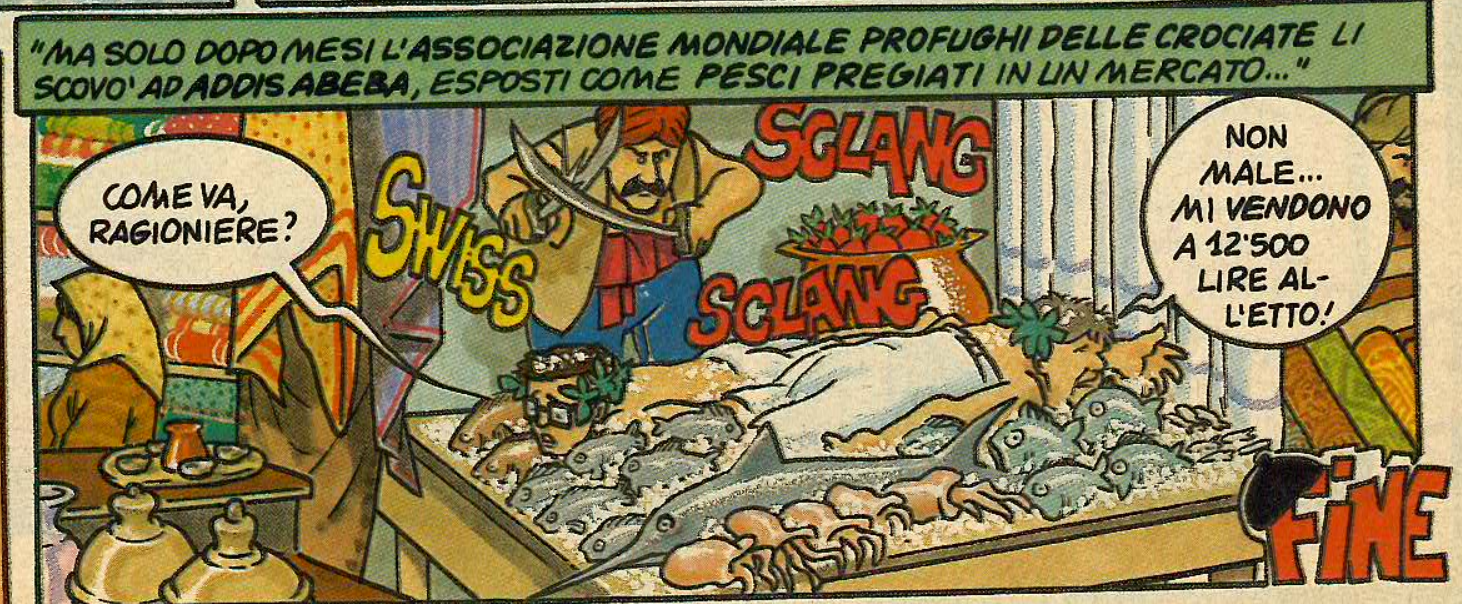


14. बeristano
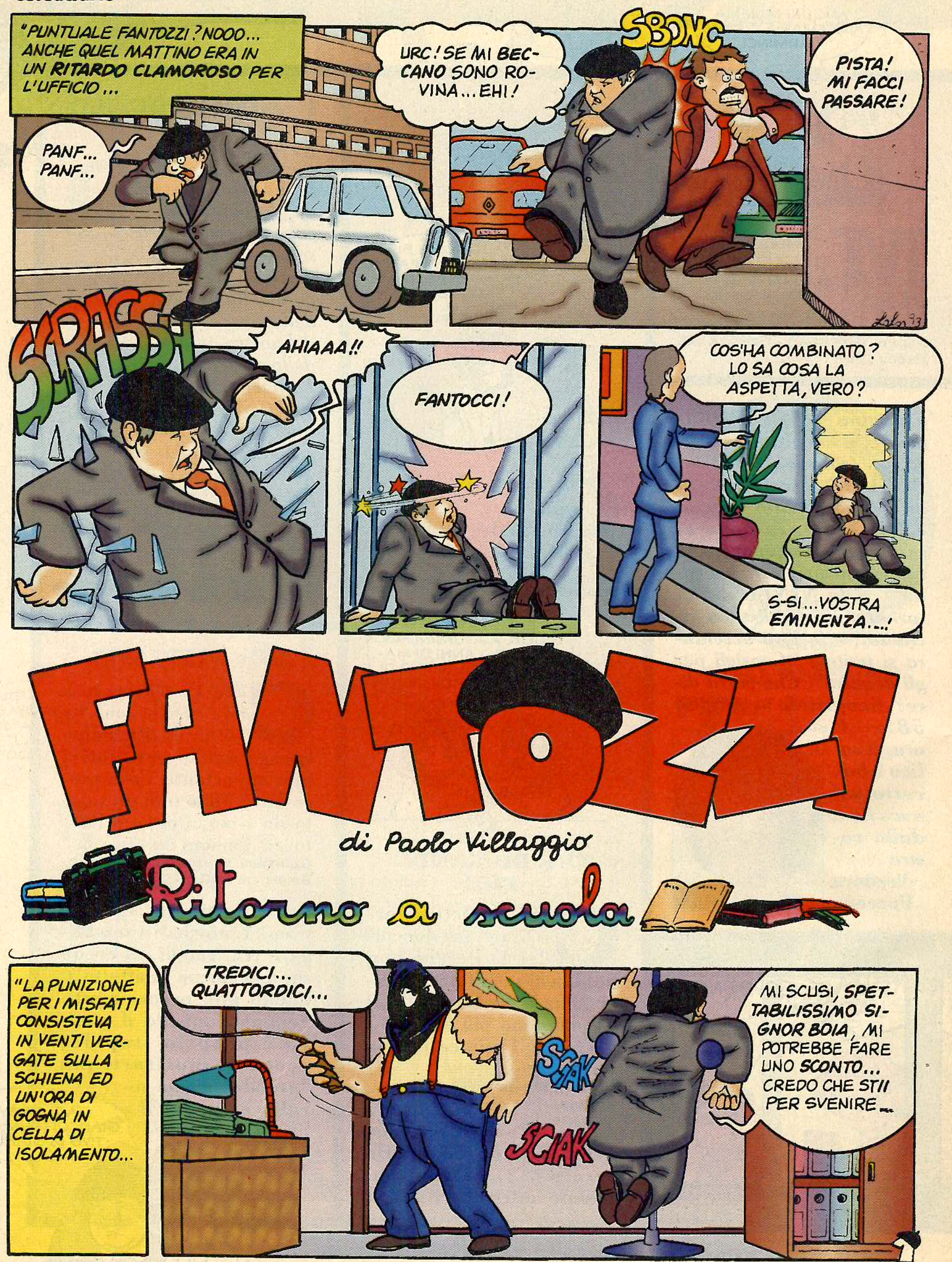

TESTO di ANTONIO ORECCHIA Disegni di LOLA AIRAGHI 


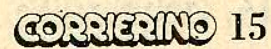
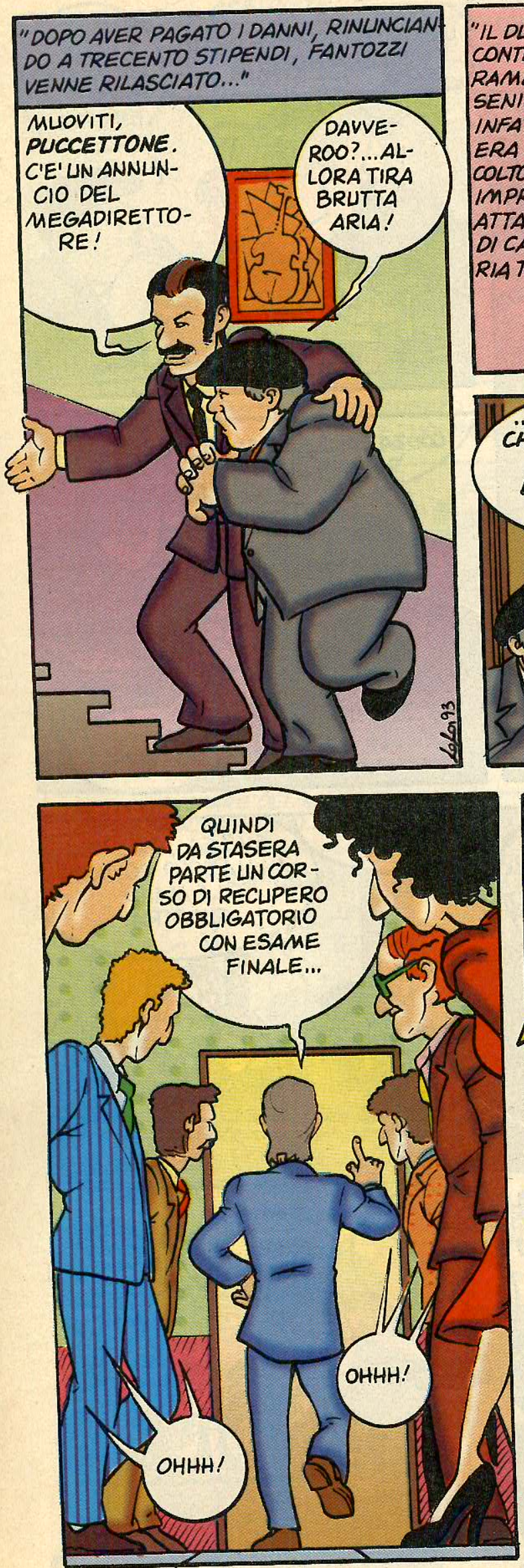
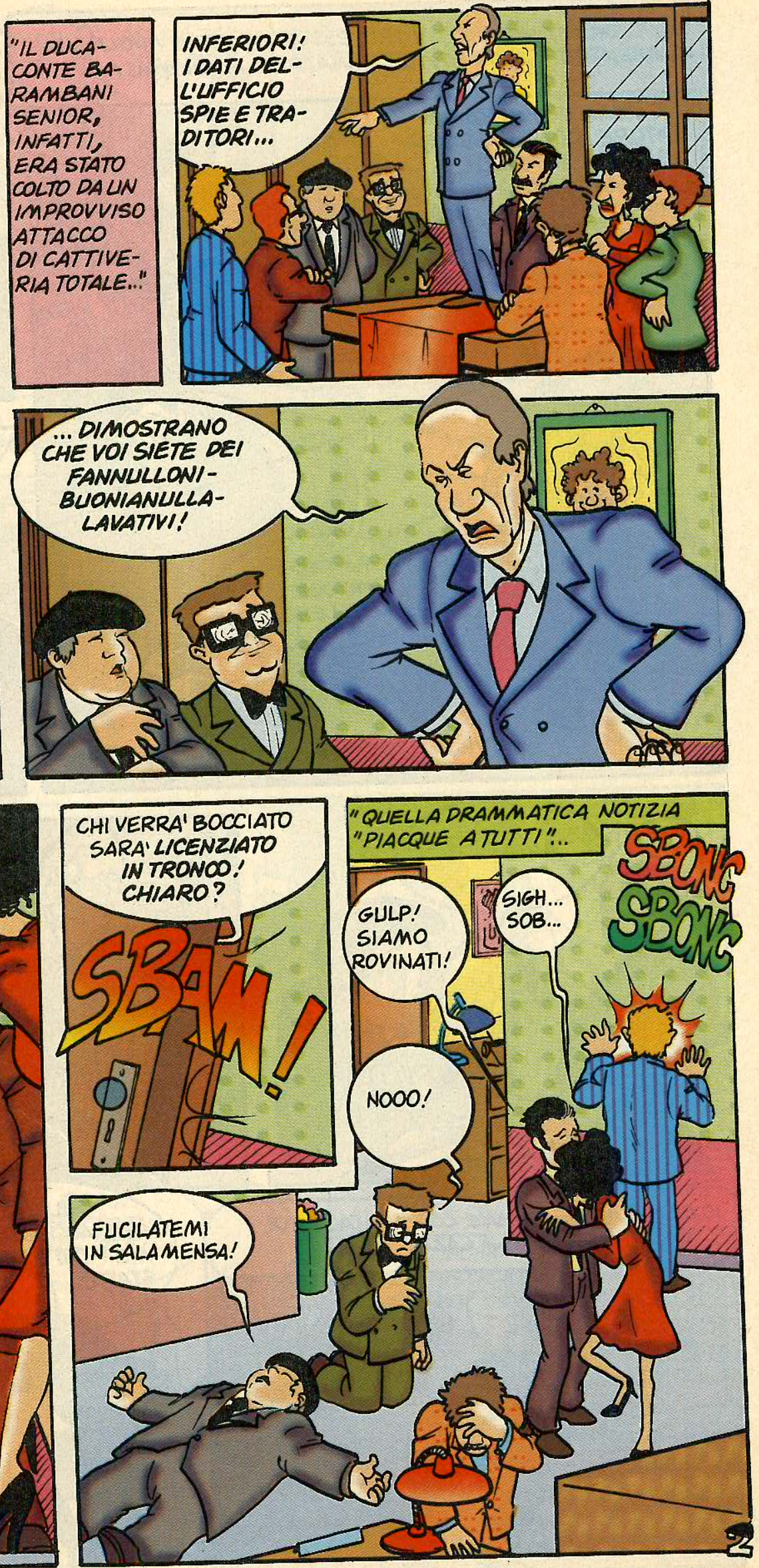
16 coriscanta

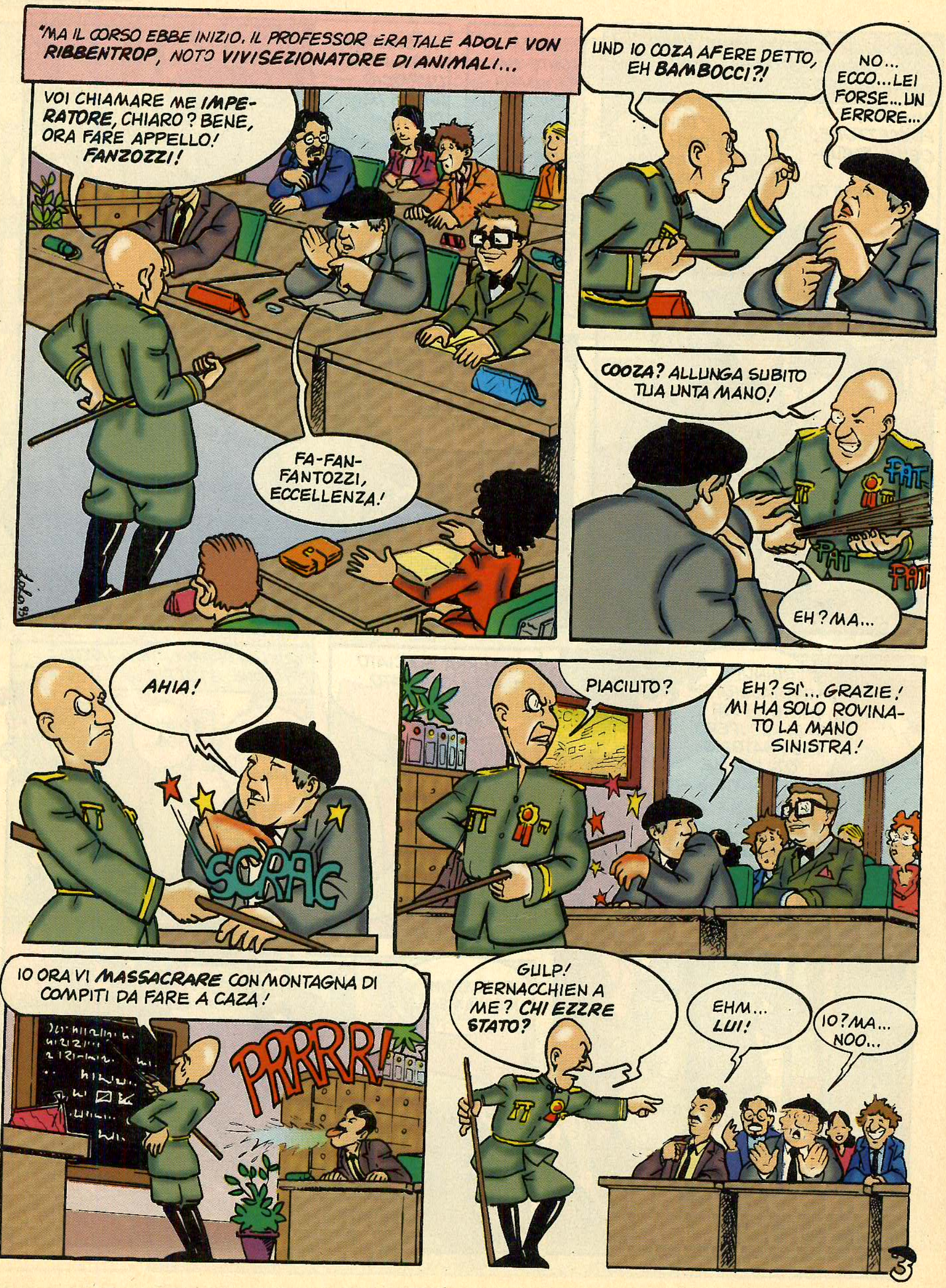




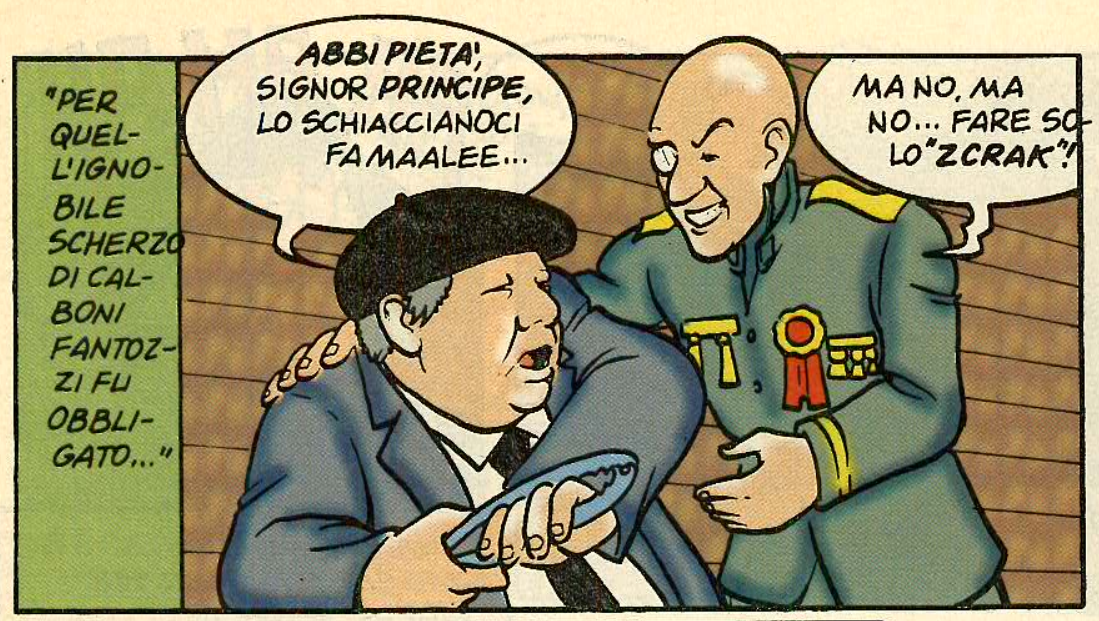

बอง? 17
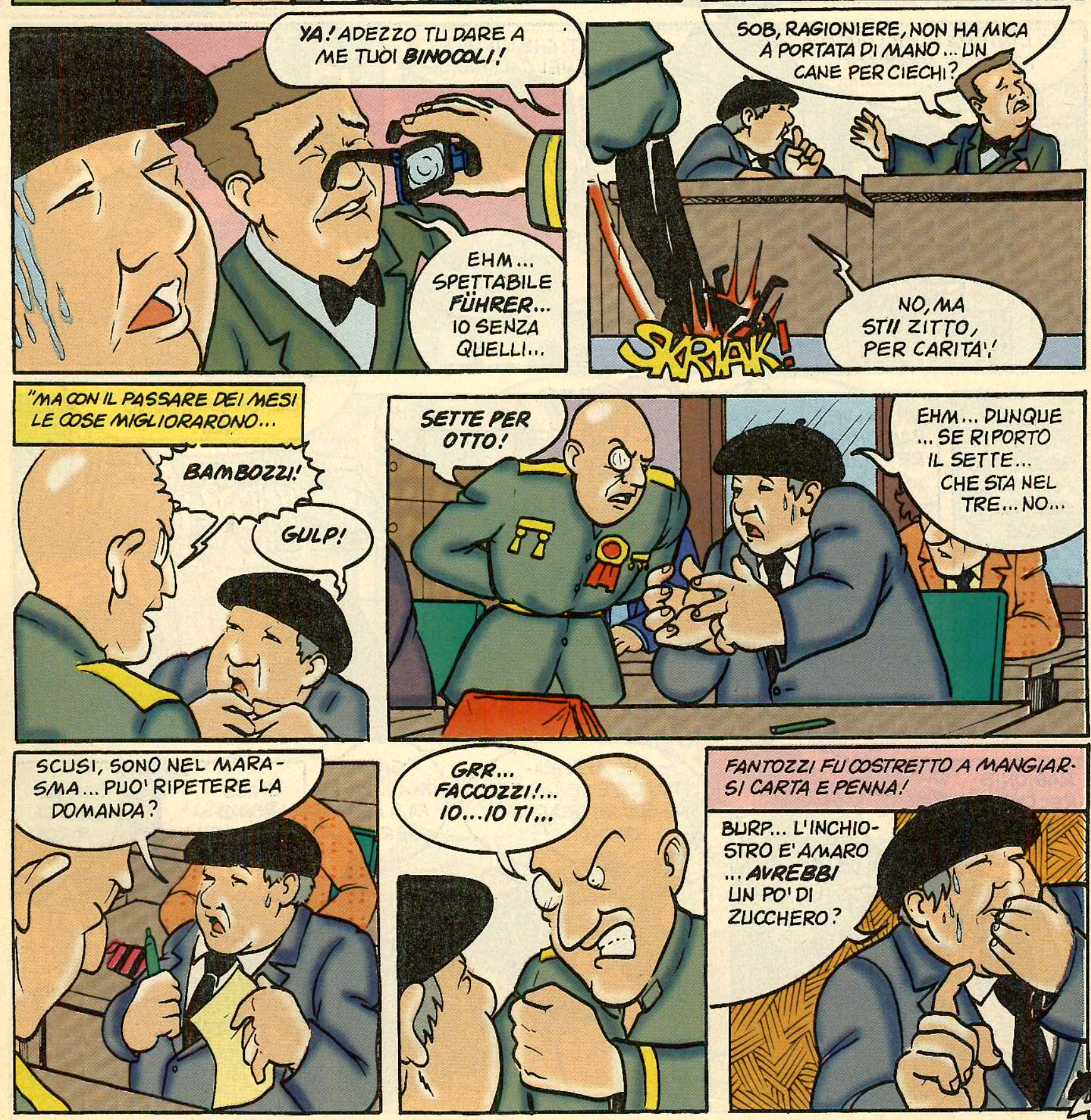

FANTOZZI FU COSTRETTO A MANGIAR. SI CARTA EPENNA!

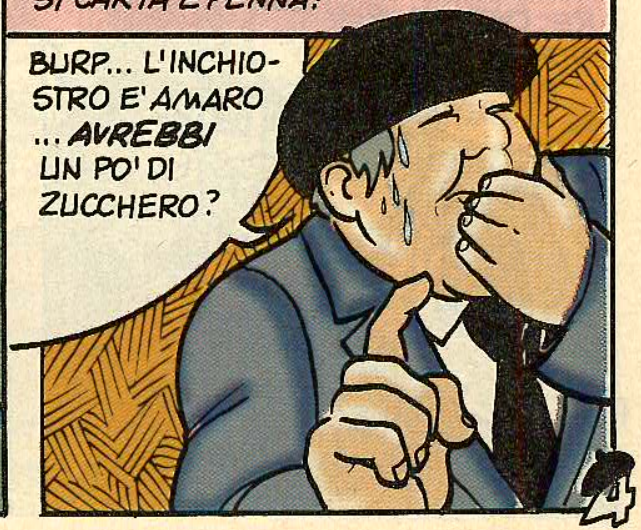




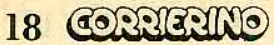
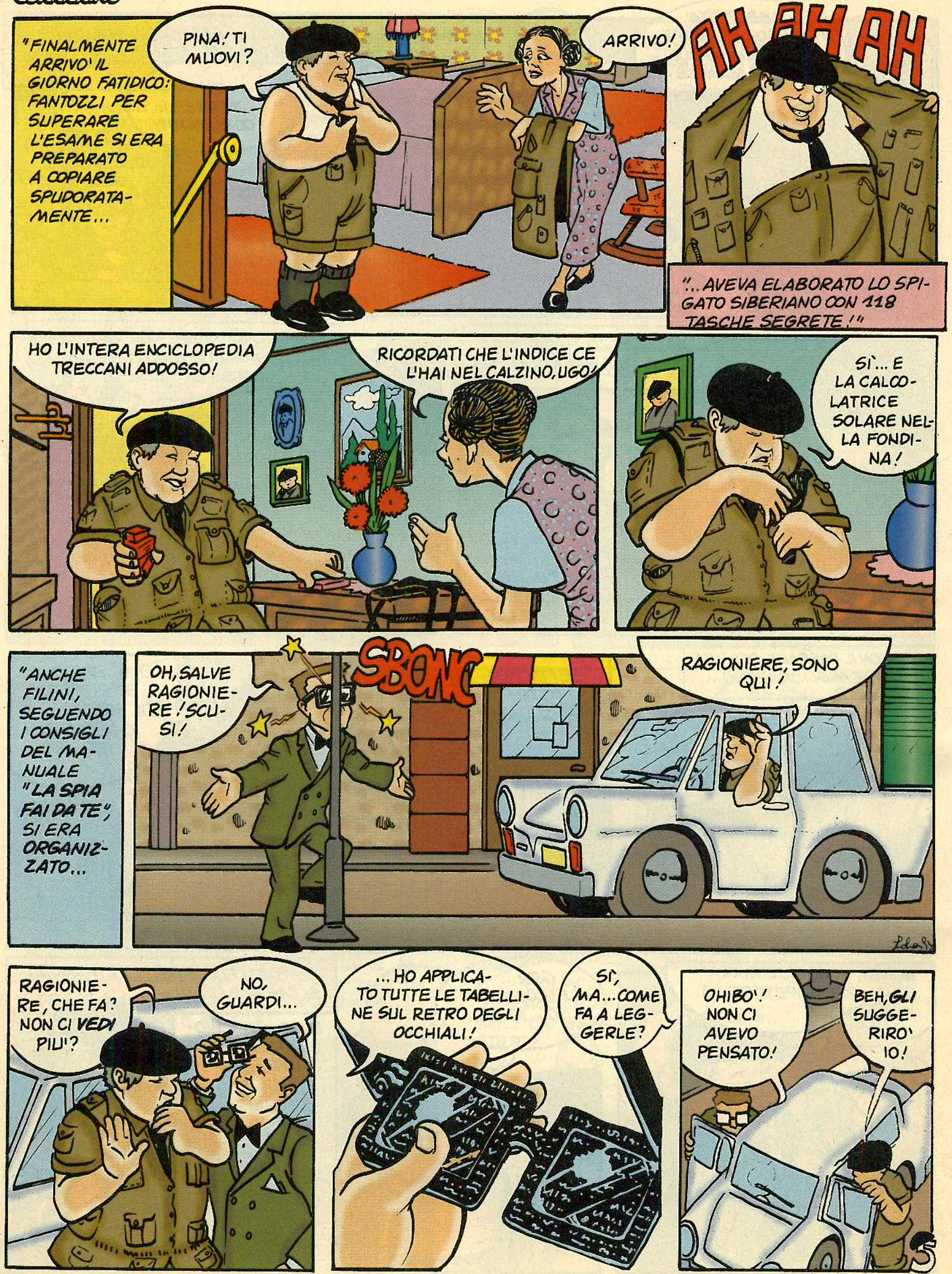

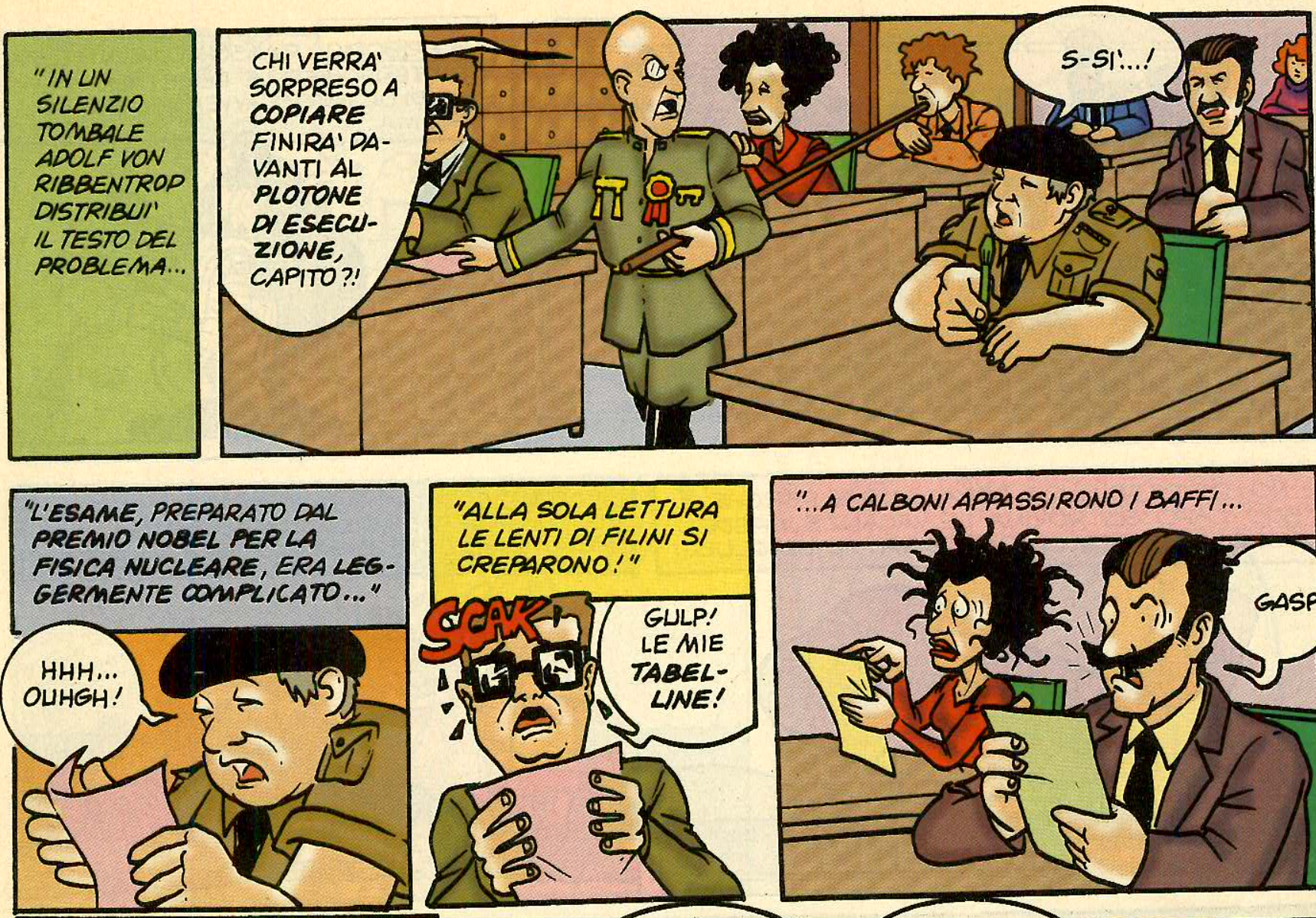

MA, ALLA FINE, USCIRONO I RISULTATI.."

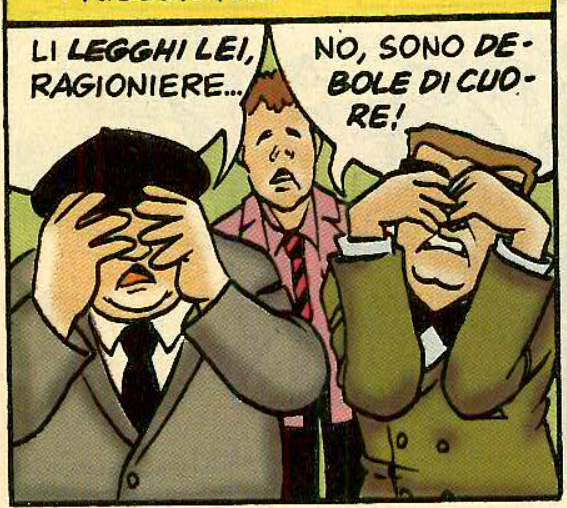

"ALLA SOLA LETTURA LE LENTI DI FILINISI CREPARONO!"

\section{GERMENTE COMPLICATO..."}
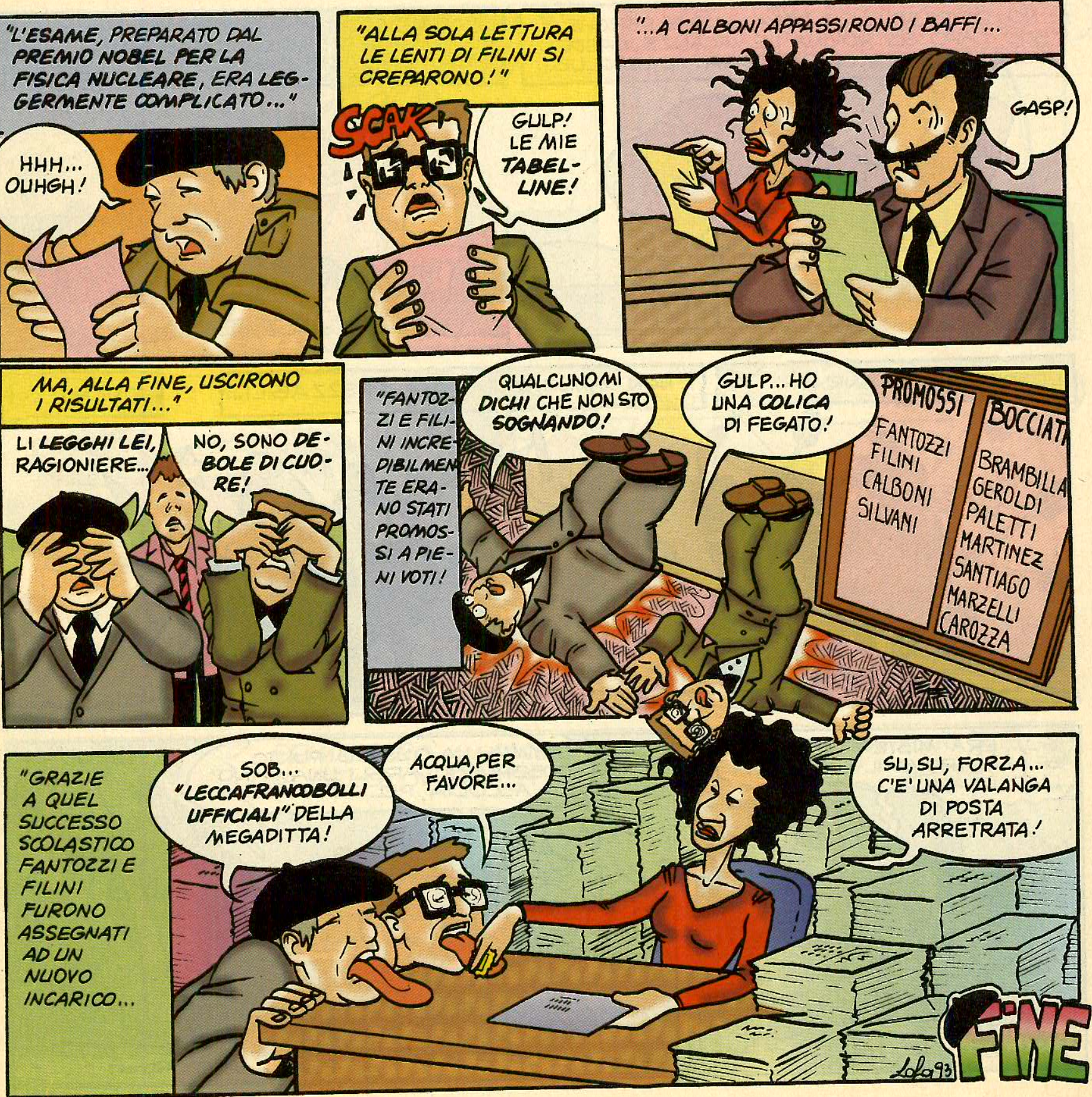


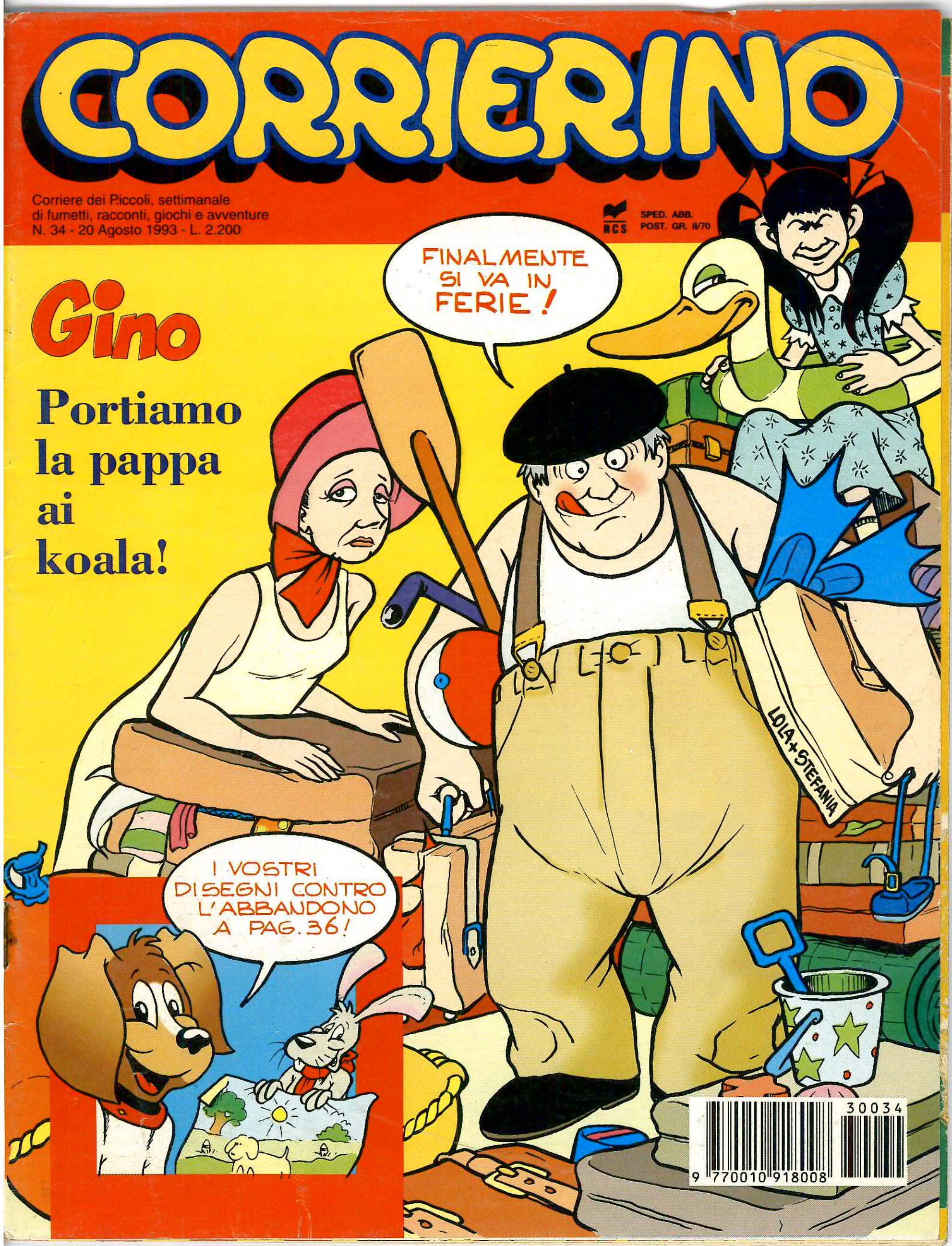




\section{4 serratino}
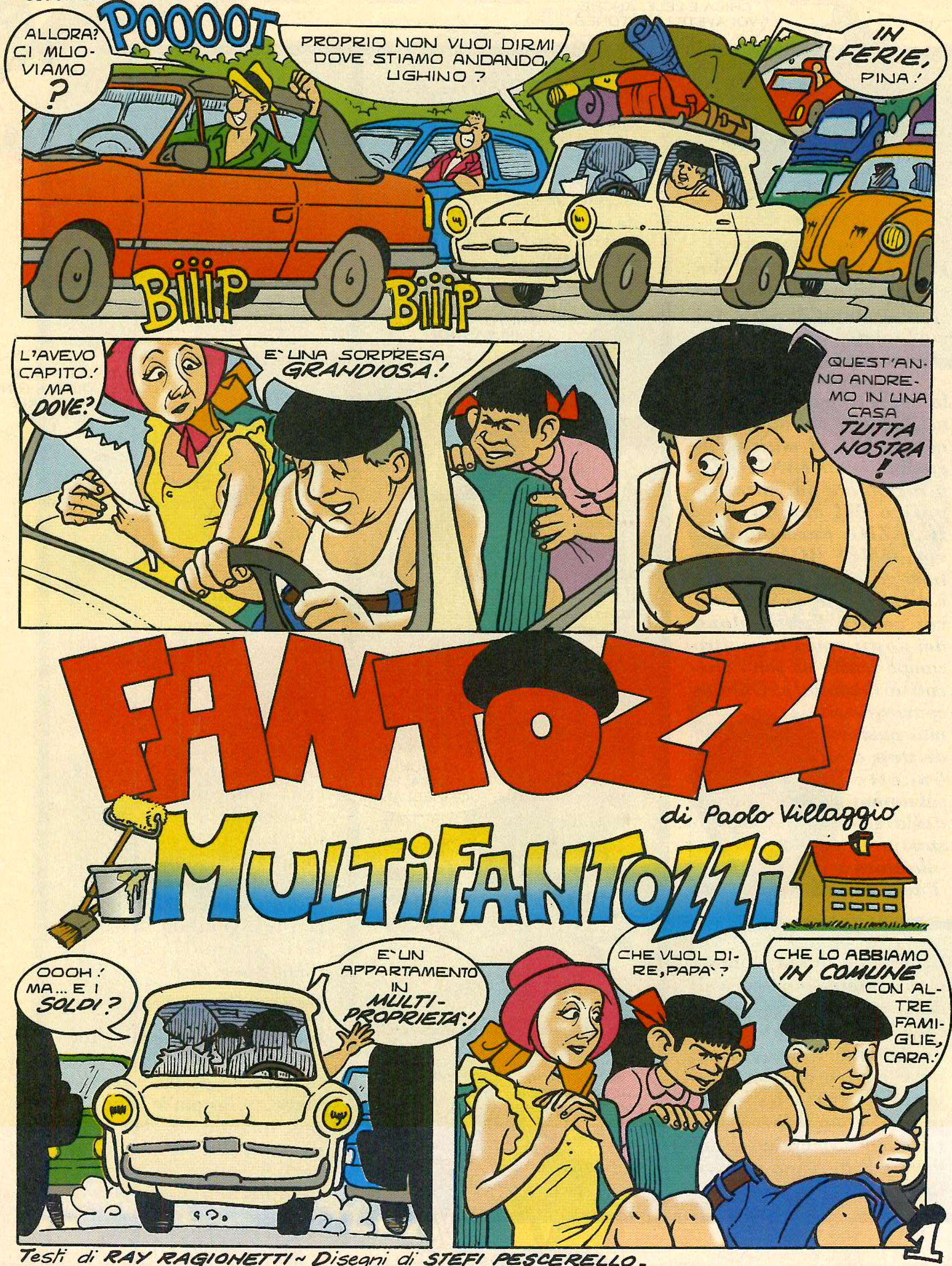


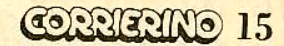
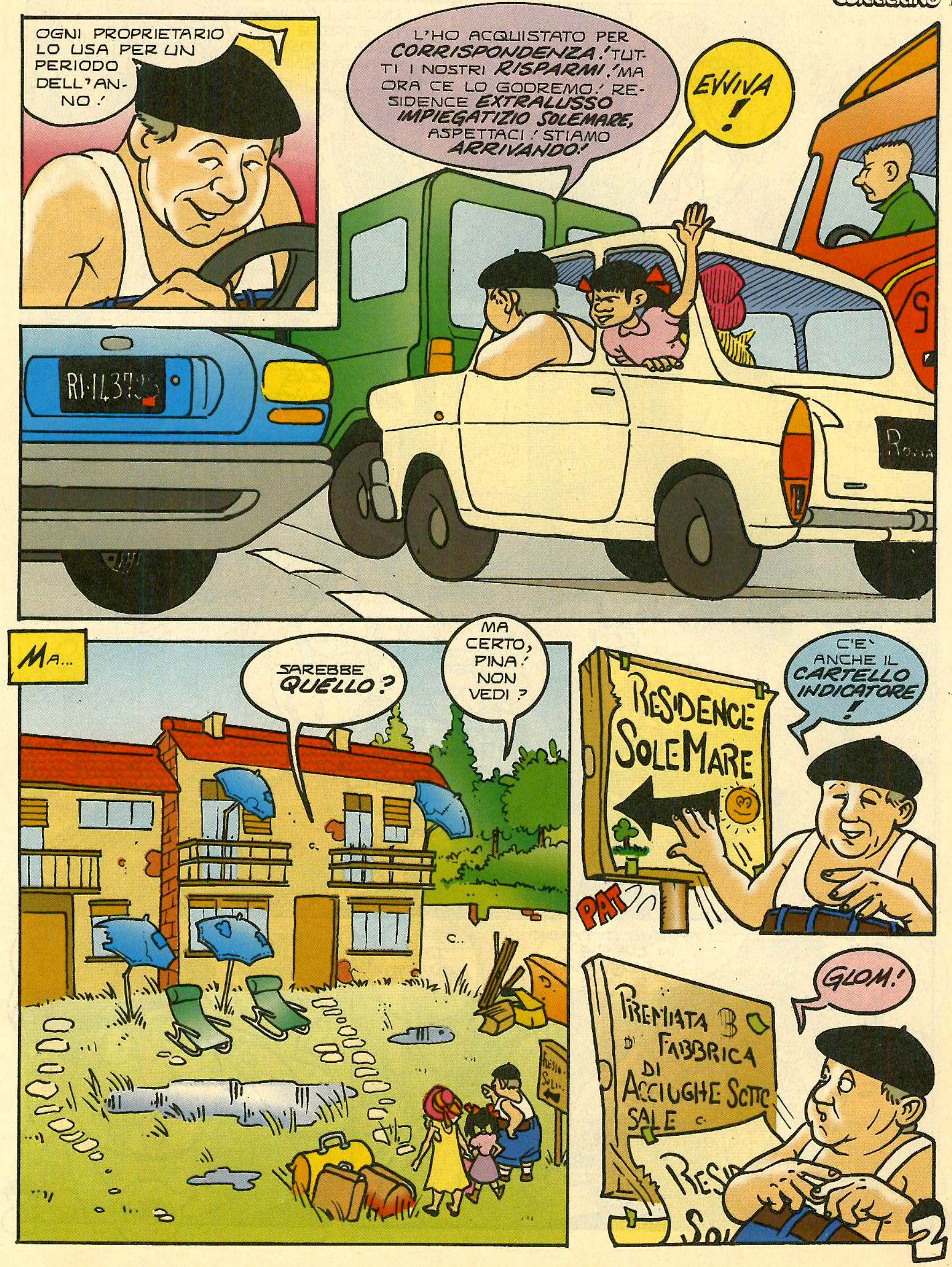
16 eristanc
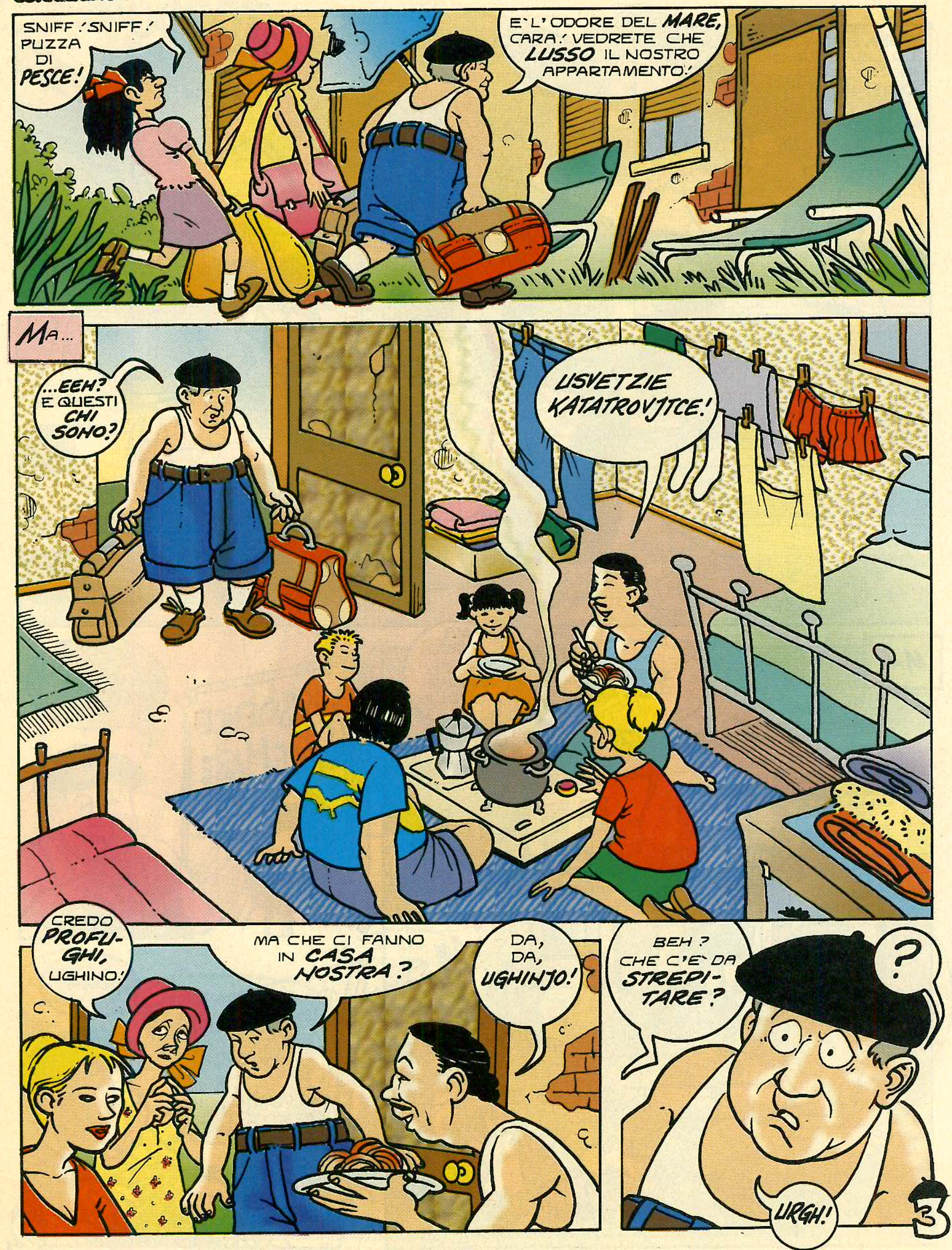


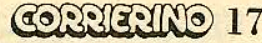

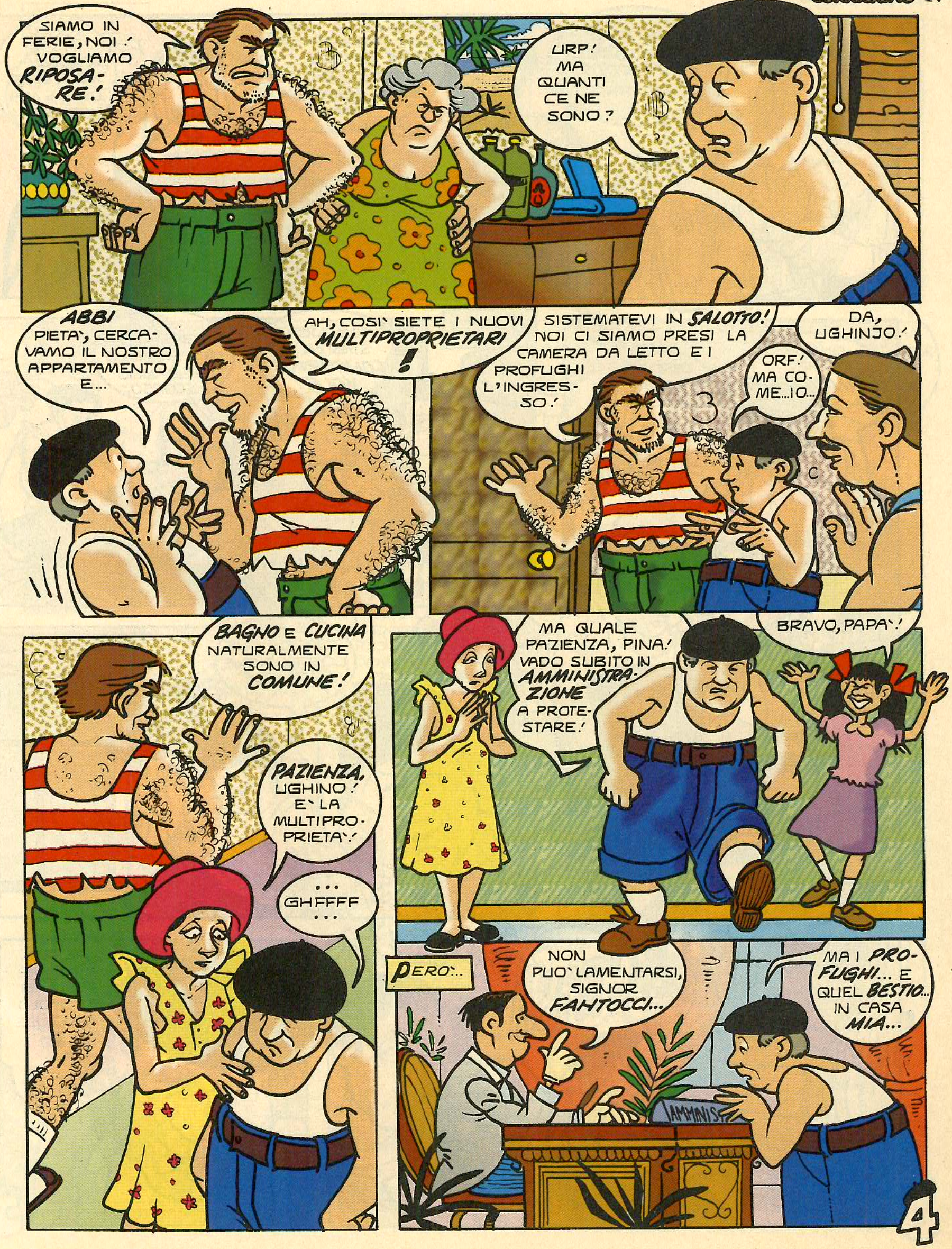


18 बอำ

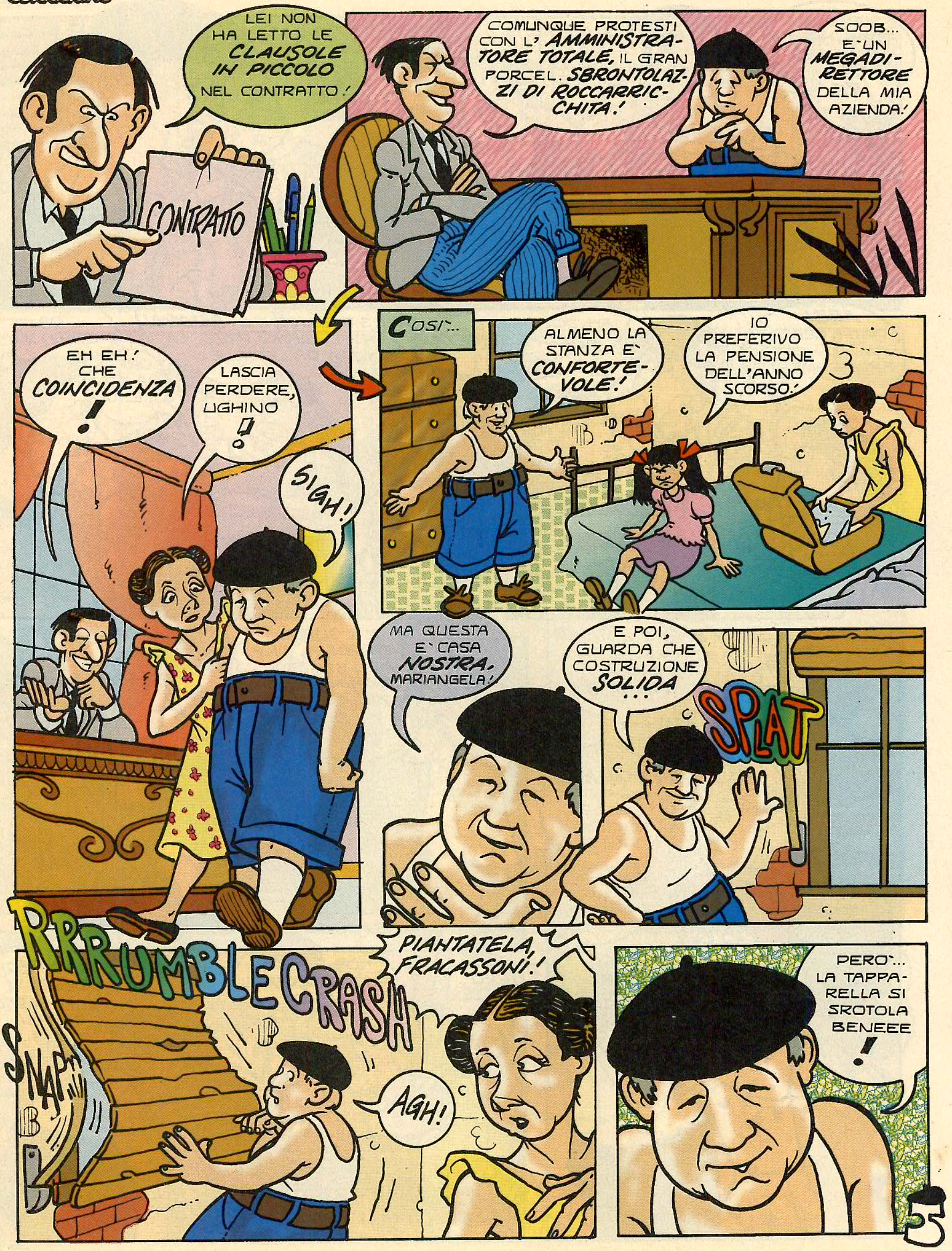




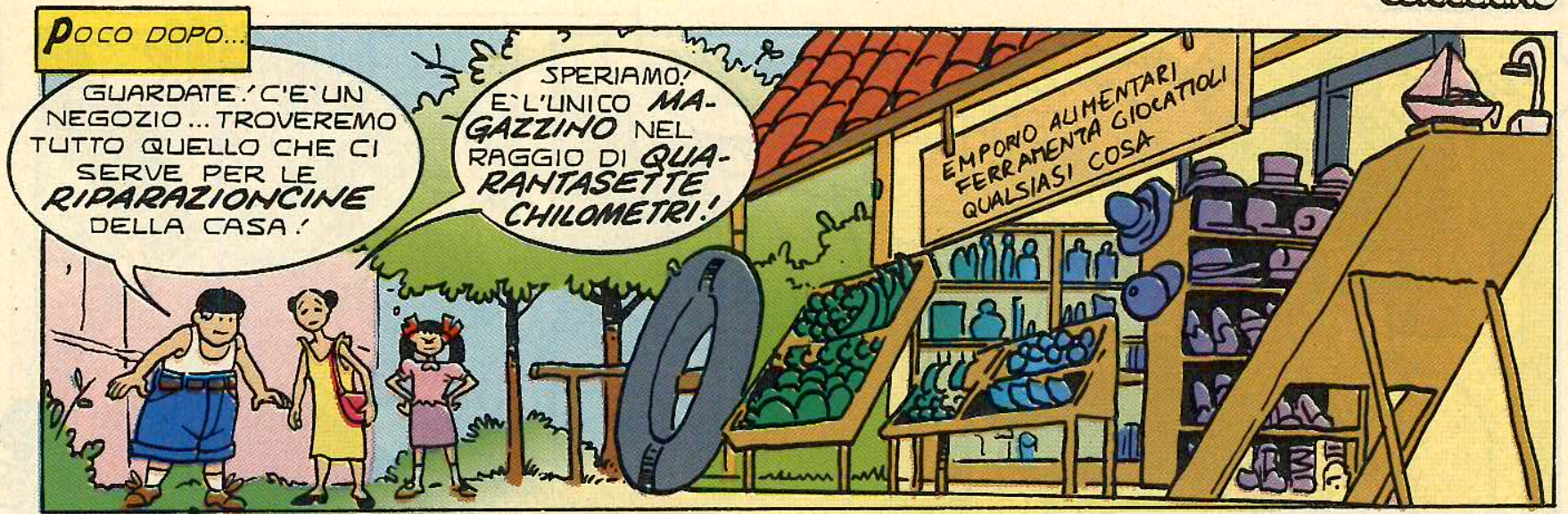

बอํำiñ 19

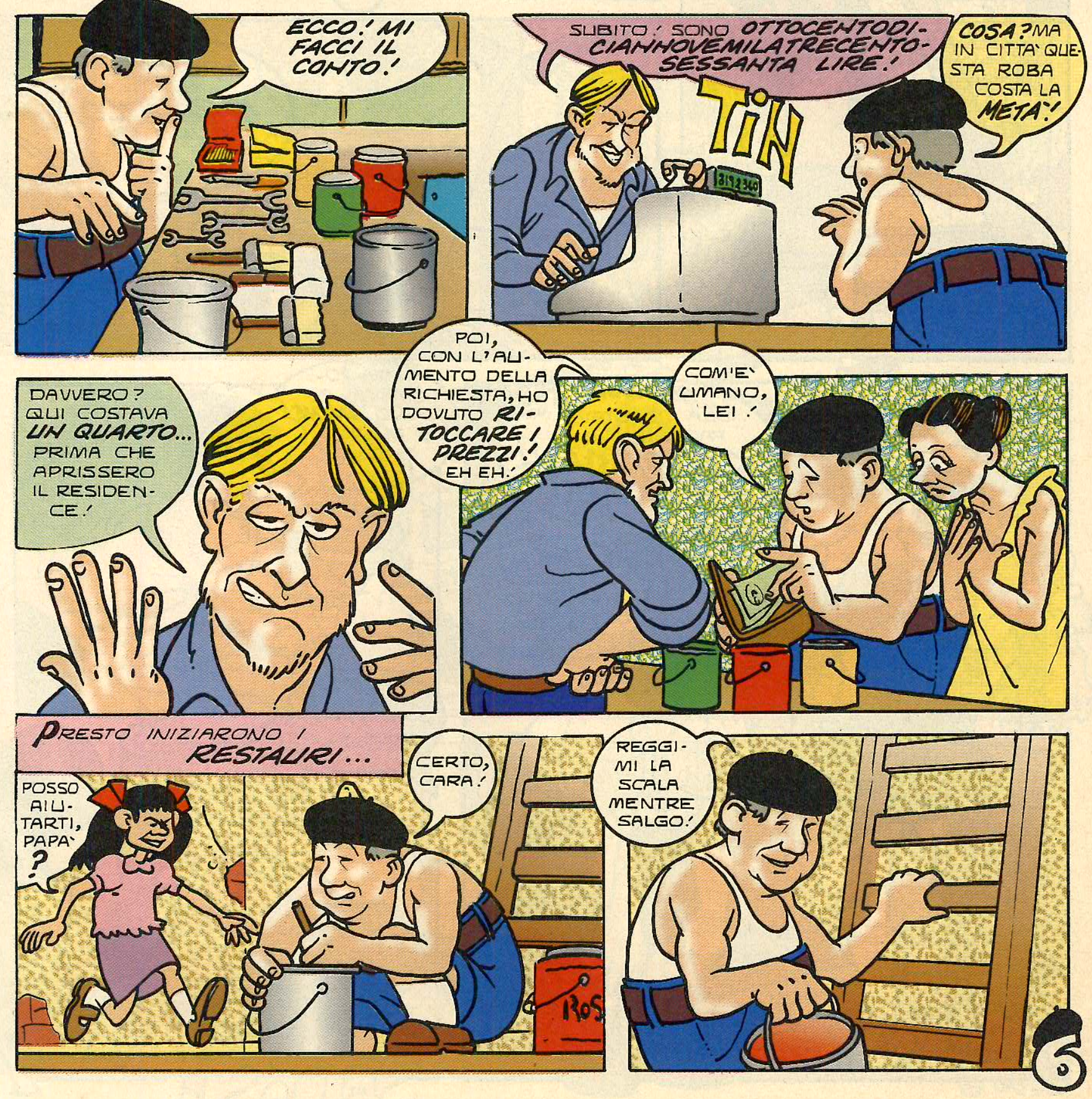




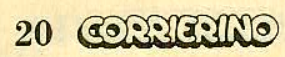

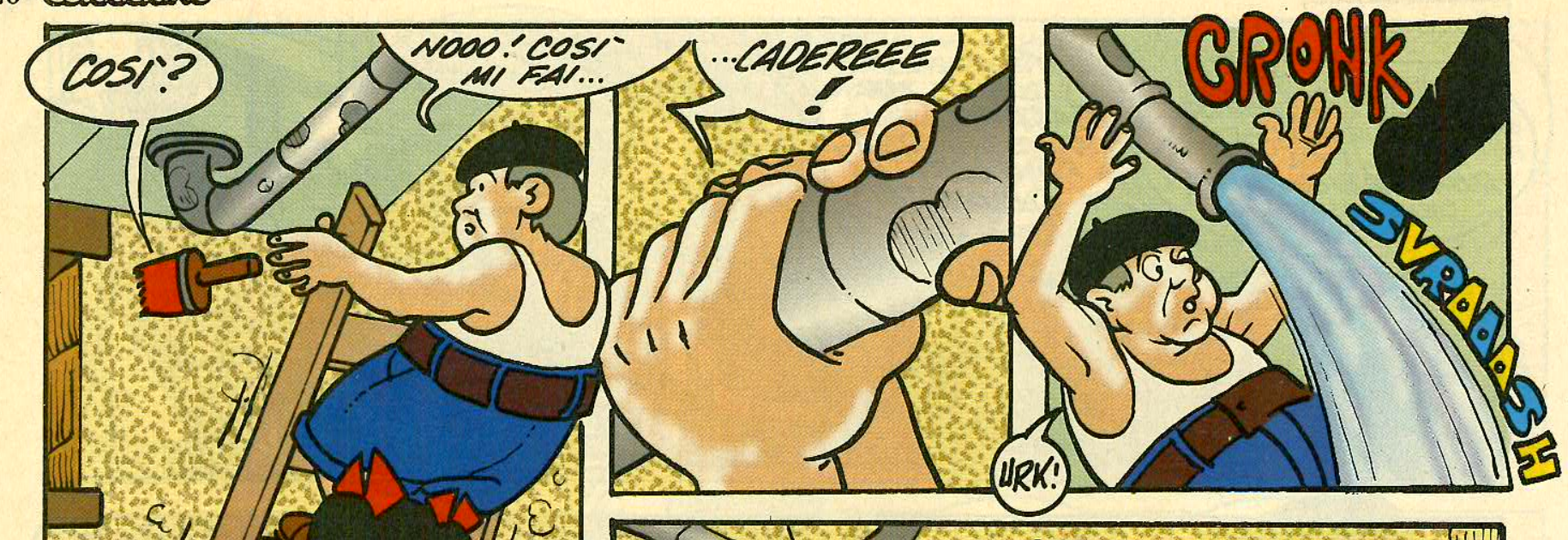

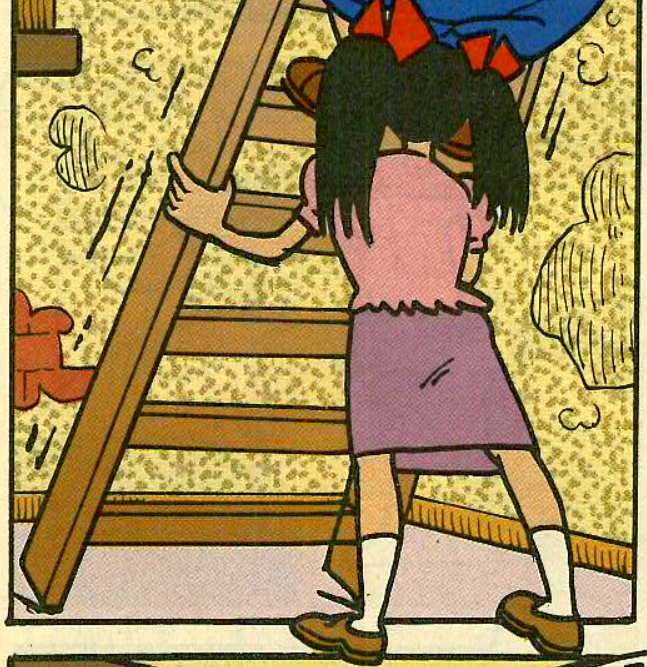

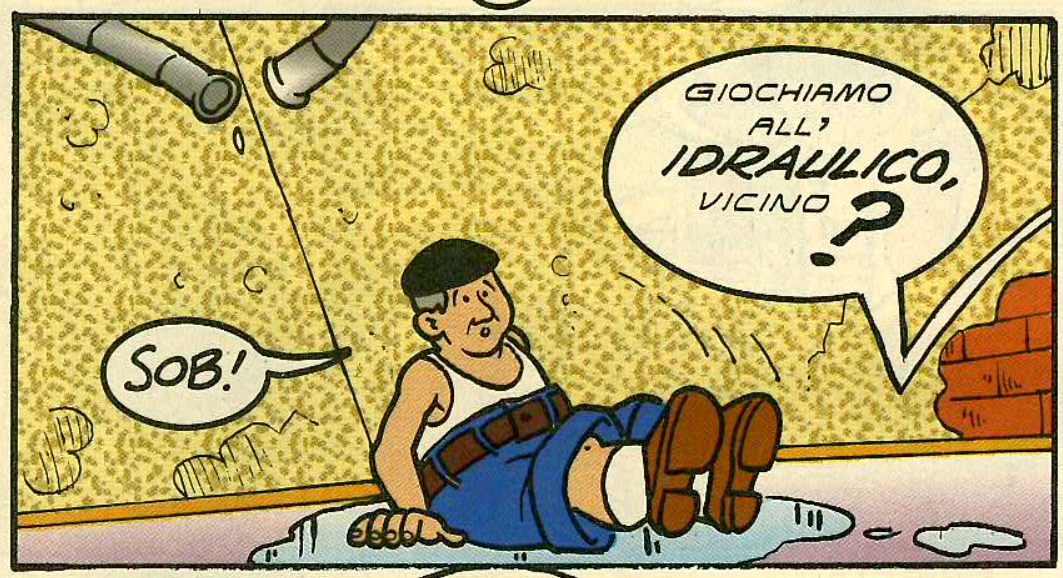
PESSIMA
: 10 STAVO
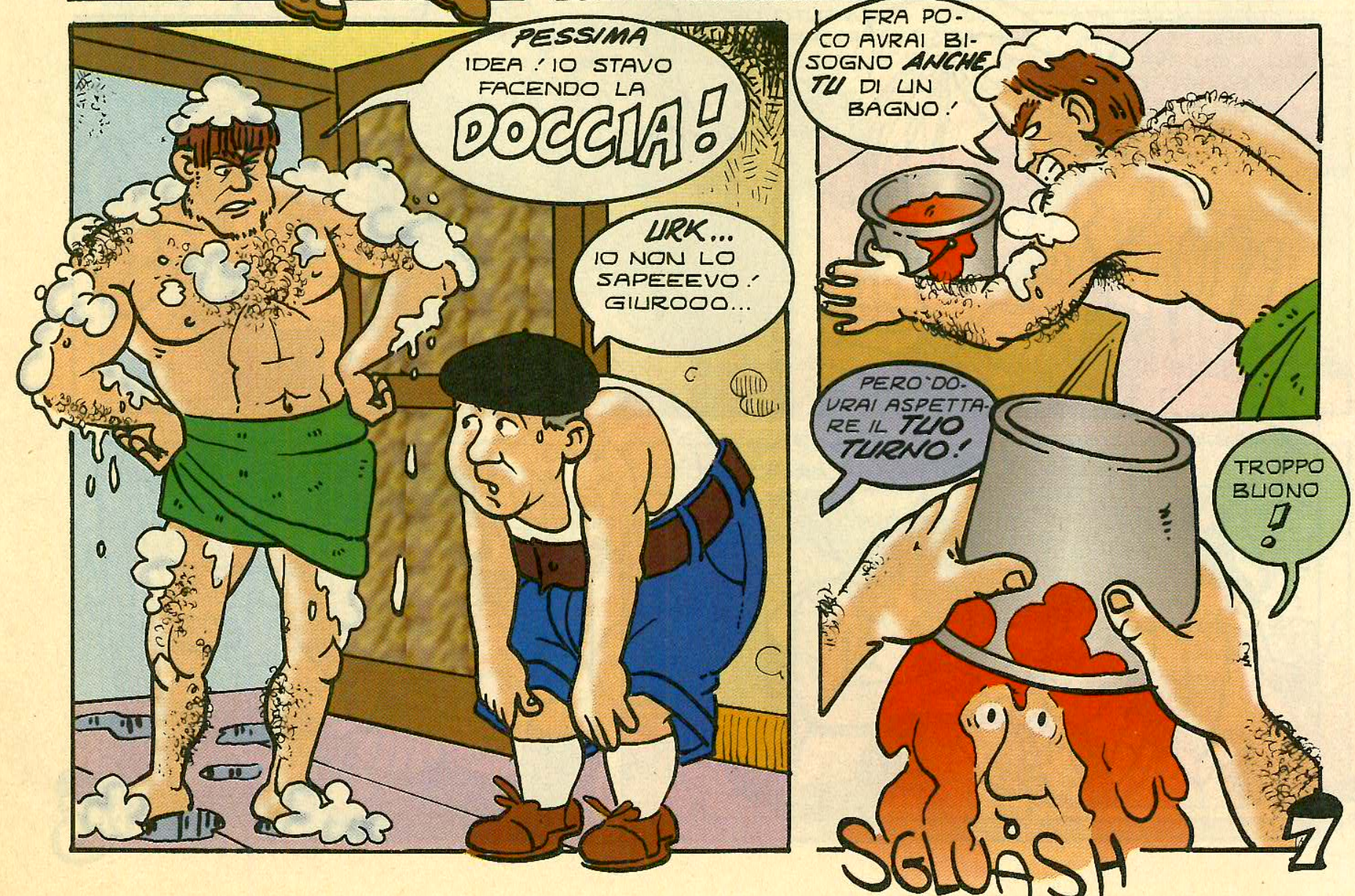


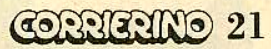
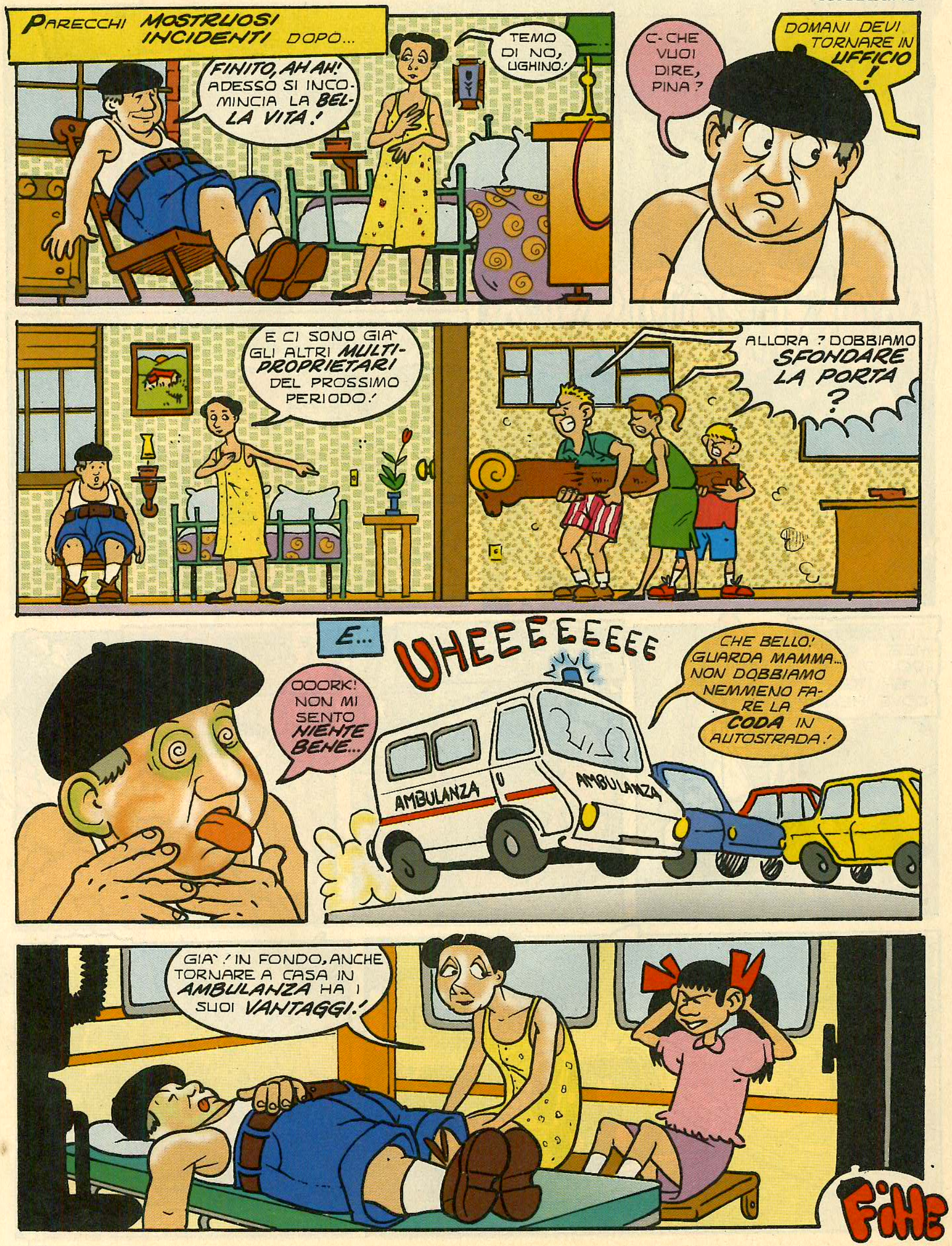